JOINT TRANSPORTATION RESEARCH PROGRAM

FHWA/IN/JTRP-2010/6

Final Report

IDENTIFICATION OF LABORATORY TECHNIQUE TO OPTIMIZE SUPERPAVE HMA SURFACE

FRICTION CHARACTERISTICS

Karol J. Kowalski

Rebecca S. McDaniel

Jan Olek

October 2010 


\section{TECHNICAL Summary}

INDOT Research

Technology Transfer and Project Implementation Information

TRB Subject Code: $31-1$ Bituminous Aggregate Bases

October 2010

Publication No.: FHWA/IN/JTRP-2010/6, SPR-2413

Final Report

\section{Identification of Laboratory Techniques to Optimize Superpave HMA Surface Friction Characteristics}

\section{Introduction}

When designing a pavement surface, engineers must strive to balance several competing parameters, including load capacity, durability, ride quality, construction and maintenance costs, as well as safety and traffic noise. While most of the above parameters can be achieved by using proper materials and construction techniques, current design methodologies do not routinely address friction and texture control. Adequate pavement friction is of paramount importance in reducing the number of collisions, especially on wet pavements.

Pavement friction is primarily a function of the surface texture, which includes both micro- and macrotexture. Pavement microtexture is a function of the surface texture of the aggregate particles and provides a gritty surface that disrupts the continuity of the water film and produces frictional resistance between the tire and pavement. Macrotexture is determined by the overall properties of the pavement surface and provides surface drainage channels for water expulsion from the contact area between the tire and pavement.

Due to limited availability of high friction aggregates in some areas, there is a need to combine them with locally available materials that may have lower polishing resistance. The main objective of this research was to evaluate various blends of aggregates to optimize the combination of micro- and macrotexture to achieve a desired level of friction. The goal was to maintain the currently provided level of friction while reducing the reliance on the microtexture provided by special friction aggregates, if possible, by increasing the mixture macrotexture.

To achieve this primary objective, a secondary objective was necessary; that is, to identify an accelerated method for polishing or abrading samples and measuring their surface friction characteristics.

Another objective was the development of preliminary procedure for determination of an International Friction Index (IFI)-based flag value that can be used as a baseline indicator for laboratory friction measurements. In addition, field investigation of the relationship between traffic volume and changes in the friction values was also undertaken in this study.

The scope of this study included the investigation of the relationship between mixture composition and the following pavement characteristics: surface texture, friction and polishing resistance. Based on the relationship between texture and friction, an International Friction Index (IFI)based flag friction value was developed to serve as a reference point for laboratory type testing.

This study involved laboratory testing of various aggregate gradations (fine, s-shaped and coarse) and aggregate sizes $(9.5 \mathrm{~mm}$ and $19 \mathrm{~mm}$ Nominal Maximum Aggregate Size, NMAS) of Superpave mixtures. Aggregates commonly used in HMA in the north central region of the USA (natural sand, dolomite and two types of limestones) were combined with different percentages (from 0 to $70 \%$ ) of two high friction aggregates (quartzite and steel slag) to produce the mixes used in the 
study. In addition, one stone matrix asphalt (SMA) and one porous friction course (PFC) mix were also tested.

Friction and texture measurements were conducted on 50 laboratory-prepared and polished HMA slabs. These included 46 slabs prepared using Superpave mixtures, two slabs prepared using an SMA mixture and two slabs prepared using a PFC mixture. In order to obtain frictional resistance curves, measurements were performed after compaction of the slabs and periodically during the slab polishing cycle. Laboratory texture and friction tests were conducted using the Circular Track Meter (CTM)

\section{Findings}

- A new laboratory testing methodology was developed and refined during this study that allows for determination of two crucial properties for characterizing and predicting pavement friction: polishing rate and terminal friction value. The Circular Track Machine (CTM) together with a Dynamic Friction Tester (DFT) can be used as a tool to assess the micro- and macrotexture of a mix and then to calculate the frictional properties (F60) of various pavement surfaces. A Circular Track Polishing Machine (CTPM), refined in this study, may be used for the laboratory simulation of the polishing action of highway traffic. In the future, a mixture approval procedure involving determination of the predicted polishing rate and terminal friction values for a given mixture could be used to evaluate the mixture frictional properties.

- $\quad$ The research proved that it is possible to modify frictional properties of the pavement by changing the aggregate type and HMA composition.

- Increasing the friction aggregate (quartzite or steel slag) content substantially improved the polishing resistance of HMA mixes. In general, mixes with steel slag generally exhibited slightly higher polishing resistance (lower polishing rate) than mixes with quartzite.

- When the carbonate aggregates used in this study were blended with high friction aggregates (steel slag and quartzite), the overall friction level generally increased. Increasing the friction aggregate content from 10 to $20 \%$ had relatively little effect on the friction level, but friction increase dramatically when the friction aggregate content was increased to 40 or $70 \%$. If friction is to be improved only by adding high and Dynamic Friction Tester (DFT) devices, respectively.

In addition to the laboratory tested slabs, the field friction and texture data were collected from 25 sites on existing highways and test track sections. These data were used to obtain the friction baseline values. Field measurements were conducted using the CTM, DFT and ASTM E 274 towed friction trailer. The field test sites included Superpave designed HMAs, Marshall designed HMAs, PFC, SMA and concrete (tined and smooth) pavements. Using these field data and recommendations found in the literature, the IFI flag value was determined.

friction aggregates to the local carbonate aggregates, the friction aggregate content should be $20 \%$ or greater, depending on the amount of improvement needed. There may be, however, other ways of increasing the overall surface friction.

- Larger NMAS sizes are desirable from a frictional point of view, and they should be used where other considerations (such as layer thickness and smoothness, among others) allow.

- An International Friction Index (IFI) based model using the parameters measured with the CTM and DFT during polishing in the CPTM was developed to describe the change in frictional properties under traffic/polishing.

- The International Friction Index parameters (F60 and $S_{\mathrm{p}}$ ) can be improved by increasing the pavement macrotexture.

- $\quad$ The value of the fineness modulus (FM) of the aggregate blend correlates well with the pavement macrotexture and thus has a great influence on the pavement frictional properties. Pavement frictional properties can be improved either by using highly polish resistant aggregate (such as quartzite or steel slag) blended with the locally available carbonate rocks or by modifying the aggregate blend in such a way that the FM will be increased. Mixes with FM values of 4.6 or higher generally had high macrotexture and friction levels.

- $\quad$ Based on the literature findings and field measurements using the ASTM E 274 towed friction trailer (equipped with both rib and smooth tires) and using the CTM/DFT devices, the approximate frictional flag value (F60) was determined.

- Comparison of the range of friction values 
obtained during the laboratory part of this study with results of the field measurements suggested good correlation between the laboratory measurements and actual highways conditions. Similarly, measurements with different friction measuring devices showed the same trends in the data.

\section{Implementation}

- The results of this study resulted in the development of a polishing model. Application of this model to the frictional properties allows for the determination of two crucial polishing parameters for a given mixture: a4 (polishing rate) and F60@X1 (terminal friction level). Frictional parameters of a mixture can then be assessed and the decision may be made whether the given mixture meets the desired friction criteria.

- $\quad$ An F60 flag value was estimated based on the current Indiana DOT practice, measurements with CTM/DFT devices and a towed friction trailer, as well as conclusions from a PIARC study [Wambold et al. 1996]. While this value should be further verified, it can be used as a starting point for the lab evaluation of the frictional properties of various HMA mixtures.

- A CTPM machine and test protocol developed in this study are very promising tools to evaluate frictional properties of various HMAs, however, additional field verification of that method is needed.

- The lab compaction method should be further improved so it more closely simulates field construction processes.

- If the use of an aggregate blend consisting mostly of polish susceptible aggregates is desired, the high friction coarse aggregate content should be $20 \%$ or greater in the overall aggregate blend. This finding should be further verified by performing field friction
- Further work is needed to improve the compaction technique for laboratory slabs and to correlate the number of wheelpasses in the CPTM to actual traffic levels. The proposed polishing model should be validated by testing more types of materials under actual field conditions; work has already begun on this effort under another research project involving extensive field testing.

\section{measurements of pavements with varying} percentages of polishing aggregates.

- $\quad$ Both steel slag and quartzite were found to improve the frictional characteristics of HMA mixes in which they are used. The choice of which high friction aggregate to use should be used based on availability and cost.

- In addition to substituting high friction aggregates for a portion of the polish susceptible aggregates, however, this study showed that the frictional characteristics of the surface can also be improved by changing the value of the fineness modulus (FM) of the surface mix. Based on the limited field observation, in general, HMA blends with a FM of about 4.6 or greater should provide a relatively "deep" pavement macrotexture (MPD above about 0.6 $\mathrm{mm})$.

- In general, the s-shaped gradation resulted in higher MPD ("deeper" texture) and thus improved friction at high speeds.

- One other way to increase the macrotexture of the surface is to use aggregates with larger NMAS sizes. Such mixtures are desirable from a frictional point of view and should be used where other considerations (such as tire pavement/noise, layer thickness and smoothness, among others) allow. 


\section{Contact}

For more information:

Dr. Rebecca McDaniel

Principal Investigator

Technical Director

North Central Superpave Center

Purdue University

West Lafayette IN 47907

Phone: (765) 463-2317

E-mail: rsmcdani@.purdue.edu
Indiana Department of Transportation

Division of Research \& Development

1205 Montgomery Street

P.O. Box 2279

West Lafayette, IN 47906

Phone: (765) 463-1521

Fax: (765) 497-1665

\section{Purdue University}

Joint Transportation Research Program

School of Civil Engineering

West Lafayette, IN 47907-1284

Phone: (765) 494-9310

Fax: (765) 496-7996

E-mail: jtrp@ecn.purdue.edu

http://www.purdue.edu/jtrp 
Final Report

\title{
Identification of Laboratory Technique to Optimize Superpave HMA Surface Friction Characteristics
}

\author{
By \\ Karol J. Kowalski \\ Former Graduate Research Assistant \\ Assistant Professor and Scholar of Foundation for Polish Science \\ Warsaw University of Technology \\ Rebecca S. McDaniel \\ Principal Investigator \\ Jan Olek \\ Professor \\ School of Civil Engineering \\ Purdue University
}

Joint Transportation Research Program

Project No. C-36-56K

File No. 2-13-11

SPR-2413

IHRB Project TR-450

Prepared in Cooperation with the Indiana Department of Transportation, Iowa Highway Research Board (IHRB) and the U.S. Department of Transportation

Federal Highway Administration

The contents of this report reflect the views of the authors who are responsible for the facts and the accuracy of the data presented herein. The contents do not necessarily reflect the official views or polices of the Federal Highway Administration, the Indiana Department of Transportation nor the Iowa Department of Transportation. This report does not constitute a standard, specification or regulation.

Purdue University

West Lafayette, Indiana 47907

October 2010 


\section{Acknowledgements}

The authors wish to express their gratitude to Dr. Eyal Levenberg, Dr. Ayesha Shah, Stephen Bowman, Yu-Min Su and Kevin Brower for their assistance in preparation of test specimens, data analysis and interesting discussions. 


\begin{tabular}{|c|c|c|}
\hline $\begin{array}{l}\text { 1. Report No. } \\
\text { FHWA/IN/JTRP-2010/6 }\end{array}$ & 2. Government Accession No. & 3. Recipient's Catalog No. \\
\hline \multirow{2}{*}{\multicolumn{2}{|c|}{$\begin{array}{l}\text { 4. Title and Subtitle } \\
\text { Identification of Laboratory Technique to Optimize Superpave HMA Surface } \\
\text { Friction Characteristics }\end{array}$}} & $\begin{array}{l}\text { 5. } \quad \text { Report Date } \\
\text { October } 2010\end{array}$ \\
\hline & & 6. Performing Organization Code \\
\hline \multicolumn{2}{|c|}{$\begin{array}{l}\text { 7. Author(s) } \\
\text { Karol J. Kowalski, Rebecca S. McDaniel, and Jan Olek }\end{array}$} & $\begin{array}{l}\text { 8. Performing Organization Report No. } \\
\text { FHWA/IN/JTRP-2010/6 }\end{array}$ \\
\hline \multirow{2}{*}{\multicolumn{2}{|c|}{$\begin{array}{l}\text { 9. Performing Organization Name and Address } \\
\text { Joint Transportation Research Program } \\
\text { 1284 Civil Engineering Building } \\
\text { Purdue University } \\
\text { West Lafayette, IN 47907-1284 }\end{array}$}} & 10. Work Unit No. \\
\hline & & $\begin{array}{l}\text { 11. Contract or Grant No. } \\
\text { SPR-2413 }\end{array}$ \\
\hline \multirow{2}{*}{\multicolumn{2}{|c|}{$\begin{array}{l}\text { 12. Sponsoring Agency Name and Address } \\
\text { Federal Highway Administration } \\
575 \text { N. Pennsylvania, Room } 254 \\
\text { Indianapolis, IN } 46204\end{array}$}} & $\begin{array}{l}\text { 13. Type of Report and Period Covered } \\
\text { Final Report }\end{array}$ \\
\hline & & 14. Sponsoring Agency Code \\
\hline
\end{tabular}

15. Supplementary Notes

Prepared in cooperation with the Federal Highway Administration and the Indiana Department of Transportation.

\section{Abstract}

Wet pavement friction is known to be one of the most important roadway safety parameters. In this research, frictional properties of flexible (asphalt) pavements were investigated.

As a part of this study, a laboratory device to polish asphalt specimens was refined and a procedure to evaluate mixture frictional properties was proposed. Following this procedure, 46 different Superpave mixtures, one stone matrix asphalt (SMA) mixture and one porous friction course (PFC) mixture were tested. In addition, 23 different asphalt and two concrete field sections were also tested for friction and noise.

The results of both field and laboratory measurements were used to develop an International Friction Index (IFI)-based protocol for measurement of the frictional characteristics of asphalt pavements for laboratory friction measurements.

Based on the results of the study, it appears the content of high friction aggregate should be $20 \%$ or more of the total aggregate blend when used with other, polish susceptible coarse aggregates; the frictional properties increased substantially as the friction aggregate content increased above $20 \%$. Both steel slag and quartzite were found to improve the frictional properties of the blend, though steel slag had a lower polishing rate. In general, mixes containing soft limestone demonstrated lower friction values than comparable mixes with hard limestone or dolomite.

Larger nominal maximum aggregate size mixes had better overall frictional performance than smaller sized mixes. In addition, mixes with higher fineness moduli generally had higher macrotexture and friction.

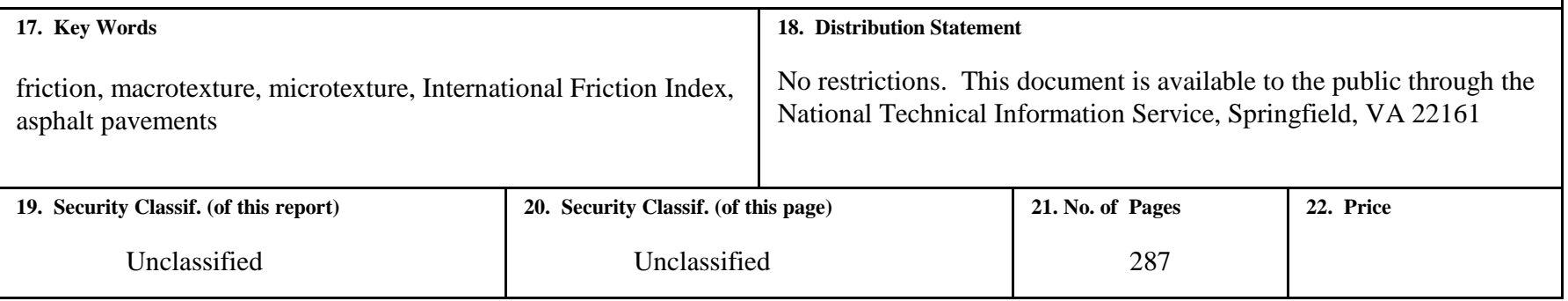


TABLE OF CONTENTS

Page

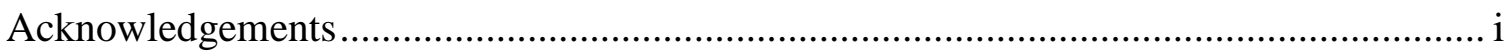

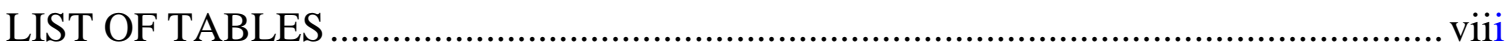

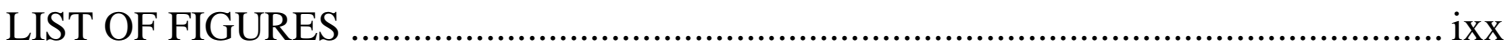

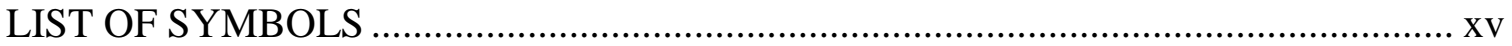



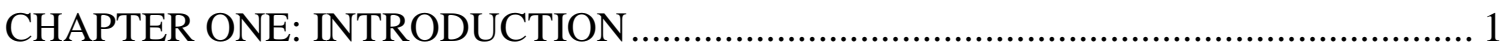

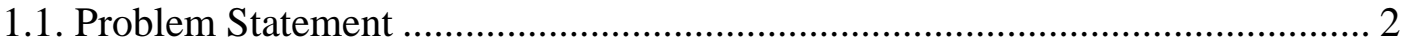

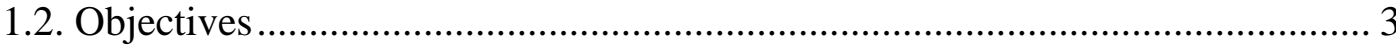

1.3. Hypothesis and Scope of the Study ………………..................................... 3

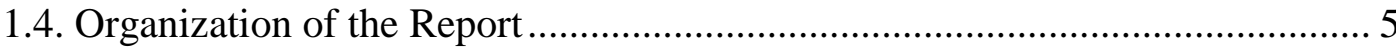

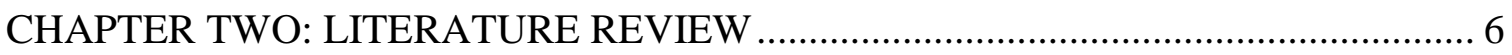

2.1. Fundamentals of Pavement Texture and Friction.............................................. 6

2.1.1. Pavement Texture and Friction .............................................................. 6

2.1.2. Mechanism of Friction ...................................................................... 7

2.1.3. Friction and Texture Relationships ........................................................ 11

2.1.4. Changes in Tire/Pavement Friction.......................................................... 13

2.2. Pavement Characteristics Contributing to Friction .......................................... 15 


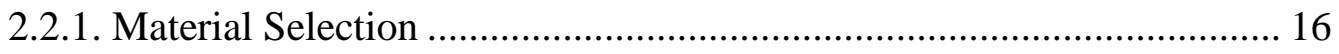

2.2.2. Mix Proportioning ............................................................................... 19

2.2.3. Pavement Construction and Maintenance Practices ................................ 21

2.3. Friction / Texture Measurements and Polishing Methods ................................ 22

2.3.1. Friction Measurement Techniques ....................................................... 22

2.3.2. Texture Measurement Techniques ........................................................ 24

2.3.3. Harmonization of Friction and Texture Test Results ............................. 25

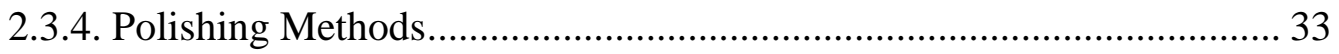

2.4. Pavement Quality: Friction Requirements ....................................................... 35

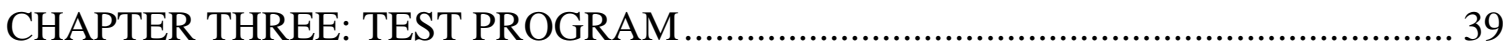

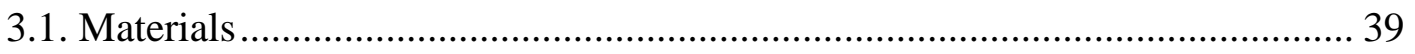

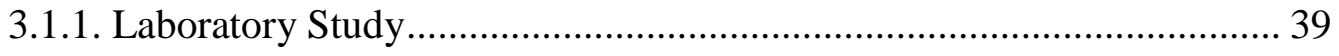

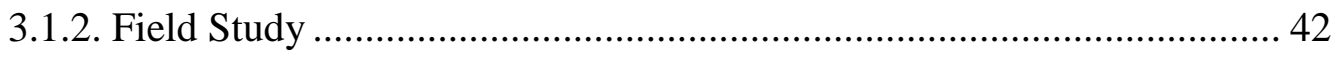

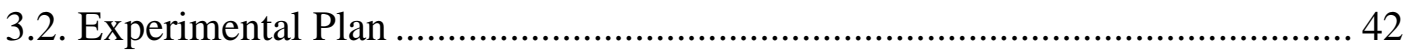

3.3. Sample Properties Characterization ................................................................. 48

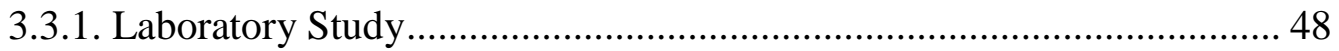

3.3.1.1 Aggregate Blend Gradation ........................................................... 48

3.3.1.2 Binder Content Determination ................................................... 55

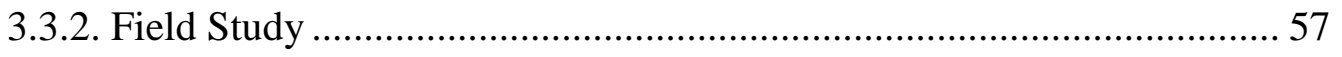

3.3. Equipment and Testing Procedures Used in the Friction Study....................... 64



3.3.1.1. Specimen Preparation .................................................................... 64

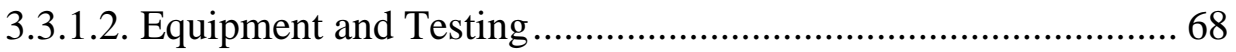


3.3.1.3. Sample Volumetric Measurement ....................................... 74

3.3.2. Field Friction Study ................................................................. 78

3.3.2.1. Field Section Construction .............................................. 78

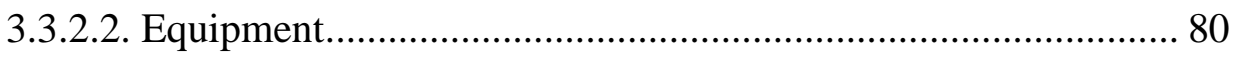

3.3.2.3. Testing Schedule, Weather and Traffic Conditions ................. 84

CHAPTER FOUR: EXPERIMENTAL RESULTS AND DISCUSSIONS ................... 92



4.1.1. Proposed Polishing Model ............................................................. 95

4.1.2. Application and Evaluation of the Proposed Polishing Model .............. 99

4.1.2.1 Aggregate Size, Gradation and Type of Carbonate Aggregate 102

4.1.2.2 Friction Aggregate Type................................................... 108

4.1.2.3 Friction Aggregate Content ............................................... 109

4.1.2.4 General Summary of the Influence of Different Factors on Frictional Properties ............................................................... 112

4.1.3. Proposed Friction Predictive Model ............................................. 112

4.1.4. Application of the Proposed Polishing Model to Non-Superpave Mixes

4.1.5. Volumetric Properties .............................................................. 125

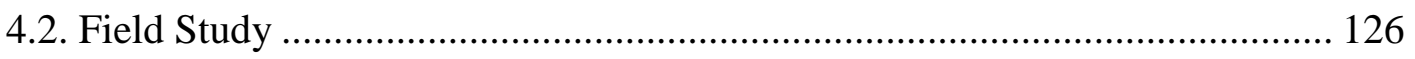

4.2.1. Establishment of Baseline Friction Values and F60 Requirements .... 127

4.2.1.1 Theoretical Study: Friction Simulation using the PIARC Model

4.2.1.2 Development of Baseline Frictional Values .......................... 131

4.2.1.3 Frictional Requirements.............................135

4.2.2. Variations in Frictional Properties ................................................. 139 
4.2.2.1 Periodic Tests of Highway Test Sections ................................ 139

4.2.2.2 Periodic Tests of Test Track Sections........................144

4.2.2.3 Transverse Variation of Frictional Properties .......................... 149

4.3. Comparison of Frictional Characteristics: Lab vs. Field................................. 155

CHAPTER FIVE: FINDINGS AND CONCLUSIONS .............................................. 157



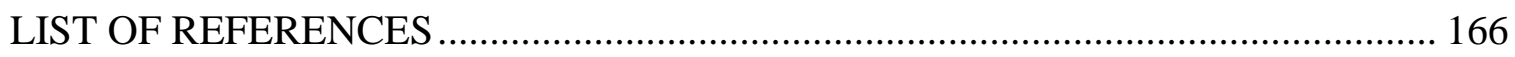

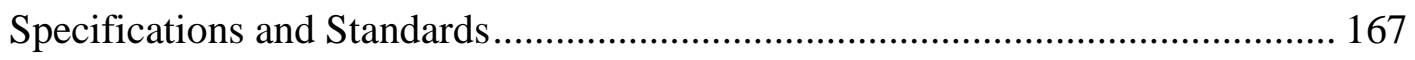

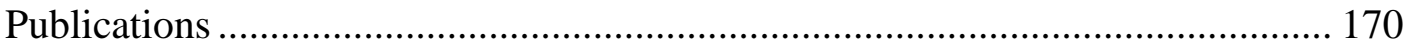

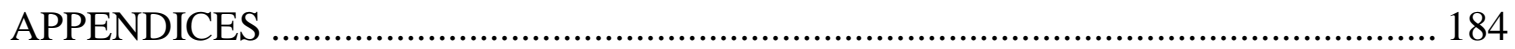

Appendix A: Laboratory Friction, Texture and Polishing Methods ...................... 185

Appendix B: Representation of the Laboratory and Field Specimens ................... 188

Appendix C: Summary of Mixture Design ........................................................ 200

Appendix D: Laboratory Polishing Data............................................................ 203

Appendix E: Summary of the Laboratory Polishing Data and Model Parameters 217

Appendix F: Summary of the Friction Predictive Model Development ................ 222

Appendix G: Laboratory Noise Measurements...................................................... 228

G.1 Selection of the Specimens for Noise Tests in TPTA ............................. 228

G.2. Specimen Preparation........................................................................ 230

G.3. Equipment and Testing ..................................................................... 237

G.4. Sample Volumetric Properties .............................................................. 245 
G.5. Results and Discussion...

G.6. Summary

G.7. Additional Test Results .. 254

Appendix H: Summary of the Field CTM/DFT Measurements. 


\section{LIST OF TABLES}

Table

Table 1. Recommended minimum friction requirements [after Kummer and Meyer 1967]

Table 2. Physical properties of aggregates used in the laboratory part of the study

Table 3. Number of variables and cells compared during the laboratory tests (only laboratory mixtures are shown).

Table 4. Fineness modulus and proportions (\% by weight) of aggregate blends used in Matrix II .....

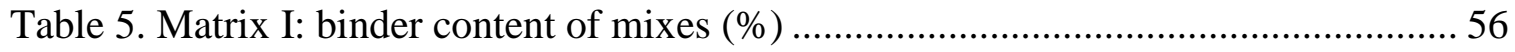

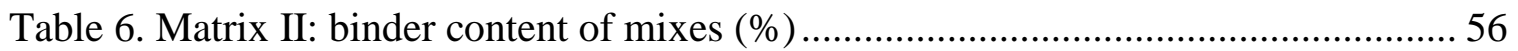

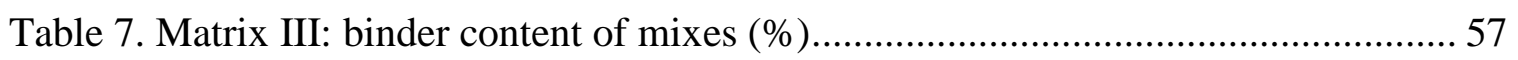

Table 8. Material type, quantity and volumetric data of asphalt sections tested periodically......

Table 9. Material type, quantity and volumetric data for Indiana sections tested one time.

Table 10. Fineness modulus and aggregate gradation (percent passing per sieve size) for Indiana sections tested one time

Table 11. Material type, quantity and volumetric data for Iowa sections tested one time 63

Table 12. Fineness modulus and aggregate gradation (percent passing per sieve size) for Iowa sections tested one time

Table 13. Information about the traffic, construction year and testing time of pavement sections tested periodically with CTM/DFT

Table 14. Information traffic, construction year and testing time of Indiana sections tested one time.

Table 15. Information about the traffic, construction year and testing time of Iowa sections tested one time. 
Table 16. Summary of the test results: laboratory friction measurements (test Matrices I-III)........

Table 17. Summary of $\mathrm{x}_{1}$ values (no. of wheel passes corresponding to the terminal friction level) ................................................................................ 100

Table 18. ANOVA table and parameter estimates for the polishing rate $\left(\mathrm{a}_{4}\right)$................ 117

Table 19. ANOVA table and parameter estimates for the terminal polishing level $\left(\mathrm{F} 60 @ \mathrm{x}_{1}\right)$

Table 20. Summary of the polishing prediction applied to the PFC and SMA mixtures tested in the laboratory

Table 21. Baseline: summary of the frictional properties of the tested section

Table 22. Influence of the transverse location and the date of test on the frictional properties of PFC, SMA and DGA sections

Table 23. Influence of the transverse location on the frictional properties for HM-IN and HM-IA sections

Table 24. Comparison of the typical macrotexture (MPD), dynamic friction $\left(\mathrm{DF}_{20}\right)$ and calibrated wet friction (F60) values obtained in the lab and during the field tests.

Table A 1. Comparison of laboratory friction measurement methods [after McDaniel and Coree 2003].

Table A 2. Comparison of portable texture measurement methods [after McDaniel and Coree 2003].

Table A 3. Comparison of accelerated polishing methods [after McDaniel and Coree 2003]. 


\section{LIST OF FIGURES}

Figure

Page

Figure 1. Principal components of pavement/tire friction [after Kummer 1966] .............. 8

Figure 2. Speed dependence of the tire/pavement friction components [Kummer 1962]; 10

Figure 3. Influence on the calibrated wet friction (F60) of: (a) $\mathrm{DF}_{20}$ and (b) MPD values. 30

Figure 4. General plan for the field and laboratory study of the HMA mixtures 43

Figure 5. Schematic of the experimental design: Matrices I to III ................................ 45

Figure 6. Aggregate gradations for mixes with $9.5 \mathrm{~mm}$ NMAS .................................. 49

Figure 7. Aggregate gradations for mixes with 19 mm NMAS .................................. 49

Figure 8. Blend composition for mixes with $9.5 \mathrm{~mm}$ NMAS and coarse gradation......... 52

Figure 9. Blend composition for mixes with $9.5 \mathrm{~mm}$ NMAS and fine gradation ............ 52

Figure 10. Blend composition for mixes with $9.5 \mathrm{~mm}$ NMAS and s-shaped gradation... 53

Figure 11. Blend composition for mixes with $19 \mathrm{~mm}$ NMAS and coarse gradation........ 53

Figure 12. Blend composition for mixes with $19 \mathrm{~mm}$ NMAS and fine gradation............ 54

Figure 13. Blend composition for mixes with $19 \mathrm{~mm}$ NMAS and s-shaped gradation.... 54

Figure 14. Gradation of HMA-TT, DGA, PFC and SMA sections tested periodically.... 58

Figure 15. Stages of the friction slab compaction process: (a) preheating roller, (b) mix placing and pre-densification, (c) initial compaction and (d) final compaction ....

Figure 16. Polishing, friction and texture equipment: (a) CTPM, (b) DFT and (c) CTM 69

Figure 17. Bottom view of the friction and texture devices: (a) DFT and (b) CTM ....... 71

Figure 18. Details of the CTPM device: (a) alignment and level device, (b) level device and actuator, (c) force control bolt and (d) rubbers washed with water. 73

Figure 19. Air void content distribution 76 


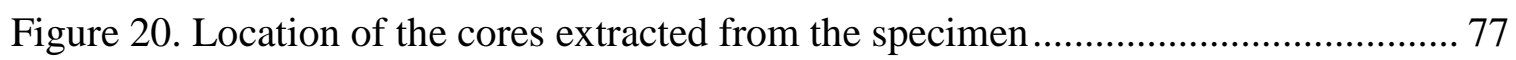

Figure 21. ASTM E-274 towed friction trailer: (a) oblique and (b) side views .............. 81

Figure 22. ASTM E-274 towed friction trailer, water nozzle system: (a) general view

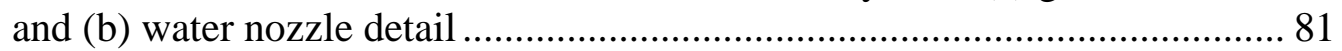

Figure 23. Tires used for the friction measurements: (a) smooth and (b) rib ................. 83

Figure 24. Location of the CTM/DFT devices during the highway tests ........................ 88

Figure 25. Mean profile depth (MPD) and dynamic friction $\left(\mathrm{DF}_{20}\right)$ data (a) and calibrated wet friction (F60) data (b) as a function of no. of wheel passes for one of the laboratory tested specimens (two repeated measurements) ............ 93

Figure 26. Plot of the typical calibrated wet friction relationship with no. of wheel passes for the 19SS20S_SL laboratory tested specimen.............................. 96

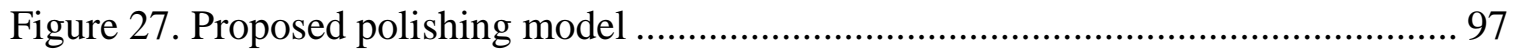

Figure 28. Polishing model fitted to the laboratory data for 19SS20S_SL specimen .... 100

Figure 29. Test Matrix I; distribution of polishing rate, general comparison................ 103

Figure 30. Test Matrix I, mixes with 20\% SS; distribution of polishing rate................ 103

Figure 31. Test Matrix I, mixes with 20\% Q; distribution of polishing rate ................. 104

Figure 32. Test Matrix I; distribution of polishing rate averaged for mixes with $\mathrm{Q}$ and SS (each point is an average of two cells).............................................. 104

Figure 33. Test Matrix I; distribution of friction terminal value (F60@ $\left.\mathrm{x}_{1}\right)$, general

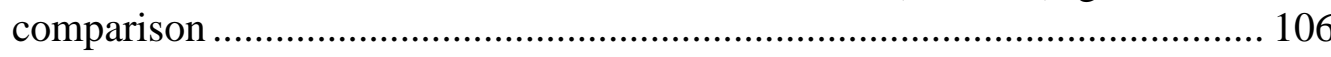

Figure 34. Test Matrix I, mixes with 20\% SS; distribution of friction terminal value ... 106

Figure 35. Test Matrix I, mixes with 20\% Q; distribution of friction terminal value .... 106

Figure 36. Test Matrix I; distribution of friction terminal values averaged for mixes with Q and SS (each point is an average of two samples)

Figure 37. Influence of type of the friction aggregate type (FAT) on the frictional properties of mixes evaluated in test Matrix I: (a) polishing rate $\left(\mathrm{a}_{4}\right)$ and (b)

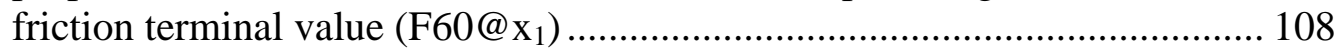

Figure 38. Distribution of polishing rate $\left(\mathrm{a}_{4}\right)$ for test Matrix II mixes ......................... 109

Figure 39. Distribution of terminal friction values $\left(\mathrm{F} 60 @ \mathrm{x}_{1}\right)$ for test Matrix II mixes.. 110 
Figure 40. Distribution of polishing rate $\left(\mathrm{a}_{4}\right)$ for test Matrix III mixes

Figure 41. Distribution of terminal friction values (F60@ $\left.\mathrm{x}_{1}\right)$ for test Matrix III mixes. 111

Figure 42. Overview of the general friction predictive model.

Figure 43. Polishing rate $\left(\mathrm{a}_{4}\right)$ distribution

Figure 44. Residual distribution for the polishing rate $\left(a_{4}\right)$ prediction model: (a) residual vs. sample no. and (b) q-q plot

Figure 45. Terminal polishing level (F60@ $\left.\mathrm{x}_{1}\right)$ distribution

Figure 46. Residual distribution for the terminal polishing level (F60@ $\mathrm{x}_{1}$ ) prediction model: (a) residual vs. sample no. and (b) q-q plot.

Figure 47. Changes in the macrotexture of the field mixtures polished in the laboratory 122

Figure 48. Changes in the dynamic friction $\left(\mathrm{DF}_{20}\right)$ values of the field mixtures polished in the laboratory.....

Figure 49. Frictional prediction model applied to the field mixtures polished in the laboratory

Figure 50. Theoretical relationship between MPD, SN and $\mathrm{DF}_{20}$ (for ASTM E 274 friction trailer and DFT device, respectively)

Figure 51. Theoretical relationship between MPD (and F60) for ASTM E 274 friction trailer: (a) $\mathrm{SN}(64)$ values for rib tire are fixed and for smooth are adjusted and (b) SN(64) values for smooth tire are fixed and for rib are adjusted ...... 130

Figure 52. Comparison of calibrated wet friction values (F60) developed from DFT device with F60 calculated using friction trailer data: using (a) smooth (F60 TST) and (b) rib (F60 TRT) tire.

Figure 53. Comparison of calibrated wet friction (F60) developed from friction trailer tests using smooth (F60 TST) and rib (F60 TRT) tire

Figure 54. Development of the F60 flag values for friction trailer with: (a) rib (F60 TRT) and (b) smooth (F60 TST) tire

Figure 55. Changes in macrotexture (MPD) over time and traffic for PFC, SMA and DGA sections

Figure 56. Changes in dynamic friction $\left(\mathrm{DF}_{20}\right)$ as a function of time and traffic for PFC, SMA and DGA sections. 
Figure 57. Changes in calibrated wet friction (F60) over time and traffic for PFC,

SMA and DGA sections

Figure 58. Changes in ASTM E-274 friction trailer measurements (SN(64)S) as a function of traffic for PFC, SMA and DGA sections

Figure 59. Macrotexture (MPD) values collected from the INDOT test track for three different surfaces: TC, HMA-TT and SC

Figure 60. Dynamic friction $\left(\mathrm{DF}_{20}\right)$ values collected from the INDOT test track for three different surfaces: TC, HMA-TT and SC 146

Figure 61. Calibrated wet friction (F60) values observed for the INDOT test track...... 146

Figure 62. Skid number (measured by (ASTM E 274) friction trailer) on the INDOT test track monitoring: results for tests with smooth tire

Figure 63. Skid number (measured by (ASTM E 274) friction trailer) on the INDOT test track monitoring: results for tests with rib tire 148

Figure 64. Influence of the transverse location and time of the test on the macrotexture (MPD) of the PFC, SMA and DGA sections .

Figure 65. Influence of the transverse location on the dynamic friction $\left(\mathrm{DF}_{20}\right)$ of the PFC, SMA and DGA sections.

Figure 66. Influence of the transverse location on the macrotexture (MPD) of the HMIN and HM-IA sections 150

Figure 67. Influence of the transverse location on the dynamic friction $\left(\mathrm{DF}_{20}\right)$ of the HM-IN and HM-IA sections . 151

Figure B 1. Laboratory mixture 95SS20C_D: before and after the polishing.... 189

Figure B 2. Laboratory mixture 95SS20F_D: before and after the polishing 190

Figure B 3. Laboratory mixture 95SS20S_D: before and after the polishing 191

Figure B 4. Laboratory mixture 19SS20C_D: before and after the polishing 192

Figure B 5. Laboratory mixture 19SS20F_D: before and after the polishing 193

Figure B 6. Laboratory mixture 19SS20S_D: before and after the polishing 194 
Figure B 7. Laboratory sample of SMA (slab $38 \mathrm{~mm}$ thick): before and after the polishing 195

Figure B 8. Laboratory sample of PFC (slab $38 \mathrm{~mm}$ thick): before and after the polishing...... 196

Figure B 9. HMA sections (interstate highway) tested periodically: (a) PFC and (b) SMA

Figure B 10. HMA sections tested periodically: (a) INDOT Test Track (HMA-TT) and

(b) state highway (DGA). 198

Figure B 11. Concrete pavement sections located in the INDOT Test Track: (a) tined (TC) and (b) smooth (SC).

Figure G 1. Concrete molds used in the study: (a) top and (b) side views 231

Figure G 2. Heating process: (a) concrete mold and (b) compaction tools 232

Figure G 3. Roller load distribution during the TPTA samples preparation: (a) roller in the "fixed" position and (b) free rolling down.... 233

Figure G 4. . Stages of the TPTA segments compaction process: (a) spraying thin metal sheet with an anti-adhesive agent, (b) first stage: pre-densification, (c) specimen before the first stage of compaction, (d) first stage: compaction, (e) removing mixture covering top of the walls, (f) second stage: predensification and $(\mathrm{g})$ second stage: compaction. 236

Figure G 5. Tire/Pavement Testing Apparatus (TPTA) 239

Figure G 6. TPTA during the operation: (a) general view and (b) transition zone between the specimens

Figure G 7. Microphones set-up: (a) side and (b) oblique view (protective windscreens removed for the picture)

Figure G 8. Tires used during the noise tests in TPTA: non standard (a) Uniroyal Tiger Paw (UTP) and (c) Goodyear Aquatred (GA) and (b) standard Radial Standard Reference Test Tire (RSRTT). 244

Figure G 9. Location of the cores extracted from the specimens 246

Figure G 10. General comparison of sound intensity levels (SIL) at test speed of 48 $\mathrm{km} / \mathrm{h}$ 
Figure G 11. Sound intensity levels of concrete specimens with various surfaces tested in TPTA [after Dare et al. 2007] ............................................................ 250

Figure G 12. 1/3 Octave frequency spectra for RSRTT SCD_3, test speed $48 \mathrm{~km} / \mathrm{h} . . . .251$

Figure G 13. Sound intensity level vs. speed relationship and SIL extrapolated to 97 $\mathrm{km} / \mathrm{h}$ for tests with RSRTT SCD_3 ....................................................... 252

Figure G 14. General comparison of predicted sound intensity levels at test speed of $97 \mathrm{~km} / \mathrm{h}$ 253

Figure G 15. 1/3 Octave frequency spectra for RSRTT SCD_1, test speed $48 \mathrm{~km} / \mathrm{h} \ldots . .254$

Figure G 16. 1/3 Octave frequency spectra for RSRTT SCD_2, test speed $48 \mathrm{~km} / \mathrm{h} \ldots . .255$

Figure G 17. 1/3 Octave frequency spectra for RSRTT SCD_3, test speed $16 \mathrm{~km} / \mathrm{h} \ldots . .255$

Figure G 18. 1/3 Octave frequency spectra for RSRTT SCD_3, test speed $32 \mathrm{~km} / \mathrm{h} \ldots . .256$

Figure G 19. 1/3 Octave frequency spectra for UTP tire, test speed $16 \mathrm{~km} / \mathrm{h}, \mathrm{SCD} \_3$.. 256

Figure G 20. 1/3 Octave frequency spectra for UTP tire, test speed $32 \mathrm{~km} / \mathrm{h}, \mathrm{SCD} \_3$.. 257

Figure G 21. 1/3 Octave frequency spectra for UTP tire, test speed $48 \mathrm{~km} / \mathrm{h}, \mathrm{SCD} \_3$.. 257

Figure G 22. 1/3 Octave frequency spectra for GA tire, test speed $16 \mathrm{~km} / \mathrm{h}, \mathrm{SCD} \_3 \ldots .258$

Figure G 23. 1/3 Octave frequency spectra for GA tire, test speed $32 \mathrm{~km} / \mathrm{h}, \mathrm{SCD} \_3 \ldots .258$

Figure G 24. 1/3 Octave frequency spectra for GA tire, test speed $48 \mathrm{~km} / \mathrm{h}, \mathrm{SCD} \_3 \ldots .259$

Figure G 25. Sound intensity level vs. speed relationship and SIL extrapolated to 97 $\mathrm{km} / \mathrm{h}$ for tests with UTP tire, SCD_3 ..................................................... 259

Figure G 26. Sound intensity level vs. speed relationship and SIL extrapolated to 97 $\mathrm{km} / \mathrm{h}$ for tests with GA tire, SCD_3 ........................................................ 260 


\section{LIST OF SYMBOLS}

\begin{tabular}{|c|c|}
\hline${ }^{\circ} \mathrm{C}$ & degrees Celsius \\
\hline A & friction device calibration constant \\
\hline $\mathrm{a}$ & texture device calibration constant \\
\hline $\mathrm{a}_{4}$ & polishing rate \\
\hline AADT & Average Annual Daily Traffic \\
\hline AASHTO & American Association of State Highway and Transportation Officials \\
\hline $\mathrm{AC}$ & viscosity graded binder \\
\hline ANOVA & analysis of variance \\
\hline ASTM & American Society for Testing and Materials \\
\hline $\mathrm{B}$ & friction device calibration constant \\
\hline $\mathrm{b}$ & texture device calibration constant \\
\hline $\mathrm{BPN}$ & British Pendulum Number \\
\hline BSRT & British (Pendulum) Skid Resistance Tester \\
\hline $\mathrm{C}$ & aggregate gradation: coarse \\
\hline $\mathrm{C}$ & friction device calibration constant (differentiate from above by context) \\
\hline CAT & carbonate aggregate type \\
\hline $\mathrm{CCD}$ & charged couple device \\
\hline CTM & Circular Track Meter \\
\hline CTPM & Circular Track Polishing Machine \\
\hline $\mathrm{D}$ & dolomite \\
\hline $\mathrm{DF}_{20}$ & dynamic friction, wet friction number measured at $20 \mathrm{~km} / \mathrm{h}$ \\
\hline DFT & Dynamic Friction Tester \\
\hline DGA & dense graded asphalt \\
\hline DOT & Department of Transportation \\
\hline ESAL & Equivalent Standard Axle Load \\
\hline $\mathrm{F}$ & aggregate gradation: fine \\
\hline $\mathrm{F}(\mathrm{S})$ & wet friction at any slip speed $\mathrm{S}$ \\
\hline $\mathrm{F}_{0}$ & function of microtexture (friction) \\
\hline & calibrated wet friction at $60 \mathrm{~km} / \mathrm{h}$ \\
\hline
\end{tabular}




$\begin{array}{ll}\mathrm{F}_{\mathrm{a}} & \text { adhesion force } \\ \text { FAA } & \text { Federal Aviation Administration } \\ \text { FAT } & \text { friction aggregate type } \\ \mathrm{F}_{\mathrm{h}} & \text { hysteresis force } \\ \text { FHWA } & \text { Federal Highway Administration } \\ \text { FM } & \text { fineness modulus } \\ \text { FN } & \text { friction number } \\ \text { FR60 } & \text { friction measured at } 60 \mathrm{~km} / \mathrm{h} \\ \text { FRS } & \text { friction measured at slip speed } \\ \mathrm{F}_{\mu} & \text { friction force } \\ \mathrm{G} & \text { gradation } \\ \mathrm{G}_{\mathrm{mb}} & \text { bulk specific gravity of compacted hot mix asphalt } \\ \mathrm{G}_{\mathrm{mm}} & \text { maximum theoretical specific gravity of hot mix asphalt } \\ \mathrm{G}_{\mathrm{sb}} & \text { aggregate blend bulk specific gravity } \\ \mathrm{HL} & \text { "hard” limestone } \\ \mathrm{HMA} & \text { hot mix asphalt } \\ \mathrm{HMA}-\mathrm{TT} & \text { hot mix asphalt, test track section } \\ \mathrm{I} & \text { interstate highways } \\ \mathrm{IA} & \text { state of Iowa } \\ \mathrm{IFI} & \text { International Friction Index } \\ \mathrm{IN} & \text { state of Indiana } \\ \mathrm{INDOT} & \text { Indiana Department of Transportation } \\ \mathrm{ITM} & \text { Indiana Test Method } \\ \mathrm{kN} & \text { kilo Newton } \\ \mathrm{kPa} & \text { kilo Pascal } \\ \text { LA } & \text { Los Angeles Abrasion test method } \\ \text { MPa } & \text { mega Pascal } \\ \text { MPD } & \text { mean profile depth, mm } \\ \text { mph } & \text { speed, miles per hour } \\ \mathrm{MS} & \text { manufactured sand } \\ \text { MTD } & \text { mean texture depth, mm } \\ \mathrm{NCAT} & \text { National Center for Asphalt Technology } \\ & \end{array}$


NCHRP National Cooperative Highway Research Program

$\mathrm{N}_{\text {design }}$ design number of the Superpave Gyratory Compactor gyrations

NMAS nominal maximum aggregate size

NS natural sand

NVA number of vehicle axles passing over tested section

OGFC open graded friction course

OFT outflow time

p-value significance of correlation

$\mathrm{P}_{0.075} / \mathrm{P}_{\text {be }} \quad$ dust-to-binder ratio

$\mathrm{Pa} \quad$ Pascal (unit of pressure)

$\mathrm{P}_{\mathrm{b}} \quad$ specific gravity of binder

PCC portland cement concrete

PFC porous friction course (also called OGFC or porous European mix (PEM)

or, mainly in Europe, porous asphalt)

PG binder performance grade

PIARC Permanent International Association of Road Congress

PMS Pavement Monitoring System

Q quartzite

R-value coefficient of correlation

$\mathrm{R}^{2} \quad$ coefficient of determination

RPM number of rotations per minute

S aggregate gradation: s-shape

S slip speed (differentiate from above by context)

SC test section: smooth concrete

SGC Superpave Gyratory Compactor

SL "soft" (oolitic) limestone

SMA stone matrix asphalt (called also in Europe "stone mastic asphalt")

SN skid number

$\mathrm{SN}(64) \mathrm{R} \quad$ skid number measured at speed of $64 \mathrm{~km} / \mathrm{h}$ using rib tire

$\mathrm{SN}(64) \mathrm{S} \quad$ skid number measured at speed of $64 \mathrm{~km} / \mathrm{h}$ using smooth tire

$\mathrm{S}_{\mathrm{p}} \quad$ speed constant of wet pavement friction

SPR skid performance rating 


$\begin{array}{ll}\text { SR } & \text { state roads } \\ \text { SS } & \text { steel slag } \\ \text { TC } & \text { test section: tined concrete } \\ \text { Tx } & \text { texture measure } \\ \text { US } & \text { United States designated highways } \\ \mathrm{V}_{\mathrm{a}} & \text { air voids content } \\ \mathrm{VFA} & \text { voids filled with asphalt, } \% \\ \mathrm{VMA} & \text { voids in the mineral aggregate, } \% \\ \mathrm{x}_{1} & \text { final polishing level } \\ \alpha & \text { significance level } \\ \mu & \text { friction coefficient }\end{array}$




\begin{abstract}
Wet pavement friction is known to be one of the most important roadway safety parameters. The microtexture, macrotexture and friction of the pavement surface are integrally related. Current widely used test methods allow evaluation of the frictional properties and polishing resistance of the aggregate only. There is no generally accepted standardized laboratory test method to address the overall pavement frictional properties. In this research, which included both laboratory and field components, frictional properties of flexible (asphalt) pavements were investigated.
\end{abstract}

As a part of this study, a laboratory device to polish asphalt specimens was refined and a procedure to evaluate mixture frictional properties was proposed. Following this procedure, 46 different Superpave mixtures (each utilizing a different aggregate blend), one stone matrix asphalt (SMA) mixture and one porous friction course (PFC) mixture were tested.

In addition, field sections constructed using Superpave, Marshall, SMA and PFC mixtures were also tested for friction and noise. Field measurements included testing a total of 23 different asphalt and two concrete pavements.

The field friction testing was performed using both portable Circular Track Meter (CTM) and Dynamic Friction Tester (DFT) devices and the ASTM E 274 locked wheel friction trailer. The laboratory friction testing was performed using the CTM and DFT devices only.

The results of both field and laboratory measurements were used to develop an International Friction Index (IFI)-based protocol for measurement of the frictional 
characteristics of asphalt pavements for laboratory friction measurements. The results collected in the course of the study indicate that the IFI-based flag values could be successfully used in place of SN-based flag values to characterize frictional characteristics of pavements.

Based on the results of the study, it appears the content of high friction aggregate should be $20 \%$ or more of the total aggregate blend when used with other, polish susceptible coarse aggregates; the frictional properties increased substantially as the friction aggregate content increased above $20 \%$. Both steel slag and quartzite were found to improve the frictional properties of the blend, though steel slag had a lower polishing rate. In general, mixes containing soft limestone demonstrated lower friction values than comparable mixes with hard limestone or dolomite.

Larger nominal maximum aggregate size mixes had better overall frictional performance than smaller sized mixes. In addition, mixes with higher fineness moduli generally had higher macrotexture and friction. 


\section{CHAPTER ONE: INTRODUCTION}

When designing a pavement surface, engineers must strive to balance several competing parameters, including load capacity, durability, ride quality, construction and maintenance costs, as well as safety and traffic noise. While most of the above parameters can be achieved by using proper materials and construction techniques, current design methodologies do not routinely address friction and texture control [Goodman et al. 2006]. Adequate pavement friction is of paramount importance in reducing the number of collisions, especially on wet pavements. A relationship between low wet pavement friction and the probability of an accident was proposed by Burchet and Rizenbergs [1982] and by Kamel and Gartshore [1982].

Pavement friction is primarily a function of the surface texture, which includes both micro- and macrotexture. Pavement microtexture is defined as "a deviation of a pavement surface from a true planar surface with characteristic dimensions along the surface of less than $0.5 \mathrm{~mm}$ " while the pavement macrotexture is defined as "a deviation of $0.5-50 \mathrm{~mm}$ " [Henry 1996, Wambold et al. 1995]. According to the same authors, these deviations correspond to texture wavelengths with one-third octave bands lower than $0.4 \mathrm{~mm}$ for microtexture and $0.5-50 \mathrm{~mm}$ for macrotexture. Microtexture (a function of the surface texture of the aggregate particles) provides a gritty surface that disrupts the continuity of the water film and produces frictional resistance between the tire and pavement. Macrotexture (determined by the overall properties of the pavement 
surface) provides surface drainage channels for water expulsion from the contact area between the tire and pavement. This expulsion prevents hydroplaning and improves wet frictional resistance by enhancing the tire/pavement contact [Fulop et al. 2000, Hanson and Prowell 2004].

While efforts to increase the mechanical durability of pavements are at the core of Superpave technology, none of the existing mix design methods specifically focuses on addressing their frictional characteristics. This property is typically ensured by using quality coarse aggregate with a history of good frictional performance [West et al. 2001].

The material properties, including aggregate types and mixture composition, were studied as a part of this project and an attempt was made to develop a relationship between them and the frictional characteristics of the pavement.

\subsection{Problem Statement}

Due to limited availability of high friction aggregates in some areas, there is a need to combine them with locally available materials that may have lower polishing resistance. There is also a need to assess and optimize the combined effects of pavement micro- and macrotexture on the level of pavement friction. In order to achieve this optimization, it is first necessary to identify an accelerated method to polish test samples and test their frictional properties. 


\subsection{Objectives}

The main objective of this research was to evaluate various blends of aggregates to optimize the combination of micro- and macrotexture to achieve a desired level of friction. The goal was to maintain the currently provided level of friction while reducing the reliance on the microtexture provided by special friction aggregates, if possible, by increasing the mixture macrotexture.

To achieve this primary objective, a secondary objective was necessary; to identify an accelerated method for polishing or abrading samples and measuring their surface friction characteristics.

Another objective was the development of preliminary procedure for determination of an International Friction Index (IFI)-based flag value that can be used as a baseline indicator for laboratory friction measurements. In addition, field investigation of the relationship between traffic volume and changes in the friction values was also undertaken in this study.

\subsection{Hypothesis and Scope of the Study}

Based on the literature survey, mixture composition seems to affect the frictional and noise properties of flexible pavements. As a result, it was hypothesized that it may be possible to predict and modify the frictional properties of the pavement by changing the aggregate type and HMA composition.

The scope of this study included the investigation of the relationship between mixture composition and the following pavement characteristics: surface texture, friction and polishing resistance. Based on the relationship between texture and friction, an 
International Friction Index (IFI)-based flag friction value was developed to serve as a reference point for laboratory type testing.

This study included both laboratory and field measurements. The overall scope of the research plan included a literature study, material (aggregate and binder) characterization, test site selection, mix design, sample preparation, testing and data analysis.

This study involved laboratory testing of various aggregate gradations (fine, sshaped and coarse) and aggregate sizes (9.5 $\mathrm{mm}$ and $19 \mathrm{~mm}$ Nominal Maximum Aggregate Size, NMAS) of Superpave mixtures. Aggregates commonly used in HMA in the north central region of the USA (natural sand, dolomite and two types of limestones) were combined with different percentages (from 0 to $70 \%$ ) of two high friction aggregates (quartzite and steel slag) to produce the mixes used in the study. In addition, one stone matrix asphalt (SMA) and one porous friction course (PFC) mix were also tested.

Friction and texture measurements were conducted on 50 laboratory-prepared and polished HMA slabs. These included 46 slabs prepared using Superpave mixtures, two slabs prepared using an SMA mixture and two slabs prepared using a PFC mixture. In order to obtain frictional resistance curves, measurements were performed after compaction of the slabs and periodically during the slab polishing cycle. Laboratory texture and friction tests were conducted using the Circular Track Meter (CTM) and Dynamic Friction Tester (DFT) devices, respectively.

In addition to the laboratory tested slabs, the field friction and texture data were collected from 25 sites on existing highways and test track sections. These data were 
used to obtain the friction baseline values. Field measurements were conducted using the CTM, DFT and ASTM E 274 towed friction trailer. The field test sites included Superpave designed HMAs, Marshall designed HMAs, PFC, SMA and concrete (tined and smooth) pavements. Using these field data and recommendations found in the literature, the IFI flag value was determined.

\subsection{Organization of the Report}

This report is divided into three parts; in the first part (Chapter 2), the current knowledge on pavement friction and noise is summarized. In the second part (Chapter 3) materials, equipments and test methods used in this study are described. The third part (Chapters 4 to 6) presents the test results, conclusions and recommendations. 


\section{CHAPTER TWO: LITERATURE REVIEW}

In this chapter, the terms pavement friction, micro- and macrotexture are defined, and general issues related to measurements of those parameters are discussed. In addition, flexible pavement characteristics contributing to friction are summarized.

\subsection{Fundamentals of Pavement Texture and Friction}

\subsubsection{Pavement Texture and Friction}

Tire/pavement friction is defined as the relationship between the vertical and horizontal forces developed as a tire slides along a pavement surface. To the vehicle operator, friction is an indicator of safety, a measure of how quickly a vehicle can be stopped [MEPDG 2004, Roberts et al. 1996]. Friction resistance is defined by the ASTM E 867 (2006) specification as "the ability of the traveled surface to prevent the loss of traction."

Loss of traction on a wet pavement surface can be attributed to the presence of a water film in the pavement/tire contact area. As the vehicle speed increases, the tire/pavement contact surface area decreases. This happens because there is less time for the water film to escape from the tire/pavement interface, resulting in a thicker film between the tire and pavement. Moreover, this relationship depends on the pavement texture [Kulakowski and Harwood 1990]. 
In this study, a great emphasis was placed on studying the wet friction phenomenon. This term should not be confused with the hydroplaning problem. Hydroplaning occurs when direct tire/pavement contact is lost due to the presence of a water film on the surface of the road. More in-depth discussion of the hydroplaning problem can be found elsewhere [Browne 1975, Ong and Fwa 2007]. General wet friction problems could be considered partial, but not full, hydroplaning.

\subsubsection{Mechanism of Friction}

The mechanism of friction can be theoretically described by the five classical Euler's laws of friction, which can be stated as follows:

1. The coefficient of friction is material dependent,

2. The coefficient of friction is independent of apparent contact area,

3. The coefficient of friction is independent of load,

4. The static coefficient of friction is higher than the sliding coefficient of friction, and

5. The coefficient of friction is independent of sliding speed.

Sometimes, incorrectly, the classical Euler's laws are applied to rubber materials. However, viscoelastic materials (including rubber) do not completely obey these laws. Moreover, tire/pavement friction depends on the normal force that the tire exerts on the pavement. This force, in turn, depends on tire inflation pressure, contact area and load, velocity and temperature [Kummer 1962, Kummer and Meyer 1962]. Probably the most fundamental tire/pavement friction study was conducted in the 1960s [Kummer 1962, Kummer and Meyer 1962] and resulted in the so-called "Unified Theory of Rubber and Tire Friction," which includes considerations of these facts. 
As shown in equation 1 and in Figure 1, conventional friction theories agree that friction at the tire/pavement interface has two principal components [Kummer 1962, Kummer and Meyer 1962, French 1989]: hysteresis, developed when the tire rubber deforms due to pavement surface irregularities; and adhesion, molecular bonds generated when the tire slides over the aggregate surface.

$$
F_{\mu}=F_{a}+F_{h}
$$

where:

$$
\mathrm{F}_{\mu}=\text { friction force, }
$$

$\mathrm{F}_{\mathrm{a}}=$ adhesion force depending on the interface shear strength and the contact area, $\mathrm{F}_{\mathrm{h}}=$ hysteresis force generated due to the damping losses within the rubber.



Figure 1. Principal components of pavement/tire friction [after Kummer 1966]

Note: The symbol used in this document to represent the friction force is " $\mathrm{F}_{\mu}$ ", rather than " $F$ " as shown in Figure 1). 
It should be noted that the hysteresis component would be present even if the pavement surface were perfectly lubricated [Bazlamit and Reza 2005, Flitsch et al. 2005, Kummer and Meyer 1962]. During typical road experiments it is not possible to distinguish between hysteresis and adhesion. Two extreme conditions where either one or the other component would play a significant role are: a clean, dry plate of glass, where there will be no repetitive deformations in the contact area so the hysteresis component may be considered negligible; or a wavy (irregular) well-lubricated surface, where the adhesion component would be greatly reduced. However, even under these conditions the adhesion component may be negligible only at low normal pressure since high pressure would cause discontinuities in the film of lubricant [Kummer and Meyer 1962].

The adhesion component, a result of interface shear forces between the tire and pavement, can be expressed as follows [Kummer 1962]:

$$
F_{a}=S \sum_{i=1}^{n} A_{i}=S \cdot A
$$

where:

$$
\begin{aligned}
& S=\text { friction force, } \\
& A=\text { actual tire/pavement contact area, } \\
& A_{i}=\text { tire/pavement contact area at a specific irregularity (asperity }{ }_{i} \text { ). }
\end{aligned}
$$

The hysteresis component is the result of damping losses within the rubber. The hysteresis component can be expressed in terms of energy as follows [Kummer 1962]:

$$
F_{h}=\frac{1}{b} S \sum_{i=1}^{n} E_{h i}=\frac{1}{b} E_{h}
$$


where:

$\mathrm{b}=$ the average spacing between asperities,

$\mathrm{E}_{\mathrm{h}}=$ lumped energy dissipated within the rubber due to the tire deformation,

$\mathrm{E}_{\mathrm{hi}}=$ energy dissipated within the rubber due to the tire deformation at a specific irregularity (asperity $\left.{ }_{i}\right)$.

As stated earlier, tire/pavement friction is both speed and temperature dependent (mainly due to the damping properties of the rubber). Kummer [1962] investigated the effect of speed and temperature and concluded that at low speeds ( 0 to $16 \mathrm{~km} / \mathrm{h}$ ), the adhesion force component of the friction is significantly speed dependent and the hysteresis force component shows a little speed dependence (refer to Figure 2). At high speeds (above about $80 \mathrm{~km} / \mathrm{h}$ ), however, the adhesion force remains relatively stable and the hysteresis force component increases substantially. In the range of typical pavement temperatures, temperature variation has a similar impact on both adhesion and hysteresis components. As temperature increases, both components decrease [Kummer and Meyer 1962].

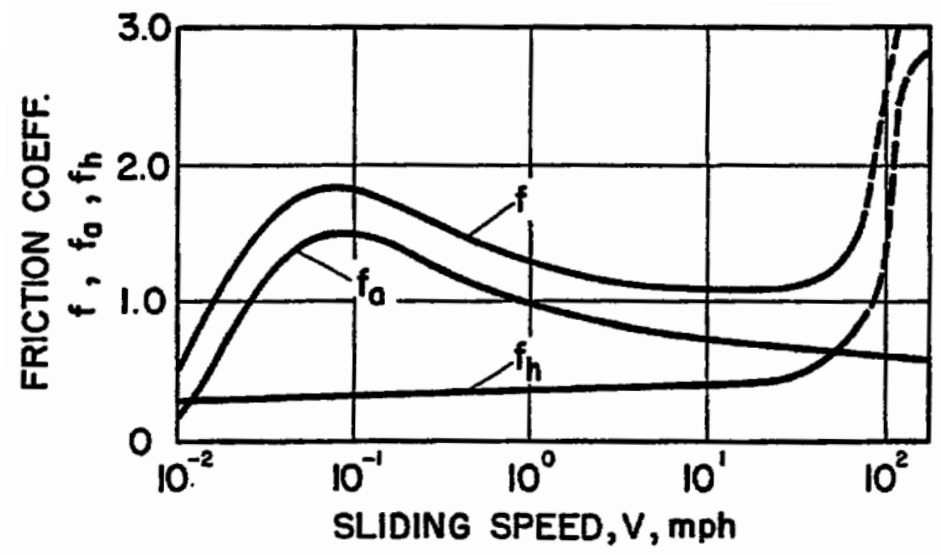

Figure 2. Speed dependence of the tire/pavement friction components [Kummer 1962]; Note: In the current document the symbol " $\mu$ " (rather than " $\mathrm{f}$ " as used in this figure) is consistently used to denote the coefficient of friction; $1 \mathrm{mph}=1.6 \mathrm{~km} / \mathrm{h}$. 
When a tire slides over a rough surface, the tread of the tire will experience continuing deformation (composed of compression and relaxation phases). In the compression phase, recoverable deformation energy is stored within the rubber tread. In the relaxation phase, part of the stored energy is recovered and part of the stored energy is lost in the form of heat. The loss of energy as heat is irreversible and may be identified as a hysteresis loss.

Li et al. [2003] created a finite element model to investigate the energy changes during braking. The energy changes caused by braking action on a wet surface were also studied by Obertop [1962]. Based on the investigation of heat energy produced in the tire/pavement contact area, the author concluded that "the production of steam by the transformation of energy causes the decrease of skid-resisting properties of wet surfaces at high speeds."

\subsubsection{Friction and Texture Relationships}

Macrotexture has wavelengths of the same magnitude as tire tread elements at the tire/pavement interface, and the typical peak to peak amplitudes are about 0.01-20 mm. Microtexture makes the surface feel more or less harsh, but is normally too small to be observed by the human eye. The typical peak to peak amplitudes for microtexture are about $0.001-0.5 \mathrm{~mm}$. In addition, megatexture and unevenness are typically reported when pavement texture is discussed. Megatexture is the "deviation of a pavement surface from a true planar surface with the characteristic dimension along the surface of 50-500 $\mathrm{mm}$;" the peak-to peak amplitudes are usually in the range of $0.1-50 \mathrm{~mm}$. Unevenness is defined as "the deviation of a pavement surface from a true planar surface with the 
characteristic dimension along the surface of more than 0.5 m." [Henry 1996, Wambold et al. 1995]

An attempt to mathematically describe pavement using a combination of simple shapes (cubes, square/pyramids and hemispherical asperities) to better understand the tire wearing mechanism was shown by Moore [1975]. Another approach [Vallejo 2001] utilized the concept of fractals theory (a mathematical concept used to describe the geometry of irregularly shaped objects) and fractal dimensions as a measure of texture.

Surface texture and friction are integrally related. In general, microtexture contributes to friction at all speeds, but dominates at low speeds (about $50 \mathrm{~km} / \mathrm{h}$ ). The relative influence of microtexture on wet friction is reduced as the speed increases [Cenek et al. 1997]. Macrotexture generally controls friction at high speeds [Ergun et al. 2005]. Only one author was found who reported that macrotexture could significantly affect low-speed friction [Liu et al. 2003, Liu et al. 2004]. Pavement texture can be used indirectly to determine surface friction [Gunaratne et al. 2000, Wayson 1998]. However, using macrotexture alone cannot define the frictional properties of a pavement [Yager and Buhlman 1982].

A relationship between the adhesion component of friction and microtexture size was developed by Britton et al. [1975], who found that as the microtexture increases, the wet friction will first increase, then stabilize, then finally decrease. Moreover, the relationship between the microtexture size and wet friction resistance also depends on the macrotexture characteristics.

A complex mechanical model to predict tire/pavement friction based on the surface texture and tread rubber properties was developed in 1983 [Yandell et al. 1983]. 
The authors verified the proposed model on coarse and fine textured roads and found a good relationship between predicted and measured coefficients of friction. A friction model applicable to aircraft tires was proposed in the 1970's [Wahi 1979]. Model parameters included tire characteristics, surface macrotexture depth and fluid characteristics (the fluid was assumed to create a film between the tire and pavement).

Another attempt to predict friction based on texture analysis was presented by Ergun et al. [2005]. The authors developed a friction prediction model based on image analysis of core samples drilled from pavements and reported a good relationship between the predicted values and field measurements.

\subsubsection{Changes in Tire/Pavement Friction}

The frictional characteristics of pavements usually change over time and under traffic. The plot of friction values versus the number of wheel passes is commonly called the polishing curve. The slope of this curve is called the polishing rate. The polishing curve generally follows a logarithmic trend to a certain value, frequently reported as the terminal friction level, after which the curve flattens and the friction level remains essentially constant [Diringer and Barros 1990, Emery et al. 1982, Liang 2007, Shahin 2005, Wang and Flintsch 2007]. Sometimes, however, the frictional resistance may continue to drop because wear continues to reduce the pavement macrotexture, resulting in decreasing numbers of channels available for clearing the water from the tire/pavement contact area [Roberts et al. 1996].

It is commonly reported that the frictional properties of a newly paved surface improve markedly over the first few months of service. One study, for example, showed 
substantial increases in the friction value of a new HMA surface from the time it was opened to traffic to 35 weeks later [Saito et al. 1996]. This phenomenon was most likely caused by improvement of the surface microtexture resulting from wearing off of the binder film coating the aggregates on the surface of the pavement (due to tire action).

Results of a laboratory study [Vollor and Hanson 2006] showed that the shape of the polishing curve may sometimes exhibit a high rate of initial friction loss after the binder film is worn off. After this initial loss, the friction may continue to decrease at a lower rate, eventually leveling off. The high initial rate of friction loss may be due to polishing of the originally sharp edges of the exposed aggregate; as these edges are worn off or reoriented, the friction decreases.

Seasonal and short-term variations in pavement friction have also been reported and widely studied [Bazlamit and Reza 2005, Flintsch et al. 2005, Gargett 1990, Henry et al. 1984, Hill and Henry 1982, Wambold et al. 1989, Wang and Flintsch 2007]. Depending on the geographical location, the lowest values of wet pavement friction typically occur towards the end of summer and the highest occur during the winter. Short-term variations are typically caused by temperature fluctuations and precipitation. A nonlinear model has been proposed to describe these seasonal variations [Diringer and Barros 1990]. Other authors [Huihua and Henry 1990] have proposed using a fuzzy clustering approach as a mathematical tool to describe seasonal friction variation. Friction changes were also found to be affected by seasonal changes in the grading of abrasive material lying on the road or embedded in vehicle tires [Donbavand and Cook 2005, Woods et al. 1960]. 
As proved in research conducted in the early 1960s [Mahone 1962], friction varies across a highway surface. Typically, the lowest friction is observed in the wheelpaths while other portions of the pavement exhibit higher friction. In addition, due to the variations in forces resulting from vehicles decelerating, turning, etc., friction varies longitudinally (between straightaways, curves, intersections, and so forth). The author concluded that those differences are more significant for pavements constructed with polish-susceptible materials.

Differences in the friction value between the driving and passing lanes have also been reported. Typically lower friction values are associated with the driving lane [Schulze and Beckman 1962]. For some asphalt interstate highways, researchers found differences up to $13 \mathrm{SN}$ (skid number, as explained later in section 2.3.3), when measured with the ASTM E 274 friction trailer [Li et al. 2003].

\subsection{Pavement Characteristics Contributing to Friction}

The surface texture depth, porosity and surface friction are key parameters in pavement safety [Wayson 1998]. Overall, texture is affected by the aggregate size and size distribution as well as by the aggregate shape. The macrotexture is determined by the overall properties of the pavement surface and the microtexture is mainly a function of the surface texture of the aggregate particles. "Pavement macrotexture can be modified by changing the size and size distribution of the aggregates, while microtexture can only be modified by changing the types of aggregates used in the mixture" [McDaniel and Coree 2003, also Gardziejczyk 2002]. 
General recommendations for material selection and blend proportioning to ensure sufficient frictional properties are summarized elsewhere by Li et al. [2007].

\subsubsection{Material Selection}

Different aggregates vary in their ability to resist undesirable microtexture changes. When worn by traffic, different aggregates polish or become smoother at different rates [McDaniel and Coree 2003]. To provide pavement frictional resistance, aggregates are commonly selected based on historical data of pavement skid resistance or based on laboratory testing. However, the reliability of predicting aggregate field polishing resistance using a single laboratory test is poor [Prasanna et al. 1999].

From the friction point of view, four aggregate characteristics should be evaluated during selection of aggregates for pavement surface courses: microtexture, shape, size, and resistance to wear and polishing. To improve pavement frictional resistance, while accommodating the use of locally available low friction aggregate sources, those local materials may be combined with aggregates with higher frictional characteristics [Gee 2005, West et al. 2001]. In many states, the acceptance procedure for friction resistant aggregates is based on historical data. In some cases, the construction and evaluation of road test sections over a period of time can also be used; for example, Indiana requires monitoring for two years [ITM 214]. Methods to evaluate frictional properties of various carbonate aggregates sources were thoroughly investigated by researchers in Indiana [West and Cho 2001].

"Generally, igneous and metamorphic rock constituents polish to a lesser extent than sedimentary rocks and should improve the overall frictional resistance of an HMA 
pavement" [West et al. 2001]. Synthetic aggregates, like slag or expanded lightweight aggregate (fabricated by heating natural clay), have also been found to improve pavement frictional resistance [Roberts et al. 1996, Wasilewska and Gardziejczyk 2005].

The Iowa DOT recognizes five different types of aggregates with respect to their frictional characteristics [Iowa DOT 2006]. These types are as follows:

- Type 1: Heterogeneous combination of minerals with coarse grained microstructure of very hard particles (generally, a Mohs hardness range of 7 to 9) bonded together by a slightly softer matrix

- Type 2: Crushed quartzite, granites and air-cooled steel furnace slag, having a Mohs hardness of 5-7

- Type 3: Synthetic aggregates (expanded shales) with LA abrasion loss below 35\%, crushed traprocks and gravels ("gravels shall contain at least 40\% igneous particles")

- Type 4: Natural gravels (in which carbonate particles shall not exceed the noncarbonate particles by more than 20\%) and "aggregates crushed from dolomitic or limestone ledges in which $80 \%$ of grains are 20 microns or larger"

- Type 5: Aggregates crushed from dolomitic or limestone ledges in which $20 \%$ of the grains are 30 microns or smaller”.

An extensive survey comparing states' requirements for aggregate selection to provide pavements that are resistant to polishing was conducted by Liang [2003]. The author proposed recommendations for blending high- and low-friction resistant aggregates to improve the polish resistance of locally available low friction resistant aggregates. 
It is commonly assumed that aggregates with lower Los Angeles (LA) abrasion loss, lower sulfate soundness loss, lower freeze-thaw (F-T) loss, lower absorption and higher specific gravity are more desirable from the frictional point of view. However, the LA abrasion loss and other physical tests may not yield good predictions of field friction [West and Cho 2001]. Several authors have shown that some aggregates that, based on physical tests, may be considered to be of "lower" quality may also (surprisingly) provide better frictional resistance. This may be due to the mineralogy of the aggregate, which perhaps influences frictional resistance the most [O'Brien 2004, West and O'Brien 2005]. For some types of carbonate aggregates (e.g., dolomite) polish susceptibility was found to decrease with an increase of clay content (and decrease in specific gravity) [West et al. 2001]. The authors of another study [Liang and Chyi 2000] found that as the calcite and dolomite contents increase, the polish susceptibility of aggregates decreases to the certain value. Further increases in the calcite and dolomite contents result in a loss of polish resistance. The authors of another study observed that there is no relationship between polish resistance and the LA abrasion test [Wasilewska and Gardziejczyk 2005].

An interesting study to compare the influence of the sand source on the frictional properties of HMA was presented by Dames [1990]. The author investigated five manufactured sand sources (basalt, granite, blast furnace slag, limestone and alpine moraine) and one natural sand source. Dames concluded that highly polished sand (limestone) can significantly reduce the frictional resistance of the pavement. Moreover, the influence of the sand properties (occupying typically about $30 \%$ of the mixture volume) on the frictional characteristics of the pavement was found to be more significant than commonly assumed. 


\subsubsection{Mix Proportioning}

Aggregate gradation affects nearly all asphalt mixture properties -- from modulus to stability. Gradations that plot further away from the maximum density line typically have higher voids in the mineral aggregate (VMA) contents and thus give higher surface texture for a given asphalt volume [Sullivan 2005].

Three types of HMA mixtures are typically used as surface layers of high traffic volume highways: dense graded asphalt (DGA), stone matrix asphalt (SMA, also called "stone mastic asphalt" in Europe) and porous friction course (PFC), also called "open graded friction course" (OGFC), "porous European mix" (PEM). Each type of mixture has various advantages and limitations. Within the wide group of conventional dense graded HMAs, three sub-groups of mixes can be identified depending on the aggregate gradation: fine, coarse and s-shaped.

Macrotexture of porous friction courses (PFC) is usually higher than that of typical dense graded asphalt (DGA), thus enhancing surface friction [Hassan et al. 2005]. In addition, it is also easier to drain water off such surfaces, thus improving wet friction [Ahe 2005, Crocker et al. 2004, Bernhard and Wayson 2005-1].

The influence of the mix design method on pavement texture was also investigated [Stroup-Gardiner et al. 2004]. The authors compared different mixes (nine Marshall and nine Superpave mix designs) and road sections (located in Alabama) and found that the macrotexture is not statistically different.

For typically used aggregate sizes, as aggregate spacing (taken as aggregate center to center distance or aggregate (texture) wavelength) increases, the coefficient of friction 
generally decreases [Fwa et al. 2003]. In other research, the same authors stated that decreasing the aggregate size would often result in the increase of friction [Liu et al. 2004].

In addition to the gradation, higher (than optimum) binder contents of asphalt mixtures will fill some of the voids in mineral aggregate (VMA) and thus lower texture [Sullivan 2005]. This would result in reducing friction as well [AASHTO 1976, Woods et al. 1960]. Nitta et al. [1990] noticed that changes of mixture components such as asphalt content, source of fine aggregate and coarse aggregate gradation do not significantly affect the polish susceptibility. Furthermore the same authors concluded that the most important factor affecting the polishing properties of mixtures is the exposed area of coarse aggregate on the specimen surface; as the exposed area of coarse aggregate increases, the susceptibility to polishing also increases (BPN decreases).

A relationship between the initial field friction and several mix properties (asphalt content, fineness modulus, bulk density and percent of aggregate passing the $4.75 \mathrm{~mm}$ sieve) was proposed by Goodman et al. [2006]. These authors further concluded that there is a good correlation between macrotexture (measured using the sand patch method) of field and gyratory compacted specimens. However, another author [Sullivan 2005] stated that there is currently no laboratory test method to predict in-service texture.

One of the first pavement friction prediction models was developed in the 1980's [Emery et al. 1982]. This model relates friction number with pavement age, accumulated traffic level and mix properties (aggregate polish resistance, Marshall sample air content, Marshall sample stability and flow). The authors conducted a field verification of the 
model and found good correlation between laboratory predicted values and field measurements of friction.

One of the most current models to predict pavement friction and polish susceptibility was recently proposed by Luce et al. [2007]. This model is based on the mix gradation and changes in aggregate microtexture. The changes in microtexture were evaluated using the Micro-Deval test. In this test, coarse aggregates are tumbled together in a drum with steel balls in the presence of water. The prediction model was successfully verified on nine field test sections.

\subsubsection{Pavement Construction and Maintenance Practices}

Although the influence of pavement maintenance, rehabilitation, or reconstruction practices on the noise and frictional properties of pavements is known to exist, these are not discussed here since the main focus of this study is on techniques to optimize friction characteristics. Non-standard solutions to improve pavement friction (e.g., placing of steel patterned elements into the asphalt pavement) are also discussed elsewhere [Clark 1975]. In addition, only the wet friction phenomenon on flexible pavement is discussed here. Such issues as friction on unpaved roads, friction on compacted snow-covered or ice-covered roads and issues related to "black ice" are considered elsewhere [e.g. by Lea and Jones 2006, Yager 1990, Trevino et al. 2007].

There are no special finishing techniques associated with asphalt pavement construction which would significantly influence future frictional properties of the pavement. Typical good engineering practice has to be followed, especially with proper mixture temperature and compaction level. 
One publication was found [Crispino et al. 2007] which related pavement macrotexture with the roller type and rolling technique used during the construction process. Based on tests using three different mixture types and four different roller types, the authors found that the total static load (sum of all static linear loads from the roller drums over a fixed section) correlates well with the surface macrotexture of the compacted pavement.

\subsection{Friction / Texture Measurements and Polishing Methods}

\subsubsection{Friction Measurement Techniques}

The locked wheel friction trailer described in ASTM E 274 is routinely used to monitor wet pavement frictional characteristics in most states [Murad and Abaza 2006] and is widely described in the literature [e.g. Wambold et al. 1990]. It is commonly assumed that the friction measurements conducted with a smooth tire are sensitive to changes in both micro- and macrotexture while measurements with a rib tire are dependent on the microtexture only [Henry 2000, Wambold et al. 1996]. Typically measurements are conducted at a speed of $64 \mathrm{~km} / \mathrm{h}(40 \mathrm{mph})$. However, due to safety issues, tests on some roads are conducted at $81 \mathrm{~km} / \mathrm{h}(50 \mathrm{mph})$ or at $48 \mathrm{~km} / \mathrm{h}(30 \mathrm{mph})$. It should be noted that there is no unique friction/speed correlation and that the existing correlations vary with pavement surface texture [ $\mathrm{Li}$ et al. 2003, Li et al. 2005]. An attempt to determine such a friction/speed correlation is presented in section 2.3.3.

Other commonly used types of full scale friction measuring devices are as follows [Henry 2000]: side force (ASTM E 670), variable slip (ASTM E 1859 and ASTM E 1337) and fixed slip. In addition, field measurements can be conducted by measuring the 
stopping distance of a passenger vehicle (ASTM E 503/E 503M and ASTM E 445/E $445 \mathrm{M})$.

Currently, the two most popular portable devices, which can be used either in the lab or in the field, are the British (Pendulum) Skid Resistance Tester (BSRT) and Dynamic Friction Tester (DFT). The BSRT (standardized in ASTM E 303) is traditionally the most popular, widely described [e.g. Giles et al. 1962, Liu et al. 2003] test method for pavement friction measurements; however the calibration procedure for this device is complex and time consuming [Kulakowski et al. 1990]. It should be remembered, however, that even when properly calibrated the BP test only gives relative comparisons for frictional properties of surfaces. The results obtained with it may not compare to other test methods [ASTM E 303].

Introduced in the 1990's and also standardized [ASTM E 1911], the DFT is a device that is relatively simple to use. It should be noted that this machine operates in a similar way to the laboratory skid testing machine used at Berlin Technical University from the 1960's [Dames 1990]. Berlin's machine, however, could be used in the laboratory only. A strong relationship between the coefficient of friction measured with DFT and BSRT devices has been reported [Saito et al. 1996]. Both the DFT and BSRT devices were used in the present study. These are described in detail in sections 3.3.1.2 and 3.3.2.2 respectively.

Friction measurement methods which can be conducted in the laboratory were compared in the Interim Report [McDaniel and Coree 2003] and are also repeated here in Appendix A in Table A 1. A list of the laboratory friction measurement devices used in the past (in the 1960's) is shown elsewhere [Woods et al. 1960]. 


\subsubsection{Texture Measurement Techniques}

There are three general types of texture measurement techniques [Abe et al. 2001, Wambold et al. 1995]: volumetric (measures mean texture depth, MTD), outflow meter (measures outflow time, OFT) and profilometer (mostly used for the measurement of the mean profile depth, MPD).

Historically, the most common technique used for macrotexture measurements was the sand patch method, a volumetric technique which is standardized in ASTM E 965. This is a relatively simple and inexpensive method, however, poor repeatability in the measurements has been reported [Doty 1975, Yager and Buhlman 1982].

The outflow meter, standardized in ASTM E 2380, measures the drainage capability of pavements as affected by macrotexture and porosity. It has been widely studied elsewhere [Henry and Hegmon 1975]. The outflow meter is highly correlated with the MPD and MTD for nonporous pavements. For porous surfaces, comparison of the OFT and MTD can potentially be used for assessment of the effectiveness of the porous structure for draining water [Henry and Hegmon 1975].

In general, there are three major types of profilometers: laser based, light sectioning and stylus contact followers (the last two are currently infrequently used in general highway applications). Some profilometers allow for texture measurement at traffic speeds using a mobile device attached to a vehicle (high-speed measurement); such a technique is widely used in Pavement Management Systems (PMS). A feasibility study of laser based pavement texture measurements was published as early as in the 1970’s [Gee et al. 1975]. 
One of the laser-based profilometers currently used is the Circular Track Meter (CTM), which was utilized in this study and is described in detail in Chapter 3. The CTM (standardized in ASTM E 2157) offers a quick and simple way to conduct texture measurements. The variability of the sand patch test and CTM methods was compared by Hanson and Prowell [2004, 2006, Prowell and Hanson 2005]. The researchers found that both techniques produce comparable results. However, the CTM offers the advantage of characterizing the variation in texture over an area rather than just measuring the average texture over an area, as the sand patch does.

Other scientists [McGhee and Flintsch 2003] conducted an extensive study to compare the reliability of different texture measurement techniques: sand patch method, CTM and two different mobile laser based profilometers (called also high speed or dynamic devices). They found a very good correlation between those various techniques.

Texture measurement methods which can be conducted in the laboratory are compared in Appendix A in Table A 2.

Measurement of aggregate shape (e.g. image analysis) is discussed elsewhere [Masad et al. 2004]).

\subsubsection{Harmonization of Friction and Texture Test Results}

It is recognized that the methods and systems used for measuring texture and friction throughout the world vary significantly. Frictional resistance can be reported in terms of friction coefficient $(\mu)$, British Pendulum Number (BPN), the International Friction Index (IFI), skid number (SN) or friction number (FN) [Henry 2000]. 
The International Friction Index (IFI) was developed in an attempt to harmonize texture and friction measured using different test methods [Henry 1996, Henry et al. 2000-1, Wambold et al. 1989, Wambold et al. 1995, Wambold 2005, Yeaman 2005]. During an international PIARC study [Wambold et al. 1995], 47 different measuring systems from 16 countries were used to measure 54 pavement sections located in Spain and Belgium. These measurements resulted in 15,000 numerical values that were included in the database for the PIARC study and were subsequently used to develop the PIARC friction model.

The PIARC model developed in this study is a modification of the Penn State Model, which relates friction $\left(\mathrm{F}_{\mu}\right)$ to slip speed $(\mathrm{S})$. As reported by Wambold et al. [1996], the "Penn State model utilizes two constants, where one is a function of microtexture $\left(\mathrm{F}_{0}\right)$ and other, called the speed constant $\left(\mathrm{S}_{\mathrm{p}}\right)$, is a function of macrotexture." This relation is given below (Eq. 4) as:

$$
F_{\mu}(S)=F_{0} \cdot e^{\frac{-S}{s_{p}}}
$$

The slip speed (S) is defined as the difference between the traveling speed of the measuring device and the speed of a point on the perimeter of the rotating measuring wheel [ASTM E1859].

Based on the measurements conducted during the international PIARC study, a friction-slip speed curve was established for each site tested (nicknamed a "golden curve"). Then, a model was developed in such a way that data from each type of equipment that participated in the study can be used to predict the golden curve values (by applying a specific calibration factor or factors). It was decided for harmonization 
purposes that all measured friction values be reported at a speed of $60 \mathrm{~km} / \mathrm{h}$. The steps used to evaluate friction using the PIARC model procedure are shown below [Wambold et al. 1996].

1. First, based on the texture measurements, the speed constant $\left(S_{p}\right)$ is calculated using Equation 5:

$$
S_{p}=a+b \cdot T x
$$

where "a" and "b" are unique calibration constants developed for each texture measuring device participating in the study and Tx is a measure.

2. Then, the value of the friction measured at any slip speed (FRS) is converted to friction at a slip speed of $60 \mathrm{~km} / \mathrm{h}$ (FR60); note that various friction devices operate at different speeds:

$$
F R 60=F R S \cdot e^{\frac{S-60}{S_{p}}}
$$

3. Finally, the FR60 is recalculated to the unified value (value located on the golden curve) to give the estimated value of friction at a speed of $60 \mathrm{~km} / \mathrm{h}(\mathrm{F} 60)$ :

$$
F 60=A+B \cdot F R 60+C \cdot T x
$$

where "A," "B," and "C" are unique calibration constants developed for each friction device participating in the study; constant $\mathrm{C}$ is equal to 0 for most devices except those using rib or patterned tires.

Based on the PIARC model, an International Friction Index (IFI) was proposed. The IFI (currently standardized in ASTM E 1960) consists of two parameters: the calibrated wet friction at $60 \mathrm{~km} / \mathrm{h}(\mathrm{F} 60)$ and the speed constant of wet pavement friction $\left(\mathrm{S}_{\mathrm{p}}\right)$. The International Friction Index is simply reported as the values of these two parameters, i.e. 
IFI $\left(\mathrm{F} 60, \mathrm{~S}_{\mathrm{p}}\right)$. Both $\mathrm{F} 60$ and $\mathrm{S}_{\mathrm{p}}$ are estimates of the golden curve. These parameters are defined as follows:

The F60 represents the average wet coefficient of friction experienced by a passenger car during a locked-wheel slide at speed of $60 \mathrm{~km} / \mathrm{h}$. The $S_{p}$ is a measure of how strongly the pavement wet friction depends on the sliding speed of a passenger car (high $S_{p}$ value indicates a low sensitivity to slip speed). [Cenek et al. 1997].

In addition to allowing calculation of IFI, the PIARC model can also be used to predict friction values at speeds other than that at which the friction was measured. Using the two IFI parameters (F60 and $S_{p}$ ), the wet friction at any slip speed can be estimated as follows:

$$
F(S)=F 60 \cdot e^{\frac{60-S}{S_{p}}}
$$

As shown in the ASTME 1960 specification, the IFI parameters can be determined using the DFT and CTM devices. Moreover, using those two devices and following ASTME 1960, other devices (which did not participate in the international PIARC study) could also be calibrated to predict the IFI parameters.

An example of how the DFT and CTM machines were calibrated to predict the IFI parameters is shown below. During the international PIARC study, unique calibration constants were developed for the CTM $(a=14.235$ and $b=89.719746)$ and the DFT (when the device measures the friction at $20 \mathrm{~km} / \mathrm{h}, \mathrm{A}=0.08114$ and $\mathrm{B}=0.73158$ ). Note that neither rib nor patterned tires are used in this device, so the constant $\mathrm{C}$ is equal to zero. Applying those constants, the $\mathrm{F} 60$ and $\mathrm{S}_{\mathrm{p}}$ can be expressed as follows: 


$$
\begin{aligned}
& F 60=0.081+0.732 D F_{20} e^{\frac{-40}{s_{p}}} \\
& S_{p}=14.2+89.7 M P D
\end{aligned}
$$

where:

$\mathrm{DF}_{20}=$ wet friction number measured at the speed of $20 \mathrm{~km} / \mathrm{h}$, $\mathrm{MPD}=$ mean profile depth $(\mathrm{mm})$.

These are the same equations as used in the ASTM E 1960 (2007) specification. The influence of changes in $\mathrm{DF}_{20}$ and MPD values on the calculated calibrated wet friction (F60) is shown in Figure 3, as determined using equations 9 and 10. It should be noted that in the range of typical friction and texture values observed on asphalt highways, changes in the wet friction $\left(\mathrm{DF}_{20}\right)$ influence the calibrated wet friction $(\mathrm{F} 60)$ much more than changes in macrotexture (MPD). Moreover, in a low range of $\mathrm{DF}_{20}$ or MPD values, the influence of changes in the second parameter (MPD or $\mathrm{DF}_{20}$, respectively) is less significant than in the high range of $\mathrm{DF}_{20}$ or MPD values. 
(a)

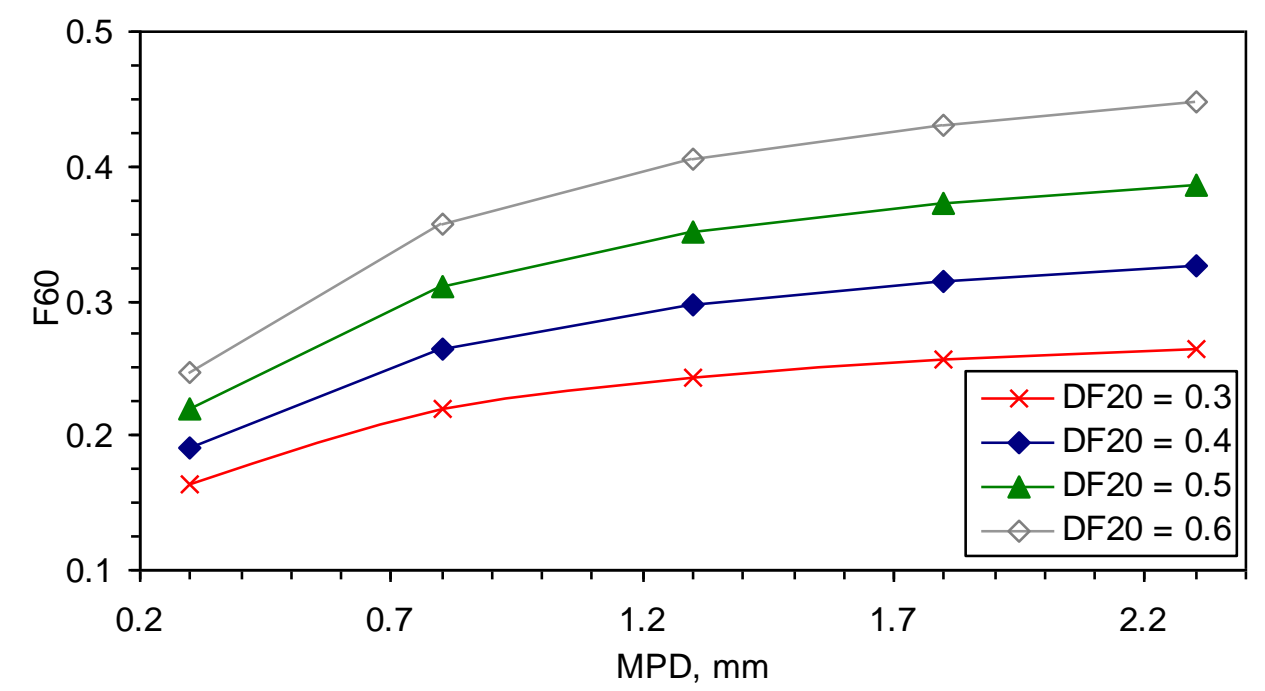

(b)

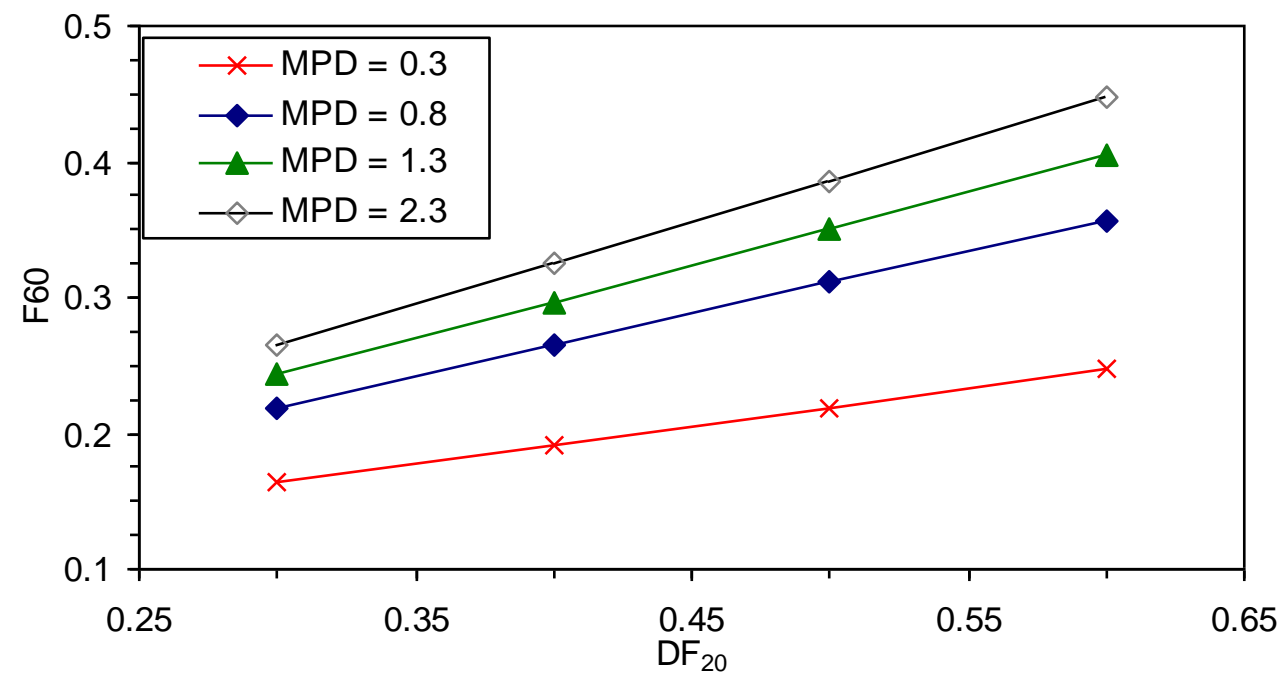

Figure 3. Influence on the calibrated wet friction (F60) of: (a) $\mathrm{DF}_{20}$ and (b) MPD values Note: the ranges of the typical highway pavement values of $\mathrm{DF}_{20}$ and MPD are shown, as determined in a baseline study described in section 4.2.1.2.

It should be noted that the calculation of the value of the speed constant of wet pavement friction is related to the texture measurement device. There were several attempts to relate the $S_{p}$ to MPD. Some of the relations came from a calculation based on the relationship between MPD and MTD (measured using volumetric sand patch method) [Henry et al. 2000, Henry 2006]. The ASTM E 1960 (2007) version of the $S_{p}$ equation 
used consistently in this study is shown above in equation 10. As previously mentioned, this ASTM version was developed based on the international PIARC study [Wambold et al. 1995]. The ASTM E 1845 (2005) specification defines the MPD as "the average of all of the mean segment depths of all of the segments of the profile," where mean segment depth is "the average value of the profile depth of the two halves of a segment having a given baselength," and profile depth is "the difference between the amplitude measurements of pavement macrotexture and a horizontal line through the top of the highest peak within a given baseline."

Although, according to ASTM E 1960, all other friction measuring devices have to be related to the DFT to determine the constants, the international PIARC study determined those constants directly for other machines. Results of the international PIARC study are especially useful if one would like to determine the IFI parameters for pavement friction tested using the ASTM E 274 (2006) locked wheel friction trailer (which, as mentioned before, is routinely used in the USA). For the friction trailer using a smooth tire, the unique calibration constants would be: $\mathrm{A}=0.04461$ and $\mathrm{B}=0.92549$; for the friction trailer using a rib tire, the unique calibration constants would be different: $\mathrm{A}=$ $-0.02283, \mathrm{~B}=0.60682$ and $\mathrm{C}=0.097589$ [Wambold et al., 1995].

Thus, the F60 value for the locked wheel friction trailer using smooth tire would be:

$$
F 60=0.045+0.925 \cdot 0.01 \cdot S N(64) S \cdot e^{\frac{4}{s_{p}}}
$$

where:

$\mathrm{SN}(64) \mathrm{S}=$ skid number measured at test speed of $64 \mathrm{~km} / \mathrm{h}$ using smooth tire. 
Note that the $\mathrm{SN}$ value is reported in a range between 1 to 100 while typically friction is reported in a range between 0 to 1 ; thus, the $\mathrm{SN}$ value in the equation should be divided by 100 .

Obviously, if the test were conducted at another speed, a more general form of this equation would have to be used:

$$
F 60=0.045+0.925 \cdot 0.01 \cdot S N(S) S \cdot e^{\frac{S-60}{S_{p}}}
$$

For the locked wheel friction trailer using a rib tire, the F60 would be calculated as follows:

$$
F 60=-0.023+0.607 \cdot 0.01 \cdot S N(64) R \cdot e^{\frac{4}{S_{p}}}+0.098 \cdot M P D
$$

where:

$\mathrm{SN}(64) \mathrm{R}=$ skid number measured at test speed of $64 \mathrm{~km} / \mathrm{h}$ using rib tire.

Note again that the $\mathrm{SN}$ value is reported in a range between 1 to 100 while typically friction is reported in a range between 0 to 1 ; thus, the $\mathrm{SN}$ value in the equation should be divided by 100 .

It should be noted, however, that during standard operations with a friction trailer, the texture is not measured. Thus, the $S_{p}$ value, which is applied to the model, is not determined. However, if the same section were tested at the same speed with both rib and smooth tires, equations 11 and 13 should be equal. Moreover, if those equations were compared, there would be only two unknowns present: MPD and $S_{p}$. Since, as shown in equation $10, \mathrm{~S}_{\mathrm{p}}$ is truly the MPD modified by some constants, only one unknown is actually present. Then, the $S_{p}$ (or MPD) value could be obtained and both IFI 
parameters could be reported. Finally, one additional comment has to be added: the constants were empirically determined and during any kind of test an error is always incorporated. Using mathematical transformations, the errors could be multiplied. Thus, although theoretically correct, this potential MPD determination method should be further verified.

It should also be recognized that when using a calibrated friction device to report friction values at a range of slip-speeds (e.g., DFT machine), the $S_{p}$ (or MPD) also could be determined. Thus, the additional texture measuring device would not be necessary. Similarly, the earlier comment about the mathematical transformation and introduced errors is applicable here as well.

\subsubsection{Polishing Methods}

The most widely used device to accelerate the polishing of coarse aggregates is the British Polishing Wheel, standardized in ASTM D 3319, then the changes in the microtexture are typically measured using a BSRT device. "However, this method evaluates only loss of microtexture of the coarse aggregate fraction, neglecting any contributions of the fine aggregate or the pavement macrotexture" [McDaniel and Coree 2003]. Another method to evaluate changes in microtexture caused by accelerated polishing was developed by Cafiso and Taormina [2007], who used a scanning procedure able to capture aggregate texture with resolution as fine as one micron.

Currently, there is no widely accepted method which would allow observing changes in both micro- and macrotexture of HMA specimens as a result of the polishing action of tires. Machines used in the past include the Penn State Reciprocating Polishing 
Machine, North Carolina State Wear and Polishing Machine and Michigan Wear Track [McDaniel and Coree, 2003]. The first of these was standardized in ASTM E 1393, but since it was never widely used the specification was discontinued in 1997 . The North Carolina State University (NCSU) Wear and Polishing Machine, standardized in ASTM E 660, is also not very widely used. The Michigan Wear Track is reportedly still used for aggregate evaluations, but only in Michigan. A machine similar to the Michigan device is the Wehner/Schulze polishing machine located at Berlin Technical University. This machine operates with flat, circular specimens $(22.5 \mathrm{~cm}$ in diameter $)$ and the polishing effect of tires is simulated by three conical rubber rollers. In order to accelerate the polishing process, water containing an abrasive additive (quartz powder) is used [Dames 1990].

Another polishing machine which could be used to study changes in both microand macrotexture was proposed by the National Center of Asphalt Technology (NCAT) [Vollor and Hanson 2006]. The NCAT polishing machine is similar to that used in this study and is described in detail in section 3.3.1.2 of this report.

Various accelerated methods for polishing of aggregates and mixes are compared in Appendix A in Table A 3. A list of the polishing devices used in the past (in the 1960's) is given elsewhere [Woods et al. 1960].

Finally, although it is not a polishing method, a recently presented [Ech et al. 2007] test procedure offers a rather unique technique to evaluate changes in road surface macrotexture. The authors introduced a mechanical device which, via a rubber cylindrical membrane, transmits repeated vertical stresses to the tested specimen. Then, using a laser technique, changes in the macrotexture can be evaluated. 


\subsection{Pavement Quality: Friction Requirements}

Pavement quality is a function of highway structural and functional performance. The functional performance of a pavement concerns how well the pavement serves the user. For drivers, good quality pavement should provide safe and comfortable riding while for highway neighborhoods noise coming from the roadway should be minimized. As a part of pavement quality monitoring, surface roughness and distresses (cracking, patching, rutting, etc.) are typically investigated. Functional performance, mainly affected by the pavement texture (micro-, macro-, and mega- texture), has a major impact on the tire/pavement interaction. This interaction affects both friction and noise properties of the pavement. Moreover, agencies and highway users are concerned about the frequency of repairs needed when the pavement performance is not adequate; the period between highway rehabilitations defines its durability [Larson et al. 2005].

As stated earlier, the frictional characteristics of pavements usually change over time and traffic. The deterioration of tire/pavement friction below a minimum acceptable (safe) level prevents the pavement from serving its desired function [Roberts 1996]. The need for a minimum friction number has been recognized by different interest groups, including law enforcement [Hutchinson 1975]. However, due the various legal issues, minimum acceptable friction level requirements have not been published or universally adopted. Instead, most agencies are currently using a so-called "friction flag value." The flag value is defined as the friction number at or below which a site investigation needs to be conducted [Li et al. 2003]. 
Murad and Abaza [2006] proposed a mathematical method (based on the friction and accident history of pavements) to determine sections with possible skidding accident potential. .

For example, in the State of Indiana, the INDOT pavement inventory friction test program covers all interstates, state routes and U.S. routes. Inventory tests are conducted annually on interstates and every three years on other roads [Li et al. 2005]. Highways with friction numbers at or below the flag value are field inspected by the appropriate INDOT district to evaluate the pavement conditions and to determine if resurfacing is necessary [Li et al. 2003].

A similar concept of the investigatory level of frictional resistance has also been proposed and followed in Great Britain. In Great Britain, however, the pavement friction flag value depends on the road category and location. A total of 13 investigatory levels have been defined. "Frictional demand will be greater at sites which are geometrically substandard and also at high-conflict sites, such as intersections or pedestrian crossings, where emergency situations frequently arise, resulting in the need for the vehicle to brake sharply or swerve to avoid collision" [Garett 1990, Kennedy 1990].

An extensive review to identify currently followed friction requirements was conducted by Henry [2000]. Neither national nor state requirements were reported; however, the existence of friction flag values was identified in most states. Although the friction flag values vary from state to state, it appears that most of them were developed based on the findings from an NCHRP study to determine the recommended minimum friction value conducted in the 1960's [Kummer and Meyer 1967]. During this study researchers analyzed a large group of factors, including driver behavior, friction level 
versus wet accidents, and friction level versus highway maintenance costs. They recommended minimum requirements for $\mathrm{BPN}$ and $\mathrm{SN}$ values (at $64 \mathrm{~km} / \mathrm{h}$ with a rib tire). In the final conclusions, the authors relate the recommended values of SN and BPN to the mean traffic speed on the investigated highways, as shown in Table 1.

Table 1. Recommended minimum friction requirements [after Kummer and Meyer 1967]

\begin{tabular}{ccc}
\hline $\begin{array}{c}\text { Mean Traffic Speed, } \\
\mathrm{km} / \mathrm{h}\end{array}$ & $\begin{array}{c}\text { Skid Number, } \\
\mathrm{SN}^{\mathrm{a}}\end{array}$ & $\begin{array}{c}\text { British Pendulum Number, } \\
\text { BPN }\end{array}$ \\
\hline 48 & 31 & 35 \\
64 & 33 & 40 \\
81 & 37 & 45 \\
97 & 41 & 50 \\
113 & 46 & --- \\
129 & 51 & --- \\
\hline
\end{tabular}

${ }^{\text {a }}$ ASTM E-274 friction trailer test conducted at $64 \mathrm{~km} / \mathrm{h}$ using rib tire

In the State of Indiana, INDOT utilized Kummer and Meyer's findings to determine the friction flag value for the trailer using a smooth tire (at $64 \mathrm{~km} / \mathrm{h}$ ). During the analysis, the recommended skid number for roads with a mean traffic speed of 81 $\mathrm{km} / \mathrm{h}$ was selected $(\mathrm{SN}=37)$ and then this recommended $\mathrm{SN}$ value was converted to the average results which could be expected with the smooth tire (instead of the rib). INDOT conducted tests and concluded that "the average friction difference for slick concrete, asphalt surface and network pavements is 18 ," when results from smooth and rib tires are compared. Then, subtracting 18 from the $\mathrm{SN}=37$ results in the flag value of 19 . Therefore INDOT recommended and follows a friction flag value of 20 (for smooth tire test conducted at $64 \mathrm{~km} / \mathrm{h}$ ) during all inventory tests. In addition, INDOT conducted other statistical analyses and concluded that such a flag value is economically reasonable for the INDOT pavement program [Li et al. 2003]. 
During the international PIARC study [Wambold et al. 1996] it was concluded that friction requirements could be developed based on IFI parameters. Once the minimum values of $\mathrm{F} 60$ and $\mathrm{S}_{\mathrm{p}}$ were determined, based on the friction/texture relationship, pavements could easily be classified in one of four groups, as: (1) good, (2) with low microtexture but with a satisfactory level of macrotexture, (3) with a satisfactory level of microtexture but with low macrotexture and (4) with both low microand macrotexture. Based on this evaluation, the appropriate maintenance action could be determined. This study did not, however, provide any suggestions with respect to the required friction values.

New Zealand is one country which was found to implement the IFI method to monitor and evaluate the highway friction level. Similar to the practice existing in Great Britain (as explained earlier), the requirements depend on the investigated section characteristics (type of traffic, geometric parameters, etc.). For each section type, investigatory and threshold levels of IFI are defined using both F60 and $S_{p}$ parameters [Yeaman 2005]. The investigatory level is defined by F60 values of 0.22 to 0.30 and $S_{p}$ values of about 76 to $95 \mathrm{~km} / \mathrm{h}$. The threshold level is defined by F60 values of 0.15 to 0.20 and $S_{p}$ values of 60 to $75 \mathrm{~km} / \mathrm{h}$.

Although airport runways are not a main subject of this study, those pavements are a good example of a complete friction policy (including testing frequency and the minimum friction requirements) [Advisory Circular 1997]. It should be noted, however, that in the case of runways the need for maintenance is not only due to friction loss resulting from surface polishing, but also due to friction loss caused by tire rubber deposits. 


\section{CHAPTER THREE: TEST PROGRAM}

The following sections describe the materials studied, experimental plan, equipment used, specimen preparation and testing techniques employed in various parts of this study.

\subsection{Materials}

Currently, a common practice for improving polishing resistance of pavements with carbonate aggregates is to substitute a portion of the carbonate aggregate with a "high friction" aggregate. During this study, mixes with different types of aggregates combined in various proportions were studied. These mixes were evaluated either during the field or laboratory part of the study, as described below.

\subsubsection{Laboratory Study}

Four types of coarse aggregate and two types of fine aggregate were used in the laboratory mix preparation. The coarse aggregates were selected based on their frictional characteristics and an attempt was made to include in this study a wide range of aggregates commonly used in the north central part of the USA. As shown in Table 2, the coarse aggregates included two friction aggregate types (called FAT): quartzite and steel slag. Quartzite, called Q, was imported from South Dakota, and steel slag, called SS, was supplied by a source located in northern Indiana. Coarse aggregates also included two carbonate aggregate types (called CAT): dolomite and limestone. In this study, dolomite from the Wabash formation (steeply inclined dolostone, called D), and two sources of limestone were used. To differentiate these two sources in this research, 
the higher quality (i.e., higher friction and polishing resistant) limestone is called "hard" limestone (HL) and the lower frictional quality limestone is called "soft" limestone (SL). The HL limestone is a "normal" limestone from the Louisville formation, and the SL limestone comes from the Salem formation; SL contains oolitic bodies, has high LA abrasion values and polishes substantially when exposed to traffic.

All the aggregate blends used in this study contained two types of fine aggregates: natural (siliceous) sand (NS) and manufactured (crushed dolomite) fine sand (MS). These two sands were used in every mix, although in varying amounts (see Table 4 in section 3.3.1.1).

Table 2. Physical properties of aggregates used in the laboratory part of the study

\begin{tabular}{|c|c|c|c|c|c|c|}
\hline & Type & & Aggregate & Symbol & $\begin{array}{c}\text { Bulk Spec. } \\
\text { Grav. }\end{array}$ & $\begin{array}{l}\text { Absorp- } \\
\text { tion [\%] }\end{array}$ \\
\hline \multirow{5}{*}{ 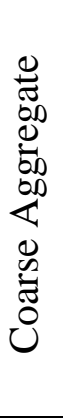 } & \multirow{2}{*}{$\begin{array}{l}\text { Polishing } \\
\text { Resistant }\end{array}$} & \multirow{2}{*}{ FAT } & Quartzite & Q & 2.63 & 0.2 \\
\hline & & & Steel Slag & SS & 3.60 & 1.1 \\
\hline & \multirow{3}{*}{ Polishable } & \multirow{3}{*}{ CAT } & Dolomite & $\mathrm{D}$ & 2.68 & 1.1 \\
\hline & & & $\begin{array}{c}\text { Hard } \\
\text { Limestone }\end{array}$ & HL & 2.63 & 1.5 \\
\hline & & & $\begin{array}{c}\text { Soft } \\
\text { Limestone }\end{array}$ & SL & 2.47 & 3.3 \\
\hline \multirow{2}{*}{\multicolumn{2}{|c|}{ Fine Aggregate }} & & Natural Sand & NS & 2.57 & 1.7 \\
\hline & & & $\begin{array}{c}\text { Manufactured } \\
\text { Sand }\end{array}$ & MS & 2.74 & 1.2 \\
\hline
\end{tabular}

Specific gravity and absorption test results (obtained according to AASHTO T 85 (2004) for coarse aggregates and AASHTO T 84 (2004) for fine aggregates) are shown in Table 2. Specific gravities of the natural aggregates ranged from 2.47 to 2.74 ; the specific gravity of the steel slag was found to be about 3.61. The quartzite had the lowest 
absorption $(0.2 \%)$ while the absorption of the soft limestone was the highest (3.3\%). Absorption of all other aggregates was between $1.1 \%$ and $1.7 \%$.

The uncompacted air void content (determined according to AASHTO T 304 (2004)) was $40.9 \%$ for the natural sand and $49.0 \%$ for the fine manufactured sand. All coarse aggregates used in this study contained more than 95\% particles with two fractured faces (tested according to ASTM D 5821 (2006)). The sand equivalent values (tested according to AASHTO T 176 (2002)) of all of the aggregates used in the study were higher than the required $45 \%$ for roadways with 3 to 10 or 10 to 30 ESAL (as per AASHTO M 323 (2004). All the coarse aggregates used in this study met the flat and elongated particles requirement (maximum 10\% of these particles present per AASHTO M 323 (2004)) with less than 5\% actually present, when tested according to ASTM D 4791 (2005).

The gradation of each of the individual types of aggregate was determined following the ASTM C 136 (2006) specification. The final gradation used in the production of the HMA mixes was obtained by blending individual fractions in proportions specified in the mix design. Each aggregate source was sieved and separated into individual size fractions so the final combined gradation could be tightly controlled.

An unmodified PG 64-22 binder was used to prepare the laboratory mixtures. The specific gravity of this binder, tested according to AASHTO T $228(2006)$ at $25^{\circ} \mathrm{C}$ $\left(77^{\circ} \mathrm{F}\right)$, was $\mathrm{P}_{\mathrm{b}}=1.028$. Although none of the other properties of the binder were tested in this study, this binder was obtained from an INDOT certified asphalt supplier and thus it was assumed that it met all Superpave requirements for a PG 64-22 grade specified in AASHTO M 320 (2005). 


\subsubsection{Field Study}

During this study, different existing road sections were investigated. The composition of the two concrete and 23 HMA mixes used in the construction of these sections varied with respect to the aggregate and binder sources and mixture proportions. The mineral components of the HMAs included steel slag, blast furnace slag, dolomite, limestone, quartzite, gravel, crushed gravel, natural and manufactured (crushed dolomite and crushed quartzite) sands, mineral fillers and cellulose. Binders used during this portion of the study included: PG 58-28, PG 64-22, PG 64-28, PG 70-22, PG 76-22, AC5, AC-10 and AC-20; the PG 76-22 was an SBS modified binder.

The concrete mixture was composed of type I portland cement, class C fly ash, fine aggregate (washed manufactured sand), coarse aggregate (dolomite), air entraining agent, water reducing admixture and potable water.

\subsection{Experimental Plan}

As mentioned in the first chapter, the main purpose of this study was to evaluate the influence of various blends of aggregates on the resulting micro- and macrotexture of HMA. In order to do this it was necessary to develop a laboratory device (and testing procedure) to accelerate the polishing of pavement surfaces and to evaluate their frictional characteristics. Based on the literature study and field measurements, the International Friction Index (IFI), IFI flag value and IFI baseline values (for the laboratory tests) were developed. The general plan of the study is shown in Figure 4. 


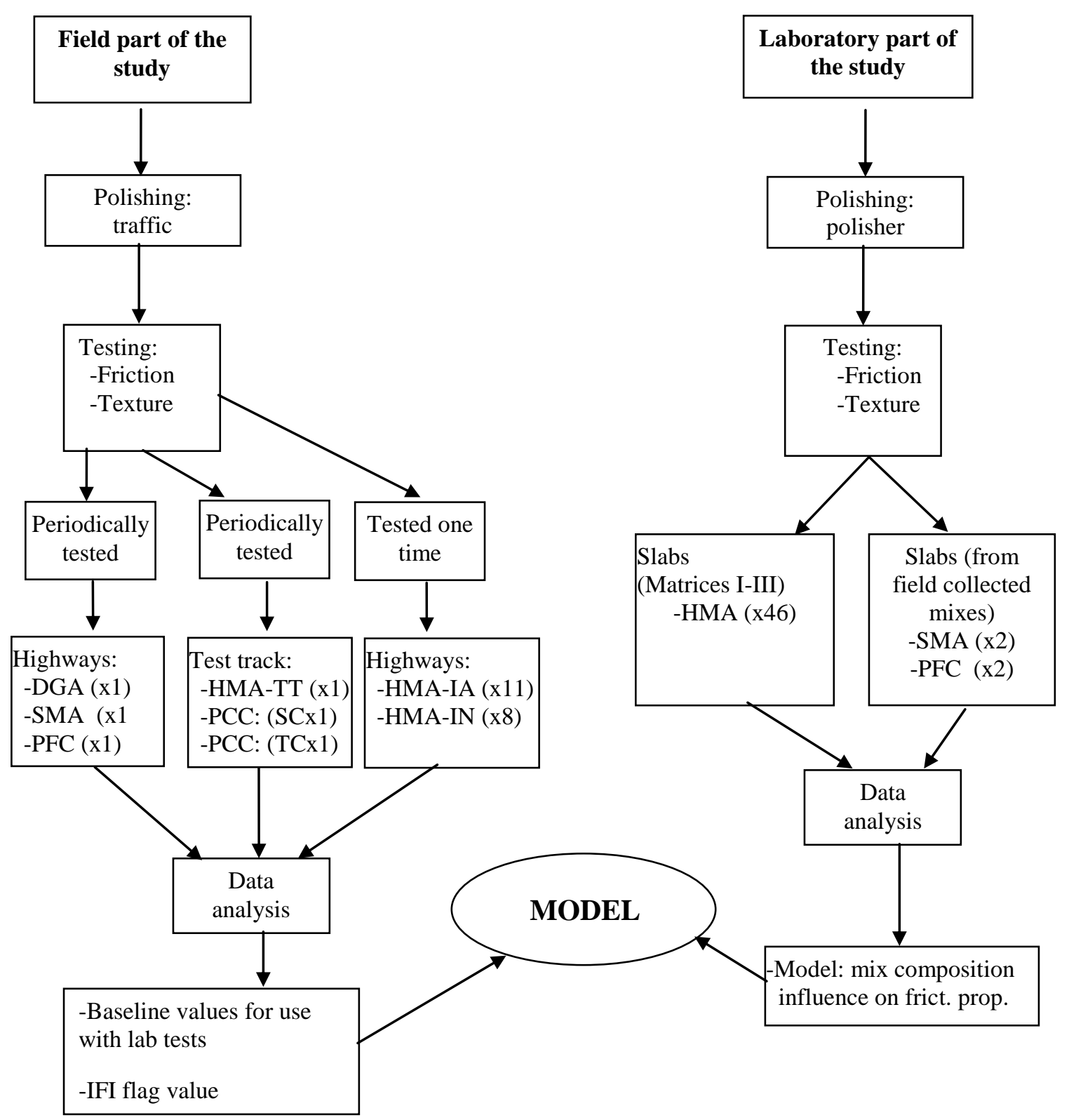

Figure 4. General plan for the field and laboratory study of the HMA mixtures

During the laboratory part of the study, conventional dense graded HMA (Superpave) mixes were fabricated and tested using slabs produced from these mixes. The following factors, potentially influencing frictional properties, were investigated:

- two high friction resistance aggregate types (FAT = Q and SS), 
- three carbonate aggregate types $(\mathrm{CAT}=\mathrm{D}, \mathrm{HL}$ and SL),

- five high friction resistance aggregate contents (called FAC; FAC $=0 \%, 10 \%$, $20 \%, 40 \%$ and $70 \%)$,

- three mixture gradations $(\mathrm{G}=\mathrm{C}, \mathrm{F}$ and $\mathrm{S})$,

- two aggregate sizes (NMAS $=9.5 \mathrm{~mm}$ and $19 \mathrm{~mm})$.

For practical reasons (due to budget and time limitations), testing of all possible combinations of materials was not possible as the full test matrix would contain 180 cells (2 FAT x 3 CAT x 5 FAC x 3 G x 2 NMAS). Therefore, a partial factorial design was implemented. Cells to be tested were selected considering the need to evaluate different combinations of the factors (FAT, CAT, FAC, G and NMAS) mentioned above.

In the adopted test program, the frictional properties of 46 laboratory prepared samples were evaluated based on three test matrices (see Figure 5). In the main matrix (Matrix I), 36 different mixes (2 FAT x 3 CAT x 3 G x 2 NMAS) were tested (refer to Table 3). All mixes contained the same friction aggregate content (FAC) equal to $20 \%$. 

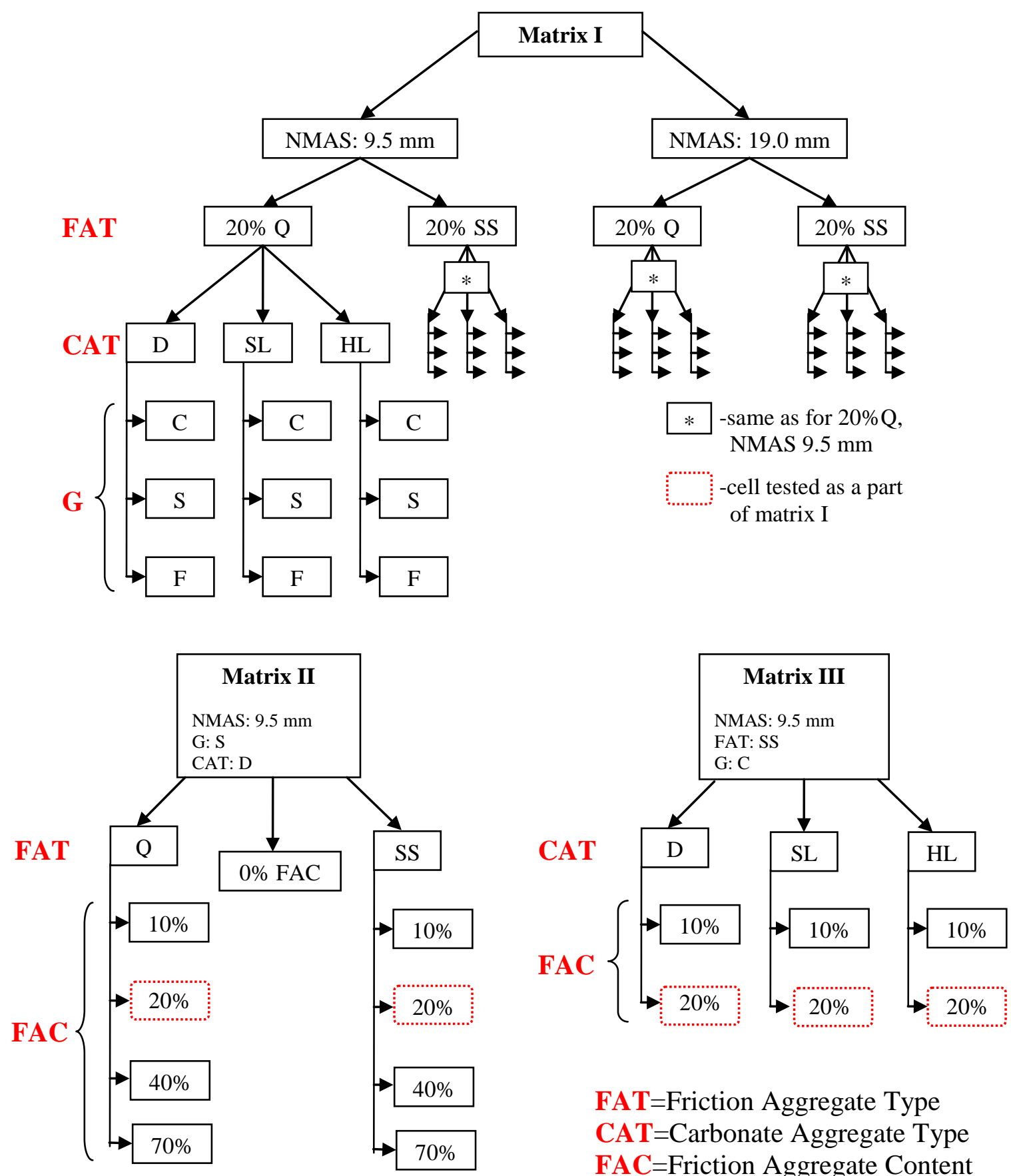

FAT=Friction Aggregate Type CAT $=$ Carbonate Aggregate Type FAC $=$ Friction Aggregate Content $\mathrm{G}=$ Gradation

Figure 5. Schematic of the experimental design: Matrices I to III

Samples included in Matrix II contained two cells previously tested in Matrix I and seven other mixes. All samples tested in Matrix II had the same CAT (D), G (S) and 
NMAS $(9.5 \mathrm{~mm})$ but differed in the FAC and FAT. Five levels of FAC $(0 \%, 10 \%, 20 \%$, $40 \%$ and $70 \%$ ) and two types of FAT (Q and SS) were studied (refer to Figure 5 and Table 3).

Table 3. Number of variables and cells compared during the laboratory tests (only laboratory mixtures are shown)

\begin{tabular}{|c|c|c|c|c|}
\hline Factor & Symbol & Matrix I & Matrix II & Matrix III \\
\hline Aggregate Size & NMAS & 2 & 1 & 1 \\
\hline Friction Aggregate Type & FAT & 2 & $2^{a}$ & 1 \\
\hline Friction Aggregate Content & FAC & 1 & $5^{\mathrm{b}}$ & $2^{\mathrm{b}}$ \\
\hline Carbonate Aggregate Type & CAT & 3 & 1 & 3 \\
\hline Gradation & G & 3 & 1 & 1 \\
\hline \multicolumn{2}{|c|}{ Total Number of Cells } & 36 & $9^{b}$ & $6^{b}$ \\
\hline
\end{tabular}

${ }^{a}$ At the FAC level of 0\%, no FAT was used.

${ }^{\mathrm{b}}$ Specimens with $\mathrm{FAC}=20 \%$ were tested in Matrix I

During the sample preparation stage, laboratory produced HMAs were compacted into wooden molds and polished in a specially developed laboratory polisher. In order to obtain the frictional resistance curve, friction and texture measurements were performed directly after sample compaction and periodically during the polishing process.

In addition to the conventional HMA mixes (shown in Figure 5), plant produced SMA and PFC mixes were also tested in the lab in the same way as the lab produced mixes. Those two mixes were collected during field construction.

Due to time and cost limitations, only one specimen (per cell) was tested in the laboratory except for the plant produced SMA and PFC mixes. Based on laboratory observation, a relationship between all the factors mentioned above (FAT, CAT, FAC, G and NMAS) was developed. Because of the number of factors being evaluated, there are many comparisons that can be drawn. The null hypothesis in each case was that the 
friction levels for different factor combinations were equal $\left(\mu_{1}=\mu_{2}=\mu_{3}=\ldots\right)$. The alternative hypothesis was that the friction levels were not equal. It was anticipated that the null hypothesis would be rejected for many of the possible comparisons.

In addition to the laboratory tests conducted in this study, field experiments were also performed. The main purpose of the field tests was to develop a baseline for the friction requirements for the laboratory measurements. The Indiana Department of Transportation (INDOT), for example, compares the skid number (SN) obtained from the ASTM towed friction trailer to a so-called friction "flag value" to identify potentially low friction pavements; a study to convert the SN-based flag value to an International Friction Index (IFI)-based flag value is reported here. The IFI-based flag value is more universal and allows for easier comparison of laboratory and field-generated friction data. During the study, mostly HMA pavements were tested in Indiana and Iowa. However, two concrete sections were also investigated. Pavements in different conditions (from one to 23 years old), subjected to different traffic levels (county roads, state roads and interstate highways) were tested. During the selection of the test sections, an attempt was made to investigate the frictional properties of pavements with low, medium and high friction values, constructed at different times and from different materials.

The second purpose of the field tests was to investigate changes in friction and texture taking place in the pavement over the time. One mix of each type (Superpave HMA, SMA and PFC) was tested periodically, about three times per year, beginning from the initial point around the time when the road was opened to the traffic for four years. 


\subsection{Sample Properties Characterization}

In this section the composition (aggregate gradation and binder content) of the laboratory and field tested mixtures is described.

\subsubsection{Laboratory Study}

\subsubsection{Aggregate Blend Gradation}

Dense graded Superpave mixes were tested during the laboratory part of this study. Three aggregate gradations $(\mathrm{G}=\mathrm{C}, \mathrm{F}$ and $\mathrm{S}))$ and two NMAS $(9.5 \mathrm{~mm}$ and 19 $\mathrm{mm}$ ) were examined. The aggregate gradations met the gradation control points as required by AASHTO M 323 (2004). In addition, they avoided the gradation restricted zone, which is not required by the current AASHTO standards but was formerly used in Superpave mix design.

Target gradations of six types of aggregate blends are shown Figures 6 and 7 . Gradation types included fine, coarse and s-shaped gradations (called 95_F, 95_C, 95_S, respectively, for aggregate with $9.5 \mathrm{~mm}$ NMAS and called 19_F, 19_C, 19_S, respectively, for aggregate with $19.0 \mathrm{~mm}$ NMAS). Mixes with such gradations are commonly used as surface layers in various parts of the USA. 


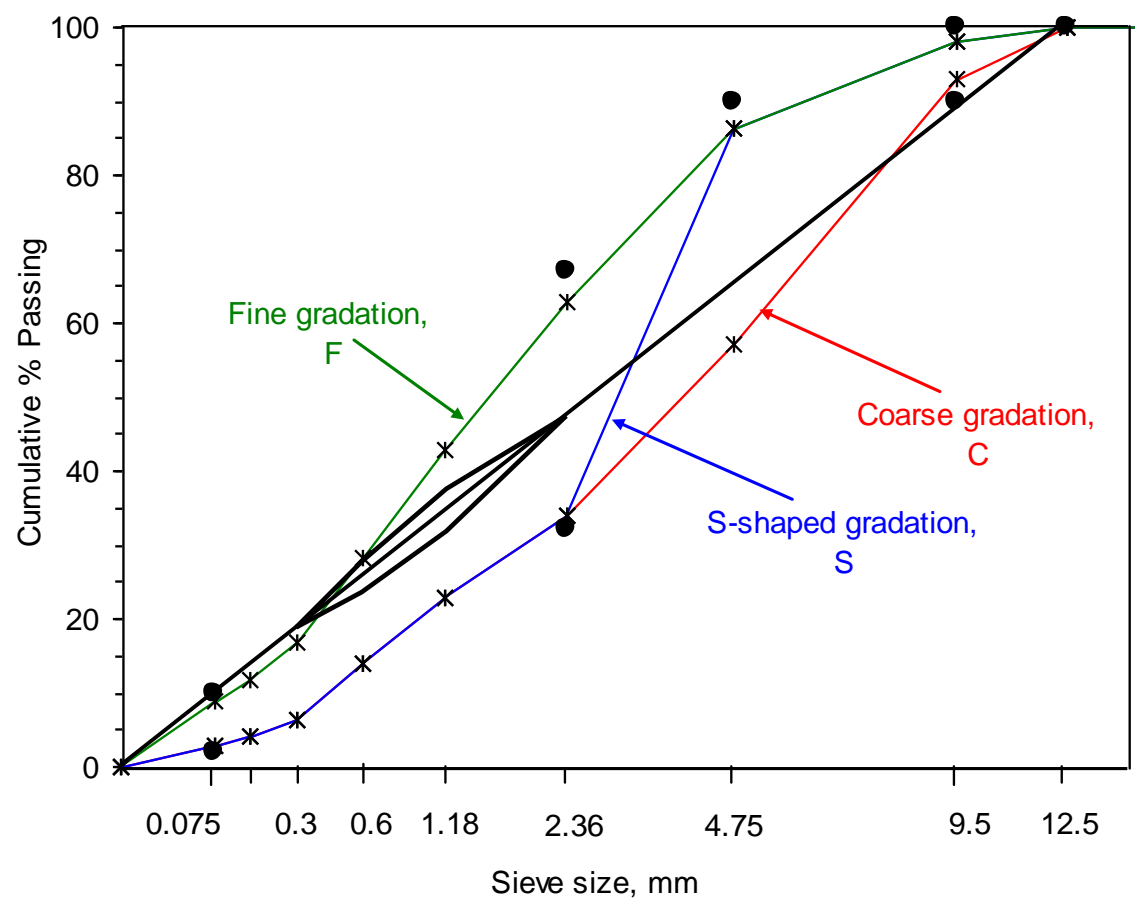

Figure 6. Aggregate gradations for mixes with $9.5 \mathrm{~mm}$ NMAS



Figure 7. Aggregate gradations for mixes with $19 \mathrm{~mm}$ NMAS 
One of the methods used to quantify and to compare aggregate gradation is the fineness modulus (FM). Generally, the fineness modulus is used in the design of portland cement concrete (PCC) mixtures to describe a weighted average of the gradation of the aggregate being analyzed. The fineness modulus is obtained by adding the sum of the cumulative percentages (by mass) of a sample aggregate retained on each of a specified series of sieves and dividing the sum by 100. The specified sieves are: No. 100 (150 $\mu \mathrm{m})$, No. $50(300 \mu \mathrm{m})$, No. $30(600 \mu \mathrm{m})$, No. $16(1.18 \mathrm{~mm})$, No. $8(2.36 \mathrm{~mm})$, and No. 4 $(4.75 \mathrm{~mm})$, and $3 / 8$ in. $(9.5 \mathrm{~mm}), 3 / 4-$ in. $(19.0 \mathrm{~mm}), 1-1 / 2$ in. $(37.5 \mathrm{~mm})$, and larger, increasing at a ratio of 2 to 1 [ASTM C 125 (2006)]. The FM calculated for mixes tested in Matrix I is shown in Table 4. For mixes with 9.5 mm NMAS the FM is smaller than for those with 19 mm NMAS. In addition, for both groups of mixes (with NMAS of 9.5 and $19 \mathrm{~mm}$ ), the smallest FM was noticed for mixes with fine type of gradation, medium for mixes with s-shaped gradation and the highest for mixes with coarse type of gradation, as expected.

For each sample evaluated in Matrix I, the aggregate blend was composed of the FAT, CAT, NS and MS in the proportions shown in Table 4. For Matrix II, only one aggregate blend (95_S) was evaluated while for Matrix III only the 95_C blend was investigated. (Steel slag was used as the friction aggregate in Matrix II because of the limited amount of imported quartzite available.) The proportioning of the blends tested in Matrices II and III was similar to the corresponding blends from Matrix I. For mixes with less than $20 \%$ FAC, part (or all) of the friction aggregate was replaced with carbonate aggregate. For mixes with more than $20 \%$ of FAC, part (or all) of the 
carbonate aggregate was replaced with friction aggregate, while keeping the gradation essentially constant (see note below Table 4).

Table 4. Fineness modulus and proportions (\% by weight) of aggregate blends used in Matrix II

\begin{tabular}{|c|c|c|c|c|c|c|}
\hline NMAS & & $9.5 \mathrm{ml}$ & & & $19 \mathrm{mr}$ & \\
\hline Gradation & Coarse & Fine & S-shaped & Coarse & Fine & S-shaped \\
\hline Symbol & 95_C & 95_F & 95_S & 19_C & 19_F & 19_S \\
\hline FAT $^{*}$ & 20 & 20 & 20 & 20 & 20 & 20 \\
\hline CAT $^{*}$ & 51 & 20 & 51 & 59 & 35 & 59 \\
\hline NS & 17 & 10 & 17 & 10 & 8 & 10 \\
\hline MS & 12 & 50 & 12 & 11 & 37 & 11 \\
\hline FM & 4.68 & 3.53 & 4.34 & 5.56 & 4.38 & 5.27 \\
\hline
\end{tabular}

*Note: actual proportions of FAT and CAT aggregates on a given sieve size varied slightly from the overall target value shown above due to the limited availability of certain fractions in the aggregates used (refer to page 56).

The final gradations of the six different blends used in the study were shown previously in Figure 6 for the $9.5 \mathrm{~mm}$ NMAS mixtures and in Figure 7 for $19 \mathrm{~mm}$ NMAS mixtures. The gradations of individual fractions used to prepare those blends are shown in Figures 8 to 13 . 


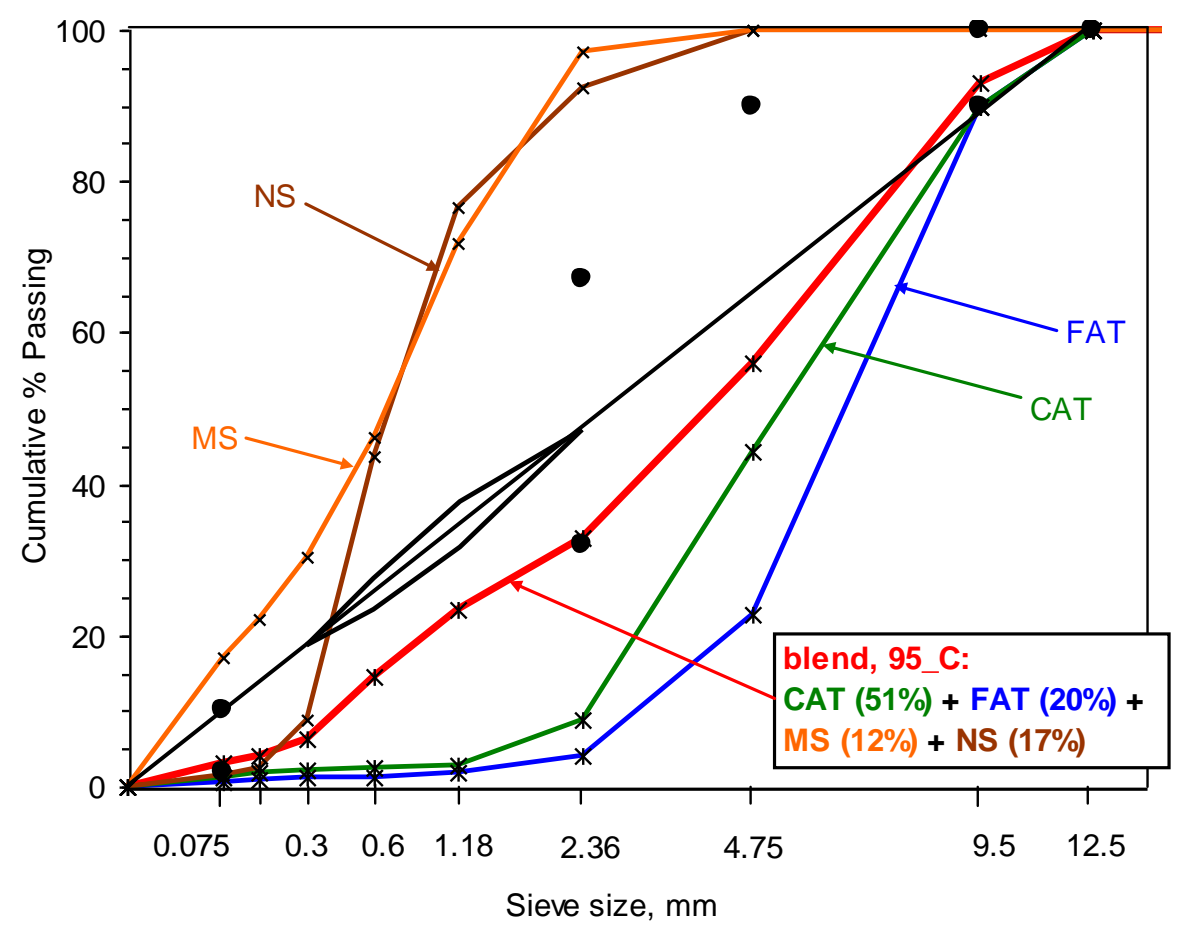

Figure 8. Blend composition for mixes with 9.5 mm NMAS and coarse gradation

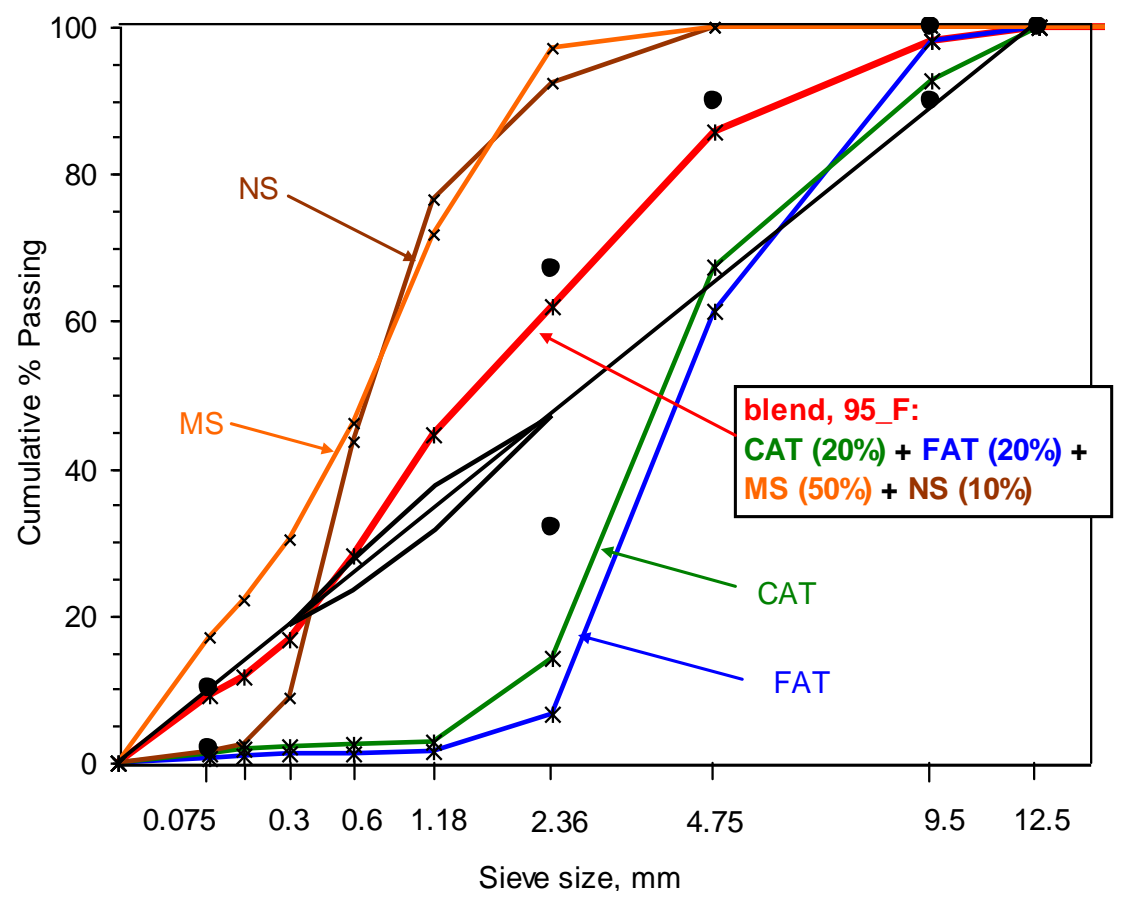

Figure 9. Blend composition for mixes with $9.5 \mathrm{~mm}$ NMAS and fine gradation 


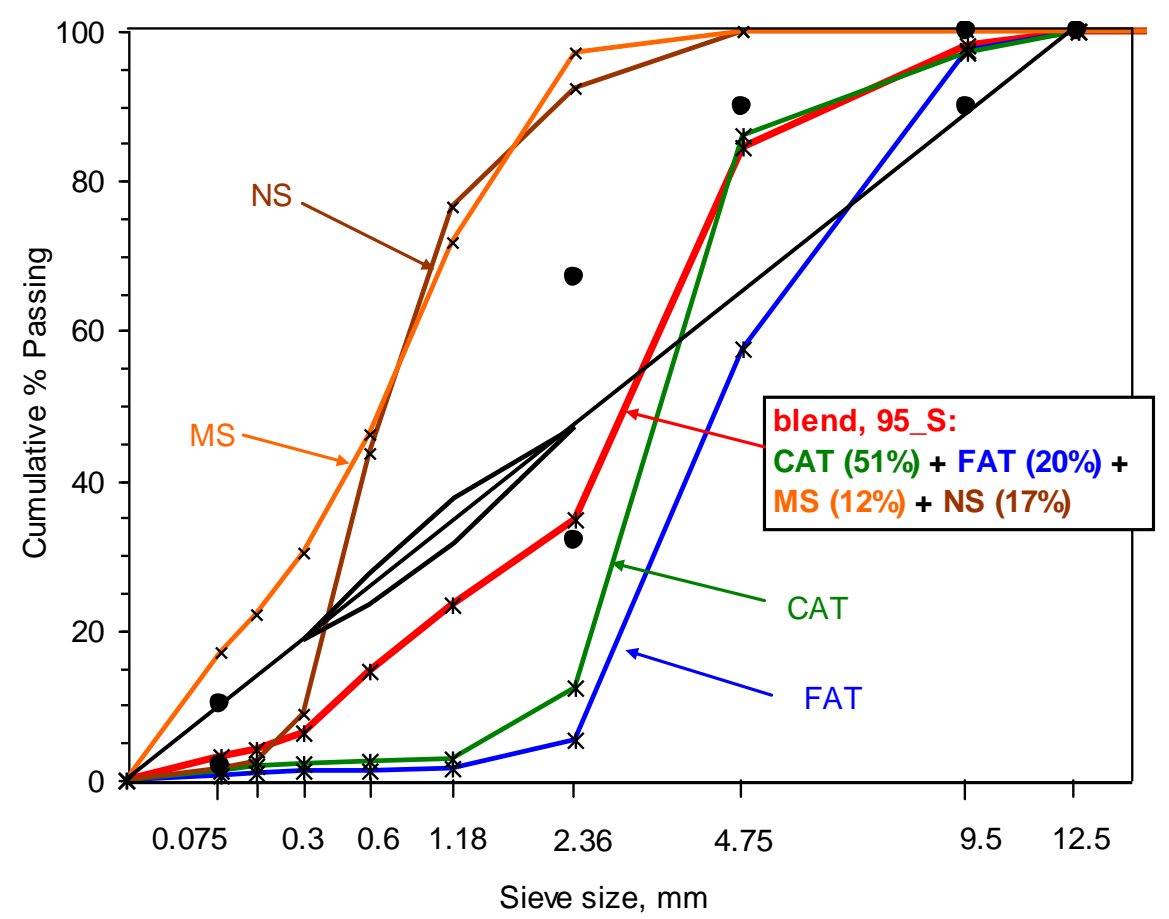

Figure 10. Blend composition for mixes with $9.5 \mathrm{~mm}$ NMAS and s-shaped gradation

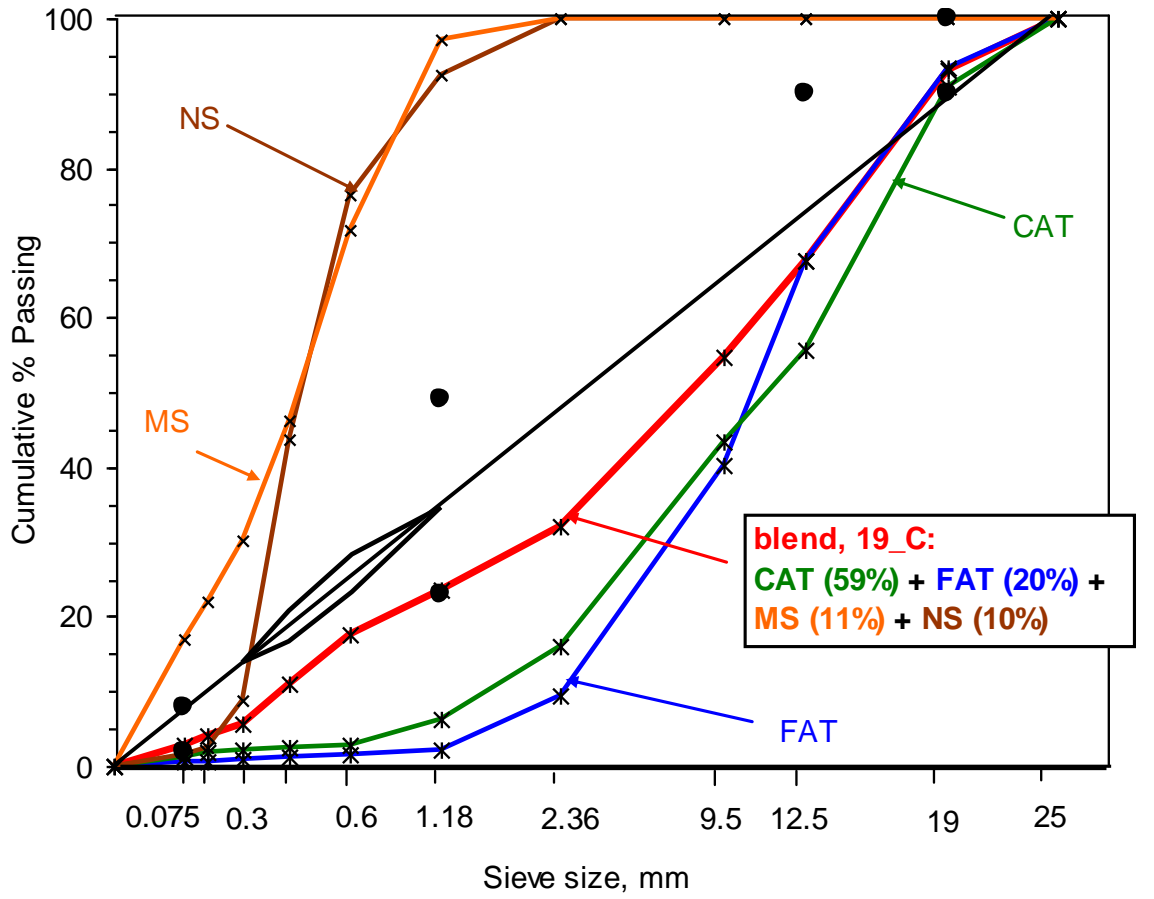

Figure 11. Blend composition for mixes with $19 \mathrm{~mm}$ NMAS and coarse gradation 


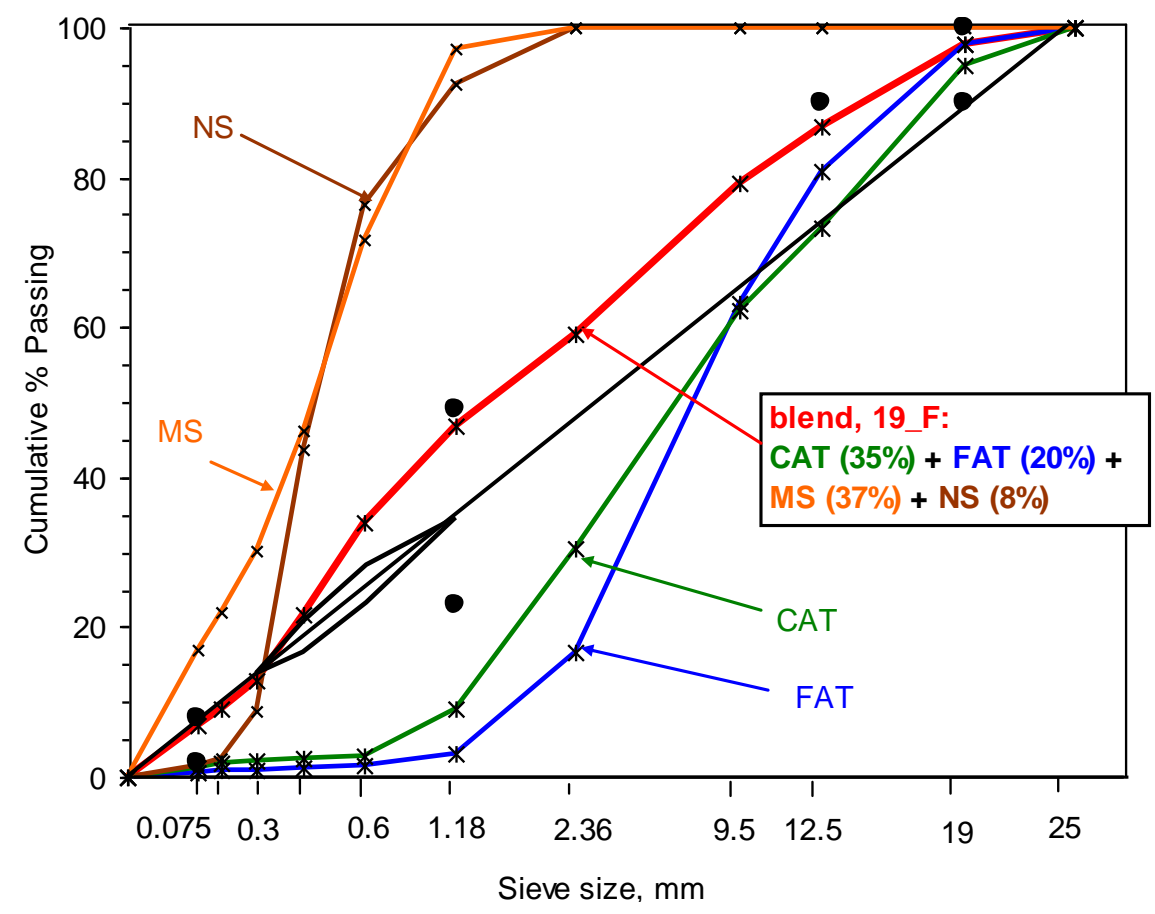

Figure 12. Blend composition for mixes with 19 mm NMAS and fine gradation

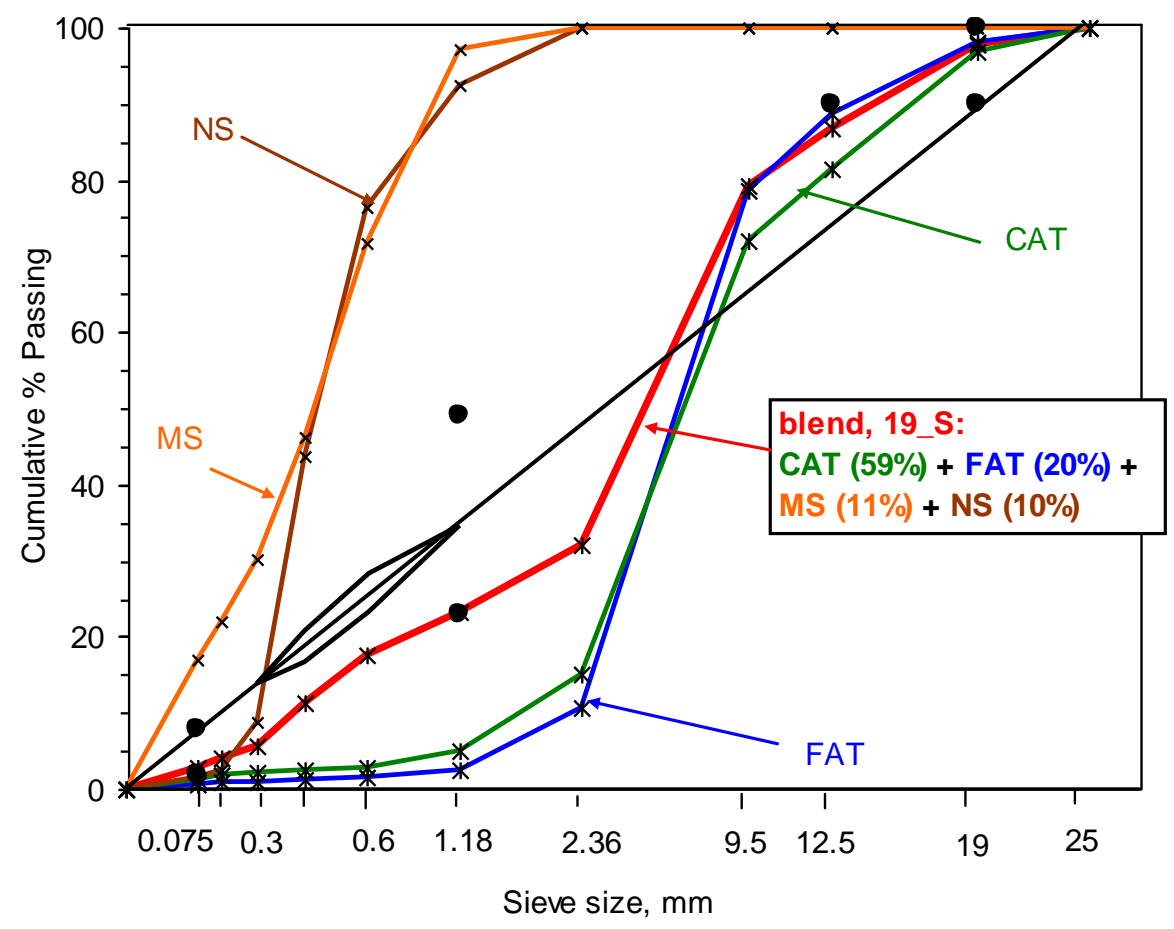

Figure 13. Blend composition for mixes with $19 \mathrm{~mm}$ NMAS and s-shaped gradation 
The following constraints were taken into account when preparing the aggregate blend for a given test cell:

- The final blend should not differ from the target gradation by more than $\pm 2.5 \%$ (when checked on each sieve size),

- The final blend's FM should not differ from the target gradation FM by more than \pm 0.025 ,

- For each of the target gradations (used to prepare six different mixes), the maximum difference in the amount of specific FAT fraction should not be higher than 5\% (when checked on each sieve size),

- For each of the target gradations (used to prepare six different mixes), the maximum difference in the amount of specific CAT fraction should not be higher than 5\% (when checked on each sieve size).

\subsubsection{Binder Content Determination}

The main criterion in designing the mixes used in this study was the air void content in the compacted sample. All other parameters (voids in the mineral aggregate (VMA), voids filled with asphalt (VFA) and dust-to-binder ratio $\left.\left(\mathrm{P}_{0.075} / \mathrm{P}_{\text {be }}\right)\right)$ were also determined but they were not included as mix design factors (refer to Appendix C, Table $\mathrm{C} 1$ for detailed information). Because of the need to tightly control the aggregate gradations and blends (above) it was not feasible to also control all the other, usual design parameters.

The design binder content for each mix was that which provided $4 \%$ air voids $\left(\mathrm{V}_{\mathrm{a}}\right)$ in the mix compacted in the Superpave Gyratory Compactor (SGC). Compaction 
effort was determined based on the anticipated (over a 20-year period) traffic level of 330 million ESAL (design Equivalent Standard Axle Loads). Many two-lane, multilane divided, and partially or completely controlled access roadways have design traffic levels in this range. These types of roadways may include medium to high traffic city streets, many state routes, U.S. highways, and some rural Interstates. Following AASHTO M 323 (2004) and AASHTO R 35 (2004), the number of SGC gyrations corresponding to the 3-30 million ESAL traffic level is $100\left(\mathrm{~N}_{\text {design }}=100\right)$. The binder contents for specimens tested in Matrices I-III are shown in Tables 5 through 7, respectively.

Table 5. Matrix I: binder content of mixes (\%)

\begin{tabular}{cccccccc}
\hline \multicolumn{2}{c}{ NMAS } & \multicolumn{3}{c}{$9.5 \mathrm{~mm}$} & \multicolumn{3}{c}{$19 \mathrm{~mm}$} \\
\hline \multicolumn{2}{c}{ Gradation } & Coarse & Fine & S-shaped & Coarse & Fine & S-shaped \\
\hline \multirow{2}{*}{ FAT } & SS & 5.7 & 5.4 & 7.0 & 4.5 & 4.2 & 5.4 \\
& Q & 5.9 & 4.9 & 7.0 & 4.9 & 4.0 & 5.3 \\
\hline
\end{tabular}

Note: each cell contains three different mixes, each with addition of different carbonate aggregate type (CAT): D, HL and SL. FAC was equal to $20 \%$.

Table 6. Matrix II: binder content of mixes (\%)

\begin{tabular}{ccc}
\hline \multirow{2}{*}{ FAC $[\%]$} & \multicolumn{2}{c}{ FAT } \\
\cline { 2 - 3 } & $\mathrm{Q}$ & $\mathrm{SS}$ \\
\hline 0 & $*$ & $*$ \\
10 & 7.3 & 7.3 \\
20 & 7.0 & 7.0 \\
40 & 7.0 & 6.5 \\
70 & 6.8 & 6.1 \\
\hline
\end{tabular}

* Binder content $7.7 \%, \mathrm{FAC}=0 \%$. 
Table 7. Matrix III: binder content of mixes (\%)

\begin{tabular}{cccc}
\hline \multirow{2}{*}{ FAC $[\%]$} & \multicolumn{4}{c}{ CAT } \\
\cline { 2 - 4 } & D & HL & SL \\
\hline 10 & 5.9 & 5.9 & 5.9 \\
20 & 5.7 & 5.7 & 5.7 \\
\hline
\end{tabular}

\subsubsection{Field Study}

Existing pavement sections constructed from four types of mixes were tested in the field part of this study: These existing PCC concrete, dense graded HMAs (both Superpave and Marshall designed mixes), PFC and SMA mixes were designed by contractors or the states in which they were located.. For detailed information refer to Appendix C, Table C 1. All the HMAs (except PFC) were designed for the same air void content.

Three field sections that are closed to public traffic were tested periodically in this study to investigate changes in friction and texture taking place in the pavement over the time without traffic. Three other field sections tested were on public roads and therefore show changes in frictional properties over the time and traffic. The three sections without traffic were located on the INDOT test track: these were HMA, called HMA-TT (for test track); tined concrete (TC) and smooth concrete (SC). The other three sections were located on an interstate highway and a U.S. road; these were conventional dense graded Superpave asphalt (DGA), SMA and PFC. 
Aggregate blend gradations of the asphalt mixes tested periodically in this study are shown in Figure 14 and fineness modulus, material type, quantity and volumetric data are shown in Table 8. The value of fineness modulus was the highest for the PFC (5.54), intermediate for the SMA (4.82) and the lowest for the HMA-TT and DGA (4.43 and 4.28, respectively). Steel slag aggregate was used in the DGA, SMA and PFC (though combined in different proportions with various other aggregates and additives), while blast furnace slag was used in the HMA-TT. SBS-modified PG 76-22 binder, from two different sources, was used in all four mixes. The binder content by weight appears to be fairly similar for all of the four mixes: $6.3 \%$ for HMA-TT, 5.5\% for SMA and 5.7\% for PFC and DGA. However, the binder content by volume was much higher for the SMA and PFC due to the quantity of dense steel slag used in these mixes.

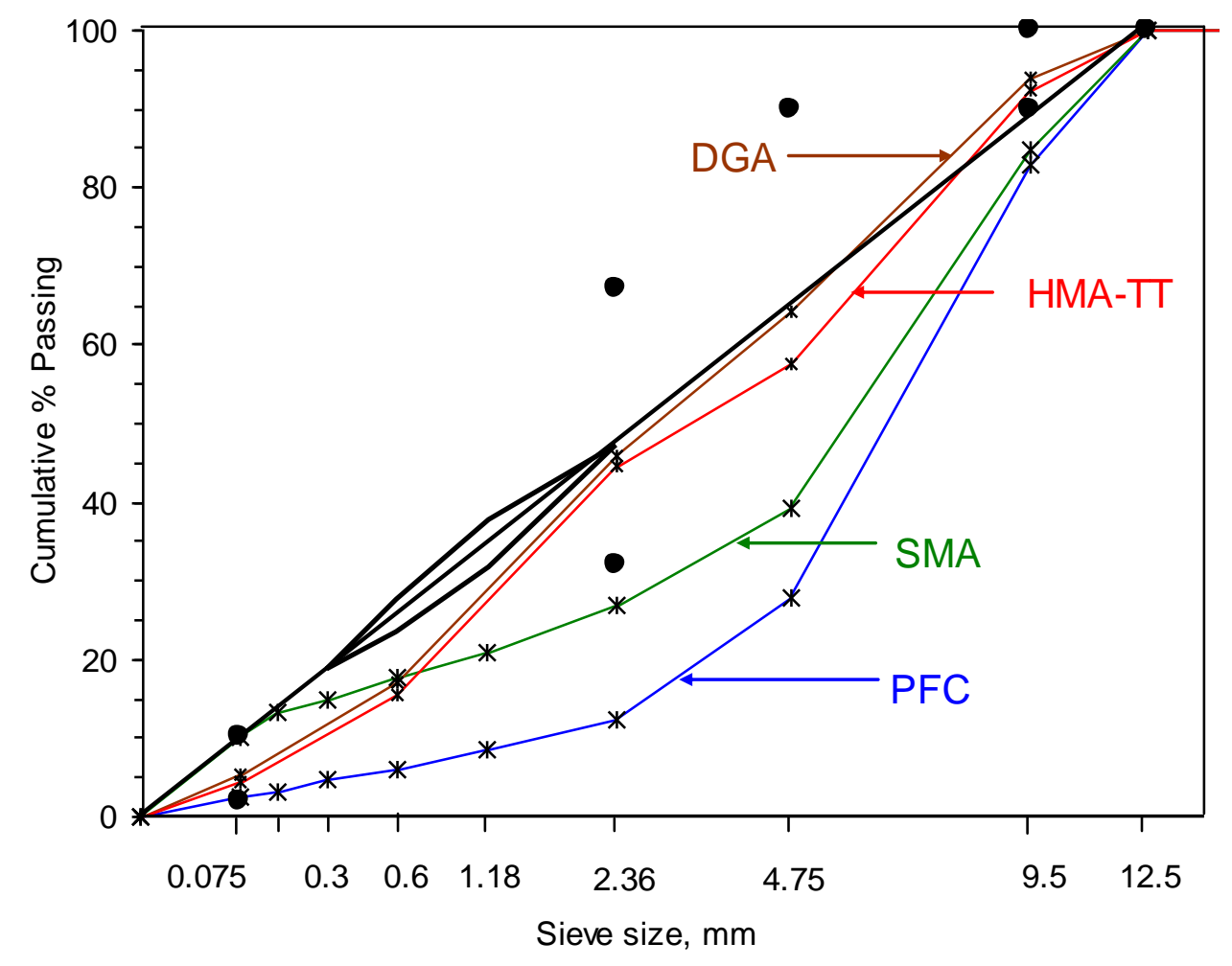

Figure 14. Gradation of HMA-TT, DGA, PFC and SMA sections tested periodically 
The same concrete mixture was used during construction of both of the concrete test sections (TC and SC). The only difference between those two sections was in the concrete finishing method. Detailed information about the concrete mixture proportions is not available, however the concrete composition was typical for those used in pavement construction in Indiana: cement $\left(260-285 \mathrm{~kg} / \mathrm{m}^{3}\right)$, class $\mathrm{C}$ fly ash $(58-70$ $\mathrm{kg} / \mathrm{m}^{3}$ ), water to cement ratio about 0.42 , fine aggregate about $35-50 \%$ of total weight of aggregates, air entraining agent in the amount needed to obtain about $6.5 \%$ air and water reducing admixture.

Table 8. Material type, quantity and volumetric data of asphalt sections tested periodically

\begin{tabular}{lcccc}
\hline Section / Mixture & DGA & HMA-TT & SMA & PFC \\
\hline Natural Sand, \% & \multicolumn{1}{c}{10} & & \\
$\begin{array}{l}\text { Manufactured Sand } \\
\text { (Dolomite), \% }\end{array}$ & 10 & 35 & 10 & 10 \\
Dolomite, \% & 40 & 28 & & \\
Blast Furnace Slag, \% & & 27 & & \\
Steel Slag, \% & 50 & & 80 & 90 \\
Mineral Filler, \% & & & 10 & \\
\hline Total, \% & 100 & 100 & 100 & 100 \\
\hline FM & 4.28 & 4.43 & 4.82 & 5.54 \\
\hline $\mathrm{G}_{\mathrm{sb}}$ & 2.95 & 2.64 & 3.48 & 3.57 \\
$\mathrm{G}_{\mathrm{mm}}$ & 2.75 & 2.45 & 3.15 & 3.19 \\
\hline Binder Type & PG 76-22 & PG 76-22 & PG 76-22 & PG 76-22 \\
Binder Content (\%) & 5.7 & 6.3 & 5.5 & 5.7 \\
\hline
\end{tabular}


Material type, quantity and volumetric data of the field asphalt sections tested only once are shown in Tables 9 and 11 (for sections located in Indiana and in Iowa, respectively). The binder content for those mixes varied from $4.3 \%$ to $6.8 \%$. Note that sections HM-IN-6, HM-IN-7 and HM-IN-8 were constructed from the same mixture.

The fineness moduli and blend gradations of sections tested once are shown in Tables 10 and 12 (for sections located in Indiana and in Iowa, respectively). Mixes with three different NMAS $(9.5,12.5$ and $19 \mathrm{~mm})$ were used. The FM of mixes HM-IN varied between 4.19 and 4.58, while the FM of mixes HM-IA varied between 3.48 and 5.30. 
Table 9. Material type, quantity and volumetric data for Indiana sections tested one time

\begin{tabular}{lcccccccc}
\hline \multirow{2}{*}{ Section / Mixture } & HM- & HM- & HM- & HM- & HM- & HM- & HM- & HM- \\
& IN-1 & IN-2 & IN-3 & IN-4 & IN-5 & IN-6 & IN-7 & IN-8 \\
\hline \multirow{2}{*}{ Natural Sand, \% } & 9 & 11 & 25 & & & 20 & 20 & 20
\end{tabular}

Manufactured Sand

(Limestone), \%

$23 \quad 18$

Manufactured Sand

(Dolomite), \%

$\begin{array}{lllll}40 & 31 & 11 & 11 & 11\end{array}$

Manufactured Sand

(Gravel), \%

$12 \quad 12 \quad 12$

Limestone, \%

$\begin{array}{lll}53 & 46 \quad 34\end{array}$

Dolomite, \%

$30 \quad 27$

Gravel, \%

16

Crushed Gravel, \%

Blast Furnace Slag, $\%$

$30 \quad 27$

\begin{tabular}{lcccccccc} 
RAP, \% & 15 & 25 & 25 & & 15 & 15 & 15 & 15 \\
\hline Total, \% & 100 & 100 & 100 & 100 & 100 & 100 & 100 & 100 \\
\hline $\mathrm{G}_{\mathrm{sb}}$ & 2.70 & 2.70 & 2.66 & 2.57 & 2.58 & 2.68 & 2.68 & 2.68 \\
$\mathrm{G}_{\mathrm{mm}}$ & 2.53 & 2.52 & 2.49 & 2.45 & 2.49 & 2.49 & 2.49 & 2.49 \\
\hline Binder Type & PG & PG & PG & PG & PG & PG & PG & PG \\
Binder Content (\%) & $70-22$ & $58-28$ & $58-28$ & $62-28$ & $62-28$ & $64-22$ & $64-22$ & $64-22$ \\
& 5.0 & 5.0 & 5.2 & 6.8 & 6.7 & 5.4 & 5.4 & 5.4
\end{tabular}


Table 10. Fineness modulus and aggregate gradation (percent passing per sieve size) for Indiana sections tested one time

\begin{tabular}{|c|c|c|c|c|c|c|c|c|c|}
\hline \multicolumn{2}{|c|}{$\begin{array}{l}\text { Section / } \\
\text { Mixture }\end{array}$} & $\begin{array}{l}\text { HM- } \\
\text { IN-1 }\end{array}$ & $\begin{array}{l}\text { HM- } \\
\text { IN-2 }\end{array}$ & $\begin{array}{l}\text { HM- } \\
\text { IN-3 }\end{array}$ & $\begin{array}{l}\text { HM- } \\
\text { IN-4 }\end{array}$ & $\begin{array}{l}\text { HM- } \\
\text { IN-5 }\end{array}$ & $\begin{array}{l}\text { HM- } \\
\text { IN-6 }\end{array}$ & $\begin{array}{l}\text { HM- } \\
\text { IN-7 }\end{array}$ & $\begin{array}{l}\text { HM- } \\
\text { IN-8 }\end{array}$ \\
\hline \multicolumn{2}{|c|}{ NMAS, mm } & 9.5 & 9.5 & 9.5 & 9.5 & 12.5 & 9.5 & 9.5 & 9.5 \\
\hline \multicolumn{2}{|l|}{$\mathrm{FM}$} & 4.30 & 4.31 & 4.19 & 4.51 & 4.58 & 4.30 & 4.30 & 4.30 \\
\hline \multirow{11}{*}{  } & 25 & 100 & 100 & 100 & 100 & 100 & 100 & 100 & 100 \\
\hline & 19 & 100 & 100 & 100 & 100 & 100 & 100 & 100 & 100 \\
\hline & 12.5 & 100 & 100 & 100 & 100 & 100 & 100 & 100 & 100 \\
\hline & 9.5 & 93 & 93 & 98 & 91 & 89 & 93 & 93 & 93 \\
\hline & 4.75 & 60 & 59 & 73 & 50 & 50 & 62 & 62 & 62 \\
\hline & 2.36 & 48 & 47 & 44 & 41 & 39 & 45 & 45 & 45 \\
\hline & 1.16 & 31 & 31 & 30 & 29 & 27 & 32 & 32 & 32 \\
\hline & 0.6 & 21 & 20 & 20 & 19 & 18 & 19 & 19 & 19 \\
\hline & 0.3 & 12 & 12 & 10 & 12 & 11 & 12 & 12 & 12 \\
\hline & 0.15 & 6.5 & 7.3 & 6.8 & 7.5 & 6.7 & 7.4 & 7.4 & 7.4 \\
\hline & 0.075 & 4.5 & 5.2 & 4.9 & 5.0 & 4.1 & 5.5 & 5.5 & 5.5 \\
\hline
\end{tabular}


Table 11. Material type, quantity and volumetric data for Iowa sections tested one time

\begin{tabular}{|c|c|c|c|c|c|c|c|c|c|c|c|}
\hline Section / Mixture & $\begin{array}{l}\text { HM- } \\
\text { IA-1 }\end{array}$ & $\begin{array}{l}\text { HM- } \\
\text { IA-2 }\end{array}$ & $\begin{array}{l}\text { HM- } \\
\text { IA-3 }\end{array}$ & $\begin{array}{l}\text { HM- } \\
\text { IA-4 }\end{array}$ & $\begin{array}{l}\text { HM- } \\
\text { IA-5 }\end{array}$ & $\begin{array}{l}\text { HM- } \\
\text { IA-6 }\end{array}$ & $\begin{array}{l}\text { HM- } \\
\text { IA-7 }\end{array}$ & $\begin{array}{l}\text { HM- } \\
\text { IA-8 }\end{array}$ & $\begin{array}{l}\text { HM- } \\
\text { IA-9 }\end{array}$ & $\begin{array}{l}\text { HM- } \\
\text { IA-10 }\end{array}$ & $\begin{array}{l}\text { HM- } \\
\text { IA-11 }\end{array}$ \\
\hline Natural Sand, \% & 40 & 20 & 25 & 22 & & 15 & 25 & 25 & 35 & 15 & 25 \\
\hline $\begin{array}{l}\text { Manufactured Sand } \\
\text { (Limestone), \% }\end{array}$ & 10 & 10 & 17 & & & & & & 45 & & 20 \\
\hline $\begin{array}{l}\text { Manufactured Sand } \\
\text { (Quartzite), } \%\end{array}$ & & & & & & & 5 & 10 & & & \\
\hline Limestone, \% & 45 & 30 & 58 & 43 & 25 & 30 & & & & 53 & 40 \\
\hline Limestone Chips, $\%$ & 5 & & & & 45 & & & & 20 & & \\
\hline Quartzite, \% & & & & & & & & & & 32 & 15 \\
\hline Gravel, \% & & & & & 30 & 10 & 25 & 20 & & & \\
\hline Crushed Gravel, \% & & 40 & & & & 45 & 45 & 45 & & & \\
\hline RAP, $\%$ & & & & 35 & & & & & & & \\
\hline Total, \% & 100 & 100 & 100 & 100 & 100 & 100 & 100 & 100 & 100 & 100 & 100 \\
\hline $\mathrm{G}_{\mathrm{sb}}$ & 2.62 & $*$ & 2.61 & $*$ & $*$ & $*$ & $*$ & $*$ & $*$ & $*$ & $*$ \\
\hline $\mathrm{G}_{\mathrm{mm}}$ & 2.43 & $*$ & 2.43 & $*$ & $*$ & $*$ & $*$ & $*$ & $*$ & $*$ & $*$ \\
\hline Binder Type & $\begin{array}{c}\text { PG } \\
64-22\end{array}$ & $\begin{array}{l}\text { AC- } \\
10\end{array}$ & $\begin{array}{c}\mathrm{PG} \\
58-28\end{array}$ & $\begin{array}{c}\mathrm{AC}- \\
5\end{array}$ & $*$ & $\begin{array}{c}\text { AC- } \\
10\end{array}$ & $\begin{array}{c}\text { AC- } \\
10\end{array}$ & $\begin{array}{c}\text { AC- } \\
10\end{array}$ & $\begin{array}{c}\text { AC- } \\
10\end{array}$ & $\begin{array}{l}\text { AC- } \\
20\end{array}$ & $\begin{array}{c}\mathrm{PG} \\
58-28\end{array}$ \\
\hline Binder Content (\%) & 6.2 & 5.0 & 5.8 & 5.5 & 4.9 & 4.3 & 5.8 & 5.2 & 6.2 & 5.1 & $*$ \\
\hline
\end{tabular}

* Data not available. 
Table 12. Fineness modulus and aggregate gradation (percent passing per sieve size) for Iowa sections tested one time

\begin{tabular}{|c|c|c|c|c|c|c|c|c|c|c|c|c|}
\hline \multicolumn{2}{|c|}{ Section / Mixture } & $\begin{array}{l}\text { HM- } \\
\text { IA-1 }\end{array}$ & $\begin{array}{l}\text { HM- } \\
\text { IA-2 }\end{array}$ & $\begin{array}{l}\text { HM- } \\
\text { IA-3 }\end{array}$ & $\begin{array}{l}\text { HM- } \\
\text { IA-4 }\end{array}$ & $\begin{array}{l}\text { HM- } \\
\text { IA-5 }\end{array}$ & $\begin{array}{l}\text { HM- } \\
\text { IA-6 }\end{array}$ & $\begin{array}{l}\text { HM- } \\
\text { IA-7 }\end{array}$ & $\begin{array}{l}\text { HM- } \\
\text { IA-8 }\end{array}$ & $\begin{array}{l}\text { HM- } \\
\text { IA-9 }\end{array}$ & $\begin{array}{l}\text { HM- } \\
\text { IA-10 }\end{array}$ & $\begin{array}{l}\text { HM- } \\
\text { IA-11 }\end{array}$ \\
\hline \multicolumn{2}{|c|}{ NMAS, mm } & 12.5 & 19 & 12.5 & 12.5 & 19 & 19 & 19 & 19 & 9.5 & 19 & 12.5 \\
\hline \multicolumn{2}{|l|}{ FM } & 4.16 & 4.76 & 4.36 & 3.88 & 4.62 & 4.91 & 4.31 & 4.49 & 3.48 & 5.30 & 4.24 \\
\hline \multirow{11}{*}{ 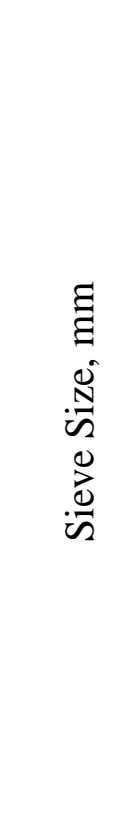 } & 25 & 100 & 100 & 100 & 100 & 100 & 100 & 100 & 100 & 100 & 100 & 100 \\
\hline & 19 & 100 & 100 & 100 & 100 & 100 & 100 & 100 & 100 & 100 & 100 & 100 \\
\hline & 12.5 & 97 & 88 & 95 & 95 & 88 & 84 & 89 & 86 & 100 & 88 & 91 \\
\hline & 9.5 & 87 & 70 & 84 & 86 & 74 & 65 & 74 & 70 & 100 & 69 & 77 \\
\hline & 4.75 & 65 & 51 & 63 & 69 & 55 & 45 & 61 & 56 & 83 & 39 & 65 \\
\hline & 2.36 & 52 & 40 & 45 & 59 & 41 & 35 & 52 & 48 & 66 & 24 & 52 \\
\hline & 1.16 & 39 & 29 & 33 & 43 & 31 & 26 & 38 & 37 & 48 & 16 & 37 \\
\hline & 0.6 & 25 & 19 & 22 & 31 & 20 & 19 & 24 & 23 & 30 & 11 & 25 \\
\hline & 0.3 & 11 & 9.1 & 11 & 16 & 10 & 12 & 13 & 11 & 16 & 6.7 & 13 \\
\hline & 0.15 & 4.6 & 6.1 & 5.9 & 8.4 & 7 & 6.9 & 7.4 & 5.7 & 9.4 & 4.6 & 6.7 \\
\hline & 0.075 & 3.7 & 4.4 & 4.3 & 7.0 & 5.1 & 4.2 & 4.8 & 3.8 & 6.5 & 3.6 & 4.1 \\
\hline
\end{tabular}

\subsection{Equipment and Testing Procedures Used in the Friction Study}

This section summarizes the specimen preparation method, equipment and test procedures used during the friction study

\subsubsection{Laboratory Friction Study}

\subsubsection{Specimen Preparation}

The experimental design for the friction study included collecting and processing relatively large amount of materials. The HMA used in this study was prepared in about 270 mix batches. The amount of the HMA used for slab preparation was about $1600 \mathrm{~kg}$ 
and for the mix design about $500 \mathrm{~kg}$. About $150 \mathrm{~kg}$ of binder was used in the laboratory part of the study. All mixes were produced from laboratory batched aggregates; about $1950 \mathrm{~kg}$ of aggregates was mixed in order to prepare the HMAs, however the amount of the aggregate collected and sieved was around $5,000 \mathrm{~kg}$.

During the process of laboratory mix preparation, all the aggregate was oven dried at $105^{\circ} \mathrm{C}$ and cooled to room temperature prior to being sieved and sorted into individual size fractions. Then the aggregate was batched. Prior to mixing, the batched aggregate blends and binder were heated to a mixing temperature of $150^{\circ} \mathrm{C}$. The mixing was performed in a five-gallon "bucket type" laboratory mixer, which was first primed with a "butter" mixture in order to avoid binder loss during preparation of the test specimens. Each slab required about $24 \mathrm{~kg}$ or $44 \mathrm{~kg}$ of mix, depending on the NMAS, which determined the slab thickness. Due to this large sample size, several mix batches (of about $6 \mathrm{~kg}$ of mix) were required to produce each slab. After mixing, the HMA samples from separate batches were combined and placed in an oven. Next, the mix was conditioned for two hours at the compaction temperature $\left(145^{\circ} \mathrm{C}\right)$ according to the AASHTO R 30 (2002) standard. The mixes were cooled and stored in closed buckets until slab preparation time.

In addition to the laboratory produced mixes, plant produced SMA and PFC mixes (collected during the construction of field sections) were also stored in closed buckets for about three years until slab preparation time. It should be noted that due to the difference in the binder used in those two mixes (SBS modified PG 76-22 instead of unmodified PG 64-22 used in laboratory produced HMAs), a higher compaction temperature was selected $\left(150^{\circ} \mathrm{C}\right)$. 
At the time of slab fabrication, mixes were reheated to the compaction temperature and compacted into square wooden molds with dimensions (width and length) of 508 x $508 \mathrm{~mm}$. The $19 \mathrm{~mm}$ NMAS mixes were compacted into $64 \mathrm{~mm}$ thick molds, and the $9.5 \mathrm{~mm}$ NMAS mixes were compacted into $38 \mathrm{~mm}$ thick molds. Plant produced mixes (SMA and PFC) were compacted into both types of molds (64 and $38 \mathrm{~mm}$ ). For all mixtures (except the PFC) the amount of mix needed to be compacted was calculated based on the mold volume and HMA bulk specific gravity $\left(\mathrm{G}_{\mathrm{mb}}\right)$ corresponding to $7 \%$ air voids (or corresponding to $\mathrm{V}_{\mathrm{a}}=22 \%$ for PFC).

It is recognized that laboratory compaction methods produce specimens different than samples extracted from field pavement sections; moreover, various laboratory compaction methods (commonly used during the mix design process) produce specimens that could vary in properties (they mostly differ by air void content) [Abo-Qudais and Qudah 2007, Iwama et al. 2007]. A specially developed laboratory compaction procedure was adopted to prepare the specimens in this study. To compact the mixes, a static "rolling pin" (with dimensions of 76 x $28 \mathrm{~cm}$ (length x diameter)) attached to a fork lift was used. To prevent the HMA mixture from losing heat, the roller was first warmed using two infrared lamps to a temperature of $75^{\circ} \mathrm{C}$ (see Figure $15 \mathrm{a}$ ). The total load of the roller and frame of the forklift applied to the sample was approximately $4.4 \mathrm{kN}$. Compaction was accomplished in two perpendicular directions in three stages, as shown in Figure $15 \mathrm{~b}$ to d. First (pre-densification), mix was placed into the mold and tamped with a spatula to prevent loss of material during initial compaction. Next (initial compaction stage), the mixture was tamped with the roller (without rolling) at ten different places to densify the structure. In the third (final compaction) stage, the roller 
was used to compact the mix after a flexible $3 \mathrm{~mm}$ thick aluminum plate was placed between the sample and roller to provide a larger contact area between the surface of the slab and the roller, thus ensuring more uniform load distribution. (The bottom surface of the plate was sprayed with an anti-adhesive agent.) Typical field roller diameters are much greater than the $28 \mathrm{~cm}$ roller used in this study; the thin plate deflected under the weight of the roller, simulating a larger diameter roller. Rolling was continued (using two directional passes) until a smooth surface was obtained and no further densification was noticed. The typical compaction process took about 10-12 minutes. This process was developed though trial and error and was found to minimize loss of material and to yield an acceptable (smooth) surface profile. The procedure was intended to simulate field compaction to the extent possible. (The air void contents in the compacted slabs are discussed in sections 3.3.1.3 and 4.3.)

Views of surface texture of the eight most characteristic (in terms of the texture) specimens compacted into the wooden molds are shown in Figures B 1 through B 8 in Appendix B; mixes with three different gradations (C, S and F) and two different NMAS (9.5 $\mathrm{mm}$ and $19 \mathrm{~mm}$ ) are shown in those figures. In addition, the plant produced SMA and PFC used in the laboratory are also illustrated there. 

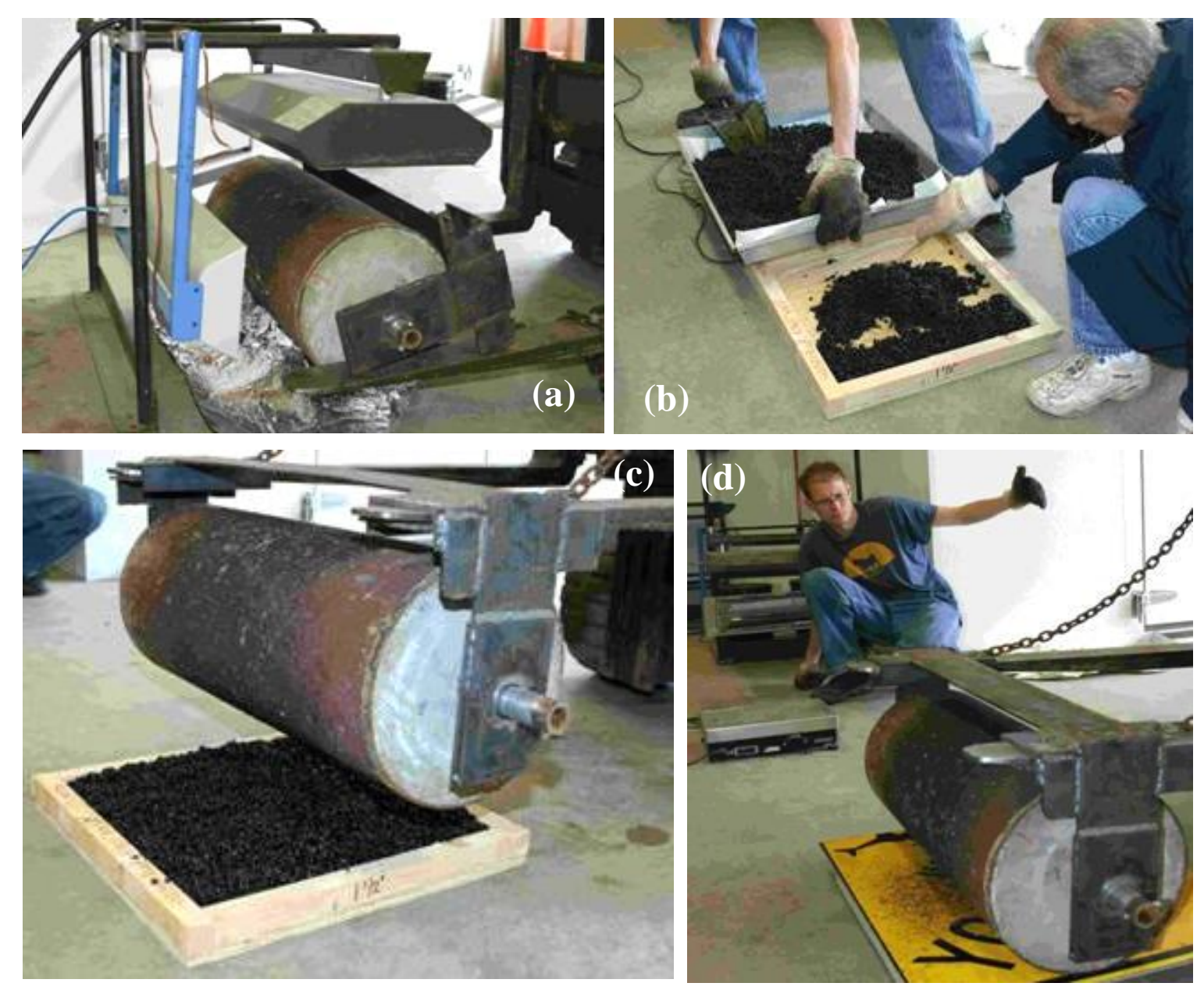

Figure 15. Stages of the friction slab compaction process: (a) preheating roller, (b) mix placing and pre-densification, (c) initial compaction and (d) final compaction

\subsubsection{Equipment and Testing}

Based on the literature review and a survey of users, as well as experience and limited data collected in an initial phase of this project, the DFT and CTM devices appeared to be the best available devices for measurement of friction and texture both in the laboratory and in the field. Therefore, these devices were selected for use in this project. In addition to these measurement devices, a specially-developed polishing 
machine was used in this study. All three devices shown in Figure 16, were designed to operate over the same circular footprint with a diameter of $284 \mathrm{~mm}$.
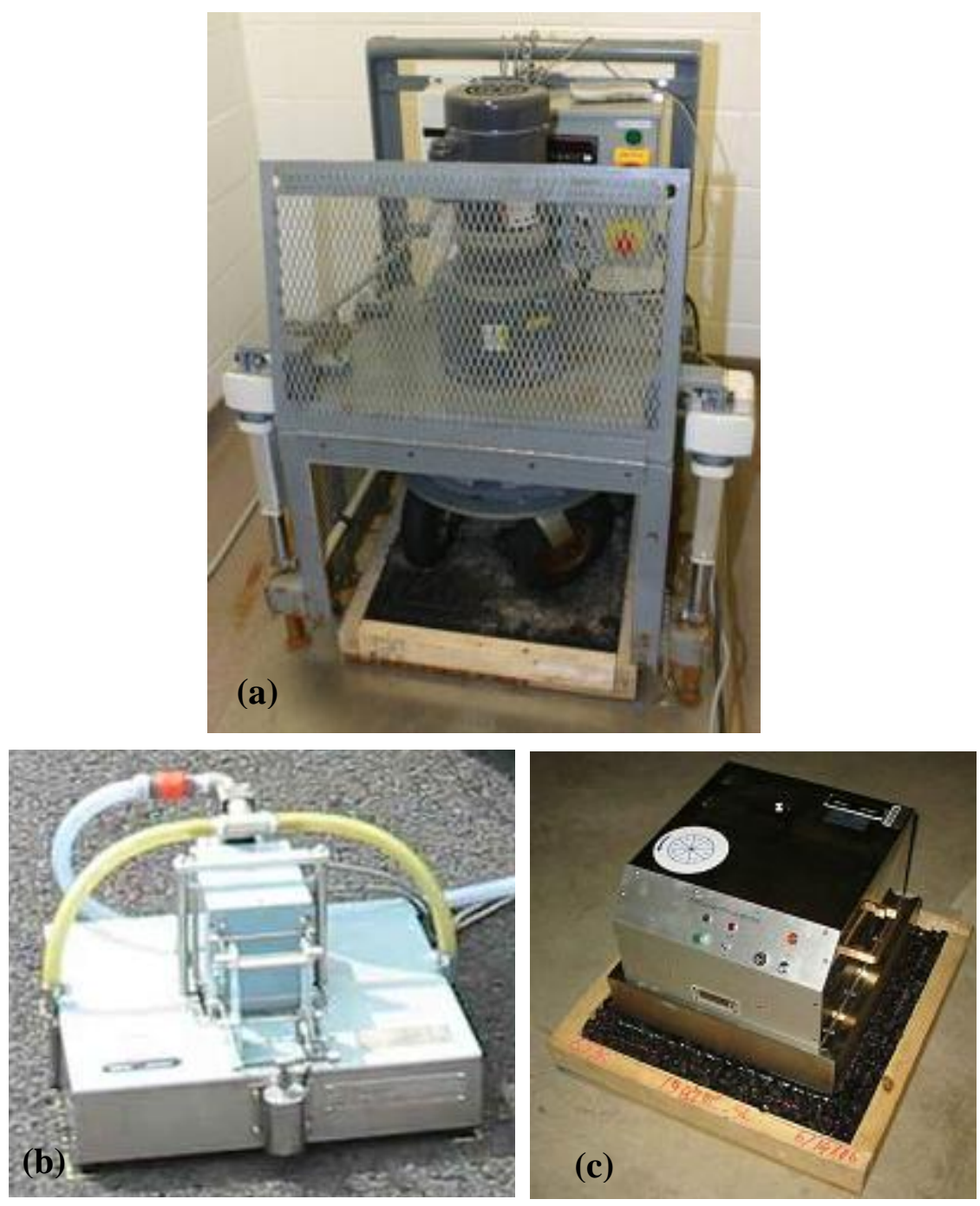

Figure 16. Polishing, friction and texture equipment: (a) CTPM, (b) DFT and (c) CTM

During the Dynamic Friction Tester (DFT) device operation, ASTM E 1911 (2002) and an operation manual [Florida DOT 2006] were followed. The DFT is a portable device consisting of a horizontal spinning disk fitted with three spring-loaded rubber sliders. The rubber sliders are located at a distance of $142 \mathrm{~mm}$ from the disk 
center, resulting in a measured surface diameter of $284 \mathrm{~mm}$ (refer to Figure 17a). The standard sliders are made of the same type of rubber used in friction test tires, though other materials are available for other applications. During a typical test with the DFT, the horizontal spinning disk is accelerated to a linear speed of $60 \mathrm{~km} / \mathrm{h}$. In addition, during the disk acceleration process, a film of water is sprayed on the measured surface. Once the designated speed is reached, the disk drops down and the rubber sliders come in contact with the surface. Due to the frictional forces generated between the sliders and surface, the disk rotational speed decreases. The torque generated by the sliders is then used to develop the friction / speed relationship. The coefficient of friction is calculated by converting the torque into the horizontal force on the sliders and dividing by the applied vertical load. Test data are collected on an attached personal computer and the wet friction number measured at a linear speed of $20 \mathrm{~km} / \mathrm{h}\left(\mathrm{DF}_{20}\right)$ is reported. The DFT measurements are affected by both the microtexture and macrotexture of the surface.

During the operation of the Circular Track Meter (CTM) device, ASTM E 2157 (2005) was followed. The CTM is a portable device allowing for macrotexture measurements. In the CTM device, a charged couple device (CCD) laser displacement sensor mounted on a rotating arm (as shown in Figure 17b) rotates around a central point at a fixed distance above the pavement and measures the change in elevation of points on the surface. The dimension of the motor driven arm allows the sensor to follow the same path as the DFT sliders. During a typical measurement, the CCD sensor rotates at a speed of 7.5 RPM and collects texture information. During the measurement, data are collected by a personal computer attached to the tester and the mean profile depth (MPD) is determined. In addition, the collected data can be separated into eight arcs. Then, if 
desired, macrotexture can be analyzed in the two arcs parallel to traffic, and two arcs perpendicular to traffic, to investigate directional effects of the texture [Nippo, web page 2006].


Figure 17. Bottom view of the friction and texture devices: (a) DFT and (b) CTM

The Circular Track Polishing Machine (CTPM) used in this study (shown in Figures 16a and

18) simulates the polishing effect of traffic and was fabricated by the Purdue Machine Shop. The main design concept was adopted from the NCAT polishing machine [Vollor and Hanson 2006], and the CTPM device is similar (in some aspects) to the NCSU Wear and Polishing Machine (ASTM E 660 (2002)). The main differences between the NCAT polisher and the CTPM device are in the form of additional features installed on the latter. Those included:

- mechanical devices to precisely align the sample in the testing spot (refer to Figure 18a), 
- electronically controlled actuators to allow easy, precise and consistent sample placement in the same testing spot (refer to Figure 18b),

- mechanical device to level the CTPM (refer to Figures 18a and b) and

- mechanical devices to control the total force applied to the sample even when samples with various thicknesses are tested (refer to Figure 18c).

The CTPM polishes the surface with three patterned pneumatic tires with a standard tire size designation of $2.80 / 2.50$ and pressure maintained at $240 \mathrm{kPa}$. Water is used to wash off abraded rubber particles from the surface during polishing as well as to cool the rubber and to simulate wet surface conditions (refer to Figure 18d). During polishing, a total load of $0.65 \mathrm{kN}$ is applied through the tires to the surface.

The drive mechanism for the vertical central shaft is an electric motor geared to rotate the shaft and wheels assembly at a speed of 47 rotations per minute (RPM) resulting in a linear tire speed of $0.7 \mathrm{~m} / \mathrm{s}$. 

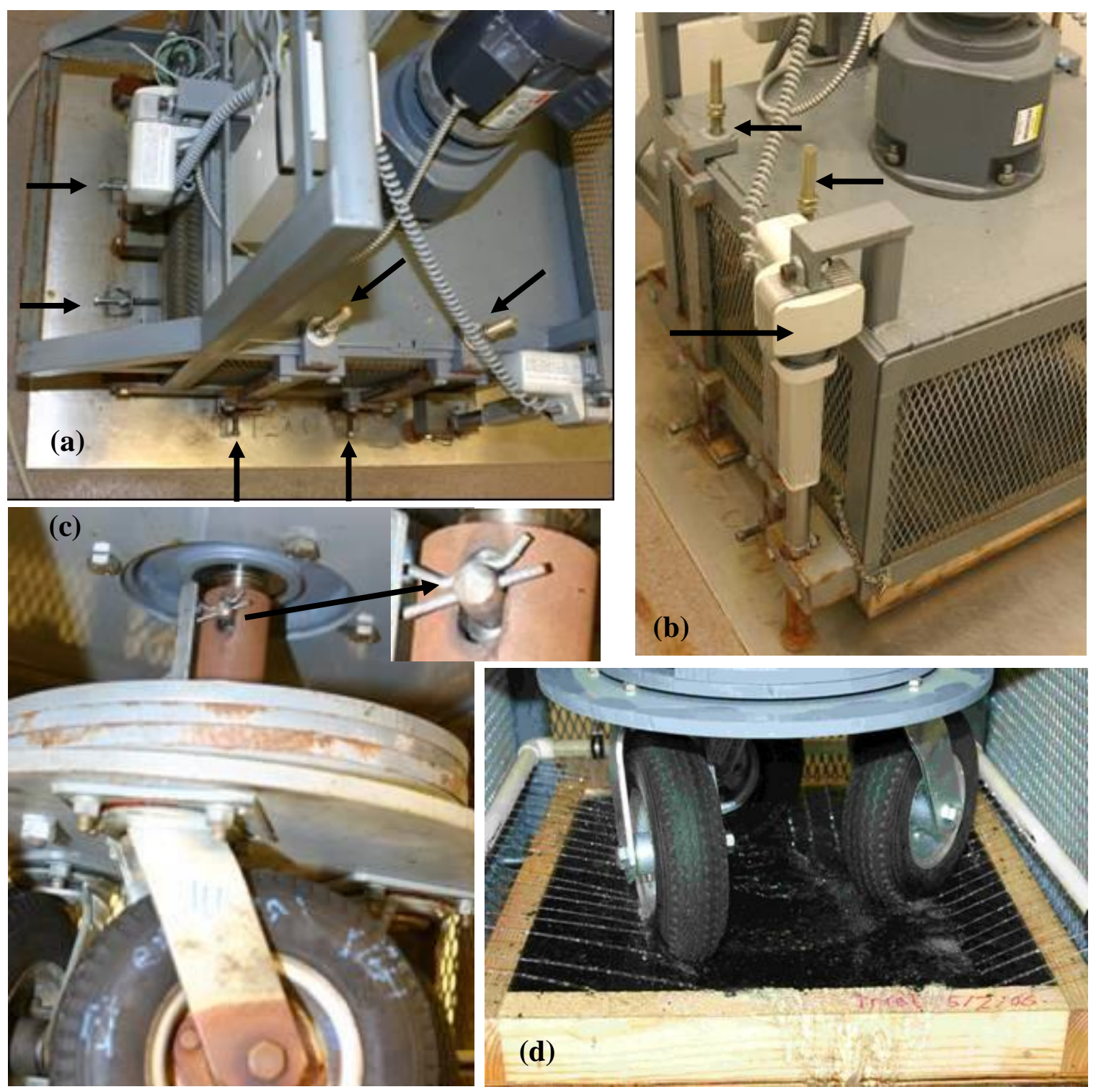

Figure 18. Details of the CTPM device: (a) alignment and level device, (b) level device and actuator, (c) force control bolt and (d) rubbers washed with water

During testing the polisher was stopped periodically so the measurement of friction and texture could be performed. It was stopped after the following cumulative number of thousands of wheel passes: $1.5,3.6,9,18,30,45,75,120,165$. Previous work at NCAT [Vollor and Hanson 2006] suggested that the friction would have reached the terminal level after about 120,000 wheel passes, so continuing to 165,000 passes was 
assumed to be sufficient; this was later verified. When the specified number of wheel passes had been applied, the specimen was removed from the polisher and allowed to dry overnight as water in the pores could affect the texture readings. Following drying, the specimen was tested for texture and friction according to ASTM E 1911 (2002) and ASTM E 2157 (2005), respectively. Each of these tests was conducted twice without moving the device. After testing, the specimen was placed back in the polisher. Alignment devices in the polisher ensured that the specimen was properly positioned so that the polishing was performed in the same path. Note: each specimen was also tested for texture and friction prior to polishing (at zero wheel passes).

Based on the average values of texture (MPD) and friction $\left(\mathrm{DF}_{20}\right)$, the IFI (F60 and $S_{p}$ ) was calculated, following the ASTM E 1960 (2007) specification.

The total cumulative time to polish a given specimen was about $19.5 \mathrm{~h}$. During polishing, the three wheels of CTPM traveled about $147 \mathrm{~km}$ (total combined distance).

It should be noted that all laboratory tests were conducted at an ambient temperature of $20 \pm 2^{\circ} \mathrm{C}$.

\subsubsection{Sample Volumetric Measurement}

As mentioned previously, the desirable air void content of the laboratory compacted slabs was $7 \%$ (and $22 \%$ for PFC). However, due to the compaction technique adopted in this project, this target could not be reached without crushing the aggregate. Loss of heat and friction between the mix and mold likely played a role. The air void content was not uniform either, i.e., the minimum air void content was in the center of the slab and increased radially with distance from the center of the specimen (refer to 
Figure 19). Based on preliminary tests and trial compactions (for the typical Superpave mixture), the air content in the center of the slab was determined to be about $9.7 \%$ and about $12.3 \%$ in the corners of the slab. In the area where polishing and testing was performed, the air void content was about $10.4 \%$, which is not considered unreasonable for the purposes of this study. In addition, the air void content likely varied from top to bottom of the slab, though this was not quantified in this study. The surface texture of the slabs appeared to be "tighter" than deeper in the slab, which is likely due to friction against the plywood bottom of the mold. Macrotexture measurements of the surfaces were compared to field measured macrotexture values to determine if the texture was reasonably representative of field conditions; see section 4.3. It also should be noted that, based on the trial tests, no densification caused by the polisher action was observed. In addition, polishing did not cause rutting in the tested samples. 


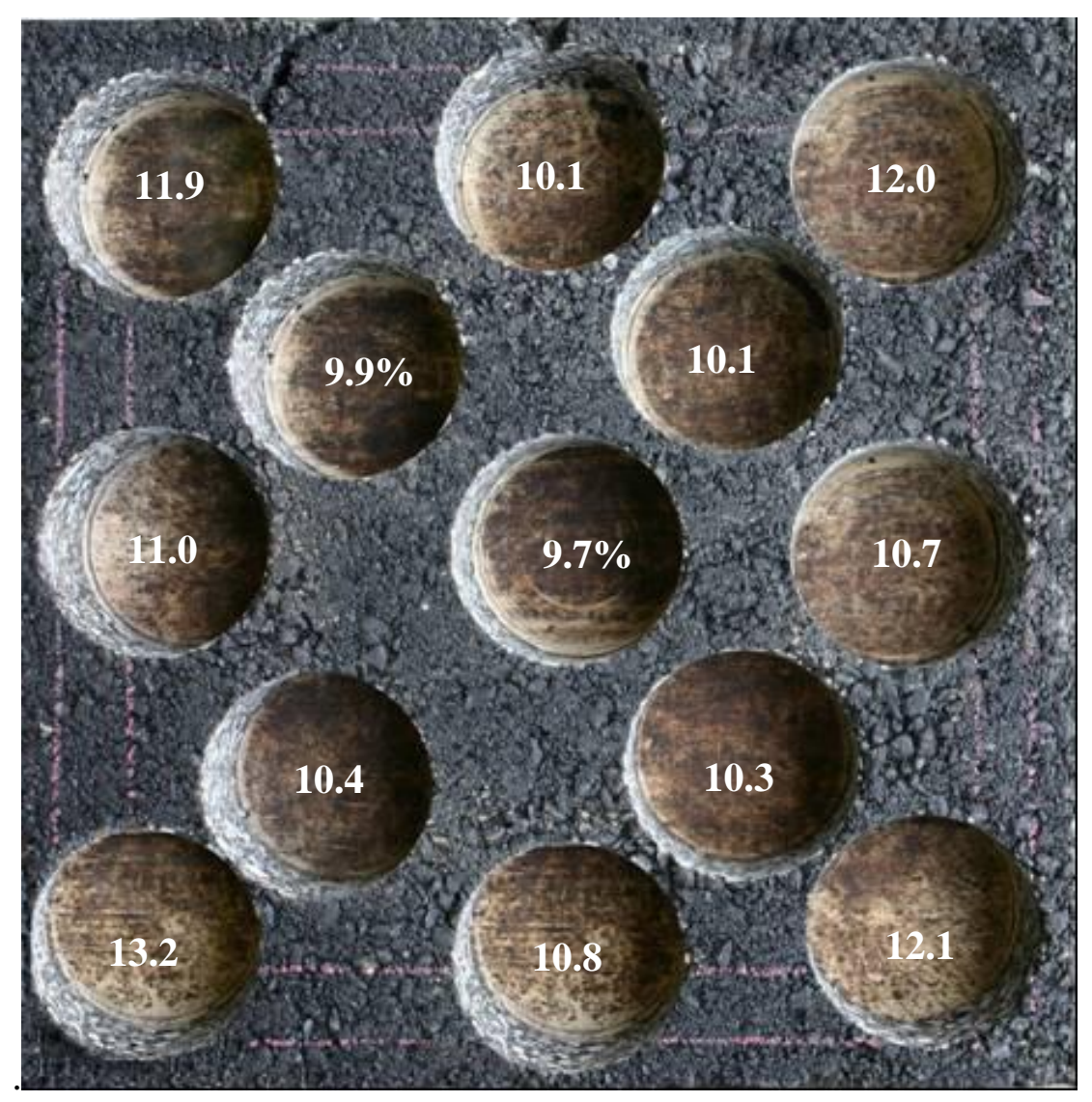

Figure 19. Air void content distribution

At the end of the laboratory part of this study, two cores were drilled from each slab. Cores with a diameter of $152 \mathrm{~mm}$ were extracted from two locations in such a way that in both cases the center of the core was positioned in the track over which the polisher, CTM and DFT devices operated. As shown in Figure 20, one core was extracted from a location on the diagonal of the slab (referred to as the "corner" core) while the other was extracted from a place located between the center of the slab and the middle of its side (referred to as the "side" core). Using those two cores, air void contents were determined for all tested specimens. 


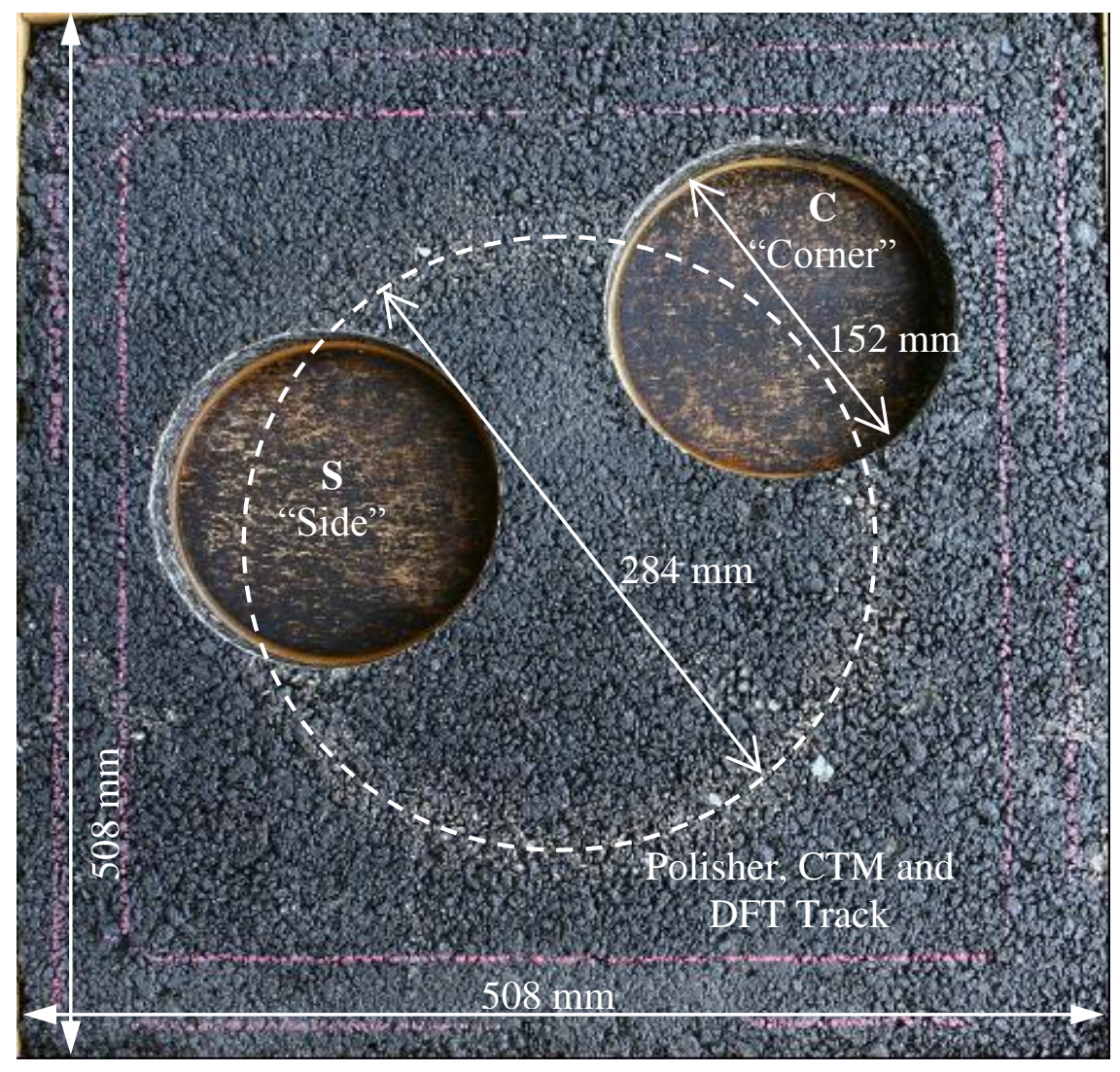

Figure 20. Location of the cores extracted from the specimen

A relatively large diameter of the core $(152 \mathrm{~mm})$ was selected to allow specimens for water permeability testing; however, this testing was later deemed to be unnecessary and was not conducted. Due to the sample internal structure (presence of open or interconnecting voids) and high probability of greater than $2.0 \%$ water absorption (by volume), AASHTO T 166 (2007), using saturated- surface dry specimens, was not applicable. ASTM D 6752 (2004), Standard Test Method for Bulk Specific Gravity and Density of Compacted Bituminous Mixtures Using Automatic Vacuum Sealing Method, was used instead, and the bulk specific gravity $\left(\mathrm{G}_{\mathrm{mb}}\right)$ was measured using the CoreLok apparatus. Based on this $\mathrm{G}_{\mathrm{mb}}$ and the previously measured $\mathrm{G}_{\mathrm{mm}}$, the air void content was calculated for each specimen. 


\subsubsection{Field Friction Study}

During field testing, the towed friction trailer, CTM/DFT and British (Pendulum) Skid Resistance Tester (BSRT) devices were used to test the same part of the pavement section. The CTM and DFT devices were positioned in the wheelpath where the towed friction trailer runs. When used, the BSRT was also placed in the same spot as the DFT and CTM.

\subsubsection{Field Section Construction}

Both the HMA and PCC field sections were constructed under the supervision of the Indiana or Iowa DOTs (depending on the location of the test section). Asphalt mixture compaction temperature, roller compactor type and number of roller passes were the same as those typically used in the states of Indiana and Iowa, respectively; however specific data are not available. In this study 22 existing sections located on public roads (interstate highways, state and U.S. roads as well as county roads) and three test sections (HMA-TT, TC and SC) located on the INDOT test track, which is closed to public traffic, were investigated.

All three sections located on the INDOT test track were long enough to allow the locked wheel friction trailer to test the surface for 1.0 second at the anticipated speed. Each section was about 40 meters long. INDOT's test track consists of three different zones: the approach zone, the test zone and the exit zone. The approach and exit zones allow the operator to adjust and maintain test speed [Li et al. 2006]. This track was 
specifically designed and used to verify the calibration and operation of INDOT's friction trailer.

Both concrete test sections (TC and SC) were constructed from the same mixture, however, they were finished in different ways. The surface of the tined concrete (TC) section was finished with transverse grooves spaced 18-20 mm apart; the grooves were around $3 \mathrm{~mm}$ wide and $3 \mathrm{~mm}$ deep. The smooth concrete section (SC, also called "slick" concrete) was finished to obtain a fine surface texture.

The PFC and SMA mixes were placed on an interstate highway as part of another study using a material transfer device (MTD) to improve ride quality and to reduce mix segregation. The MTD transferred mix from the trucks into the hopper of a conventional paver. Compaction was accomplished with two steel wheeled rollers. Only one pass with each roller was needed to seat the PFC and SMA, since relatively little compaction effort is needed to bring the coarse aggregates into contact and because over-rolling can lead to aggregate breakdown. Due to the gap-graded nature of those mixes, there is extensive stone to stone contact between the coarse aggregate particles with very little mastic or fine material to "cushion" the coarse aggregates [McDaniel et al. 2004, McDaniel and Thornton 2005]. Other sections were placed using typical construction equipment and operations.

Before opening to traffic, the air void content was determined for the PFC and SMA sections (following ASTM D 3203 (2005)) to be $22.5 \%$ for the PFC and $7-8 \%$ for the SMA. The air content in six cores extracted from the PFC section varied from $22.0 \%$ to $24.9 \%$. No information is available about the air void content in other field test 
sections, however the quality of all sections was approved by either the Indiana or Iowa DOT's.

Views of the surface texture of the pavement sections tested periodically in this study are provided in Figures B 9 through B 11 in Appendix B.

\subsubsection{Equipment}

Various types of testing equipment were used in the field part of the study; the previously described portable DFT and CTM devices and ASTM E 274 (2006) locked wheel friction trailers were used throughout. In addition, for a limited number of tests, a British (Pendulum) Skid Resistance Tester (BSRT) was also used.

During a typical measurement, the friction trailer (shown in Figure 21) is towed at a constant speed over the tested pavement. When the test is initiated, water is sprayed ahead of the tire so the wet pavement friction can be determined (refer to Figure 22). The wheel is fully locked, and the resulting torque is recorded. Based on the measured torque (converted to the horizontal force) and dynamic vertical load on the test wheel, the wet coefficient of friction between the test tire and pavement surface can be calculated. The skid number (SN) is then reported as the coefficient of friction multiplied by 100 . It should be noted that the same speed should be maintained before the test and when the wheel is locked. 

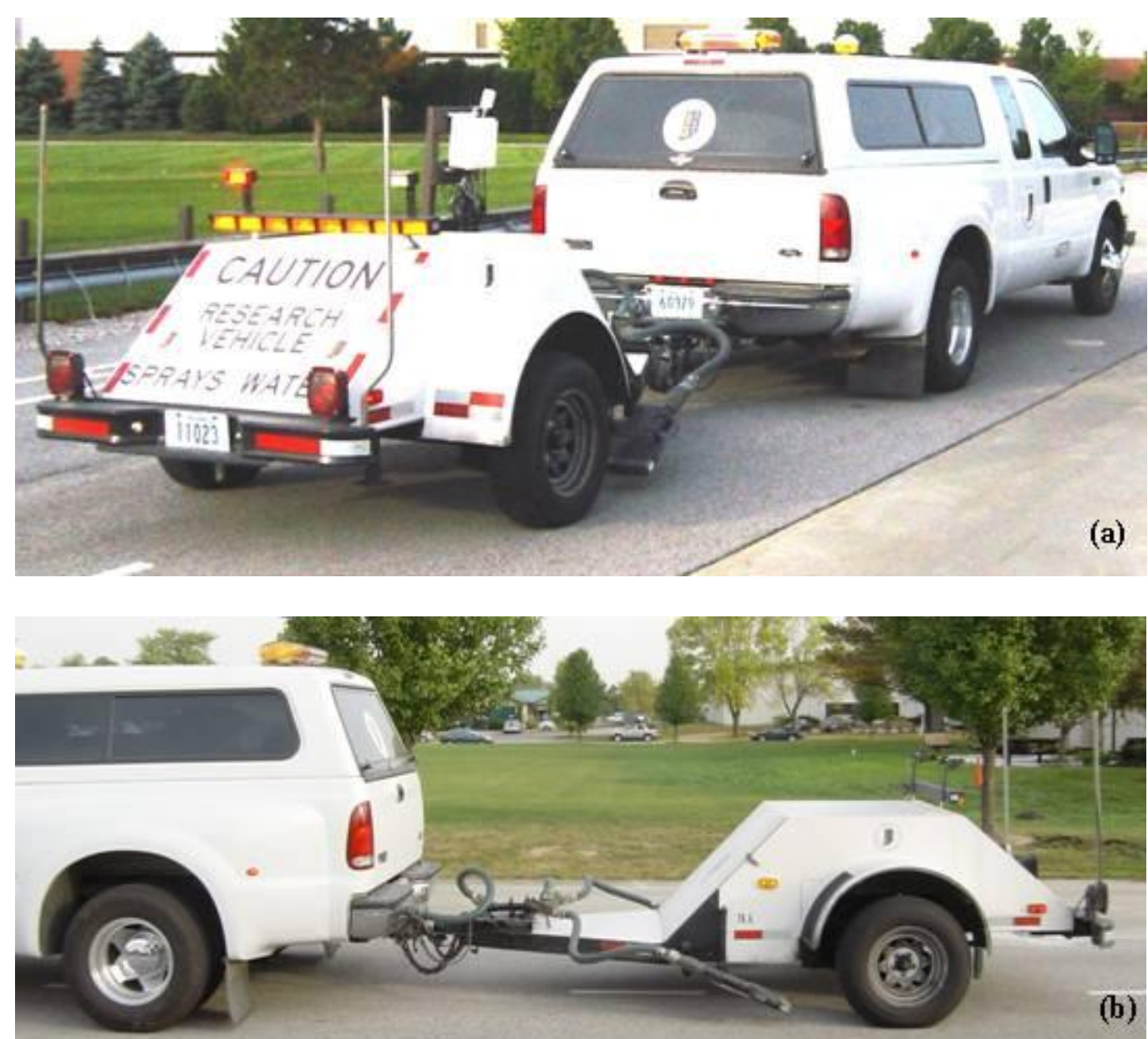

Figure 21. ASTM E-274 towed friction trailer: (a) oblique and (b) side views
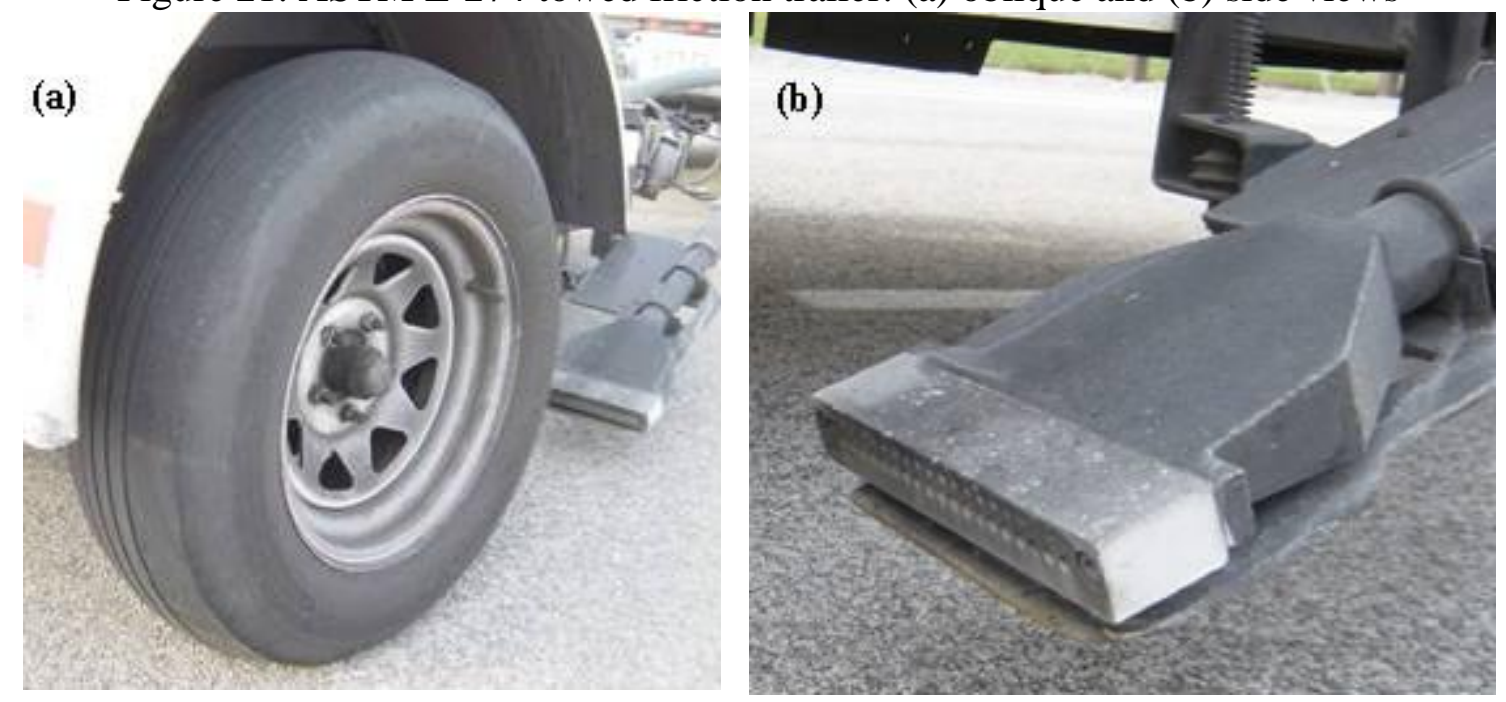

Figure 22. ASTM E-274 towed friction trailer, water nozzle system: (a) general view and (b) water nozzle detail 
As shown in Figure 23, the friction trailer used in Indiana is typically equipped with two types of tires: ASTM E 501 (2006) rib tire (on the right side) and ASTM E 524 (2006) smooth tire (on the left side).

Following the recommendations of the ASTM E274 (2006) specification, the test speed $(48,64$ or $85 \mathrm{~km} / \mathrm{h}$ ) and type of tire (rib (R) and smooth (S)) are stated when the obtained skid number ( $\mathrm{SN})$ is reported. The typical reporting format is as follows: $\mathrm{SN}$ (speed in SI units)tire type. As an example, $\mathrm{SN}(48) \mathrm{S}$ indicates that the test was performed at a speed of $48 \mathrm{~km} / \mathrm{h}$ with the smooth type of tire. (When the speed is reported in miles per hour, the parentheses around the speed are not used, thus SN30S signifies the test was conducted with the smooth tire at $30 \mathrm{mph}$.) During tests, five measurements (as required by the ASTM E274 (2006) specification) were conducted. 

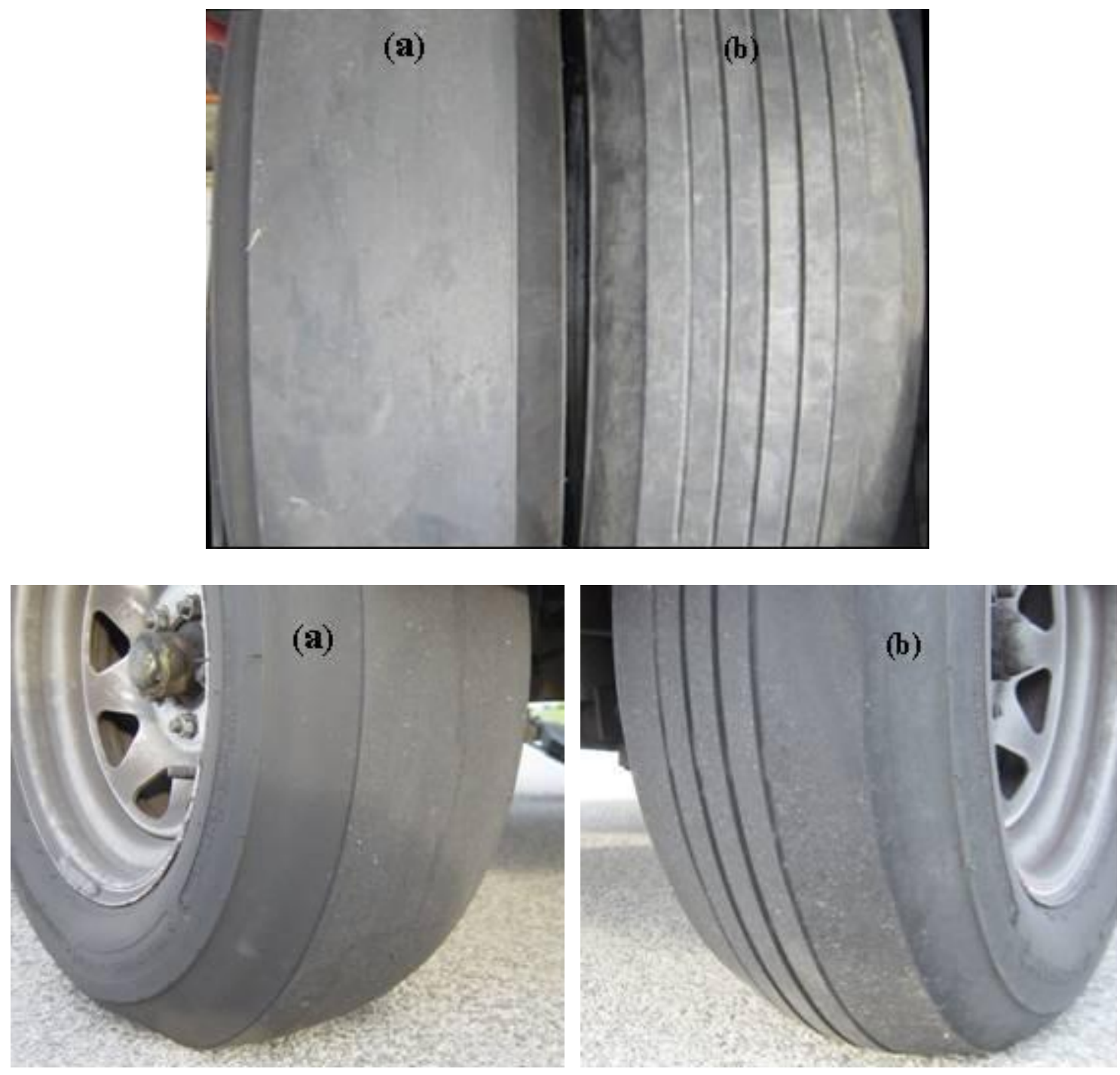

Figure 23. Tires used for the friction measurements: (a) smooth and (b) rib

For a limited number of tests, the BSRT device was also used. The BSRT consists of a pendulum and rubber slider. During the test, the slider (located at the end of the pendulum) is positioned to be in contact with the measured surface for a specific distance (from $124 \mathrm{~mm}$ to $127 \mathrm{~mm}$ ). Then the pendulum is raised to the locked position, the surface is wetted, and the pendulum is released. Due to the frictional forces generated 
between the slider and tested pavement, the pendulum decelerates when propelled over the pavement. The relative amount of energy lost during the deceleration is indicated by the drag pointer attached to the pendulum; the scale for the pointer is calibrated to read the British Pendulum Number (BPN).

Note that tests with the BSRT, DFT and CTM devices require traffic control arrangements and lane closure. Due to this reason, only the driving (right) lane was investigated during this study. Corresponding tests with the friction trailer were also conducted only on the driving lane.

\subsubsection{Testing Schedule, Weather and Traffic Conditions}

A wide range of pavements was tested in this study. All test sections are located in the central region of Indiana and in eastern Iowa. The pavement ages were between two and 25 years, and pavements were tested for up to four years, though most were tested only once. The testing was conducted between early March and the middle of November, when temperatures were significantly above the freezing temperature of water (above $5^{\circ} \mathrm{C}$ ).

For the pavements monitored periodically, an attempt was made to capture a wide range of weather conditions and to conduct tests during different seasons. Pavements were tested at air temperatures between $5^{\circ} \mathrm{C}$ and $34^{\circ} \mathrm{C}$ (pavement surface temperatures between $5^{\circ} \mathrm{C}$ and $50^{\circ} \mathrm{C}$ ) and on both cloudy and sunny days. The relative humidity during testing also varied from $40 \%$ up to $80 \%$.

The test track sections are unique and were included in this study because they are not exposed to traffic; the only vehicles allowed to drive on the track are the pick-ups 
towing the friction trailers. The test track was also designed to provide three ranges of friction numbers on the asphalt, tined concrete and smooth concrete sections. Using this trailer, weekly (using only the left, smooth tire) and monthly calibration tests (using both tires: left - smooth and right - rib) were conducted a multiple number of times (from early March until late October). Five measurements (per tire) were performed per each section at each calibration. Due to the length of the approach and exit sections and due to safety restrictions, tests were only conducted at $48 \mathrm{~km} / \mathrm{h}$. Information about the date of construction, traffic and test schedule for three test sections located on the INDOT test track (HMA-TT, SC and TC) is shown in Table 13, along with information on the other field sections.

During all tests conducted with the CTM/DFT devices on the test track, the machines were positioned in the left tire path. In addition, during tests conducted in 2005, 2006 and two times in 2007, pavement in the right (rib) tire path was also tested (compare with Table 13). Three spots were tested per each section. One DFT and two CTM readings were performed in each spot (the DFT machine was positioned in the same location as the CTM) following ASTM E 2157 (2005) and ASTM E 1911 (2002), respectively.

The BSRT device was used only once on the test track (during tests on 9/14/2007). The BSRT device was positioned in the same spots as CTM/DFT devices had been previously placed and four measurements were performed (following ASTM E 303 (2003)). Measurements were conducted in three directions: longitudinal, transverse and diagonal to the direction of traffic. 
The test schedule for the DGA, SMA and PFC sections tested with the friction trailer and DFT/CTM devices is also shown in Table 13. During tests with the CTM/DFT devices, machines were positioned in the left $(\mathrm{L})$ and right $(\mathrm{R})$ wheelpaths of the driving (right) lane and in the center of the lane (C), as shown in Figure 24. Three sets of tests ( $\mathrm{L}, \mathrm{C}$ and $\mathrm{R}$ ) were conducted, resulting in observations at nine locations. The DFT and CTM tests followed the method previously described for the INDOT test track. Using both smooth and rib tires, towed friction trailer tests were conducted at a speed of $64 \mathrm{~km} / \mathrm{h}$.

The BSRT device was used only once on the DGA, DMA and PFC sections when they were tested in July 2007 (on 7/10/2007 for the DGA and on 7/25/07 for the SMA and PFC). The BSRT device was positioned in the exactly same locations where the CTM/DFT devices were previously placed and tests followed the previously described methods. Again, measurements were conducted in three directions: longitudinal, transverse and diagonal to the direction of traffic. 
Table 13. Information about the traffic, construction year and testing time of pavement sections tested periodically with CTM/DFT

\begin{tabular}{|c|c|c|c|c|}
\hline Section / Mixture & DGA & $\begin{array}{l}\text { PFC } \\
\text { SMA }\end{array}$ & HMA-TT & $\begin{array}{l}\mathrm{TC} \\
\mathrm{SC} \\
\end{array}$ \\
\hline Road Category $^{\mathrm{a}}$ & US & I & \multicolumn{2}{|c|}{ INDOT Test Track } \\
\hline $\begin{array}{l}\text { AADT }\left(\times 10^{3}\right) \\
\% \text { Trucks }\end{array}$ & $\begin{array}{c}31.5 \\
5 \%\end{array}$ & $\begin{array}{l}37.3 \\
26 \%\end{array}$ & \multicolumn{2}{|c|}{ Closed to Traffic } \\
\hline NVA/month (x 10 $\left.{ }^{3}\right)$ & 451 & 980 & \multicolumn{2}{|c|}{ Closed to Traffic } \\
\hline $\begin{array}{l}\text { Open to Traffic, } \\
\text { Year }\end{array}$ & 2003 & 2003 & 2002 & $\begin{array}{c}\text { Before } \\
1997\end{array}$ \\
\hline $\begin{array}{l}\text { Date of DFT/CTM } \\
\text { Test }\end{array}$ & $\begin{array}{c}9 / 6 / 2003 \\
5 / 5 / 2006 \\
10 / 30 / 2006 \\
4 / 10 / 2007 \\
7 / 10 / 2007 \\
9 / 25 / 2007\end{array}$ & $\begin{array}{c}9 / 10 / 2003^{\mathrm{b}} \\
8 / 1 / 2005 \\
11 / 16 / 2005 \\
10 / 3 / 2006 \\
4 / 10 / 2007 \\
7 / 25 / 2007 \\
9 / 25 / 2007\end{array}$ & \multicolumn{2}{|c|}{$\begin{array}{c}8 / 31 / 2005^{\mathrm{c}} \\
10 / 5 / 2005^{\mathrm{c}} \\
11 / 10 / 2005^{\mathrm{c}} \\
5 / 4 / 2006^{\mathrm{c}} \\
3 / 30 / 2007^{\mathrm{c}} \\
6 / 1 / 2007 \\
6 / 15 / 2007 \\
6 / 29 / 2007 \\
7 / 6 / 2007^{\mathrm{c}} \\
7 / 13 / 2007 \\
8 / 10 / 2007 \\
9 / 14 / 2007 \\
9 / 21 / 07\end{array}$} \\
\hline $\begin{array}{l}\text { Date of Friction } \\
\text { Trailer Test } \\
\text { (Month, Year) }\end{array}$ & $\begin{array}{l}8 / 2004 \\
9 / 2007\end{array}$ & $\begin{array}{l}9 / 2004 \\
9 / 2005 \\
9 / 2006 \\
9 / 2007\end{array}$ & $*$ & \\
\hline
\end{tabular}

${ }^{a}$ Road category: US - U.S. highway, I - interstate highway.

${ }^{\mathrm{b}}$ Before opening to traffic.

${ }^{\mathrm{c}}$ Dates when pavement in rib tire path was also tested

* During the test season (from early March until late October), tests were conducted weekly (smooth tire) and monthly (rib tire), if the weather permitted. 
Passing Lane Driving Lane

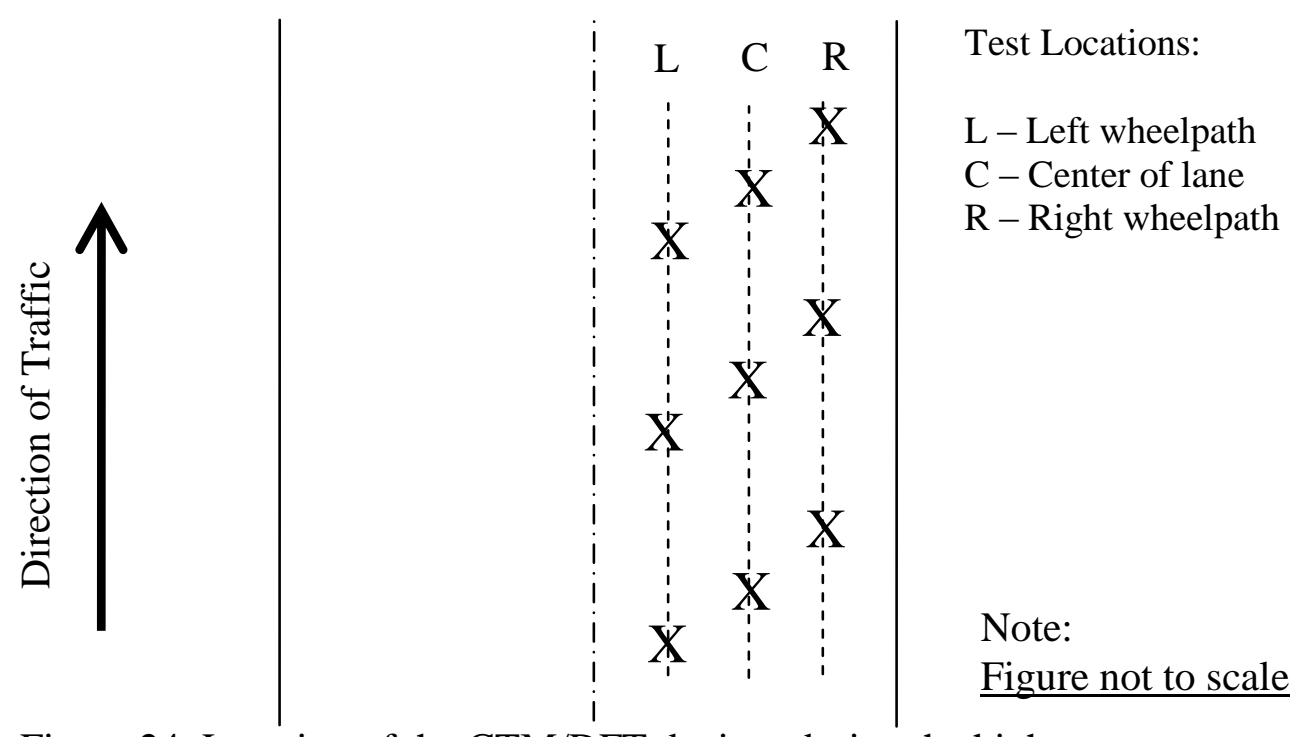

Figure 24. Location of the CTM/DFT devices during the highway tests

Information about the traffic on the Indiana sections tested one time (HM-IN-1 to HM-IN-8) is shown in Table 14. Based on the AADT traffic information, the number of vehicle axles passes (NVA) on the test section (per month) was calculated. During the calculation, several simplifications were applied. It was assumed that an equal number of vehicles travelled in both directions (AADT was divided by two) and that $55 \%$ of the vehicles were using the driving lane on interstate highways (I) and U.S. highways. In the case of state roads (SR), there was only one lane in each direction, so the $55 \%$ "lane dividing" factor was not used. It was assumed that the average truck has 4.5 axles and the average car has 2 axles. No information about the number of trucks within the total traffic was available; it was assumed that $15 \%$ of vehicles were trucks. Results were multiplied by 30 , which is the number of days per month. No traffic growth adjustment 
factors were employed. This simplified equation for NVA for interstate and state highways had the following form:

$$
\mathrm{NVA}=(\mathrm{AADT}) \cdot 0.5 \cdot 0.55 \cdot[\% \text { Trucks } \cdot 4.5+(100 \%-\% \text { Trucks }) \cdot 2] \cdot 30(14)
$$

The test schedule for sections HM-IN-1 to HM-IN-8 is also shown in Table 14. During testing with the CTM/DFT devices, machines were positioned as shown in Figure 24 and described previously. The only difference was that five (instead of three) sets of locations (L, C and R) were tested (i.e., 15 locations were tested per section). Friction tests were conducted at a speed of $64 \mathrm{~km} / \mathrm{h}$ using both smooth and rib tires.

Table 14. Information traffic, construction year and testing time of Indiana sections tested one time

\begin{tabular}{|c|c|c|c|c|c|c|c|c|c|}
\hline \multicolumn{2}{|c|}{$\begin{array}{l}\text { Section / } \\
\text { Mixture }\end{array}$} & $\begin{array}{l}\text { HM- } \\
\text { IN-1 }\end{array}$ & $\begin{array}{l}\text { HM- } \\
\text { IN-2 }\end{array}$ & $\begin{array}{l}\text { HM- } \\
\text { IN-3 }\end{array}$ & $\begin{array}{l}\text { HM- } \\
\text { IN-4 }\end{array}$ & $\begin{array}{l}\text { HM- } \\
\text { IN-5 }\end{array}$ & $\begin{array}{l}\text { HM- } \\
\text { IN-6 }\end{array}$ & $\begin{array}{l}\text { HM- } \\
\text { IN-7 }\end{array}$ & $\begin{array}{l}\text { HM- } \\
\text { IN-8 }\end{array}$ \\
\hline \multicolumn{2}{|c|}{ Road Category ${ }^{\mathrm{a}}$} & SR & US & SR & I & I & SR & SR & US \\
\hline \multicolumn{2}{|c|}{$\begin{array}{l}\text { AADT }\left(x 10^{3}\right) \\
\% \text { Trucks }\end{array}$} & $\begin{array}{c}5.8 \\
*\end{array}$ & $\begin{array}{c}3.0 \\
*\end{array}$ & $\begin{array}{c}1.9 \\
*\end{array}$ & $\begin{array}{c}41.7 \\
*\end{array}$ & $\begin{array}{c}41.7 \\
*\end{array}$ & $\begin{array}{c}1.4 \\
*\end{array}$ & $\begin{array}{c}1.0 \\
*\end{array}$ & $\begin{array}{c}3.5 \\
*\end{array}$ \\
\hline \multicolumn{2}{|l|}{ Year } & 2002 & 2002 & 2002 & 2002 & 2002 & 2002 & 2001 & 2001 \\
\hline \multicolumn{2}{|c|}{$\begin{array}{l}\text { NVA/month } \\
\left(\mathrm{x} 10^{3}\right)\end{array}$} & 206 & 59 & 68 & 817 & 817 & 50 & 36 & 69 \\
\hline \multicolumn{2}{|c|}{$\begin{array}{l}\text { Open to Traffic } \\
\text { Year }\end{array}$} & 2005 & 2006 & 2006 & 1997 & 1997 & 2006 & 2006 & 2006 \\
\hline \multirow{2}{*}{ 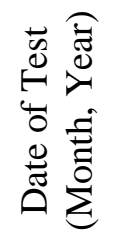 } & $\begin{array}{l}\text { CTM/ } \\
\text { DFT }\end{array}$ & \multicolumn{8}{|c|}{ Late August and Early September, 2007} \\
\hline & $\begin{array}{l}\text { Friction } \\
\text { Trailer }\end{array}$ & \multicolumn{8}{|c|}{ Late August and Early September, 2007} \\
\hline
\end{tabular}

${ }^{\text {a }}$ Road category: US - U.S. highway, I - interstate highway, SR - state road.

* Data not available. 
Information about the traffic on the Iowa sections tested one time (HM-IA-1 to HM-IA-11) is shown in Table 15. The NVA was calculated utilizing a similar procedure as previously described for the Indiana sections (HM-IN-1 to HM-IN-8). For sections in Iowa, information about the number of trucks in the total traffic was available, thus the estimate could be more accurate.

The test schedule for sections HM-IA-1 to HM-IA-11 is shown in Table 15. In addition to tests conducted during this study, historical friction data (measured using a friction trailer) also exists; tests were conducted during late summer/early fall months during the test years shown in Table 15. During testing with the CTM/DFT devices, the machines were positioned as shown in Figure 24 and described previously. The only difference was that, in the interests of time, only two sets of locations (L, C and R) were tested (i.e., a total of six locations were tested per section). All the Iowa DFT/CTM tests were conducted on two consecutive days. This was under windy and chilly weather conditions; air and surface temperatures were about $5-10^{\circ} \mathrm{C}$. Tests with the friction trailer were conducted at a speed of $64 \mathrm{~km} / \mathrm{h}$ using a rib tire only. 
Table 15. Information about the traffic, construction year and testing time of Iowa sections tested one time

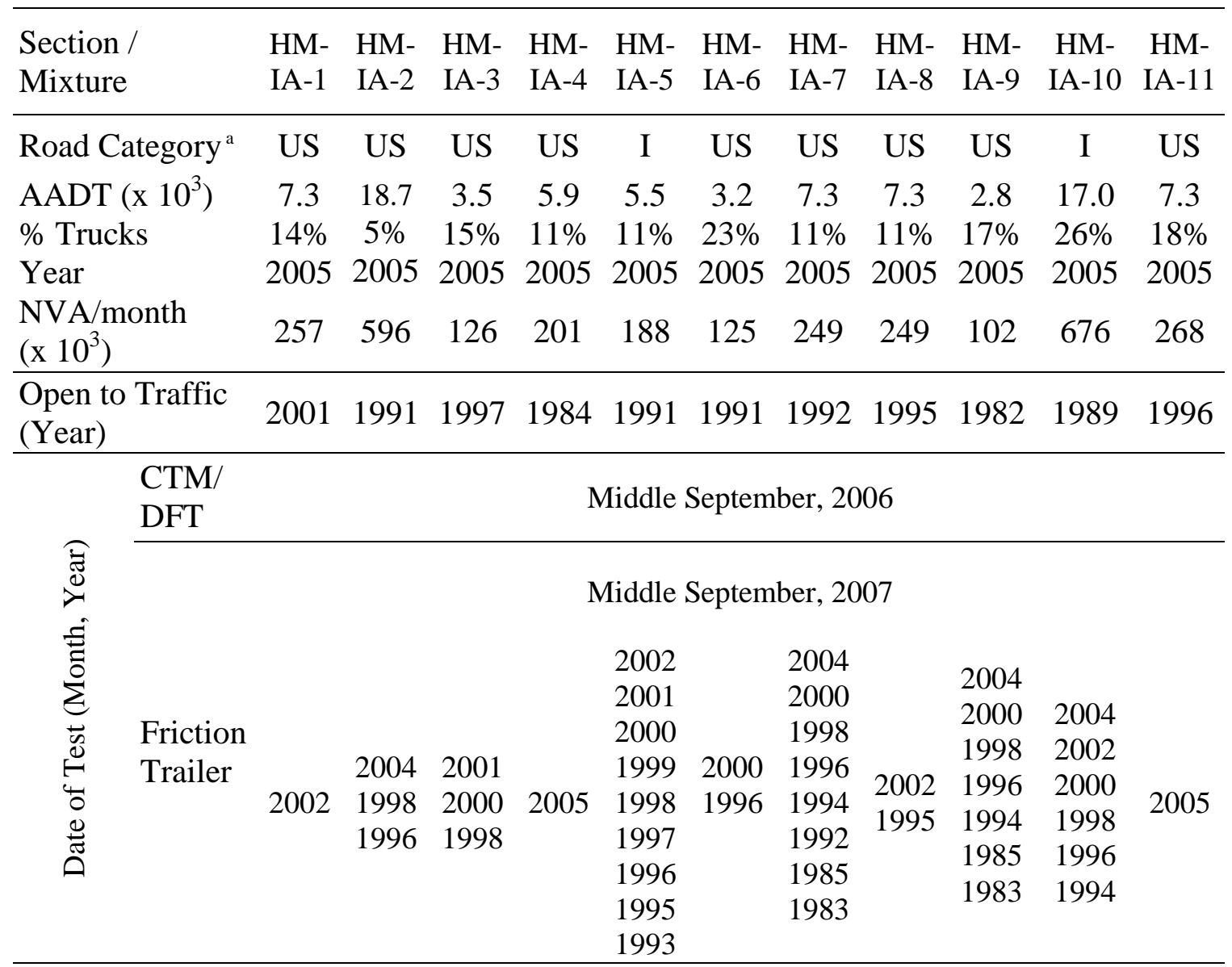

\footnotetext{
${ }^{\mathrm{a}}$ Road category: US - U.S. highway, I - interstate highway.
} 


\section{CHAPTER FOUR: EXPERIMENTAL RESULTS AND DISCUSSIONS}

This chapter contains the results of the laboratory and field part of this study. Discussion of the various factors influencing polishing resistance of the HMA is also shown here. Note that all laboratory friction tests were conducted by the same operator under stable laboratory conditions. On the other hand, the field tests were conducted at different temperatures and by a multiple operators.

\subsection{Laboratory Study}

This section presents some general observations about the measured laboratory parameters; specific details about the different aggregate and mixture variables studied are presented later in the report (4.1.3.1 and following) and are presented in the appendix. Typical friction (F60 and $\mathrm{DF}_{20}$ ) and texture (MPD) values obtained from two repeated measurements on same slab without moving the device are shown in Figure 25 (using the 19 mm NMAS, s-shaped mixture with $20 \%$ steel slag and soft limestone as an example). In most cases, the replicate mean profile depth (MPD) values were similar (at a specific number of wheel passes). 



Figure 25. Mean profile depth (MPD) and dynamic friction $\left(\mathrm{DF}_{20}\right)$ data (a) and calibrated wet friction (F60) data (b) as a function of no. of wheel passes for one of the laboratory tested specimens (two repeated measurements)

The values of dynamic friction $\left(\mathrm{DF}_{20}\right)$ (from duplicate measurements) were also close to each other, at specific numbers of wheel passes. The first two $\mathrm{DF}_{20}$ values (measured for the unpolished samples) were significantly lower than those measured after 1,500 wheel passes, where the friction peaked. This initial increase in friction is the result of the process of abrading the binder from the surface of the aggregate, which 
exposed its microtexture (as is typically observed in the field). After achieving this peak value, the $\mathrm{DF}_{20}$ plot decreased gradually and leveled off at about 120,000 wheel passes.

As implied by equations 9 and 10 (and discussed in section 2.3.3), the changes in MPD had less of an impact on F60 than on the $\mathrm{DF}_{20}$ values; for the convenience of the reader those equations are repeated below. Thus, as expected, the distribution of the calculated F60 is similar to that observed for $\mathrm{DF}_{20}$.

$$
\begin{array}{ll}
F 60=0.081+0.732 D F_{20} e^{\frac{-40}{s_{p}}} & \text { (repeated eq. 9) } \\
S_{p}=14.2+89.7 M P D & \text { (repeated eq. 10) }
\end{array}
$$

The trends in the MPD, $\mathrm{DF}_{20}$ and F60 changes observed for other specimens are similar to those shown in Figure 25 with some differences in the individual values related to gradation and aggregate type (at a given number of wheel passes) and slopes. A summary of the average values of the MPD, $\mathrm{DF}_{20}$ and $\mathrm{F} 60$ results for all 46 specimens tested (test matrices I to III) is shown in Table 16. Due to the objectives of this study, the main effort was directed towards comparing the frictional properties of various Superpave mixtures. In addition, PFC and SMA specimens were also tested; these results are presented separately in section 4.1.3.4. A complete set of laboratory polishing data (including CTM and DFT tests results) is shown in Appendix D (in Table D 1), while the data summary is shown in Appendix E, in Table E 1. 
Table 16. Summary of the test results: laboratory friction measurements (test Matrices I-

\begin{tabular}{lcll}
\hline & MPD, mm & $\mathrm{DF}_{20}$ & F60 \\
\hline Minimum Value & 0.31 & 0.28 & 0.21 \\
Maximum Value & 2.42 & 0.85 & 0.57 \\
Average Value & 1.08 & 0.54 & 0.35 \\
\hline Minimum Range $^{\mathrm{a}}$ & 0.11 & 0.21 & 0.05 \\
Maximum Range $^{\mathrm{a}}$ & 1.24 & 0.50 & 0.25 \\
Average Range $^{\mathrm{a}}$ & 0.30 & 0.34 & 0.15 \\
\hline${ }^{\mathrm{a}}$ range within the sample & & &
\end{tabular}

\subsubsection{Proposed Polishing Model}

Previously discussed similarities in the calibrated wet friction (F60) data distribution (in 4.1 and illustrated in Figure 25b) suggest that a general model incorporating all the test results could be developed. Such a model would be useful to compare and to evaluate specimen frictional properties.

The frictional resistance evaluation (polishing) model proposed here is somewhat similar to so called "skid performance rating (SPR)" procedure originally proposed by Prasanna et al. [1999]. In the SPR evaluation procedure, the relationship between friction performance and cumulative vehicle passes was plotted for various pavements. The authors concluded that most of the plots were parallel to each other. Based on this finding, different zones corresponding to the pavement frictional properties were arbitrarily selected for rating using the SPR; each of these zones matched the pavement section with specific polishing performance (graded from excellent to poor). 
In an attempt to apply the SPR procedure approach to the analysis of polishing data from this study, the F60 results from Figure 25b have been re-plotted in Figure 26, but this time using a logarithmic scale to represent the number of wheel passes (horizontal axis). This data presentation allows for "magnification" of the initial portion of the curve (up to 3,000 passes), thus making the analysis process easier. As shown in Figure 26, the F60 data can be assigned to three different zones: initial pavement life (zone I), decreasing friction (zone II) and friction stabilization (zone III).

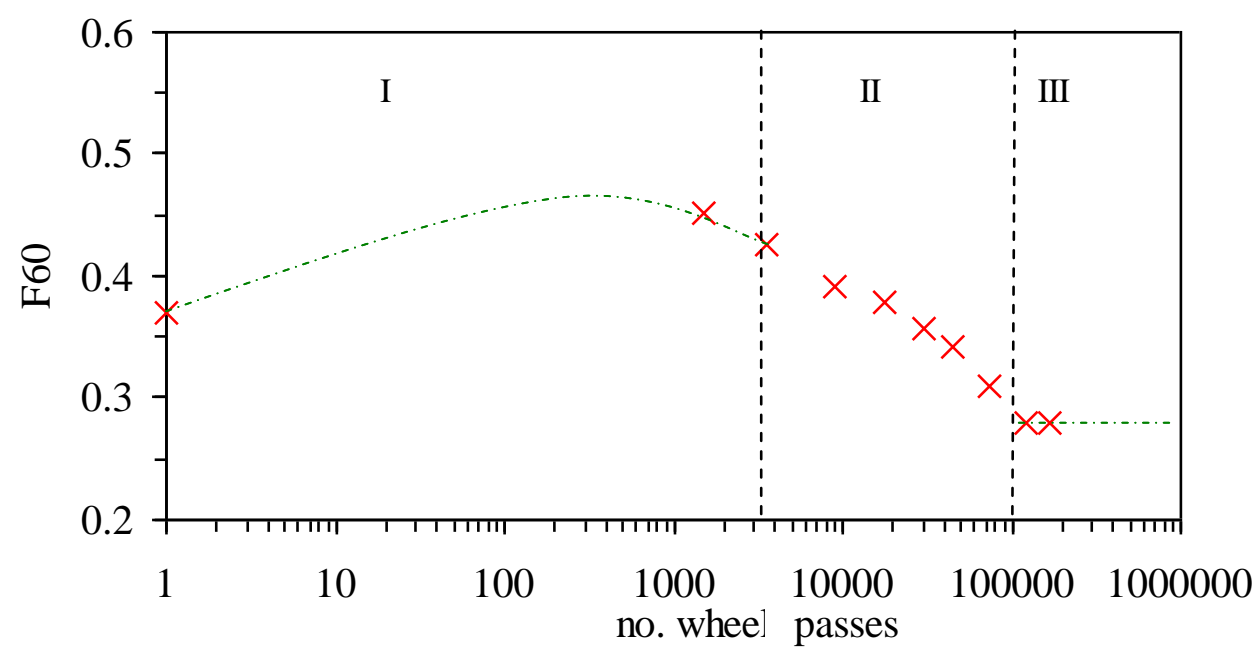

Figure 26. Plot of the typical calibrated wet friction relationship with no. of wheel passes for the 19SS20S_SL laboratory tested specimen

Following these findings, a polishing model containing all three of the above is proposed as shown in Figure 27. 


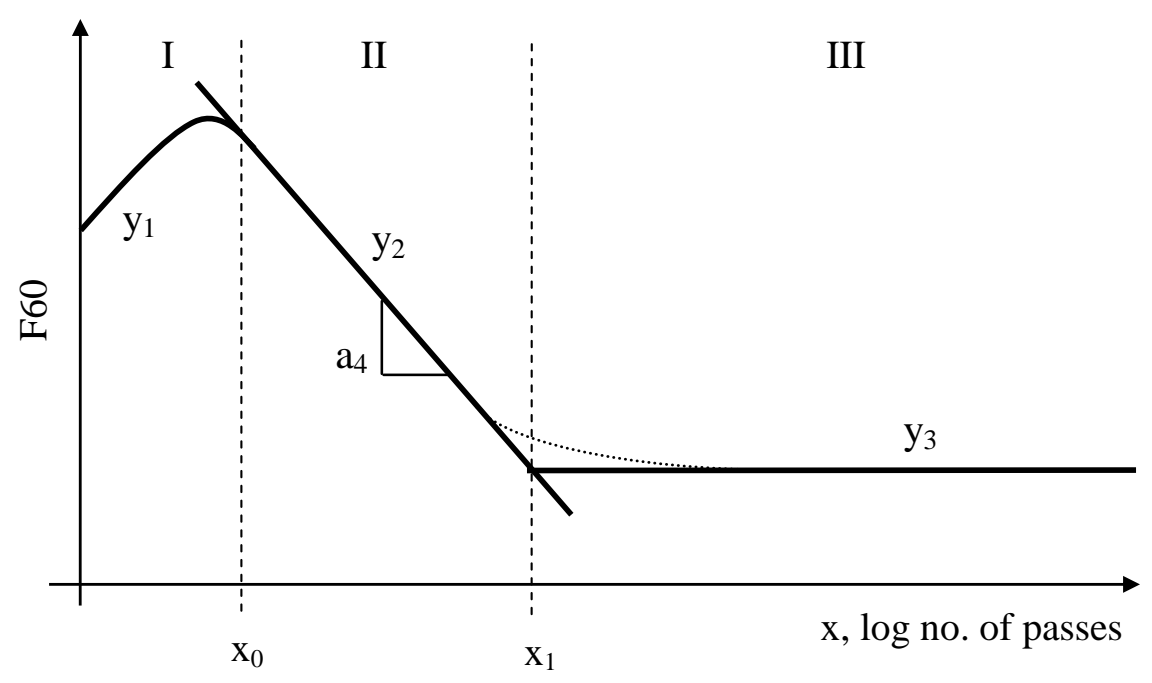

Figure 27. Proposed polishing model

The proposed model includes three functions: polynomial $\left(\mathrm{y}_{1}\right)$, logarithmic $\left(\mathrm{y}_{2}\right)$ and linear $\left(y_{3}\right)$, corresponding, respectively, to zones I, II and III of the model. These functions can be expressed as follows (equations 15-17):

$$
\begin{gathered}
y_{1}(x)=a_{0}+a_{1} x+a_{2} x^{2}+a_{3} x^{3} \\
0<x \leq x_{0} \\
y_{2}(x)=a_{4} \ln \left(x+1-x_{0}\right)+a_{5} \\
x_{0}<x \leq x_{1} \\
y_{3}(x)=a_{4} \ln \left(x_{1}+1-x_{0}\right)+a_{5} \\
x>x_{1}
\end{gathered}
$$

The proposed model, therefore, contains eight parameters: $\mathrm{x}_{0}, \mathrm{x}_{1}, \mathrm{a}_{0}-\mathrm{a}_{5}$. However, since functions $\mathrm{y}_{1}$ and $\mathrm{y}_{2}$ are equal at $\mathrm{x}_{0}$, the number of unknown parameters can be reduced. Substituting $\mathrm{x}=\mathrm{x}_{0}$ in equations 18 and 19 yields:

$$
\begin{aligned}
& \left.y_{1}\right|_{x=x_{0}}=a_{0}+a_{1} x_{0}+a_{2} x_{0}{ }^{2}+a_{3} x_{0}{ }^{3} \\
& \left.y_{2}\right|_{x=x_{0}}=a_{5}
\end{aligned}
$$

And since $\mathrm{y}_{1}$ and $\mathrm{y}_{2}$ are equal at $\mathrm{x}=\mathrm{x}_{0}$, then

$$
a_{5}=a_{0}+a_{1} x_{0}+a_{2} x_{0}^{2}+a_{3} x_{0}^{3}
$$


In addition, since the continuity of the slopes at $\mathrm{x}_{0}$ is also required, at $\mathrm{x}=\mathrm{x}_{0}$ the first derivatives of functions $\mathrm{y}_{1}$ and $\mathrm{y}_{2}$ also have to be equal. As a result, the number of unknown parameters can be further reduced as follows:

$$
\begin{aligned}
& \left.\frac{d y_{1}}{d x}\right|_{x=x_{0}}=a_{1}+2 a_{2} x_{0}+3 a_{3} x_{0}{ }^{2} \\
& \left.\frac{d y_{2}}{d x}\right|_{x=x_{0}}=\frac{a_{4}}{x_{0}}
\end{aligned}
$$

And since $\mathrm{dy}_{1}$ and $\mathrm{dy}_{2}$ are equal at $\mathrm{x}=\mathrm{x}_{0}$, then

$$
a_{4}=a_{1} x_{0}+2 a_{2} x_{0}^{2}+3 a_{3} x_{0}^{3}
$$

By combining equations 22 and 23, the number of parameters required for the model is thus reduced to six $\left(\mathrm{x}_{0}, \mathrm{x}_{1}, \mathrm{a}_{0}-\mathrm{a}_{3}\right)$.

Ideally, the transition curve should follow the dotted (smooth change) curve shown in Figure 27. In the simplified model, this smooth curve was replaced by a constant linear function $\left(\mathrm{y}_{3}\right)$ intercepting the logarithmic function $\left(\mathrm{y}_{2}\right)$ at $\mathrm{x}_{1}$. Such a simplification helps to further reduce the number of parameters in the model without producing significant error. Substituting $\mathrm{a}_{4}$ and $\mathrm{a}_{5}$ in equations 16 and 17 yields:

$$
\begin{aligned}
y_{2}(x)= & \left(a_{1} x_{0}+2 a_{2} x_{0}{ }^{2}+3 a_{3} x_{0}^{3}\right) \ln \left(x+1-x_{0}\right)+ \\
& +\left(a_{0}+a_{1} x_{0}+a_{2} x_{0}{ }^{2}+a_{3} x_{0}{ }^{3}\right) \\
& x_{0}<x \leq x_{1} \\
y_{3}(x)= & \left(a_{1} x_{0}+2 a_{2} x_{0}{ }^{2}+3 a_{3} x_{0}^{3}\right) \ln \left(x_{1}+1-x_{0}\right)+ \\
& +\left(a_{0}+a_{1} x_{0}+a_{2} x_{0}{ }^{2}+a_{3} x_{0}{ }^{3}\right) \\
x & >x_{1}
\end{aligned}
$$

The model parameters are found by minimizing the sum of square errors (SSE) as shown in equation 26. This operation was performed assuming that the minimum SSE would result in the model that best fits the measured data:

$$
S S E=\sum \text { nodel }- \text { data }
$$


From the engineering (practical) point of view, the polishing rate (corresponding to the $\mathrm{a}_{4}$ parameter) is one of the most important factors for the evaluation of pavement frictional properties. The higher (less negative) the $\mathrm{a}_{4}$ value, the more resistant the specimen is to polishing. The other important parameter of the model is the $y_{3}(x)$ value, equivalent to the terminal friction value F60 (at number of polisher wheel passes greater than or equal to $\mathrm{x}_{1}$ ). A high F60 value corresponds to high pavement terminal friction.

\subsubsection{Application and Evaluation of the Proposed Polishing Model}

Models generated for the specimens tested in all three matrices yielded a relatively high coefficient of determination $\left(\mathrm{R}^{2}\right)$ values with an average of 0.92 (refer to Table E 2 in Appendix E for detailed information). Of the 46 specimens tested, one mix had an $\mathrm{R}^{2}$ value equal to 0.50 , three mixes had $\mathrm{R}^{2}$ values of about 0.75 , seven mixes had $\mathrm{R}^{2}$ values of about 0.8 and all the others were greater than 0.9 . Note that due to the focus of this study on Superpave mixes, the results of polishing of PFC and SMA mixes are summarized separately (see section 4.1.3.4).

The proposed model was used to determine the number of wheel passes corresponding to the final $\left(\mathrm{x}_{1}\right)$ polishing level for each of the test specimens. A summary of the resulting $\mathrm{x}_{1}$ values is shown in Table 17 . The complete summary of all model parameters for each sample is shown in Appendix E, in Table E 2. 
Table 17. Summary of $\mathrm{x}_{1}$ values (no. of wheel passes corresponding to the terminal friction level)

\begin{tabular}{lccc}
\hline Matrix & I & II & III \\
\hline Min $x_{1}$ Value & 109,000 & 96,000 & 125,000 \\
Max $x_{1}$ Value & 200,000 & 166,000 & 165,000 \\
Average $x_{1}$ Value & 162,000 & 147,000 & 141,000 \\
\hline
\end{tabular}

The $\mathrm{x}_{1}$ values were determined to be between 96,000 and 200,000 wheel passes. The average value (from 46 specimens tested in Matrices I to III) of 157,000 indicates that preliminary selection of the maximum number of wheel passes $(165,000)$ applied to each specimen during polishing was a reasonable choice.

To illustrate the goodness of fit of the proposed polishing model, the model was fitted to the actual test data for an example specimen (19SS20S_SL). The results shown in Figure 28 indicate that for this particular test specimen there was good agreement between the actual and predicted F60 values.

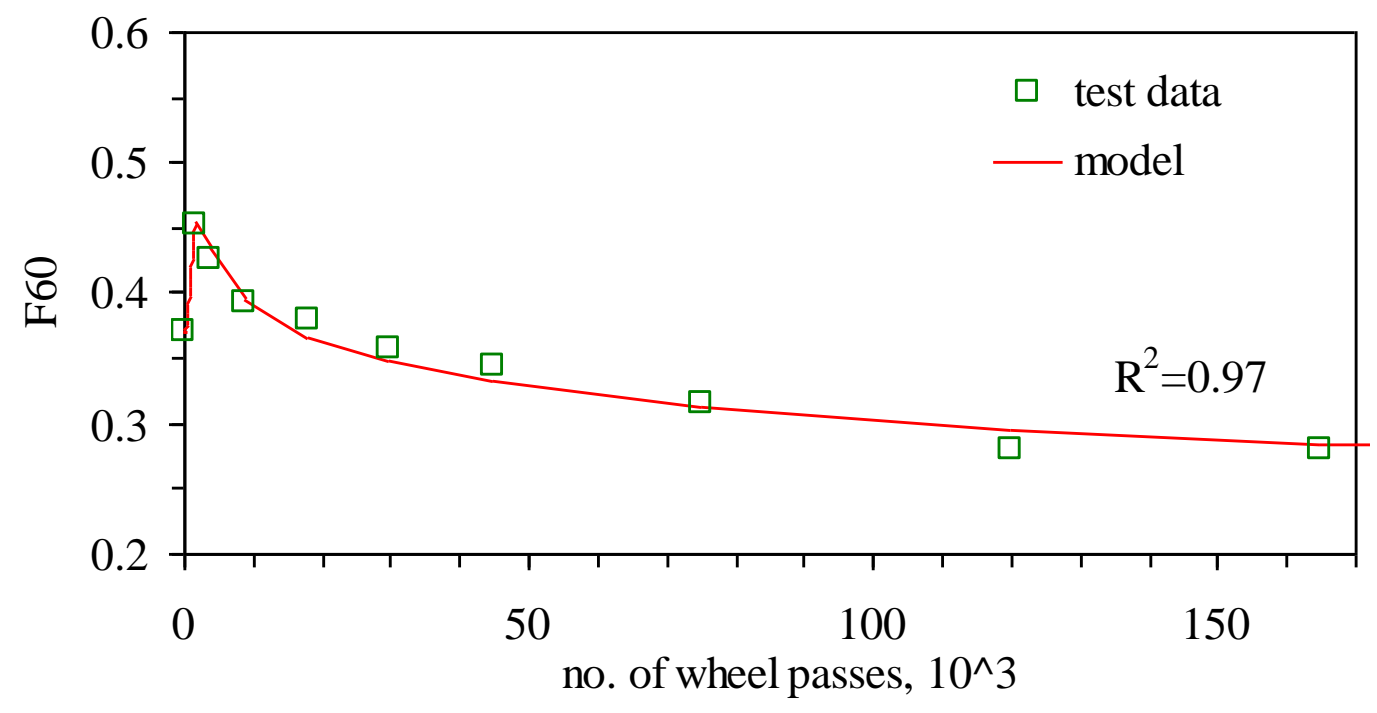

Figure 28. Polishing model fitted to the laboratory data for 19SS20S_SL specimen 
In subsequent sections, the influences of aggregate types, sizes and blend gradations on the frictional properties are discussed. The frictional properties of 46 laboratory produced and two plant produced mixes are investigated.

Two parameters of the model, the polishing rate $\left(a_{4}\right)$ and the terminal friction value F60 (at number of polisher wheel passes equal to $\mathrm{x}_{1}, \mathrm{~F} 60 @ \mathrm{x}_{1}$ ), were selected to compare the influence of the mixture composition on the frictional properties. A statistical correlation analysis was performed to check whether or not these two parameters are correlated.

A correlation analysis was conducted using the SAS 9.1 statistical package software. Two parameters characterize the degree of correlation: coefficient of correlation (R-value) and significance of correlation ( $\mathrm{p}$-value). The former describes the degree of linear relationship among variables and the latter is determined from hypothesis testing. A low p-value (below significance level; an arbitrarily selected significance level of $\alpha=0.05$ was used in this study) indicates great significance of the test (variables are correlated). A p-value greater than the selected $\alpha$ value indicates a lack of significance or correlation. An R-value between 0.5 and 0.8 implies fair correlation while below 0.5 is considered to be low.

The correlation analysis resulted in an R-value of 0.07016 and p-value of 0.6432 , implying that the polishing rate $\left(\mathrm{a}_{4}\right)$ and the terminal friction value F60 (F60@ $\left.\mathrm{x}_{1}\right)$ are not correlated and are thus statistically independent. 


\subsubsection{Aggregate Size, Gradation and Type of Carbonate Aggregate}

In order to facilitate comparison of the F60 values for different specimens tested in this study, their polishing rates (a $a_{4}$ parameter of the model) calculated for the different specimens tested in Matrix I are presented in four different figures. Figure 29 shows a general comparison. Figures 30 and 31 show mixes with steel slag and with quartzite, respectively. And Figure 32 shows a comparison where each point is the average of two similar mixes, which differed only with respect to the friction aggregate type (FAT); one mix contained quartzite (Q) and the other contained steel slag (SS).

As seen in Figure 29, the average polishing rate for specimens with $9.5 \mathrm{~mm}$ NMAS was -0.022 while for specimens with a 19 mm NMAS it was -0.037 . In addition, the average value of $\mathrm{a}_{4}$ for samples (for $9.5 \mathrm{~mm}$ NMAS) with a coarse gradation was similar to that of samples with an s-shaped gradation and less negative for those samples with fine gradation. The same general trend was observed for the three types of gradations in the 19 mm NMAS mixtures.

In general, it could be noticed that mixes with a 19 mm NMAS have higher MPD values (refer to Appendix D, Tables D1 and D2 for detailed information) than those with a 9.5 NMAS; for mixes with a 19 mm NMAS the FM was also higher than for those with a $9.5 \mathrm{~mm}$, as it was discussed in section 3.3.1.1, Table 4. Most likely due to the higher MPDs, mixes with a 19 mm NMAS shown also higher F60@ $\mathrm{x}_{1}$ values (as it is shown in Figures 33 to 36 ). Generally less negative $a_{4}$ values for mixes with a $9.5 \mathrm{~mm}$ NMAS is of the secondary importance, since the terminal friction level for those mixtures is lower than for mixtures with a 19 mm NMAS. 


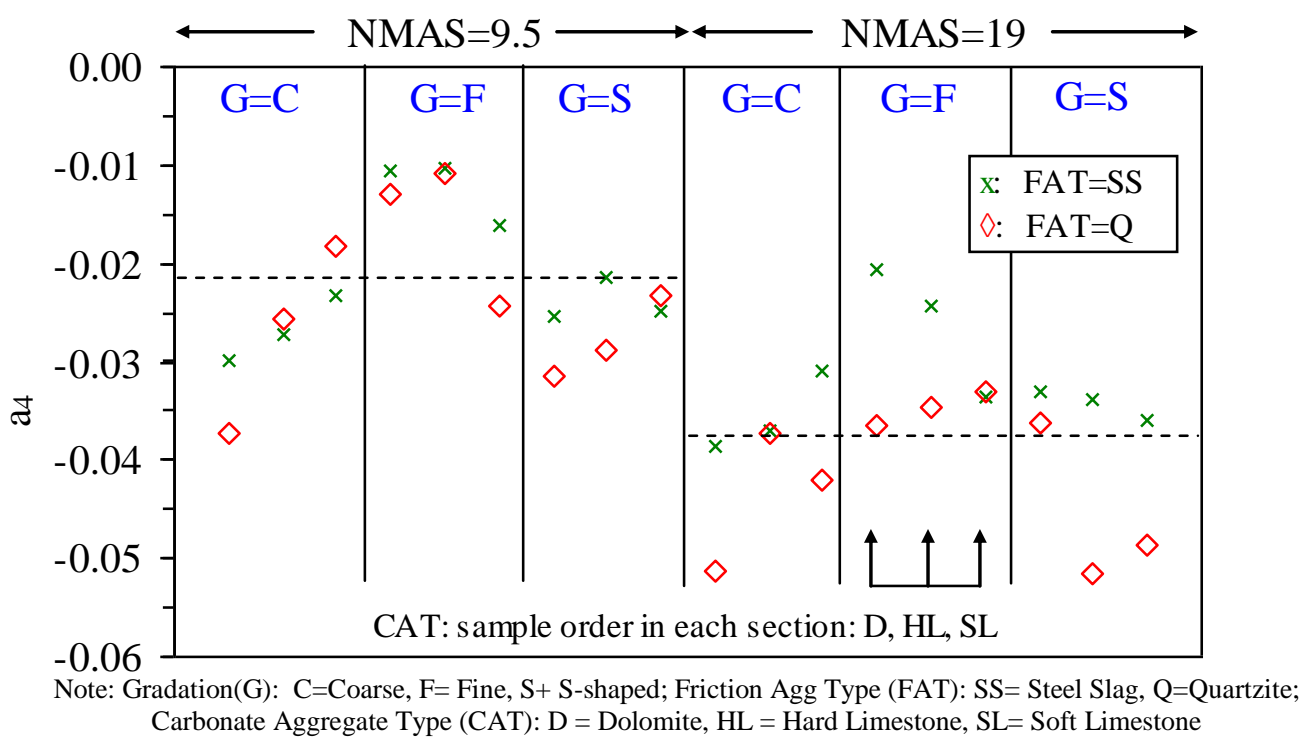

Figure 29. Test Matrix I; distribution of polishing rate, general comparison

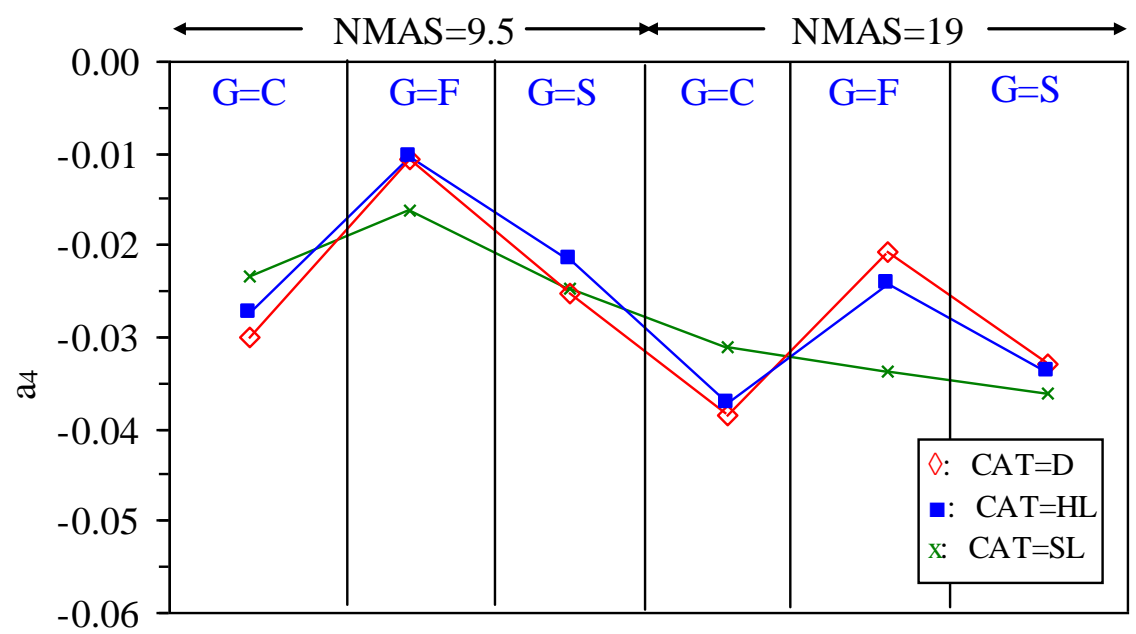

Note: Gradation(G): C=Coarse, F= Fine, S+ S-shaped; Friction Agg Type (FAT): SS= Steel Slag, Q=Quartzite; Carbonate Aggregate Type (CAT): $\mathrm{D}=$ Dolomite, $\mathrm{HL}=$ Hard Limestone, $\mathrm{SL}=\mathrm{Soft}$ Limestone

Figure 30. Test Matrix I, mixes with 20\% SS; distribution of polishing rate 




Note: Gradation(G): C=Coarse, F= Fine, S+ S-shaped; Friction Agg Type (FAT): SS= Steel Slag, Q=Quartzite; Carbonate Aggregate Type (CAT): $\mathrm{D}=$ Dolomite, $\mathrm{HL}=$ Hard Limestone, $\mathrm{SL}=$ Soft Limestone

Figure 31. Test Matrix I, mixes with 20\% Q; distribution of polishing rate

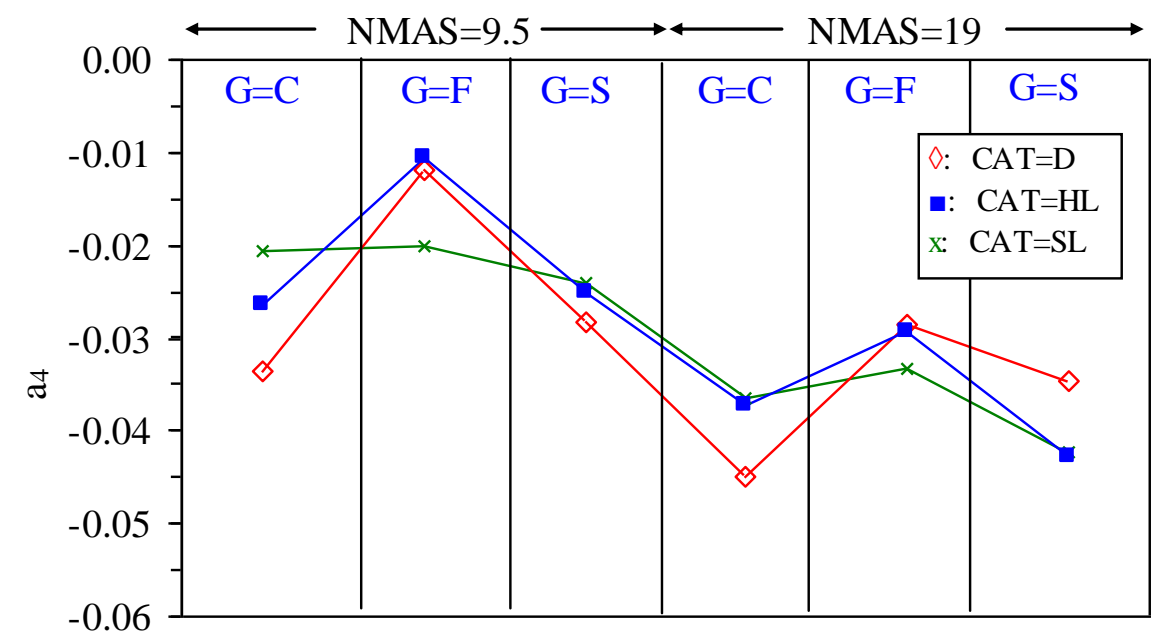

Note: Gradation(G): C=Coarse, F= Fine, S+ S-shaped; Friction Agg Type (FAT): SS= Steel Slag, Q=Quartzite; Carbonate Aggregate Type $(\mathrm{CAT}): \mathrm{D}=$ Dolomite, $\mathrm{HL}=$ Hard Limestone, $\mathrm{SL}=$ Soft Limestone

Figure 32. Test Matrix I; distribution of polishing rate averaged for mixes with Q and SS (each point is an average of two cells)

It should also be noted that the lower (less negative) polishing rate for the $9.5 \mathrm{~mm}$ NMAS mixes does not necessarily mean that pavements with this size of aggregates would have better frictional properties as discussed in more detail later in this section. 
To explain this, the distribution of the F60 values at a number of wheel passes equal to $\mathrm{x}_{1}$ calculated for the different specimens tested in Matrix I are presented in four different figures: Figure 33 (which shows general comparison), Figures 34 and 35 (separately for mixes with steel slag (SS) and with quartzite (Q), respectfully) and Figure 36 (where each point is an average of two similar mixes, differ with respect to the friction aggregate type: one with Q and the other with SS). As seen in Figure 33, the average terminal F60 value for specimens with $9.5 \mathrm{~mm}$ NMAS was equal to 0.26 and was lower than that observed for the specimens with 19 mm NMAS (equal to 0.31). It could be concluded that for the $9.5 \mathrm{~mm}$ mixes smaller changes in friction occurred (refer to Figure 29). The overall frictional resistance of the $9.5 \mathrm{~mm}$ mixes, however, was also lower. In addition, the average values of F60 for the 9.5 NMAS samples with fine gradations are similar to those with s-shaped gradations and slightly lower than those for the coarse gradations. For the 19 mm NMAS mixes, the average F60 for the coarse $(\mathrm{C})$ and fine $(\mathrm{F})$ gradations are similar and slightly higher than those for the s-shaped (S) gradation.

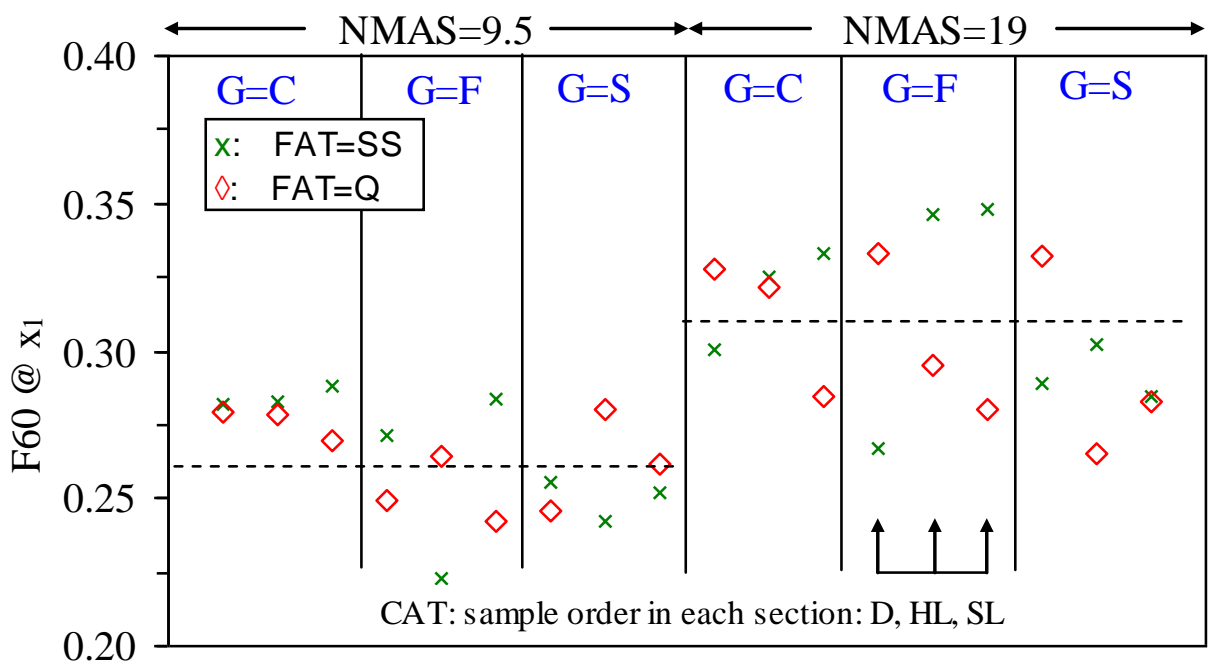

Note: Gradation(G): C=Coarse, F= Fine, S+ S-shaped; Friction Agg Type (FAT): SS= Steel Slag, Q=Quartzite; Carbonate Aggregate Type $(\mathrm{CAT}): \mathrm{D}=$ Dolomite, $\mathrm{HL}=$ Hard Limestone, $\mathrm{SL}=$ Soft Limestone 
Figure 33. Test Matrix I; distribution of friction terminal value $\left(\mathrm{F} 60 @ \mathrm{x}_{1}\right)$, general comparison

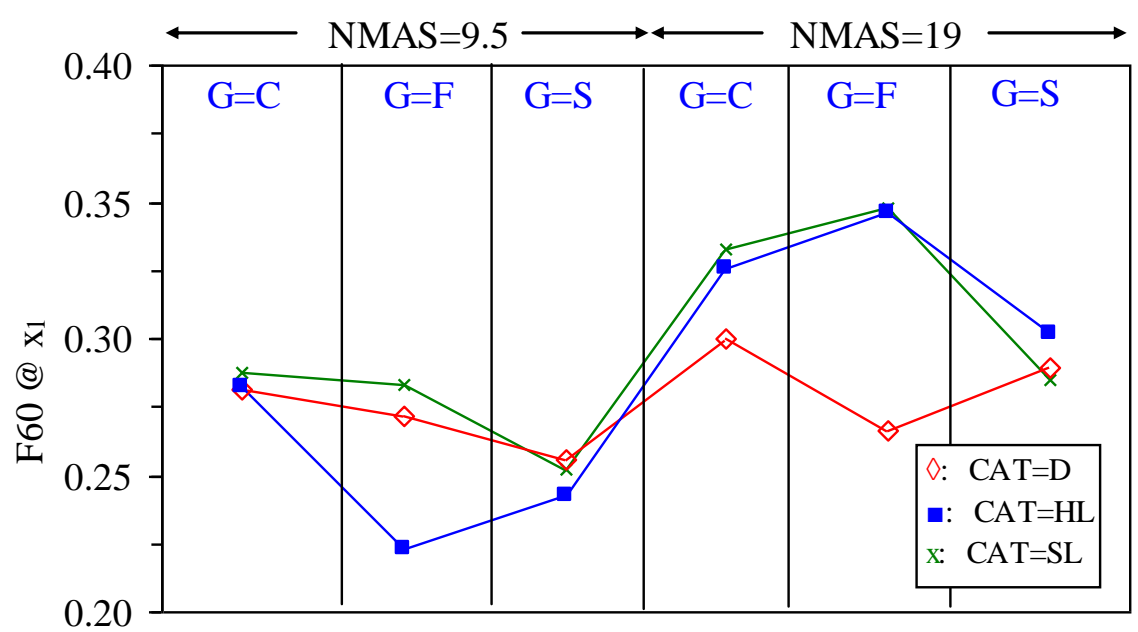

Note: Gradation(G): C=Coarse, F= Fine, S+ S-shaped; Friction Agg Type (FAT): SS= Steel Slag, Q=Quartzite; Carbonate Aggregate Type (CAT): D = Dolomite, HL = Hard Limestone, $\mathrm{SL}=$ Soft Limestone

Figure 34. Test Matrix I, mixes with 20\% SS; distribution of friction terminal value

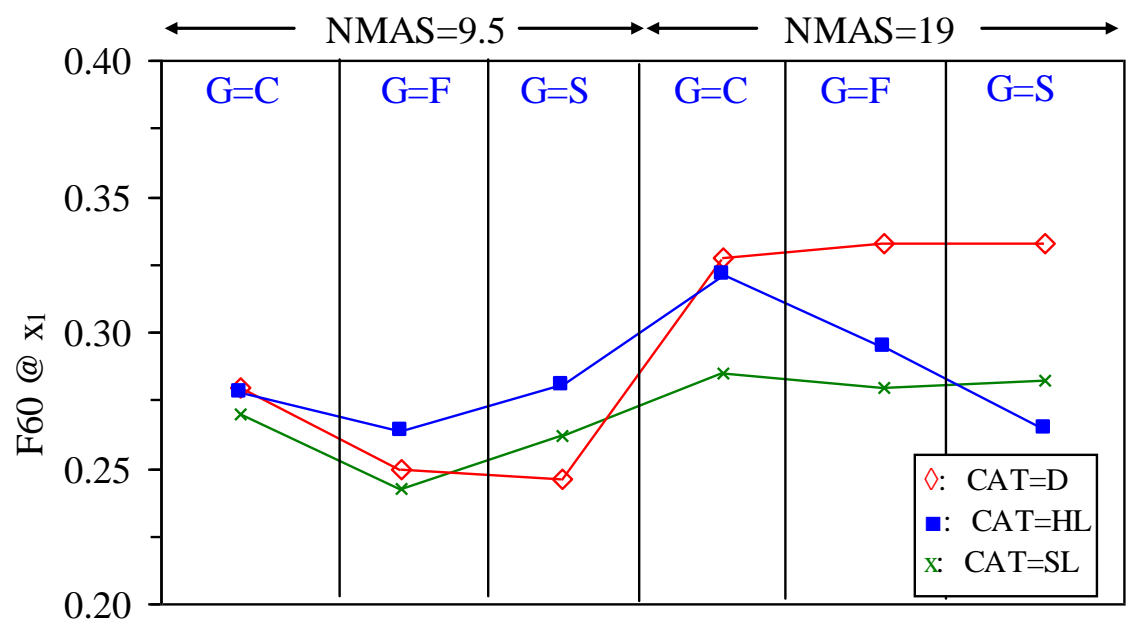

Note: Gradation $(\mathrm{G})$ : C=Coarse, F= Fine, S+ S-shaped; Friction Agg Type (FAT): SS= Steel Slag, Q=Quartzite; Carbonate Aggregate Type $(\mathrm{CAT}): \mathrm{D}=$ Dolomite, $\mathrm{HL}=$ Hard Limestone, $\mathrm{SL}=$ Soft Limestone

Figure 35. Test Matrix I, mixes with 20\% Q; distribution of friction terminal value 




Note: Gradation(G): C=Coarse, F= Fine, S+ S-shaped; Friction Agg Type (FAT): SS= Steel Slag, Q=Quartzite; Carbonate Aggregate Type $(\mathrm{CAT}): \mathrm{D}=$ Dolomite, $\mathrm{HL}=$ Hard Limestone, $\mathrm{SL}=\mathrm{Soft}$ Limestone

Figure 36. Test Matrix I; distribution of friction terminal values averaged for mixes with $\mathrm{Q}$ and SS (each point is an average of two samples)

When comparing the polishing rates $\left(a_{4}\right)$ of specimens with various types of carbonate aggregates (CAT), similarities between the dolomite (D) and hard limestone (HL) could be found, especially for mixtures blended with steel slag (SS) (see Figures 31 and 32). The terminal F60 values were similar for the dolomite and soft limestone (SL) blended with steel slag for 9.5 mm NMAS and between soft and hard limestone for the 19 mm NMAS (see Figures 34 and 35). No clearly visible similarities were observed for the blends with quartzite. A possible explanation of this phenomenon could be related to the fact that the steel slag may have more dominant effect on the overall frictional properties than quartzite (at this specific addition level) thus masking the differences between various types of CAT; differences between specimens with various CAT are more visible in mixes containing Q type of FAT. 


\subsubsection{Friction Aggregate Type}

In this section, the influence of the FAT (steel slag and quartzite) on the frictional properties of the mixture is presented. The influence of the FAT on the polishing rate $\left(a_{4}\right)$ is shown in Figure 37a while the influence of the FAT on the terminal friction value $\left(\mathrm{F} 60 @ \mathrm{x}_{1}\right)$ is shown in Figure 37b. Generally, specimens with steel slag exhibit slightly better (less negative, thus specimen is more resistant to polishing) $a_{4}$ values than specimens with quartzite ( $\mathrm{R}^{2}$ for this comparison is equal to 0.71 ). These differences decrease as the $\mathrm{a}_{4}$ value increases. No correlation $\left(\mathrm{R}^{2}\right.$ equal to 0.10$)$ was noticed when the terminal friction value $\left(\mathrm{F} 60 @ \mathrm{x}_{1}\right)$ for mixes with two different types of friction aggregate were compared.
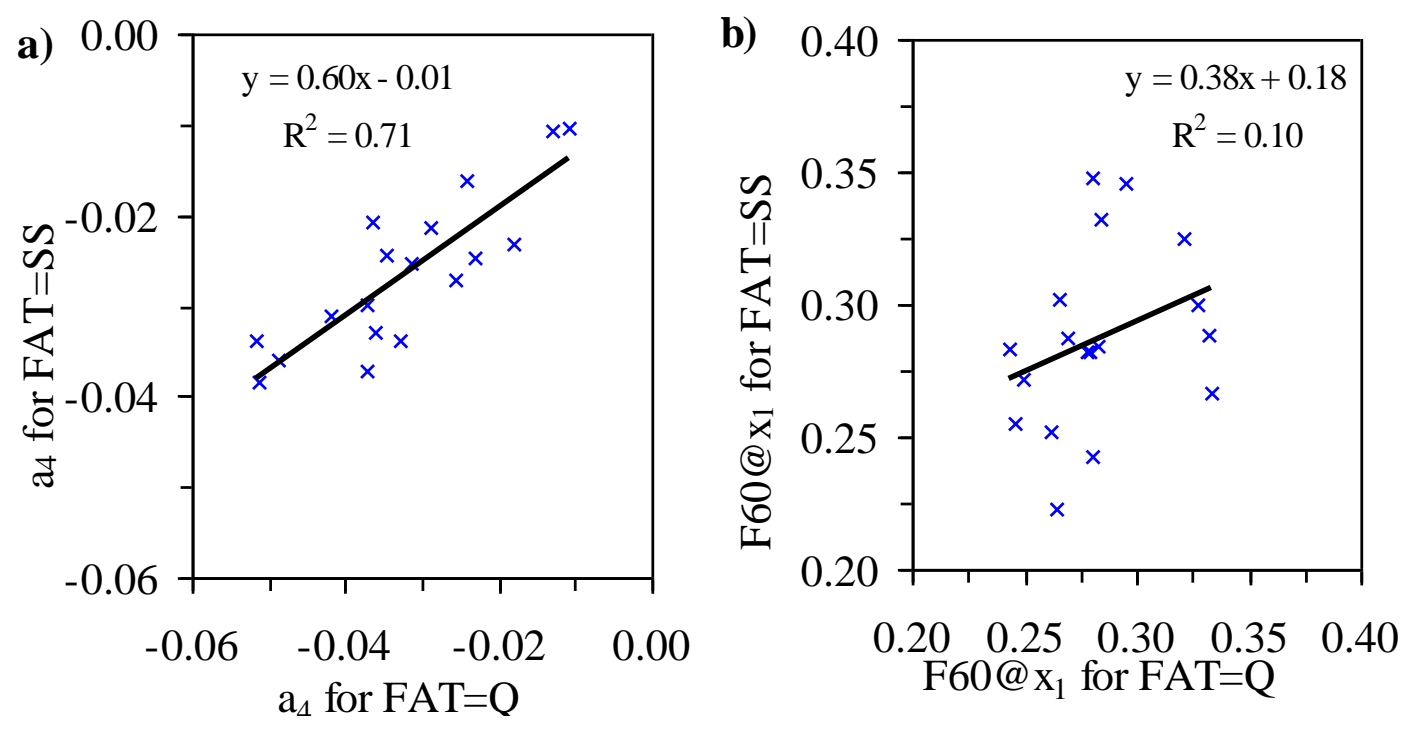

Figure 37. Influence of type of the friction aggregate type (FAT) on the frictional properties of mixes evaluated in test Matrix I: (a) polishing rate $\left(a_{4}\right)$ and (b) friction terminal value $\left(\mathrm{F} 60 @ \mathrm{x}_{1}\right)$ 


\subsubsection{Friction Aggregate Content}

Specimens with various amounts of friction aggregate (FAC between $0 \%$ and 70\%) were tested in test Matrix II. The improvement of mixture resistance to polishing (resulting with less negative value of the polishing rate $\left(\mathrm{a}_{4}\right)$ ) associated with the increase in the amount of friction aggregate could be clearly observed (see Figure 38). In two cases $(\mathrm{FAC}=20$ and $40 \%$ ), specimens with steel slag showed better polishing resistance than quartzite specimens (lower polishing rate) and in two cases (FAC $=10$ and $70 \%)$ the specimens with quartzite showed more favorable response.

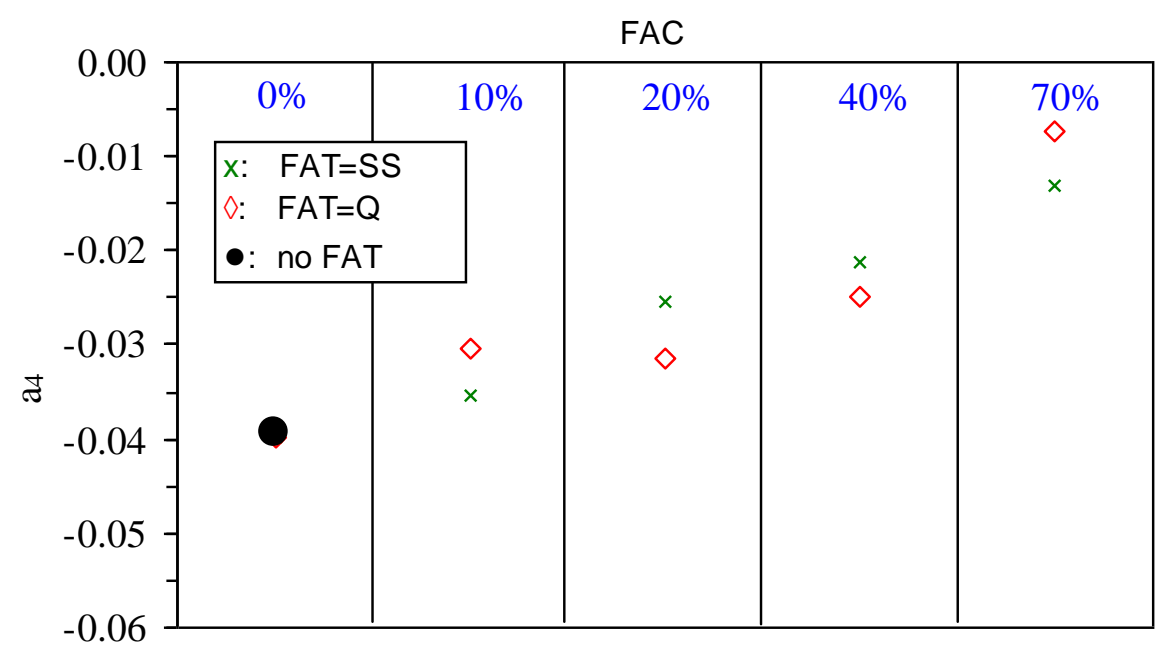

Figure 38. Distribution of polishing rate $\left(\mathrm{a}_{4}\right)$ for test Matrix II mixes

A comparison of the terminal friction values $\left(\mathrm{F} 60 @ \mathrm{x}_{1}\right)$ for mixtures with various FAC is shown in Figure 39. For specimens with FAC between 0\% and $20 \%$ no significant changes in the F60 values were noticed. However, for specimens with $40 \%$ and $70 \%$ of FAC the F60 values increased considerably. The F60 value increased more for the specimens with quartzite than for those with steel slag. 


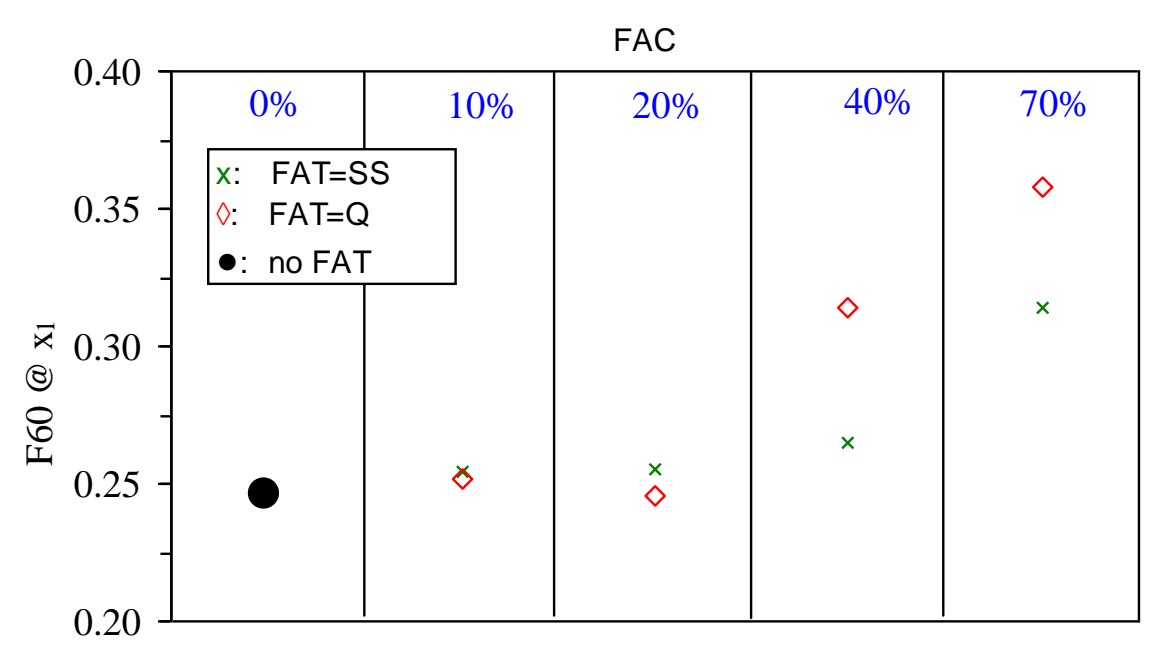

Figure 39. Distribution of terminal friction values $\left(\mathrm{F} 60 @ \mathrm{x}_{1}\right)$ for test Matrix II mixes

Specimens with all three types of carbonate aggregate (CAT=D, SL and HL) and two addition levels of steel slag $(\mathrm{FAC}=10 \%$ and $20 \%)$ were incorporated in test Matrix III. Again, the improvement in resistance to polishing (polishing rate, $\mathrm{a}_{4}$ ) associated with the increased content of FAC could be clearly observed when comparing each pair of the mix with same CAT (refer to Figure 40). From the group of carbonate aggregates tested in this study (dolomite, "hard" limestone and "soft" limestone), the highest improvement of polishing rate $\left(\mathrm{a}_{4}\right)$ caused by addition of steel slag was noticed for mixes with SL. Moreover, for specimens with $10 \% \mathrm{FAC}$, the lowest (most negative) $\mathrm{a}_{4}$ value was observed for mix with "soft" limestone. In other words, the mix with "soft" limestone and only $10 \%$ steel slag yielded the worst polishing rate. However, the addition of $10 \%$ more steel slag improved the polishing rate of this mix more than in the case of mixes with dolomite or "hard" limestone. 


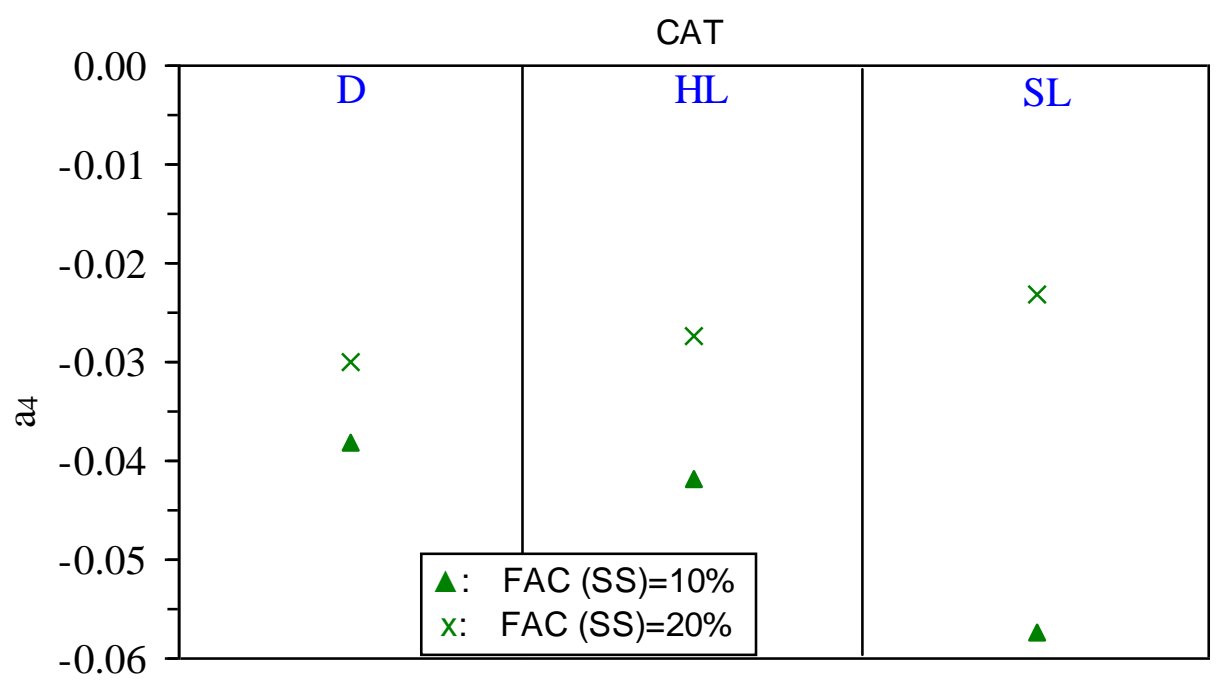

Figure 40. Distribution of polishing rate $\left(a_{4}\right)$ for test Matrix III mixes

Comparison of the terminal friction values $\left(\mathrm{F} 60 @ \mathrm{x}_{1}\right)$ for mixes with the same carbonate aggregate type and at the same addition levels as previously shown in Figure 40, is presented in Figure 41. When compared at the same level of coarse aggregate content $(\mathrm{CAT}=10 \%$ and $20 \%)$ the values of $\mathrm{F} 60 @ \mathrm{x}_{1}$ were very similar for all three types of coarse aggregate (CAT - D, HL and SL). Higher F60@ $\mathrm{x}_{1}$ values were observed for specimens with $20 \%$ of FAC than for specimens with $10 \%$ FAC.



Figure 41. Distribution of terminal friction values $\left(\mathrm{F} 60 @ \mathrm{x}_{1}\right)$ for test Matrix III mixes 


\subsubsection{General Summary of the Influence of Different Factors on Frictional Properties}

It was found in this study that increasing the friction aggregate (quartzite or steel slag) content substantially improved polishing resistance. In general, the polishing resistance of the smaller NMAS $(9.5 \mathrm{~mm})$ mixes was better than that of the larger NMAS (19 $\mathrm{mm}$ ) mixes, however, the $19 \mathrm{~mm}$ mixes exhibited higher levels of friction than the 9.5 mm mixes. In addition, mixes with steel slag generally exhibited slightly higher polishing resistance than mixes with quartzite. However, for those mixes no correlation between the influences of friction aggregate type and the terminal friction value (F60@ $\mathrm{x}_{1}$ ) was clearly identified.

The influence of the carbonate aggregate type (dolomite, "hard" limestone or "soft" limestone) on the frictional properties was also observed. This influence, however, varied depending on the other factors being studied. In general, the mixes with soft limestone exhibited lower friction properties than those with dolomite and hard limestone.

\subsubsection{Proposed Friction Predictive Model}

One of the objectives of this study was to develop a relationship (model) to predict the F60 values (output) based on the aggregate type, size and gradation (input). The overall output of the model (shown in Figure 42) should also account for the cumulative number of wheel passes. It should be noted, however, that due to a limited number of data points obtained from SMA and PFC mixtures, only Superpave mixes from test 
Matrices I to III were used in the model development. However, to check the general applicability of the proposed model, it was used to predict the $\mathrm{a}_{4}$ and F60@ $\mathrm{x}_{1}$ values of the SMA and PFC mixtures as described in section 4.1.5. (Additional research under a separate study is continuing to investigate and potentially validate this model.



Figure 42. Overview of the general friction predictive model

The transfer function shown in Figure 42 consists of the set of equations presented in section 4.1.1 (equations 24 through 26). In order to predict the F60 value at any given polishing level, six parameters of the proposed polishing model $\left(\mathrm{x}_{0}, \mathrm{x}_{1}, \mathrm{a}_{0}-\mathrm{a}_{3}\right)$ must be determined.

These parameters have been determined using SAS 9.1 software and are presented in Appendix F. Using the same statistical software, multiple analysis of variance (ANOVA) was also performed, separately, for each estimate of the proposed polishing model parameter $\left(\mathrm{x}_{0}, \mathrm{x}_{1}, \mathrm{a}_{0}-\mathrm{a}_{3}\right)$. In general, there was a satisfactory significance level and random distribution of residuals.

It should be realized, however, that the terminal frictional properties of the mixture depend mostly on only two of the six parameters discussed earlier. These 
parameters include the polishing rate $\left(\mathrm{a}_{4}\right)$ and the friction terminal value $\left(\mathrm{F} 60 @ \mathrm{x}_{1}\right)$. As a result, the simplified friction model prediction (based on just the polishing rate $\left(\mathrm{a}_{4}\right)$ and the friction terminal value F60@ $\mathrm{x}_{1}$ was developed as discussed below.

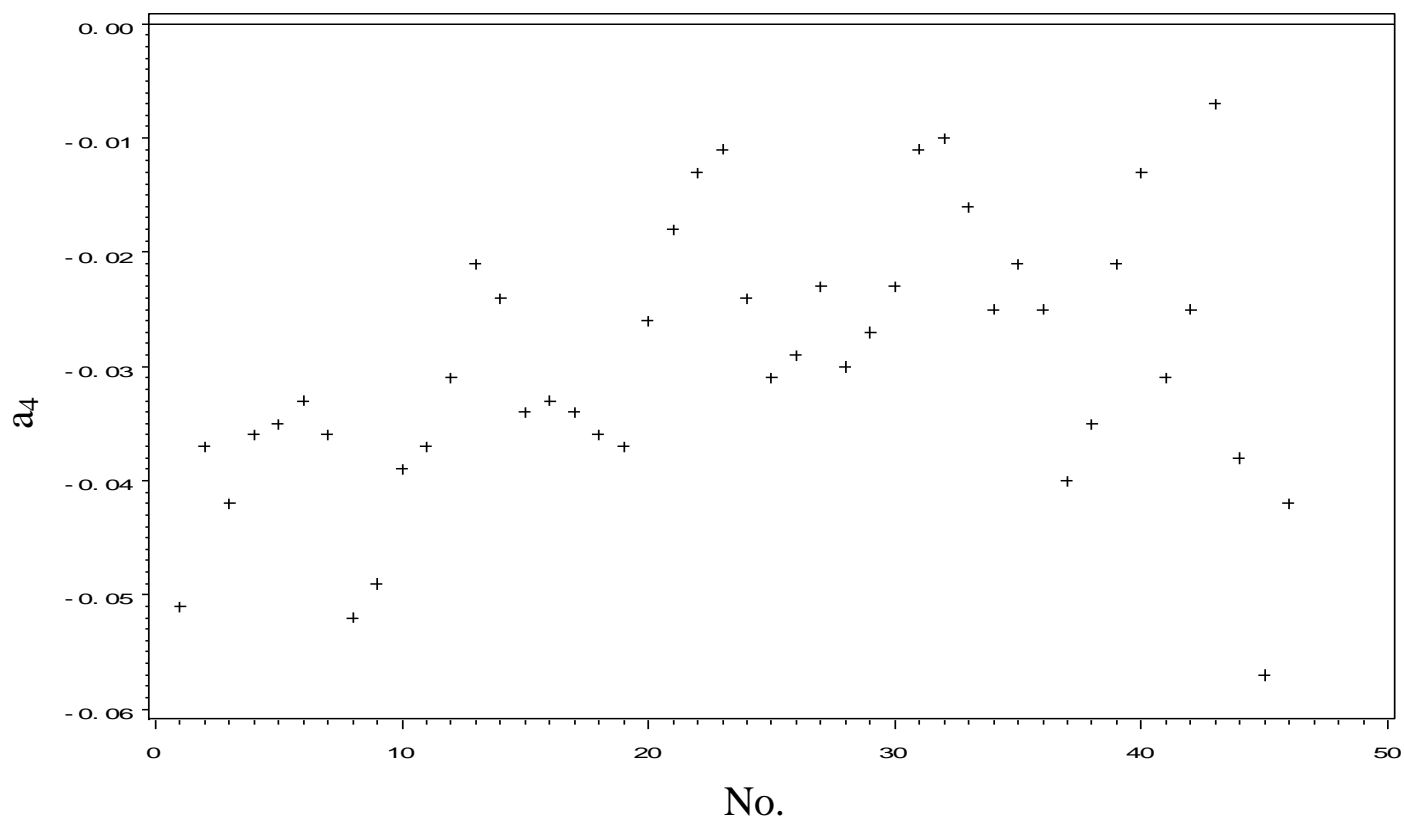

Figure 43. Polishing rate $\left(\mathrm{a}_{4}\right)$ distribution

First, the polishing rate $\left(a_{4}\right)$ parameters determined for all 46 specimens were summarized in one plot to investigate potential trends in the data distribution (as shown in Figure 43). Note that specimens are numbered in the same order as is used in Appendixes $\mathrm{C}$ to $\mathrm{E}$. Then, the multiple ANOVA table was generated and the parameter estimates for the general linear model were calculated (refer to Table 18). Since there were no replicates in this study, only one observation per cell is reported. The overall model was found to be statistically significant with a p-value lower than 0.0001 . In addition, no statistically significant trends were observed for the residuals (see Figure 44a), which appear to be normally distributed as indicated by the quantile-quantile (q-q) 
plot shown in Figure 44b. However, based on the discussion presented in sections 4.1.3.1 to 4.1.3.3, some interactions between model parameters are known to exist. Although these interactions are likely to lower the model reliability, they were not considered in model development. Changes in the FAT and CAT variables (friction and carbonate aggregate types, respectively) are not statistically significant for the overall model predictability ( $p$-values about or greater than the significance level of $\alpha=0.05$ ). 

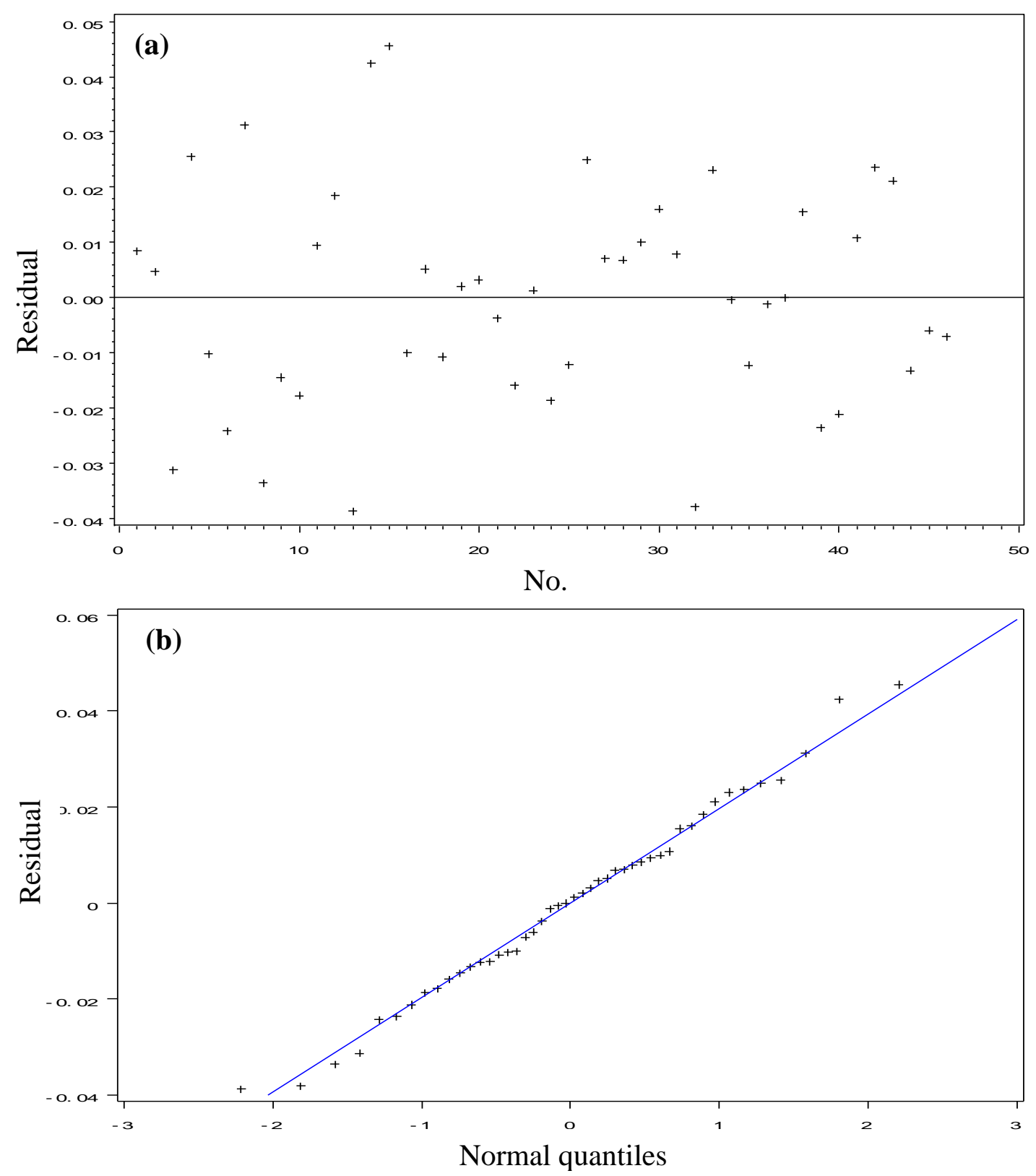

Figure 44. Residual distribution for the polishing rate $\left(a_{4}\right)$ prediction model: (a) residual vs. sample no. and (b) q-q plot 
Table 18. ANOVA table and parameter estimates for the polishing rate $\left(\mathrm{a}_{4}\right)$

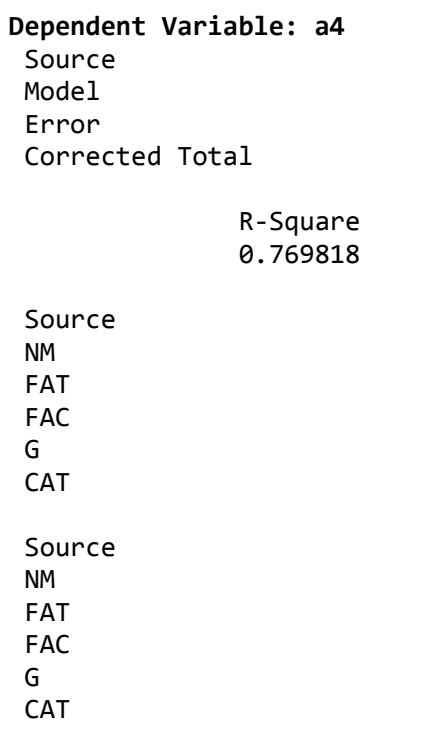

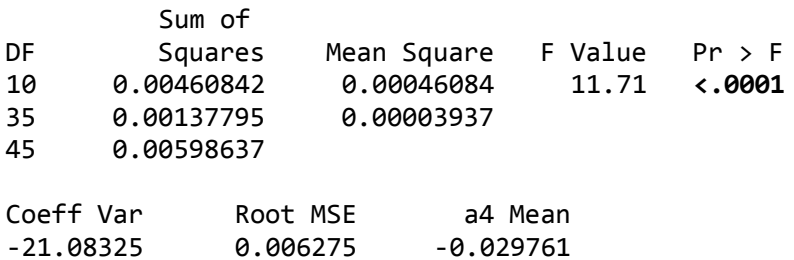



In order to use the proposed model for the prediction of the polishing rate, various parameters shown in Table 18 have to be combined as shown in the example below (for the 19SS20S_SL mixture):

$$
\begin{aligned}
& \mathrm{a}_{4}=\text { intercept }+ \text { aggregate/mixture parameters } \\
& \mathrm{a}_{4}=-0.0098+0.0144(\mathrm{NMAS}=19)+0.0000(\mathrm{FAT}=\mathrm{SS})-0.0139(\mathrm{FAC}=20 \%) \\
& +0.0000(\mathrm{G}=\mathrm{S})+0.0000(\mathrm{CAT}=\mathrm{SL})
\end{aligned}
$$


An analysis similar to that presented above for the prediction of the polishing rate $\mathrm{a}_{4}$ was also conducted for the prediction of the F60@ $\mathrm{x}_{1}$ values. Again, F60@ $\mathrm{x}_{1}$ parameters determined for all 46 specimens were compared to investigate potential trends in the data distribution (as shown in Figure 45).

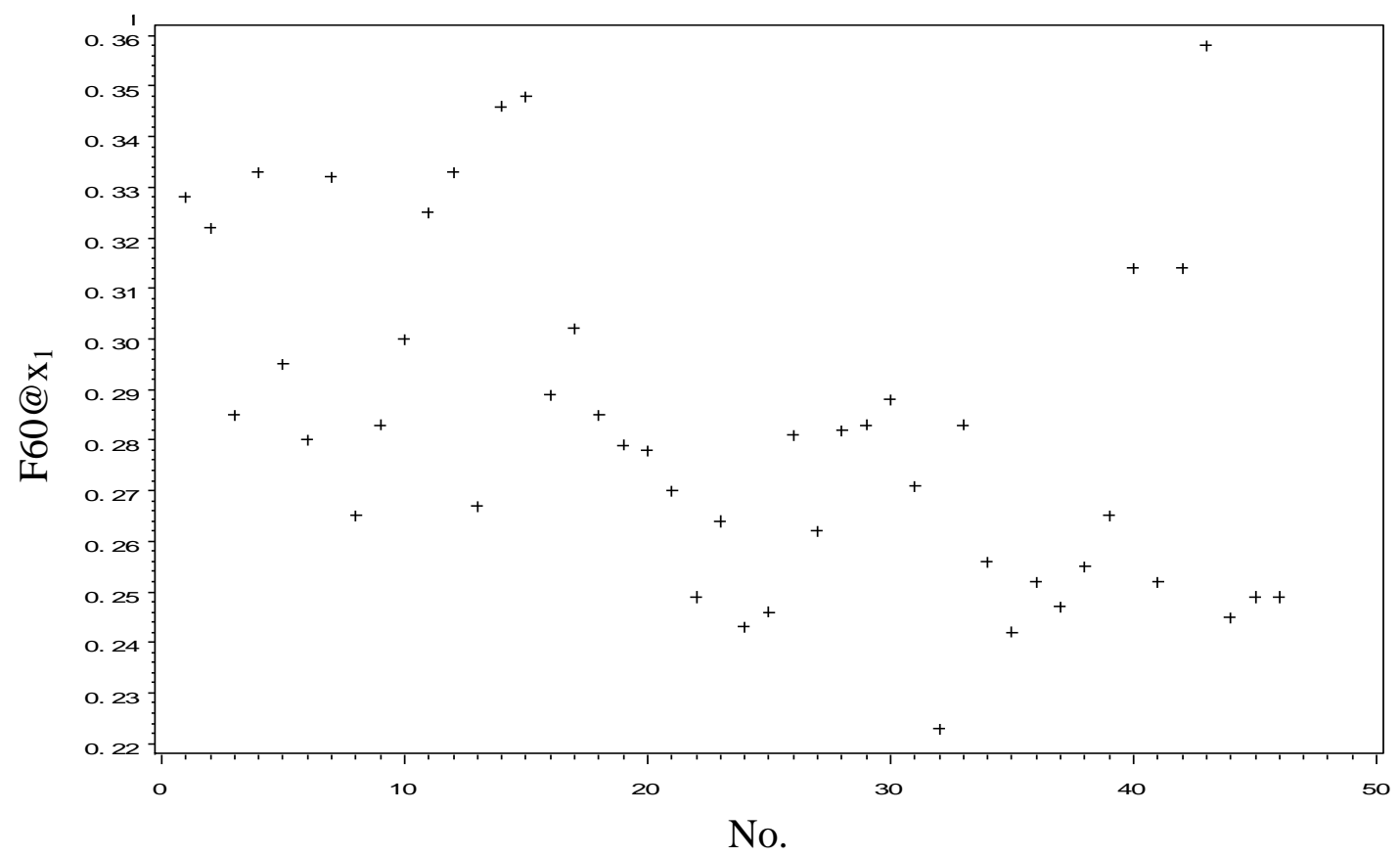

Figure 45. Terminal polishing level $\left(\mathrm{F} 60 @ \mathrm{x}_{1}\right)$ distribution

Again, the multiple ANOVA table was generated and parameter estimates for the general linear model were calculated (refer to Table 19). In general, the data shown in Table 19 indicate that the model is statistically significant with a p-value lower than 0.0001. In addition, no statistically significant trends were observed for the residuals (see Figure 46a), which appear to be normally distributed as indicated by the quantile-quantile (q-q) plot shown in Figure 46b. Again, no interactions were considered in the model development. As could be expected, changes in the FAT and CAT variables (friction and 
carbonate aggregate types, respectively) are not statistically significant for the overall model predictability ( $p$-values about or greater than the significance level of $\alpha=0.05$ ).


Figure 46. Residual distribution for the terminal polishing level $\left(\mathrm{F} 60 @ \mathrm{x}_{1}\right)$ prediction model: (a) residual vs. sample no. and (b) q-q plot 
Table 19. ANOVA table and parameter estimates for the terminal polishing level $\left(\mathrm{F} 60 @ \mathrm{x}_{1}\right)$

Dependent Variable: $F 60 @ X_{1}$
Source
Model
Error
Corrected Total

$\quad$ R-Square

Source
NM
FAT
FAC
G
CAT

Source
NM
FAT
FAC
G
CAT

$\begin{array}{crrr}\text { Sum of } & & & \\ \text { Squares } & \text { Mean Square } & \text { F Value } & \text { Pr > F } \\ 0.03151605 & 0.00315160 & 6.21 & <.0001 \\ 0.01776595 & 0.00050760 & & \\ 0.04928200 & & & \end{array}$

\begin{tabular}{|c|c|c|c|c|c|c|}
\hline \multirow{2}{*}{\multicolumn{2}{|c|}{ Parameter }} & \multicolumn{5}{|c|}{ Standard } \\
\hline & & Estimate & & Error & t Value & $\operatorname{Pr}>|t|$ \\
\hline \multicolumn{2}{|c|}{ Intercept } & 0.3318794828 & B & 0.01841701 & 18.02 & $<.0001$ \\
\hline NM & 19 & 0.0425555556 & B & 0.00750998 & 5.67 & $<.0001$ \\
\hline NM & 95 & 0.0000000000 & B & . & & 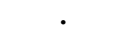 \\
\hline FAT & $Q$ & 0.0017296990 & B & 0.00687547 & 0.25 & 0.8028 \\
\hline FAT & SS & 0.0000000000 & B & . & & . \\
\hline FAC & 0 & -.0881351505 & B & 0.02780676 & -3.17 & 0.0032 \\
\hline FAC & 10 & -.0956618399 & B & 0.01973734 & -4.85 & $<.0001$ \\
\hline FAC & 20 & - .0786893337 & B & 0.01810177 & -4.35 & 0.0001 \\
\hline FAC & 40 & -.0465000000 & B & 0.02252995 & -2.06 & 0.0465 \\
\hline FAC & 70 & 0.0000000000 & B & . & • & . \\
\hline G & C & 0.0187793867 & B & 0.00845486 & 2.22 & 0.0329 \\
\hline G & $\mathrm{F}$ & 0.0067230267 & B & 0.00901782 & 0.75 & 0.4609 \\
\hline G & $\mathrm{s}$ & 0.0000000000 & B & . & $\cdot$ & . \\
\hline CAT & D & 0.0032556677 & B & 0.00867746 & 0.38 & 0.7098 \\
\hline CAT & $\mathrm{HL}$ & 0.0010769231 & B & 0.00883697 & 0.12 & 0.9037 \\
\hline CAT & SL & 0.0000000000 & B & . & $\cdot$ & . \\
\hline
\end{tabular}

The influence of various mixture parameters on the model predicted values for polishing rate $\left(\mathrm{a}_{4}\right)$ and friction terminal value $\left(\mathrm{F} 60 @ \mathrm{x}_{1}\right)$ is in general agreement with the trends summarized in section 4.1.3.4. Specifically, the influence of type of friction and carbonate aggregates (FAT and CAT) variables is less significant than the influence of friction aggregate content (FAT). 


\subsubsection{Application of the Proposed Polishing Model to Non-Superpave Mixes}

Since the development of the proposed polishing model described in section 4.1.4 was entirely based on Superpave designed mixtures, its general applicability to predict friction parameters of non-Superpave mixtures was investigated by analyzing two sets of field produced mixes with different gradations (SMA and PFC). Those mixes were used for fabrication of the laboratory testing slabs of the same dimensions as those used for mixtures from matrices I-III. Two samples of each mix were compacted and tested, which provided limited evaluation of repeatability. As mentioned in section 3.3.1.1, the same type of plant produced SMA mixture was compacted into molds with two different depths (64 and $38 \mathrm{~mm}$ ). A similar procedure was applied to PFC mixture.

Changes in the macrotexture (expressed by MPD) of the SMA and PFC specimens polished in the lab are shown in Figure 47 while changes in their dynamic friction $\left(\mathrm{DF}_{20}\right)$ are presented in Figure 48.

Although, in general, the macrotexture did not change during the polishing process, for the $38 \mathrm{~mm}$ thick PFC specimen a slight increasing trend in the MPD was noticed. It could also be observed that, for the SMA specimens, MPD values were similar for both 38 and $64 \mathrm{~mm}$ thick specimens. For the PFC mixture, however, the macrotexture varied depending on the thickness of the specimen and was about $2.3 \mathrm{~mm}$ for the $64 \mathrm{~mm}$ thick specimen and about $2.5 \mathrm{~mm}$ for the $38 \mathrm{~mm}$ thick specimen. Theoretically, the macrotexture of both $38 \mathrm{~mm}$ and $64 \mathrm{~mm}$ specimens should be similar (for the same mixture type). The observed inconsistency could possibly be related to insufficient compaction of the $38 \mathrm{~mm}$ thick PFC specimen. In addition, with an average value of about $1.7 \mathrm{~mm}$, the MPD for the SMA mixture was lower than that measured for 
the PFC mixture. For comparison of those values to the field measurements, refer to discussion in section 4.3 of this report.

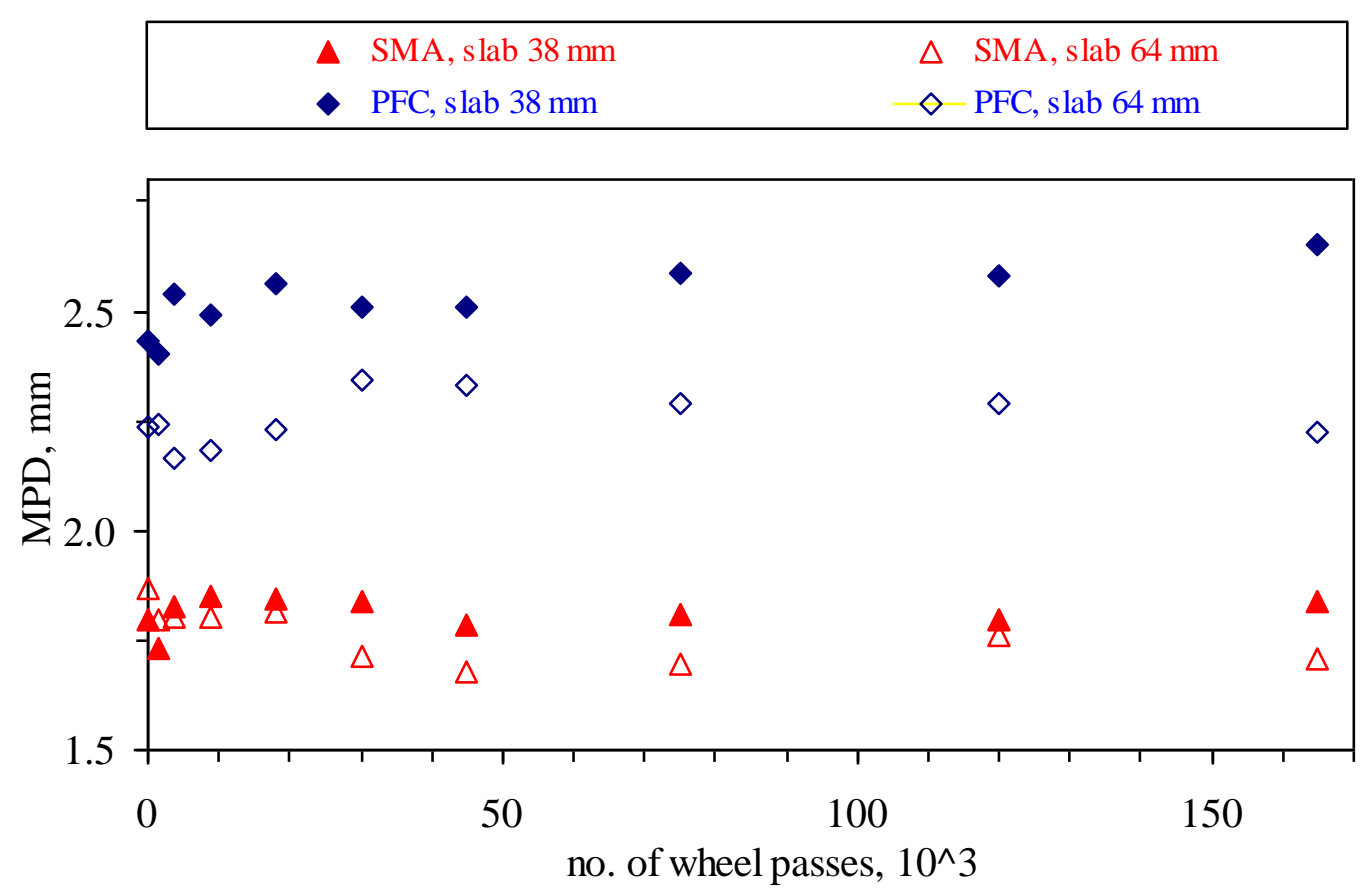

Figure 47. Changes in the macrotexture of the field mixtures polished in the laboratory

As shown in Figure 48, after the initial polishing stage was completed (end of zone I), the dynamic friction $\left(\mathrm{DF}_{20}\right)$ was found to be about 0.7 for SMA mixes and about 0.5 for the PFC specimens. The $64 \mathrm{~mm}$ thick PFC specimen experienced the highest variations in the dynamic friction values, while for the other samples those variations were less significant. Friction for both the 64 and $38 \mathrm{~mm}$ thick PFC specimens was similar. For the SMA specimens, higher values were noticed for the $64 \mathrm{~mm}$ thick specimen. Again, this difference could be related to the compaction of the specimen. 


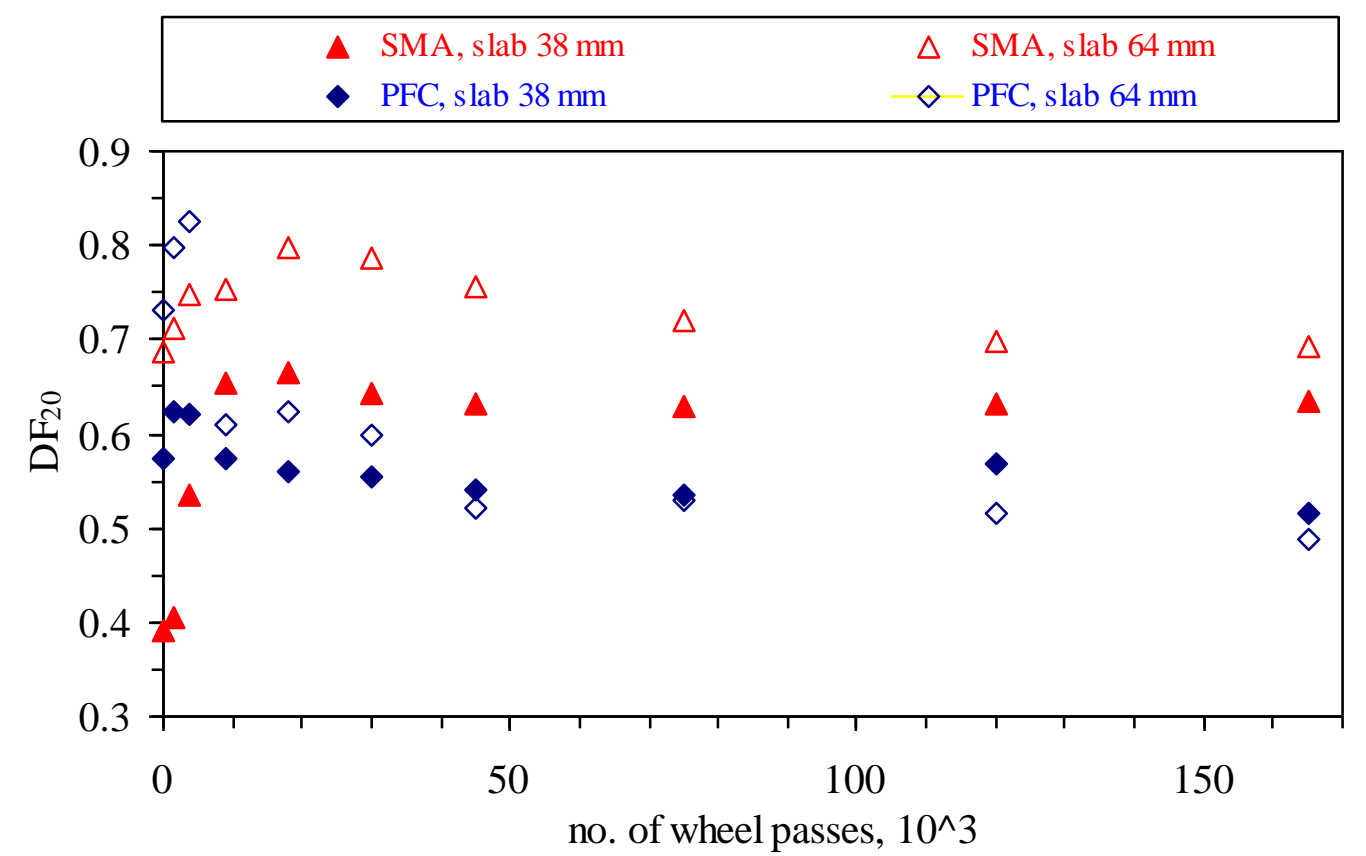

Figure 48. Changes in the dynamic friction $\left(\mathrm{DF}_{20}\right)$ values of the field mixtures polished in the laboratory

Based on the texture and friction measurements, the F60@ $\mathrm{x}_{1}$ values were calculated for each specimen and model parameters were generated. Model fitting of the PFC and SMA test data is shown in Figure 49. As expected, trends for the F60 values are similar to those observed for the $\mathrm{DF}_{20}$, as discussed in section 4.1. It should also be noticed that although the prediction model was developed for lab produced mixes tested in Matrices I to III, this model fits the results of PFC and SMA samples well (refer to $\mathrm{R}^{2}$ values presented in Table 20). 


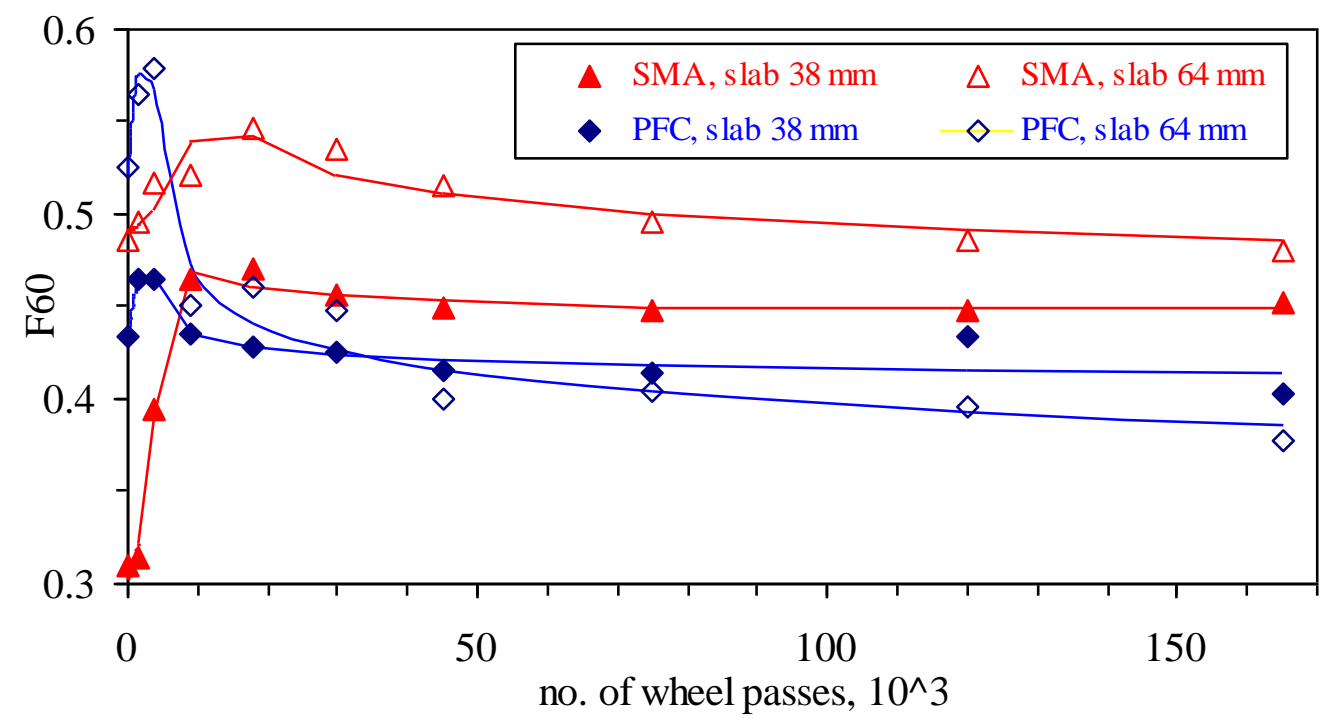

Figure 49. Frictional prediction model applied to the field mixtures polished in the laboratory

Parameters of the friction model are summarized in Table 20. It can be noticed that the polishing rate (parameter $\mathrm{a}_{4}$ ) has very low values in all four cases. Based on the specimen composition (high amount of the steel slag used) those mixtures could be compared to the mixes tested in Matrix II (mixes with 70\% steel slag). However, the polishing rates observed for the SMA and PFC specimens have even lower values (specimens are more resistant to polishing).

In addition, the terminal friction value $\left(\mathrm{F} 60 @ \mathrm{X}_{1}\right)$ for all four samples was above 0.38 , which is a high value (in comparison to the terminal friction values for specimens tested in Matrices I-III, where a maximum value of 0.36 was determined for the specimen with the $70 \%$ quartzite). 
Table 20. Summary of the polishing prediction applied to the PFC and SMA mixtures tested in the laboratory

\begin{tabular}{ccccc}
\hline $\begin{array}{c}\text { Mixture Type, } \\
\text { Specimen Thickness }\end{array}$ & SMA 3.8 cm & SMA 6.4 cm & PFC 3.8 cm & PFC 6.4 cm \\
\hline $\mathrm{R}^{2}$ & 0.99 & 0.79 & 0.86 & 0.96 \\
\hline $\mathrm{a}_{4}$ & -0.006 & -0.015 & -0.005 & -0.021 \\
$\mathrm{~F} 60 @ \mathrm{x}_{1}$ & 0.450 & 0.486 & 0.414 & 0.386 \\
$\mathrm{x}_{\mathrm{o}}$ & 7.1 & 15.0 & 7.0 & 7.5 \\
\hline
\end{tabular}

\subsubsection{Volumetric Properties}

To determine the actual air content in the test specimens, cores were extracted from friction test slabs and used for volumetric analysis. From each slab, two cores were extracted, both of them positioned in the polisher wheel track: from the "corner" and "side" locations (see Figure 20). It was found that the average absolute differences in air content between those two locations was about $1 \%$. In most cases, the "corner" location showed higher air content. It also was found that, in general, specimens with "coarse" and "fine" blend gradations were better compacted than those with the "s-shaped" gradation. Those observations could be related to the effect of the particles interlocking, which was probably more pronounced for the s-shaped mixtures. It is expected that for such mixtures a higher compactive effort would be needed to reach a compaction level similar to the one obtained in fine and coarse graded mixtures. The overall range of air contents in the actual slabs tested in Matrices I to III varied between $11.5 \%$ and $19.5 \%$, with the average value of $15 \%$ air voids. Detailed information about measured air contents is provided in the Appendix E, in Table E 1. The air content in the SMA slabs was $15.1 \%$ and $12.7 \%$ (for $38 \mathrm{~mm}$ and $64 \mathrm{~mm}$ thick slabs, respectively) and in the PFC slabs was $24.2 \%$ and $22.6 \%$ (for $38 \mathrm{~mm}$ and $64 \mathrm{~mm}$ thick slabs, respectively). 
This determined air content is higher than it was expected (about 8-10\% for all mixtures but PFC, for which about $22 \%$ voids was expected), based on the theoretical calculations which utilized the weight of the mix and the volume of the mold. Most likely reasons for this discrepancy are the relatively small mass of the compactor, relatively small height $(38$ and $64 \mathrm{~mm}$ ) and relatively small area of the molds (wall effects). It could be expected that the air voids would be preferentially generated in the contact areas between the sample and comparatively rough wooden base of the mold. The generation of these voids is more difficult to control in relatively thin specimens such as those used in this study. The void content of the top surface, however, which was in contact with the relatively smooth surface of the aluminum plate, could be expected to be lower than the bottom of the slab, so the effect on the friction and texture measurements may have been smaller than the average air void content might suggest.

The visual examination of the extracted cores revealed random orientation of particles in cores from both types of specimens and apparently higher void contents near the bottom of the slab..

\subsection{Field Study}

The focus of the field part of the study was on texture/friction related tests and properties. In addition, this section also presents development of the frictional baseline values and requirements. 


\subsubsection{Establishment of Baseline Friction Values and F60 Requirements}

In this portion of the study, the two friction measurement devices are compared and an attempt to determine the F60 friction requirement is presented. In addition, the friction value baseline, which could be used to evaluate the accuracy of the laboratory friction and polishing test method, is discussed.

\subsubsection{Theoretical Study: Friction Simulation using the PIARC Model}

The PIARC model (described in section 2.3.3.) was used to simulate the response of two different devices (the DFT and the towed friction trailer) hypothetically operated on a pavement with a certain frictional performance. This attempt was made to determine and to compare the theoretical response of those devices while operating on pavements with various macrotextures.

It should be noted that in order to generate the hypothetical speed constant $\left(S_{p}\right)$ data for those two devices, the pavement macrotexture data (MPD values) were assumed to be obtained from the CTM device and treated as a variable in the model. The assumed range of the MPD values was quite wide (from $0.3 \mathrm{~mm}$ to $2.7 \mathrm{~mm}$ ) covering the typically observed field macrotexture data. In addition, it was assumed that the friction trailer was equipped with both smooth and rib tires and that it was travelling at a speed of $64 \mathrm{~km} / \mathrm{h}$ (common speed for this device). The above assumptions allowed for an adherence to a realistic range of friction values while performing the simulations.

As implied by equations 9 to 11 and by equation 13 (discussed in section 2.3.3. and repeated below for the convenience of the reader), the $S_{p}$ value (when using the CTM device) can be calculated according to equation 10 while the F60 value (when using DFT 
device) can be calculated according to equation 9. The F60 value (when using the ASTM E 274 friction trailer) can be calculated according to equations 11 and 13 (for tests conducted using smooth and rib tires, respectively):

$$
\begin{array}{cc}
F 60=0.081+0.732 D F_{20} e^{\frac{-40}{s_{p}}} & \text { (repeated eq. 9) } \\
S_{p}=14.2+89.7 M P D & \text { (repeated eq. 10) } \\
F 60=0.045+0.925 \cdot 0.01 \cdot S N(64) S \cdot e^{\frac{4}{s_{p}}} & \text { (repeated eq. 11) } \\
F 60=-0.023+0.607 \cdot 0.01 \cdot S N(64) R \cdot e^{\frac{4}{s_{p}}}+0.098 \cdot M P D & \text { (repeated eq. 13) }
\end{array}
$$

In order to simplify the comparison of the friction trailer and DFT-based F60 values, it was first assumed that all virtually tested pavements had a relatively good calibrated wet friction with the F60 value equal to 0.35 . Since the macrotexture was used as a variable in these simulations, in order to reach the fixed value of $F 60=0.35$, the microtexture was also required to change.

Theoretical results of simulation tests conducted with both test devices (DFT and friction trailer) are shown in Figure 50. It could be noted that the friction trailer responses strongly depended on the type of test tire used. The $\mathrm{SN}(64)$ value for the smooth tire seems to steadily increase with an increase in the MPD value, while the SN decreases with an increase in the MPD for the rib tire. The rate of increase of the SN(64) value for the smooth tire was relatively low, with the overall change (over the entire range of MPD value) not exceeding $5 \mathrm{SN}$ units. At the same time, the rate of $\mathrm{SN}$ change 
for rib tire was higher as was the overall friction change (about $30 \mathrm{SN}$ units). The curves corresponding to these two types of tires intercept at an MPD value of about $1.75 \mathrm{~mm}$. At this MPD value the common SN number for both tires was about 32. For the DFT device, relatively large changes were noticed for virtual tests on a surface with poor and average macrotexture (MPD below $1 \mathrm{~mm}$ ) while for the surfaces with higher macrotexture, changes in the $\mathrm{DF}_{20}$ values were relatively small.

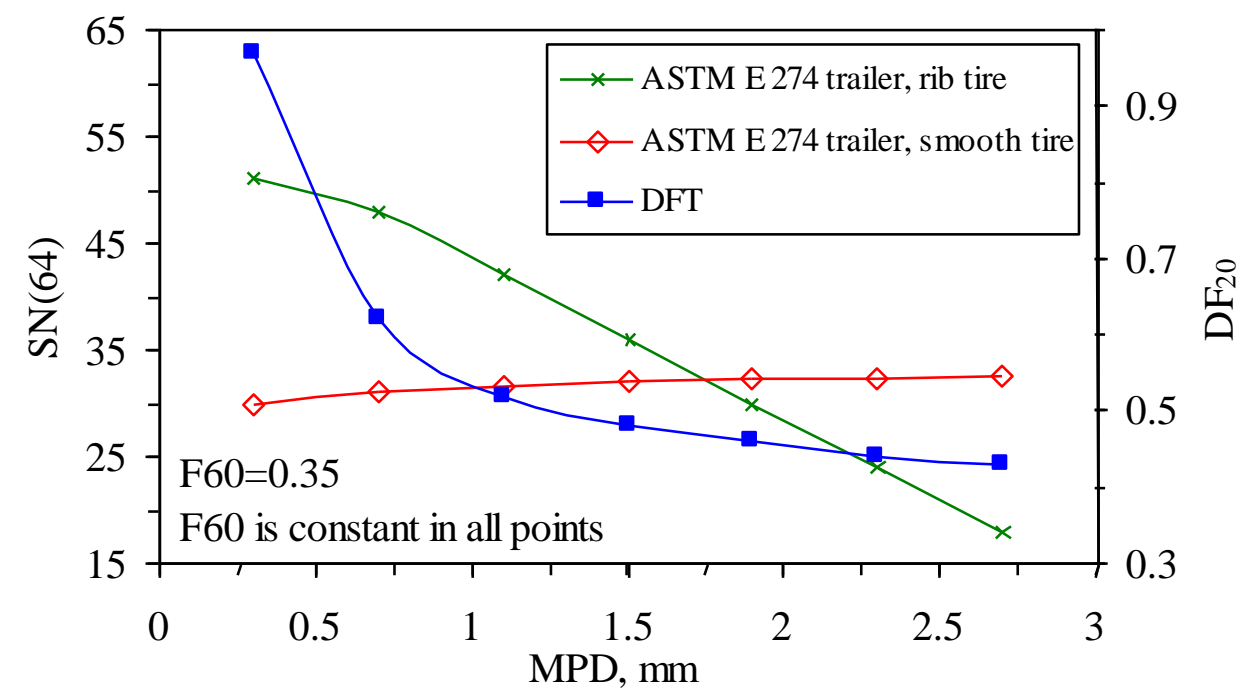

Figure 50. Theoretical relationship between MPD, SN and DF 20 (for ASTM E 274 friction trailer and DFT device, respectively)

Simulations showing the influence of $\mathrm{DF}_{20}$ and MPD values on the F60 values were described earlier (see section 2.3.3) and are shown in Figure 3. Similar simulations, this time applied to the friction trailer, are presented below. During the first case of this simulation (for the rib tire), $\mathrm{SN}(64) \mathrm{R}$ values were fixed $(\mathrm{SN}=35)$ and MPD data were changed in a range from 0.3 to $2.7 \mathrm{~mm}$. Corresponding F60 values were then calculated using the previously introduced equation 13 (for the friction trailer). (Note: In order to use equation 13, the MPD values were used to calculate Sp using equation 10.) Then, for 
these F60 and MPD values, the smooth tire $\mathrm{SN}(64) \mathrm{S}$ values were determined using equations 11 and 10 .

During the second case of this simulation, the smooth tire $\mathrm{SN}(64) \mathrm{S}$ values were fixed ( $\mathrm{SN}=35)$ and MPD data were again changed in a range from 0.3 to $2.7 \mathrm{~mm}$. Corresponding F60 values were then calculated using previously introduced equations 11 and 10. Then, for these F60 and MPD values, the $\mathrm{SN}(64) \mathrm{R}$ values were determined using equations 13 and 10. The results of simulations conducted for the friction trailer are shown in Figure 51. In the first case (Figure 51a) the $\mathrm{SN}(64)$ results for virtual tests with rib tire remain constant (MPD and thus F60 change) and virtual test results for the smooth tire are recalculated to fit the MPD and F60. In the second case (Figure 51b), the $\mathrm{SN}(64)$ results for virtual tests with the smooth tire remain constant while the results for the rib tire are adjusted.

(a)
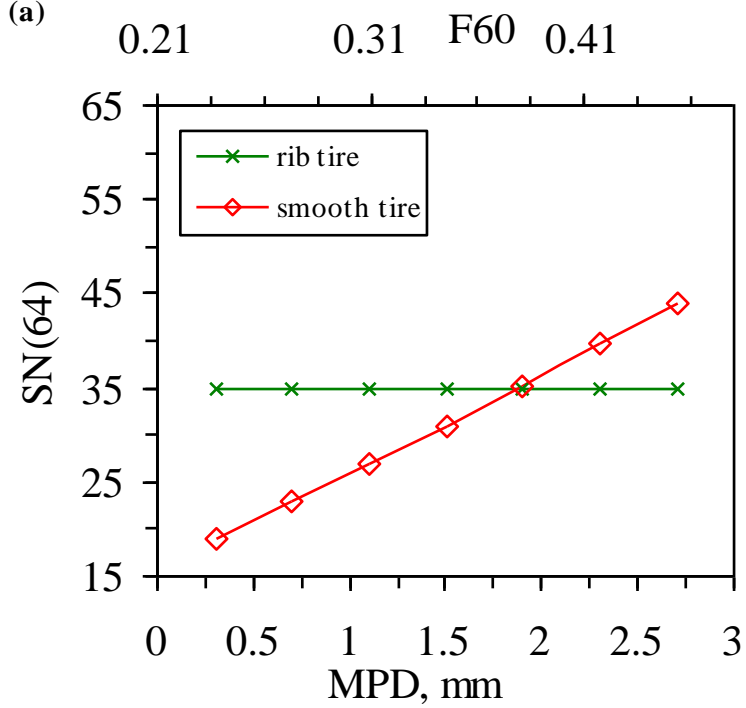

(b)


Figure 51. Theoretical relationship between MPD (and F60) for ASTM E 274 friction trailer: (a) SN(64) values for rib tire are fixed and for smooth are adjusted and (b) $\mathrm{SN}(64)$ values for smooth tire are fixed and for rib are adjusted Note: the reversed orientation of the F60 axis in Figure (b) 
From this simulation it could be noted that if the rib tire $\mathrm{SN}(64)$ results are fixed and macrotexture change from 0.3 to $2.7 \mathrm{~mm}$, the F60 will change from about 0.23 to 0.44. In addition, if the macrotexture and F60 change in this range, the $\mathrm{SN}$ for the smooth tire will change from about 20 to 43 . On the other hand, if the smooth tire $\mathrm{SN}(64)$ results are fixed and macrotexture changes from 0.3 to $2.7 \mathrm{~mm}$, the F60 will change from about 0.40 to 0.38 (will decrease). In addition, if the macrotexture and F60 change in this range, the $\mathrm{SN}$ for rib tire will change from about 54 to 20 . In other words, depending on the type of tire, there is a completely different trend on the sensitivity of the $\mathrm{SN}(64)$ values to changes in microtexture.

Finally, it could be observed that no single number could be employed to correlate between the results of tests conducted with the ASTM E 274 friction trailer using different tires.

\subsubsection{Development of Baseline Frictional Values}

In order to determine a baseline of the frictional properties for existing pavements, a total of 25 different sections were tested. Due to the commonly acknowledged seasonal changes in the frictional values, an attempt was made to compare different sections using data collected within about three months (August/October). The data presented in Table 21 were obtained in the following way:

- For DGA, SMA and PFC sections, the CTM/DFT tests were conducted in August and in October. Results were averaged to predict values in September. Friction trailer tests were conducted in September. 
- For test track sections (SC, TC and HMA-TT), the CTM/DFT and friction trailer tests were conducted several times in August and September. Data from this time period were averaged.

- For the HM-IN sections (sections 1 to 8), the CTM/DFT and friction trailer tests were conducted in a similar time period.

- For the HM-IA sections (sections 1 to 11 ), the CTM/DFT data were obtained in September 2006 and friction trailer tests were conducted in August/September 2007. The friction trailer SN values were interpolated from tests conducted in 2007 and from the historical data provided by Iowa DOT (sections were periodically tested every other year during the late summer/early fall season) to predict frictional values for the same time period as CTM/DFT tests were conducted.

Note that the CTM/DFT values may vary, depending on the tested spot's transverse location (as discussed in section 2.3.3). As used here, data from the left and right wheelpaths were averaged (data from the centerline path were excluded). For the complete sets of MPD and $\mathrm{DF}_{20}$ data refer to Appendix H, Table $\mathrm{H} 1$. 
Table 21. Baseline: summary of the frictional properties of the tested section

\begin{tabular}{cccccccc}
\hline Section & $\begin{array}{c}\text { Years in } \\
\text { Service } \\
\text { at Test }\end{array}$ & $\begin{array}{c}\text { Cumulative } \\
\text { Traffic, } \\
\text { NVA, } 10^{\wedge} 6\end{array}$ & $\begin{array}{c}\mathrm{MPD}, \\
\mathrm{mm}\end{array}$ & $\mathrm{DF}_{20}$ & $\begin{array}{c}\text { F60 (from } \\
\text { CTM/DFT) }\end{array}$ & $\mathrm{SN}(64) \mathrm{S}^{\mathrm{a}}$ & $\mathrm{SN}(64) \mathrm{R}^{\mathrm{a}}$ \\
PFC & 3 & 46.7 & 1.41 & 0.57 & 0.40 & 48 & $*$ \\
SMA & 3 & 46.7 & 1.03 & 0.68 & 0.42 & 51 & $*$ \\
DGA & 3 & 22.8 & 0.57 & 0.46 & 0.26 & 29 & $*$ \\
\hline HMA-TT & $\mathrm{b}$ & $\mathrm{b}$ & 0.84 & 0.58 & 0.35 & $45(38)^{\mathrm{a}}$ & $52(43)^{\mathrm{a}}$ \\
SC & $\mathrm{b}$ & $\mathrm{b}$ & 0.06 & 0.40 & 0.12 & $11(5)^{\mathrm{a}}$ & $33(15)^{\mathrm{a}}$ \\
TC & $\mathrm{b}$ & $\mathrm{b}$ & 1.36 & 0.88 & 0.56 & $67(60)^{\mathrm{a}}$ & $68(60)^{\mathrm{a}}$ \\
\hline HM-IN-1 & 2 & 6.1 & 0.40 & 0.52 & 0.25 & 47 & 54 \\
HM-IN-2 & 1 & 1.1 & 0.30 & 0.50 & 0.22 & 33 & 54 \\
HM-IN-3 & 1 & 1.3 & 0.33 & 0.55 & 0.24 & 45 & 57 \\
HM-IN-4 & 10 & 54.9 & $*$ & $*$ & $*$ & 22 & $*$ \\
HM-IN-5 & 10 & 54.9 & $*$ & $*$ & $*$ & 28 & $*$ \\
HM-IN-6 & 1 & 1.0 & 0.37 & 0.61 & 0.27 & 37 & 58 \\
HM-IN-7 & 1 & 0.7 & 0.35 & 0.69 & 0.29 & 58 & 62 \\
HM-IN-8 & 1 & 1.3 & 0.38 & 0.65 & 0.29 & 45 & 58 \\
\hline HM-IA-1 & 5 & 17.2 & 0.66 & 0.48 & 0.28 & $*$ & 54 \\
HM-IA-2 & 15 & 119.7 & 0.67 & 0.38 & 0.25 & $*$ & 47 \\
HM-IA-3 & 9 & 15.2 & 0.49 & 0.25 & 0.17 & $*$ & 28 \\
HM-IA-4 & 22 & 59.3 & 0.94 & 0.32 & 0.24 & $*$ & 34 \\
HM-IA-5 & 15 & 37.7 & 0.87 & 0.22 & 0.19 & $*$ & 30 \\
HM-IA-6 & 15 & 25.1 & 1.27 & 0.66 & 0.44 & $*$ & 63 \\
HM-IA-7 & 14 & 38.2 & 0.63 & 0.62 & 0.34 & $*$ & 54 \\
HM-IA-8 & 11 & 36.7 & 0.79 & 0.51 & 0.32 & $*$ & 58 \\
HM-IA-9 & 24 & 32.8 & 0.23 & 0.27 & 0.14 & $*$ & 34 \\
HM-IA-10 & 17 & 153.7 & 1.26 & 0.27 & 0.22 & $*$ & 33 \\
HM-IA-11 & 11 & 35.9 & 0.69 & 0.41 & 0.26 & $*$ & 46 \\
\hline & & & & & & & \\
\hline
\end{tabular}

* data not available,

${ }^{a}$ for test track, tests were conducted at $48 \mathrm{~km} / \mathrm{h}$ (instead of $64 \mathrm{~km} / \mathrm{h}$ used for all other sections); using equations 6 and 8, SN values could be adjusted to the speed of $64 \mathrm{~km} / \mathrm{h}$ (shown in parentheses);

${ }^{\mathrm{b}}$ test track is closed to traffic other than the friction test vehicles.

When available, information on the section age and traffic volume was also collected but no direct correlation between these variables and frictional properties was found. 
As expected, the SC (smooth concrete) section exhibited the lowest values of macrotexture while the SMA, PFC and TC (tined concrete) sections had MPD values in the upper range. In addition to these "less common" surfaces, two dense graded asphalt sections (namely HM-IA-6 and HM-IA-10) had macrotexture with MPD values greater than $1 \mathrm{~mm}$. It should also be noted that all asphalt sections with high macrotexture values also had relatively high $(\mathrm{FM}>4.6)$ values of fineness modulus (refer to Tables 10 and 12 for details). The typical MPD values of sections constructed after the middle 1990s were in the range between 0.6 to $0.9 \mathrm{~mm}$. Low MPD was observed for section HM-IA-9; for this mixture the FM value was also low (FM=3.48).

Similarly, high $\mathrm{DF}_{20}$ values (which were about 0.6 to 0.9 ) were observed for the PFC, SMA and TC sections. For typical dense graded asphalt sections, those values were about 0.4 to 0.6. In most cases, sections with low $\mathrm{DF}_{20}$ values $\left(\mathrm{DF}_{20}\right.$ below 0.3$)$ were typically constructed a long time ago $(15+$ years $)$ with mixes containing significant amounts of limestone.

For the limited number of asphalt sections where SN values using the smooth tire were determined, those values generally ranged between 23 and 50 (at $64 \mathrm{~km} / \mathrm{h}$ ). For tests with the rib tire, the common range was between 35 and 60 (at $64 \mathrm{~km} / \mathrm{h}$ ). Comparison of $\mathrm{SN}$ and $\mathrm{DF}_{20}$ values is presented in the subsequent section.

It should be noted that for the pavements tested in this study, the usual difference between the highest and lowest results for the various tests (when tests were conducted on the same day by one operator) were in the range of $0.08 \mathrm{~mm}$ (when MPD was determined), 0.05 (when $\mathrm{DF}_{20}$ was determined), 5-6 (when $\mathrm{SN}(64) \mathrm{S}$ was determined) and 
5-6 (when SN(64)R was determined). These values, then, could be considered representative of typical single-operator testing variability.

\subsubsection{Frictional Requirements}

In this study, an attempt was made to determine the calibrated wet friction (F60) flag value. The analysis shown here utilizes the $\mathrm{SN}$ requirement of $\mathrm{SN}(64) \mathrm{R}=38$ [Kummer and Meyer 1967] and SN(64)S=20 [Li et al. 2003] (discussed in detail in chapter 2.5) to develop the recommended value of F60.

The MPD, $\mathrm{DF}_{20}, \mathrm{SN}(64) \mathrm{S}$ and $\mathrm{SN}(64) \mathrm{R}$ data shown in Table 21 were used as inputs to the previously discussed friction prediction model to develop the F60 values (see section 2.3.3). For the convenience of the reader, the formulas needed to determine F60 values are repeated below:

$$
\begin{array}{cc}
S_{p}=14.2+89.7 M P D & \text { (repeated eq. 10) } \\
F 60=0.081+0.732 D F_{20} e^{\frac{-40}{s_{p}}} & \text { (repeated eq. 9) } \\
F 60=0.045+0.925 \cdot 0.01 \cdot S N(64) S \cdot e^{\frac{4}{s_{p}}} & \text { (repeated eq. 11) } \\
F 60=-0.023+0.607 \cdot 0.01 \cdot S N(64) R \cdot e^{\frac{4}{s_{p}}}+0.098 \cdot M P D & \text { (repeated eq. 13) }
\end{array}
$$

To differentiate F60 values determined using the two different friction measurement devices (DFT and friction trailer), F60 values calculated using DFT data are referred to as "F60" but the F60 values calculated using friction trailer test results are 
referred to as "F60 TST" and "F60 TRT" for tests conducted with smooth and rib tires, respectively.

As shown in Figure 52, there is a fair correlation $\left(\mathrm{R}^{2}=0.61\right)$ between $\mathrm{F} 60$ and F60 TST. Better $\left(\mathrm{R}^{2}=0.79\right)$, but still not perfect, is the correlation between F60 and F60 TRT. It could also be noticed that the F60 values developed from the DFT measurements are lower than those developed from tests with the friction trailer.
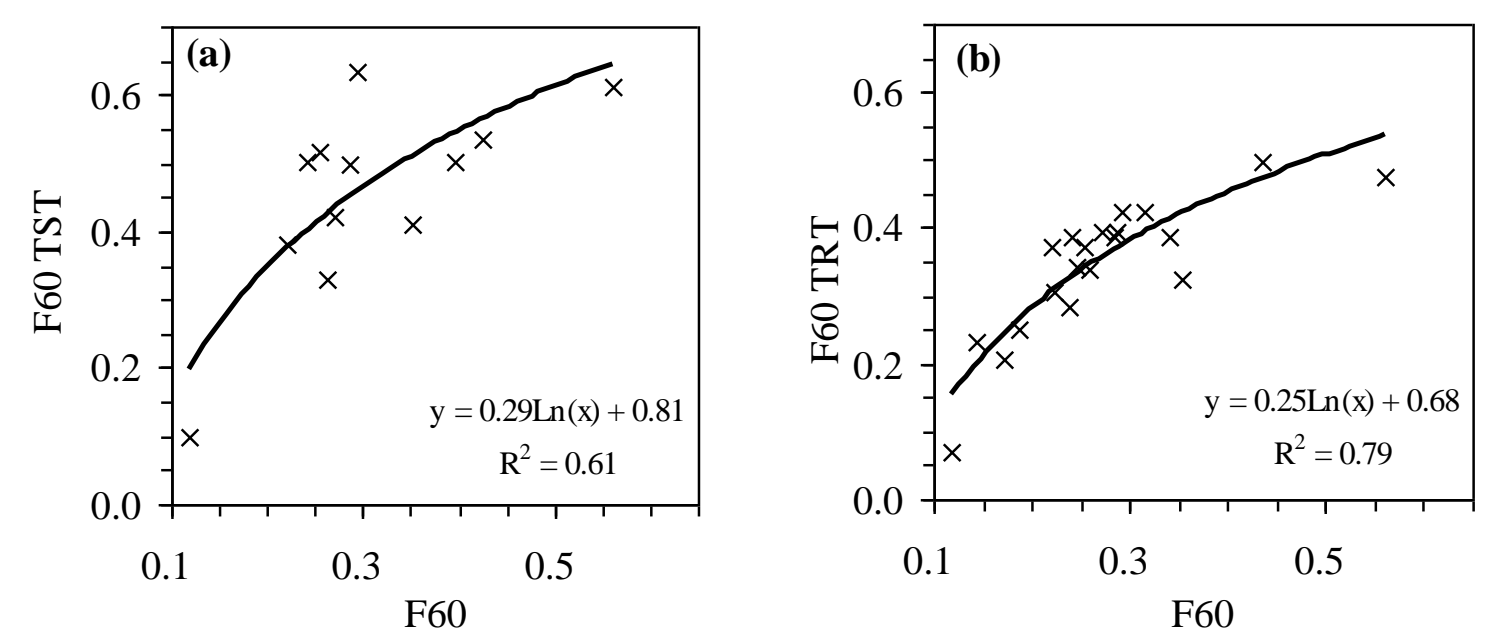

Figure 52. Comparison of calibrated wet friction values (F60) developed from DFT device with F60 calculated using friction trailer data: using (a) smooth (F60 TST) and (b) rib (F60 TRT) tire

Due to the limited number of sites (eight sites only) where tests with the friction trailer using both the rib and smooth tire were conducted, the relationship between F60 TST and F60 TRT is, from the statistical point of view, limited. However, as shown in Figure 53, a linear relationship with a relatively high $\mathrm{R}^{2}$ equal to 0.87 exists. 


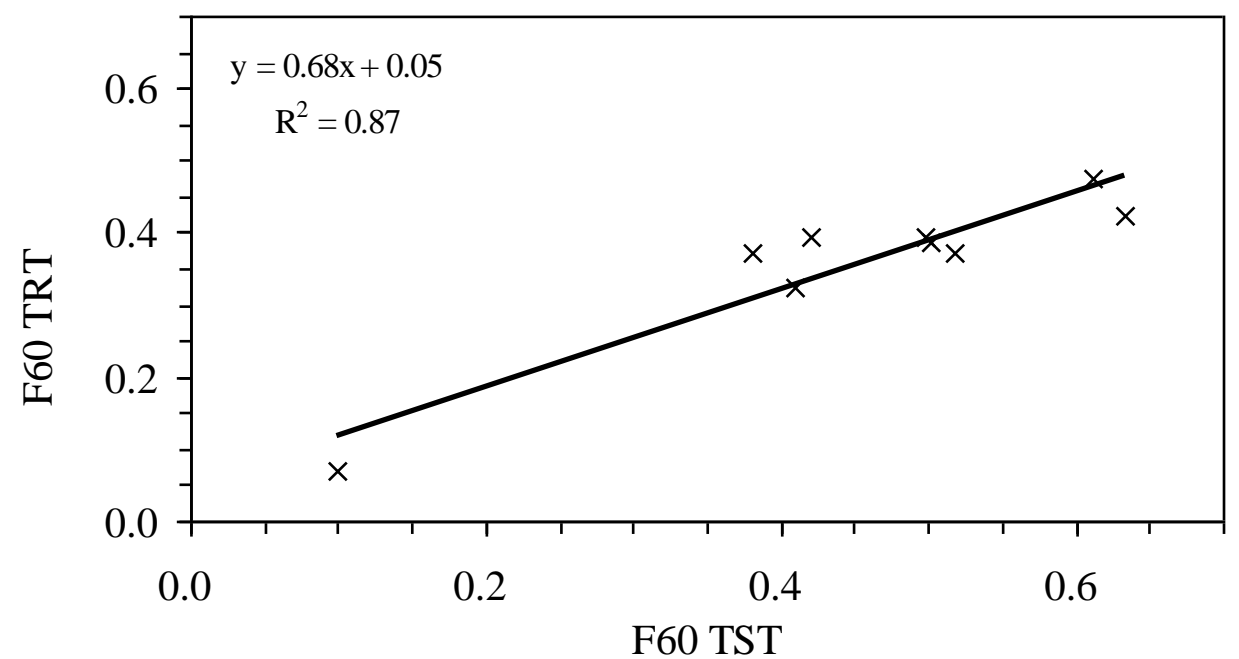

Figure 53. Comparison of calibrated wet friction (F60) developed from friction trailer tests using smooth (F60 TST) and rib (F60 TRT) tire

Although the calibration factors developed by the international PIARC study (Wambold et al. 1996) were applied to current data used for calculation of the F60 values, thus theoretically ensuring equivalency of results from different devices, the analysis shown above indicates that there were some differences. The possible reasons are discussed later in this section.

The relationship between friction trailer (using rib tire) test results and the corresponding F60 value is shown in Figure 54a, while a similar relationship, but for the smooth tire, is shown in Figure 54b. Based on those relationships, the requirement of $\mathrm{SN}(64) \mathrm{R}=37$ [Kummer and Meyer 1967] could be correlated with the F60 value; the corresponding number would be $\mathrm{F} 60=0.27$.

Next, the $F 60=0.27$ could be correlated with the trailer test with smooth tire; the corresponding value would be $\mathrm{SN}(64) \mathrm{S}=22$. This value of 22 is close to the flag value accepted by INDOT [Li et al. 2003], where SN(64)S=20. 



Figure 54. Development of the F60 flag values for friction trailer with: (a) rib (F60 TRT) and (b) smooth (F60 TST) tire

The impression is that $\mathrm{F} 60=0.27$ could also represent the flag value for tests conducted with the CTM and DFT devices. However, based on the relationships shown in Figure 52, F60 values developed from the various types of equipment are not equal. Based on those plots, the flag value should be lowered to 0.17-0.20 (note that this lowered F60 value corresponds well with the friction threshold level accepted for highways in New Zealand [Yeaman 2005]).

It should be noted that the F60 values developed during the international PIARC study [Wambold et al. 1996] were an attempt to harmonize various friction measuring devices. Data presented here shows only a limited relationship between the various friction measuring techniques. As is always the case during testing, equipment and human inconsistency may play an important role. It is also possible that the equipment used during the international PIARC study had slightly different characteristics than that used here, so those applied correction factors may contain an error. Note also that the 
same devices were used for all the CTM and DFT measurements; moreover, only three operators conducted those tests. However, four different ASTM E 274 friction trailers and multiple operators conducted the towed friction tests.

\subsubsection{Variations in Frictional Properties}

In this part of the report, the influence of traffic, seasons of the year and transverse location within the driving lane is discussed.

\subsubsection{Periodic Tests of Highway Test Sections}

Three highway test sections (DGA, PFC and SMA) were periodically tested for friction. The cumulative traffic level on these sections was expressed as the number of vehicle axles. This number was calculated based on the previously defined number of vehicle axles (NVA) passing the test section multiplied by the period of time when the sections were exposed to traffic (see section 3.3.2.3). Note that this is only an approximate calculation and thus it may contain errors. However, for the purpose of this study, this approximated calculation should be precise enough. Test data shown in this section were averaged over the specific section and transverse position (left, center and right wheel path) for the DFT/CTM devices and over the specific section for the friction trailer (tests were conducted in left wheel path only).

Changes in the macrotexture (expressed by MPD values) are shown in Figure 55 (dashed lines are used to express the MPD range for each section). The MPD value for the PFC section was about $1.4 \mathrm{~mm}$ while for the SMA and DGA sections it was about 1.1 $\mathrm{mm}$ and $0.5 \mathrm{~mm}$, respectively. For the SMA and PFC sections, the MPDs obtained from 
the pavements before they were opened to traffic were similar to those collected at later ages. However, for DGA the macrotexture increased after the initial tests (conducted soon after opening to traffic).

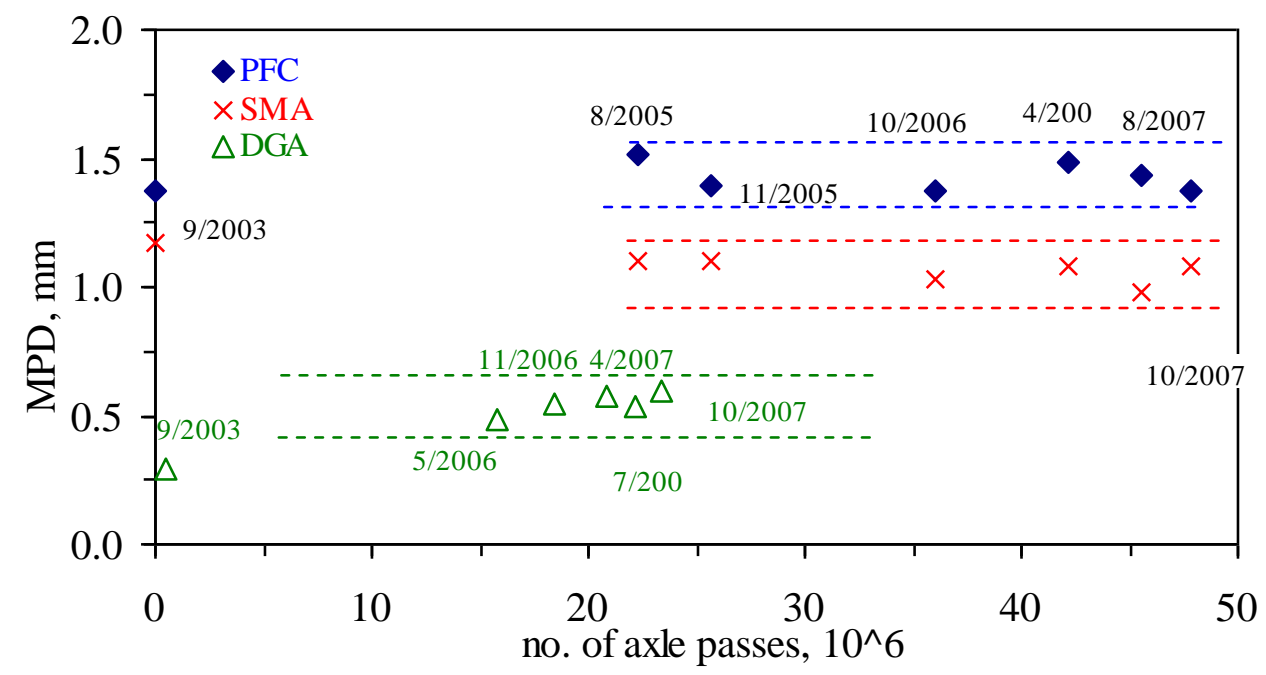

Figure 55. Changes in macrotexture (MPD) over time and traffic for PFC, SMA and DGA sections

Changes in the dynamic friction $\left(\mathrm{DF}_{20}\right)$ values are shown in Figure 56. It can be observed that the $\mathrm{DF}_{20}$ values for the PFC and SMA pavements not yet opened to traffic were lower than those measured at later ages. This finding is not surprising, as a new pavement typically has lower microtexture due to the film of binder coating the aggregate surface. This film is typically quickly removed by the polishing action of tires, resulting in increased friction. For the DGA section, however, no significant changes in the $\mathrm{DF}_{20}$ values were observed when initial test results were compared with those collected at later ages. The DGA surface layer was placed about six weeks before the friction test and was exposed to moderate car traffic and limited truck traffic prior to the first test. 


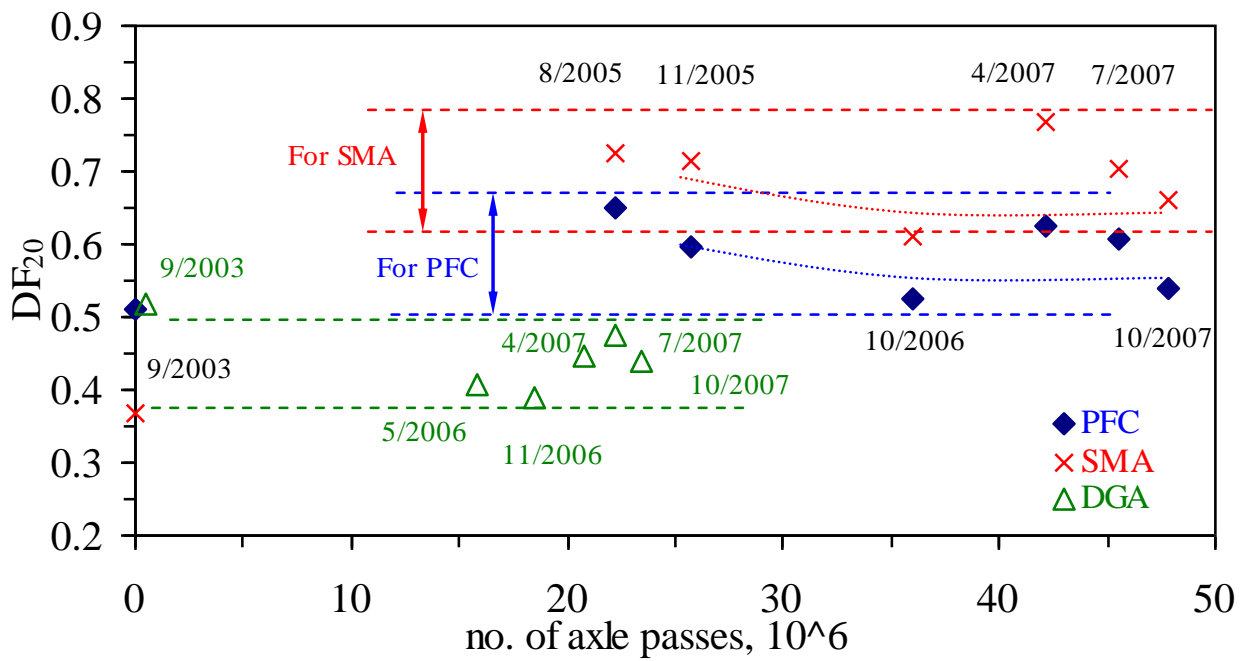

Figure 56. Changes in dynamic friction $\left(\mathrm{DF}_{20}\right)$ as a function of time and traffic for PFC, SMA and DGA sections

For the analysis of the variations in $\mathrm{DF}_{20}$ values, not only the total cumulative traffic should be taken into account, but also the date when the tests were conducted. Indeed, the seasonal variations reported in the literature could be observed in the test results reported here. For example, when comparing the test results obtained during different months of 2007 it can be observed that $\mathrm{DF}_{20}$ values recorded in August were higher than those recorded in October.

When only the friction data of tests conducted in a similar season (in October and November) of 2005-2007 are compared for the same section, average $\mathrm{DF}_{20}$ values would be about 0.60 for PFC, 0.73 for SMA and 0.42 for DGA.

It was noticed that results of tests conducted in the Fall of 2006 (for all three sections) were lower than the average values. This can be most likely explained either as a seasonal variation phenomenon or as a measurement error. 
Results of the calibrated wet friction F60 values (calculated using both the DFT and the CTM) are shown in Figure 57. The general trend in this data followed previously mentioned results of tests with the DFT device alone ( $\mathrm{DF}_{20}$ values). Again, when only the results of tests conducted during similar months of different years (from September to November) are compared, a decreasing logarithmic line between results (for the same section/pavement type) can be drawn. The F60 value would then be about 0.41 for PFC, 0.46 for SMA and 0.24 for DGA.

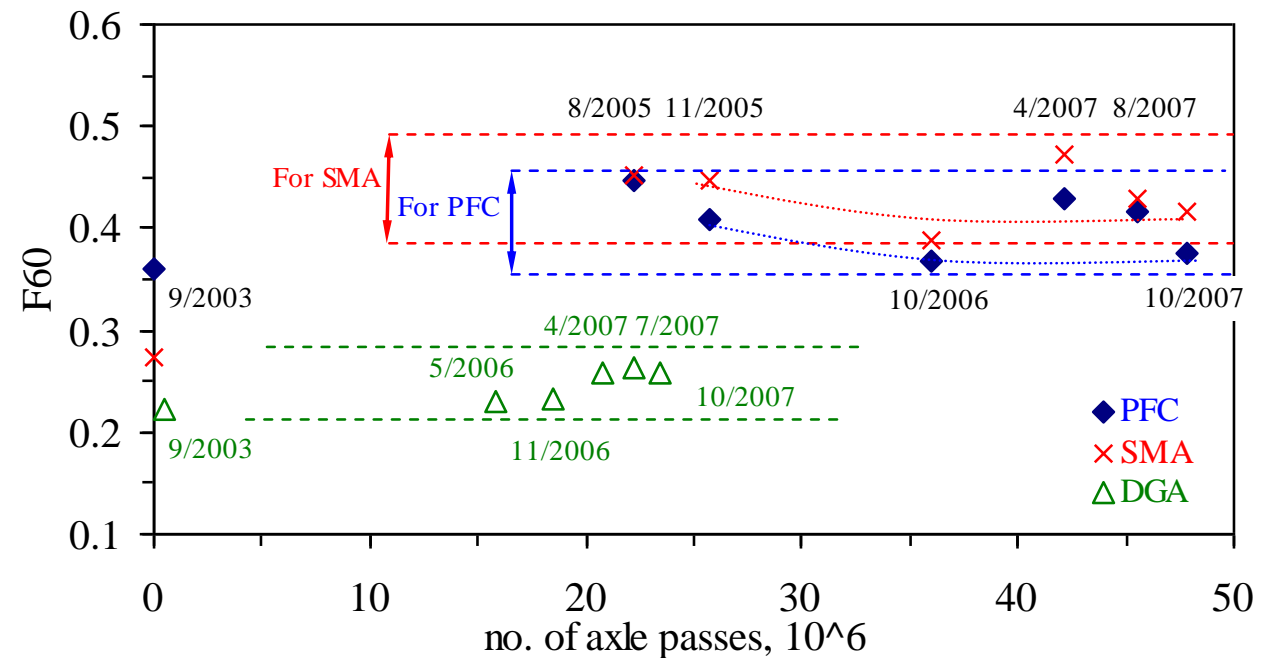

Figure 57. Changes in calibrated wet friction (F60) over time and traffic for PFC, SMA and DGA sections

In addition to the friction measurements conducted with the DFT device, changes in friction were also monitored using the ASTM E 274 friction trailer. The test results from the friction trailer, presented in Figure 58, seem to correspond well with those presented previously for the DFT device (see Figure 57). For example, for the PFC and SMA sections, the parabolic trend in the data is clearly visible (expressed as dotted curves). Note that for the PFC and SMA sections, the friction tests were conducted during the Fall season only to avoid seasonal variations. 
Although the presented set of data is limited to four years only, friction data presented in Figures 57 and 58 for the SMA section appears to have stabilized. The distribution of data for the PFC section suggests that the friction may continue to decrease, however only slightly. The maximum friction value overall was observed for the PFC section $(\mathrm{SN}=57)$ while for the SMA section the maximum SN was 54.

A wider range of changes of the friction value $(\mathrm{SN})$ was observed for the PFC section than for the SMA. For tests conducted with the CTM and DFT devices during similar seasons of different years, the relative changes in F60 values were similar for both the SMA and PFC sections. F60 values for the SMA section were higher.

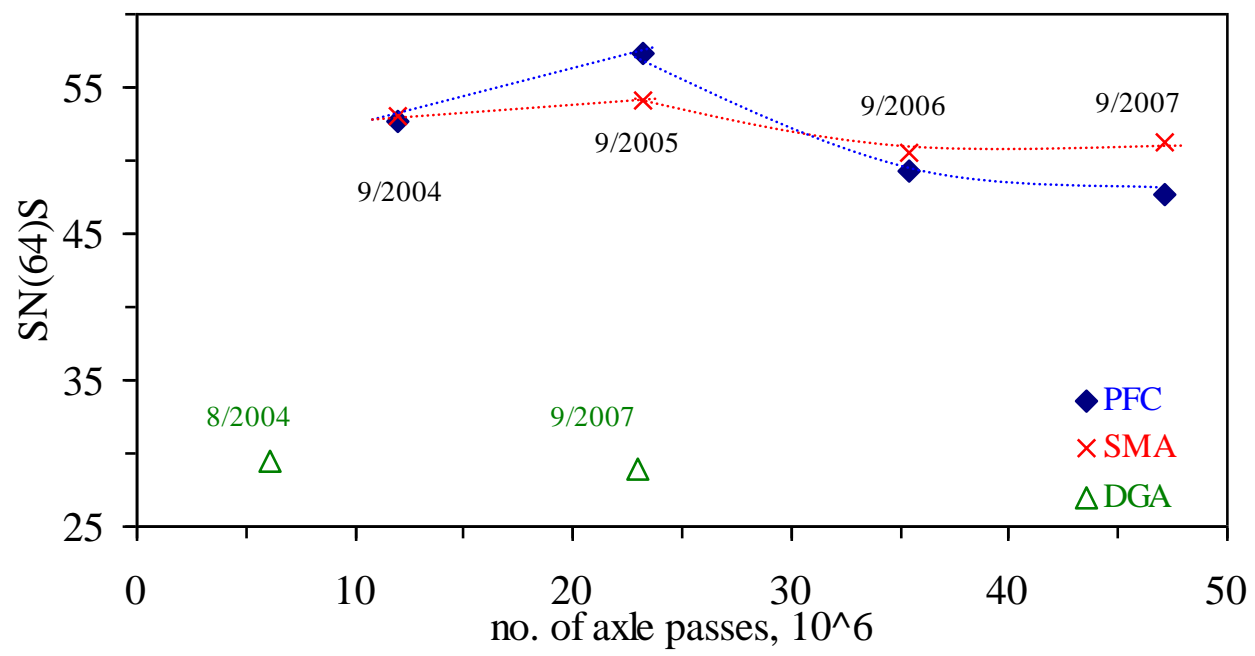

Figure 58. Changes in ASTM E-274 friction trailer measurements (SN(64)S) as a function of traffic for PFC, SMA and DGA sections

Significantly lower frictional values were observed for the DGA section with both the DFT and friction trailer. The friction of this section was tested by the trailer only two times, in 2004 and in 2007. Friction values obtained from these two sets of measurements were similar (SN about 27). With such limited data, it is hard to conclude whether the friction values of this section followed any trend. 
The data presented here confirmed that environmental factors indeed influence the friction. Both short-term (seasonal) and long-term (year-to-year) frictional variations were observed.

In order to provide an additional type of friction data, which could be particularly useful for those researchers who are still using the BSRT device, a limited number of tests with this equipment were also conducted. During those measurements (conducted in the same spots and at the same time as the last friction tests with the DFT/CTM devices), all three sections were tested. Test results were averaged accounting for the device orientation (transverse, longitudinal and diagonal) and for location of the test spot within the specific section. Again, the SMA section had the highest friction $(\mathrm{BPN}=60)$ while the DGA section had the lowest $(\mathrm{BPN}=51)$. For PFC section the average BPN value was equal to 57.

\subsubsection{Periodic Tests of Test Track Sections}

Frictional properties of the INDOT test track were investigated from June 2005 for a period of two years. The data presented here reflects the average properties for each given section.

Results of the macrotexture measurements are shown in Figure 59. For the SC section, the MPD values were found to be very low and relatively constant. However, for HMA-TT and for TC section, variations in the results could be observed. MPD values for TC section oscillated about the MPD of $1.36 \mathrm{~mm}$, while for HMA-TT section, a slight increase in the MPD value could be observed. A visual examination of the HMA-TT section suggested that in some spots some of the aggregate particles appeared to have 
been mechanically detached from the surface (possibly due to the forces caused by the trailer braking action).



Figure 59. Macrotexture (MPD) values collected from the INDOT test track for three different surfaces: TC, HMA-TT and SC

Changes in the dynamic friction $\left(\mathrm{DF}_{20}\right)$ value for the same surfaces are shown in Figure 60. A relatively large variation in the test results can be observed. Variations were possibly influenced by the weather conditions. It is expected that seasonal variations would be more significant for the test track, which is closed to traffic, than for highways. For such roads, dust can accumulate on the surface of the road, especially in the absence of "suction" provided by the tires. This dust layer may significantly change the frictional properties of the surface.

Tests conducted during the summer of 2007 were more frequent than earlier. The upward trend in the $\mathrm{DF}_{20}$ values (observed for all three sections tested in the 2007) could be related to weather changes during the summer (humidity, temperature) which caused changes in the dust accumulation on the surface. 


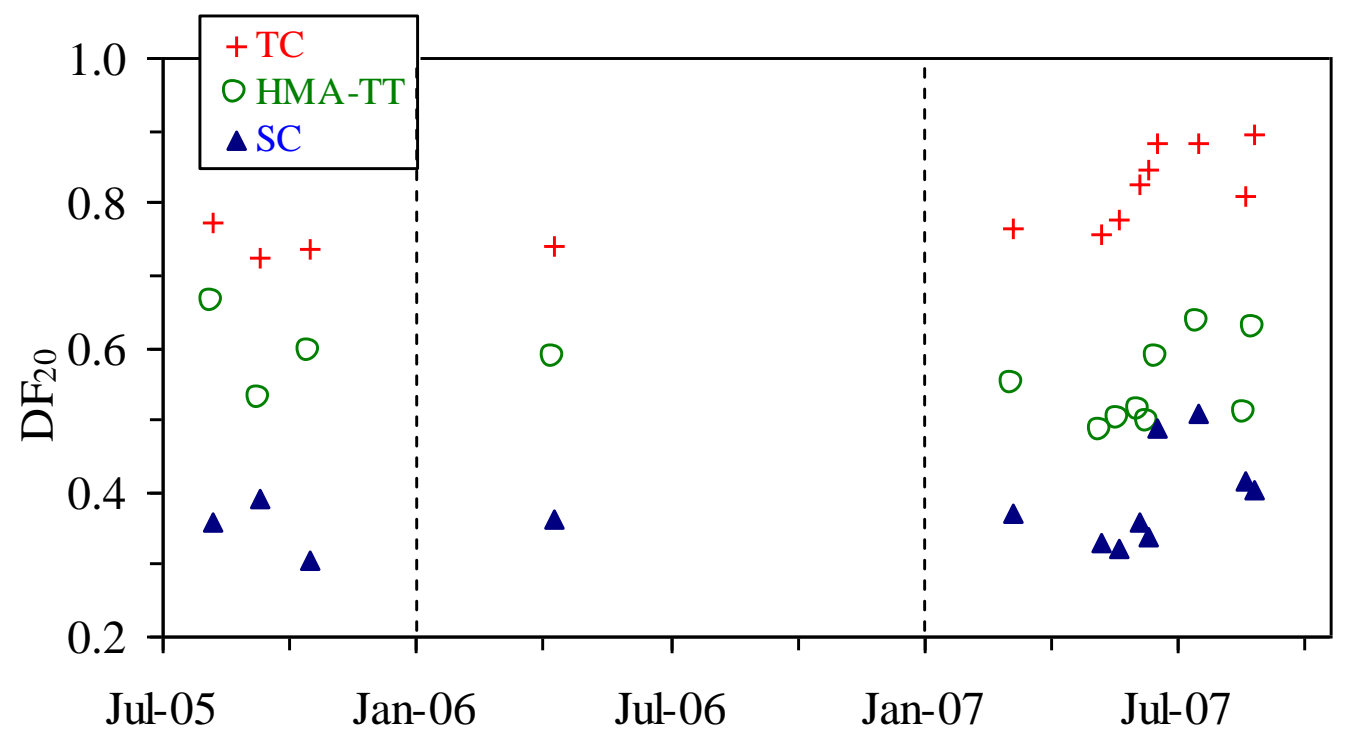

Figure 60. Dynamic friction $\left(\mathrm{DF}_{20}\right)$ values collected from the INDOT test track for three different surfaces: TC, HMA-TT and SC

As expected, the general trend of the wet pavement friction F60 (presented in Figure 61) corresponds well with the trend for $\mathrm{DF}_{20}$.

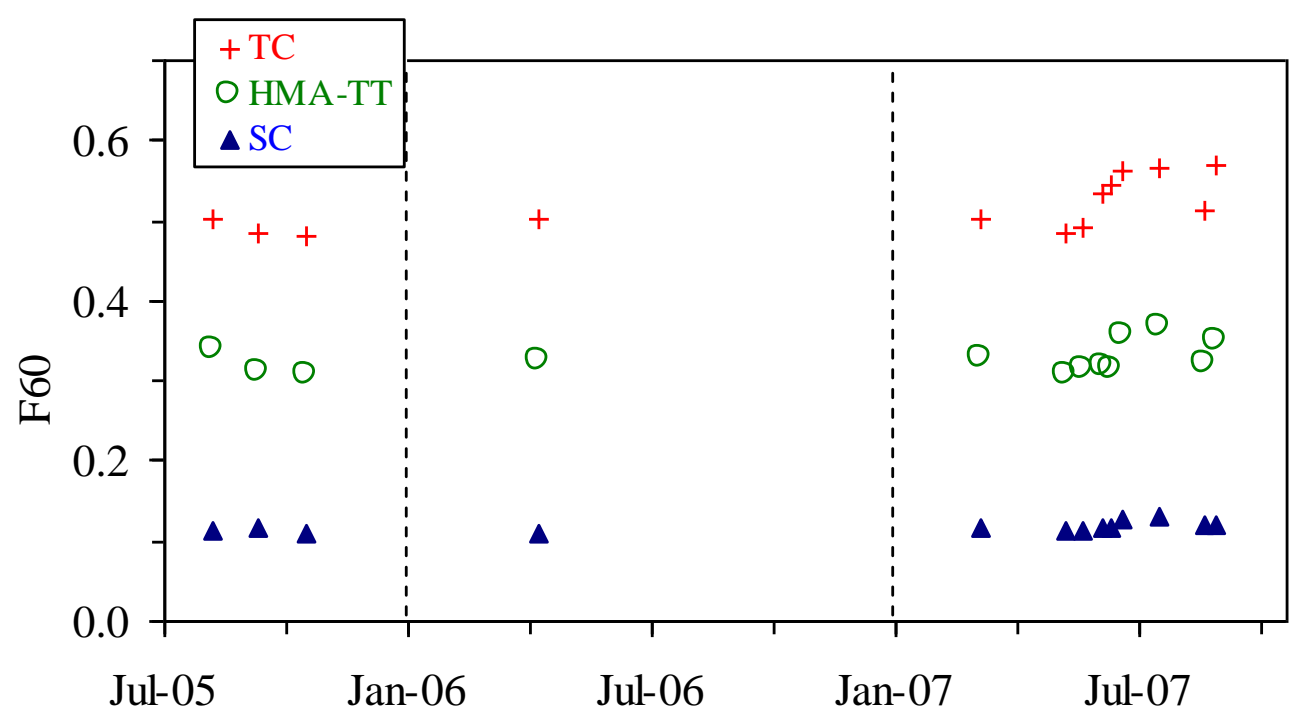

Figure 61. Calibrated wet friction (F60) values observed for the INDOT test track 
Results of the friction measurements conducted with the ASTM E 274 towed friction trailer using the smooth tire are presented in Figure 62. It can be observed that for three sections tested (SC, TC and HMA-TT), the changes in the friction follow a similar trend (within a given time period). Due to much lower values of friction for the SC section, no definite trends were observed.

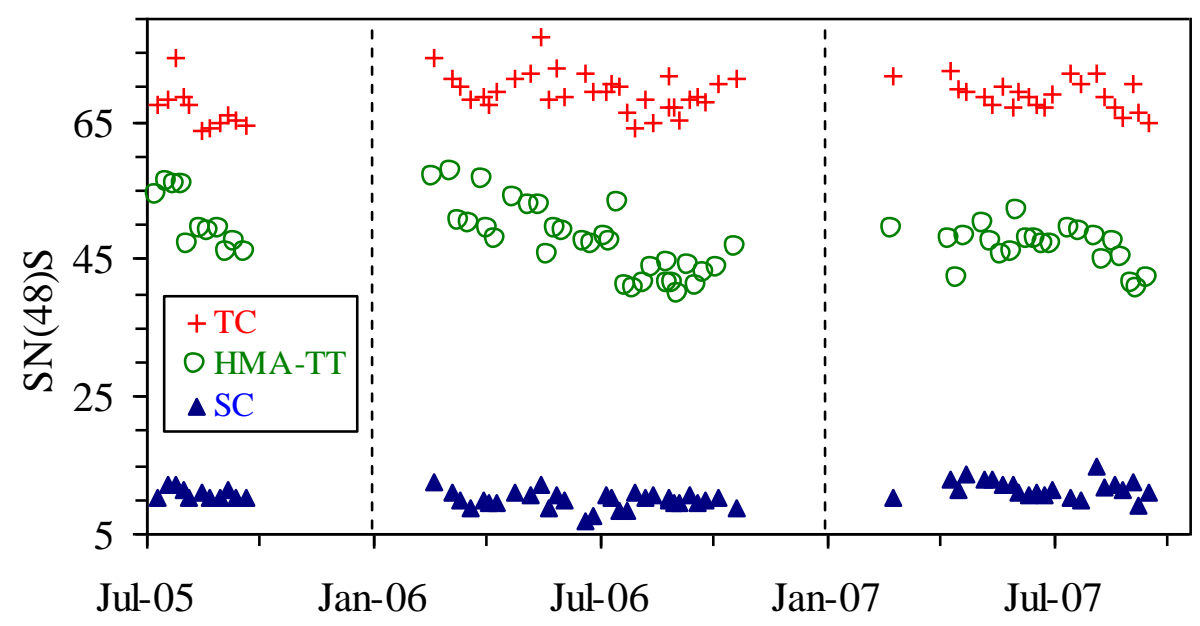

Figure 62. Skid number (measured by (ASTM E 274) friction trailer) on the INDOT test track monitoring: results for tests with smooth tire

For the HMA-TT section, a slight decrease in the skid number is observed, when results from three different years are compared. This observation could be related to changes in the macrotexture of the HM-TT section (refer to Figure 59).

Results of the friction tests conducted with the friction trailer using a rib tire are presented in Figure 63. This set of data is more limited, since tests with the rib tire were conducted only monthly as opposed to tests with a smooth tire that were conducted weekly. Although the results of tests with those two tires have different values (especially for the smooth concrete section), the general trend remains the same (compare 
Figure 62 and 63). Relative differences between results of tests with rib and smooth tires are discussed in section 4.2.1.1.

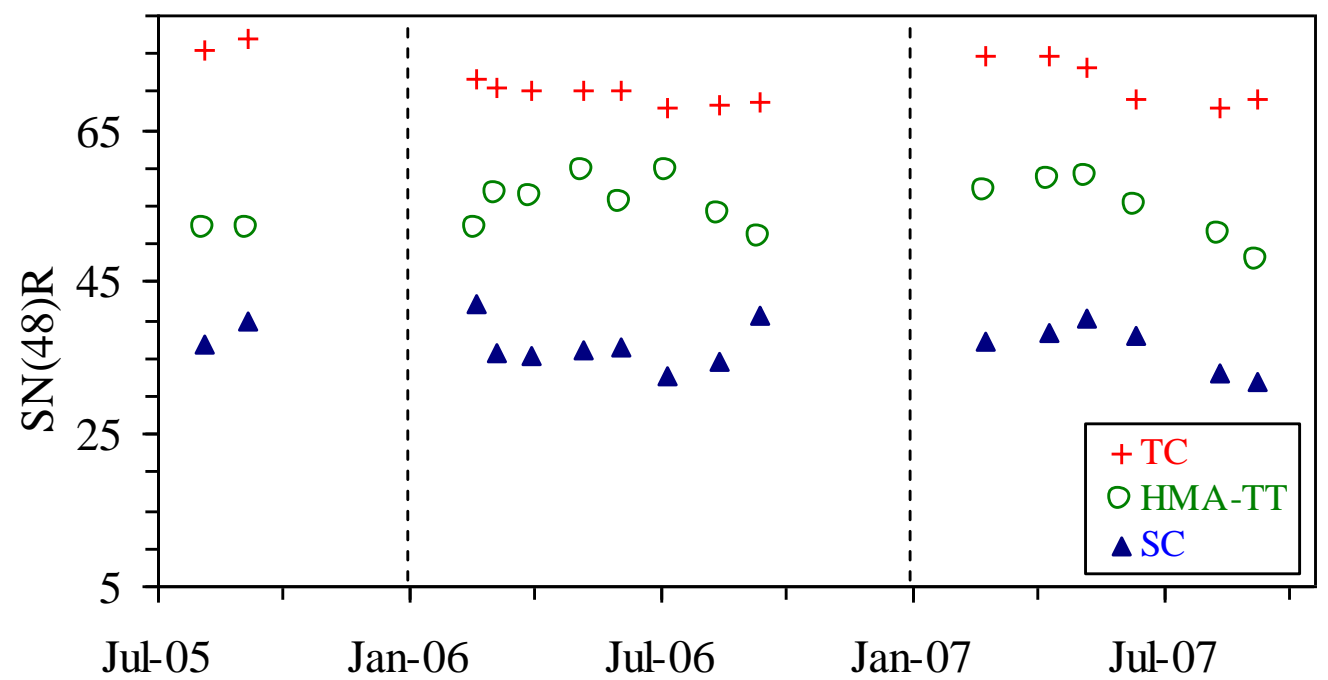

Figure 63. Skid number (measured by (ASTM E 274) friction trailer) on the INDOT test track monitoring: results for tests with rib tire

When comparing the friction trailer and DFT data it can be observed that, in general, the plots showing the changes in friction have similar shape. Similarly, when analyzed during similar periods, both data sets show the influence of seasonal variations.

As on highway test sections tested periodically, limited testing was conducted with the BSRT device on the test track. During those measurements (conducted in the same spots and at the same time as the last friction tests with the DFT/CTM devices), all three test track sections were tested. These tests result were averaged over the device orientation (transverse, longitudinal and diagonal) and over different spots tested within each specific section. Again, the highest friction was measured on the TC section (BPN=76) while the SC section exhibited the lowest friction $(\mathrm{BPN}=58)$. For HMA-TT section, the average BPN value was equal to 69. 


\subsubsection{Transverse Variation of Frictional Properties}

A comparison of the MPD and $\mathrm{DF}_{20}$ values obtained from measurements conducted at three different transverse locations of the test devices (left wheel path, center of the lane and right wheel path) is shown in Figures 64 to 67 . Visual assessment of the plots shown in those figures implies that the frictional properties depended on the transverse location of the test spot. To investigate this hypothesis, analysis of variance (ANOVA) was performed using the SAS 9.1 software. For the macrotexture data, one way ANOVA was used to reject either the null hypothesis (MPD values are the same, $\mathrm{H}_{0}=\mu_{\text {left }}=\mu_{\text {center }}=\mu_{\text {right }}$ ) or the alternative hypothesis (the MPD values are not equal). Similar analysis was performed for the $\mathrm{DF}_{20}$ values.

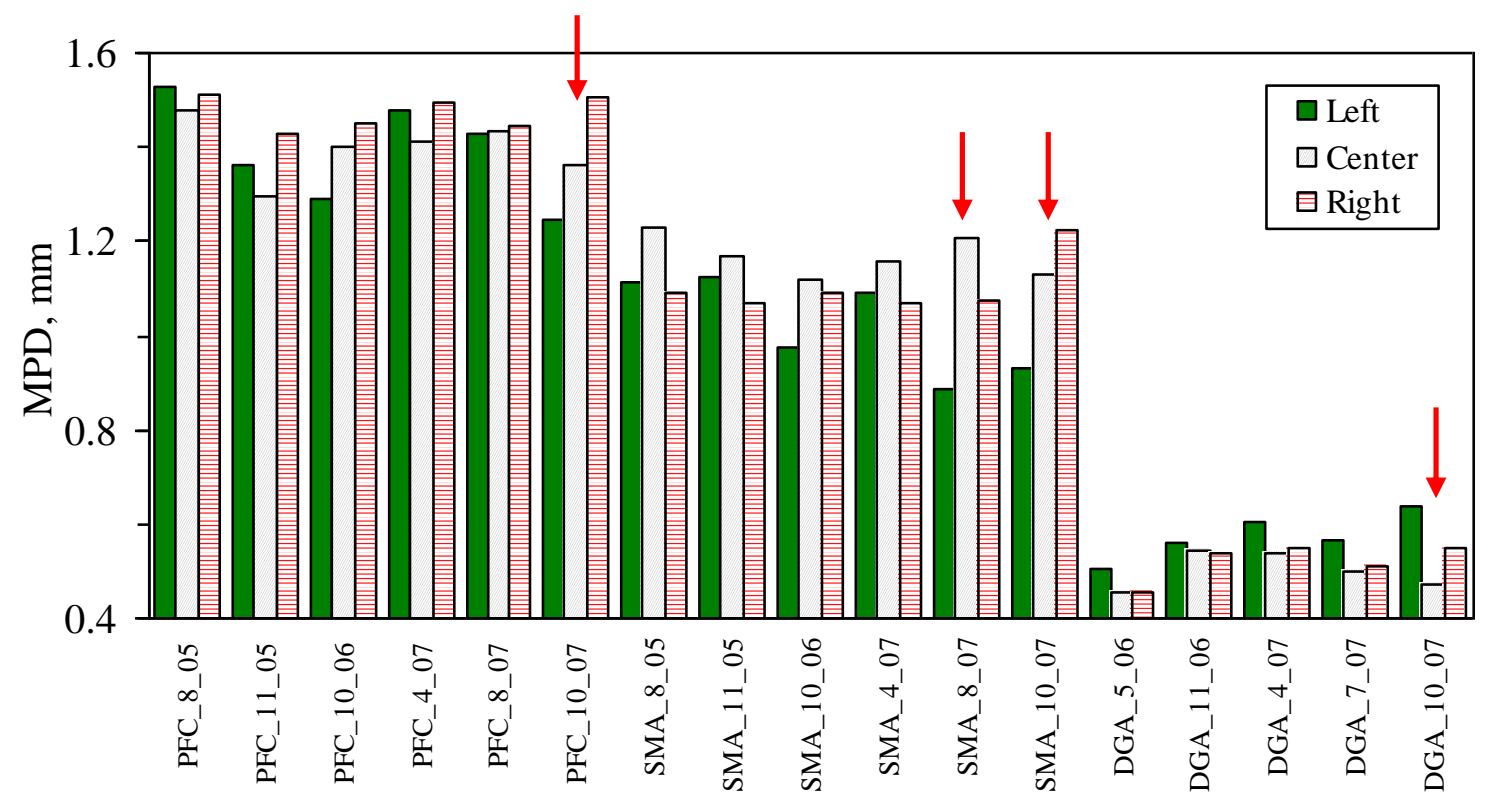

Figure 64. Influence of the transverse location and time of the test on the macrotexture (MPD) of the PFC, SMA and DGA sections

Note: Arrows indicate sections where location was a significant factor. 


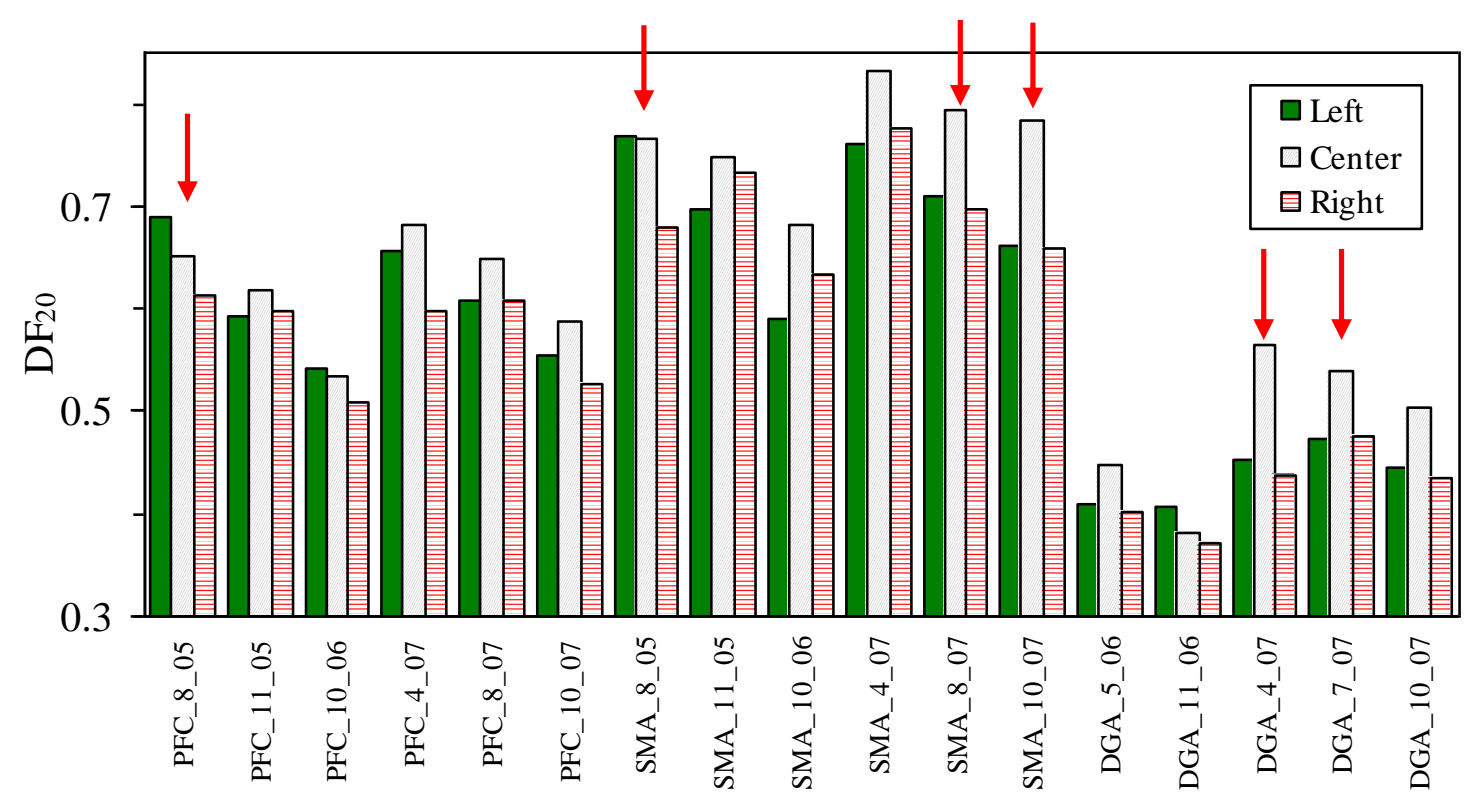

Figure 65. Influence of the transverse location on the dynamic friction $\left(\mathrm{DF}_{20}\right)$ of the PFC, SMA and DGA sections

Note: Arrows indicate sections where location was a significant factor.

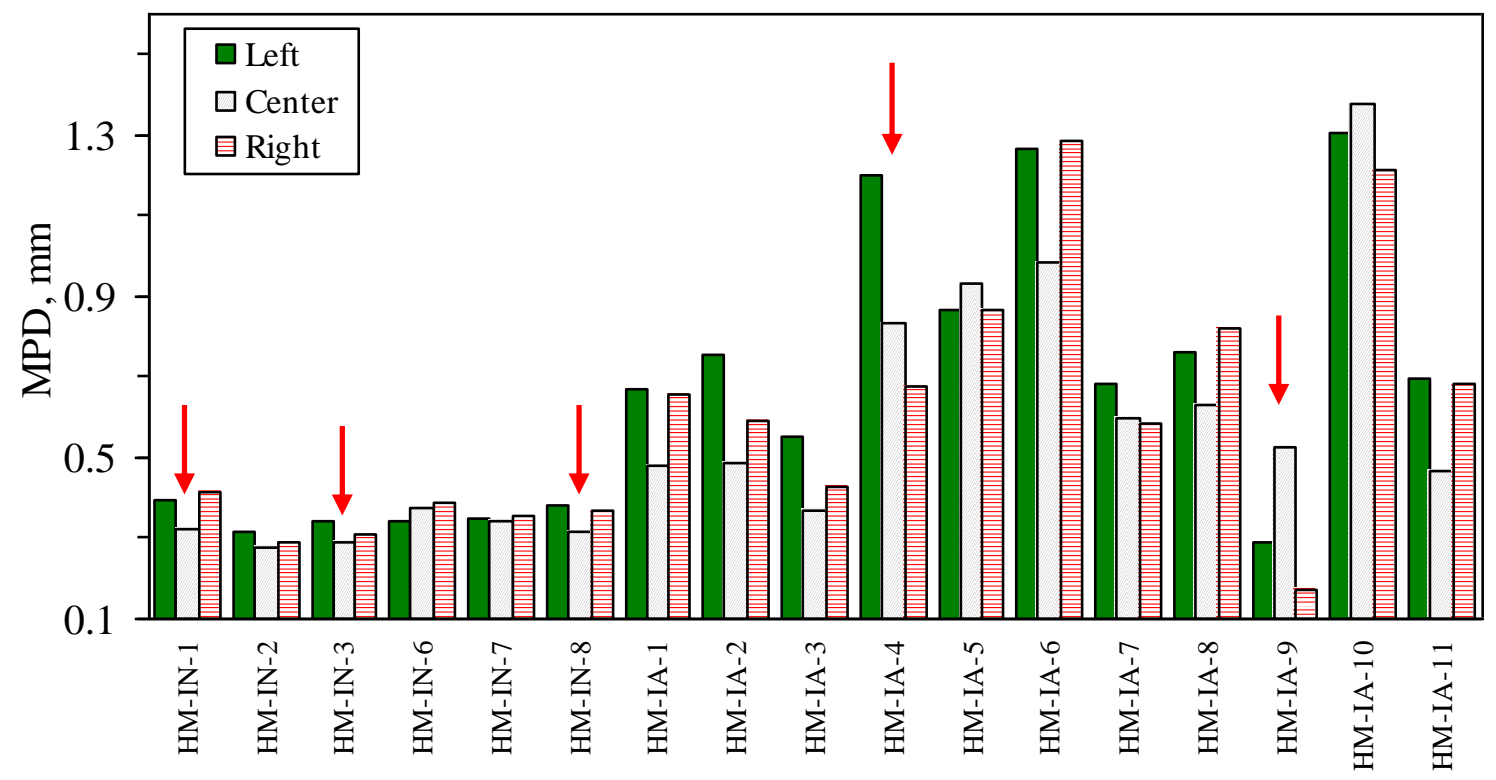

Figure 66. Influence of the transverse location on the macrotexture (MPD) of the HM-IN and HM-IA sections

Note: Arrows indicate sections where location was a significant factor. 


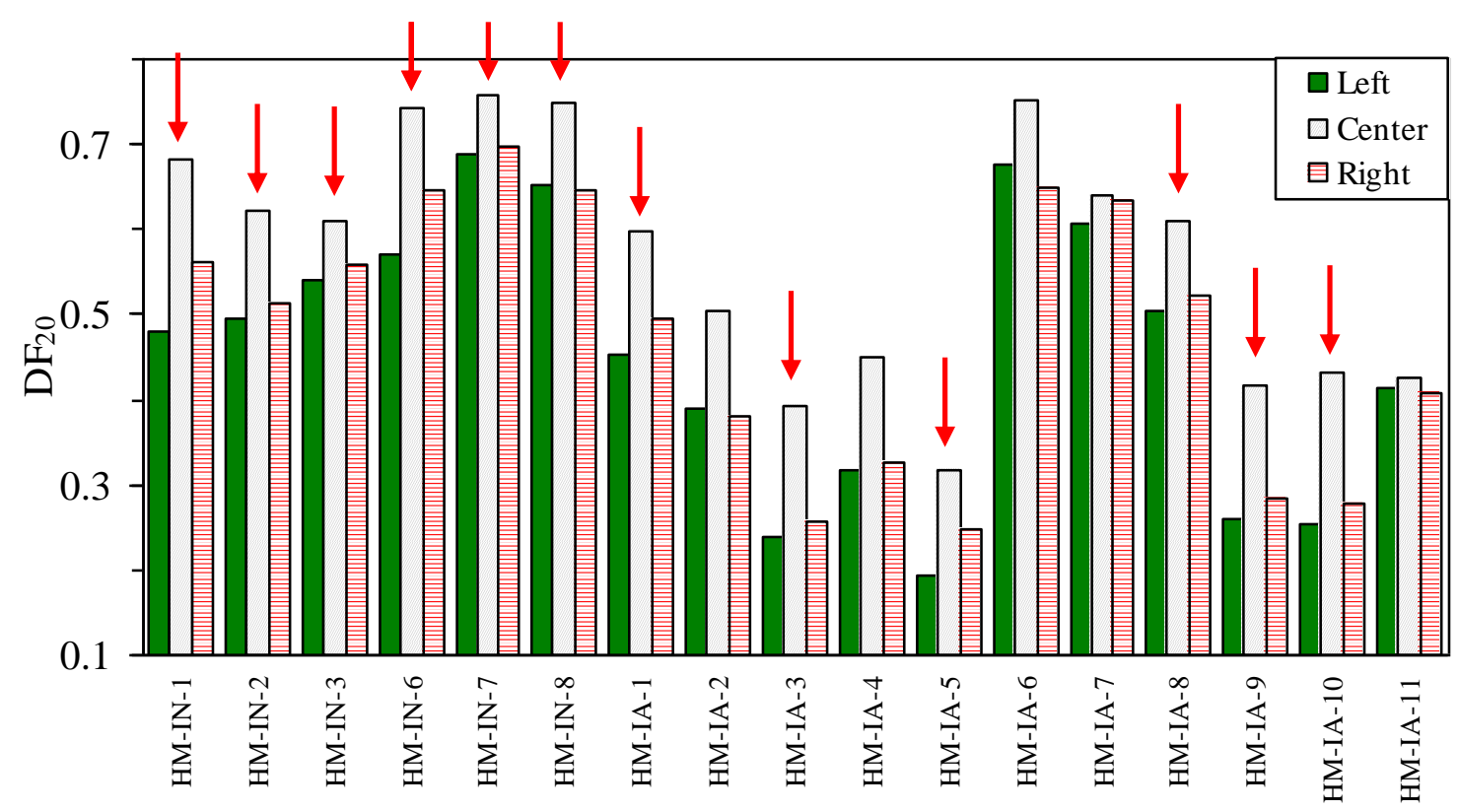

Figure 67. Influence of the transverse location on the dynamic friction $\left(\mathrm{DF}_{20}\right)$ of the HM-IN and HM-IA sections

Note: Arrows indicate sections where location was a significant factor.

An ANOVA table was determined for each test section (and test time) separately. The p-values obtained are summarized in Table 22 for the PFC, SMA, DGA sections and in Table 23 for the HM-IN and HM-IA sections. For those sections where the p-value was lower than the significance level $(\alpha=0.05)$, an additional analysis (multiple comparison of means) was performed. Bonferroni comparison of means was used to determine if differences in the MPD (or $\mathrm{DF}_{20}$ ) values in the left, right and center locations were statistically significant. Locations that were similar (at a significance level of $\alpha=0.05$ ) fall in the same grouping. These results are presented in Tables 22 and 23. 
Table 22. Influence of the transverse location and the date of test on the frictional properties of PFC, SMA and DGA sections

\begin{tabular}{|c|c|c|c|c|c|c|c|}
\hline Section & $\begin{array}{c}\text { Time of } \\
\text { Test }\end{array}$ & $\begin{array}{l}\text { Section } \\
\text { Age, } \\
\text { Years }\end{array}$ & $\begin{array}{l}\text { No. of } \\
\text { Axle } \\
\text { Passes, } \\
10^{\wedge} 6\end{array}$ & $\begin{array}{c}\text { MPD: } \\
\text { Location } \\
\text { Comparison } \\
\text { p-value }\end{array}$ & $\begin{array}{l}\text { Locations } \\
\text { Stat. } \\
\text { Different }^{\mathrm{b}}\end{array}$ & $\begin{array}{c}\mathrm{DF}_{20}: \\
\text { Location } \\
\text { Comparison } \\
\text { p-value }\end{array}$ & $\begin{array}{l}\text { Locations } \\
\text { Stat. } \\
\text { Different }^{b}\end{array}$ \\
\hline PFC & $8 / 2005$ & 2.0 & 23.3 & 0.815 & & 0.008 & L vs. R \\
\hline PFC & $11 / 2005$ & 2.3 & 25.7 & 0.250 & & 0.614 & \\
\hline PFC & $10 / 2006$ & 3.2 & 36.0 & 0.566 & & 0.295 & \\
\hline PFC & $4 / 2007$ & 2.7 & 42.1 & 0.752 & & 0.042 & Not Signif. \\
\hline PFC & $8 / 2007$ & 3.0 & 45.5 & 0.995 & & 0.375 & \\
\hline PFC & $10 / 2007$ & 3.2 & 47.8 & 0.003 & R vs. L & 0.087 & \\
\hline SMA & $8 / 2005$ & 2.0 & 23.3 & 0.275 & & $<.001$ & $\begin{array}{c}\mathrm{L} \text { and } \mathrm{C} \text { vs. } \\
\mathrm{R}\end{array}$ \\
\hline SMA & $11 / 2005$ & 2.3 & 25.7 & 0.775 & & 0.486 & \\
\hline SMA & $10 / 2006$ & 3.2 & 36.0 & 0.103 & & 0.211 & \\
\hline SMA & $4 / 2007$ & 2.7 & 42.1 & 0.116 & & 0.110 & \\
\hline SMA & $8 / 2007$ & 3.0 & 45.5 & 0.004 & L vs. C & 0.011 & $\begin{array}{c}\mathrm{L} \text { and } \mathrm{R} \text { vs. } \\
\mathrm{C}\end{array}$ \\
\hline SMA & $10 / 2007$ & 3.2 & 47.8 & 0.008 & R vs. L & $<.001$ & $\begin{array}{c}\mathrm{L} \text { and } \mathrm{R} \text { vs. } \\
\mathrm{C}\end{array}$ \\
\hline DGA & $5 / 2006$ & 1.8 & 15.8 & 0.236 & & 0.040 & Not Signif. \\
\hline DGA & $11 / 2006$ & 2.3 & 18.5 & 0.968 & & 0.207 & \\
\hline DGA & 4/2007 & 2.8 & 20.8 & 0.178 & & $<.001$ & $\begin{array}{c}\mathrm{L} \text { and } \mathrm{R} \text { vs. } \\
\mathrm{C}\end{array}$ \\
\hline DGA & $7 / 2007$ & 3.0 & 22.2 & 0.233 & & $<.001$ & $\begin{array}{l}\mathrm{L} \text { and } \mathrm{R} \text { vs. } \\
\mathrm{C}\end{array}$ \\
\hline DGA & $10 / 2007$ & 3.3 & 23.4 & 0.004 & L vs. C & 0.072 & \\
\hline
\end{tabular}

a p-value generated using ANOVA table,

${ }^{b}$ locations statistically different based on the Bonferroni comparison of means method. 
Table 23. Influence of the transverse location on the frictional properties for HM-IN and HM-IA sections

\begin{tabular}{|c|c|c|c|c|c|c|}
\hline Section & $\begin{array}{l}\text { Section } \\
\text { Age, } \\
\text { Years }\end{array}$ & $\begin{array}{c}\text { No. of } \\
\text { Axle } \\
\text { Passes, } \\
10^{\wedge} 6 \\
\end{array}$ & $\begin{array}{c}\text { MPD: } \\
\text { Location } \\
\text { Comparison }^{\mathrm{a}} \\
\text { p-value }\end{array}$ & $\begin{array}{l}\text { Locations } \\
\text { Stat. } \\
\text { Different }^{\text {b }}\end{array}$ & $\begin{array}{c}\mathrm{DF}_{20}: \\
\text { Location } \\
\text { Comparison }^{\mathrm{a}} \\
\text { p-value }\end{array}$ & $\begin{array}{l}\text { Locations } \\
\text { Stat. } \\
\text { Different }^{\mathrm{b}}\end{array}$ \\
\hline HM-IN-1 & 2 & 6.4 & 0.011 & R vs. C & $<.001$ & $\begin{array}{l}\text { L vs. C vs. } \\
\mathrm{R}\end{array}$ \\
\hline HM-IN-2 & 1 & 1.1 & 0.085 & & 0.004 & $\begin{array}{c}\mathrm{L} \text { and } \mathrm{R} \text { vs. } \\
\mathrm{C}\end{array}$ \\
\hline HM-IN-3 & 1 & 1.3 & 0.002 & L vs. C & 0.014 & L vs. C \\
\hline HM-IN-6 & 1 & 1.0 & 0.134 & & $<.001$ & $\begin{array}{c}\text { LVs. C vs. } \\
\text { R }\end{array}$ \\
\hline HM-IN-7 & 1 & 0.7 & 0.857 & & 0.025 & L vs. C \\
\hline HM-IN-8 & 1 & 1.3 & 0.019 & L vs. C & $<.001$ & $\begin{array}{c}\mathrm{L} \text { and } \mathrm{R} \text { vs. } \\
\mathrm{C}\end{array}$ \\
\hline HM-IA-1 & 5 & 17.2 & 0.092 & & 0.026 & L vs. C \\
\hline HM-IA-2 & 15 & 119.7 & 0.082 & & 0.081 & \\
\hline HM-IA-3 & 9 & 15.2 & 0.231 & & $<.001$ & $\begin{array}{c}\text { L vs. C vs. } \\
\text { R }\end{array}$ \\
\hline HM-IA-4 & 22 & 59.3 & 0.031 & L vs. R & 0.323 & \\
\hline HM-IA-5 & 15 & 37.7 & 0.908 & & 0.005 & $\begin{array}{c}\mathrm{L} \text { and } \mathrm{R} \text { vs. } \\
\mathrm{C}\end{array}$ \\
\hline HM-IA-6 & 15 & 25.1 & 0.128 & & 0.042 & \\
\hline HM-IA-7 & 14 & 38.2 & 0.411 & & 0.491 & \\
\hline HM-IA-8 & 11 & 36.7 & 0.063 & & 0.018 & $\begin{array}{c}\mathrm{L} \text { and } \mathrm{R} \text { vs. } \\
\mathrm{C}\end{array}$ \\
\hline HM-IA-9 & 24 & 32.8 & 0.027 & R vs. C & 0.002 & $\begin{array}{c}\mathrm{L} \text { and } \mathrm{R} \text { vs. } \\
\qquad \mathrm{C}\end{array}$ \\
\hline HM-IA-10 & 17 & 153.7 & 0.395 & & $<.001$ & $\begin{array}{c}\mathrm{L} \text { and } \mathrm{R} \text { vs. } \\
\mathrm{C}\end{array}$ \\
\hline HM-IA-11 & 10 & 35.9 & 0.134 & & 0.892 & \\
\hline
\end{tabular}

${ }^{a}$ p-value generated using ANOVA table,

${ }^{\mathrm{b}}$ locations statistically different based on the Bonferroni comparison of means method.

As shown in Table 22 and in Figure 64 (for mean profile depth, MPD) and in Table 22 and Figure 65 (for dynamic friction, $\mathrm{DF}_{20}$ ), the frictional properties of the PFC, SMA and DGA sections are, in general, similarly sensitive to the transverse location. Based on the series of measurements, significant differences between friction measured at the center of the lane and either wheel path were observed in three cases for the SMA and 
DGA sections and in two cases for the PFC section. Most likely, this is the effect of unequal wear of the pavement surface due to the different intensity of traffic. It is expected that most vehicles travel with their left and right wheels in the appropriate wheel path. Under typical traffic conditions, the center of the lane would be exposed to tire traffic only during lane changing operations. As a result, friction in the center of the lane location should be higher than in the left or right wheelpaths. Significant differences between the macrotexture measured in the center of the lane and either wheelpath were observed in one case for the SMA and DGA sections and between the left and right wheelpaths in one case for the PFC and SMA sections. Again, this is most likely related to the unequal number of wheel passes.

Similar to the SMA, PFC and DGA sections, for the HM-IA and HM-IN sections the frictional properties also vary by transverse location, as shown in Figure 66 (for MPD), Figure 67 (for $\mathrm{DF}_{20}$ ) and in Table 23. Again, friction in the center of the lane typically was the highest. In addition, the macrotexture in the center of the lane was typically the lowest.

It was observed that in all the HM-IN sections the $\mathrm{DF}_{20}$ values for the center of the lane were higher than those from the left or right wheelpaths, which was not always the case for the HM-IA sections. Possibly this is related to differences in the pavement age and number of wheel passes over the sections. Note that all the HM-IN sections compared here were opened to the traffic one to two years before the tests were conducted while, on average, the HM-IA sections were about 14 years old. 
It can also be noticed that the MPD values for the HM-IN sections were typically lower than for the HM-IA sections and different than those for the PFC and SMA sections. Refer to the previous discussion in section 4.2.1.2 for further explanation.

\subsection{Comparison of Frictional Characteristics: Lab vs. Field}

During this study, a large group of specimens was tested in the laboratory and a relatively high amount of data was also collected from the field tests. It was observed that for mixes from test Matrices I to III, in general, the macrotexture (MPD) of laboratory specimens, their dynamic friction $\left(\mathrm{DF}_{20}\right)$ values and their wet pavement friction (F60) values were comparable to those obtained from field measurements (see Table 24). It should be noted, however, that these values were obtained for specimens with different compositions than those tested in the field; more details regarding the compositions of the laboratory specimens are provided in Appendices D and E.

Table 24. Comparison of the typical macrotexture (MPD), dynamic friction $\left(\mathrm{DF}_{20}\right)$ and calibrated wet friction (F60) values obtained in the lab and during the field tests

\begin{tabular}{lccc}
\hline & MPD, mm & $\mathrm{DF}_{20}$ & $\mathrm{~F} 60$ \\
\hline Specimens from Matrices I-III, & $0.7-1.1^{\mathrm{a}, \mathrm{b}}$ & $0.3-0.5^{\mathrm{a}, \mathrm{b}}$ & $0.2-0.4^{\mathrm{a}, \mathrm{b}}$ \\
Laboratory Tests & $2.4^{\mathrm{a}}$ & $0.6^{\mathrm{a}}$ & $0.4^{\mathrm{a}}$ \\
PFC, Laboratory Tests & $1.8^{\mathrm{a}}$ & $0.7^{\mathrm{a}}$ & $0.5^{\mathrm{a}}$ \\
SMA, Laboratory Tests & $0.6-0.9$ & $0.4-0.6$ & $0.2-0.4$ \\
\hline Typical Highways Sections & 1.4 & 0.6 & 0.4 \\
PFC, Field Tests & 1.0 & 0.7 & 0.4 \\
SMA, Field Tests & & \\
\hline
\end{tabular}

${ }^{a}$ Values obtained for specimens after the initial phase of the polishing

${ }^{\mathrm{b}}$ Note the wide range of samples being tested (mixtures with fine, coarse and s-shaped gradation)

The macrotexture of the laboratory compacted specimens was slightly higher than that observed in the field. The air content measurements confirmed that the compaction 
method adopted in the laboratory did not provide the same densification level as that obtained in the field. Most likely, the slightly higher macrotexture of the laboratory specimens reflects their lower level of compaction.

In addition to the mixtures from test Matrices I to III, the same PFC and SMA mixtures were also tested both in the laboratory and (periodically) in the field. It was observed that laboratory tested SMA and PFC specimens showed higher MPD values than those tested in the field. Again, most likely this discrepancy is the result of differences in the compaction efforts. For specimens with "stiff" skeletons, such as SMA and PFC, the limitations of the laboratory adopted compaction method were reflected in higher values of MPD. It is interesting to note, however, that for the PFC mixture the air content of the laboratory and field specimens was similar. In the field, the PFC mixture required only one or two roller passes to seat the aggregates; apparently this level of compaction could be applied easily in the lab as well.

Due to the insufficient amount of data, it was not possible to verify the proposed polishing model based on field tests. However, the general trend of changes in the fielddetermined F60 values corresponded well with the model predictions for the laboratory polished specimens. 


\section{CHAPTER FIVE: FINDINGS AND CONCLUSIONS}

During this study, the influence of mixture composition on the frictional properties of flexible (asphalt) pavements was investigated. The research proved that it is possible to modify frictional properties of the pavement by changing the aggregate type and HMA composition.

This study also found that increasing the friction aggregate (quartzite or steel slag) content substantially improved the polishing resistance of HMA mixes. In general, mixes with steel slag generally exhibited slightly higher polishing resistance (lower polishing rate) than mixes with quartzite.

The influence of the carbonate aggregate type (dolomite, limestone or soft limestone) on the frictional properties of mixes was also studied. In general, the mixes with soft limestone exhibited lower friction values than those with dolomite and hard limestone. The influence of the carbonate aggregate type, however, depended on other factors as well; for example, steel slag appeared to dominate the mixtures' frictional performance (both polishing rate and terminal friction value) and overwhelmed the influence of the carbonate aggregate type.

When the carbonate aggregates used in this study were blended with high friction aggregates (steel slag and quartzite), the overall friction level generally increased. Increasing the friction aggregate content from 10 to $20 \%$ had relatively little effect on the friction level, but friction increased dramatically when the friction aggregate content was increased to 40 or $70 \%$. If friction is to be improved only by adding high friction 
aggregates to the local carbonate aggregates, the friction aggregate content should be $20 \%$ or greater, depending on the amount of improvement needed. There may be, however, other ways of increasing the overall surface friction.

In general, mixes with $9.5 \mathrm{~mm}$ NMAS had a lower polishing rate than mixes with a $19 \mathrm{~mm}$ NMAS. However, the overall frictional resistance of the $9.5 \mathrm{~mm}$ NMAS mixes was also lower than that of mixes with a 19 mm NMAS. Therefore, larger NMAS sizes are desirable from a frictional point of view, and they should be used where other considerations (such as layer thickness and smoothness, among others) allow.

The sensitivity analysis of friction/texture influence based on the theoretical relationship between macrotexture (expressed by mean profile depth (MPD)), microtexture (expressed by $\mathrm{DF}_{20}$ ) and International Friction Index parameters (F60 and $\mathrm{S}_{\mathrm{p}}$ ) shows that the IFI characteristics can be improved by increasing the pavement macrotexture, since the friction at high speed is mainly macrotexture dependent. It was found in the field and laboratory part of this study that the value of the fineness modulus (FM) of the aggregate blend correlates well with the pavement macrotexture and thus has a great influence on the pavement frictional properties. Moreover, the analysis confirmed that pavement frictional properties could be improved either by using highly polish resistant aggregate (such as quartzite or steel slag) blended with the locally available carbonate rocks or by combining both methods, such as blending both types of aggregates (low and high friction polishing resistant) and modifying the aggregate blend in such a way that the FM will be increased. Mixes with FM values of 4.6 or higher generally had high macrotexture and friction levels. 
During this study, the baseline values (for the laboratory tests) for the macrotexture (expressed by MPD), dynamic friction $\left(\mathrm{DF}_{20}\right)$ and calibrated wet friction (F60) values for typical asphalt pavements were determined. Based on the literature findings and field measurements using the ASTM E 274 towed friction trailer (equipped with both rib and smooth tires) and using the CTM/DFT devices, the approximate frictional flag value (F60) was determined. This flag value (when determined using the CTM and DFT devices) was in the range of 0.17-0.20.

The influence of the traffic volume on changes in the frictional properties of the pavement was also analyzed in this study. It was confirmed that, when exposed to traffic, the friction values of new pavement initially increase, most likely due to wearing-off of the binder film from the surface of the aggregate particles. After this initial increase, the values decrease and then stabilize at a lower level (terminal level). Once the terminal level is reached, no further traffic-associated changes in the friction are observed. However, it was also confirmed that seasonal variations in temperature and precipitation may influence the frictional characteristics of the surface.

In addition, it was confirmed that for many pavement sections, the left and right wheelpaths had lower friction than the center of the lane. Most likely, this phenomenon is related to the unequal exposure of these areas to the tires of the passing vehicles.

Comparison of the range of friction values obtained during the laboratory part of this study with results of the field measurements suggested good correlation between the laboratory measurements and actual highways conditions. Similarly, measurements with different friction measuring devices showed the same trends in the data. 
A new laboratory testing methodology was developed and refined during this study that allows for determination of two crucial properties for characterizing and predicting pavement friction: polishing rate and terminal friction value. The use of the CTM and DFT to measure pavement texture and friction in both the laboratory and the field was shown to be a feasible approach to determining frictional characteristics. When used in conjunction with the CTPM, the CTM and DFT can be used to detect changes in the surface properties of the mixtures under the effects of polishing. In the future, a mixture approval procedure involving determination of the predicted polishing rate and terminal friction values for a given mixture could be used to evaluate the mixture frictional properties.

Further work is needed to improve the compaction technique for laboratory slabs and to correlate the number of wheelpasses in the CPTM to actual traffic levels. The proposed polishing model should be validated by testing more types of materials under actual field conditions; work has already begun on this effort under another research project involving extensive field testing. 


\section{CHAPTER SIX: RECOMMENDATIONS}

The focus of this study was on investigating frictional properties of selected group of hot mix asphalt (HMA) mixes and correlating these properties with the frictional aggregate type and content. In order to accomplish this, suitable test methods needed to be identified or developed.

Laboratory testing method and evaluation criteria were, therefore, developed in this study to allow for the accelerated estimation of the frictional properties of various HMA mixes consisting of different aggregate types and gradations. It was found in this study that the Circular Track Machine (CTM) together with a Dynamic Friction Tester (DFT) can be used as a tool to assess the micro- and macrotexture of a mix and then to calculate the frictional properties (F60) of various pavement surfaces. A Circular Track Polishing Machine (CTPM), refined in this study, may be used for the laboratory simulation of the polishing action of highway traffic.

The test protocol developed in this study consists of preparing square slabs and determining their macrotexture (MPD) and dynamic friction $\left(\mathrm{DF}_{20}\right)$ using the CTM and DFT at various stages of polishing in the CPTM. Polishing should be continued until a steady level of friction is achieved (i.e., the measured texture and frictional properties remain reasonably constant while the specimen is subjected to additional polishing). It was found that 55,000 revolutions of the polishing assembly (resulting in 165,000 passes 
of the polishing wheels) should allow for the determination of the terminal friction level $\left(\mathrm{F} 60 @ \mathrm{x}_{1}\right)$.

The results of this study resulted in the development of a polishing model. Application of this model to the frictional properties allows for the determination of two crucial polishing parameters for a given mixture: $\mathrm{a}_{4}$ (polishing rate) and $\mathrm{F} 60 @ \mathrm{x}_{1}$ (terminal friction level). Frictional parameters of a mixture can then be assessed and the decision may be made whether the given mixture meets the desired friction criteria.

An F60 flag value was estimated based on the current INDOT practice, measurements with CTM/DFT devices and a towed friction trailer as well as conclusions from the PIARC study [Wambold et al. 1996]. The friction trailer flag value (SN-based) in Indiana is equal to 20 for smooth tire tests conducted at $64 \mathrm{~km} / \mathrm{h}$. The IFI-based friction flag value recommended in this study is $\mathrm{F} 60=0.20$. While this value should be further verified, it can be used as a starting point for the lab evaluation of the frictional properties of various HMA mixtures. Furthermore, it would be desired to determine the limit values which could potentially be used for the evaluation of mixture frictional properties under accelerated polishing, namely $\mathrm{a}_{4}$ (polishing rate) and F60@ $\mathrm{x}_{1}$ (terminal friction level) parameters.

A CTPM machine and test protocol developed in this study is a very promising test method to evaluate frictional properties of various HMAs, however, additional field verification of that method is needed. As of now, a promising validation has been conducted for two mixtures (SMA and PFC) which were the subjects of long-term field monitoring and which were also tested in the laboratory using the proposed test protocol. For those mixtures, similarities in the trends of plots of F60-value vs. number of 
wheelpasses were observed. Some differences between the values of F60 determined under field and lab conditions were found, however. Similar observations also apply to the MPD values in the sense that although the plots of MPD vs. number of wheelpasses were similar for both lab and field cases, there were some differences in the actual numerical values of the MPD. It is believed that those differences are mainly caused by the limitations of the existing laboratory specimen compaction method, which does not densify the material to the same extent as occurs in the field. It is recognized that the compaction method should be further improved so it more closely simulates field construction processes. It has to be mentioned, however, that those differences in macrotexture are of secondary importance if only the relative changes in the micro- and macrotexture occurring during polishing under field and lab conditions are compared. The previously mentioned $\mathrm{a}_{4}$ and F60@ $\mathrm{x}_{1}$ parameters developed in this study should serve work well for such relative comparisons and evaluations. Relative comparisons could be used, for example, to compare a selected standard mix to new, untried mixture compositions or aggregate blends.

Regarding the main objective of this study, it was confirmed here that pavement frictional properties can be improved either by using highly polish resistant aggregate blended with the locally available carbonate rocks or by increasing the macrotexture of the surface layer. The best approach would be to combine both methods, such as blending both types of aggregates (low and high friction polishing resistant) and also modifying the aggregate blend in such a way that the macrotexture and fineness modulus (FM) are increased (by using appropriate aggregate gradations and maximum aggregate sizes). 
Based on the test results obtained in this study, it can be concluded that the use of up to $20 \%$ of polish susceptible coarse aggregate (in the overall aggregate blend) does not negatively affect the frictional properties of the HMA. On the other hand, if the use of an aggregate blend consisting mostly of polish susceptible aggregates is desired, the high friction coarse aggregate content should be $20 \%$ or greater in the overall aggregate blend, depending on the amount of improvement needed, mixture type (fine, s-shaped or coarse) and nominal maximum aggregate size used. This finding should be further verified by performing field friction measurements of pavements with varying percentages of polishing aggregates.

Both steel slag and quartzite were found to improve the frictional characteristics of HMA mixes in which they are used. The choice of which high friction aggregate to use should be used based on availability and cost.

In addition to substituting high friction aggregates for a portion of the polish susceptible aggregates, however, this study showed that the frictional characteristics of the surface can also be improved by changing the value of the fineness modulus (FM) of the surface mix. The fineness modulus (FM) of the aggregate blend highly influences the pavement macrotexture (together with the compaction method and compaction effort, HMA temperature, etc.). Since the pavement frictional properties, especially at the high speeds (above about $50 \mathrm{~km} / \mathrm{h}$ ) are closely related to the pavement macrotexture, changing the FM should allow for improvement of the frictional properties of mixtures for which the aggregate type may not be favorable (high amount of the polishable aggregate type). Based on the limited field observation, in general, HMA blends with a FM of about 4.6 or greater should provide a relatively "deep" pavement macrotexture (MPD above about 0.6 
$\mathrm{mm})$. Such a texture improves wet weather friction by helping to evacuate water that may potentially lead to hydroplaning and helping to reduce braking distance. In general, the s-shaped gradation resulted in higher MPD (“deeper" texture) and thus improved friction at high speeds.

One other way to increase the macrotexture of the surface is to use aggregates with larger NMAS sizes. Such mixtures are desirable from a frictional point of view and should be used where other considerations (such as tire pavement/noise, layer thickness and smoothness, among others) allow. 
LIST OF REFERENCES 


\section{$\underline{\text { Specifications and Standards }}$}

AASHTO M 320 (2005): Standard Specification for Performance-Graded Asphalt Binder.

AASHTO M 323 (2004): Standard Specification for Superpave Volumetric Mix Design.

AASHTO R 30 (2002): Mixture Conditioning of Hot-Mix Asphalt (HMA).

AASHTO R 35 (2004): Standard Practice for Superpave Volumetric Design for Hot-Mix Asphalt (HMA).

AASHTO T 166 (2007): Standard Method of Test for Bulk Specific Gravity of Compacted Hot Mix Asphalt (HMA) Using Saturated Surface-Dry Specimens.

AASHTO T 176 (2002): Standard Method of Test for Plastic Fines in Graded Aggregates and Soils by Use of the Sand Equivalent Test.

AASHTO T 228 (2006): Standard Method of Test for Specific Gravity of Semi-Solid Bituminous Materials (ASTM Designation: D 70-03).

AASHTO T 304 (2004): Standard Method of Test for Uncompacted Void Content of Fine Aggregate.

AASHTO T 84 (2004): Standard Method of Test for Specific Gravity and Absorption of Fine Aggregate.

AASHTO T 85 (2004): Standard Method of Test for Specific Gravity and Absorption of Coarse Aggregate.

ASTM C 125 (2007): Standard Terminology Relating to Concrete and Concrete Aggregates.

ASTM C 136 (2006): Standard Test Method for Sieve Analysis of Fine and Coarse Aggregates.

ASTM D 2419 (2002): Standard Test Method for Sand Equivalent Value of Soils and Fine Aggregate. 
ASTM D 3203 (2005): Standard Test Method for Percent Air Voids in Compacted Dense and Open Bituminous Paving Mixtures.

ASTM D 3319 (2006): Standard Practice for the Accelerated Polishing of Aggregates Using the British Wheel.

ASTM D 4791 (2005): Standard Test Method for Flat Particles, Elongated Particles, or Flat and Elongated Particles in Coarse Aggregate.

ASTM D 5821 (2006): Standard Test Method for Determining the Percentage of Fractured Particles in Coarse Aggregate.

ASTM D 6752 (2004): Standard Test Method for Bulk Specific Gravity and Density of Compacted Bituminous Mixtures Using Automatic Vacuum Sealing Method.

ASTM E 1337 (2002): Standard Test Method for Determining Longitudinal Peak Braking Coefficient of Paved Surfaces Using a Standard Reference Test Tire.

ASTM E 1393 (1997): Standard Test Method for Determining the Polishability of Bituminous Pavement Surfaces and Specimens by Means of the Penn State Reciprocating Polishing Machine, Discontinued 1997; No Replacement.

ASTM E 1845 (2005): Standard Practice for Calculating Pavement Macrotexture Mean Profile Depth.

ASTM E 1859 (2006): Standard Test Method for Friction Coefficient Measurements between Tire and Pavement Using a Variable Slip Technique.

ASTM E 1911 (2002): Standard Test Method for Measuring Paved Surface Frictional Properties Using the Dynamic Friction Tester.

ASTM E 1960 (2005): Standard Practice for Calculating International Friction Index of a Pavement Surface.

ASTM E 2157 (2005): Standard Test Method for Measuring Pavement Macrotexture Properties Using the Circular Track Meter.

ASTM E 2380 (2005): Standard Test Method for Measuring Pavement Texture Drainage Using an Outflow Meter. 
ASTM E 274 (2006): Standard Test Method for Skid Resistance of Paved Surfaces Using a Full-Scale Tire.

ASTM E 303 (2003): Standard Test Method for Measuring Surface Frictional Properties Using the British Pendulum Tester.

ASTM E 445/E 445M (2001): Standard Test Method for Stopping Distance on Paved Surfaces Using a Passenger Vehicle Equipped with Full-Scale Tires.

ASTM E 501 (2006): Standard Specification for Standard Rib Tire for Pavement SkidResistance Tests.

ASTM E 503/E 503M (2004): Standard Test Methods for Measurement of Skid Resistance on Paved Surfaces Using a Passenger Vehicle Diagonal Braking Technique.

ASTM E 524 (2006): Standard Specification for Standard Smooth Tire for Pavement Skid-Resistance Tests.

ASTM E 660 (2002): Standard Practice for Accelerated Polishing of Aggregates or Pavement Surfaces Using a Small-Wheel, Circular Track Polishing Machine.

ASTM E 670 (2000): Standard Test Method for Side Force Friction on Paved Surfaces Using the Mu-Meter.

ASTM E 707 (2002): Standard Test Method for Skid Resistance Measurements Using the North Carolina State University Variable-Speed Friction Tester.

ASTM E 867 (2006): Standard Terminology Relating to Vehicle-Pavement Systems.

ASTM E 965 (2006): Standard Test Method for Measuring Pavement Macrotexture Depth Using a Volumetric Technique.

ITM 214 (2006): Acceptance Procedures for Polish Resistant Aggregates, Indiana Test Method. 


\section{$\underline{\text { Publications }}$}

AASHTO 1976, "Guidelines for Skid Resistant Pavement Design," American Association of State Highway and Transportation Officials, Washington D.C., 1976.

Abe, H., A. Tamai, J.J. Henry and J. Wambold, "Measurement of Pavement Macrotexture using the CTMeter," Transportation Research Record 1764, 2001, pp. 201-209.

Abo-Qudais, S., and M. Qudah, "Effect of compaction method on bituminous mixtures voids distribution and magnitude," Loizos, Scarpas \& Al-Qadi eds., "Advanced Characterisation of Pavement and Soil Engineering Materials," London, 2007, pp. 817-829.

Advisory Circular, "Measurement, Construction, and Maintenance of Skid-Resistant Airport Pavement Surfaces," FAA Advisory Circular, 150/5320-12C, 1997.

Ahe, G., "European Design of Low-Noise Pavements," A Tire/Pavement Noise Symposium Quiet Asphalt, Lafayette, Indiana, 2005.

Augustin, H., "Skid Resistance and Road-Surface Texture," Surface Characteristics of Roadways: International Research and Technology, ASTM STP 1031. W. E. Meyer and J. Reichert, Eds., American Society for Testing and Materials, Philadelphia, Pennsylvania, 1990, pp. 5-13.

Barrett, M. E., and C. B. Shaw, "Stormwater Quality Benefits of a Porous Asphalt Overlays," Proceedings of the $86^{\text {th }}$ Transportation Research Board Annual Meeting (CD), Washington D.C., January 2007.

Bazlamit, S. M., and F. Reza, "Changes in Asphalt Pavement Friction Components and Adjustments of Skid Number for Temperature," ASCE Journal of Transportation Engineering, vol. 131 no. 6., 2005, pp. 470-476.

Bernhard, R. J., and R. L. Wayson, "An Introduction to Tire/Pavement Noise," Final Research Report, SQDH 2005-1, West Lafayette, IN, 2005. 
Britton, S. C., W. B. Ledbetter and B. M. Gallaway, "Estimation of Skid Numbers from Surface Texture Parameters in the Rational Design of Standard Reference Pavements for Test Equipment Calibration," Surface Texture Skidding: Measurements, Frictional Aspects, and Safety Features of Tire-Pavement Interactions, ASTM STP 583, J. G. Rose editor, American Society for Testing and Materials, Washington D.C., 1975, pp. 112-115.

Brown, A. L., "Mathematical Analysis for Pneumatic Tire Hydroplaning," Surface Texture Skidding: Measurements, Frictional Aspects, and Safety Features of TirePavement Interactions, ASTM STP 583, J. G. Rose editor, American Society for Testing and Materials, Washington D.C., 1975, pp. 75-94.

Burchet, J. L., and R. L. Rizenbergs, "Frictional Performance of Pavements and Estimates of Accident Probability," Pavement Surface Characteristics and Materials, ASTM STP 763, C.M. Hayden, Ed., American Society of Civil Engineering, Washington D.C., 1982, pp. 73-97.

Burge, P. L., K. Travis and Z. Rado., "Transverse Tined and Longitudinal Diamond Ground Texturing for Newly Constructed Concrete Pavement: A Comparison," Transportation Research Record 1792, 2002, pp. 75-82.

Cafiso, S, and S. Taormina, "Texture analysis of aggregates for wearing courses in asphalt pavements," International Journal of Pavement Engineering, Vol. 8, No. 1, 2007, pp. 45-54.

Cenek, P. D., N. J. Jamieson and J. I. Towler, "The Influence of Texture Depth on Skidding Resistance," Transit New Zealand Conference, New Zealand, 1997.

Clark, S. K., "Interactive Tire Design," Surface Texture Skidding: Measurements, Frictional Aspects, and Safety Features of Tire-Pavement Interactions, ASTM STP 583, J. G. Rose editor, American Society for Testing and Materials, Washington D.C., 1975, pp. 95-111. 
Crispino, M., R. Rampini and M. Pozzi, “An experimental analysis of the effects of compaction on asphalt pavement macrotexture," Loizos, Scarpas \& Al-Qadi eds., “Advanced Characterisation of Pavement and Soil Engineering Materials," London, 2007, pp. 205-217.

Crocker, M. J., D. Hanson, Z. Li, R. Karjatkar and K. S. Vissamraju, "Measurement of the Acoustical and Mechanical Properties of Porous Road Surfaces and Tire/Road Noise," Transportation Research Record 1891, 2004, pp. 16-22.

Dames, J., "The Influence of Polishing Resistance of Sand on Skid Resistance of Asphalt Concrete," Surface Characteristics of Roadways: International Research and Technologies, ASTM STP 1031, W. E. Meyer and J. Reichert, Eds., American Society for Testing and Materials, Philadelphia, Pennsylvania, 1990, pp. 14-29.

Diringer, K. T., and R. T. Barros, "Predicting the Skid Resistance of Bituminous Pavements Through Accelerated Laboratory Testing of Aggregates," Surface Characteristics of Roadways: International Research and Technologies, ASTM STP 1031, W. E. Meyer and J. Reichert, Eds., American Society for Testing and Materials, Philadelphia, Pennsylvania, 1990, pp. 61-73.

Donbavand, J., and D. Cock, "Procedure for Correcting Seasonal Variations," proceedings from the International Conference on Surface Friction, Christchurch, New Zealand, May 1-4 2005.

Doty, R. N., "Study of the Sand Patch and Outflow Meter Methods of Pavement Surface Texture Measurement," Surface Texture Skidding: Measurements, Frictional Aspects, and Safety Features of Tire-Pavement Interactions, ASTM STP 583, J. G. Rose editor, American Society for Testing and Materials, Washington D.C., 1974, pp. 4261.

Ech, M., S. Morel, S. Yotte, D. Breysse and B. Pouteau, "Laboratory evaluation of road surface macrotexture durability," Loizos, Scarpas \& Al-Qadi eds., "Advanced Characterisation of Pavement and Soil Engineering Materials," London, 2007, pp. 1483-1493. 
Emery, J. J., M. A. Lee and N. Kamel, "Skid Resistance Predictive Models for Asphaltic Concrete Surface Courses," Pavement Surface Characteristics and Materials, ASTM STP 763, C. M. Hayden, Ed., American Society of Civil Engineering, Washington D.C., 1982, pp. 61-72.

Ergun, M., S. Iyinam and A. F. Iyinam, "Prediction of Road Surface Friction Coefficient Using Only Macro- and Microtexture Measurements," ASCE Journal of Transportation Engineering, vol. 131 no. 4., 2005, pp. 311-319.

FHWA Traffic Monitoring Guide, FHWA publication, 2001, available online, http://www.fhwa.dot.gov/ohim/ tmguide/, accessed August 2007.

Flintsch, G. W., Y. Luo and I. L. Al-Qadi, "Analysis of the Effect of Pavement Temperature on the Frictional Properties of Flexible Pavement Surfaces," Proceedings (CD) from the $84^{\text {th }}$ Transportation Research Board Annual Meeting, Washington D.C., January 2005.

Florida DOT, "Dynamic Friction Tester (DFT) Operations \& Reference Manual," State of Florida Materials Office publication, August 2006.

French, T., "Tyre Technology," Adam Hilger, Bristol and New York publisher, New York, NY, 1989.

Fulop, I. A., I. Bogardi, A. Gulyas and M. Csicsely-Tarpay, "Use of Friction and Trxture in Pavement Performance Modelling," ASCE Journal of Transportation Engineering, vol. 126 no. 3,2000 , pp. 243-248.

Fwa, T. F., Y. S. Choo and Y. Liu, "Effect of Aggregate Spacing on Skid Resistance of Asphalt Pavement," ASCE Journal of Transportation Engineering, vol. 129 no. 4, 2003, pp. 420-426.

Gardziejczyk, W., "Texture of Road Surfaces - Methods of Measurement, Parameters Evaluation and its Influence on the Tire/Road Noise," Drogi i Mosty, no. 2, Poland, 2002, pp. 5-29. 
Gargett, T., "The Introduction of a Skidding-Resistance Policy in Great Britain," Surface Characteristics of Roadways: International Research and Technologies, ASTM STP 1031, W. E. Meyer and J. Reichert, Eds., American Society for Testing and Materials, Philadelphia, Pennsylvania, 1990, pp. 30-38.

Gee, S., W. L. King Jr. and R. R. Hegmon, "Pavement Texture Measurement by Laser; A Feasibility Study," Surface Texture Skidding: Measurements, Frictional Aspects, and Safety Features of Tire-Pavement Interactions, ASTM STP 583, J. G. Rose editor, American Society for Testing and Materials, Washington D.C., 1975, pp. 29-41.

Giles, C. G., B. E. Sabey and K. H. F. Cardew, "Development and Performance of the Portable Skid-Resistance Tester," Symposium on Skid Resistance, ASTM STP 326, American Society for Testing and Materials, Philadelphia, Pennsylvania, 1962, pp. $50-74$.

Goodman, S. N., Y. Hassan and A. O. Abd El Halim, "Preliminary Estimation of Asphalt Pavement Frictional Properties from Superpave Gyratory Specimen and Mix Parameters," Transportation Research Record 1949, 2006, pp. 173-180.

Gunaratne, M., N. Bandara, J. Medzorian, M. Chawla and P. Ulrich, "Correlation of Tire Wear and Friction to Texture of Concrete Pavements," ASCE Journal of Materials in Civil Engineering, vol. 12 no. 1., 2000, pp. 46-54.

Gustafson, M., Traffic Monitoring Section, Indiana Department of Transportation (INDOT), personal communication and provided materials: "Interstate System Flow Map \& Annual Average Daily Traffic (AADT) County Flow Map," http://www.in.gov/dot/div/traffic/count/index.html, October 2006.

Hall Jr., H. W., "Findings from NCHRP Projects on Surface Texturing and Pavement Friction," presentation to "AASHTO Highway Subcommittee on Construction," August 2, 2006.

Hanson, D. I., and B. D. Prowell, "Circular Texture Meter Proves Useful for Measuring Pavement Surface Texture," North Central Superpave Center News, vol. 5, 2006, pp. 8-9. 
Hanson, D. I., and B. D. Prowell, "Evaluation of Circular Texture Meter for Measuring Surface Texture of Pavements," NCAT Report No. 04-05, National Center for Asphalt Technology, Auburn, Alabama, 2004.

Hassan, H. F., S. Al-Oraimi and R. Taha, "Evaluation of Open-Graded Friction Course Mixtures Containing Cellulose Fibers and Styrene Butadiene Rubber Polymer," ASCE Journal of Materials in Civil Engineering, vol. 17 no. 4, 2005, pp. 416-422.

Henry, J. J., NCHRP Synthesis 291, "Evaluation of Pavement Friction Characteristics," Transportation Research Board, National Academy Press, Washington D. C., 2000.

Henry, J. J., "Overview of the International PIARC Experiment to Compare and Harmonize Texture and Skid Resistance Measurements: The International Friction Index," proceedings of the Third International Symposium on Pavement Surface Characteristics: Christchurch, New Zealand, 3-4 September 1996.

Henry, J. J., "Personal Communication”, October 2006.

Henry, J. J., H. Abe, S. Kameyama, A. Tamai, A. Kasahara and K. Saito, "Determination of the International Friction Index (IFI) using the Circular Texture Meter (CTM) and the Dynamic Friction Tester (DFT)," $4^{\text {th }}$ PIARC Symposium on Surface Characteristics, Nante, France, 2000.

Henry, J. J., and R. R. Hegmon, "Pavement Texture Measurement and Evaluation," Surface Texture Skidding: Measurements, Frictional Aspects, and Safety Features of Tire-Pavement Interactions, ASTM STP 583, J. G. Rose editor, American Society for Testing and Materials, Washington D.C., 1975, pp. 3-17.

Henry, J. J., K. Saito and R. Blackburn, "Predictor Model for Seasonal Variations in Skid Resistance," vol. I: Summary Report and vol. II: Comprehensive Report, FHWA reports, FHWA/RD-83/004 and FHWA/RD-83/005, Washington D.C., 1984.

Hill, B. J., and J. J. Henry, "Surface Materials and Properties Related to Seasonal Variations in Skid Resistance," Pavement Surface Characteristics and Materials, ASTM STP 763, C. M. Hayden, Ed., American Society of Civil Engineering, Washington D.C., 1982, pp. 45-60. 
Horne, W. B., and F. Buhlmann, "A Method for Rating the Skid Resistance and Micro/Macrotexture Characteristics of Wet Pavements," Frictional Interaction of Tire and Pavement, ASTM STP 793, W. E. Meyer and J. D. Walter, Ed., American Society of Civil Engineering, Washington D.C., 1983, pp. 191-218.

Huihua, X., and J. J. Henry, "The Use of Fuzzy-Sets Mathematics for Analysis of Pavement Skid Resistance," Surface Characteristics of Roadways: International Research and Technologies, ASTM STP 1031, W. E. Meyer and J. Reichert, Eds., American Society for Testing and Materials, Philadelphia, Pennsylvania, 1990, pp. $39-49$.

Hutchinson, J. W., N. G. Tsongos, R. C. Bennett and W. J. Fogarty, "Pavement Surface Information Needs in Accident Investigation," Surface Texture Skidding: Measurements, Frictional Aspects, and Safety Features of Tire-Pavement Interactions, ASTM STP 583, J. G. Rose editor, American Society for Testing and Materials, Washington D.C., 1975, pp. 75-94.

Iowa DOT, "General Aggregate Source Information, Matls. IM T203," Iowa DOT publication, Office of Materials, October 17, 2006.

Iwama, M., G. D. Airey and A. E. Hunter, "Influence of asphalt mixture compaction method and specimen size on internal structure and mechanical properties," Loizos, Scarpas \& Al-Qadi eds., "Advanced Characterisation of Pavement and Soil Engineering Materials," London, 2007, pp. 1063-1073.

Kamel, N., and T. Gartshore, "Ontario's Wet Pavement Accident Reduction Program," Pavement Surface Characteristics and Materials, ASTM STP 763, C. M. Hayden, Ed., American Society of Civil Engineering, Washington D.C., 1982, pp. 98-117.

Kennedy, C. K., A. E. Young and I. C. Butler., "Measurement of Skidding Resistance and Surface Texture and the Use of Results in the United Kingdom," Surface Characteristics of Roadways: International Research and Technologies, ASTM STP 1031, W. E. Meyer and J. Reichert, Eds., American Society for Testing and Materials, Philadelphia, Pennsylvania, 1990, pp. 87-102. 
Kulakowski, B. T., and D. W. Harwood, "Effect of Water-Film Thickness on TirePavement Friction," Surface Characteristics of Roadways: International Research and Technologies, ASTM STP 1031, W. E. Meyer and J. Reichert, Eds., American Society for Testing and Materials, Philadelphia, Pennsylvania, 1990, pp. 50-60.

Kulakowski, B. T., J. J. Henry and C. Lin, “A Closed-Loop Calibration Procedure for a British Pendulum Tester," Surface Characteristics of Roadways: International Research and Technologies, ASTM STP 1031, W. E. Meyer and J. Reichert, Eds., American Society for Testing and Materials, Philadelphia, Pennsylvania, 1990, pp. 103-112.

Kummer, H. W., "Unified Theory of Rubber and Tire Friction," Engineering Research Bulletin B-94, The Pennsylvania State University, College of Engineering, University Park, Pennsylvania, July 1962.

Kummer, H. W., and W. E. Meyer, "Measurement of Skid Resistance," Symposium on Skid Resistance, $65^{\text {th }}$ Annual Meeting ASTM, New York, 1962.

Kummer, H. W., and W. E. Meyer, "Tentative Skid-Resistance Requirements for Main Rural Highways,” NCHRP Report 37, Washington D.C., 1967.

Larson, R. M., L. Scofield and J. Sorenson, "Providing Durable, Safe and Quiet Highways," proceeding from the $8^{\text {th }}$ International Conference on Concrete Pavements, Colorado Springs, Colorado, August 14-18 2005, pp. 500-522.

Lea, J. D., and D. J. Jones, "Initial Findings on the Skid Resistance of unpaved Roads," Proceedings (CD) from the $86^{\text {th }}$ Transportation Research Board Annual Meeting, Washington D.C., January 2007.

Li, S., S. Noureldin and K. Zhu, "Evaluation of Friction Performance of Coarse Aggregates and Hot Mix Asphalt Pavements," Journal of Testing and Evaluation, vol. 35 , no. 6,2007 , pp. 30-40.

Li, S., S. Noureldin and K. Zhu, "Realistic Approach for Enhancing Reliability of Pavement Surface Friction Testing," Journal of ASTM International, vol. 3 no. 9., 2006, pp. 50-58. 
Li, S., S. Noureldin and K. Zhu, "Upgrading the INDOT Pavement Friction Testing Program," final report FHWA/IN/JTRP-2003/23, West Lafayette, Indiana, October 2003.

Li, S., K. Zhu and S. Noureldin, "Considerations in Developing a Network Pavement Inventory Test Program for a State Highway Agency," ASTM Journal of Testing and Evaluation, vol. 33 no. 5., 2005, pp. 1-8.

Liang, R. Y., "Blending Proportions of High Skid and Low Skid Aggregate," FHWA report, FHWA/OH-2003/014, Columbus, OH, August 2003.

Liang, R., "Pavement Friction Investigation," Ohio Asphalt Paving Conference, February 2007.

Liang, R. Y., and L. L. Chyi, "BPolishing and Friction Characteristics of Aggregates Produced in Ohio," FHWA report, FHWA/OH-2000/001, Columbus, OH, April 2000.

Liu, Y., T. F. Fwa and Y. S. Choo, "Effects of Surface Macrotexture on Skid Resistance Measurements by the British Pendulum Test," ASTM International, Journal of Testing and Evaluation, vol. 32 no. 4., 2004, pp. 304-309.

Liu, Y., T. F. Fwa and Y. S. Choo, "Finite Element Modelling of Skid Resistance Test," ASCE Journal of Transportation Engineering, vol. 129 no. 3, 2003, pp. 316-321.

Luce, A., E. Mahmud, E. Masad, A. Chowdhury and D. Little, "Relationship of Aggregate Texture to Asphalt Pavement Skid Resistant," Proceedings (CD) from the $86^{\text {th }}$ Transportation Research Board Annual Meeting, Washington D.C., January 2007.

Mahone, D. C., "Variation in Highway Slipperiness Characteristics with Location," Symposium on Skid Resistance, ASTM STP 326, American Society for Testing and Materials, Philadelphia, Pennsylvania, 1962, pp. 75-101.

Masad, E., D. Little and R. Sukhwani, "Sensitivity of HMA Performance to Aggregate Shape Measured Using Conventional and Image Analysis Methods," Road Materials and Pavement Design Journal, Vol. 5, No. 4, 2004, pp. 477-498. 
MEPDG, Guide for Mechanistic-Empirical Design of New and Rehabilitated Pavement Structures, part 2 Design Inputs, Washington D.C., 2004.

McDaniel, R. S., and B. J. Coree., "Identification of Laboratory Techniques to Optimize Superpave HMA Surface Friction Characterization," Phase I: Final Report SQDH 2003-6, West Lafayette, Indiana, 2003.

McGhee, K. K., and G. W. Flintsch, "High-Speed Texture Measurement of Pavements," Final Report, Charlotsville, Virginia, VTRC 03-R9, February 2003.

Moore, D. F., "Design of Texture in Standard Surfaces," Surface Texture Skidding: Measurements, Frictional Aspects, and Safety Features of Tire-Pavement Interactions, ASTM STP 583, J. G. Rose editor, American Society for Testing and Materials, Washington D.C., 1975, pp. 75-94.

Murad, M. M., and K. A. Abaza, "Pavement Friction in a Program to Reduce Wet Weather Traffic Accidents at the Network Level," Transportation Research Record 1949, 2006, pp. 126-136.

Nippo Sangyo Co. LTD, information from web page http://www.nippou.com, accessed October 2006

Nitta, N., K. Saito and S. Isozaki, "Evaluating the Polishing Properties of Aggregates and Bituminous Pavement Surfaces by Means of the Penn State Reciprocating Polishing Machine," Surface Characteristics of Roadways: International Research and Technologies, ASTM STP 1031, W. E. Meyer and J. Reichert, Eds., American Society for Testing and Materials, Philadelphia, Pennsylvania, 1990, pp. 113-126.

O’Brien, J. P., "Frictional Resistance of Aggregates for Hot-Mix Asphalt Pavements," MS thesis, Purdue University, Indiana, December 2004.

Obertop, D. H. F., "Decrease of Skid-Resisting Properties of Wet Road Surfaces at High Speeds," Symposium on Skid Resistance, ASTM STP 326, American Society for Testing and Materials, Philadelphia, Pennsylvania, 1962, pp. 102-114. 
Ong, G. P., and T. F. Fwa, "Prediction of Wet-Pavement Skid Resistance and Hydroplaning Potential," Proceedings (CD) from the $86^{\text {th }}$ Transportation Research Board Annual Meeting, Washington D.C., January 2007.

Prasanna, R., B. Nageswaran and P. W. Jayawickrama, "Use of Relation Database Management Systems Principles in Reliable Prediction of Pavement Skid Resistance," Transportation Research Record 1655, 1999, pp. 192-199.

Prowell, B. D., and D. I. Hanson, "Evaluation of Circular Texture Meter for Measuring Surface Texture of Pavements," Transportation Research Record 1929, 2005, pp. 8896.

Roberts, F. L., P. S. Kandhal, E. R. Brown, D. Lee and T. W. Kennedy, "Hot Mix Asphalt Materials, Mixture Design, and Construction”, $2^{\text {nd }}$ ed.., NAPA Education Foundation, Lanham, Maryland, 1996.

Saito, K., T. Horiguchi, A. Kasahara, H. Abe and J. J. Henry, "Development of Portable Tester for Measuring Skid Resistance and its Speed Dependency on Pavement Surfaces," Transportation Research Record 1536, 1996, pp. 45-51.

Schulze, K. H., and L. Beckman, "Friction Properties of Pavements at Different Speeds," Symposium on Skid Resistance, ASTM STP 326, American Society for Testing and Materials, Philadelphia, Pennsylvania, 1962, pp. 42-49.

Shahin, M. Y., "Pavement Management for Airports, Roads, and Parking Lots," $2^{\text {nd }}$ edition, Springer, New York, 2005.

Stroup-Gardiner, M., J. Studdard and C. Wagner, "Evaluation of Hot Mix Asphalt Macro- and Microtexture," ASTM Journal of Testing and Evaluation, vol. 32 no. 1, 2004, pp. 1-10.

Sullivan, B. W., "Development of a Fundamental Skid Resistance Asphalt Mix Design Procedure," proceedings of the International Conference on Surface Friction, Christchurch, New Zealand, May 1-4 2005. 
Timm, D., R. West, A. Priest, B. Powell, Im. Selvaraj, J. Zhang and R. Brown, "Phase II NCAT Test Track Results," NCAT Report No. 06-05, National Center for Asphalt Technology, Auburn, Alabama, 2006.

Trevino, M., T. Dossey, and Y. Yildrim, "Black Ice Detection in Open-Graded Friction Courses," Proceedings (CD) from the $86^{\text {th }}$ Transportation Research Board Annual Meeting, Washington D.C., January 2007.

Vallejo, L. E., "Fractal Assessment of the Surface Texture of Pavements," International Journal of Pavement Engineering, Vol. 2, No. 2, 2001, pp. 149-156.

Vollor, T. W., and D. I. Hanson, Development of Laboratory Procedure for Measuring Friction of HMA Mixtures - Phase I," NCAT Report No. 06-06, National Center for Asphalt Technology, Auburn, Alabama, 2006.

Wahi, M. K., "A Tire Interface Friction Prediction Model Concept," Journal of Aircraft, vol. 16, no. 6, article no. 79-4086, June 1979, pp. 407-416.

Wambold, J. C., "International Friction Index," TESC Conference, Penn State Conference Center, Pennsylvania, December 52005.

Wambold, J. C., C. E. Antle, J. J. Henry and Z. Rado, PIARC (Permanent International Association of Road Congress) report, International PIARC Experiment to Compare and Harmonize Texture and Skid Resistance Measurement, C-1 PIARC Technical Committee on Surface Characteristics, France, 1995.

Wambold, J. C., J. J. Henry, C. E. Antle, B. T. Kulakowski, W. E. Meyer, A. J. Stocker, J. W. Button, and D. A. Anderson, "Pavement Friction Measurement Normalized for Operational, Seasonal, and Weather Effects.” FHWA report, FHWA-RD-88-069, Washington D.C., 1989.

Wambold, J. C., W. E. Meyer, and J. J. Henry, "New-Generation Skid Testers for the 1990s," Surface Characteristics of Roadways: International Research and Technologies, ASTM STP 1031, W. E. Meyer and J. Reichert, Eds., American Society for Testing and Materials, Philadelphia, Pennsylvania, 1990, pp. 138-153. 
Wang, H., and G. W. Flintsch, "Investigation of Short and Long-Term Variations of Pavement Surface Characteristics at the Virginia Smart Road," Proceedings (CD) from the $86^{\text {th }}$ Transportation Research Board Annual Meeting, Washington D.C., January 2007.

Wasilewska, M., and W. Gardziejczyk, Polishing Resistance of Road Aggregates Applied in Wearing Courses," proceedings from $3^{\text {rd }}$ International Conference „Modern Technologies in Highway Engineering”, Poznan, Poland, 8-9 September 2005.

Wayson, R. L., "Relationship Between Pavement Surface Texture and Highway Traffic Noise," NCHRP Synthesis 268, Transportation Research Board, National Academy Press, Washington D. C., 1998.

West, T. R., and K. H. Cho, "Development of a Procedure to Identify Aggregates for Bituminous Surfaces in Indiana," Joint Transportation Research Program, report FHWA/IN/JTRP-2000/28, West Lafayette, September 2001.

West, T.R., and J. O'Brien, "A Coarse Aggregate Paradox for Indiana Highway Pavements, Less is Better," proceedings from the $56^{\text {th }}$ Highway Geology Symposium, Wilmington, North Carolina, June. 2005.

West, T. R., J. C. Choi, D. W. Bruner, H. J. Park and K. H. Cho, "Evaluation of Dolomite and Related Aggregates Used in Bituminous Overlays for Indiana Pavements," Transportation Research Record 1757, 2001, pp. 137-147.

Woods, K. B., D. S. Berry and W. H. Goetz, editors, "Highway engineering handbook," section 20 by Shupe J. W., "Pavement Slipperines," 1 st edition, New York, McGrawHill, 1960.

Woodward, W. D., A. Woodside and J. H. Jellie, "Higher PSV and other Aggregate Properties," Proceedings of the International Conference on Surface Friction, Christchurch, New Zealand, May 1-4, 2005. 
Yager, T. J., “Aircraft/Ground-Vehicle Friction Measurement Study," Surface Characteristics of Roadways: International Research and Technologies, ASTM STP 1031, W. E. Meyer and J. Reichert, Eds., American Society for Testing and Materials, Philadelphia, Pennsylvania, 1990, pp. 154-167.

Yager, T. J., and F. Buhlman, "Macrotexture and Drainage Measurements on a Variety of Concrete and Asphalt Surfaces," Pavement Surface Characteristics and Materials, ASTM STP 763, C.M. Hayden, Ed., American Society of Civil Engineering, Washington D.C., 1982, pp. 16-30.

Yandell, W. O., P. Taneerananon and V. Zankin, "Prediction of Tire-Road Friction from Surface Texture and Tread Rubber Properties," Frictional Interaction of Tire and Pavement, ASTM STP 793, W. E. Meyer and J. D. Walter, Ed., American Society of Civil Engineering, Washington D.C., 1983, pp. 304-322.

Yeaman, J., "Are We Afraid of the IFI?," Proceedings of the International Conference on Surface Friction, Christchurch, New Zealand, May 1-4 2005. 
APPENDICES 
Appendix A: Laboratory Friction, Texture and Polishing Methods

Table A 1. Comparison of laboratory friction measurement methods [after McDaniel and Coree 2003].

\begin{tabular}{|c|c|c|c|c|c|}
\hline \multicolumn{6}{|c|}{ Devices for the testing and evaluation of the surface friction of aggregates and mixes } \\
\hline Device & $\begin{array}{l}\text { About } \\
\text { Device }\end{array}$ & Properties & Strengths & Weaknesses & $\begin{array}{c}\text { Specs/used } \\
\text { by }\end{array}$ \\
\hline $\begin{array}{l}\text { British } \\
\text { Pendulum } \\
\text { Tester }\end{array}$ & $\begin{array}{l}\text { Pendulum } \\
\text { arm }\end{array}$ & $\begin{array}{c}\text { Evaluates the amount of } \\
\text { Kinetic Energy lost } \\
\text { when a rubber slider } \\
\text { attached to the pendulum } \\
\text { arm is propelled over the } \\
\text { test surface }\end{array}$ & $\begin{array}{l}\text { Portable. Very simple. } \\
\text { Widely used. }\end{array}$ & $\begin{array}{l}\text { Variable quality of results. } \\
\text { Cumbersome and sometimes } \\
\text { ineffective calibration. } \\
\text { Pendulum only allows for a } \\
\text { small area to be tested. }\end{array}$ & $\begin{array}{l}\text { ASTM } \\
\text { E303 }\end{array}$ \\
\hline $\begin{array}{l}\text { Michigan } \\
\text { Laboratory } \\
\text { Friction Tester }\end{array}$ & $\begin{array}{l}\text { Rotating } \\
\text { Wheel }\end{array}$ & $\begin{array}{l}\text { One wheel is brought to } \\
\text { a speed of } 40 \mathrm{mph} \text { and } \\
\text { dropped onto the surface } \\
\text { of the sample. Torque } \\
\text { measurement is recorded } \\
\text { before wheel stops }\end{array}$ & $\begin{array}{l}\text { Good measure of the } \\
\text { tire/surface interaction. } \\
\text { Similar to towed } \\
\text { friction trailer. }\end{array}$ & $\begin{array}{l}\text { Poor measurement of pavement } \\
\text { macrotexture. History of use on } \\
\text { aggregate only. }\end{array}$ & MDOT \\
\hline $\begin{array}{l}\text { Dynamic } \\
\text { Friction Tester }\end{array}$ & $\begin{array}{l}\text { Rotating } \\
\text { slide }\end{array}$ & $\begin{array}{c}\text { Measures the coefficient } \\
\text { of friction }\end{array}$ & $\begin{array}{l}\text { Laboratory or field } \\
\text { measurements of } \\
\text { microtexture }\end{array}$ & N/A & $\begin{array}{l}\text { ASTM } \\
\text { E1911 }\end{array}$ \\
\hline $\begin{array}{l}\text { North Carolina } \\
\text { Variable Speed } \\
\text { Friction Tester }\end{array}$ & $\begin{array}{l}\text { Pendulum } \\
\text { Type } \\
\text { Testing } \\
\text { Device } \\
\end{array}$ & $\begin{array}{c}\text { Pendulum with locked } \\
\text { wheel smooth rubber tire } \\
\text { at its lower end }\end{array}$ & $\begin{array}{l}\text { Can simulate different } \\
\text { vehicle speeds }\end{array}$ & $\begin{array}{c}\text { Uneven pavement surfaces in } \\
\text { the field may provide inaccurate } \\
\text { results }\end{array}$ & $\begin{array}{c}\text { ASTM } \\
\text { E707 }\end{array}$ \\
\hline $\begin{array}{l}\text { PTI Friction } \\
\text { Tester }\end{array}$ & $\begin{array}{l}\text { Rubber } \\
\text { slider }\end{array}$ & $\begin{array}{c}\text { Rubber slider is } \\
\text { propelled linearly along } \\
\text { surface by falling weight }\end{array}$ & $\begin{array}{l}\text { Tests in linear } \\
\text { direction }\end{array}$ & $\begin{array}{c}\text { Companion to Penn State } \\
\text { Reciprocating Polisher. Fallen } \\
\text { into disuse. }\end{array}$ & $\begin{array}{c}\text { Formerly by } \\
\text { PTI }\end{array}$ \\
\hline
\end{tabular}


Table A 2. Comparison of portable texture measurement methods [after McDaniel and Coree 2003].

\begin{tabular}{|c|c|c|c|c|c|}
\hline \multicolumn{6}{|c|}{ Devices for the evaluation of the surface texture of mixes } \\
\hline Device & About device & Properties & Strengths & Weaknesses & $\begin{array}{l}\text { Specs/used } \\
\text { by }\end{array}$ \\
\hline Sand Patch & $\begin{array}{l}\text { Sand spread over } \\
\text { circular area to } \\
\text { fill surface voids }\end{array}$ & $\begin{array}{l}\text { Measures mean texture } \\
\text { depth over covered area }\end{array}$ & Simple & $\begin{array}{l}\text { Cumbersome. Poor } \\
\text { repeatability. Average depth } \\
\text { only. }\end{array}$ & ASTM E965 \\
\hline Grease Patch & $\begin{array}{l}\text { Grease spread } \\
\text { over surface }\end{array}$ & $\begin{array}{l}\text { Measures mean texture } \\
\text { depth over covered area }\end{array}$ & Simple & $\begin{array}{l}\text { Cumbersome. Poor } \\
\text { repeatability. Average depth } \\
\text { only. Not widely used. }\end{array}$ & NASA \\
\hline $\begin{array}{l}\text { Silicone } \\
\text { Putty } \\
\text { Method }\end{array}$ & $\begin{array}{l}\text { Silicone putty } \\
\text { pressed onto } \\
\text { surface }\end{array}$ & $\begin{array}{l}\text { Measures mean texture } \\
\text { depth over covered area }\end{array}$ & Simple & $\begin{array}{l}\text { Cumbersome. Poor } \\
\text { repeatability. Average depth } \\
\text { only. Not widely used. }\end{array}$ & TTI \\
\hline $\begin{array}{l}\text { Outflow } \\
\text { Meter }\end{array}$ & $\begin{array}{c}\text { Water flows } \\
\text { from cylinder } \\
\text { through surface } \\
\text { voids } \\
\end{array}$ & Estimates average texture & Simple. Quick. & $\begin{array}{l}\text { For non-porous surfaces } \\
\text { only. }\end{array}$ & FHWA \\
\hline Dromometer & $\begin{array}{l}\text { Stylus traces } \\
\text { surface }\end{array}$ & $\begin{array}{l}\text { Lowers a tracing pin, that } \\
\text { creates a profile of the } \\
\text { specimen surface }\end{array}$ & $\begin{array}{c}\text { Can measure } \\
\text { both micro and } \\
\text { macro texture }\end{array}$ & $\begin{array}{l}\text { Can only be used on small } \\
\text { areas of pavement }\end{array}$ & $\begin{array}{c}\text { Ref. } \\
\text { [Augustin } \\
\text { 1990] }\end{array}$ \\
\hline $\begin{array}{l}\text { Surtronix 3+ } \\
\text { Profilometer }\end{array}$ & $\begin{array}{l}\text { Stylus traces } \\
\text { profiles }\end{array}$ & $\begin{array}{l}\text { Horiz Res }=1 \text { micrometer } \\
\text { Vert Res }=0.001 \text { micrometer } \\
\text { Traverse Length }=25.4 \mathrm{~mm}\end{array}$ & $\begin{array}{l}\text { Can read micro } \\
\text { and macrotexture }\end{array}$ & $\begin{array}{l}\text { Can only be used on small } \\
\text { areas of pavement }\end{array}$ & $\begin{array}{c}\text { Ref. } \\
\text { [Gunaratne } \\
\text { 2000] }\end{array}$ \\
\hline $\begin{array}{l}\text { Circular } \\
\text { Track Meter }\end{array}$ & Laser based & $\begin{array}{l}\text { Laser mounted on an arm } \\
\text { that rotates on a } \\
\text { circumference of } 142 \mathrm{~mm} \\
\text { and measures the texture }\end{array}$ & $\begin{array}{c}\text { Used with DFT } \\
\text { can calculate IFI. } \\
\text { Fast. Portable. } \\
\text { Repeatable. }\end{array}$ & $\begin{array}{l}\text { Measures small area. } \\
\text { Relatively new. }\end{array}$ & $\begin{array}{l}\text { ASTM } \\
\text { E2157 }\end{array}$ \\
\hline
\end{tabular}


Table A 3. Comparison of accelerated polishing methods [after McDaniel and Coree 2003].

\begin{tabular}{|c|c|c|c|c|c|}
\hline \multicolumn{6}{|c|}{ Devices for the accelerated polishing of aggregates and mixes } \\
\hline Device & $\begin{array}{l}\text { About } \\
\text { device }\end{array}$ & Properties & Strengths & Weaknesses & $\begin{array}{c}\text { Specs/used } \\
\text { by }\end{array}$ \\
\hline $\begin{array}{l}\text { British } \\
\text { Polishing } \\
\text { Wheel }\end{array}$ & $\begin{array}{l}\text { Wheel for } \\
\text { polishing } \\
\text { away } \\
\text { macro- } \\
\text { texture }\end{array}$ & $\begin{array}{l}\text { Curved aggregates } \\
\text { specimens polished by a } \\
\text { rotating wheel. }\end{array}$ & $\begin{array}{l}\text { Accelerated polishing } \\
\text { for lab testing. Bench } \\
\text { sized. }\end{array}$ & $\begin{array}{c}\text { Coarse aggregate coupons only. } \\
\text { Does not affect macrotexture or } \\
\text { mix properties }\end{array}$ & $\begin{array}{r}\text { ASTM } \\
\text { D3319 }\end{array}$ \\
\hline $\begin{array}{l}\text { Michigan } \\
\text { Indoor Wear } \\
\text { Track }\end{array}$ & $\begin{array}{l}\text { Large } \\
\text { circular } \\
\text { track }\end{array}$ & $\begin{array}{l}\text { Wheels centered around } \\
\text { pivot point, move in } \\
\text { circle around track }\end{array}$ & Close to real world. & $\begin{array}{l}\text { Track is very large and } \\
\text { cumbersome. Time consuming } \\
\text { sample preparation. Used for } \\
\text { aggregate only. }\end{array}$ & MDOT \\
\hline $\begin{array}{l}\text { NCSU } \\
\text { Polishing } \\
\text { Machine }\end{array}$ & $\begin{array}{l}4 \text { wheels } \\
\text { rotate } \\
\text { around } \\
\text { central } \\
\text { pivot } \\
\end{array}$ & $\begin{array}{l}\text { Four pneumatic tires are } \\
\text { adjusted for camber and } \\
\text { toe-out to provide } \\
\text { scrubbing action for } \\
\text { polishing }\end{array}$ & $\begin{array}{c}\text { No need for water or } \\
\text { grinding compounds, } \\
\text { can polish aggregate or } \\
\text { mixes }\end{array}$ & $\begin{array}{l}\text { Polishes a relatively small area } \\
\text { or few number of samples }\end{array}$ & ASTM E660 \\
\hline $\begin{array}{l}\text { NCAT } \\
\text { Polishing } \\
\text { Machine }\end{array}$ & $\begin{array}{l}3 \text { wheels } \\
\text { rotate } \\
\text { around } \\
\text { central } \\
\text { pivot } \\
\end{array}$ & $\begin{array}{l}\text { Three pneumatic tires } \\
\text { are adjusted for camber } \\
\text { and toe-out to provide } \\
\text { scrubbing action for } \\
\text { polishing }\end{array}$ & $\begin{array}{l}\text { Sized to match DFT } \\
\text { and CTM. }\end{array}$ & $\begin{array}{l}\text { New device developed by } \\
\text { NCAT based on older devices. }\end{array}$ & NCAT \\
\hline $\begin{array}{l}\text { Penn State } \\
\text { Reciprocating } \\
\text { Polishing } \\
\text { Machine }\end{array}$ & $\begin{array}{l}\text { Recipro- } \\
\text { cating pad }\end{array}$ & $\begin{array}{l}\text { Reciprocates rubber pad } \\
\text { under pressure against } \\
\text { specimen surface while } \\
\text { slurry of water and } \\
\text { abrasive is fed to } \\
\text { surface. }\end{array}$ & $\begin{array}{l}\text { Portable. Can be used } \\
\text { to polish aggregate or } \\
\text { mix in lab or field. }\end{array}$ & $\begin{array}{l}\text { Polishes a relatively small area. } \\
\text { Oscillation obliterates } \\
\text { directional polishing. Fallen } \\
\text { into disuse. }\end{array}$ & $\begin{array}{r}\text { ASTM } \\
\text { E1393 }\end{array}$ \\
\hline
\end{tabular}




\section{Appendix B: Representation of the Laboratory and Field Specimens}

Figures in the following 11 pages are presented in the subsequent order:

Figure B 1. Laboratory mixture 95SS20C_D: before and after the polishing

Figure B 2. Laboratory mixture 95SS20F_D: before and after the polishing

Figure B 3. Laboratory mixture 95SS20S_D: before and after the polishing

Figure B 4. Laboratory mixture 19SS20C_D: before and after the polishing

Figure B 5. Laboratory mixture 19SS20F_D: before and after the polishing

Figure B 6. Laboratory mixture 19SS20S_D: before and after the polishing

Figure B 7. Laboratory sample of SMA (slab $38 \mathrm{~mm}$ thick): before and after the polishing

Figure B 8. Laboratory sample of PFC (slab $38 \mathrm{~mm}$ thick): before and after the polishing

Figure B 9. HMA sections (interstate highway) tested periodically: (a) PFC and (b) SMA

Figure B 10. HMA sections tested periodically: (a) INDOT Test Track (HMA-TT) and (b) state highway (DGA)

Figure B 11. Concrete pavement sections located in the INDOT Test Track: (a) tined (TC) and (b) smooth (SC)

Note, that some pictures were taken with the flash while for the rest only ambient light was used. This influenced the picture brightness and contrast. 

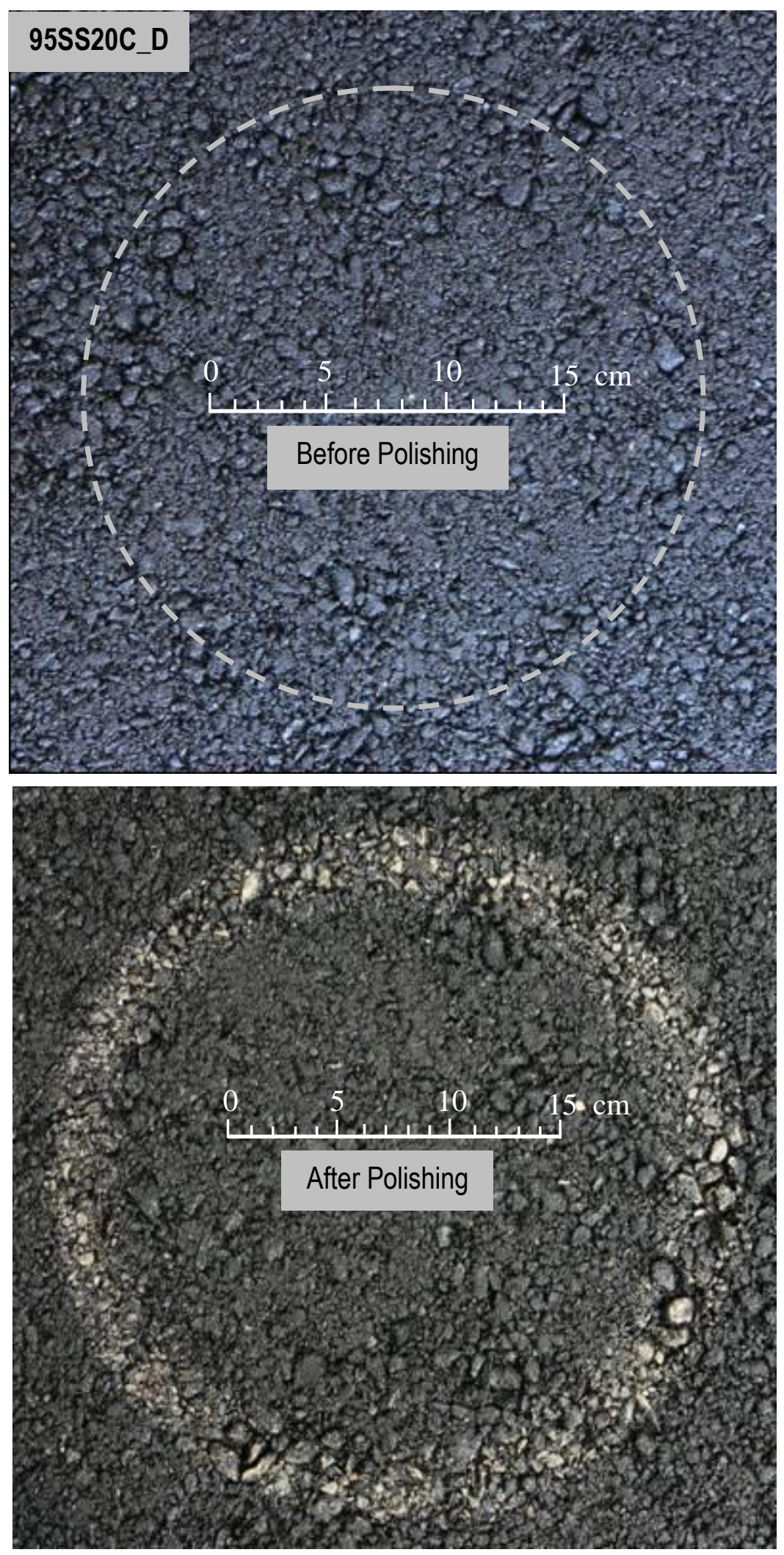

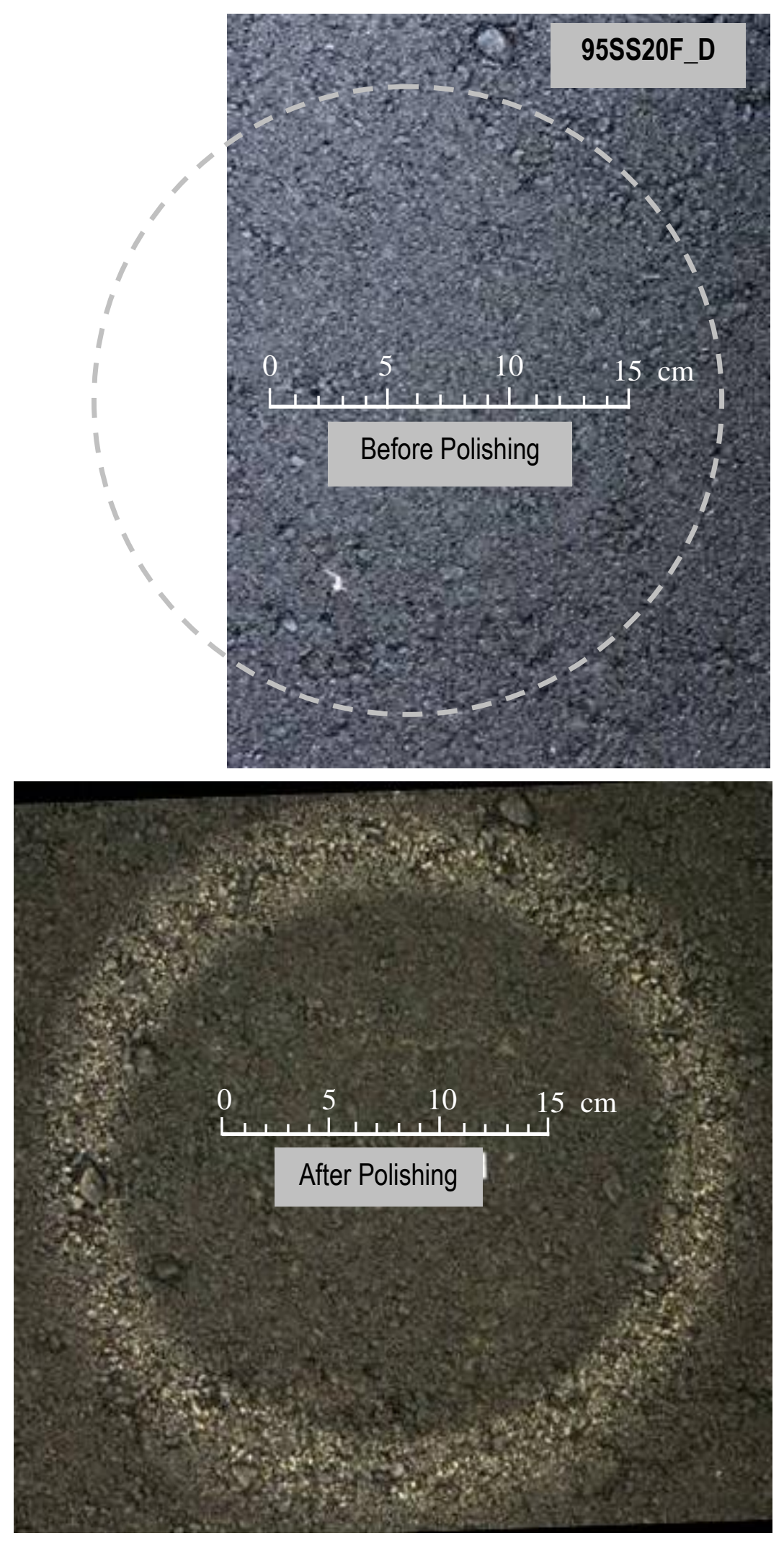

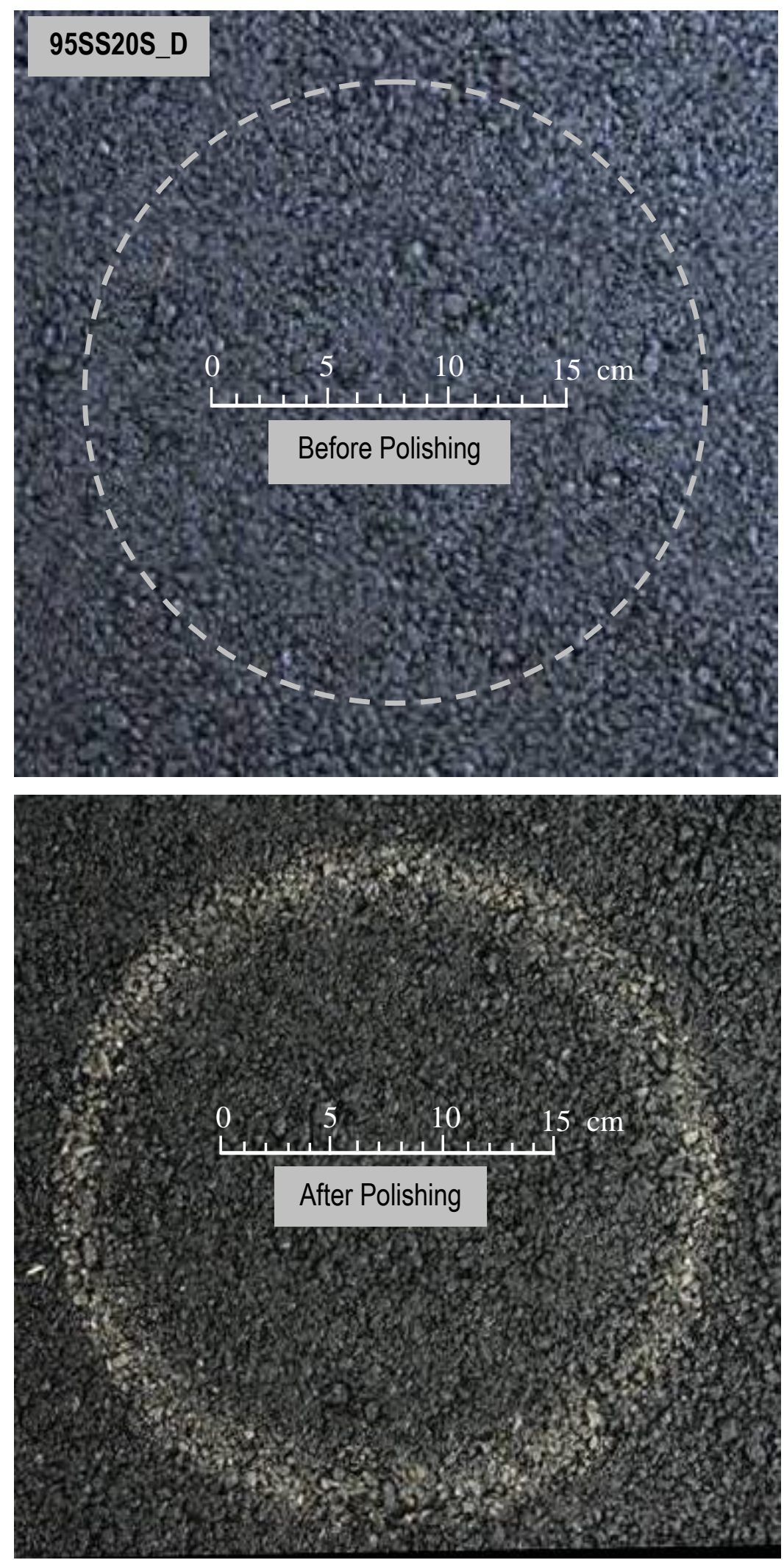



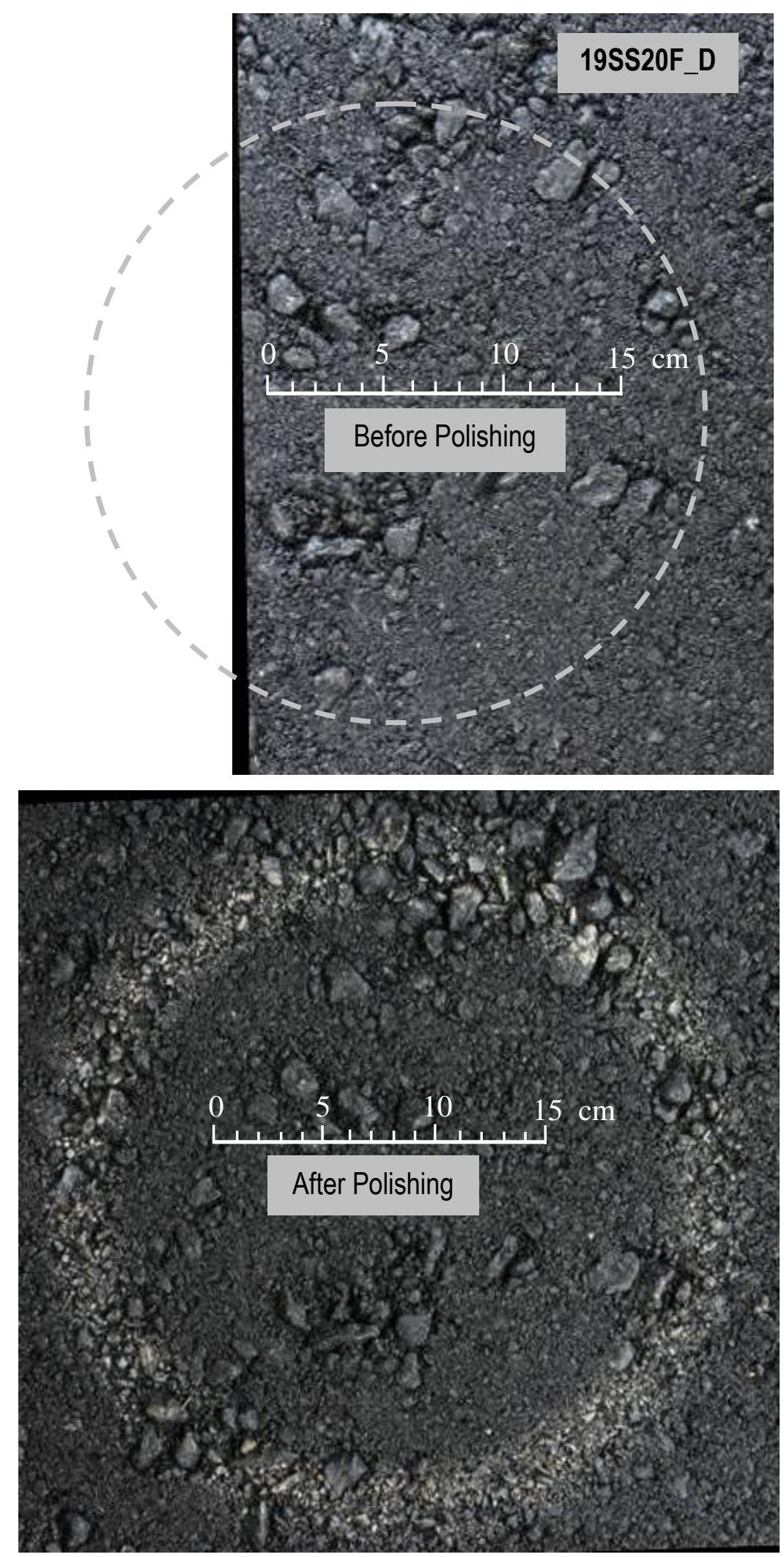



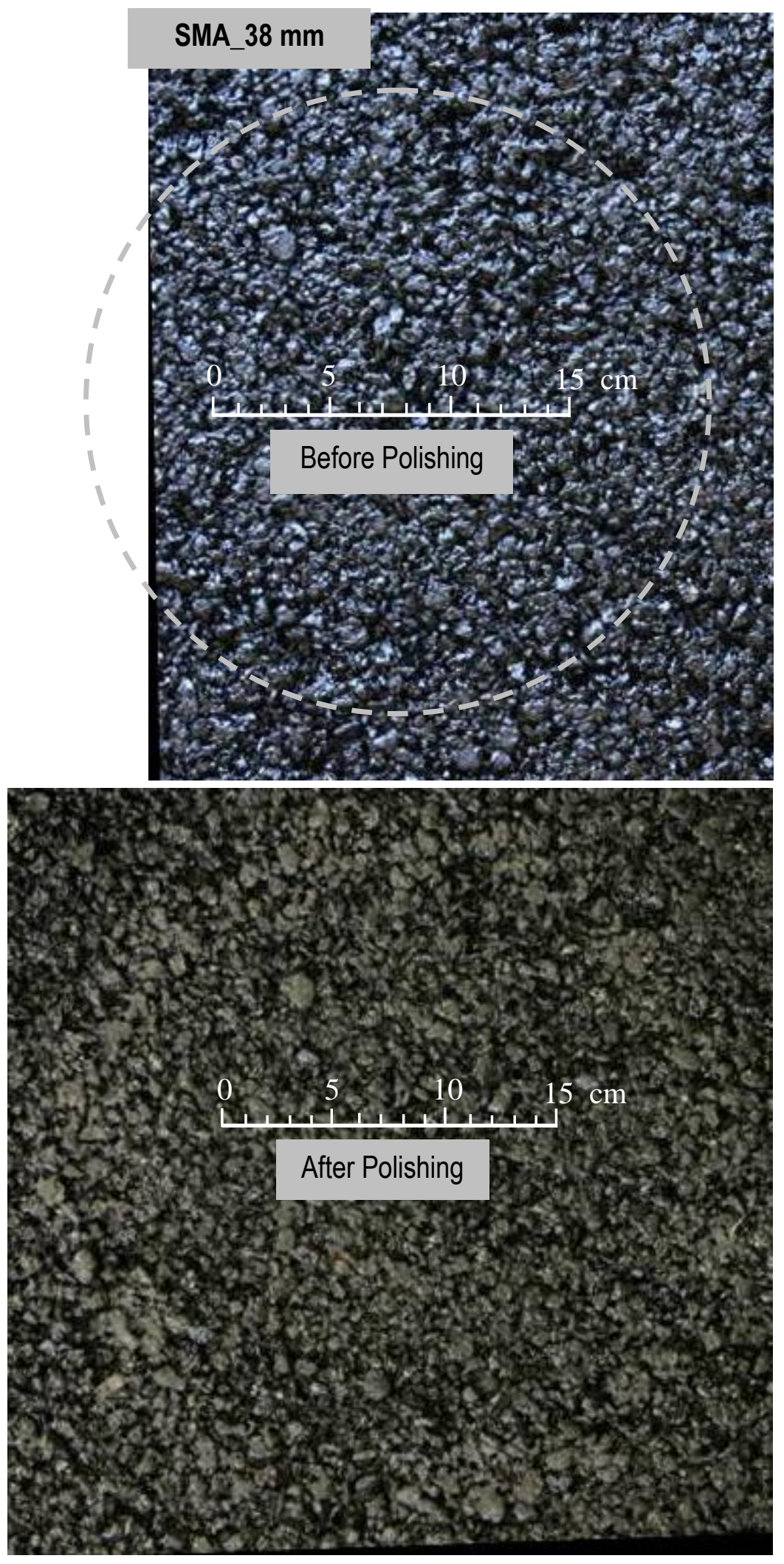

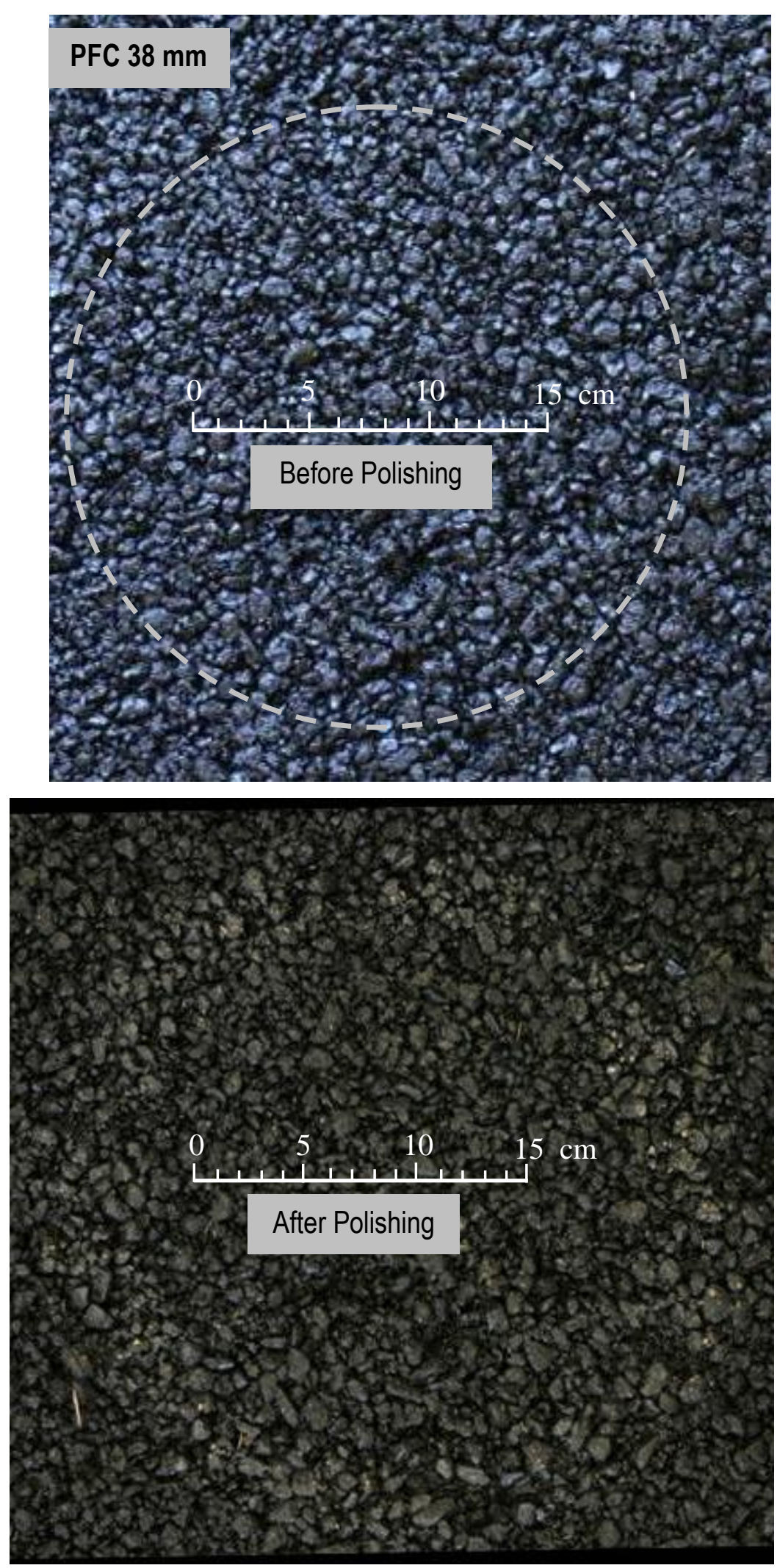

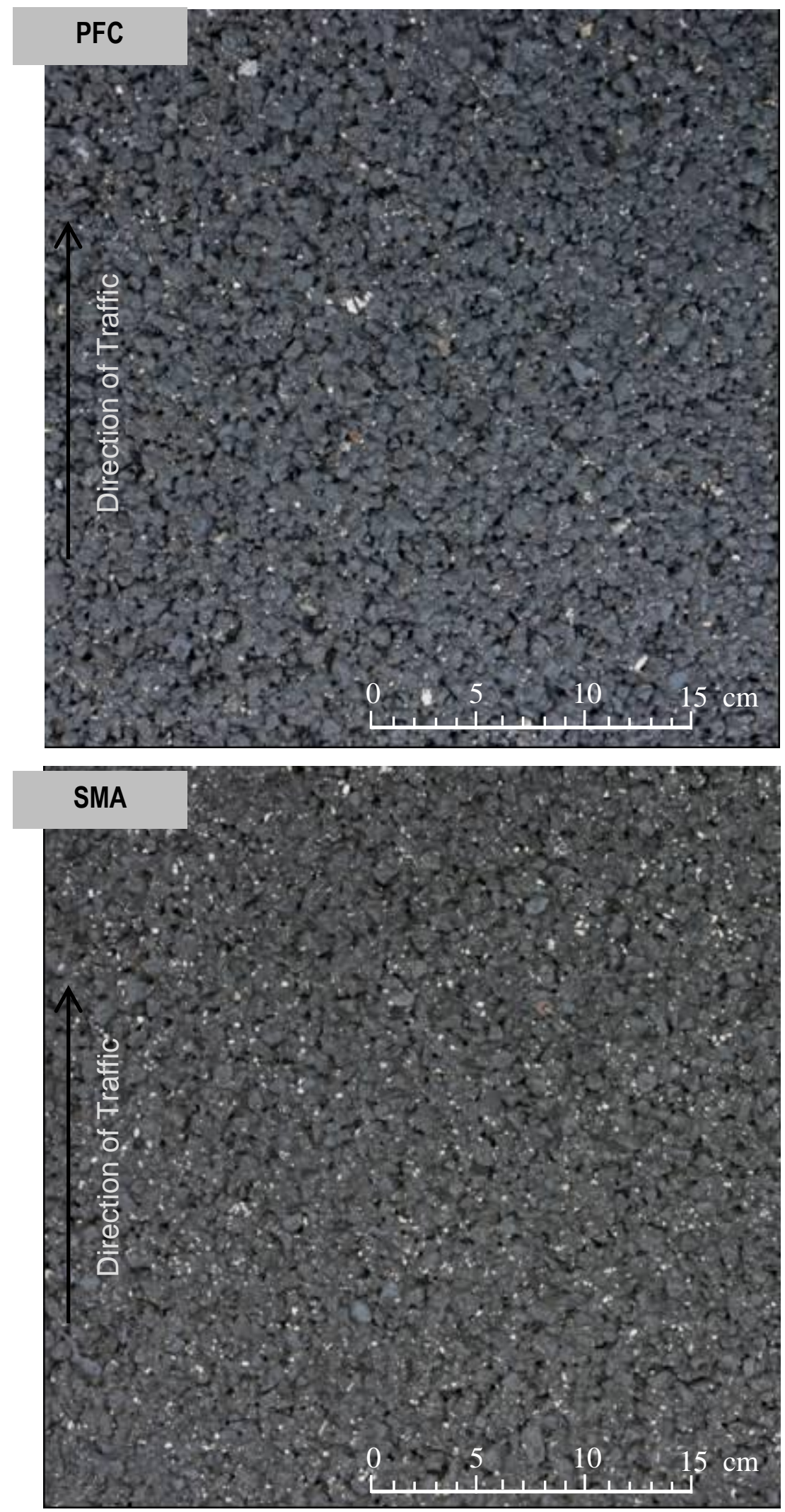




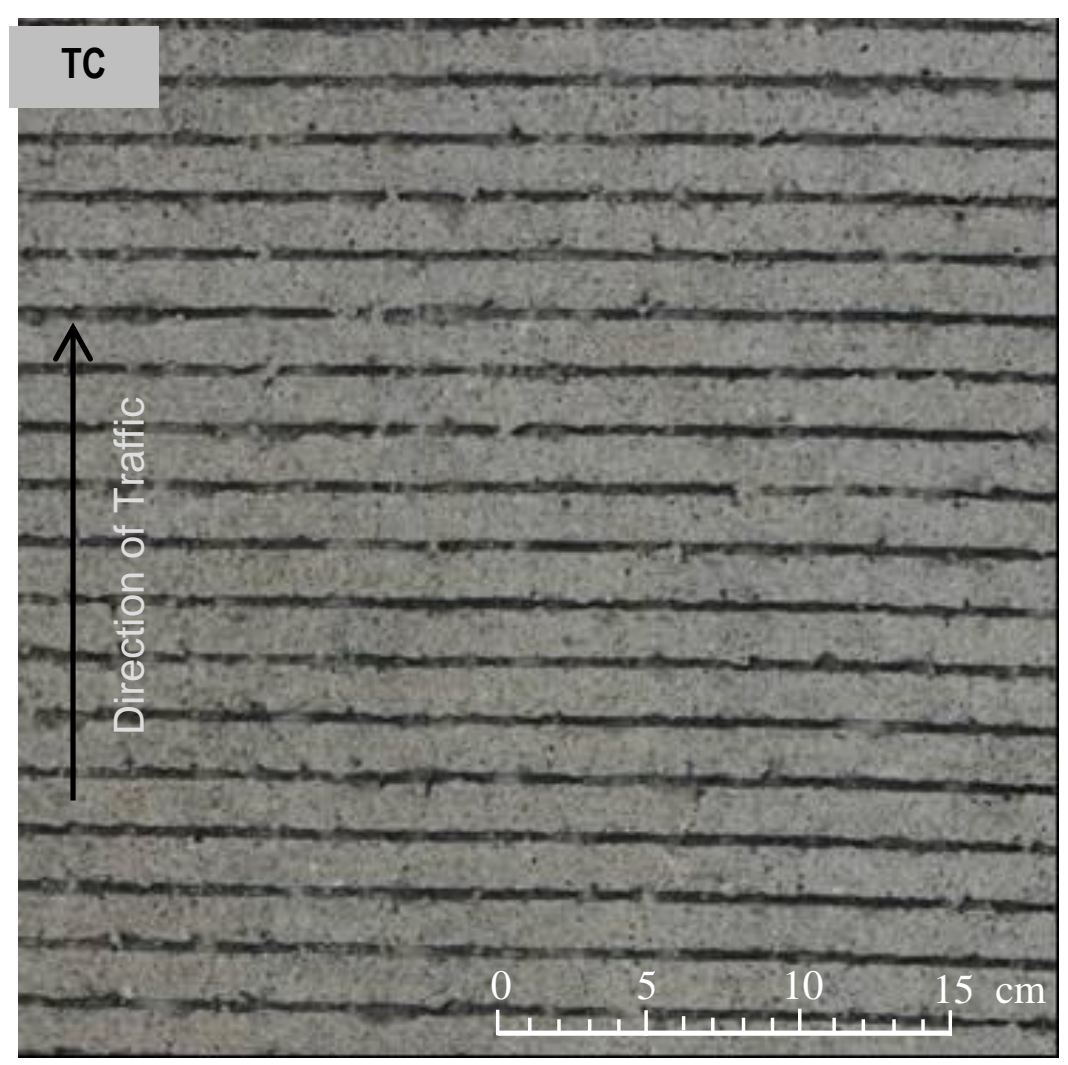

\section{SC}

凡 


\section{Appendix C: Summary of Mixture Design}

This appendix contains a summary of the mixture design data. Data for mixes tested in the laboratory is shown in Table $\mathrm{C} 1$ in the following order: mix number (No.), the mix label, mixture maximum theoretical specific gravity $\left(\mathrm{G}_{\mathrm{mm}}\right)$, aggregate blend specific gravity $\left(\mathrm{G}_{\mathrm{sb}}\right)$, percent of voids in the mineral aggregate (VMA), percent of voids filled with asphalt (VFA) and dust to binder ratio $\left(\mathrm{P}_{0.075} / \mathrm{P}_{\text {be }}\right)$. The mix label includes the the mixture nominal maximum aggregate size (NMAS, mm), friction aggregate type (FAT), friction aggregate content (FAC), aggregate gradation type $(\mathrm{G})$ and common aggregate type (CAT),

Plant produced mixes tested in the lab (PFC and SMA) are identified by the mix type; for those two mixtures only $\mathrm{G}_{\mathrm{mm}}$ and $\mathrm{G}_{\mathrm{sb}}$ values are reported. 
Table C 1 . Summary of the mixture design properties

\begin{tabular}{|c|c|c|c|c|c|c|c|c|c|c|}
\hline No. & \multicolumn{5}{|c|}{ Mix Label } & $\mathrm{G}_{\mathrm{mm}}$ & $\mathrm{G}_{\mathrm{sb}}$ & VMA & VFA & $\mathrm{P}_{0.075} / \mathrm{P}_{\mathrm{be}}$ \\
\hline 1 & 19 & $Q$ & 20 & C & D & 2.54 & 2.66 & 12.8 & 68.8 & 0.7 \\
\hline 2 & 19 & Q & 20 & $\mathrm{C}$ & HL & 2.49 & 2.64 & 13.8 & 71.0 & 0.7 \\
\hline 3 & 19 & Q & 20 & $\mathrm{C}$ & SL & 2.44 & 2.54 & 12.2 & 67.2 & 0.8 \\
\hline 4 & 19 & Q & 20 & $\mathrm{~F}$ & $\mathrm{D}$ & 2.57 & 2.68 & 11.6 & 65.6 & 2.2 \\
\hline 5 & 19 & Q & 20 & $\mathrm{~F}$ & $\mathrm{HL}$ & 2.55 & 2.66 & 11.9 & 66.3 & 2.1 \\
\hline 6 & 19 & Q & 20 & $\mathrm{~F}$ & SL & 2.52 & 2.61 & 10.7 & 62.7 & 2.5 \\
\hline 7 & 19 & Q & 20 & $S$ & $\mathrm{D}$ & 2.51 & 2.66 & 14.3 & 72.0 & 0.6 \\
\hline 8 & 19 & Q & 20 & $S$ & $\mathrm{HL}$ & 2.49 & 2.64 & 14.3 & 72.0 & 0.6 \\
\hline 9 & 19 & Q & 20 & $S$ & SL & 2.43 & 2.54 & 12.9 & 69.1 & 0.7 \\
\hline 10 & 19 & SS & 20 & $\mathrm{C}$ & $\mathrm{D}$ & 2.69 & 2.82 & 12.3 & 67.6 & 0.9 \\
\hline 11 & 19 & SS & 20 & $\mathrm{C}$ & $\mathrm{HL}$ & 2.68 & 2.79 & 11.8 & 66.0 & 1.0 \\
\hline 12 & 19 & SS & 20 & $\mathrm{C}$ & SL & 2.61 & 2.68 & 10.7 & 62.6 & 1.1 \\
\hline 13 & 19 & SS & 20 & $\mathrm{~F}$ & $\mathrm{D}$ & 2.69 & 2.84 & 12.8 & 68.8 & 2.0 \\
\hline 14 & 19 & SS & 20 & $\mathrm{~F}$ & $\mathrm{HL}$ & 2.69 & 2.82 & 12.3 & 67.4 & 2.2 \\
\hline 15 & 19 & SS & 20 & $\mathrm{~F}$ & SL & 2.64 & 2.75 & 11.9 & 66.3 & 2.2 \\
\hline 16 & 19 & SS & 20 & $S$ & $\mathrm{D}$ & 2.65 & 2.82 & 14.6 & 72.6 & 0.7 \\
\hline 17 & 19 & SS & 20 & $S$ & $\mathrm{HL}$ & 2.61 & 2.79 & 14.9 & 73.2 & 0.7 \\
\hline 18 & 19 & SS & 20 & $S$ & SL & 2.55 & 2.68 & 13.5 & 70.3 & 0.7 \\
\hline 19 & 95 & $\mathrm{Q}$ & 20 & $\mathrm{C}$ & $\mathrm{D}$ & 2.50 & 2.66 & 15.1 & 73.5 & 0.6 \\
\hline 20 & 95 & $\mathrm{Q}$ & 20 & $\mathrm{C}$ & $\mathrm{HL}$ & 2.46 & 2.63 & 15.6 & 74.3 & 0.6 \\
\hline 21 & 95 & Q & 20 & $\mathrm{C}$ & SL & 2.42 & 2.55 & 14.2 & 71.8 & 0.7 \\
\hline 22 & 95 & $\mathrm{Q}$ & 20 & $\mathrm{~F}$ & $\mathrm{D}$ & 2.54 & 2.69 & 13.6 & 70.6 & 2.2 \\
\hline 23 & 95 & $\mathrm{Q}$ & 20 & $\mathrm{~F}$ & $\mathrm{HL}$ & 2.52 & 2.68 & 13.9 & 71.2 & 2.2 \\
\hline 24 & 95 & $\mathrm{Q}$ & 20 & $\mathrm{~F}$ & SL & 2.51 & 2.64 & 13.1 & 69.6 & 3.8 \\
\hline 25 & 95 & Q & 20 & $S$ & $\mathrm{D}$ & 2.47 & 2.66 & 16.9 & 76.4 & 0.5 \\
\hline 26 & 95 & $\mathrm{Q}$ & 20 & $S$ & $\mathrm{HL}$ & 2.46 & 2.63 & 16.6 & 75.9 & 0.6 \\
\hline 27 & 95 & $\mathrm{Q}$ & 20 & $S$ & SL & 2.42 & 2.55 & 15.4 & 74.0 & 0.4 \\
\hline 28 & 95 & SS & 20 & $\mathrm{C}$ & $\mathrm{D}$ & 2.65 & 2.81 & 14.6 & 72.6 & 0.7 \\
\hline 29 & 95 & SS & 20 & $\mathrm{C}$ & HL & 2.60 & 2.78 & 15.3 & 73.8 & 0.7 \\
\hline 30 & 95 & SS & 20 & $\mathrm{C}$ & SL & 2.61 & 2.69 & 12.3 & 67.4 & 0.9 \\
\hline 31 & 95 & SS & 20 & $\mathrm{~F}$ & $\mathrm{D}$ & 2.66 & 2.84 & 15.2 & 73.6 & 2.0 \\
\hline 32 & 95 & SS & 20 & $\mathrm{~F}$ & HL & 2.66 & 2.83 & 14.7 & 72.9 & 2.1 \\
\hline
\end{tabular}




\begin{tabular}{|c|c|c|c|c|c|c|c|c|c|}
\hline No. & \multicolumn{4}{|c|}{ Mix Label } & $\mathrm{G}_{\mathrm{mm}}$ & $\mathrm{G}_{\mathrm{sb}}$ & VMA & SL & $\mathrm{P}_{0.075} / \mathrm{P}_{\mathrm{be}}$ \\
\hline 33 & 95 & 20 & $\mathrm{~F}$ & SL & 2.65 & 2.80 & 14.0 & 71.5 & 2.3 \\
\hline 34 & 95 & 20 & $\mathrm{~S}$ & D & 2.58 & 2.81 & 17.9 & 77.7 & 0.5 \\
\hline 35 & 95 & 20 & $\mathrm{~S}$ & HL & 2.56 & 2.78 & 18.1 & 77.8 & 0.6 \\
\hline 36 & 95 & 20 & $\mathrm{~S}$ & SL & 2.53 & 2.69 & 16.1 & 75.2 & 0.6 \\
\hline 37 & 95 & 0 & $\mathrm{~S}$ & $\mathrm{D}$ & 2.45 & 2.67 & 17.9 & 77.7 & 0.5 \\
\hline 38 & 95 & 10 & $\mathrm{~S}$ & $\mathrm{D}$ & 2.52 & 2.74 & 18.1 & 77.9 & 0.5 \\
\hline 39 & 95 & 40 & $\mathrm{~S}$ & $\mathrm{D}$ & 2.71 & 2.97 & 18.2 & 78.1 & 0.6 \\
\hline 40 & 95 & 70 & $\mathrm{~S}$ & $\mathrm{D}$ & 2.89 & 3.25 & 19.7 & 79.7 & 0.5 \\
\hline 41 & 95 & 10 & $\mathrm{~S}$ & $\mathrm{D}$ & 2.45 & 2.66 & 18.0 & 77.7 & 0.5 \\
\hline 42 & 95 & 40 & $\mathrm{~S}$ & $\mathrm{D}$ & 2.43 & 2.65 & 17.9 & 77.7 & 0.5 \\
\hline 43 & 95 & 70 & $\mathrm{~S}$ & $\mathrm{D}$ & 2.41 & 2.63 & 18.1 & 77.9 & 0.5 \\
\hline 44 & 95 & 10 & $\mathrm{C}$ & $\mathrm{D}$ & 2.56 & 2.74 & 15.6 & 74.4 & 0.6 \\
\hline 45 & 95 & 10 & $\mathrm{C}$ & SL & 2.53 & 2.71 & 14.3 & 72.0 & 0.7 \\
\hline 46 & 95 & 10 & $\mathrm{C}$ & $\mathrm{HL}$ & 2.47 & 2.60 & 15.7 & 74.5 & 0.7 \\
\hline 47 & PFC & & & & 3.19 & 3.57 & & & \\
\hline 48 & PFC & & & & 3.19 & 3.57 & & & \\
\hline 49 & SMA & & & & 3.15 & 3.57 & & & \\
\hline 50 & SMA & & & & 3.15 & 3.57 & & & \\
\hline
\end{tabular}

Note: Only one plant produced mixture of PFC (no. 47 and 48) and SMA (no. 49 and 50) was used in the laboratory part of this study. 


\section{Appendix D: Laboratory Polishing Data}

This appendix contains a complete summary of the laboratory polishing data. Data are presented in Table D 1 in groups separately for each tested specimen. Data are presented in the following order: sample number (No.), mix label, measurement repetition number (R), number of wheel passes (\#) corresponding to the time at which the measurement was conducted, dynamic friction value $\left(\mathrm{DF}_{20}\right)$, mean profile depth $(\mathrm{MPD}, \mathrm{mm})$ and calibrated wet friction (F60) value. Note that the F60 value was calculated based on the average MPD and $\mathrm{DF}_{20}$ data at a given number of wheel passes. As before, the mix label includes mixture nominal maximum aggregate size (NMAS, mm), friction aggregate type (FAT), friction aggregate content (FAC), aggregate gradation type $(\mathrm{G})$, and common aggregate type (CAT).

Samples of the plant produced mixes tested in the lab (PFC and SMA) are identified by the mix type and sample height (in $\mathrm{mm}$ ). 
Table D 1. Laboratory polishing data

\begin{tabular}{|c|c|c|c|c|c|c|c|c|c|c|c|c|c|c|c|c|c|c|}
\hline No & $\mathrm{Mi}$ & $\mathrm{X}$ & abel & & $\mathrm{R}$ & \# & MPD & $\mathrm{DF}_{20}$ & F6 & No & & X & & & $\#$ & MPD & $\mathrm{DF}_{20}$ & F6 \\
\hline 1 & $19 \mathrm{Q}$ & 2 & $\mathrm{C}$ & $\mathrm{D}$ & 1 & 0000 & 2.08 & 0.74 & 0.5 & 2 & $19 \mathrm{Q}$ & 2 & $\mathrm{CH}$ & 1 & 0000 & 1.19 & 0.63 & 0.4 \\
\hline 1 & $19 \mathrm{Q}$ & 2 & $\mathrm{C}$ & D & 2 & 0000 & 2.05 & 0.72 & 0.5 & 2 & $19 \mathrm{Q}$ & 2 & $\mathrm{CH}$ & 2 & 0000 & 1.32 & 0.68 & 0.4 \\
\hline 1 & $19 \mathrm{Q}$ & $\hat{2}$ & $\mathrm{C}$ & $\mathrm{D}$ & 1 & 0015 & 2.40 & 0.82 & 0.5 & 2 & $19 \mathrm{Q}$ & $\hat{2}$ & $\mathrm{CH}$ & 1 & 0015 & 1.33 & 0.79 & 0.5 \\
\hline 1 & $19 \mathrm{Q}$ & 2 & $\mathrm{C}$ & D & 2 & 0015 & 2.42 & 0.77 & 0.5 & 2 & $19 \mathrm{Q}$ & $\hat{2}$ & $\mathrm{CH}$ & 2 & 0015 & 1.23 & 0.76 & 0.5 \\
\hline 1 & $19 \mathrm{Q}$ & $\hat{2}$ & $\mathrm{C}$ & D & 1 & 0036 & 2.38 & 0.71 & $\overline{0} .5$ & 2 & $19 \mathrm{Q}$ & $\hat{2}$ & $\mathrm{C} \mathrm{H}$ & 1 & 0036 & 1.33 & 0.72 & 0.4 \\
\hline 1 & $19 \mathrm{Q}$ & $\hat{2}$ & $\mathrm{C}$ & $\mathrm{D}$ & 2 & 0036 & 2.30 & 0.68 & 0.5 & 2 & $19 \mathrm{Q}$ & $\hat{2}$ & $\mathrm{CH}$ & 2 & 0036 & 1.23 & 0.70 & 0.4 \\
\hline 1 & $19 \mathrm{Q}$ & $\hat{2}$ & $\mathrm{C}$ & D & 1 & 0090 & 2.40 & 0.65 & 0.4 & 2 & $19 \mathrm{Q}$ & $\hat{2}$ & $\mathrm{CH}$ & 1 & 0090 & 1.24 & 0.68 & 0.4 \\
\hline 1 & $19 \mathrm{Q}$ & $\hat{2}$ & $\mathrm{C}$ & D & 2 & 0090 & 2.38 & 0.63 & $\overline{0} .4$ & 2 & $19 \mathrm{Q}$ & $\hat{2}$ & $\mathrm{CH}$ & 2 & 0090 & 1.25 & 0.65 & 0.4 \\
\hline 1 & 19 Q & $\hat{2}$ & $\mathrm{C}$ & D & 1 & 0180 & 2.33 & 0.62 & $\overline{0} .4$ & 2 & $19 \mathrm{Q}$ & $\hat{2}$ & $\mathrm{C} \mathrm{H}$ & 1 & 0180 & 1.30 & 0.56 & 0.3 \\
\hline 1 & $19 \mathrm{Q}$ & $\hat{2}$ & $\mathrm{C}$ & D & 2 & 0180 & 2.30 & 0.59 & $\overline{0} .4$ & 2 & $19 \mathrm{Q}$ & $\hat{2}$ & $\mathrm{C} \mathrm{H}$ & 2 & 0180 & 1.27 & 0.57 & 0.3 \\
\hline 1 & $19 \mathrm{Q}$ & $\hat{2}$ & $\mathrm{C}$ & D & 1 & 0300 & 2.39 & 0.56 & $\overline{0} .4$ & 2 & $19 \mathrm{Q}$ & $\hat{2}$ & $\mathrm{CH}$ & 1 & 0300 & 1.31 & 0.58 & 0.3 \\
\hline 1 & $19 \mathrm{Q}$ & $\hat{2}$ & $\mathrm{C}$ & $\mathrm{D}$ & 2 & 0300 & 2.31 & 0.56 & 0.4 & 2 & $19 \mathrm{Q}$ & $\hat{2}$ & $\mathrm{CH}$ & 2 & 0300 & 1.37 & 0.56 & 0.3 \\
\hline 1 & $19 \mathrm{Q}$ & $\hat{2}$ & $\mathrm{C}$ & D & 1 & 0450 & 2.23 & 0.54 & 0.4 & 2 & $19 \mathrm{Q}$ & $\hat{2}$ & $\mathrm{CH}$ & 1 & 0450 & 1.43 & 0.56 & 0.3 \\
\hline 1 & $19 \mathrm{Q}$ & $\hat{2}$ & $\mathrm{C}$ & D & 2 & 0450 & 2.22 & 0.52 & 0.4 & 2 & $19 \mathrm{Q}$ & $\hat{2}$ & $\mathrm{CH}$ & 2 & 0450 & 1.45 & 0.53 & 0.3 \\
\hline 1 & $19 \mathrm{Q}$ & $\hat{2}$ & $\mathrm{C}$ & D & 1 & 0750 & 2.22 & 0.49 & 0.3 & 2 & $19 \mathrm{Q}$ & $\hat{2}$ & $\mathrm{C} \mathrm{H}$ & 1 & 0750 & 1.37 & 0.50 & 0.3 \\
\hline 1 & $19 \mathrm{Q}$ & $\hat{2}$ & $\mathrm{C}$ & D & 2 & 0750 & 2.32 & 0.46 & $\overline{0} .3$ & 2 & $19 \mathrm{Q}$ & $\hat{2}$ & $\mathrm{CH}$ & 2 & 0750 & 1.38 & 0.50 & 0.3 \\
\hline 1 & 19 Q & $\hat{2}$ & $\mathrm{C}$ & D & 1 & 1200 & 2.32 & 0.42 & $\overline{0} .3$ & 2 & $19 \mathrm{Q}$ & $\hat{2}$ & $\mathrm{CH}$ & 1 & 1200 & 1.39 & 0.48 & $\overline{0} .3$ \\
\hline 1 & $19 \mathrm{Q}$ & $\hat{2}$ & $\mathrm{C}$ & $\mathrm{D}$ & 2 & 1200 & 2.35 & 0.41 & 0.3 & 2 & $19 \mathrm{Q}$ & $\hat{2}$ & $\mathrm{CH}$ & 2 & 1200 & 1.39 & 0.48 & 0.3 \\
\hline 1 & $19 \mathrm{Q}$ & 2 & $\mathrm{C}$ & $\mathrm{D}$ & 1 & 1650 & 2.24 & 0.40 & 0.3 & 2 & $19 \mathrm{Q}$ & $\hat{2}$ & $\mathrm{CH}$ & 1 & 1650 & 1.35 & 0.42 & 0.3 \\
\hline 1 & $19 \mathrm{Q}$ & 2 & $\mathrm{C}$ & D & 2 & 1650 & 2.28 & 0.40 & 0.3 & 2 & $19 \mathrm{Q}$ & $\hat{2}$ & $\mathrm{C}$ & 2 & 1650 & 1.37 & 0.41 & 0.3 \\
\hline 3 & $19 \mathrm{Q}$ & 2 & $\mathrm{C}$ & S & 1 & 0000 & 1.73 & 0.69 & 0.4 & 4 & $19 \mathrm{Q}$ & 2 & $\mathrm{~F} D$ & 1 & 0000 & 0.91 & 0.81 & 0.4 \\
\hline 3 & 19 Q & 2 & $\mathrm{C}$ & $S$ & 2 & 0000 & 1.73 & 0.71 & 0.4 & 4 & $19 \mathrm{Q}$ & $\hat{2}$ & $\mathrm{~F} \mathrm{D}$ & 2 & 0000 & 0.93 & 0.75 & 0.4 \\
\hline 3 & $19 \mathrm{Q}$ & 2 & $\mathrm{C}$ & S & 1 & 0015 & 1.76 & 0.63 & 0.4 & 4 & $19 \mathrm{Q}$ & 2 & & 1 & 0015 & 1.18 & 0.79 & 0.4 \\
\hline 3 & $19 \mathrm{Q}$ & 2 & $\mathrm{C}$ & S & 2 & 0015 & 1.75 & 0.62 & 0.4 & 4 & $19 \mathrm{Q}$ & $\hat{2}$ & & 2 & 0015 & 1.18 & 0.75 & 0.4 \\
\hline 3 & $19 \mathrm{Q}$ & 2 & $\mathrm{C}$ & $S$ & 1 & 0036 & 1.63 & 0.63 & 0.4 & 4 & $19 \mathrm{Q}$ & $\hat{2}$ & $\mathrm{~F} D$ & 1 & 0036 & 1.28 & 0.73 & 0.4 \\
\hline 3 & $19 \mathrm{Q}$ & $\hat{2}$ & $\mathrm{C}$ & $S$ & 2 & 0036 & 1.59 & 0.61 & 0.4 & 4 & $19 \mathrm{Q}$ & $\hat{2}$ & $\mathrm{~F} \mathrm{D}$ & 2 & 0036 & 1.21 & 0.70 & 0.4 \\
\hline 3 & $19 \mathrm{Q}$ & $\hat{2}$ & $\mathrm{C}$ & S & 1 & 0090 & 1.73 & 0.59 & 0.4 & 4 & $19 \mathrm{Q}$ & $\hat{2}$ & & 1 & 0090 & 1.30 & 0.67 & 0.4 \\
\hline 3 & $19 \mathrm{Q}$ & 2 & $\mathrm{C}$ & $S$ & 2 & 0090 & 1.73 & 0.57 & 0.4 & 4 & $19 \mathrm{Q}$ & 2 & $\mathrm{~F} D$ & 2 & 0090 & 1.28 & 0.65 & 0.4 \\
\hline 3 & $19 \mathrm{Q}$ & 2 & $\mathrm{C}$ & $S$ & 1 & 0180 & 1.67 & 0.54 & 0.3 & 4 & $19 \mathrm{Q}$ & $\hat{2}$ & & 1 & 0180 & 1.39 & 0.64 & 0.4 \\
\hline 3 & $19 \mathrm{Q}$ & 2 & $\mathrm{C}$ & $S$ & 2 & 0180 & 1.74 & 0.51 & 0.3 & 4 & $19 \mathrm{Q}$ & $\hat{2}$ & $\mathrm{~F} D$ & 2 & 0180 & 1.39 & 0.62 & 0.4 \\
\hline 3 & $19 \mathrm{Q}$ & $\hat{2}$ & $\mathrm{C}$ & S & 1 & 0300 & 1.72 & 0.52 & 0.3 & 4 & $19 \mathrm{Q}$ & $\hat{2}$ & $\mathrm{~F} \mathrm{D}$ & 1 & 0300 & 1.46 & 0.59 & 0.4 \\
\hline 3 & $19 \mathrm{Q}$ & $\hat{2}$ & $\mathrm{C}$ & S & 2 & 0300 & 1.75 & 0.52 & 0.3 & 4 & $19 \mathrm{Q}$ & $\hat{2}$ & & 2 & 0300 & 1.48 & 0.58 & 0.4 \\
\hline 3 & $19 \mathrm{Q}$ & 2 & $\mathrm{C}$ & $S$ & 1 & 0450 & 1.84 & 0.51 & 0.3 & 4 & $19 \mathrm{Q}$ & 2 & F D & 1 & 0450 & 1.71 & 0.53 & 0.3 \\
\hline 3 & $19 \mathrm{Q}$ & 2 & $\mathrm{C}$ & $\mathrm{S}$ & 2 & 0450 & 1.86 & 0.50 & 0.3 & 4 & $19 \mathrm{Q}$ & $\hat{2}$ & $\mathrm{~F} D$ & 2 & 0450 & 1.68 & 0.54 & 0.3 \\
\hline 3 & $19 \mathrm{Q}$ & 2 & $\mathrm{C}$ & $S$ & 1 & 0750 & 1.82 & 0.49 & 0.3 & 4 & $19 \mathrm{Q}$ & 2 & $\mathrm{~F} D$ & 1 & 0750 & 1.58 & 0.50 & 0.3 \\
\hline 3 & $19 \mathrm{Q}$ & $\hat{2}$ & $\mathrm{C}$ & & 2 & 0750 & 1.85 & 0.48 & 0.3 & 4 & $19 \mathrm{Q}$ & $\hat{2}$ & $\mathrm{~F} \mathrm{D}$ & 2 & 0750 & 1.59 & 0.49 & 0.3 \\
\hline 3 & $19 \mathrm{Q}$ & 2 & $\mathrm{C}$ & 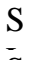 & 1 & 1200 & 1.77 & 0.31 & 0.2 & 4 & $19 \mathrm{Q}$ & $\hat{2}$ & $\mathrm{~F} D$ & & 1200 & 1.59 & 0.47 & 0.3 \\
\hline 3 & $19 \mathrm{Q}$ & 2 & $\mathrm{C}$ & 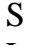 & 2 & 1200 & 1.73 & 0.31 & 0.2 & 4 & $19 \mathrm{Q}$ & 2 & $\mathrm{~F} D$ & 2 & 1200 & 1.59 & 0.47 & 0.3 \\
\hline 3 & $19 \mathrm{Q}$ & 2 & $\mathrm{C}$ & C & 1 & 1650 & 1.82 & 0.30 & 0.2 & 4 & $19 \mathrm{Q}$ & $\hat{2}$ & $\mathrm{~F} D$ & & 1650 & 1.62 & 0.43 & 0.3 \\
\hline 3 & $19 \mathrm{Q}$ & 2 & $\mathrm{C}$ & & 2 & 1650 & 1.89 & 0.31 & 0.2 & 4 & $19 \mathrm{Q}$ & $\hat{2}$ & $\mathrm{~F} \mathrm{D}$ & & 1650 & 1.61 & 0.43 & 0.3 \\
\hline
\end{tabular}




\begin{tabular}{|c|c|c|c|c|c|c|c|c|c|c|c|c|c|c|c|c|c|c|}
\hline No & & iix & abel & K & $\#$ & MPD & $\mathrm{DF}_{20}$ & F6 & No & & li & el & & $\mathrm{R}$ & $\#$ & MPD & $\mathrm{DF}_{20}$ & F6 \\
\hline 5 & 19 Q & 2 & $\mathrm{~F} \mathrm{I}$ & \begin{tabular}{l|l}
$\mathrm{H}$ & 1
\end{tabular} & 0000 & 0.65 & 0.79 & 0.4 & 6 & $19 Q$ & 2 & $\mathrm{~F}$ & S & 1 & 0000 & 0.69 & 0.85 & 0.4 \\
\hline 5 & 19 Q & 2 & F 1 & $\mathrm{H} 2$ & 0000 & 0.67 & 0.71 & 0.4 & 6 & 19 Q & 2 & $\mathrm{~F}$ & S & 2 & 0000 & 0.69 & 0.74 & 0.4 \\
\hline 5 & 19 Q & $\hat{2}$ & $\mathrm{~F}$ & $\begin{array}{ll}H \\
H\end{array}$ & 0015 & 0.90 & 0.78 & 0.4 & 6 & $19 \mathrm{Q}$ & 2 & $\mathrm{~F}$ & S & 1 & 0015 & 1.10 & 0.64 & 0.4 \\
\hline S & 19 Q & 2 & $\mathrm{~F}$ & 2 & 0015 & 0.89 & 0.73 & 0.4 & 6 & 19 Q & 2 & $\mathrm{~F}$ & S & 2 & 0015 & 1.08 & 0.62 & 0.4 \\
\hline P & 19 Q & 2 & $\mathrm{~F}$ & \begin{tabular}{l|l}
$\mathrm{H}$ & 1
\end{tabular} & 0036 & 1.09 & 0.71 & 0.4 & 6 & $19 \mathrm{Q}$ & 2 & $\mathrm{~F}$ & S & 1 & 0036 & 1.20 & 0.61 & 0.4 \\
\hline 5 & 19 Q & 2 & $\mathrm{~F}$ & H 2 & 0036 & 1.08 & 0.68 & 0.4 & 6 & 19 Q & 2 & $\mathrm{~F}$ & S & 2 & 0036 & 1.19 & 0.60 & 0.4 \\
\hline 5 & 19 Q & 2 & $\mathrm{~F} I$ & $\mathrm{H}$ & 0090 & 1.08 & 0.64 & 0.4 & 6 & 19 Q & 2 & $\mathrm{~F}$ & S & 1 & 0090 & 1.27 & 0.59 & 0.3 \\
\hline 5 & 19 Q & 2 & $\mathrm{~F}$ & $\mathrm{H} 2$ & 0090 & 1.08 & 0.62 & 0.4 & 6 & $19 \mathrm{Q}$ & $\hat{2}$ & $\mathrm{~F}$ & S & 2 & 0090 & 1.33 & 0.56 & 0.3 \\
\hline 5 & 19 Q & 2 & $\mathrm{~F}$ & $\begin{array}{ll}\mathrm{H} & 1\end{array}$ & 0180 & 1.24 & 0.60 & 0.3 & 6 & $19 \mathrm{Q}$ & 2 & $\mathrm{~F}$ & S & 1 & 0180 & 1.47 & 0.54 & 0.3 \\
\hline 5 & 19 Q & 2 & $\mathrm{~F} I$ & $\mathrm{H} 2$ & 0180 & 1.20 & 0.58 & 0.3 & 6 & 19 Q & 2 & $\mathrm{~F}$ & S & 2 & 0180 & 1.46 & 0.52 & 0.3 \\
\hline 5 & 19 Q & 2 & $\mathrm{~F}$ & $\begin{array}{ll}H \\
H\end{array}$ & 0300 & 1.31 & 0.54 & 0.3 & 6 & & $\hat{2}$ & $\mathrm{~F}$ & S & 1 & 0300 & 1.41 & 0.48 & $\overline{0} .3$ \\
\hline 5 & 19 Q & 2 & $\mathrm{~F}$ & $\mathrm{H} 2$ & 0300 & 1.31 & 0.52 & 0.3 & 6 & 19 Q & 2 & $\mathrm{~F}$ & S & 2 & 0300 & 1.40 & 0.47 & 0.3 \\
\hline 5 & 19 Q & 2 & $\mathrm{~F}$ & H 1 & 0450 & 1.27 & 0.52 & 0.3 & 6 & $19 \mathrm{Q}$ & 2 & $\mathrm{~F}$ & S & 1 & 0450 & 1.51 & 0.44 & 0.3 \\
\hline 5 & 19 Q & 2 & $\mathrm{~F}$ & $\mathrm{H} 2$ & 0450 & 1.30 & 0.50 & 0.3 & 6 & 19 Q & 2 & $\mathrm{~F}$ & S & 2 & 0450 & 1.51 & 0.44 & 0.3 \\
\hline 5 & 19 Q & 2 & $\mathrm{~F} I$ & $\begin{array}{ll}\mathrm{H} & 1\end{array}$ & 0750 & 1.34 & 0.45 & 0.3 & 6 & 19 Q & 2 & $\mathrm{~F}$ & S & 1 & 0750 & 1.52 & 0.39 & 0.3 \\
\hline 5 & 19 Q & 2 & $\mathrm{~F}$ & $\mathrm{H} 2$ & 0750 & 1.30 & 0.44 & 0.3 & 6 & 19 Q & 2 & $\mathrm{~F}$ & S & 2 & 0750 & 1.51 & 0.39 & 0.3 \\
\hline 5 & 19 Q & 2 & $\mathrm{~F}$ & $\begin{array}{ll}\mathrm{H} & 1\end{array}$ & 1200 & 1.32 & 0.40 & 0.2 & 6 & 19 Q & 2 & $\mathrm{~F}$ & S & 1 & 1200 & 1.47 & 0.38 & 0.2 \\
\hline 5 & 19 Q & 2 & $\mathrm{~F}$ & $\mathrm{H} 2$ & 1200 & 1.31 & 0.39 & 0.2 & 6 & $19 \mathrm{Q}$ & 2 & $\mathrm{~F}$ & S & 2 & 1200 & 1.45 & 0.38 & 0.2 \\
\hline 5 & 19 Q & 2 & $\mathrm{~F}$ & H 1 & 1650 & 1.27 & 0.39 & 0.2 & 6 & 19 Q & 2 & $\mathrm{~F}$ & S & 1 & 1650 & 1.42 & 0.36 & 0.2 \\
\hline 5 & 19 Q & 2 & $\mathrm{~F}$ & $\mathrm{H} \quad 2$ & 1650 & 1.30 & 0.38 & 0.2 & 6 & $19 \mathrm{Q}$ & 2 & $\mathrm{~F}$ & S & 2 & 1650 & 1.40 & 0.35 & 0.2 \\
\hline 7 & 19 Q & 2 & S I & \begin{tabular}{l|l} 
D & 1
\end{tabular} & 0000 & 1.34 & 0.67 & 0.4 & 8 & 19 Q & 2 & $\mathrm{~S}$ & 11 & 1 & 0000 & 1.32 & 0.66 & 0.4 \\
\hline 7 & 19 Q & 2 & $S \quad I$ & D 2 & 0000 & 1.38 & 0.63 & 0.4 & 8 & 19 Q & 2 & $S$ & $=$ & 2 & 0000 & 1.30 & 0.65 & 0.4 \\
\hline 7 & 19 Q & 2 & $S \quad I$ & \begin{tabular}{l|l}
$\mathrm{D}$ & 1
\end{tabular} & 0015 & 1.37 & 0.82 & 0.5 & 8 & 19 Q & 2 & $\mathrm{~S}$ & $\begin{array}{l}\mathrm{H} \\
-\end{array}$ & 1 & 0015 & 1.41 & 0.75 & 0.4 \\
\hline 7 & 19 Q & 2 & $S \quad I$ & D 2 & 0015 & 1.41 & 0.78 & 0.5 & 8 & $19 \mathrm{Q}$ & 2 & $\mathrm{~S}$ & H & 2 & 0015 & 1.45 & 0.70 & 0.4 \\
\hline 7 & 19 Q & 2 & $S \quad I$ & D 1 & 0036 & 1.37 & 0.75 & 0.4 & 8 & 19 Q & 2 & $S$ & $\begin{array}{l}\mathrm{H} \\
-\end{array}$ & 1 & 0036 & 1.30 & 0.65 & 0.4 \\
\hline 7 & 19 Q & 2 & $S \quad I$ & D 2 & 0036 & 1.40 & 0.72 & 0.4 & 8 & 19 Q & 2 & $S$ & H & 2 & 0036 & 1.28 & 0.63 & 0.4 \\
\hline 7 & 19 Q & 2 & $S \quad I$ & D 1 & 0090 & 1.41 & 0.68 & 0.4 & 8 & 19 Q & 2 & $\mathrm{~S}$ & $\begin{array}{ll}\mathrm{H} \\
-\end{array}$ & 1 & 0090 & 1.32 & 0.64 & .4 \\
\hline 7 & 19 Q & 2 & $S \quad I$ & D 2 & 0090 & 1.35 & 0.67 & 0.4 & 8 & 19 Q & 2 & $S$ & $\mathrm{H}$ & 2 & 0090 & 1.37 & 0.62 & 0.4 \\
\hline 7 & 19 Q & 2 & $S \quad I$ & D 1 & 0180 & 1.28 & 0.61 & 0.4 & 8 & $19 \mathrm{Q}$ & 2 & $S$ & $\begin{array}{l}\mathrm{H} \\
-\end{array}$ & 1 & 0180 & 1.34 & 0.52 & 0.3 \\
\hline 7 & 19 Q & 2 & $S \quad I$ & $\mathrm{D} 2$ & 0180 & 1.32 & 0.60 & 0.4 & 8 & 19 Q & 2 & $S$ & $\mathrm{H}$ & 2 & 0180 & 1.34 & 0.50 & 0.3 \\
\hline 7 & 19 Q & 2 & S I & $\begin{array}{ll}\mathrm{D} & 1\end{array}$ & 0300 & 1.38 & 0.57 & 0.3 & 8 & $19 \mathrm{Q}$ & 2 & $S$ & II & 1 & 0300 & 1.31 & 0.47 & 0.3 \\
\hline 7 & 19 Q & 2 & S I & D 2 & 0300 & 1.37 & 0.56 & 0.3 & 8 & 19 Q & 2 & $\mathrm{~S}$ & U & 2 & 0300 & 1.25 & 0.45 & 0.3 \\
\hline 7 & 19 Q & 2 & $S \quad I$ & D 1 & 0450 & 1.41 & 0.55 & 0.3 & 8 & 19 Q & 2 & $S$ & - & 1 & 0450 & 1.34 & 0.45 & 0.3 \\
\hline 7 & 19 Q & 2 & $S \quad I$ & D 2 & 0450 & 1.47 & 0.53 & 0.3 & 8 & $19 \mathrm{Q}$ & 2 & $S$ & - & 2 & 0450 & 1.32 & 0.45 & 0.3 \\
\hline 7 & 19 Q & 2 & S I & D 1 & 0750 & 1.46 & 0.53 & 0.3 & 8 & 19 Q & 2 & $\mathrm{~S}$ & $=$ & 1 & 0750 & 1.34 & 0.41 & 0.3 \\
\hline 7 & 19 Q & 2 & $S \quad I$ & D 2 & 0750 & 1.48 & 0.52 & 0.3 & 8 & 19 Q & 2 & $S$ & -1 & & 0750 & 1.32 & 0.40 & 0.3 \\
\hline 7 & 19 Q & 2 & S I & D 1 & 1200 & 1.45 & 0.48 & 0.3 & 8 & $19 \mathrm{Q}$ & 2 & $\mathrm{~S}$ & 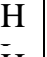 & & 1200 & 1.39 & 0.34 & 0.2 \\
\hline 7 & 19 Q & 2 & $S \quad I$ & D 2 & 1200 & 1.42 & 0.48 & 0.3 & 8 & 19 Q & 2 & $S$ & 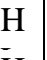 & & 1200 & 1.35 & 0.34 & 0.2 \\
\hline 7 & 19 Q & 2 & $S \quad I$ & D 1 & 1650 & 1.55 & 0.44 & 0.3 & 8 & $19 \mathrm{Q}$ & 2 & $S$ & -1 & & 1650 & 1.32 & 0.34 & 0.2 \\
\hline 7 & 19 Q & 2 & $\mathrm{~S} \quad \mathrm{I}$ & $\mathrm{D} 2$ & 1650 & 1.49 & 0.43 & 0.3 & 8 & $19 \mathrm{Q}$ & 2 & $\mathrm{~S}$ & $=1$ & 12 & 1650 & 1.33 & 0.34 & 0.2 \\
\hline
\end{tabular}




\begin{tabular}{|c|c|c|c|c|c|c|c|c|c|c|c|c|c|c|c|c|c|c|}
\hline $\mathrm{No}$ & & iix & abel & & R & \# & MPD & $\mathrm{DF}_{20}$ & F6 & No & & lix L & bel & R & \# & MPD & $\mathrm{DF}_{20}$ & F6 \\
\hline 9 & 19 Q & 2 & S & $S$ & 1 & 0000 & 1.53 & 0.67 & 0.4 & 10 & $19 \mathrm{~S}$ & 2 & $\mathrm{CD}$ & 1 & 0000 & 1.58 & 0.70 & 0.4 \\
\hline 9 & 19 Q & 2 & $S$ & $S$ & 2 & 0000 & 1.47 & 0.66 & 0.4 & 10 & $19 \stackrel{S}{S}$ & 2 & C D & $J^{2}$ & 0000 & 1.64 & 0.65 & 0.4 \\
\hline 9 & 19 Q & $\hat{2}$ & $S$ & $S$ & 1 & 0015 & 1.49 & 0.78 & 0.5 & 10 & $19 \tilde{\mathrm{S}}$ & $\hat{2}$ & C D & 1 & 0015 & 1.61 & 0.73 & 0.4 \\
\hline 9 & 19 Q & 2 & $S$ & $S$ & 2 & 0015 & 1.46 & 0.76 & 0.5 & 10 & $19 \mathrm{~S}$ & 2 & C D & 2 & 0015 & 1.61 & 0.69 & 0.4 \\
\hline 9 & 19 Q & 2 & $\mathrm{~S}$ & $S$ & 1 & 0036 & 1.38 & 0.66 & 0.4 & 10 & $19 \mathrm{~S}$ & 2 & C D & ]$^{1}$ & 0036 & 1.65 & 0.66 & 0.4 \\
\hline 9 & 19 Q & 2 & $S$ & $S$ & 2 & 0036 & 1.46 & 0.64 & 0.4 & 10 & $19 \stackrel{\tilde{S}}{\sim}$ & 2 & C D & 2 & 0036 & 1.68 & 0.63 & 0.4 \\
\hline 9 & 19 Q & 2 & $\mathrm{~S}$ & $S$ & 1 & 0090 & 1.44 & 0.62 & 0.4 & 10 & $19 \stackrel{S}{S}$ & 2 & C D & 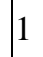 & 0090 & 1.63 & 0.63 & 0.4 \\
\hline 9 & 19 Q & 2 & $\mathrm{~S}$ & $\mathrm{~S}$ & 2 & 0090 & 1.46 & 0.58 & 0.4 & 10 & $19 \stackrel{\tilde{S}}{\mathcal{S}}$ & 2 & C D & 2 & 0090 & 1.70 & 0.61 & 0.4 \\
\hline 9 & 19 Q & 2 & $S$ & $S$ & 1 & 0180 & 1.49 & 0.60 & 0.4 & 10 & $19 \underset{\sim}{S}$ & 2 & C D & 1 & 0180 & 1.67 & 0.58 & 0.4 \\
\hline 9 & 19 Q & 2 & $S$ & S & 2 & 0180 & 1.45 & 0.59 & 0.4 & 10 & $19 \stackrel{S}{S}$ & 2 & C D & 2 & 0180 & 1.66 & 0.55 & 0.4 \\
\hline 9 & $19 \mathrm{Q}$ & 2 & $\mathrm{~S}$ & S & 1 & 0300 & 1.44 & 0.55 & 0.3 & 10 & $19 \stackrel{\tilde{S}}{\mathrm{~S}}$ & 2 & C D & T. & 0300 & 1.66 & 0.52 & 0.3 \\
\hline 9 & 19 Q & 2 & $S$ & S & 2 & 0300 & 1.50 & 0.53 & 0.3 & 10 & $19 \stackrel{S}{S}$ & 2 & C D & 2 & 0300 & 1.66 & 0.51 & 0.3 \\
\hline 9 & 19 Q & 2 & S & $S$ & 1 & 0450 & 1.45 & 0.55 & 0.3 & 10 & 19 & 2 & C D & 1 & 0450 & 1.71 & 0.49 & 0.3 \\
\hline 9 & 19 Q & 2 & $S$ & $S$ & 2 & 0450 & 1.41 & 0.53 & 0.3 & 10 & $19 \stackrel{\tilde{S}}{\sim}$ & 2 & C D & $\mathrm{I}^{-}$ & 0450 & 1.74 & 0.47 & 0.3 \\
\hline 9 & 19 Q & 2 & $S$ & S & 1 & 0750 & 1.58 & 0.48 & 0.3 & 10 & $19 \tilde{\mathrm{S}}$ & 2 & C D & $a^{1}$ & 0750 & 1.67 & 0.42 & 0.3 \\
\hline 9 & 19 Q & 2 & $\mathrm{~S}$ & S & 2 & 0750 & 1.58 & 0.49 & 0.3 & 10 & 19 & $\hat{2}$ & C D & 2 & 0750 & 1.61 & 0.41 & 0.3 \\
\hline 9 & 19 Q & 2 & $S$ & $S$ & 1 & 1200 & 1.54 & 0.38 & 0.3 & 10 & $19 \stackrel{S}{S}$ & 2 & C D & 11 & 1200 & 1.74 & 0.41 & 0.3 \\
\hline 9 & $19 \mathrm{Q}$ & $\hat{2}$ & $S$ & $\mathrm{~S}$ & 2 & 1200 & 1.54 & 0.40 & 0.3 & 10 & $19 \tilde{S}$ & 2 & C D & 2 & 1200 & 1.78 & 0.39 & 0.3 \\
\hline 9 & $19 \mathrm{Q}$ & 2 & $\mathrm{~S}$ & S & 1 & 1650 & 1.57 & 0.32 & 0.2 & 10 & $19 \stackrel{\tilde{S}}{\mathrm{~S}}$ & 2 & C D & 1 & 1650 & 1.74 & 0.36 & 0.2 \\
\hline 9 & 19 Q & 2 & $S$ & S & 2 & 1650 & 1.59 & 0.32 & 0.2 & 10 & $19 \stackrel{\tilde{S}}{\sim}$ & $\hat{2}$ & C D & 1 & 1650 & 1.77 & 0.36 & 0.2 \\
\hline 11 & $19 \stackrel{\mathrm{S}}{\sim}$ & 2 & C & $\mathrm{H}$ & 1 & 0000 & 1.84 & 0.63 & 0.4 & 12 & 192 & 2 & $\mathrm{C}^{\mathrm{S}}$ & 1 & 0000 & 1.36 & 0.80 & 0.4 \\
\hline 11 & $19 \stackrel{\widetilde{S}}{\sim}$ & 2 & $\mathrm{C}$ & H & 2 & 0000 & 1.76 & 0.61 & 0.4 & 12 & $19 \stackrel{\tilde{S}}{\sim}$ & 2 & C $\stackrel{S}{S}$ & 2 & 0000 & 1.36 & 0.72 & 0.4 \\
\hline 11 & $19 \stackrel{\mathrm{S}}{\sim}$ & 2 & $\mathrm{C}$ & $\mathrm{H}$ & 1 & 0015 & 1.82 & 0.67 & 0.4 & 12 & $19 \tilde{S}$ & 2 & 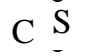 & 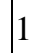 & 0015 & 1.35 & 0.68 & 0.4 \\
\hline 11 & $19 \stackrel{\tilde{S}}{\tilde{N}}$ & 2 & $\mathrm{C}$ & $\begin{array}{l}\mathrm{H} \\
-\end{array}$ & 2 & 0015 & 1.78 & 0.66 & 0.4 & 12 & 19 & 2 & $C \stackrel{S}{S}$ & 2 & 0015 & 1.44 & 0.65 & .4 \\
\hline 11 & $19 \underset{\tilde{\sigma}}{\mathrm{S}}$ & 2 & $\mathrm{C}$ & $\begin{array}{l}\mathrm{H} \\
\end{array}$ & 1 & 0036 & 1.81 & 0.65 & 0.4 & 12 & $19 \stackrel{\tilde{S}}{-}$ & 2 & $\mathrm{C}^{\mathrm{S}}$ & 1 & 0036 & 1.41 & 0.70 & 0.4 \\
\hline 11 & $19 \tilde{\mathrm{S}}$ & 2 & $\mathrm{C}$ & $\mathrm{H}$ & 2 & 0036 & 1.83 & 0.62 & 0.4 & 12 & $19 \tilde{\mathrm{S}}$ & 2 & $\mathrm{C} \stackrel{S}{\mathrm{~S}}$ & 2 & 0036 & 1.36 & 0.67 & 0.4 \\
\hline 11 & $19 \stackrel{\mathrm{S}}{\mathrm{S}}$ & 2 & C & H & 1 & 0090 & 1.84 & 0.65 & 0.4 & 12 & $19 \stackrel{\tilde{S}}{\sim}$ & 2 & $\mathrm{C}^{\mathrm{S}}$ & 1 & 0090 & 1.39 & 0.62 & 0.4 \\
\hline 11 & $19 \stackrel{\mathrm{S}}{ }$ & 2 & $\mathrm{C}$ & $\mathrm{H}$ & 2 & 0090 & 1.88 & 0.63 & 0.4 & 12 & $19 \tilde{\mathrm{S}}$ & 2 & $\mathrm{C}^{\mathrm{S}}$ & 2 & 0090 & 1.41 & 0.61 & 0.4 \\
\hline 11 & $19 \underset{\tilde{\sim}}{\mathrm{S}}$ & 2 & $\mathrm{C}$ & $\mathrm{H}$ & 1 & 0180 & 1.88 & 0.63 & 0.4 & 12 & $19 \stackrel{\tilde{S}}{\sim}$ & 2 & $\mathrm{C}^{\mathrm{S}}$ & 1 & 0180 & 1.42 & 0.60 & 0.4 \\
\hline 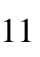 & $19 \stackrel{\widetilde{S}}{\sim}$ & 2 & $\mathrm{C}$ & $\mathrm{H}$ & 2 & 0180 & 1.94 & 0.62 & 0.4 & 12 & $19 \stackrel{\tilde{S}}{\sim}$ & 2 & C $\stackrel{S}{S}$ & t & 0180 & 1.35 & 0.58 & 0.4 \\
\hline 11 & $19 \tilde{\sim}$ & 2 & $\mathrm{C}$ & $\mathrm{H}$ & 1 & 0300 & 2.00 & 0.60 & 0.4 & 12 & $19 \tilde{\mathrm{S}}$ & 2 & $\mathrm{C}^{\mathrm{S}}$ & $\mathrm{J}^{2}$ & 0300 & 1.38 & 0.59 & 0.3 \\
\hline 11 & $19 \stackrel{\tilde{S}}{\sim}$ & 2 & C & $\mathrm{H}$ & 2 & 0300 & 1.92 & 0.59 & 0.4 & 12 & $19 \stackrel{\tilde{S}}{\mathrm{~S}}$ & 2 & C $\stackrel{S}{S}$ & 2 & 0300 & 1.37 & 0.56 & 0.3 \\
\hline 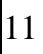 & $19 \stackrel{\widetilde{S}}{\sim}$ & 2 & $\mathrm{C}$ & $\mathrm{H}$ & 1 & 0450 & 1.85 & 0.53 & 0.3 & 12 & $19 \stackrel{\tilde{S}}{\sim}$ & 2 & $\mathrm{C}^{\mathrm{S}}$ & 1 & 0450 & 1.37 & 0.56 & 0.3 \\
\hline 11 & $19 \stackrel{\mathrm{S}}{\tilde{\tilde{n}}}$ & 2 & $\mathrm{C}$ & $\mathrm{H}$ & 2 & 0450 & 1.90 & 0.53 & 0.3 & 12 & $19 \stackrel{\tilde{S}}{\tilde{S}}$ & 2 & C $\stackrel{S}{S}$ & 2 & 0450 & 1.34 & 0.53 & 0.3 \\
\hline 11 & $19 \stackrel{\mathrm{S}}{\sim}$ & 2 & C & H & 1 & 0750 & 1.92 & 0.49 & 0.3 & 12 & 19 & 2 & C $\stackrel{S}{S}$ & 1 & 0750 & 1.45 & 0.48 & 0.3 \\
\hline $1 *$ & $19 \stackrel{\widetilde{S}}{ }$ & 2 & C & H & 2 & 0750 & 1.94 & 0.46 & 0.3 & 12 & 19 & 2 & $\mathrm{C}^{\mathrm{S}}$ & 2 & 0750 & 1.43 & 0.47 & 0.3 \\
\hline 11 & $19 \stackrel{\mathrm{S}}{\sim}$ & 2 & C & H & 1 & 1200 & 1.97 & 0.36 & 0.2 & 12 & $19 \mathrm{~S}$ & 2 & $\mathrm{C}^{\mathrm{S}}$ & 1 & 1200 & 1.45 & 0.50 & 0.3 \\
\hline 11 & $19 \stackrel{\sim}{\mathrm{S}}$ & 2 & C & t & 2 & 1200 & 1.95 & 0.34 & 0.2 & 12 & $19 \stackrel{\tilde{S}}{ }$ & 2 & C $\stackrel{S}{S}$ & 2 & 1200 & 1.48 & 0.48 & 0.3 \\
\hline 11 & $19 \stackrel{\mathrm{S}}{ }$ & 2 & $\mathrm{C}$ & I & 1 & 1650 & 1.97 & 0.37 & 0.3 & 12 & 19 & $\overline{2}$ & $C \stackrel{S}{S}$ & 1 & 1650 & 1.43 & 0.44 & 0.3 \\
\hline 11 & $19 \mathrm{~S}$ & 2 & $\mathrm{C}$ & 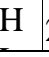 & 2 & 1650 & 2.02 & 0.38 & 0.3 & 12 & $19 \mathrm{~S}$ & 2 & $\mathrm{C} S$ & $1^{2}$ & 1650 & 1.45 & 0.43 & 0.3 \\
\hline
\end{tabular}




\begin{tabular}{|c|c|c|c|c|c|c|c|c|c|c|c|c|c|c|c|c|c|}
\hline No & Mix I & abel & & $\mathrm{R}$ & \# & MPD & $\mathrm{DF}_{20}$ & F6 & No & & Iix & & $\mathrm{R}$ & $\#$ & MPD & $\mathrm{DF}_{20}$ & F6 \\
\hline \begin{tabular}{|l|ll}
13 & $19 \mathrm{~S}$ \\
\end{tabular} & 2 & $\mathrm{~F}$ & D & 1 & 0000 & 0.72 & 0.65 & 0.3 & 14 & $19 \mathrm{~S}$ & 2 & $\mathrm{FH}$ & 1 & 0000 & 0.73 & 0.78 & 0.4 \\
\hline $1319 \overline{\mathrm{S}}$ & 2 & $\mathrm{~F}$ & D & 2 & 0000 & 0.71 & 0.67 & 0.3 & 14 & $19 \bar{S}$ & 2 & $\mathrm{~F} \overline{\mathrm{H}}$ & 2 & 0000 & 0.69 & 0.72 & 0.4 \\
\hline $1319 \tilde{\mathrm{S}}$ & 2 & $\mathrm{~F}$ & D & 1 & 0015 & 0.77 & 0.56 & $\overline{0} .3$ & 14 & $19 \tilde{S}$ & 2 & $\mathrm{~F}$ & 1 & 0015 & 0.93 & 0.81 & 0.4 \\
\hline $1319 \tilde{\mathrm{S}}$ & $\hat{2}$ & $\mathrm{~F}$ & D & 2 & 0015 & 0.77 & 0.53 & 0.3 & 14 & $19 \tilde{S}$ & 2 & $\mathrm{~F}$ & 2 & 0015 & 0.89 & 0.77 & 0.4 \\
\hline $13 \mid 19 \mathrm{~S}$ & 2 & $\mathrm{~F}$ & D & 1 & 0036 & 0.85 & 0.58 & 0.3 & 14 & $19 \tilde{S}$ & $\hat{2}$ & $\mathrm{~F}$ & 1 & 0036 & 0.93 & 0.75 & 0.4 \\
\hline $1319 \underset{\sim}{\tilde{S}}$ & $\hat{2}$ & $\mathrm{~F}$ & D & 2 & 0036 & 0.84 & 0.56 & 0.3 & 14 & $19 \tilde{\mathrm{S}}$ & $\hat{2}$ & $\mathrm{~F}$ & 2 & 0036 & 0.89 & 0.71 & 0.4 \\
\hline $1319 \stackrel{\tilde{S}}{\mathrm{~S}}$ & 2 & $\mathrm{~F}$ & D & 1 & 0090 & 0.90 & 0.51 & 0.3 & 14 & $19 \underset{\sim}{\tilde{S}}$ & 2 & $\mathrm{~F}$ & 1 & 0090 & 0.88 & 0.72 & 0.4 \\
\hline $1319 \tilde{\mathrm{S}}$ & $\hat{2}$ & $\mathrm{~F}$ & D & 2 & 0090 & 0.88 & 0.51 & 0.3 & 14 & $19 \tilde{S}$ & $\hat{2}$ & $\mathrm{~F} \mathrm{H}$ & 2 & 0090 & 0.92 & 0.69 & 0.4 \\
\hline $1319 \tilde{S}$ & $\hat{2}$ & $\mathrm{~F}$ & D & 1 & 0180 & 0.99 & 0.51 & 0.3 & 14 & $19 \tilde{\sim}$ & 2 & $\mathrm{~F}$ & 1 & 0180 & 1.05 & 0.64 & 0.4 \\
\hline $1319 \mathrm{~S}$ & 2 & $\mathrm{~F}$ & D & 2 & 0180 & 0.98 & 0.51 & 0.3 & 14 & $19 \tilde{S}$ & $\hat{2}$ & $\mathrm{~F} \mathrm{H}$ & 2 & 0180 & 1.10 & 0.62 & 0.4 \\
\hline $1319 \stackrel{\tilde{S}}{\tilde{S}}$ & $\hat{2}$ & $\mathrm{~F}$ & D & 1 & 0300 & 1.06 & 0.48 & 0.3 & 14 & $19 \underset{\sim}{\tilde{S}}$ & $\hat{2}$ & $\mathrm{~F} \stackrel{\mathrm{H}}{\mathrm{H}}$ & 1 & 0300 & 1.03 & 0.60 & 0.3 \\
\hline $1319 \underset{\sim}{\mathrm{S}}$ & 2 & $\mathrm{~F}$ & D & 2 & 0300 & 1.06 & 0.47 & 0.3 & 14 & $19 \underset{\sim}{\tilde{S}}$ & 2 & $\mathrm{~F}$ & 2 & 0300 & 1.07 & 0.58 & 0.3 \\
\hline $1319 \tilde{\mathrm{S}}$ & $\hat{2}$ & $\mathrm{~F}$ & D & 1 & 0450 & 1.06 & 0.44 & 0.3 & 14 & $19 \tilde{S}$ & $\hat{2}$ & $\mathrm{~F}$ & 1 & 0450 & 1.04 & 0.60 & 0.3 \\
\hline $1319 \tilde{\sim}$ & $\hat{2}$ & $\mathrm{~F}$ & D & 2 & 0450 & 1.06 & 0.44 & 0.3 & 14 & $19 \tilde{\sim}$ & 2 & $\mathrm{~F}$ & 2 & 0450 & 1.06 & 0.58 & 0.3 \\
\hline $1319 \mathrm{~S}$ & 2 & F & D & 1 & 0750 & 1.10 & 0.37 & 0.2 & 14 & $19 \tilde{S}$ & $\hat{2}$ & $\mathrm{~F}$ & 1 & 0750 & 1.23 & 0.57 & 0.3 \\
\hline $1319 \stackrel{\tilde{S}}{\tilde{S}}$ & $\hat{2}$ & $\mathrm{~F}$ & D & 2 & 0750 & 1.06 & 0.37 & 0.2 & 14 & $19 \tilde{\sim}$ & $\hat{2}$ & $\mathrm{~F}$ & 2 & 0750 & 1.30 & 0.57 & 0.3 \\
\hline $1319 \mathrm{~S}$ & 2 & $\mathrm{~F}$ & $\mathrm{D}$ & 1 & 1200 & 1.09 & 0.36 & 0.2 & 14 & $19 \stackrel{S}{S}$ & 2 & $\mathrm{~F} \mathrm{H}$ & 1 & 1200 & 1.29 & 0.49 & 0.3 \\
\hline $1319 \tilde{\mathrm{S}}$ & $\hat{2}$ & $\mathrm{~F}$ & D & 2 & 1200 & 1.13 & 0.36 & $\overline{0} .2$ & 14 & $19 \tilde{S}$ & $\hat{2}$ & $\mathrm{~F}$ & 2 & 1200 & 1.27 & 0.49 & 0.3 \\
\hline $1319 \stackrel{\tilde{\sim}}{\mathrm{S}}$ & 2 & $\mathrm{~F}$ & D & 1 & 1650 & 1.14 & 0.33 & 0.2 & 14 & $19 \underset{\sim}{\tilde{S}}$ & 2 & $\mathrm{~F} \stackrel{\mathrm{H}}{-}$ & 11 & 1650 & 1.43 & 0.47 & 0.3 \\
\hline $13 \mid 19 \underset{\sim}{\mathrm{S}}$ & 2 & $\mathrm{~F}$ & D & 2 & 1650 & 1.08 & 0.34 & 0.2 & 14 & $19 \underset{\sim}{\tilde{S}}$ & $\hat{2}$ & $\mathrm{~F} \stackrel{\mathrm{H}}{-}$ & 2 & 1650 & 1.40 & 0.47 & 0.3 \\
\hline $1519 \underset{\sim}{\mathrm{S}}$ & 2 & $\mathrm{~F}$ & $S$ & 1 & 0000 & 1.08 & 0.87 & 0.5 & 16 & $19 \stackrel{S}{S}$ & 2 & & 1 & 0000 & 1.43 & 0.67 & 0.4 \\
\hline $1519 \underset{\tilde{z}}{\mathrm{~S}}$ & 2 & $\mathrm{~F}$ & $S$ & 2 & 0000 & 1.05 & 0.79 & 0.5 & 16 & $19 \underset{\sim}{\tilde{S}}$ & $\hat{2}$ & & 2 & 0000 & 1.43 & 0.64 & 0.4 \\
\hline $1519 \tilde{\mathrm{S}}$ & $\hat{2}$ & $\mathrm{~F}$ & S & 1 & 0015 & 1.40 & 0.78 & 0.5 & 16 & $19 \tilde{S}$ & 2 & S D & 1 & 0015 & 1.49 & 0.64 & 0.4 \\
\hline $1519 \stackrel{\widetilde{\sim}}{\mathrm{S}}$ & 2 & $\mathrm{~F}$ & S & 2 & 0015 & 1.37 & 0.75 & 0.5 & 16 & $19 \underset{\sim}{\tilde{S}}$ & 2 & & 2 & 0015 & 1.45 & 0.61 & 0.4 \\
\hline $1519 \stackrel{\mathrm{S}}{\mathrm{S}}$ & 2 & $\mathrm{~F}$ & S & 1 & 0036 & 1.66 & 0.73 & 0.4 & 16 & $19 \mathrm{~S}$ & 2 & $S \quad D$ & 1 & 0036 & 1.42 & 0.67 & 0.4 \\
\hline $15 \mid 19 \stackrel{\tilde{S}}{\mathrm{~S}}$ & 2 & $\mathrm{~F}$ & S & 2 & 0036 & 1.64 & 0.71 & 0.4 & 16 & $19 \stackrel{\tilde{S}}{\sim}$ & 2 & $S \quad D$ & 2 & 0036 & 1.38 & 0.65 & 0.4 \\
\hline $1519 \underset{\tilde{a}}{\mathrm{~S}}$ & 2 & $\mathrm{~F}$ & $S$ & 1 & 0090 & 1.81 & 0.66 & 0.4 & 16 & $19 \stackrel{\widetilde{S}}{\tilde{S}}$ & 2 & $S \quad D$ & 1 & 0090 & 1.38 & 0.60 & 0.4 \\
\hline $1519 \tilde{S}$ & $\hat{2}$ & $\mathrm{~F}$ & $S$ & 2 & 0090 & 1.80 & 0.64 & 0.4 & 16 & $19 \tilde{S}$ & 2 & $S \quad D$ & 2 & 0090 & 1.35 & 0.58 & 0.4 \\
\hline $1519 \stackrel{\text { S }}{\mathrm{S}}$ & 2 & $\mathrm{~F}$ & S & 1 & 0180 & 2.00 & 0.62 & 0.4 & 16 & $19 \stackrel{\tilde{S}}{\sim}$ & 2 & $S \quad D$ & 1 & 0180 & 1.36 & 0.55 & 0.3 \\
\hline $1519 \mathrm{~S}$ & 2 & $\mathrm{~F}$ & S & 2 & 0180 & 2.06 & 0.60 & 0.4 & 16 & $19 \underset{\sim}{S}$ & 2 & $S D$ & 2 & 0180 & 1.37 & 0.54 & 0.3 \\
\hline $1519 \stackrel{\mathrm{S}}{\mathrm{S}}$ & 2 & $\mathrm{~F}$ & S & 1 & 0300 & 2.03 & 0.56 & 0.4 & 16 & $19 \stackrel{\mathrm{S}}{\mathrm{S}}$ & 2 & $S \quad D$ & 1 & 0300 & 1.35 & 0.52 & 0.3 \\
\hline $15 \mid 19 \underset{\text { S }}{\mathrm{S}}$ & 2 & $\mathrm{~F}$ & S & 2 & 0300 & 2.02 & 0.54 & 0.4 & 16 & $19 \mathrm{~S}$ & 2 & $S D$ & 2 & 0300 & 1.41 & 0.50 & 0.3 \\
\hline $1519 \tilde{\sim}$ & $\hat{2}$ & $\mathrm{~F}$ & S & 1 & 0450 & 1.92 & 0.54 & 0.4 & 16 & $19 \tilde{\sim}$ & 2 & S D & 1 & 0450 & 1.39 & 0.47 & 0.3 \\
\hline $1519 \underset{\sim}{\mathrm{S}}$ & 2 & $\mathrm{~F}$ & $S$ & 2 & 0450 & 1.98 & 0.53 & 0.4 & 16 & $19 \underset{\sim}{\tilde{S}}$ & 2 & S D & 2 & 0450 & 1.43 & 0.46 & 0.3 \\
\hline $1519 \mathrm{~S}$ & 2 & $\mathrm{~F}$ & S & 1 & 0750 & 1.91 & 0.49 & 0.3 & 16 & $19 \stackrel{\mathrm{S}}{\sim}$ & 2 & $S \quad D$ & 1 & 0750 & 1.47 & 0.41 & 0.3 \\
\hline \begin{tabular}{l|l}
15 & $19 \stackrel{\mathrm{S}}{ }$
\end{tabular} & 2 & $\mathrm{~F}$ & $\underline{S}$ & 2 & 0750 & 1.87 & 0.48 & 0.3 & 16 & $19 \stackrel{S}{S}$ & 2 & $S \quad D$ & 2 & 0750 & 1.48 & 0.40 & 0.3 \\
\hline $1519 \stackrel{\mathrm{S}}{\mathrm{S}}$ & 2 & $\mathrm{~F}$ & S & 1 & 1200 & 2.31 & 0.46 & 0.3 & 16 & $19 \stackrel{\mathrm{S}}{\mathrm{S}}$ & 2 & $S \quad D$ & 1 & 1200 & 1.30 & 0.39 & 0.2 \\
\hline $1519 \stackrel{\tilde{S}}{\sim}$ & 2 & $\mathrm{~F}$ & $\underline{S}$ & 2 & 1200 & 2.27 & 0.44 & 0.3 & 16 & $19 \underset{\sim}{\tilde{S}}$ & 2 & $S D$ & 2 & 1200 & 1.30 & 0.38 & 0.2 \\
\hline $1519 \underset{\sim}{S}$ & 2 & F & S & 1 & 1650 & 2.30 & 0.42 & 0.3 & 16 & $19 \stackrel{\mathrm{S}}{\mathrm{S}}$ & 2 & $S \quad D$ & 1 & 1650 & 1.49 & 0.37 & 0.2 \\
\hline \begin{tabular}{|l|l}
15 & $19 \mathrm{~S}$ \\
\end{tabular} & 2 & $\mathrm{~F}$ & S & 2 & 1650 & 2.31 & 0.43 & 0.3 & 16 & $19 \mathrm{~S}$ & 2 & $S D$ & 2 & 1650 & 1.39 & 0.38 & 0.2 \\
\hline
\end{tabular}




\begin{tabular}{|c|c|c|c|c|c|c|c|c|c|c|c|c|c|c|c|c|c|c|}
\hline No & Iix L & $a b$ & & $\mathrm{R}$ & & MPD & $\mathrm{DF}_{20}$ & & No & & ix L & & & & & MPD & $\mathrm{DF}_{20}$ & F6 \\
\hline \begin{tabular}{|l|l}
17 & $19 \mathrm{~S}$ \\
\end{tabular} & 2 & S & $\mathrm{H}$ & 1 & 0000 & 1.37 & 0.70 & 0.4 & 18 & $19 \mathrm{~S}$ & 2 & & $S$ & 1 & 0000 & 1.12 & 0.55 & 0.3 \\
\hline $1719 \overline{\mathrm{S}}$ & 2 & S & $\overline{\mathrm{H}}$ & 2 & 0000 & 1.42 & 0.67 & 0.4 & 18 & $19 \overline{\mathrm{S}}$ & 2 & $S$ & $S$ & 2 & 0000 & 1.13 & 0.57 & 0.3 \\
\hline \begin{tabular}{|l|l|l}
17 & $\tilde{\mathrm{S}}$
\end{tabular} & $\hat{2}$ & S & $\mathrm{H}$ & 1 & 0015 & 1.42 & 0.62 & 0.4 & 18 & $19 \tilde{\mathrm{S}}$ & 2 & $S$ & S & 1 & 0015 & 1.10 & 0.73 & $\overline{0} .4$ \\
\hline $1719 \tilde{\mathrm{S}}$ & 2 & S & $\mathrm{H}$ & 2 & 0015 & 1.40 & 0.59 & 0.4 & 18 & $19 \tilde{\mathrm{S}}$ & 2 & $S$ & S & 2 & 0015 & 1.11 & 0.71 & 0.4 \\
\hline \begin{tabular}{|l|l}
17 & $19 \tilde{\mathrm{S}}$
\end{tabular} & $\hat{2}$ & S & $\dot{\mathrm{H}}$ & 1 & 0036 & 1.28 & 0.66 & 0.4 & 18 & $19 \tilde{\mathrm{S}}$ & $\hat{2}$ & $\mathrm{~S}$ & S & 1 & 0036 & 1.08 & 0.69 & $\overline{0} .4$ \\
\hline $1719 \tilde{\mathrm{S}}$ & 2 & S & $\mathrm{H}$ & 2 & 0036 & 1.30 & 0.64 & 0.4 & 18 & $19 \tilde{\mathrm{S}}$ & $\hat{2}$ & $S$ & S & 2 & 0036 & 1.10 & 0.66 & 0.4 \\
\hline \begin{tabular}{|l|l}
17 & $19 \tilde{\mathrm{S}}$
\end{tabular} & $\hat{2}$ & S & $\mathrm{H}$ & 1 & 0090 & 1.37 & 0.63 & 0.4 & 18 & $19 \tilde{\mathrm{S}}$ & $\hat{2}$ & $S$ & S & 1 & 0090 & 1.08 & 0.62 & 0.3 \\
\hline $1719 \tilde{\mathrm{S}}$ & 2 & S & $\mathrm{H}$ & 2 & 0090 & 1.30 & 0.61 & 0.4 & 18 & $19 \tilde{\mathrm{S}}$ & $\hat{2}$ & $S$ & S & 2 & 0090 & 1.06 & 0.60 & 0.3 \\
\hline $1719 \tilde{S}$ & 2 & S & $\mathrm{H}$ & 1 & 0180 & 1.31 & 0.59 & 0.3 & 18 & $19 \tilde{\sim}$ & 2 & $S$ & S & 1 & 0180 & 1.11 & 0.59 & 0.3 \\
\hline \begin{tabular}{l|l}
17 & $19 \tilde{\mathrm{S}}$
\end{tabular} & $\hat{2}$ & S & $\mathrm{H}$ & 2 & 0180 & 1.33 & 0.56 & 0.3 & 18 & $19 \tilde{\mathrm{S}}$ & $\hat{2}$ & $S$ & S & 2 & 0180 & 1.15 & 0.56 & 0.3 \\
\hline $1719 \tilde{\mathrm{S}}$ & 2 & S & $\mathrm{H}$ & 1 & 0300 & 1.28 & 0.56 & 0.3 & 18 & $19 \underset{\sim}{\tilde{S}}$ & $\hat{2}$ & $S$ & S & 1 & 0300 & 1.14 & 0.54 & 0.3 \\
\hline $1719 \tilde{\mathrm{S}}$ & $\hat{2}$ & S & $\mathrm{H}$ & 2 & 0300 & 1.28 & 0.54 & 0.3 & 18 & $19 \tilde{\mathrm{S}}$ & 2 & $S$ & S & 2 & 0300 & 1.15 & 0.52 & 0.3 \\
\hline $1719 \tilde{\mathrm{S}}$ & 2 & S & $\mathrm{H}$ & 1 & 0450 & 1.33 & 0.51 & 0.3 & 18 & $19 \tilde{\mathrm{S}}$ & $\hat{2}$ & $S$ & S & 1 & 0450 & 1.17 & 0.50 & 0.3 \\
\hline $1719 \tilde{S}$ & 2 & S & $\mathrm{H}$ & 2 & 0450 & 1.28 & 0.48 & 0.3 & 18 & $19 \tilde{\mathrm{S}}$ & $\hat{2}$ & $S$ & S & 2 & 0450 & 1.20 & 0.49 & 0.3 \\
\hline $17 \mid 19 \tilde{\mathrm{S}}$ & 2 & S & $\mathrm{H}$ & 1 & 0750 & 1.29 & 0.45 & 0.3 & 18 & $19 \tilde{\mathrm{S}}$ & $\hat{2}$ & $S$ & S & 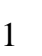 & 0750 & 1.14 & 0.46 & 0.3 \\
\hline $1719 \underset{\sim}{\tilde{S}}$ & 2 & S & $\mathrm{H}$ & 2 & 0750 & 1.25 & 0.43 & 0.3 & 18 & $19 \underset{\sim}{\tilde{S}}$ & 2 & $S$ & S & 2 & 0750 & 1.17 & 0.44 & 0.3 \\
\hline $1719 \tilde{\mathrm{S}}$ & 2 & S & $\mathrm{H}$ & 1 & 1200 & 1.33 & 0.42 & 0.3 & 18 & $19 \tilde{\mathrm{S}}$ & $\hat{2}$ & $\mathrm{~S}$ & S & 1 & 1200 & 1.19 & 0.38 & 0.2 \\
\hline $1719 \underset{\sim}{\tilde{S}}$ & 2 & S & $\mathrm{H}$ & 2 & 1200 & 1.29 & 0.42 & 0.3 & 18 & $19 \underset{\sim}{\tilde{S}}$ & $\hat{2}$ & $S$ & S & 2 & 1200 & 1.09 & 0.38 & 0.2 \\
\hline \begin{tabular}{|l|l}
17 & $19 \stackrel{\tilde{S}}{\sim}$
\end{tabular} & 2 & S & $\mathrm{H}$ & 1 & 1650 & 1.38 & 0.39 & 0.2 & 18 & $19 \underset{\sim}{\tilde{S}}$ & $\hat{2}$ & $S$ & S & 1 & 1650 & 1.16 & 0.39 & 0.2 \\
\hline $1719 \underset{\sim}{\tilde{S}}$ & 2 & S & $\mathrm{H}$ & 2 & 1650 & 1.33 & 0.38 & 0.2 & 18 & $19 \underset{\sim}{\tilde{S}}$ & $\hat{2}$ & S & S & 2 & 1650 & 1.14 & 0.38 & 0.2 \\
\hline 1995 Q & 2 & C & $\mathrm{D}$ & 1 & 0000 & 0.98 & 0.63 & 0.3 & 20 & $95 \mathrm{Q}$ & 2 & C & & 1 & 0000 & 0.77 & 0.54 & 0.3 \\
\hline 1995 Q & 2 & $c$ & $D$ & 2 & 0000 & 1.02 & 0.59 & 0.3 & 20 & $95 \mathrm{Q}$ & $\hat{2}$ & $\mathrm{C}$ & & 2 & 0000 & 0.78 & 0.57 & 0.3 \\
\hline 1995 Q & 2 & C & D & 1 & 0015 & 1.02 & 0.76 & 0.4 & 20 & $95 \mathrm{Q}$ & 2 & C & $\mathrm{H}$ & 1 & 0015 & 0.88 & 0.52 & 0.3 \\
\hline 1995 Q & 2 & C & $D$ & 2 & 0015 & 1.05 & 0.73 & 0.4 & 20 & $95 \mathrm{Q}$ & 2 & $\mathrm{C}$ & & 2 & 0015 & 0.82 & 0.51 & 0.3 \\
\hline 1995 Q & 2 & C & $D$ & 1 & 0036 & 1.03 & 0.70 & 0.4 & 20 & $95 \mathrm{Q}$ & 2 & C & 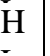 & 1 & 0036 & 0.80 & 0.65 & 0.3 \\
\hline $1995 \mathrm{Q}$ & 2 & C & $D$ & 2 & 0036 & 0.98 & 0.68 & 0.4 & 20 & $95 \mathrm{Q}$ & $\hat{2}$ & C & 5 & 2 & 0036 & 0.78 & 0.64 & $\overline{0} .3$ \\
\hline $1995 \mathrm{Q}$ & 2 & C & $D$ & 1 & 0090 & 1.04 & 0.63 & 0.3 & 20 & $95 \mathrm{Q}$ & $\hat{2}$ & $\mathrm{C}$ & & 1 & 0090 & 0.75 & 0.65 & $\overline{0} .3$ \\
\hline 1995 Q & 2 & C & D & 2 & 0090 & 1.01 & 0.61 & 0.3 & 20 & $95 \mathrm{Q}$ & $\hat{2}$ & C & . & 2 & 0090 & 0.83 & 0.61 & $\overline{0} .3$ \\
\hline 1995 Q & 2 & C & $\mathrm{D}$ & 1 & 0180 & 0.97 & 0.58 & 0.3 & 20 & $95 \mathrm{Q}$ & $\hat{2}$ & $\mathrm{C}$ & 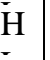 & 1 & 0180 & 0.79 & 0.60 & $\overline{0} .3$ \\
\hline 1995 Q & 2 & C & $D$ & 2 & 0180 & 0.97 & 0.57 & $\overline{0} .3$ & 20 & $95 \mathrm{Q}$ & $\hat{2}$ & C & & 2 & 0180 & 0.84 & 0.58 & 0.3 \\
\hline $1995 \mathrm{Q}$ & 2 & c & $\mathrm{D}$ & 1 & 0300 & 1.01 & 0.56 & $\overline{0} .3$ & 20 & $95 \mathrm{Q}$ & $\hat{2}$ & $\mathrm{C}$ & & 1 & 0300 & 0.83 & 0.56 & 0.3 \\
\hline 1995 Q & 2 & C & $D$ & 2 & 0300 & 1.07 & 0.55 & 0.3 & 20 & $95 \mathrm{Q}$ & $\hat{2}$ & $\mathrm{C}$ & & 2 & 0300 & 0.89 & 0.53 & 0.3 \\
\hline 1995 Q & 2 & C & $\mathrm{D}$ & 1 & 0450 & 1.12 & 0.52 & 0.3 & 20 & $95 \mathrm{Q}$ & 2 & C & & 1 & 0450 & 0.81 & 0.52 & 0.3 \\
\hline 1995 Q & 2 & c & $D$ & 2 & 0450 & 1.18 & 0.51 & 0.3 & 20 & $95 \mathrm{Q}$ & $\hat{2}$ & C & & 2 & 0450 & 0.86 & 0.50 & 0.3 \\
\hline 1995 Q & 2 & C & D & 1 & 0750 & 1.07 & 0.46 & 0.3 & 20 & $95 \mathrm{Q}$ & 2 & C & 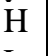 & 1 & 0750 & 0.77 & 0.47 & 0.2 \\
\hline $1995 \mathrm{Q}$ & $\hat{2}$ & C & $\mathrm{D}$ & 2 & 0750 & 1.09 & 0.45 & 0.3 & 20 & $95 \mathrm{Q}$ & $\hat{2}$ & $\mathrm{C}$ & & 2 & 0750 & 0.76 & 0.47 & 0.2 \\
\hline 1995 Q & 2 & C & $D$ & 1 & 1200 & 1.13 & 0.38 & 0.2 & 20 & $95 \mathrm{Q}$ & $\hat{2}$ & $\mathrm{C}$ & & 1 & 1200 & 0.90 & 0.43 & 0.2 \\
\hline 1995 Q & 2 & C & D & 2 & 1200 & 1.14 & 0.38 & 0.2 & 20 & $95 \mathrm{Q}$ & $\hat{2}$ & C & & 2 & 1200 & 0.84 & 0.42 & 0.2 \\
\hline 1995 Q & 2 & C & $D$ & 1 & 1650 & 1.11 & 0.36 & 0.2 & 20 & 95 & $\hat{2}$ & $\mathrm{C}$ & & 1 & 1650 & 0.89 & 0.41 & 0.2 \\
\hline $1995 \mathrm{Q}$ & 2 & C & $\mathrm{D}$ & 2 & 1650 & 1.10 & 0.36 & 0.2 & 20 & $95 \mathrm{Q}$ & 2 & $\mathrm{C}$ & & 2 & 1650 & 0.86 & 0.41 & 0.2 \\
\hline
\end{tabular}




\begin{tabular}{|c|c|c|c|c|c|c|c|c|c|c|c|c|c|c|c|c|c|c|}
\hline No & Mix & $\overline{\text { abel }}$ & & $\mathrm{R}$ & \# & MPD & $\mathrm{DF}_{20}$ & F6 & No & & 11 & & & $\mathrm{R}$ & $\#$ & MPD & $\mathrm{DF}_{20}$ & F6 \\
\hline \begin{tabular}{|l|l|}
21 & $95 \mathrm{Q}$
\end{tabular} & 2 & C & $\mathrm{S}$ & 1 & 0000 & 0.86 & 0.52 & 0.3 & 22 & $95 \mathrm{Q}$ & 2 & & $\mathrm{D}$ & 1 & 0000 & 0.34 & 0.70 & 0.2 \\
\hline $2195 \mathrm{Q}$ & 2 & $\mathrm{C}$ & S & 2 & 0000 & 0.89 & 0.51 & 0.3 & 22 & $95 \mathrm{Q}$ & 2 & $\mathrm{~F}$ & D & 2 & 0000 & 0.34 & 0.66 & 0.2 \\
\hline $2195 \mathrm{Q}$ & 2 & $\mathrm{C}$ & $\dot{S}$ & 1 & 0015 & 0.87 & 0.57 & 0.3 & 22 & $95 \mathrm{Q}$ & 2 & $\mathrm{~F}$ & D & 1 & 0015 & 0.43 & 0.67 & 0.3 \\
\hline $2195 \mathrm{Q}$ & 2 & $\mathrm{C}$ & $\mathrm{S}$ & 2 & 0015 & 0.86 & 0.55 & 0.3 & 22 & $95 \mathrm{Q}$ & 2 & $\mathrm{~F}$ & D & 2 & 0015 & 0.42 & 0.63 & 0.3 \\
\hline $2195 \mathrm{Q}$ & 2 & $\mathrm{C}$ & S & 1 & 0036 & 0.92 & 0.58 & 0.3 & 22 & $95 \mathrm{Q}$ & 2 & $\mathrm{~F}$ & D & 1 & 0036 & 0.41 & 0.65 & 0.2 \\
\hline $2195 \mathrm{Q}$ & 2 & $\mathrm{C}$ & $S$ & 2 & 0036 & 0.88 & 0.55 & 0.3 & 22 & $95 \mathrm{Q}$ & 2 & $\mathrm{~F}$ & D & 2 & 0036 & 0.43 & 0.60 & 0.2 \\
\hline $2195 \mathrm{Q}$ & 2 & $\mathrm{C}$ & $S$ & 1 & 0090 & 0.94 & 0.52 & 0.3 & 22 & $95 \mathrm{Q}$ & $\hat{2}$ & $\mathrm{~F}$ & D & 1 & 0090 & 0.49 & 0.59 & 0.3 \\
\hline $2195 \mathrm{Q}$ & 2 & $\mathrm{C}$ & $\mathrm{S}$ & 2 & 0090 & 0.92 & 0.51 & 0.3 & 22 & $95 \mathrm{Q}$ & $\hat{2}$ & $\mathrm{~F}$ & D & 2 & 0090 & 0.52 & 0.58 & 0.3 \\
\hline $2195 \mathrm{Q}$ & 2 & $\mathrm{C}$ & S & 1 & 0180 & 1.05 & 0.49 & 0.3 & 22 & $95 \mathrm{Q}$ & 2 & $\mathrm{~F}$ & D & 1 & 0180 & 0.56 & 0.52 & 0.2 \\
\hline $2195 \mathrm{Q}$ & 2 & $\mathrm{C}$ & S & 2 & 0180 & 0.94 & 0.47 & 0.3 & 22 & $95 \mathrm{Q}$ & 2 & $\mathrm{~F}$ & D & 2 & 0180 & 0.57 & 0.51 & 0.2 \\
\hline $2195 \mathrm{Q}$ & 2 & $\mathrm{C}$ & $\mathrm{S}$ & 1 & 0300 & 0.90 & 0.43 & 0.2 & 22 & $95 \mathrm{Q}$ & 2 & $\mathrm{~F}$ & D & 1 & 0300 & 0.63 & 0.50 & 0.2 \\
\hline $2195 \mathrm{Q}$ & 2 & $\mathrm{C}$ & $\mathrm{S}$ & 2 & 0300 & 0.92 & 0.43 & 0.2 & 22 & $95 \mathrm{Q}$ & 2 & $\mathrm{~F}$ & D & 2 & 0300 & 0.61 & 0.48 & 0.2 \\
\hline $2195 \mathrm{Q}$ & 2 & $\mathrm{C}$ & $\mathrm{S}$ & 1 & 0450 & 1.02 & 0.49 & 0.3 & 22 & $95 \mathrm{Q}$ & $\hat{2}$ & $\mathrm{~F}$ & D & 1 & 0450 & 0.65 & 0.50 & 0.2 \\
\hline $2195 \mathrm{Q}$ & 2 & $\mathrm{C}$ & S & 2 & 0450 & 1.02 & 0.47 & 0.3 & 22 & $95 \mathrm{Q}$ & $\hat{2}$ & $\mathrm{~F}$ & D & 2 & 50 & 0.66 & 0.47 & 0.2 \\
\hline $2195 \mathrm{Q}$ & 2 & $\mathrm{C}$ & S & 1 & 0750 & 1.00 & 0.47 & 0.3 & 22 & $95 \mathrm{Q}$ & 2 & $\mathrm{~F}$ & D & 1 & 0750 & 0.66 & 0.42 & 0.2 \\
\hline $2195 \mathrm{Q}$ & 2 & $\mathrm{C}$ & $\mathrm{S}$ & 2 & 0750 & 0.99 & 0.47 & 0.3 & 22 & $95 \mathrm{Q}$ & 2 & $\mathrm{~F}$ & D & 2 & 0750 & 0.68 & 0.42 & 0.2 \\
\hline $2195 \mathrm{Q}$ & 2 & $\mathrm{C}$ & $S$ & 1 & 1200 & 0.98 & 0.35 & 0.2 & 22 & $95 \mathrm{Q}$ & 2 & $\mathrm{~F}$ & D & 1 & 1200 & 0.65 & 0.38 & 0.2 \\
\hline $2195 \mathrm{Q}$ & 2 & $\mathrm{C}$ & $S$ & 2 & 1200 & 0.99 & 0.35 & 0.2 & 22 & $95 \mathrm{Q}$ & 2 & $\mathrm{~F}$ & D & 2 & 1200 & 0.66 & 0.39 & 0.2 \\
\hline $2195 \mathrm{Q}$ & 2 & $\mathrm{C}$ & $\mathrm{S}$ & 1 & 1650 & 0.94 & 0.34 & 0.2 & 22 & $95 \mathrm{Q}$ & 2 & $\mathrm{~F}$ & D & 1 & 1650 & 0.66 & 0.36 & 0.2 \\
\hline $2195 \mathrm{Q}$ & 2 & $\mathrm{C}$ & S & 2 & 1650 & 0.96 & 0.38 & 0.2 & 22 & $95 \mathrm{Q}$ & 2 & $\mathrm{~F}$ & $\mathrm{D}$ & 2 & 1650 & 0.66 & 0.36 & 0.2 \\
\hline $2395 \mathrm{Q}$ & 2 & $\mathrm{~F}$ & $\mathrm{H}$ & 1 & 0000 & 0.33 & 0.66 & 0.2 & 24 & $95 \mathrm{Q}$ & 2 & $\mathrm{~F}$ & $\underline{S}$ & 1 & 0000 & 0.42 & 0.67 & 0.3 \\
\hline $2395 \mathrm{Q}$ & 2 & $\mathrm{~F}$ & $\mathrm{H}$ & 2 & 0000 & 0.31 & 0.61 & 0.2 & 24 & $95 \mathrm{Q}$ & 2 & $\mathrm{~F}$ & $\underline{S}$ & 2 & 0000 & 0.42 & 0.62 & 0.3 \\
\hline $2395 \mathrm{Q}$ & 2 & $\mathrm{~F}$ & $\mathrm{H}$ & 1 & 0015 & 0.39 & 0.69 & 0.3 & 24 & $95 \mathrm{Q}$ & 2 & $\mathrm{~F}$ & $\underline{S}$ & 1 & 0015 & 0.57 & 0.65 & 0.3 \\
\hline $2395 \mathrm{Q}$ & 2 & $\mathrm{~F}$ & $\mathrm{H}$ & 2 & 0015 & 0.39 & 0.65 & 0.3 & 24 & $95 \mathrm{Q}$ & 2 & $\mathrm{~F}$ & $\underline{S}$ & 2 & 0015 & 0.54 & 0.62 & 0.3 \\
\hline $2395 \mathrm{Q}$ & 2 & $\mathrm{~F}$ & $\mathrm{H}$ & 1 & 0036 & 0.39 & 0.68 & 0.2 & 24 & $95 \mathrm{Q}$ & 2 & $\mathrm{~F}$ & $\underline{S}$ & 1 & 0036 & 0.56 & 0.65 & 0.3 \\
\hline $2395 \mathrm{Q}$ & 2 & $\mathrm{~F}$ & $\mathrm{H}$ & 2 & 0036 & 0.40 & 0.63 & 0.2 & 24 & $95 \mathrm{Q}$ & 2 & $\mathrm{~F}$ & $\underline{S}$ & 2 & 0036 & 0.53 & 0.63 & 0.3 \\
\hline $2395 \mathrm{Q}$ & 2 & $\mathrm{~F}$ & $\mathrm{H}$ & 1 & 0090 & 0.45 & 0.61 & 0.3 & 24 & $95 \mathrm{Q}$ & 2 & $\mathrm{~F}$ & $S$ & 1 & 0090 & 0.68 & 0.59 & 0.3 \\
\hline $2395 \mathrm{Q}$ & 2 & $\mathrm{~F}$ & $\mathrm{H}$ & 2 & 0090 & 0.49 & 0.60 & 0.3 & 24 & $95 \mathrm{Q}$ & 2 & $\mathrm{~F}$ & S & 2 & 0090 & 0.68 & 0.57 & 0.3 \\
\hline $2395 \mathrm{Q}$ & 2 & $\mathrm{~F}$ & $\mathrm{H}$ & 1 & 0180 & 0.52 & 0.59 & 0.3 & 24 & $95 \mathrm{Q}$ & 2 & $\mathrm{~F}$ & $\underline{S}$ & 1 & 0180 & 0.72 & 0.53 & 0.3 \\
\hline $2395 \mathrm{Q}$ & 2 & $\mathrm{~F}$ & $\mathrm{H}$ & 2 & 0180 & 0.51 & 0.55 & 0.3 & 24 & $95 \mathrm{Q}$ & 2 & $\mathrm{~F}$ & $S$ & 2 & 0180 & 0.73 & 0.52 & 0.3 \\
\hline $2395 \mathrm{Q}$ & 2 & $\mathrm{~F}$ & $\mathrm{H}$ & 1 & 0300 & 0.64 & 0.52 & 0.3 & 24 & $95 \mathrm{Q}$ & 2 & $\mathrm{~F}$ & $\underline{S}$ & 1 & 0300 & 0.82 & 0.48 & 0.3 \\
\hline $2395 \mathrm{Q}$ & 2 & $\mathrm{~F}$ & $\mathrm{H}$ & 2 & 0300 & 0.64 & 0.52 & 0.3 & 24 & $95 \mathrm{Q}$ & 2 & $\mathrm{~F}$ & $\underline{S}$ & 2 & 0300 & 0.80 & 0.50 & 0.3 \\
\hline $2395 \mathrm{Q}$ & 2 & F & H & 1 & 0450 & 0.71 & 0.53 & 0.3 & 24 & $95 \mathrm{Q}$ & 2 & $\mathrm{~F}$ & $\underline{S}$ & 1 & 0450 & 0.87 & 0.48 & 0.3 \\
\hline $2395 \mathrm{Q}$ & 2 & $\mathrm{~F}$ & $\mathrm{H}$ & 2 & 0450 & 0.70 & 0.51 & 0.3 & 24 & $95 \mathrm{Q}$ & 2 & $\mathrm{~F}$ & - & 2 & 0450 & 0.87 & 0.45 & 0.3 \\
\hline $2395 \mathrm{Q}$ & 2 & F & $\mathrm{H}$ & 1 & 0750 & 0.72 & 0.45 & 0.2 & 24 & $95 \mathrm{Q}$ & 2 & $\mathrm{~F}$ & $S$ & 1 & 0750 & 0.84 & 0.37 & 0.2 \\
\hline $2395 \mathrm{Q}$ & 2 & $\mathrm{~F}$ & $\mathrm{H}$ & 2 & 0750 & 0.74 & 0.43 & 0.2 & 24 & $95 \mathrm{Q}$ & 2 & $\mathrm{~F}$ & - & & 0750 & 0.87 & 0.37 & 0.2 \\
\hline $2395 \mathrm{Q}$ & 2 & $\mathrm{~F}$ & $\mathrm{H}$ & 1 & 1200 & 0.80 & 0.42 & 0.2 & 24 & $95 \mathrm{Q}$ & 2 & $\mathrm{~F}$ & $S$ & & 1200 & 0.86 & 0.33 & 0.2 \\
\hline $2395 \mathrm{Q}$ & 2 & F & H & 2 & 1200 & 0.75 & 0.40 & 0.2 & 24 & $95 \mathrm{Q}$ & 2 & $\mathrm{~F}$ & - & 2 & 1200 & 0.85 & 0.34 & 0.2 \\
\hline $2395 \mathrm{Q}$ & 2 & $\mathrm{~F}$ & $\mathrm{H}$ & 1 & 1650 & 0.73 & 0.34 & 0.2 & 24 & $95 \mathrm{Q}$ & 2 & $\mathrm{~F}$ & - & & 1650 & 0.88 & 0.30 & 0.2 \\
\hline $2395 \mathrm{Q}$ & 2 & $\mathrm{~F}$ & 11 & 2 & 1650 & 0.72 & 0.33 & 0.2 & 24 & $95 \mathrm{Q}$ & 2 & $\mathrm{~F}$ & 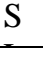 & & 1650 & 0.88 & 0.28 & 0.2 \\
\hline
\end{tabular}




\begin{tabular}{|c|c|c|c|c|c|c|c|c|c|c|c|c|c|c|c|c|c|}
\hline No & Mix & abel & & R & \# & MPD & $\mathrm{DF}_{20}$ & F6 & No & & Mix & & $\mathbb{R}$ & $\#$ & MPD & $\mathrm{DF}_{20}$ & F6 \\
\hline \begin{tabular}{|l|l|}
25 & $95 \mathrm{Q}$
\end{tabular} & 2 & S & D & 1 & 0000 & 0.70 & 0.64 & 0.3 & 26 & $95 \mathrm{Q}$ & 2 & $\begin{array}{ll}\mathrm{S} & \mathrm{H}\end{array}$ & 1 & 0000 & 0.95 & 0.67 & 0.4 \\
\hline $2595 \mathrm{Q}$ & 2 & S & $\mathrm{D}$ & 2 & 0000 & 0.70 & 0.60 & 0.3 & 26 & $95 \mathrm{Q}$ & 2 & $\mathrm{~S} \overline{\mathrm{H}}$ & $J^{2}$ & 0000 & 1.00 & 0.65 & 0.4 \\
\hline $2595 \mathrm{Q}$ & 2 & $S$ & D & 1 & 0015 & 0.69 & 0.73 & 0.4 & 26 & $95 \mathrm{Q}$ & $\hat{2}$ & $\mathrm{SH}$ & 1 & 0015 & 0.94 & 0.67 & 0.4 \\
\hline $2595 \mathrm{Q}$ & $\hat{2}$ & $\mathrm{~S}$ & $\mathrm{D}$ & 2 & 0015 & 0.73 & 0.71 & 0.4 & 26 & $95 \mathrm{Q}$ & 2 & $\mathrm{~S} \stackrel{\mathrm{H}}{\mathrm{H}}$ & 2 & 0015 & 0.94 & 0.65 & 0.4 \\
\hline $2595 \mathrm{Q}$ & 2 & $\mathrm{~S}$ & $\mathrm{D}$ & 1 & 0036 & 0.66 & 0.70 & 0.3 & 26 & $95 \mathrm{Q}$ & 2 & $\mathrm{~S}^{\mathrm{H}}$ & 1 & 0036 & 1.01 & 0.68 & 0.4 \\
\hline $2595 \mathrm{Q}$ & $\hat{2}$ & $S$ & $\mathrm{D}$ & 2 & 0036 & 0.66 & 0.66 & $\overline{0} .3$ & 26 & $95 \mathrm{Q}$ & 2 & $S \stackrel{H}{H}$ & 2 & 0036 & 1.08 & 0.65 & 0.4 \\
\hline $2595 \mathrm{Q}$ & 2 & $\mathrm{~S}$ & $\mathrm{D}$ & 1 & 0090 & 0.67 & 0.62 & 0.3 & 26 & $95 \mathrm{Q}$ & 2 & $\mathrm{~S} \mathrm{H}$ & $\left.\right|^{1}$ & 0090 & 0.96 & 0.61 & 0.3 \\
\hline $2595 \mathrm{Q}$ & 2 & $\mathrm{~S}$ & $\mathrm{D}$ & 2 & 0090 & 0.64 & 0.59 & 0.3 & 26 & $95 \mathrm{Q}$ & 2 & $\mathrm{~S}^{\mathrm{H}}$ & 2 & 0090 & 0.96 & 0.58 & $\overline{0} .3$ \\
\hline $2595 \mathrm{Q}$ & $\hat{2}$ & S & $\mathrm{D}$ & 1 & 0180 & 0.72 & 0.58 & 0.3 & 26 & $95 \mathrm{Q}$ & 2 & $S \stackrel{H}{H}$ & 1 & 0180 & 1.08 & 0.58 & 0.3 \\
\hline $2595 \mathrm{Q}$ & 2 & $\mathrm{~S}$ & $\mathrm{D}$ & 2 & 0180 & 0.72 & 0.57 & 0.3 & 26 & $95 \mathrm{Q}$ & 2 & $\mathrm{~S} \stackrel{\mathrm{H}}{ }$ & 2 & 0180 & 0.98 & 0.56 & 0.3 \\
\hline $2595 \mathrm{Q}$ & $\hat{2}$ & $S$ & $\mathrm{D}$ & 1 & 0300 & 0.69 & 0.54 & 0.3 & 26 & $95 \mathrm{Q}$ & 2 & $\mathrm{~S}^{\mathrm{H}}$ & 1 & 0300 & 0.98 & 0.56 & 0.3 \\
\hline $2595 \mathrm{Q}$ & 2 & $S$ & $\mathrm{D}$ & 2 & 0300 & 0.78 & 0.53 & 0.3 & 26 & $95 \mathrm{Q}$ & 2 & $\mathrm{~S} \mathrm{H}$ & 2 & 0300 & 1.00 & 0.53 & 0.3 \\
\hline $2595 \mathrm{Q}$ & 2 & $\mathrm{~S}$ & $\mathrm{D}$ & 1 & 0450 & 0.74 & 0.47 & 0.2 & 26 & $95 \mathrm{Q}$ & 2 & $\mathrm{SH}$ & 1 & 0450 & 1.03 & 0.51 & 0.3 \\
\hline $2595 \mathrm{Q}$ & $\hat{2}$ & $S$ & $\mathrm{D}$ & 2 & 0450 & 0.72 & 0.47 & 0.2 & 26 & $95 \mathrm{Q}$ & 2 & $S \stackrel{H}{H}$ & 2 & 0450 & 1.00 & 0.49 & 0.3 \\
\hline $2595 \mathrm{Q}$ & 2 & $\mathrm{~S}$ & $\mathrm{D}$ & 1 & 0750 & 0.76 & 0.41 & 0.2 & 26 & $95 \mathrm{Q}$ & $\hat{2}$ & $\mathrm{~S}^{\mathrm{H}}$ & $\mid 1$ & 0750 & 1.02 & 0.41 & 0.2 \\
\hline $2595 \mathrm{Q}$ & 2 & $S$ & $\mathrm{D}$ & 2 & 0750 & 0.76 & 0.41 & 0.2 & 26 & $95 \mathrm{Q}$ & 2 & $\mathrm{~S}^{\mathrm{H}}$ & 2 & 0750 & 1.05 & 0.39 & 0.2 \\
\hline $2595 \mathrm{Q}$ & $\hat{2}$ & $S$ & $\mathrm{D}$ & 1 & 1200 & 0.75 & 0.37 & 0.2 & 26 & $95 \mathrm{Q}$ & 2 & $S \stackrel{H}{H}$ & 1 & 1200 & 0.98 & 0.42 & 0.2 \\
\hline $2595 \mathrm{Q}$ & $\hat{2}$ & $\mathrm{~S}$ & $\mathrm{D}$ & 2 & 1200 & 0.76 & 0.37 & 0.2 & 26 & $95 \mathrm{Q}$ & $\hat{2}$ & $S \stackrel{H}{H}$ & 2 & 1200 & 1.02 & 0.40 & 0.2 \\
\hline $2595 \mathrm{Q}$ & $\hat{2}$ & $S$ & $\mathrm{D}$ & 1 & 1650 & 0.73 & 0.36 & 0.2 & 26 & $95 \mathrm{Q}$ & $\hat{2}$ & $\mathrm{~S}^{\mathrm{H}}$ & $a^{2}$ & 1650 & 1.04 & 0.39 & 0.2 \\
\hline $2595 \mathrm{Q}$ & 2 & $\mathrm{~S}$ & $\mathrm{D}$ & 2 & 1650 & 0.76 & 0.36 & 0.2 & 26 & $95 \mathrm{Q}$ & $\hat{2}$ & $\mathrm{~S}$ & h & 1650 & 1.06 & 0.40 & 0.2 \\
\hline $2795 \mathrm{Q}$ & 2 & $S$ & $\underline{S}$ & 1 & 0000 & 0.73 & 0.67 & 0.3 & 28 & $95 \stackrel{\mathrm{S}}{\sim}$ & 2 & C D & 1 & 0000 & 0.75 & 0.70 & 0.3 \\
\hline $2795 \mathrm{Q}$ & 2 & $\mathrm{~S}$ & $\underline{S}$ & 2 & 0000 & 0.72 & 0.63 & $\overline{0} .3$ & 28 & $95 \tilde{\mathrm{S}}$ & 2 & C D & 2 & 0000 & 0.81 & 0.67 & 0.3 \\
\hline $2795 \mathrm{Q}$ & 2 & $\mathrm{~S}$ & S & 1 & 0015 & 0.68 & 0.62 & 0.3 & 28 & $95 \stackrel{\mathrm{S}}{\sim}$ & 2 & C D & 1 & 0015 & 0.80 & 0.71 & 0.4 \\
\hline $2795 \mathrm{Q}$ & 2 & $S$ & $\underline{S}$ & 2 & 0015 & 0.68 & 0.60 & 0.3 & 28 & $95 \stackrel{\mathrm{S}}{\sim}$ & 2 & C D & 2 & 0015 & 0.79 & 0.67 & 0.4 \\
\hline $2795 \mathrm{Q}$ & 2 & $S$ & $\underline{S}$ & 1 & 0036 & 0.73 & 0.65 & 0.3 & 28 & $95 \stackrel{\mathrm{S}}{\sim}$ & 2 & C D & 1 & 0036 & 0.87 & 0.71 & 0.4 \\
\hline $2795 \mathrm{Q}$ & 2 & $\mathrm{~S}$ & $S$ & 2 & 0036 & 0.70 & 0.65 & 0.3 & 28 & $95 \tilde{\mathrm{S}}$ & 2 & C D & 2 & 0036 & 0.88 & 0.68 & 0.4 \\
\hline $27 \mid 95 \mathrm{Q}$ & 2 & $S$ & $\underline{S}$ & 1 & 0090 & 0.68 & 0.60 & 0.3 & 28 & $95 \tilde{\mathrm{S}}$ & 2 & C D & 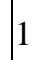 & 0090 & 0.86 & 0.67 & 0.3 \\
\hline $2795 \mathrm{Q}$ & 2 & $\mathrm{~S}$ & S & 2 & 0090 & 0.71 & 0.58 & 0.3 & 28 & $95 \stackrel{\mathrm{S}}{\sim}$ & 2 & C D & 2 & 0090 & 0.90 & 0.64 & 0.3 \\
\hline $2795 \mathrm{Q}$ & 2 & $\mathrm{~S}$ & $\underline{S}$ & 1 & 0180 & 0.74 & 0.59 & 0.3 & 28 & $95 \stackrel{\mathrm{S}}{\sim}$ & 2 & C D & 1 & 0180 & 0.88 & 0.60 & 0.3 \\
\hline $2795 \mathrm{Q}$ & 2 & $\mathrm{~S}$ & $S$ & 2 & 0180 & 0.72 & 0.57 & 0.3 & 28 & $95 \stackrel{\mathrm{S}}{\sim}$ & 2 & C D & 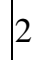 & 0180 & 0.82 & 0.58 & 0.3 \\
\hline $2795 \mathrm{Q}$ & 2 & $S$ & $\underline{S}$ & 1 & 0300 & 0.75 & 0.51 & 0.3 & 28 & $95 \tilde{\mathrm{S}}$ & $\overline{2}$ & C D & $\mathrm{J}^{2}$ & 0300 & 0.89 & 0.57 & 0.3 \\
\hline $2795 \mathrm{Q}$ & 2 & $S$ & $\mathrm{~S}$ & 2 & 0300 & 0.83 & 0.50 & 0.3 & 28 & $95 \stackrel{\tilde{S}}{\sim}$ & 2 & C D & 2 & 0300 & 0.90 & 0.56 & 0.3 \\
\hline $2795 \mathrm{Q}$ & 2 & $\mathrm{~S}$ & $\underline{S}$ & 1 & 0450 & 0.81 & 0.48 & 0.3 & 28 & $95 \mathrm{~S}$ & 2 & C D & 1 & 0450 & 0.89 & 0.53 & 0.3 \\
\hline $2795 \mathrm{Q}$ & 2 & $S$ & $\underline{S}$ & 2 & 0450 & 0.83 & 0.47 & 0.3 & 28 & $95 \stackrel{\mathrm{S}}{\sim}$ & 2 & C D & L & 0450 & 0.90 & 0.52 & 0.3 \\
\hline $2795 \mathrm{Q}$ & 2 & $\mathrm{~S}$ & $S$ & 1 & 0750 & 0.82 & 0.43 & 0.2 & 28 & $95 \stackrel{\mathrm{S}}{\mathrm{S}}$ & 2 & C D & 1 & 0750 & 0.98 & 0.48 & 0.3 \\
\hline $2795 \mathrm{Q}$ & 2 & $S$ & S & 2 & 0750 & 0.78 & 0.43 & 0.2 & 28 & $95 \stackrel{\tilde{S}}{\sim}$ & 2 & C D & 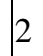 & 0750 & 1.00 & 0.46 & 0.3 \\
\hline $2795 \mathrm{Q}$ & 2 & $\mathrm{~S}$ & $\mathrm{~S}$ & 1 & 1200 & 0.79 & 0.39 & 0.2 & 28 & $95 \stackrel{\tilde{S}}{\sim}$ & 2 & C D & 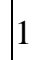 & 1200 & 0.97 & 0.43 & 0.2 \\
\hline $2795 \mathrm{Q}$ & 2 & $\mathrm{~S}$ & $\mathrm{~S}$ & 2 & 1200 & 0.84 & 0.38 & 0.2 & 28 & $95 \stackrel{\tilde{S}}{\sim}$ & 2 & C D & 2 & 1200 & 1.00 & 0.39 & 0.2 \\
\hline $2795 \mathrm{Q}$ & 2 & $S$ & $\underline{S}$ & 1 & 1650 & 0.80 & 0.35 & 0.2 & 28 & $95 \stackrel{\mathrm{S}}{\sim}$ & 2 & C D & 1 & 1650 & 0.92 & 0.39 & 0.2 \\
\hline $2795 \mathrm{Q}$ & 2 & $S$ & $S$ & 2 & 1650 & 0.82 & 0.34 & 0.2 & 28 & $95 \mathrm{~S}$ & 2 & C D & 2 & 1650 & 1.00 & 0.38 & 0.2 \\
\hline
\end{tabular}




\begin{tabular}{|c|c|c|c|c|c|c|c|c|c|c|c|c|c|c|c|c|}
\hline No & Mix I & abel & R & $\#$ & MPD & $\mathrm{DF}_{20}$ & F6 & No & & Mix & & $\mathrm{R}$ & $\#$ & MPD & $\mathrm{DF}_{20}$ & F6 \\
\hline \begin{tabular}{|l|l|}
29 & $95 \mathrm{~S}$ \\
\end{tabular} & 2 & $\mathrm{C} \mathrm{H}$ & 1 & 0000 & 0.88 & 0.65 & 0.3 & 30 & $95 \mathrm{~S}$ & 2 & $\mathrm{C} \mathrm{S}$ & 1 & 0000 & 1.03 & 0.59 & 0.3 \\
\hline $2995 \overline{\mathrm{S}}$ & 2 & $\mathrm{C}^{\overline{\mathrm{H}}}$ & 2 & 0000 & 0.81 & 0.59 & 0.3 & 30 & $95 \overline{\mathrm{S}}$ & 2 & $\mathrm{C} \overline{\mathrm{S}}$ & 2 & 0000 & 0.96 & 0.62 & 0.3 \\
\hline $2995 \tilde{\mathrm{S}}$ & $\hat{2}$ & C $\mathrm{H}$ & 1 & 0015 & 0.82 & 0.64 & $\overline{0} .3$ & 30 & $95 \tilde{\mathrm{S}}$ & $\hat{2}$ & $\mathrm{C} \stackrel{\mathrm{S}}{\mathrm{S}}$ & 1 & 0015 & 0.94 & 0.50 & 0.3 \\
\hline $2995 \tilde{S}$ & 2 & $\mathrm{C} \stackrel{\mathrm{H}}{\mathrm{H}}$ & 2 & 0015 & 0.81 & 0.67 & 0.3 & 30 & $95 \underset{\sim}{\widetilde{S}}$ & 2 & $\mathrm{C} S$ & 2 & 0015 & 0.93 & 0.49 & 0.3 \\
\hline $2995 \tilde{\mathrm{S}}$ & $\hat{2}$ & $\mathrm{C} \stackrel{\mathrm{H}}{\mathrm{H}}$ & 1 & 0036 & 0.79 & 0.71 & 0.4 & 30 & $95 \tilde{\mathrm{S}}$ & $\hat{2}$ & $\mathrm{C} S$ & 1 & 0036 & 0.94 & 0.64 & 0.3 \\
\hline $2995 \tilde{\mathrm{S}}$ & 2 & C $\mathrm{H}$ & 2 & 0036 & 0.84 & 0.68 & 0.4 & 30 & $95 \tilde{\sim}$ & $\hat{2}$ & $\mathrm{C} \stackrel{\mathrm{S}}{\mathrm{S}}$ & 2 & 0036 & 0.93 & 0.61 & 0.3 \\
\hline $2995 \mathrm{~S}$ & 2 & $\mathrm{C} \stackrel{\mathrm{H}}{ }$ & 1 & 0090 & 0.82 & 0.62 & 0.3 & 30 & $95 \stackrel{S}{S}$ & 2 & C $S$ & 1 & 0090 & 1.00 & 0.60 & 0.3 \\
\hline $2995 \tilde{S}$ & $\hat{2}$ & $\mathrm{C} \stackrel{\mathrm{H}}{\mathrm{H}}$ & 2 & 0090 & 0.85 & 0.61 & 0.3 & 30 & $95 \tilde{\mathrm{S}}$ & $\hat{2}$ & $\mathrm{C} \stackrel{\mathrm{S}}{\mathrm{S}}$ & 2 & 0090 & 1.00 & 0.59 & 0.3 \\
\hline $2995 \underset{\sim}{\mathrm{S}}$ & 2 & $\mathrm{C} \stackrel{\mathrm{H}}{\mathrm{H}}$ & 1 & 0180 & 0.91 & 0.57 & 0.3 & 30 & $95 \underset{\sim}{\tilde{S}}$ & $\hat{2}$ & $\mathrm{C} S$ & 1 & 0180 & 1.00 & 0.56 & 0.3 \\
\hline $2995 \tilde{S}$ & $\hat{2}$ & $\mathrm{C} \stackrel{\mathrm{H}}{\mathrm{H}}$ & 2 & 0180 & 0.88 & 0.55 & 0.3 & 30 & $95 \tilde{S}$ & 2 & $\mathrm{C} \dot{\mathrm{S}}$ & 2 & 0180 & 1.03 & 0.54 & 0.3 \\
\hline $2995 \mathrm{~S}$ & 2 & $\mathrm{C} \stackrel{\mathrm{H}}{ }$ & 1 & 0300 & 0.92 & 0.53 & 0.3 & 30 & $95 \underset{\sim}{\tilde{S}}$ & 2 & $\mathrm{C} S$ & 1 & 0300 & 1.02 & 0.52 & 0.3 \\
\hline $2995 \underset{\sim}{\mathrm{S}}$ & 2 & $\mathrm{C} \mathrm{H}$ & 2 & 0300 & 0.92 & 0.52 & 0.3 & 30 & $95 \underset{\sim}{\tilde{S}}$ & 2 & $\mathrm{C} S$ & 2 & 0300 & 1.00 & 0.51 & 0.3 \\
\hline $2995 \tilde{S}$ & $\hat{2}$ & $\mathrm{C} \stackrel{\mathrm{H}}{\mathrm{H}}$ & 1 & 0450 & 0.96 & 0.49 & 0.3 & 30 & $95 \tilde{\mathrm{S}}$ & $\hat{2}$ & $\mathrm{C} S$ & 1 & 0450 & 1.04 & 0.50 & 0.3 \\
\hline $2995 \underset{\sim}{\tilde{S}}$ & 2 & C $\mathrm{H}$ & 2 & 0450 & 0.98 & 0.48 & 0.3 & 30 & $95 \stackrel{\widetilde{S}}{\sim}$ & 2 & $\mathrm{C} \stackrel{\mathrm{S}}{\mathrm{N}}$ & 2 & 0450 & 1.05 & 0.48 & 0.3 \\
\hline $2995 \tilde{S}$ & $\hat{2}$ & $\mathrm{C} \stackrel{\mathrm{H}}{\mathrm{H}}$ & 1 & 0750 & 1.04 & 0.42 & 0.2 & 30 & $95 \tilde{\mathrm{S}}$ & 2 & $\mathrm{C} S$ & 1 & 0750 & 1.03 & 0.46 & 0.3 \\
\hline $2995 \stackrel{\mathrm{S}}{\tilde{\mathrm{S}}}$ & 2 & $\mathrm{C} \stackrel{\mathrm{H}}{ }$ & 2 & 0750 & 1.01 & 0.42 & 0.2 & 30 & $95 \underset{\sim}{\tilde{S}}$ & $\hat{2}$ & $\mathrm{C} \stackrel{\mathrm{S}}{\mathrm{S}}$ & 2 & 0750 & 1.01 & 0.44 & 0.3 \\
\hline $2995 \mathrm{~S}$ & 2 & $\mathrm{C} \mathrm{H}$ & 1 & 1200 & 0.96 & 0.40 & 0.2 & 30 & $95 \underset{\sim}{\tilde{S}}$ & $\hat{2}$ & $\mathrm{C} S$ & 1 & 1200 & 1.08 & 0.42 & 0.2 \\
\hline $2995 \tilde{\sim}$ & $\hat{2}$ & $\mathrm{C} \stackrel{\mathrm{H}}{\mathrm{H}}$ & 2 & 1200 & 1.00 & 0.39 & 0.2 & 30 & $95 \tilde{\mathrm{S}}$ & $\hat{2}$ & $\mathrm{C} S$ & 2 & 1200 & 1.02 & 0.41 & 0.2 \\
\hline $2995 \stackrel{\tilde{S}}{\sim}$ & 2 & $\mathrm{C} \stackrel{\mathrm{H}}{ }$ & 1 & 1650 & 1.07 & 0.40 & 0.2 & 30 & $95 \stackrel{\mathrm{S}}{\sim}$ & 2 & C $S$ & 1 & 1650 & 1.00 & 0.40 & 0.2 \\
\hline $2995 \underset{\sim}{\tilde{S}}$ & 2 & $\mathrm{C} \stackrel{\mathrm{H}}{\mathrm{H}}$ & 2 & 1650 & 0.97 & 0.41 & 0.2 & 30 & $95 \stackrel{\widetilde{S}}{\sim}$ & 2 & $\mathrm{C} \stackrel{\mathrm{S}}{\mathrm{S}}$ & 2 & 1650 & 1.02 & 0.40 & 0.2 \\
\hline $3195 \mathrm{~S}$ & 2 & F D & 1 & 0000 & 0.39 & 0.75 & 0.3 & 32 & $95 \stackrel{S}{S}$ & 2 & $\mathrm{~F}^{\mathrm{H}}$ & 1 & 0000 & 0.35 & 0.45 & 0.2 \\
\hline 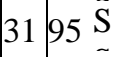 & 2 & $\mathrm{~F} \quad \mathrm{D}$ & 2 & 0000 & 0.40 & 0.64 & 0.3 & 32 & $95 \stackrel{\widetilde{S}}{\sim}$ & 2 & $\mathrm{~F}_{-}^{\mathrm{H}}$ & 2 & 0000 & 0.34 & 0.42 & 0.2 \\
\hline $3195 \mathrm{~S}$ & 2 & F D & 1 & 0015 & 0.42 & 0.71 & 0.3 & 32 & $95 \tilde{\mathrm{S}}$ & 2 & $\mathrm{~F}_{-}^{\mathrm{H}}$ & 1 & 0015 & 0.33 & 0.54 & 0.2 \\
\hline $3195 \stackrel{\tilde{S}}{\sim}$ & 2 & F D & 2 & 0015 & 0.41 & 0.70 & 0.3 & 32 & $95 \stackrel{\mathrm{S}}{\sim}$ & 2 & $\mathrm{~F}_{-}^{\mathrm{H}}$ & 2 & 0015 & 0.34 & 0.53 & 0.2 \\
\hline $3195 \underset{\tilde{\sigma}}{\mathrm{S}}$ & 2 & F D & 1 & 0036 & 0.43 & 0.68 & 0.3 & 32 & $95 \stackrel{\tilde{S}}{\tilde{S}}$ & 2 & $\mathrm{~F} \stackrel{\mathrm{H}}{-}$ & 1 & 0036 & 0.35 & 0.61 & 0.2 \\
\hline $3195 \tilde{S}$ & 2 & $\mathrm{~F} \quad \mathrm{D}$ & 2 & 0036 & 0.43 & 0.67 & 0.3 & 32 & $95 \tilde{\mathrm{S}}$ & 2 & $\mathrm{~F} \stackrel{\mathrm{H}}{\mathrm{H}}$ & 2 & 0036 & 0.35 & 0.58 & 0.2 \\
\hline $3195 \stackrel{\tilde{S}}{\sim}$ & 2 & F D & 1 & 0090 & 0.47 & 0.62 & 0.3 & 32 & $95 \stackrel{\mathrm{S}}{\sim}$ & 2 & $\mathrm{~F}_{-}^{\mathrm{H}}$ & 1 & 0090 & 0.39 & 0.58 & 0.2 \\
\hline $3195 \mathrm{~S}$ & 2 & F D & 2 & 0090 & 0.46 & 0.60 & 0.3 & 32 & $95 \stackrel{S}{S}$ & 2 & $\mathrm{~F}^{\mathrm{H}}$ & 2 & 0090 & 0.36 & 0.55 & 0.2 \\
\hline $3195 \underset{\sim}{\mathrm{S}}$ & 2 & F D & 1 & 0180 & 0.57 & 0.57 & 0.3 & 32 & $95 \underset{\sim}{\tilde{S}}$ & 2 & $\mathrm{~F}_{-}^{\mathrm{H}}$ & 1 & 0180 & 0.37 & 0.52 & 0.2 \\
\hline $3195 \stackrel{\mathrm{S}}{\mathrm{S}}$ & 2 & F D & 2 & 0180 & 0.54 & 0.55 & 0.3 & 32 & $95 \stackrel{\widetilde{S}}{\sim}$ & 2 & $\mathrm{~F}_{-}^{\mathrm{H}}$ & 2 & 0180 & 0.38 & 0.51 & 0.2 \\
\hline $3195 \tilde{S}$ & 2 & F D & 1 & 0300 & 0.58 & 0.55 & 0.3 & 32 & $95 \tilde{\mathrm{S}}$ & 2 & $\mathrm{~F}_{-}^{\mathrm{H}}$ & 1 & 0300 & 0.43 & 0.50 & 0.2 \\
\hline $3195 \stackrel{\tilde{S}}{\sim}$ & 2 & F D & 2 & 0300 & 0.59 & 0.54 & 0.3 & 32 & 95 & 2 & $\mathrm{~F}_{-}^{\mathrm{H}}$ & 2 & 0300 & 0.44 & 0.47 & 0.2 \\
\hline $3195 \stackrel{\mathrm{S}}{\sim}$ & 2 & F D & 1 & 0450 & 0.72 & 0.51 & 0.3 & 32 & 95 & 2 & $\mathrm{~F}_{-}^{\mathrm{H}}$ & 1 & 0450 & 0.51 & 0.47 & 0.2 \\
\hline $3195 \underset{\sim}{\mathrm{S}}$ & 2 & F D & 2 & 0450 & 0.67 & 0.51 & 0.3 & 32 & 95 & 2 & $\mathrm{~F}_{-}^{\mathrm{H}}$ & 2 & 0450 & 0.50 & 0.45 & 0.2 \\
\hline $3195 \stackrel{\mathrm{S}}{\mathrm{S}}$ & 2 & F D & 1 & 0750 & 0.69 & 0.47 & 0.2 & 32 & $95 \stackrel{\mathrm{S}}{\sim}$ & 2 & $\mathrm{~F}_{-}^{\mathrm{H}}$ & 1 & 0750 & 0.52 & 0.41 & 0.2 \\
\hline $3195 \tilde{S}$ & 2 & F D & 2 & 0750 & 0.70 & 0.46 & 0.2 & 32 & $95 \tilde{\mathrm{S}}$ & 2 & $\mathrm{~F} \stackrel{\mathrm{H}}{-}$ & $1^{2}$ & 0750 & 0.51 & 0.38 & 0.2 \\
\hline $3195 \underset{\sim}{\mathrm{S}}$ & 2 & F D & 1 & 1200 & 0.75 & 0.43 & 0.2 & 32 & $95 \stackrel{\mathrm{S}}{\tilde{\sigma}}$ & 2 & $\mathrm{~F} \mathrm{H}$ & 1 & 1200 & 0.51 & 0.37 & 0.2 \\
\hline $3195 \stackrel{\mathrm{S}}{\mathrm{S}}$ & 2 & F D & 2 & 1200 & 0.73 & 0.42 & 0.2 & 32 & 95 & 2 & $\mathrm{~F}_{-}^{\mathrm{H}}$ & 2 & 1200 & 0.52 & 0.37 & 0.2 \\
\hline $3195 \underset{\tilde{\sigma}}{\mathrm{S}}$ & 2 & F D & 1 & 1650 & 0.71 & 0.41 & 0.2 & 32 & 95 & 2 & $\mathrm{~F}_{-}^{\mathrm{H}}$ & 1 & 1650 & 0.54 & 0.34 & 0.2 \\
\hline $3195 \tilde{S}$ & 2 & F D & 12 & 1650 & 0.69 & 0.40 & 0.2 & 32 & $95 \mathrm{~S}$ & 2 & $\mathrm{~F} \mathrm{H}^{\mathrm{H}}$ & 2 & 1650 & 0.51 & 0.34 & 0.2 \\
\hline
\end{tabular}




\begin{tabular}{|c|c|c|c|c|c|c|c|c|c|c|c|c|c|c|c|c|c|c|}
\hline No & Mix I & abel & & R & \# & MPD & $\mathrm{DF}_{20}$ & F6 & No & & $\overline{\mathrm{Mi}}$ & & & $\mathrm{R}$ & $\#$ & MPD & $\mathrm{DF}_{20}$ & F6 \\
\hline \begin{tabular}{|l|l|}
33 & $95 \mathrm{~S}$
\end{tabular} & 2 & $\mathrm{~F}$ & $S$ & 1 & 0000 & 0.35 & 0.50 & 0.2 & 34 & $95 \mathrm{~S}$ & 2 & S & $\mathrm{D}$ & 1 & 0000 & 0.71 & 0.62 & 0.3 \\
\hline $3395 \overline{\mathrm{S}}$ & 2 & $\mathrm{~F}$ & $\mathrm{~S}$ & 2 & 0000 & 0.36 & 0.47 & 0.2 & 34 & $95 \overline{\mathrm{S}}$ & 2 & $\mathrm{~S}$ & $\mathrm{D}$ & 2 & 0000 & 0.64 & 0.61 & 0.3 \\
\hline $3395 \tilde{\mathrm{S}}$ & $\hat{2}$ & $\mathrm{~F}$ & $\dot{S}$ & 1 & 0015 & 0.43 & 0.75 & 0.3 & 34 & $95 \tilde{\mathrm{S}}$ & $\hat{2}$ & $S$ & D & 1 & 0015 & 0.64 & 0.65 & 0.3 \\
\hline $3395 \tilde{\mathrm{S}}$ & 2 & $\mathrm{~F}$ & $S$ & 2 & 0015 & 0.43 & 0.71 & 0.3 & 34 & $95 \underset{\sim}{\widetilde{S}}$ & 2 & $S$ & D & 2 & 0015 & 0.64 & 0.62 & 0.3 \\
\hline $3395 \tilde{\mathrm{S}}$ & $\hat{2}$ & $\mathrm{~F}$ & $S$ & 1 & 0036 & 0.47 & 0.72 & 0.3 & 34 & $95 \tilde{\mathrm{S}}$ & $\hat{2}$ & S & $\mathrm{D}$ & 1 & 0036 & 0.63 & 0.70 & 0.3 \\
\hline $3395 \stackrel{\widetilde{S}}{\sim}$ & 2 & $\mathrm{~F}$ & $S$ & 2 & 0036 & 0.48 & 0.68 & 0.3 & 34 & $95 \tilde{\sim}$ & $\hat{2}$ & $S$ & D & 2 & 0036 & 0.65 & 0.68 & 0.3 \\
\hline $3395 \tilde{\mathrm{S}}$ & 2 & $\mathrm{~F}$ & $S$ & 1 & 0090 & 0.57 & 0.66 & 0.3 & 34 & $95 \underset{\sim}{\widetilde{S}}$ & $\hat{2}$ & $S$ & $\mathrm{D}$ & 1 & 0090 & 0.65 & 0.63 & 0.3 \\
\hline $3395 \tilde{\mathrm{S}}$ & $\hat{2}$ & $\mathrm{~F}$ & $S$ & 2 & 0090 & 0.55 & 0.65 & 0.3 & 34 & $95 \tilde{\mathrm{S}}$ & $\hat{2}$ & $\mathrm{~S}$ & D & 2 & 0090 & 0.71 & 0.61 & 0.3 \\
\hline 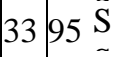 & 2 & $\mathrm{~F}$ & $S$ & 1 & 0180 & 0.61 & 0.62 & 0.3 & 34 & $95 \underset{\sim}{\tilde{S}}$ & $\hat{2}$ & $S$ & $\mathrm{D}$ & 1 & 0180 & 0.68 & 0.58 & 0.3 \\
\hline $3395 \tilde{S}$ & $\hat{2}$ & $\mathrm{~F}$ & $S$ & 2 & 0180 & 0.59 & 0.61 & 0.3 & 34 & $95 \tilde{\mathrm{S}}$ & $\hat{2}$ & $\mathrm{~S}$ & D & 2 & 0180 & 0.66 & 0.56 & 0.3 \\
\hline $3395 \stackrel{\mathrm{S}}{\mathrm{S}}$ & 2 & $\mathrm{~F}$ & $\mathrm{~S}$ & 1 & 0300 & 0.68 & 0.58 & 0.3 & 34 & $95 \underset{\sim}{\tilde{S}}$ & $\hat{2}$ & $S$ & D & 1 & 0300 & 0.72 & 0.54 & 0.3 \\
\hline $3395 \mathrm{~S}$ & 2 & $\mathrm{~F}$ & $\mathrm{~S}$ & 2 & 0300 & 0.68 & 0.56 & 0.3 & 34 & $95 \widetilde{\mathrm{S}}$ & 2 & $S$ & D & 2 & 0300 & 0.69 & 0.53 & 0.3 \\
\hline $3395 \tilde{\mathrm{S}}$ & $\hat{2}$ & $\mathrm{~F}$ & $S$ & 1 & 0450 & 0.71 & 0.55 & 0.3 & 34 & $95 \tilde{\mathrm{S}}$ & $\hat{2}$ & $\mathrm{~S}$ & D & 1 & 0450 & 0.74 & 0.48 & 0.2 \\
\hline 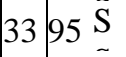 & 2 & $\mathrm{~F}$ & $S$ & 2 & 0450 & 0.70 & 0.55 & 0.3 & 34 & $95 \stackrel{\widetilde{S}}{\sim}$ & 2 & $S$ & D & 2 & 0450 & 0.76 & 0.47 & 0.2 \\
\hline $3395 \tilde{\mathrm{S}}$ & $\hat{2}$ & $\mathrm{~F}$ & S & 1 & 0750 & 0.76 & 0.47 & 0.3 & 34 & $95 \tilde{\mathrm{S}}$ & $\hat{2}$ & $S$ & D & 1 & 0750 & 0.75 & 0.41 & 0.3 \\
\hline $3395 \stackrel{\mathrm{S}}{\mathrm{S}}$ & 2 & $\mathrm{~F}$ & $S$ & 2 & 0750 & 0.77 & 0.48 & 0.3 & 34 & $95 \underset{\sim}{\tilde{S}}$ & $\hat{2}$ & $S$ & D & 2 & 0750 & 0.75 & 0.42 & 0.3 \\
\hline $3395 \mathrm{~S}$ & 2 & $\mathrm{~F}$ & S & 1 & 1200 & 0.83 & 0.44 & 0.3 & 34 & $95 \underset{\sim}{\tilde{S}}$ & 2 & $S$ & $\mathrm{D}$ & 1 & 1200 & 0.81 & 0.40 & 0.2 \\
\hline $3395 \tilde{\mathrm{S}}$ & $\hat{2}$ & $\mathrm{~F}$ & $S$ & 2 & 1200 & 0.81 & 0.42 & 0.3 & 34 & $95 \tilde{\mathrm{S}}$ & $\hat{2}$ & $S$ & D & 2 & 1200 & 0.77 & 0.42 & $\overline{0} .2$ \\
\hline $3395 \tilde{\mathrm{S}}$ & $\hat{2}$ & $\mathrm{~F}$ & $S$ & 1 & 1650 & 0.81 & 0.41 & 0.2 & 34 & $95 \tilde{\mathrm{S}}$ & $\hat{2}$ & $\mathrm{~S}$ & D & 1 & 1650 & 0.73 & 0.39 & $\overline{0} .2$ \\
\hline $3395 \underset{\sim}{\tilde{S}}$ & 2 & $\mathrm{~F}$ & $S$ & 2 & 1650 & 0.83 & 0.40 & $\overline{0} .2$ & 34 & $95 \stackrel{\widetilde{S}}{\sim}$ & 2 & $S$ & D & 2 & 1650 & 0.76 & 0.38 & 0.2 \\
\hline $3595 \mathrm{~S}$ & 2 & $\mathrm{~S}$ & $\mathrm{H}$ & 1 & 0000 & 0.67 & 0.45 & 0.2 & 36 & נין & 2 & $\mathrm{~S}$ & S & 1 & 0000 & 0.78 & 0.57 & 0.3 \\
\hline $3595 \tilde{\sim}$ & 2 & $S$ & $\mathrm{H}$ & 2 & 0000 & 0.66 & 0.42 & 0.2 & 36 & $95 \stackrel{\widetilde{S}}{\sim}$ & 2 & $S$ & S & 2 & 0000 & 0.65 & 0.54 & 0.3 \\
\hline $3595 \mathrm{~S}$ & 2 & $S$ & $\mathrm{H}$ & 1 & 0015 & 0.66 & 0.48 & 0.2 & 36 & $95 \tilde{\mathrm{S}}$ & 2 & $S$ & S & 1 & 0015 & 0.70 & 0.56 & 0.3 \\
\hline $3595 \stackrel{\tilde{S}}{\sim}$ & 2 & $\mathrm{~S}$ & $\mathrm{H}$ & 2 & 0015 & 0.69 & 0.46 & 0.2 & 36 & $95 \stackrel{\mathrm{S}}{\sim}$ & 2 & $S$ & S & 2 & 0015 & 0.73 & 0.55 & 0.3 \\
\hline $3595 \mathrm{~S}$ & 2 & $\mathrm{~S}$ & $\mathrm{H}$ & 1 & 0036 & 0.64 & 0.58 & 0.3 & 36 & $95 \tilde{\mathrm{S}}$ & 2 & $S$ & $S$ & 1 & 0036 & 0.71 & 0.65 & 0.3 \\
\hline $3595 \tilde{\mathrm{S}}$ & 2 & $\mathrm{~S}$ & $\mathrm{H}$ & 2 & 0036 & 0.70 & 0.56 & 0.3 & 36 & $95 \tilde{\mathrm{S}}$ & 2 & $\mathrm{~S}$ & $S$ & 2 & 0036 & 0.68 & 0.62 & 0.3 \\
\hline & 2 & $S$ & $\mathrm{H}$ & 1 & 0090 & 0.63 & 0.54 & 0.3 & 36 & $95 \stackrel{\mathrm{S}}{\sim}$ & 2 & $S$ & $\underline{S}$ & 1 & 0090 & 0.69 & 0.58 & 0.3 \\
\hline $3595 \mathrm{~S}$ & 2 & $S$ & $\mathrm{H}$ & 2 & 0090 & 0.72 & 0.54 & 0.3 & 36 & $95 \stackrel{\mathrm{S}}{\sim}$ & 2 & $S$ & S & 2 & 0090 & 0.69 & 0.57 & 0.3 \\
\hline $3595 \underset{\sim}{\mathrm{S}}$ & 2 & $\mathrm{~S}$ & $\mathrm{H}$ & 1 & 0180 & 0.71 & 0.48 & 0.2 & 36 & 95 & 2 & $S$ & S & 1 & 0180 & 0.75 & 0.53 & 0.3 \\
\hline $3595 \tilde{\sim}$ & 2 & S & $\mathrm{H}$ & 2 & 0180 & 0.68 & 0.49 & 0.2 & 36 & $95 \stackrel{\widetilde{S}}{\sim}$ & 2 & $S$ & $\underline{S}$ & 2 & 0180 & 0.69 & 0.53 & 0.3 \\
\hline $3595 \tilde{S}$ & 2 & $\mathrm{~S}$ & $\mathrm{H}$ & 1 & 0300 & 0.68 & 0.45 & 0.2 & 36 & $95 \tilde{\mathrm{S}}$ & 2 & $S$ & $\underline{S}$ & $1+$ & 0300 & 0.77 & 0.47 & 0.2 \\
\hline $3595 \tilde{\mathrm{S}}$ & 2 & $\mathrm{~S}$ & $\mathrm{H}$ & 2 & 0300 & 0.72 & 0.44 & 0.2 & 36 & 95 & 2 & $\mathrm{~S}$ & $S$ & 2 & 0300 & 0.71 & 0.47 & 0.2 \\
\hline $3595 \tilde{S}$ & 2 & $\mathrm{~S}$ & $\mathrm{H}$ & 1 & 0450 & 0.68 & 0.41 & 0.2 & 36 & $95 \underset{\sim}{\tilde{S}}$ & 2 & $S$ & S & 1 & 0450 & 0.83 & 0.44 & 0.2 \\
\hline $3595 \underset{\sim}{\mathrm{S}}$ & 2 & $\mathrm{~S}$ & $\mathrm{H}$ & 2 & 0450 & 0.70 & 0.43 & 0.2 & 36 & 95 & 2 & $S$ & $\underline{S}$ & 2 & 0450 & 0.72 & 0.43 & 0.2 \\
\hline $3595 \stackrel{\tilde{S}}{\sim}$ & 2 & $\mathrm{~S}$ & $\mathrm{H}$ & 1 & 0750 & 0.70 & 0.38 & 0.2 & 36 & 95 & 2 & $S$ & $\underline{S}$ & 1 & 0750 & 0.77 & 0.40 & 0.2 \\
\hline $3595 \tilde{S}$ & 2 & $S$ & $\mathrm{H}$ & 2 & 0750 & 0.74 & 0.38 & 0.2 & 36 & 95 & 2 & $S$ & $\underline{S}$ & 2 & 0750 & 0.76 & 0.40 & 0.2 \\
\hline $3595 \tilde{\mathrm{S}}$ & 2 & $\mathrm{~S}$ & $\mathrm{H}$ & 1 & 1200 & 0.69 & 0.36 & 0.2 & 36 & 95 & 2 & $\mathrm{~S}$ & $S$ & 1 & 1200 & 0.76 & 0.38 & 0.2 \\
\hline $95 \stackrel{\mathrm{S}}{\sim}$ & 2 & $S$ & $\mathrm{H}$ & 2 & 1200 & 0.71 & 0.39 & 0.2 & 36 & 95 & 2 & $S$ & - & 2 & 1200 & 0.72 & 0.38 & 0.2 \\
\hline $3595 \mathrm{~S}$ & 2 & $\mathrm{~S}$ & $\mathrm{H}$ & 1 & 1650 & 0.70 & 0.37 & 0.2 & 36 & 95 & 2 & $S$ & - & 1 & 1650 & 0.85 & 0.37 & 0.2 \\
\hline $3595 \mathrm{~S}$ & 2 & $\mathrm{~S}$ & $\mathrm{H}$ & 2 & 1650 & 0.70 & 0.37 & 0.2 & 36 & $95 \mathrm{~S}$ & 2 & $\mathrm{~S}$ & 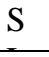 & 2 & 1650 & 0.81 & 0.37 & 0.2 \\
\hline
\end{tabular}




\begin{tabular}{|c|c|c|c|c|c|c|c|c|c|c|c|c|c|c|c|c|c|c|}
\hline No & Mix & abel & & R & \# & MPD & $\mathrm{DF}_{20}$ & F6 & No & & $\overline{\mathrm{Mi}}$ & & & $\mathrm{R}$ & $\#$ & MPD & $\mathrm{DF}_{20}$ & F6 \\
\hline \begin{tabular}{|l|l|}
37 & $95 \mathrm{~S}$
\end{tabular} & 0 & S & D & 1 & 0000 & 0.97 & 0.68 & 0.4 & 38 & $95 \mathrm{~S}$ & 1 & S & D & 1 & 0000 & 0.99 & 0.67 & 0.4 \\
\hline $3795 \overline{\mathrm{S}}$ & 0 & $S$ & D & 2 & 0000 & 1.14 & 0.64 & 0.4 & 38 & $95 \overline{\mathrm{S}}$ & 1 & $\mathrm{~S}$ & $\mathrm{D}$ & 2 & 0000 & 0.93 & 0.64 & 0.4 \\
\hline $3795 \tilde{\mathrm{S}}$ & 0 & S & D & 1 & 0015 & 0.89 & 0.68 & 0.4 & 38 & $95 \tilde{\mathrm{S}}$ & 1 & $S$ & $\mathrm{D}$ & 1 & 0015 & 0.94 & 0.64 & 0.3 \\
\hline $3795 \tilde{S}$ & 0 & S & D & 2 & 0015 & 0.93 & 0.65 & 0.4 & 38 & $95 \underset{\sim}{\widetilde{S}}$ & $\hat{1}$ & $S$ & D & 2 & 0015 & 0.91 & 0.62 & 0.3 \\
\hline $3795 \tilde{\mathrm{S}}$ & 0 & S & D & 1 & 0036 & 1.02 & 0.64 & 0.4 & 38 & $95 \tilde{\mathrm{S}}$ & $\hat{1}$ & $\mathrm{~S}$ & $\mathrm{D}$ & 1 & 0036 & 0.96 & 0.58 & 0.3 \\
\hline $3795 \stackrel{\widetilde{S}}{\sim}$ & 0 & S & $\mathrm{D}$ & 2 & 0036 & 0.98 & 0.62 & 0.4 & 38 & $95 \tilde{\sim}$ & $\hat{1}$ & $\mathrm{~S}$ & D & 2 & 0036 & 0.93 & 0.56 & 0.3 \\
\hline $3795 \mathrm{~S}$ & 0 & S & D & 1 & 0090 & 1.01 & 0.55 & 0.3 & 38 & $95 \underset{\sim}{\tilde{S}}$ & $\hat{1}$ & $S$ & $\mathrm{D}$ & 1 & 0090 & 0.95 & 0.53 & 0.3 \\
\hline $3795 \tilde{\mathrm{S}}$ & 0 & $\mathrm{~S}$ & D & 2 & 0090 & 0.96 & 0.53 & 0.3 & 38 & $95 \tilde{\mathrm{S}}$ & $\hat{1}$ & $\mathrm{~S}$ & $\mathrm{D}$ & 2 & 0090 & 0.93 & 0.51 & 0.3 \\
\hline $3795 \stackrel{\widetilde{S}}{\sim}$ & 0 & S & D & 1 & 0180 & 0.98 & 0.52 & 0.3 & 38 & $95 \underset{\sim}{\tilde{S}}$ & $\hat{1}$ & $S$ & $\mathrm{D}$ & 1 & 0180 & 0.99 & 0.49 & 0.3 \\
\hline $3795 \tilde{S}$ & 0 & S & D & 2 & 0180 & 0.97 & 0.50 & 0.3 & 38 & $95 \tilde{\mathrm{S}}$ & $\hat{1}$ & $\mathrm{~S}$ & $\mathrm{D}$ & 2 & 0180 & 1.00 & 0.48 & 0.3 \\
\hline $3795 \stackrel{\mathrm{S}}{\mathrm{S}}$ & 0 & $S$ & $\mathrm{D}$ & 1 & 0300 & 1.02 & 0.47 & 0.3 & 38 & $95 \underset{\sim}{\tilde{S}}$ & $\hat{1}$ & $S$ & $\mathrm{D}$ & 1 & 0300 & 0.99 & 0.43 & 0.2 \\
\hline $37 \underset{95}{\mathrm{~S}}$ & 0 & $S$ & D & 2 & 0300 & 0.98 & 0.45 & 0.3 & 38 & $95 \underset{\sim}{\tilde{S}}$ & 1 & $S$ & $\mathrm{D}$ & 2 & 0300 & 0.97 & 0.43 & 0.2 \\
\hline $3795 \tilde{\mathrm{S}}$ & 0 & $\mathrm{~S}$ & D & 1 & 0450 & 1.01 & 0.42 & 0.2 & 38 & $95 \tilde{\mathrm{S}}$ & $\hat{1}$ & $\mathrm{~S}$ & $\mathrm{D}$ & 1 & 0450 & 1.03 & 0.40 & 0.2 \\
\hline $3795 \stackrel{\widetilde{S}}{2}$ & 0 & S & D & 2 & 0450 & 1.05 & 0.41 & 0.2 & 38 & $95 \stackrel{\widetilde{S}}{\sim}$ & 1 & $S$ & $\mathrm{D}$ & 2 & 0450 & 0.98 & 0.39 & 0.2 \\
\hline $3795 \tilde{S}$ & 0 & S & D & 1 & 0750 & 1.03 & 0.41 & 0.2 & 38 & $95 \tilde{\mathrm{S}}$ & $\hat{1}$ & $\mathrm{~S}$ & $\mathrm{D}$ & 1 & 0750 & 1.01 & 0.37 & 0.2 \\
\hline $3795 \stackrel{\widetilde{S}}{\mathrm{~S}}$ & 0 & S & D & 2 & 0750 & 1.05 & 0.39 & 0.2 & 38 & $95 \stackrel{\mathrm{S}}{\sim}$ & 1 & $S$ & D & 2 & 0750 & 0.95 & 0.36 & 0.2 \\
\hline $3795 \underset{\tilde{a}}{\mathrm{~S}}$ & 0 & $\mathrm{~S}$ & D & 1 & 1200 & 1.08 & 0.32 & 0.2 & 38 & $95 \stackrel{\widetilde{S}}{\sim}$ & 1 & $\mathrm{~S}$ & $\mathrm{D}$ & 1 & 1200 & 0.94 & 0.35 & 0.2 \\
\hline $3795 \tilde{S}$ & 0 & S & D & 2 & 1200 & 1.12 & 0.32 & 0.2 & 38 & $95 \tilde{\mathrm{S}}$ & $\hat{1}$ & $S$ & D & 2 & 1200 & 0.98 & 0.36 & 0.2 \\
\hline $3795 \stackrel{\tilde{S}}{\sim}$ & 0 & $S$ & D & 1 & 1650 & 0.99 & 0.34 & 0.2 & 38 & $95 \stackrel{\mathrm{S}}{\sim}$ & 1 & $S$ & $\mathrm{D}$ & 1 & 1650 & 0.95 & 0.35 & 0.2 \\
\hline $3795 \stackrel{\mathrm{S}}{\sim}$ & 0 & S & $\mathrm{D}$ & 2 & 1650 & 0.98 & 0.33 & 0.2 & 38 & $95 \stackrel{\widetilde{S}}{\sim}$ & 1 & $S$ & D & 2 & 1650 & 0.94 & 0.35 & 0.2 \\
\hline $3995 \mathrm{~S}$ & 4 & S & D & 1 & 0000 & 0.77 & 0.72 & 0.3 & 40 & 95 & I & $S$ & D & 1 & 0000 & 0.97 & 0.74 & 0.4 \\
\hline 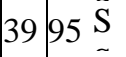 & 4 & $S$ & D & 2 & 0000 & 0.78 & 0.64 & 0.3 & 40 & $95 \stackrel{\widetilde{S}}{\sim}$ & 7 & $S$ & D & 2 & 0000 & 0.90 & 0.67 & 0.4 \\
\hline $3995 \mathrm{~S}$ & 4 & $\mathrm{~S}$ & D & 1 & 0015 & 0.73 & 0.68 & 0.3 & 40 & $95 \tilde{\mathrm{S}}$ & 7 & $S$ & $\mathrm{D}$ & 1 & 0015 & 0.79 & 0.65 & 0.3 \\
\hline $3995 \mathrm{~S}$ & 4 & S & $\mathrm{D}$ & 2 & 0015 & 0.71 & 0.66 & 0.3 & 40 & 95 & 7 & $\mathrm{~S}$ & D & 2 & 0015 & 0.78 & 0.62 & $\overline{0} .3$ \\
\hline $39 \underset{95}{\mathrm{~S}}$ & 4 & $\mathrm{~S}$ & D & 1 & 0036 & 0.71 & 0.63 & 0.3 & 40 & $95 \stackrel{\tilde{S}}{\tilde{S}}$ & 7 & $S$ & D & 1 & 0036 & 0.83 & 0.63 & 0.3 \\
\hline $3995 \tilde{\mathrm{S}}$ & 4 & $\mathrm{~S}$ & D & 2 & 0036 & 0.70 & 0.60 & 0.3 & 40 & $95 \tilde{\mathrm{S}}$ & 7 & $\mathrm{~S}$ & D & 2 & 0036 & 0.80 & 0.61 & 0.3 \\
\hline 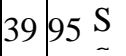 & 4 & S & D & 1 & 0090 & 0.75 & 0.56 & 0.3 & 40 & 95 & 7 & $S$ & D & 1 & 0090 & 0.86 & 0.58 & 0.3 \\
\hline $3995 \mathrm{~S}$ & 4 & $\mathrm{~S}$ & D & 2 & 0090 & 0.68 & 0.55 & 0.3 & 40 & $95 \stackrel{\mathrm{S}}{\sim}$ & 7 & $S$ & D & 2 & 0090 & 0.83 & 0.57 & 0.3 \\
\hline $39 \stackrel{95}{\mathrm{~S}}$ & 4 & $\mathrm{~S}$ & $\mathrm{D}$ & 1 & 0180 & 0.71 & 0.53 & 0.3 & 40 & 95 & $\overline{7}$ & $S$ & D & 1 & 0180 & 0.85 & 0.58 & 0.3 \\
\hline 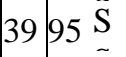 & 4 & $S$ & $\mathrm{D}$ & 2 & 0180 & 0.72 & 0.52 & 0.3 & 40 & $95 \stackrel{\widetilde{S}}{\sim}$ & 7 & $S$ & D & 2 & 0180 & 0.87 & 0.55 & 0.3 \\
\hline $3995 \tilde{\mathrm{S}}$ & 4 & $S$ & D & 1 & 0300 & 0.79 & 0.49 & 0.3 & 40 & $95 \tilde{\mathrm{S}}$ & 7 & $S$ & $\mathrm{D}$ & 1 & 0300 & 0.89 & 0.53 & 0.3 \\
\hline $3995 \stackrel{\tilde{S}}{\sim}$ & 4 & $S$ & D & 2 & 0300 & 0.76 & 0.47 & 0.3 & 40 & 95 & 7 & $S$ & D & 2 & 0300 & 0.89 & 0.53 & 0.3 \\
\hline $3995 \tilde{\sim}$ & 4 & $\mathrm{~S}$ & $\mathrm{D}$ & 1 & 0450 & 0.76 & 0.47 & 0.2 & 40 & 95 & 7 & $S$ & D & 1 & 0450 & 0.89 & 0.51 & 0.3 \\
\hline $39 \underset{95}{\mathrm{~S}}$ & 4 & $\mathrm{~S}$ & $\mathrm{D}$ & 2 & 0450 & 0.77 & 0.45 & 0.2 & 40 & 95 & 7 & $S$ & D & 2 & 0450 & 0.83 & 0.51 & 0.3 \\
\hline $3995 \stackrel{\mathrm{S}}{\mathrm{S}}$ & 4 & $\mathrm{~S}$ & $\mathrm{D}$ & 1 & 0750 & 0.80 & 0.45 & 0.2 & 40 & $95 \stackrel{\mathrm{S}}{\sim}$ & 7 & $S$ & D & 1 & 0750 & 0.85 & 0.50 & 0.3 \\
\hline $3995 \tilde{S}$ & $\overline{4}$ & $S$ & D & 2 & 0750 & 0.83 & 0.44 & 0.2 & 40 & 95 & 7 & $S$ & D & & 0750 & 0.86 & 0.49 & 0.3 \\
\hline $3995 \mathrm{~S}$ & 4 & $\mathrm{~S}$ & $\mathrm{D}$ & 1 & 1200 & 0.75 & 0.42 & 0.2 & 40 & 95 & 7 & $S$ & $\mathrm{D}$ & & 1200 & 0.89 & 0.51 & 0.3 \\
\hline $3995 \stackrel{\tilde{S}}{\sim}$ & 4 & $\mathrm{~S}$ & D & 2 & 1200 & 0.76 & 0.41 & 0.2 & 40 & 95 & 7 & $S$ & D & & 1200 & 0.88 & 0.48 & 0.3 \\
\hline $39 \underset{95}{\mathrm{~S}}$ & 4 & $\mathrm{~S}$ & D & 1 & 1650 & 0.80 & 0.40 & 0.2 & 40 & 95 & 7 & $S$ & $\mathrm{D}$ & & 1650 & 0.91 & 0.51 & 0.3 \\
\hline $3995 \mathrm{~S}$ & 4 & $\mathrm{~S}$ & D & 2 & 1650 & 0.84 & 0.39 & 0.2 & 40 & $95 \mathrm{~S}$ & 7 & $\mathrm{~S}$ & $\mathrm{D}$ & & 1650 & 0.89 & 0.50 & 0.3 \\
\hline
\end{tabular}









\begin{tabular}{|c|c|c|c|c|c|c|c|c|c|c|c|c|c|c|c|c|}
\hline No & Mix L & abel & $\mathrm{R}$ & $\#$ & MPD & $\mathrm{DF}_{20}$ & F6 & No & & Mix & & $\mathrm{R}$ & $\#$ & MPD & $\mathrm{DF}_{20}$ & F6 \\
\hline \begin{tabular}{|l|l|l|}
$455 \mathrm{~S}$ \\
\end{tabular} & 1 & C S & $\begin{array}{ll} & 1\end{array}$ & 0000 & 1.18 & 0.84 & 0.4 & 46 & $95 \mathrm{~S}$ & 1 & $\mathrm{CH}$ & 1 & 0000 & 0.98 & 0.74 & 0.4 \\
\hline $4595 \overline{\mathrm{S}}$ & 1 & $\mathrm{C} \overline{\mathrm{S}}$ & 2 & 0000 & 1.22 & 0.72 & 0.4 & 46 & $95 \bar{S}$ & 1 & $\mathrm{C} \overline{\mathrm{H}}$ & 2 & 0000 & 0.97 & 0.70 & 0.4 \\
\hline $4595 \tilde{S}$ & $\hat{1}$ & $\mathrm{C} \mathrm{S}$ & 1 & 0015 & 1.34 & 0.73 & 0.4 & 46 & $95 \tilde{S}$ & $\hat{1}$ & $\mathrm{C} \mathrm{H}$ & 1 & 0015 & 0.96 & 0.69 & 0.4 \\
\hline $4595 \tilde{S}$ & $\hat{1}$ & $\mathrm{C} \dot{\mathrm{S}}$ & 2 & 0015 & 1.46 & 0.69 & $\overline{0} .4$ & 46 & $95 \tilde{S}$ & $\hat{1}$ & $\mathrm{C} \mathrm{H}$ & 2 & 0015 & 0.96 & 0.66 & 0.4 \\
\hline $4595 \tilde{S}$ & $\hat{1}$ & $\mathrm{C} \quad \dot{S}$ & 1 & 0036 & 1.38 & 0.65 & $\overline{0} .4$ & 46 & $95 \tilde{S}$ & $\hat{1}$ & $\mathrm{C} \mathrm{H}$ & 1 & 0036 & 1.02 & 0.62 & 0.3 \\
\hline $4595 \tilde{S}$ & $\hat{1}$ & $\mathrm{C} \quad \dot{S}$ & 2 & 0036 & 1.40 & 0.62 & 0.4 & 46 & $95 \tilde{S}$ & $\hat{1}$ & $\mathrm{C} \mathrm{H}$ & 2 & 0036 & 0.98 & 0.60 & 0.3 \\
\hline $4595 \tilde{S}$ & $\hat{1}$ & $\mathrm{C} \dot{\mathrm{S}}$ & 1 & 0090 & 1.45 & 0.60 & 0.4 & 46 & $95 \tilde{S}$ & $\hat{1}$ & $\mathrm{C} \mathrm{H}$ & 1 & 0090 & 0.97 & 0.60 & 0.3 \\
\hline $4595 \tilde{S}$ & $\hat{1}$ & $\mathrm{C} \quad \dot{\mathrm{S}}$ & 2 & 0090 & 1.52 & 0.57 & 0.4 & 46 & $95 \tilde{S}$ & $\hat{1}$ & $\mathrm{C} \mathrm{H}$ & 2 & 0090 & 0.99 & 0.57 & $\overline{0} .3$ \\
\hline $4595 \tilde{S}$ & $\hat{1}$ & $\mathrm{C} \dot{\mathrm{S}}$ & 1 & 0180 & 1.46 & 0.53 & 0.3 & 46 & $95 \tilde{S}$ & $\hat{1}$ & $\mathrm{C} \mathrm{H}$ & 1 & 0180 & 1.02 & 0.53 & $\overline{0} .3$ \\
\hline $4595 \tilde{\mathrm{S}}$ & $\hat{1}$ & $\mathrm{C} \mathrm{S}$ & 2 & 0180 & 1.45 & 0.49 & 0.3 & 46 & $95 \tilde{S}$ & $\hat{1}$ & $\mathrm{C} \mathrm{H}$ & 2 & 0180 & 1.05 & 0.51 & 0.3 \\
\hline $4595 \tilde{S}$ & $\hat{1}$ & $\mathrm{C} S$ & 1 & 0300 & 1.45 & 0.47 & 0.3 & 46 & $95 \tilde{S}$ & $\hat{1}$ & $\mathrm{C} \mathrm{H}$ & 1 & 0300 & 0.97 & 0.47 & 0.3 \\
\hline $4595 \tilde{S}$ & $\hat{1}$ & $\mathrm{C} \dot{\mathrm{S}}$ & 2 & 0300 & 1.48 & 0.45 & 0.3 & 46 & $95 \tilde{S}$ & $\hat{1}$ & $\mathrm{C} \mathrm{H}$ & 2 & 0300 & 0.97 & 0.45 & 0.3 \\
\hline $4595 \tilde{S}$ & $\hat{1}$ & $\mathrm{C} \dot{\mathrm{S}}$ & 1 & 0450 & 1.42 & 0.40 & 0.3 & 46 & $95 \tilde{S}$ & $\hat{1}$ & $\mathrm{C} \mathrm{H}$ & 1 & 0450 & 0.96 & 0.44 & 0.2 \\
\hline $4595 \tilde{S}$ & $\hat{1}$ & $\mathrm{C} \quad \dot{S}$ & 2 & 0450 & 1.43 & 0.39 & 0.3 & 46 & $95 \tilde{S}$ & $\hat{1}$ & $\mathrm{C} \mathrm{H}$ & 2 & 0450 & 0.98 & 0.42 & 0.2 \\
\hline $4595 \tilde{\mathrm{S}}$ & $\hat{1}$ & $\mathrm{CS}$ & 1 & 0750 & 1.45 & 0.35 & 0.2 & 46 & $95 \tilde{\mathrm{S}}$ & $\hat{1}$ & $\mathrm{C} \mathrm{H}$ & 1 & 0750 & 0.98 & 0.40 & 0.2 \\
\hline $4595 \tilde{\mathrm{S}}$ & $\hat{1}$ & $\mathrm{C} \dot{\mathrm{S}}$ & 2 & 0750 & 1.52 & 0.34 & $\overline{0} .2$ & 46 & $95 \tilde{S}$ & $\hat{1}$ & $\mathrm{C} \mathrm{H}$ & 2 & 0750 & 0.99 & 0.38 & $\overline{0} .2$ \\
\hline $4595 \tilde{S}$ & 1 & $\mathrm{C} \dot{\mathrm{S}}$ & 1 & 1200 & 1.47 & 0.31 & $\overline{0} .2$ & 46 & $95 \tilde{\mathrm{S}}$ & $\hat{1}$ & $\mathrm{C} \mathrm{H}$ & 1 & 1200 & 0.98 & 0.33 & $\overline{0} .2$ \\
\hline $4595 \tilde{S}$ & $\hat{1}$ & $\mathrm{C} \mathrm{S}$ & 2 & 1200 & 1.47 & 0.29 & $\overline{0} .2$ & 46 & $95 \tilde{\mathrm{S}}$ & $\hat{1}$ & $\mathrm{C} \mathrm{H}$ & 2 & 1200 & 1.09 & 0.34 & $\overline{0} .2$ \\
\hline $4595 \tilde{S}$ & $\hat{1}$ & $\mathrm{CS}$ & 1 & 1650 & 1.56 & 0.30 & 0.2 & 46 & $96 \tilde{\mathrm{S}}$ & $\hat{1}$ & $\mathrm{C} \mathrm{H}$ & 1 & 1650 & 0.98 & 0.35 & $\overline{0} .2$ \\
\hline $4595 \tilde{S}$ & $\hat{1}$ & $\mathrm{CS}$ & 2 & 1650 & 1.56 & 0.30 & 0.2 & 46 & $96 \tilde{\mathrm{S}}$ & $\hat{1}$ & $\mathrm{C} \mathrm{H}$ & 2 & 1650 & 0.99 & 0.33 & 0.2 \\
\hline $47 \mathrm{PFC}$ & & 3 & 1 & 0000 & 2.43 & 0.58 & 0.4 & 48 & PFC & 64 & & 1 & 0000 & 2.22 & 0.88 & 0.5 \\
\hline $47 \mathrm{PFC}$ & & 3 & 2 & 0000 & 2.43 & 0.57 & 0.4 & 48 & PFC & 64 & & 2 & 0000 & 2.24 & 0.58 & 0.5 \\
\hline $47 \mathrm{PFC}$ & & 3 & 1 & 0015 & 2.41 & 0.63 & 0.4 & 48 & PFC & 64 & & 1 & 0015 & 2.25 & 0.94 & 0.5 \\
\hline $47 \mathrm{PFC}$ & & 3 & 2 & 0015 & 2.39 & 0.62 & 0.4 & 48 & PFC & 64 & & 2 & 0015 & 2.23 & 0.65 & $\overline{0} .5$ \\
\hline $47 \mathrm{PFC}$ & & 3 & 1 & 0036 & 2.54 & 0.63 & 0.4 & 48 & PFC & 64 & & 1 & 0036 & 2.18 & 0.92 & 0.5 \\
\hline $47 \mathrm{PFC}$ & & 3 & 2 & 0036 & 2.54 & 0.61 & 0.4 & 48 & PFC & 64 & & 2 & 0036 & 2.14 & 0.73 & 0.5 \\
\hline 47 PFC & & 3 & 1 & 0090 & 2.43 & 0.56 & 0.4 & 48 & PFC & 64 & & 1 & 0090 & 2.19 & 0.61 & 0.4 \\
\hline $47 \mathrm{PFC}$ & & 3 & 2 & 0090 & 2.56 & 0.59 & 0.4 & 48 & PFC & 64 & & 2 & 0090 & 2.17 & 0.62 & 0.4 \\
\hline $47 \mathrm{PFC}$ & & 3 & 1 & 0180 & 2.57 & 0.54 & 0.4 & 48 & PFC & 64 & & 1 & 0180 & 2.24 & 0.61 & 0.4 \\
\hline $47 \mathrm{PFC}$ & & 3 & 2 & 0180 & 2.56 & 0.58 & 0.4 & 48 & PFC & 64 & & 2 & 0180 & 2.23 & 0.64 & 0.4 \\
\hline 47 PFC & & 3 & 1 & 0300 & 2.55 & 0.52 & 0.4 & 48 & PFC & 64 & & 1 & 0300 & 2.33 & 0.61 & 0.4 \\
\hline 47 PFC & & 3 & 2 & 0300 & 2.47 & 0.59 & 0.4 & 48 & PFC & 64 & & 2 & 0300 & 2.35 & 0.59 & 0.4 \\
\hline $47 \mathrm{PFC}$ & & 3 & 1 & 0450 & 2.49 & 0.51 & 0.4 & 48 & PFC & 64 & & 1 & 0450 & 2.36 & 0.52 & 0.4 \\
\hline $47 \mathrm{PFC}$ & & 3 & 2 & 0450 & 2.54 & 0.57 & 0.4 & 48 & PFC & 64 & & 2 & 0450 & 2.30 & 0.52 & 0.4 \\
\hline $47 \mathrm{PFC}$ & & 3 & 1 & 0750 & 2.56 & 0.50 & 0.4 & 48 & PFC & 64 & & 1 & 0750 & 2.31 & 0.53 & 0.4 \\
\hline $47 \mathrm{PFC}$ & & 3 & 2 & 0750 & 2.61 & 0.57 & 0.4 & 48 & PFC & 64 & & 2 & 0750 & 2.27 & 0.53 & 0.4 \\
\hline $47 \mathrm{PFC}$ & & 3 & 1 & 1200 & 2.59 & 0.57 & 0.4 & 48 & PFC & 64 & & 1 & 1200 & 2.28 & 0.55 & 0.4 \\
\hline $47 \mathrm{PFC}$ & & 3 & 2 & 1200 & 2.57 & 0.57 & 0.4 & 48 & PFC & 64 & & 2 & 1200 & 2.29 & 0.48 & 0.4 \\
\hline $47 \mathrm{PFC}$ & & 3 & 1 & 1650 & 2.61 & 0.49 & 0.4 & 48 & PFC & 64 & & 1 & 1650 & 2.24 & 0.48 & 0.3 \\
\hline 47 PFC & & 3 & 2 & 1650 & 2.70 & 0.54 & 0.4 & 48 & PFC & 64 & & 2 & 1650 & 2.20 & 0.49 & 0.3 \\
\hline
\end{tabular}




\begin{tabular}{|c|c|c|c|c|c|c|c|c|c|c|c|c|c|c|}
\hline No & \multicolumn{2}{|c|}{ Mix Label } & \multirow{2}{*}{$\begin{array}{l}\mathrm{R} \\
1\end{array}$} & \multirow{2}{*}{$\begin{array}{l}\# \\
0000\end{array}$} & \multicolumn{2}{|c|}{$\mathrm{MPD} \mathrm{DF}_{20}$} & \multirow{2}{*}{$\begin{array}{c}\text { F6 } \\
0.3\end{array}$} & \multirow{2}{*}{\begin{tabular}{|l|} 
No \\
50
\end{tabular}} & Mix Label & \multirow[t]{2}{*}{$\mathrm{R}$} & & \multirow{2}{*}{$\begin{array}{l}\text { MPD } \\
1.86\end{array}$} & \multirow{2}{*}{$\begin{array}{l}\mathrm{DF}_{20} \\
0.71\end{array}$} & \multirow{2}{*}{$\begin{array}{c}\text { F6 } \\
0.4\end{array}$} \\
\hline 495 & SMA & 3 & & & 1.79 & 0.40 & & & SMA 64 & & & & & \\
\hline $49:$ & SMA & 3 & 2 & 0000 & 1.80 & 0.39 & 0.3 & 50 & SMA 64 & 2 & 0000 & 1.89 & 0.67 & 0.4 \\
\hline $49:$ & SMA & 3 & 1 & 0015 & 1.65 & 0.40 & 0.3 & 50 & SMA 64 & 1 & 0015 & 1.80 & 0.72 & 0.5 \\
\hline 49 & SMA & $\hat{3}$ & 2 & 0015 & 1.82 & 0.41 & 0.3 & 50 & SMA 64 & 2 & 0015 & 1.80 & 0.70 & 0.5 \\
\hline 49 s & SMA & 3 & 1 & 0036 & 1.83 & 0.54 & 0.3 & 50 & SMA 64 & 1 & 0036 & 1.84 & 0.76 & 0.5 \\
\hline $49:$ & SMA & 3 & 2 & 0036 & 1.82 & 0.53 & 0.3 & 50 & SMA 64 & 2 & 0036 & 1.76 & 0.74 & 0.5 \\
\hline $49:$ & SMA & 3 & 1 & 0090 & 1.86 & 0.66 & 0.4 & 50 & SMA 64 & 1 & 0090 & 1.83 & 0.76 & 0.5 \\
\hline $49:$ & SMA & 3 & 2 & 0090 & 1.83 & 0.65 & 0.4 & 50 & SMA 64 & 2 & 0090 & 1.78 & 0.75 & 0.5 \\
\hline 49 & SMA & $\hat{3}$ & 1 & 0180 & 1.84 & 0.67 & 0.4 & 50 & SMA 64 & 1 & 0180 & 1.85 & 0.81 & 0.5 \\
\hline $49:$ & SMA & 3 & 2 & 0180 & 1.84 & 0.67 & 0.4 & 50 & SMA 64 & 2 & 0180 & 1.78 & 0.78 & 0.5 \\
\hline & SMA & 3 & 1 & 0300 & 1.81 & 0.64 & 0.4 & 50 & SMA 64 & 1 & 0300 & 1.72 & 0.80 & 0.5 \\
\hline $49:$ & SMA & 3 & 2 & 0300 & 1.86 & 0.64 & 0.4 & 50 & SMA 64 & 2 & 0300 & 1.71 & 0.78 & 0.5 \\
\hline $49:$ & SMA & 3 & 1 & 0450 & 1.79 & 0.63 & 0.4 & 50 & SMA 64 & 1 & 0450 & 1.65 & 0.76 & 0.5 \\
\hline & SMA & 3 & 2 & 0450 & 1.78 & 0.64 & 0.4 & 50 & SMA 64 & 2 & 0450 & 1.70 & 0.75 & 0.5 \\
\hline & SMA & 3 & 1 & 0750 & 1.82 & 0.63 & 0.4 & 50 & SMA 64 & 1 & 0750 & 1.70 & 0.72 & 0.5 \\
\hline & SMA & 3 & 2 & 0750 & 1.80 & 0.62 & 0.4 & 50 & SMA 64 & 2 & 0750 & 1.69 & 0.72 & 0.5 \\
\hline $49:$ & SMA & 3 & 1 & 1200 & 1.78 & 0.64 & 0.4 & 50 & SMA 64 & 1 & 1200 & 1.81 & 0.70 & 0.4 \\
\hline $49:$ & SMA & 3 & 2 & 1200 & 1.81 & 0.62 & 0.4 & 50 & SMA 64 & 2 & 1200 & 1.79 & 0.70 & 0.4 \\
\hline $49:$ & SMA & 3 & 1 & 1650 & 1.85 & 0.64 & 0.4 & 50 & SMA 64 & 1 & 1650 & 1.72 & 0.70 & 0.4 \\
\hline $49 \mathrm{~s}$ & SMA & 3 & 2 & 1650 & 1.83 & 0.63 & 0.4 & 50 & SMA 64 & 2 & 1650 & 1.70 & 0.69 & 0.4 \\
\hline
\end{tabular}




\section{Appendix E: Summary of the Laboratory Polishing Data and Model Parameters}

This appendix contains a summary of the laboratory polishing data, air content values and model parameters. The summary of the laboratory polishing data and air content is shown in Table E 1 in the following order: sample number (No.), mix label, mean profile depth (MPD) data (minimum, maximum and range of the values per specific specimen), dynamic friction $\left(\mathrm{DF}_{20}\right)$ data (minimum, maximum and range of the values per specific specimen), calibrated wet friction (F60) data (minimum, maximum and range of the values per specific specimen) and air content information $\left(\mathrm{V}_{\mathrm{a}}\right)$ (for the "corner" core and for the "side" core). The mix label consists of the mixture nominal maximum aggregate size (NMAS, mm), friction aggregate type (FAT), friction aggregate content (FAC), aggregate gradation type $(\mathrm{G})$, and common aggregate type (CAT).

The summary of the model parameters is shown in Table E 2 in the following order: sample number (No.), mix label, parameters of the model $\left(\mathrm{x}_{0}, \mathrm{x}_{1}, \mathrm{a}_{0}, \mathrm{a}_{1}, \mathrm{a}_{2}, \mathrm{a}_{3}, \mathrm{a}_{4}, \mathrm{a}_{5}\right)$, statistical information about the model $\left(\mathrm{R}^{2}, \mathrm{SSE}\right.$ and SST) and F60 values at the wheel passes corresponding to $\mathrm{x}_{\mathrm{o}}$ and to $\mathrm{x}_{1}$ levels.

Samples of the plant produced mixes tested in the lab (PFC and SMA) are identified by the mix type and sample height (reported in $\mathrm{mm}$ ). 
Table E 1. Summary of the laboratory polishing data and air content information

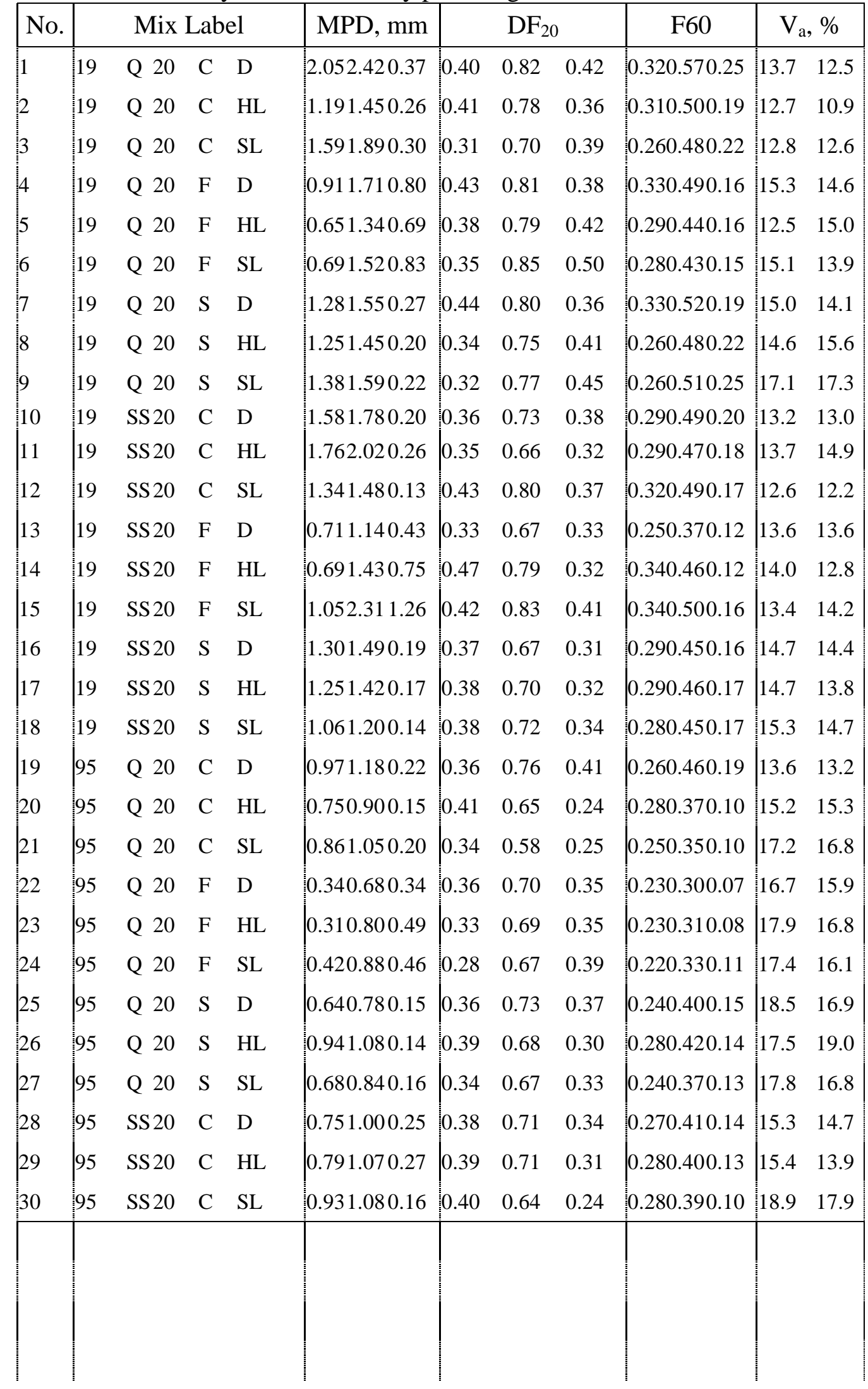




\begin{tabular}{|c|c|c|c|c|c|c|c|c|c|c|c|}
\hline No. & & Mix & & & MPD, mm & & $\mathrm{DF}_{20}$ & & F60 & & \\
\hline 31 & 95 & SS 20 & $\mathrm{~F}$ & $\mathrm{D}$ & 0.390 .750 .36 & 0.40 & 0.75 & 0.36 & 0.260 .320 .06 & 17.9 & 17.1 \\
\hline 32 & 95 & SS 20 & $\mathrm{~F}$ & HL & 0.330 .540 .21 & 0.34 & 0.61 & 0.28 & 0.210 .260 .05 & 13.9 & 12.0 \\
\hline 33 & 95 & SS 20 & $\mathrm{~F}$ & SL & 0.350 .830 .48 & 0.40 & 0.75 & 0.35 & 0.230 .340 .11 & 16.2 & 16.1 \\
\hline 34 & 95 & SS 20 & S & $\mathrm{D}$ & 0.630 .810 .18 & 0.38 & 0.70 & 0.33 & 0.250 .370 .12 & 17.6 & 16.5 \\
\hline 35 & 95 & SS 20 & S & HL & 0.630 .740 .11 & 0.36 & 0.58 & 0.22 & 0.240 .320 .08 & 17.2 & 16.9 \\
\hline 36 & 95 & SS 20 & S & SL & 0.650 .850 .19 & 0.37 & 0.65 & 0.29 & 0.250 .360 .11 & 16.3 & 16.6 \\
\hline 37 & 95 & SS 0 & $S$ & $\mathrm{D}$ & 0.891 .140 .25 & 0.32 & 0.66 & 0.34 & 0.250 .420 .17 & 16.6 & 17.5 \\
\hline 38 & 95 & SS 10 & $\mathrm{~S}$ & $\mathrm{D}$ & 0.911 .030 .13 & 0.35 & 0.65 & 0.30 & 0.250 .400 .15 & 16.5 & 17.2 \\
\hline 39 & 95 & SS 40 & $\mathrm{~S}$ & $\mathrm{D}$ & 0.680 .840 .16 & 0.40 & 0.68 & 0.28 & 0.260 .390 .12 & 16.5 & 18.3 \\
\hline 40 & 95 & SS 70 & S & $\mathrm{D}$ & 0.780 .970 .19 & 0.49 & 0.70 & 0.21 & 0.320 .420 .11 & 14.1 & 13.1 \\
\hline 41 & 95 & Q 10 & S & $\mathrm{D}$ & 0.700 .870 .17 & 0.36 & 0.67 & 0.31 & 0.250 .380 .13 & 19.2 & 19.4 \\
\hline 42 & 95 & Q 40 & S & $\mathrm{D}$ & 0.931 .220 .30 & 0.44 & 0.70 & 0.26 & 0.310 .430 .12 & 17.7 & 17.6 \\
\hline 43 & 95 & Q 70 & S & $\mathrm{D}$ & 0.951 .200 .26 & 0.51 & 0.72 & 0.21 & 50.10 & 16.5 & 16.4 \\
\hline 44 & 95 & SS 10 & $\mathrm{C}$ & $\mathrm{D}$ & 0.921 .070 .15 & 0.33 & 0.64 & 0.31 & 0.240 .400 .16 & 19.4 & 18.3 \\
\hline 45 & 95 & SS 10 & $\mathrm{C}$ & SL & 1.181 .560 .38 & 0.30 & 0.78 & 0.48 & 90.25 & 17.2 & 20.2 \\
\hline 46 & 95 & SS 10 & $\mathrm{C}$ & HL & 0.961 .090 .14 & 0.33 & 0.72 & 0.39 & 0.250 .430 .19 & 15.3 & 15.2 \\
\hline 47 & PFC & 3 & & & 2.402 .650 .25 & 0.52 & 0.62 & 0.11 & 0.400 .470 .06 & 23.8 & 24.6 \\
\hline 48 & PFC & 6 & & & 2.162 .340 .18 & 0.49 & 0.82 & 0.34 & 0.380 .580 .20 & 21.9 & 23.3 \\
\hline 49 & SMA & 3 & & & 1.731 .850 .11 & 0.39 & 0.67 & 0.27 & 0.310 .470 .16 & 15.0 & 15.1 \\
\hline 50 & SMA & 6 & & & 1.681 .870 .19 & 0.69 & 0.80 & 0.11 & 0.480 .550 .07 & 12.6 & 12.7 \\
\hline
\end{tabular}


Table E 2. Summary of the model parameters

\begin{tabular}{|c|c|c|c|c|c|c|c|c|c|c|c|c|c|c|c|}
\hline \begin{tabular}{|l|}
$\mathrm{No}$ \\
\end{tabular} & \multicolumn{4}{|c|}{ Mix Label } & & \multirow{2}{*}{$\begin{array}{l}\mathrm{x}_{1} \quad \mathrm{a}_{0} \\
1650.52\end{array}$} & \multirow{2}{*}{$\frac{\mathrm{a}_{1}}{0.20}$} & \multirow{2}{*}{$\frac{a_{2}}{-0.12}$} & \multirow{2}{*}{$\frac{a_{3}}{-0.02}$} & \multirow{2}{*}{$\begin{array}{l}\mathrm{a}_{4} \\
-0.05\end{array}$} & \multirow{2}{*}{$\frac{\mathrm{a}_{5}}{0.59}$} & \multirow{2}{*}{$\frac{\mathbf{R}^{2} \text { SSE }}{0.990 .00}$} & \multirow{2}{*}{$\begin{array}{l}\text { SST } \\
0.06\end{array}$} & \multicolumn{2}{|c|}{ F60@ $\mathrm{x}_{0} \mathrm{~F} 60 @ \mathrm{x}_{1}$} \\
\hline 1 & 19 & Q & $20 \mathrm{C}$ & C D & & & & & & & & & & 0.59 & 0.33 \\
\hline 2 & 19 & Q & $20 C$ & C HL & 0.99 & 1650.43 & 0.08 & 0.13 & -0.13 & -0.04 & 0.51 & 0.970 .00 & 0.03 & 0.51 & 0.32 \\
\hline 3 & 19 & Q & 20 & C SL & 0.00 & 1910.48 & 72 & -47039 & -1179 & -0.04 & 0.50 & 0.860 .01 & 0.05 & 0.50 & 0.28 \\
\hline 4 & 19 & Q & $20 \mathrm{~F}$ & $\mathrm{~F} \quad \mathrm{D}$ & 0.00 & 1800.46 & 57 & -12262 & -678 & -0.04 & 0.52 & 0.980 .00 & 0.03 & 0.52 & 0.33 \\
\hline 5 & 19 & Q & $20 \mathrm{~F}$ & $\mathrm{~F} \quad \mathrm{HL}$ & 2.61 & 1670.40 & 0.00 & 0.04 & -0.01 & -0.03 & 0.47 & 0.960 .00 & 0.03 & 0.47 & 0.30 \\
\hline 6 & 19 & Q & $20 \mathrm{~F}$ & F SL & 0.00 & 1800.43 & 25 & -5030 & -131 & -0.03 & 0.45 & 0.960 .00 & 0.03 & 0.45 & 0.28 \\
\hline 7 & 19 & Q & $20 \mathrm{~S}$ & $S \quad D$ & 2.07 & 1650.44 & 0.11 & -0.04 & 0.00 & -0.04 & 0.52 & 0.990 .00 & 0.03 & 0.52 & 0.33 \\
\hline 8 & 19 & Q & $20 \mathrm{~S}$ & $\mathrm{~S} \quad \mathrm{HL}$ & 0.39 & 1310.43 & 0.67 & -1.46 & 0.74 & -0.05 & 0.52 & 0.980 .00 & 0.05 & 0.52 & 0.26 \\
\hline 9 & 19 & Q & $20 \mathrm{~S}$ & S SL & 0.00 & 2000.45 & 53 & -7653 & -255 & -0.05 & 0.54 & 0.930 .00 & 0.05 & 0.54 & 0.28 \\
\hline 10 & 19 & SS & $20 \mathrm{C}$ & C D & 2.23 & 1650.48 & 0.00 & 0.02 & -0.01 & -0.04 & 0.50 & 0.970 .00 & 0.05 & 0.50 & 0.30 \\
\hline 11 & 19 & SS & $20 \mathrm{C}$ & C $\mathrm{HL}$ & 2.66 & 1650.43 & 0.00 & 0.04 & -0.01 & -0.04 & 0.51 & 0.770 .01 & 0.04 & 0.51 & 0.33 \\
\hline 12 & 19 & SS & $20 \mathrm{C}$ & C SL & 0.00 & 1650.49 & 19 & -15894 & -720 & -0.03 & 0.49 & 0.960 .00 & 0.03 & 0.49 & 0.33 \\
\hline 13 & 19 & SS & $20 \mathrm{~F}$ & F D & 0.00 & 1650.37 & 27 & -25815 & -358 & -0.02 & 0.37 & 0.830 .00 & 0.01 & 0.37 & 0.27 \\
\hline 14 & 19 & SS & $20 \mathrm{~F}$ & $\mathrm{~F} \quad \mathrm{HL}$ & 0.90 & 1650.41 & 0.00 & 0.26 & -0.20 & -0.02 & 0.47 & 0.940 .00 & 0.01 & 0.47 & 0.35 \\
\hline 15 & 19 & SS & $20 \mathrm{~F}$ & F SL & 2.89 & 1650.50 & 0.00 & 0.01 & 0.00 & -0.03 & 0.52 & 0.980 .00 & 0.03 & 0.52 & 0.35 \\
\hline 16 & 19 & SS & $20 \mathrm{~S}$ & $S \quad D$ & 3.42 & 1660.43 & 0.00 & 0.01 & 0.00 & -0.03 & 0.46 & 0.970 .00 & 0.03 & 0.46 & 0.29 \\
\hline 17 & 19 & SS & $20 \mathrm{~S}$ & $\mathrm{~S} \quad \mathrm{HL}$ & 0.01 & 1680.46 & 6 & -377 & 89 & -0.03 & 0.48 & 0.920 .00 & 0.03 & 0.48 & 0.30 \\
\hline 18 & 19 & SS & $20 \mathrm{~S}$ & S SL & 2.10 & 1650.37 & 0.00 & 0.07 & -0.03 & -0.04 & 0.47 & 0.970 .00 & 0.03 & 0.47 & 0.28 \\
\hline 19 & 95 & Q & $20 \mathrm{C}$ & C D & 2.05 & 1650.38 & 0.00 & 0.07 & -0.02 & -0.04 & 0.47 & 0.960 .00 & 0.03 & 0.47 & 0.28 \\
\hline 20 & 95 & Q & $20 \mathrm{C}$ & C $\mathrm{HL}$ & 6.68 & 1690.32 & 0.00 & 0.01 & 0.00 & -0.03 & 0.41 & 0.940 .00 & 0.01 & 0.41 & 0.28 \\
\hline 21 & 95 & Q & $20 \mathrm{C}$ & C SL & 5.74 & 1650.33 & 0.01 & 0.00 & 0.00 & -0.02 & 0.36 & 0.750 .00 & 0.01 & 0.36 & 0.27 \\
\hline 22 & 95 & Q & $20 \mathrm{~F}$ & $\mathrm{~F} \quad \mathrm{D}$ & 2.37 & 1650.28 & 0.00 & 0.02 & -0.01 & -0.01 & 0.32 & 0.760 .00 & 0.00 & 0.32 & 0.25 \\
\hline 23 & 95 & Q & $20 \mathrm{~F}$ & F HL & 2.46 & 1650.26 & 0.00 & 0.03 & -0.01 & -0.01 & 0.32 & 0.500 .00 & 0.01 & 0.32 & 0.26 \\
\hline 24 & 95 & Q & $20 \mathrm{~F}$ & F SL & 2.75 & 1650.30 & 0.00 & 0.03 & -0.01 & -0.02 & 0.37 & 0.800 .00 & 0.01 & 0.37 & 0.24 \\
\hline 25 & 95 & Q & $20 \mathrm{~S}$ & $S \quad D$ & 2.12 & 1650.35 & 0.00 & 0.04 & -0.02 & -0.03 & 0.41 & 0.960 .00 & 0.02 & 0.41 & 0.25 \\
\hline 26 & 95 & Q & $20 \mathrm{~S}$ & S HL & 5.80 & 1630.40 & 0.00 & 0.00 & 0.00 & -0.03 & 0.43 & 0.950 .00 & 0.03 & 0.43 & 0.28 \\
\hline 27 & 95 & Q & $20 \mathrm{~S}$ & S SL & 3.51 & 1500.35 & 0.00 & 0.01 & 0.00 & -0.02 & 0.38 & 0.880 .00 & 0.02 & 0.38 & 0.26 \\
\hline 28 & 95 & SS & $20 \mathrm{C}$ & C D & 6.77 & 1650.39 & 0.00 & 0.00 & 0.00 & -0.03 & 0.43 & 0.970 .00 & 0.02 & 0.43 & 0.28 \\
\hline 29 & 95 & SS & $20 \mathrm{C}$ & C $\mathrm{HL}$ & 5.57 & 1290.37 & 0.00 & 0.00 & 0.00 & -0.03 & 0.41 & 0.980 .00 & 0.02 & 0.41 & 0.28 \\
\hline 30 & 95 & SS & $20 \mathrm{C}$ & C SL & 7.26 & 1650.35 & 0.00 & 0.00 & 0.00 & -0.02 & 0.41 & 0.800 .00 & 0.01 & 0.41 & 0.29 \\
\hline 31 & 95 & SS & $20 \mathrm{~F}$ & $\mathrm{~F} \quad \mathrm{D}$ & 2.41 & 1650.31 & 0.00 & 0.01 & 0.00 & -0.01 & 0.33 & 0.800 .00 & 0.00 & 0.33 & 0.27 \\
\hline 32 & 95 & SS & $20 \mathrm{~F}$ & $\mathrm{~F} \quad \mathrm{HL}$ & 3.17 & 1500.21 & 0.00 & 0.02 & 0.00 & -0.01 & 0.27 & 0.800 .00 & 0.00 & 0.27 & 0.22 \\
\hline
\end{tabular}




\begin{tabular}{|c|c|c|c|c|c|c|c|c|c|c|c|c|c|c|c|}
\hline $\mathrm{No}$ & & $\mathrm{x} \mathrm{Lab}$ & bel & & $\mathrm{x}_{0}$ & $\begin{array}{ll}\mathrm{x}_{1} & \mathrm{a}_{0}\end{array}$ & $a_{1}$ & $\mathrm{a}_{2}$ & $a_{3}$ & $\mathrm{a}_{4}$ & $a_{5}$ & $\mathrm{R}^{2} \quad \mathrm{SSE}$ & SST & F60@ $\mathrm{x}_{0}$ & F60@ $x_{1}$ \\
\hline 3395 & $5 \mathrm{SS}$ & 20 & $\mathrm{~F}$ & SL & 2.23 & 1650.23 & 0.00 & 0.09 & -0.03 & -0.02 & 0.37 & 0.900 .00 & 0.01 & 0.37 & 0.28 \\
\hline 3495 & $5 \mathrm{SS}$ & 20 & $S$ & D & 6.30 & 1650.34 & 0.00 & 0.00 & 0.00 & -0.03 & 0.38 & 0.980 .00 & 0.01 & 0.38 & 0.26 \\
\hline 3595 & $5 \mathrm{SS}$ & 20 & $S$ & HL & 6.56 & 1090.29 & 0.00 & 0.00 & 0.00 & -0.02 & 0.34 & 0.960 .00 & 0.01 & 0.34 & 0.24 \\
\hline 3695 & $5 \mathrm{SS}$ & 20 & $S$ & SL & 5.99 & 1200.32 & 0.00 & 0.00 & 0.00 & -0.02 & 0.37 & 0.990 .00 & 0.01 & 0.37 & 0.25 \\
\hline 3795 & $5 \mathrm{SS}$ & 0 & $S$ & D & 0.00 & 1460.42 & 31 & -6991 & 6720 & -0.04 & 0.45 & 0.990 .00 & 0.04 & 0.45 & 0.25 \\
\hline 3895 & $5 \mathrm{SS}$ & 10 & $S$ & $\mathrm{D}$ & 0.01 & $\begin{array}{ll}96 & 0.40\end{array}$ & 9 & -1008 & 745 & -0.04 & 0.42 & 1.000 .00 & 0.03 & 0.42 & 0.25 \\
\hline 3995 & $5 \mathrm{SS}$ & 40 & $S$ & D & 1.73 & 1520.39 & 0.11 & -0.14 & 0.04 & -0.02 & 0.37 & 1.000 .00 & 0.02 & 0.37 & 0.26 \\
\hline 4095 & $5 \mathrm{SS}$ & 70 & $S$ & D & 0.91 & 1500.42 & 0.16 & -0.49 & 0.29 & -0.01 & 0.38 & 0.970 .00 & 0.01 & 0.38 & 0.31 \\
\hline 4195 & $5 \mathrm{Q}$ & 10 & $S$ & D & 0.00 & 1660.38 & 45 & -16025 & -1002 & -0.03 & 0.41 & 0.970 .00 & 0.02 & 0.41 & 0.25 \\
\hline 4295 & $5 \mathrm{Q}$ & 40 & $\mathrm{~S}$ & $\mathrm{D}$ & 0.24 & 1650.43 & 0.24 & -0.83 & 0.38 & -0.02 & 0.44 & 0.970 .00 & 0.02 & 0.44 & 0.31 \\
\hline 4395 & $5 \mathrm{Q}$ & 70 & $S$ & D & 6.63 & 1200.44 & 0.00 & 0.00 & 0.00 & -0.01 & 0.39 & 0.970 .00 & 0.01 & 0.39 & 0.36 \\
\hline 4495 & $5 \mathrm{SS}$ & 10 & $\mathrm{C}$ & D & 0.00 & 1280.40 & 37 & -9023 & 9884 & -0.04 & 0.43 & 0.990 .00 & 0.03 & 0.43 & 0.25 \\
\hline 4595 & $5 \mathrm{SS}$ & 10 & $\mathrm{C}$ & SL & 0.00 & 1250.49 & 49 & -14178 & 58362 & -0.06 & 0.53 & 0.990 .00 & 0.07 & 0.53 & 0.25 \\
\hline 4695 & $5 \mathrm{SS}$ & 10 & $\mathrm{C}$ & HL & 0.00 & 1370.43 & 43 & -17144 & 240 & -0.04 & 0.45 & 0.990 .00 & 0.04 & 0.45 & 0.25 \\
\hline 47 & $\mathrm{PFC}$ & 38 & & & 6.97 & 1670.43 & 0.03 & -0.01 & 0.00 & -0.01 & 0.44 & 0.860 .00 & 0.00 & 0.41 & 0.41 \\
\hline 48 & PFC & 64 & 4 & & 7.46 & 1650.52 & 0.06 & -0.02 & 0.00 & -0.02 & 0.49 & 0.960 .05 & 0.00 & 0.39 & 0.39 \\
\hline 49 & SMA & 38 & 8 & & 7.12 & $\begin{array}{ll}75 & 0.30\end{array}$ & 0.00 & 0.01 & 0.00 & -0.01 & 0.48 & 0.990 .03 & 0.00 & 0.45 & 0.45 \\
\hline 50 & SMA & 64 & & & 15.0 & 1690.49 & 0.00 & 0.00 & 0.00 & -0.02 & 0.56 & 0.790 .00 & 0.00 & 0.49 & 0.49 \\
\hline
\end{tabular}




\section{Appendix F: Summary of the Friction Predictive Model Development}

The ANOVA tables and estimates for six parameters $(\mathrm{x} 0, \mathrm{x} 1, \mathrm{a} 0-\mathrm{a} 3)$ of the proposed polishing model are presented on the following six pages.




Dependent Variable: $\mathbf{x}_{1}$

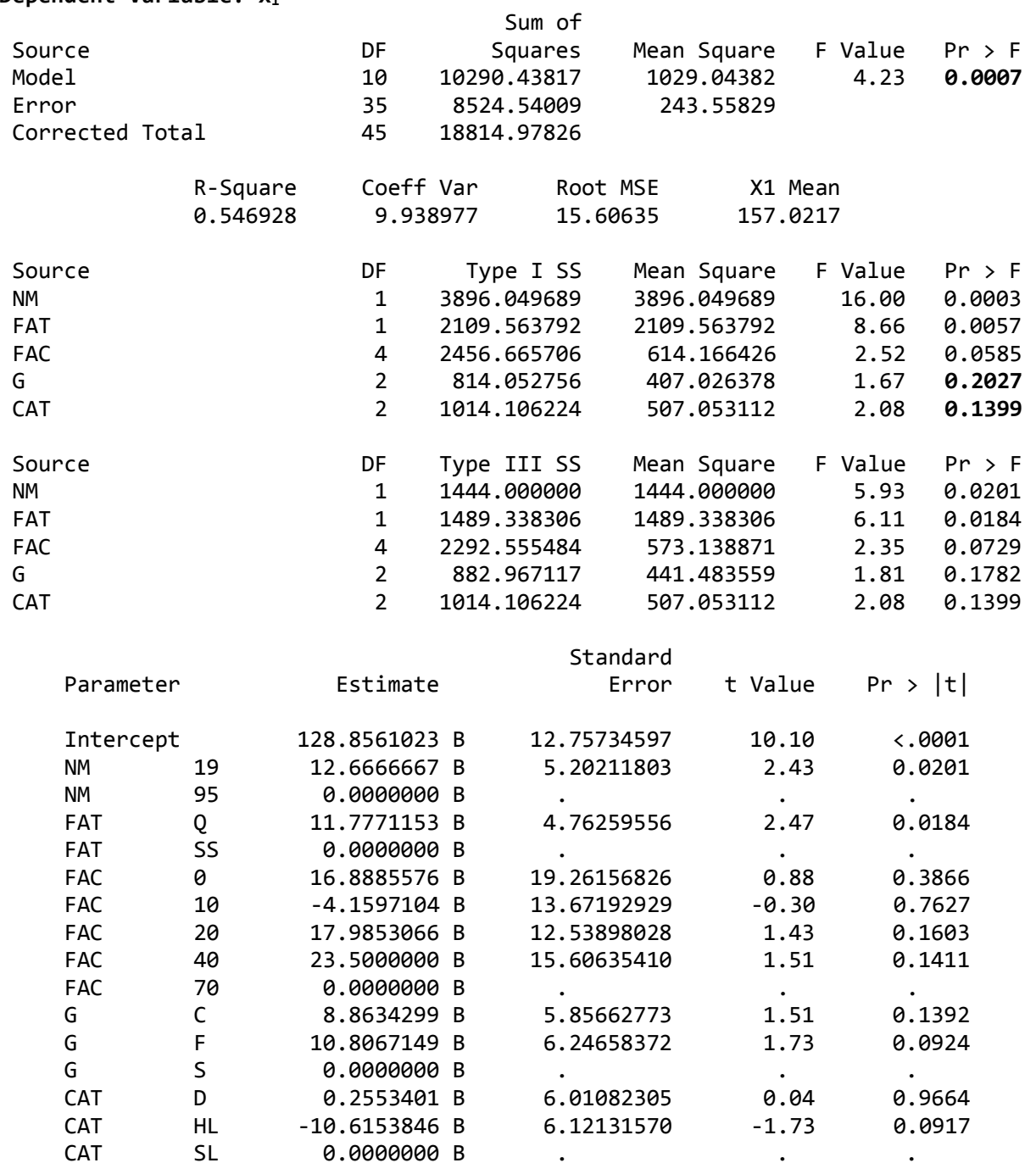


Dependent Variable: $a_{\ominus}$

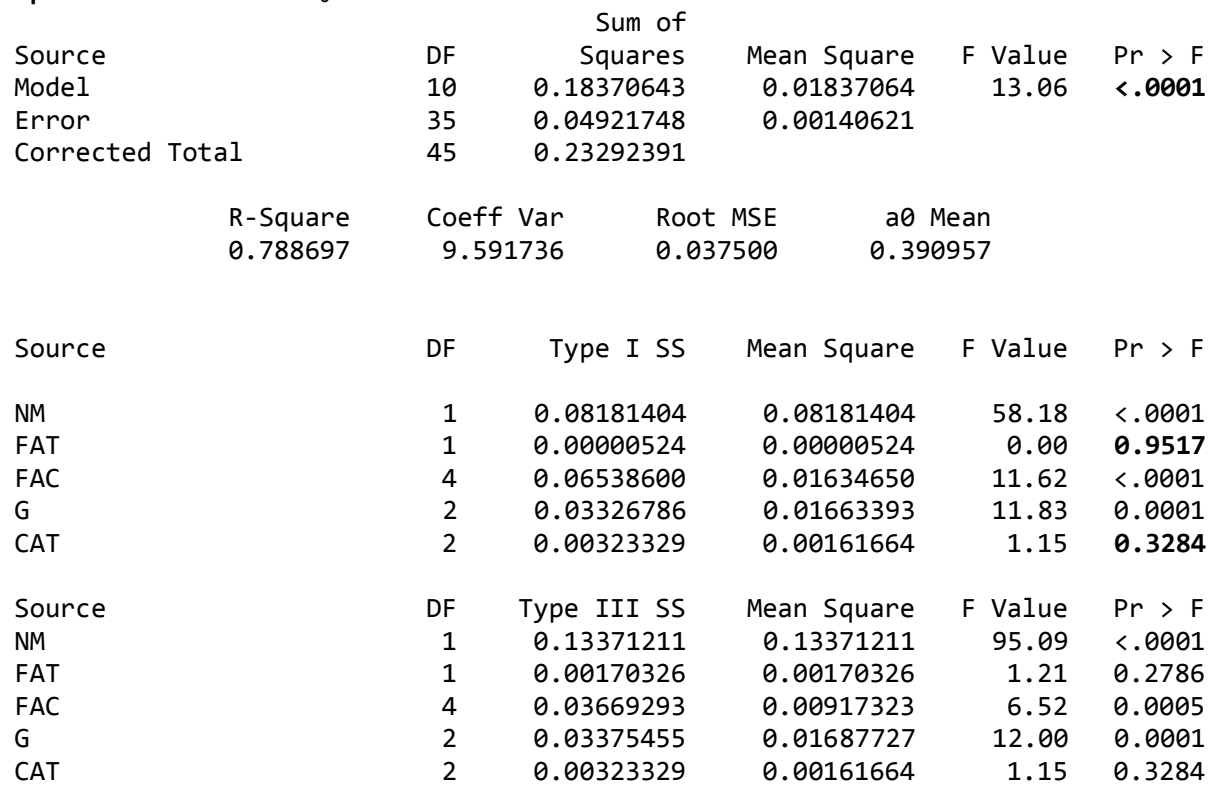

\begin{tabular}{|c|c|c|c|c|c|c|}
\hline \multicolumn{2}{|c|}{ Parameter } & \multicolumn{5}{|c|}{ Standard } \\
\hline Inte & & 0.4258576858 & B & 0.03065382 & 13.89 & $<.0001$ \\
\hline NM & 19 & 0.1218888889 & B & 0.01249984 & 9.75 & $<.0001$ \\
\hline NM & 95 & 0.0000000000 & B & . & . & . \\
\hline FAT & $Q$ & 0.0125945486 & B & 0.01144374 & 1.10 & 0.2786 \\
\hline FAT & SS & 0.0000000000 & B & . & . & . \\
\hline FAC & 0 & -.0102027257 & B & 0.04628240 & -0.22 & 0.8268 \\
\hline FAC & 10 & -.0247427598 & B & 0.03285141 & -0.75 & 0.4564 \\
\hline FAC & 20 & -.1036867783 & B & 0.03012912 & -3.44 & 0.0015 \\
\hline FAC & 40 & -.0250000000 & B & 0.03749952 & -0.67 & 0.5094 \\
\hline FAC & 70 & 0.0000000000 & B & . & . & . \\
\hline G & $C$ & 0.0350857467 & B & 0.01407252 & 2.49 & 0.0175 \\
\hline G & $\mathrm{F}$ & -.0383737933 & B & 0.01500952 & -2.56 & 0.0151 \\
\hline G & $S$ & 0.0000000000 & B & . & . & . \\
\hline CAT & $\mathrm{D}$ & 0.0013450400 & B & 0.01444303 & 0.09 & 0.9263 \\
\hline CAT & $\mathrm{HL}$ & -.0184615385 & B & 0.01470852 & -1.26 & 0.2177 \\
\hline CAT & SL & 0.0000000000 & B & . & . & . \\
\hline
\end{tabular}


Dependent Variable: $a_{1}$

Source
Model
Error
Corrected Total



Source
NM
NM
FAT
FAC
G
CAT
Source
NM
FAT
FAC
G
CAT

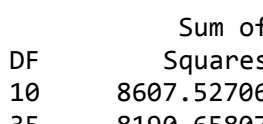

8190.65807

16798.18514

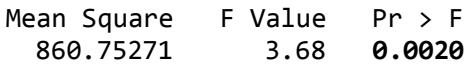

Mean Square

860.75271

F Value $\mathrm{Pr}>\mathrm{F}$

$3.68 \quad 0.0020$

234.01880

Coeff Var

Root MSE

a1 Mean

148.4835

15.29767

10.30261

$\begin{array}{rrrl}\text { Type I SS } & \text { Mean Square } & \mathrm{F} \text { Value } & \mathrm{Pr}>\mathrm{F} \\ 502.120305 & 502.120305 & 2.15 & 0.1519 \\ 89.928204 & 89.928204 & 0.38 & 0.5393 \\ 6613.319517 & 1653.329879 & 7.06 & 0.0003 \\ 277.037890 & 138.518945 & 0.59 & 0.5587 \\ 1125.121149 & 562.560575 & 2.40 & 0.1051 \\ & & & \\ \text { Type III SS } & \text { Mean Square } & \mathrm{F} \text { Value } & \mathrm{Pr}>\mathrm{F} \\ 1872.148669 & 1872.148669 & 8.00 & 0.0077 \\ 762.941530 & 762.941530 & 3.26 & 0.0796 \\ 6439.243412 & 1609.810853 & 6.88 & 0.0003 \\ 256.809910 & 128.404955 & 0.55 & 0.5826 \\ 1125.121149 & 562.560575 & 2.40 & 0.1051\end{array}$

$\begin{array}{llll}1125.121149 & 562.560575 & 2.40 & 0.1051\end{array}$

\begin{tabular}{|c|c|c|c|c|c|c|}
\hline \multicolumn{2}{|c|}{ Parameter } & \multicolumn{5}{|c|}{ Standard } \\
\hline \multicolumn{2}{|c|}{ Intercept } & 4.51159162 & B & 12.50501603 & 0.36 & 0.7204 \\
\hline NM & 19 & 14.42277778 & B & 5.09922437 & 2.83 & 0.0077 \\
\hline NM & 95 & 0.00000000 & B & . & & 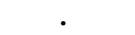 \\
\hline FAT & $Q$ & 8.42922942 & B & 4.66839529 & 1.81 & 0.0796 \\
\hline FAT & SS & 0.00000000 & B & . & . & . \\
\hline FAC & 0 & 35.19911471 & B & 18.88059008 & 1.86 & 0.0707 \\
\hline FAC & 10 & 34.95909114 & B & 13.40150964 & 2.61 & 0.0133 \\
\hline FAC & 20 & -5.08975317 & B & 12.29096943 & -0.41 & 0.6813 \\
\hline FAC & 40 & 0.09550000 & B & 15.29767310 & 0.01 & 0.9951 \\
\hline FAC & 70 & 0.00000000 & B & . & . & . \\
\hline G & C & 5.26036371 & B & 5.74078839 & 0.92 & 0.3658 \\
\hline G & $\mathrm{F}$ & 5.37609852 & B & 6.12303136 & 0.88 & 0.3859 \\
\hline G & $\mathrm{s}$ & 0.00000000 & B & & ${ }^{\circ}$ & . \\
\hline CAT & D & -8.64770633 & B & 5.89193385 & -1.47 & 0.1511 \\
\hline CAT & $\mathrm{HL}$ & -12.89661538 & B & 6.00024105 & -2.15 & 0.0386 \\
\hline CAT & SL & 0.00000000 & B & & . & \\
\hline
\end{tabular}


Dependent Variable: $a_{2}$

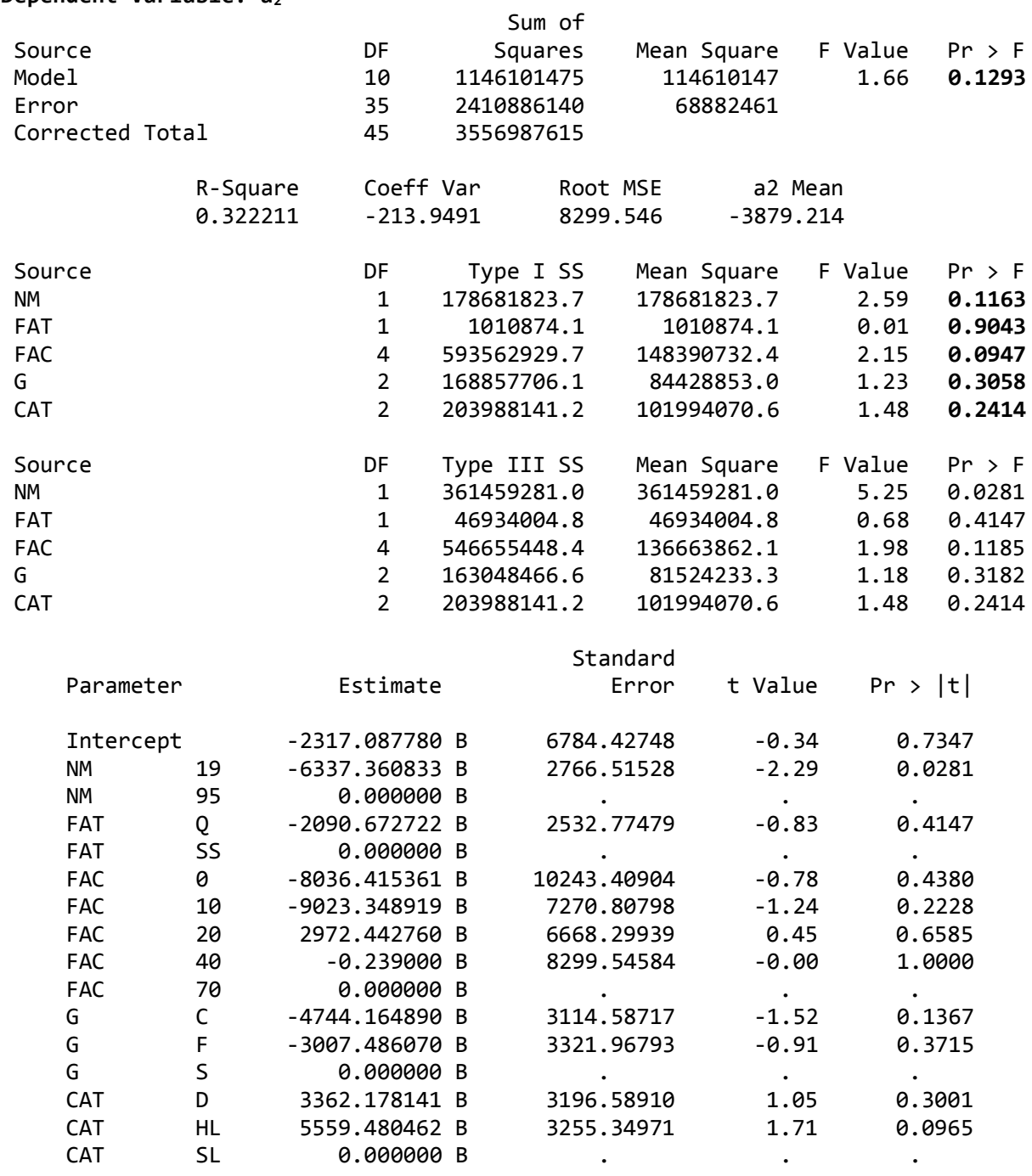


Dependent Variable: $a_{3}$

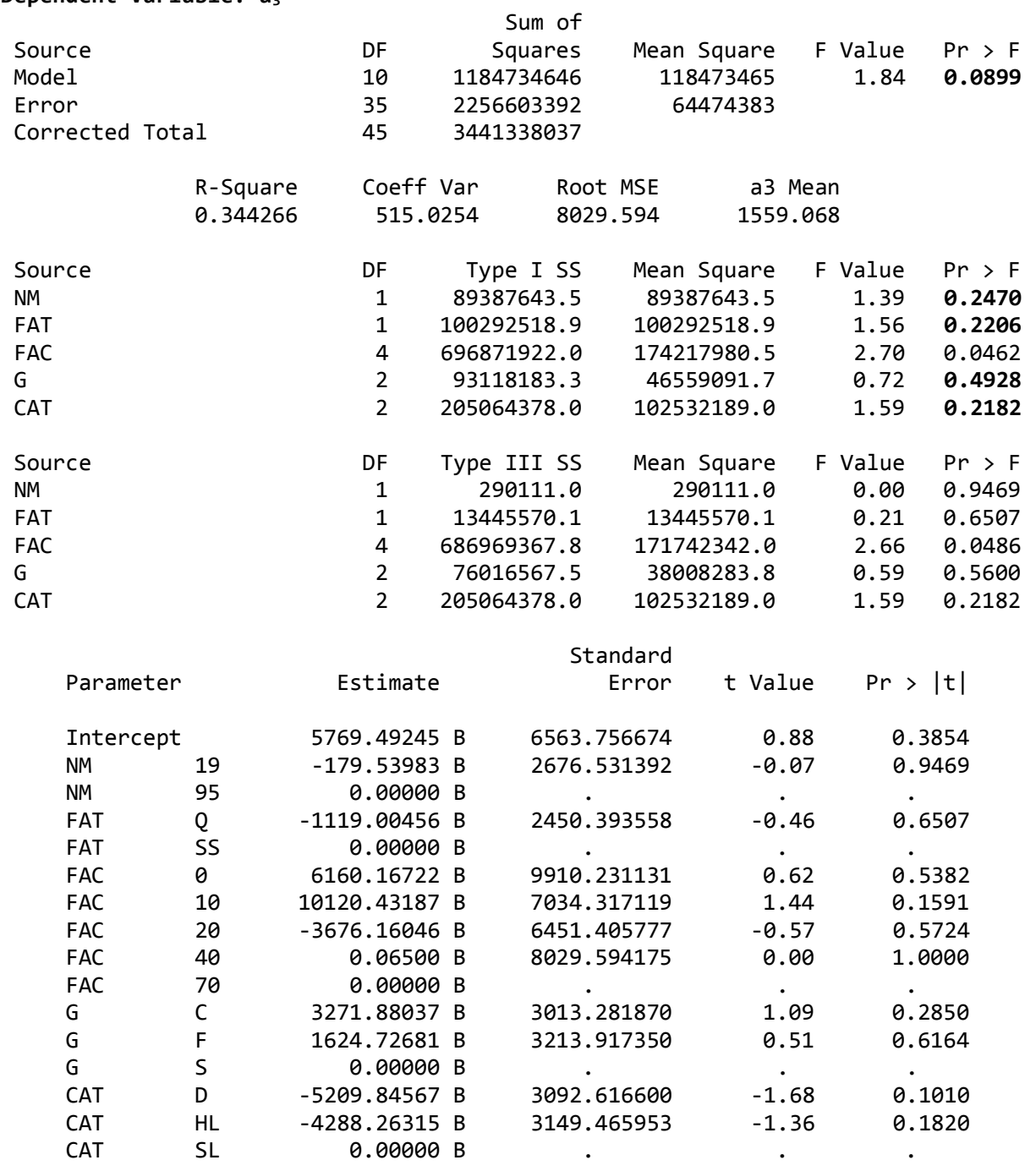




\section{Appendix G: Laboratory Noise Measurements}

This appendix contains information about the additional tests conducted in this study. Information summarized here describes the noise properties of selected specimens tested for frictional properties in the previous part of this report.

\section{G.1 Selection of the Specimens for Noise Tests in TPTA}

It is known that the texture of a pavement surface can have a significant impact on tire/pavement noise. While not a major objective of Phase I or II of this study, the research did provide an opportunity for limited study of this relationship. (Note that funding for this work came from an FHWA funded project on tire pavement noise.)

It could be assumed that the aggregate type (within the typical range of aggregates specific gravities) would not significantly influence the tire/pavement noise generation and propagation mechanisms. The macrotexture of the surface, however, will influence tire/pavement noise. Essentially, in terms of texture, there were eight different mixture types that were tested for friction in the laboratory part of this study. These are summarized in Table G 1 and views of surface texture are shown in Figures B 1 through B 8, Appendix B.

Ideally, all eight types should be tested for noise. However, due to the resource limitations, only six were chosen. (Six specimans can be mounted at one time in the Tire/Pavement Test Apparatus.) The selected mixtures included PFC, SMA and four Superpave HMA mixtures. As shown in Table G 1, the s-shaped mix (with NMAS=9.5 mm) and the coarse mix (with NMAS=19 mm) were dropped from the test matrix. This selection was dictated by the fact that the macrotexture of the 95SS20S_D mix was within the range of two other mixes with $9.5 \mathrm{~mm}$ NMAS (95SS20F_D and 95SS20C_D). In case of mixtures with NMAS=19 mm, the 
coarse mix (19SS20C_D) also had the highest Mean Profile Depth (MPD). However, the coarse mix was already selected for testing (in the group of mixes with NMAS=9.5 mm) and the goal was to capture as many different mixtures as possible.

It should also be noted that the same FAT (steel slag) and CAT (dolomite) aggregates were used in all four Superpave mixes tested for noise.

Table G 1. Composition, texture and frictional properties of mixtures considered for noise testing in TPTA

\begin{tabular}{|c|c|c|c|c|c|c|c|c|}
\hline Mix Type & \multicolumn{6}{|c|}{ Superpave } & PFC & SMA \\
\hline Gradation & Coarse & Fine & S-shaped & Coarse & Fine & S-shaped & PFC & SMA \\
\hline $\begin{array}{l}\text { NMAS, } \\
\text { mm }\end{array}$ & 9.5 & 9.5 & 9.5 & 19 & 19 & 19 & 12.5 & 12.5 \\
\hline $\begin{array}{c}\text { Steel Slag } \\
\text { Content, \% }\end{array}$ & 20 & 20 & 20 & 20 & 20 & 20 & 80 & 90 \\
\hline Label & $\begin{array}{c}\text { 95SS } \\
\text { 20C_D }\end{array}$ & $\begin{array}{l}\text { 95SS } \\
\text { 20F_D }\end{array}$ & $\begin{array}{l}\text { 95SS } \\
\text { 20S_D }\end{array}$ & $\begin{array}{c}\text { 19SS } \\
\text { 20C_D }\end{array}$ & $\begin{array}{c}\text { 19SS } \\
\text { 20F_D }\end{array}$ & $\begin{array}{c}\text { 19SS } \\
\text { 20S_D }\end{array}$ & PFC & SMA \\
\hline $\begin{array}{c}\text { Binder } \\
\text { Content, \% }\end{array}$ & 5.7 & 5.7 & 7.0 & 4.5 & 4.2 & 5.4 & 5.7 & 5.7 \\
\hline $\mathrm{G}_{\mathrm{mm}}$ & 2.65 & 2.66 & 2.58 & 2.69 & 2.69 & 2.65 & 3.15 & 3.19 \\
\hline $\mathrm{DF}_{20}{ }^{\mathrm{a}}$ & 0.57 & 0.56 & 0.53 & 0.54 & 0.48 & 0.52 & 0.66 & 0.60 \\
\hline $\mathrm{MPD}^{\mathrm{a}}, \mathrm{mm}$ & 0.89 & 0.57 & 0.70 & 1.68 & 0.96 & 1.40 & 1.79 & 2.52 \\
\hline $\begin{array}{c}\text { Selected } \\
\text { for Tests } \\
\text { in TPTA? }\end{array}$ & Yes & Yes & No & No & Yes & Yes & Yes & Yes \\
\hline
\end{tabular}

${ }^{a}$ Data from laboratory friction and texture measurements (results of this part of the study are discussed in Chapter Four); MPD and $\mathrm{DF}_{20}$ values presented here are averages from the whole slabs polishing process.

Although by definition the NMAS of SMA and PFC mixes was $12.5 \mathrm{~mm}$, and the conventional Superpave mixes had a NMAS of $9.5 \mathrm{~mm}$, all the mixes had $100 \%$ passing the 12.5 mm sieve size (refer to Figure 14). 


\section{G.2. Specimen Preparation}

As with the friction tests, the experimental design included collecting and processing a relatively large amount of material (as explained in section 3.3.1.1). Six essentially similar mixes (as tested for friction) were also tested for noise properties. The additional work to prepare mixtures for the laboratory noise tests included batching about 55 mix samples (four mixture types were prepared in the laboratory and two mixture types were collected during construction, as explained previously). The amount of HMA placed in the mold for noise testing was about 130 to $160 \mathrm{~kg}$ (depending on the mixture's $\mathrm{G}_{\mathrm{mm}}$ ). The mix preparation process followed the steps explained in chapter 3.3.1.1.

In general, the compaction procedure was similar to that presented previously for the rectangular slab preparation (refer to chapter 3.3.1.1). However, due to the differences in sample size and shape, some changes were necessary. At the time of specimen fabrication, mixes were reheated to the compaction temperature (refer to 3.3.1.1) and compacted into convex concrete molds (also called "concrete segments"). The internal dimensions of these segments (which also were the asphalt layer dimensions) were: $406 \mathrm{~mm}$ wide x $1970 \mathrm{~mm}$ long x $76 \mathrm{~mm}$ deep (note that length is not a planar dimension, but the total length of the convex asphalt layer). For detailed information regarding the mold dimensions, refer to Figure G 1. 

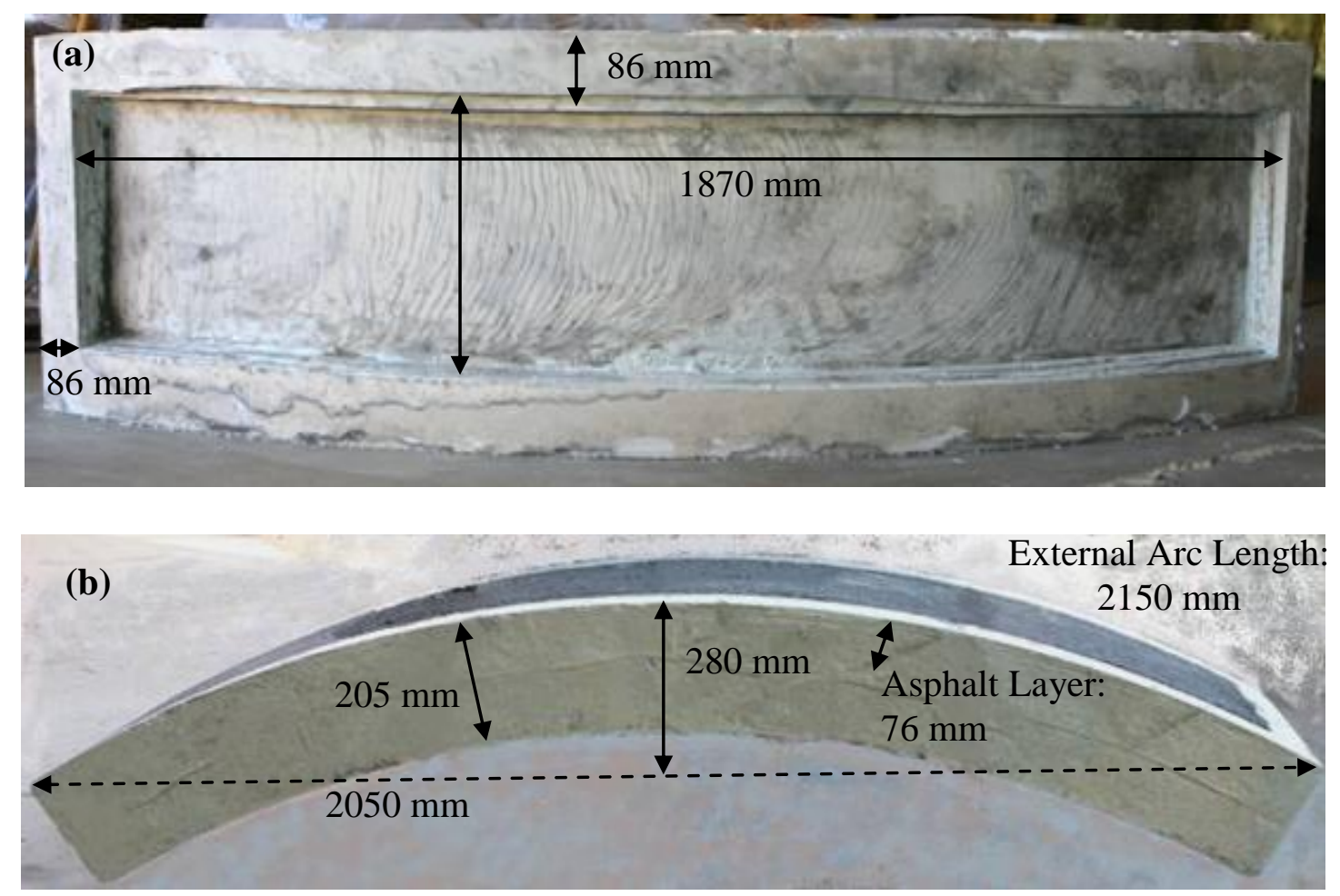

Figure G 1. Concrete molds used in the study: (a) top and (b) side views

In order to improve adhesion of the tested HMA to the concrete molds, the molds were "painted" with PG 64-22 binder 24 hours prior to compaction. Due to the relatively large mass of the roller and concrete molds (in comparison to the amount of the compacted mixture), those two elements were heated to prevent the HMA from cooling rapidly during the compaction process (refer to Figure G 2 for details). Molds were heated with eight heating lamps $(0.5 \mathrm{~kW}$ each) for about seven hours prior to compaction.

Note that the dark binder coating the internal part of the mold accelerated the heating process. At the time of compaction, the concrete temperature was about $55^{\circ} \mathrm{C}$. The roller was heated as described for the friction sample preparation. In addition to the roller, the hand tamper utilized in this part of the study was also heated. 

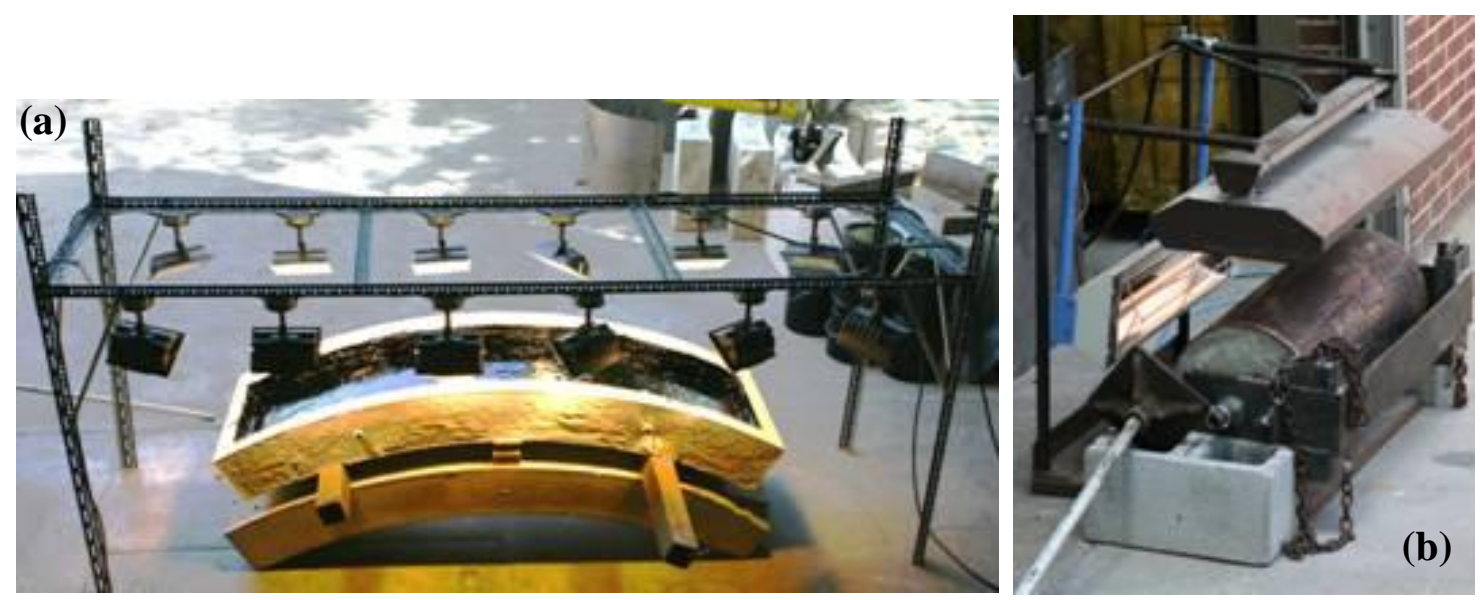

Figure G 2. Heating process: (a) concrete mold and (b) compaction tools

Due to the size of the compacted specimen, the rolling load was increased from $4.4 \mathrm{kN}$ (the load applied during the friction specimen preparation) to $8.6 \mathrm{kN}$. This load was achieved by placing an additional dead weight on the forks of the forklift used during the compaction process (the same roller was used during the preparation of specimens for both types of tests).

During trial compaction, inconsistencies in the amount of the applied load were recognized, as shown in

Figure G 3. Due to the convex shape of the sample, the load applied during compaction could not be constant; a different load was applied at the top of the mold compared to the areas close to the ends. The downward force at all points was $8.6 \mathrm{kN}$ while the total load depended on the roller speed, with the highest load differences in the areas close to the ends. Two extreme cases include the roller in the "fixed" position (not rolling because the forklift uses brakes) and the roller free rolling down. The loads normal to the surface of the specimen near the ends were about $9.9 \mathrm{kN}$ and $7.4 \mathrm{kN}$ for the "fixed" and free rolling cases, respectively. The normal load is greater for the "fixed" case because the forklift braking action applies a horizontal load to the 
specimen. During the compaction the actual normal load was in the range between $7.4 \mathrm{kN}$ and $9.9 \mathrm{kN}$.

In addition, static pressure from the mixture located in the center of the segment also influenced the load applied to the mixture located in the areas near both ends. In order to prevent the concrete segment from unnecessary stresses, the concrete mold was supported in three places (in the middle and close to both ends), as shown in Figure $\mathrm{G} 4 \mathrm{~d}$.
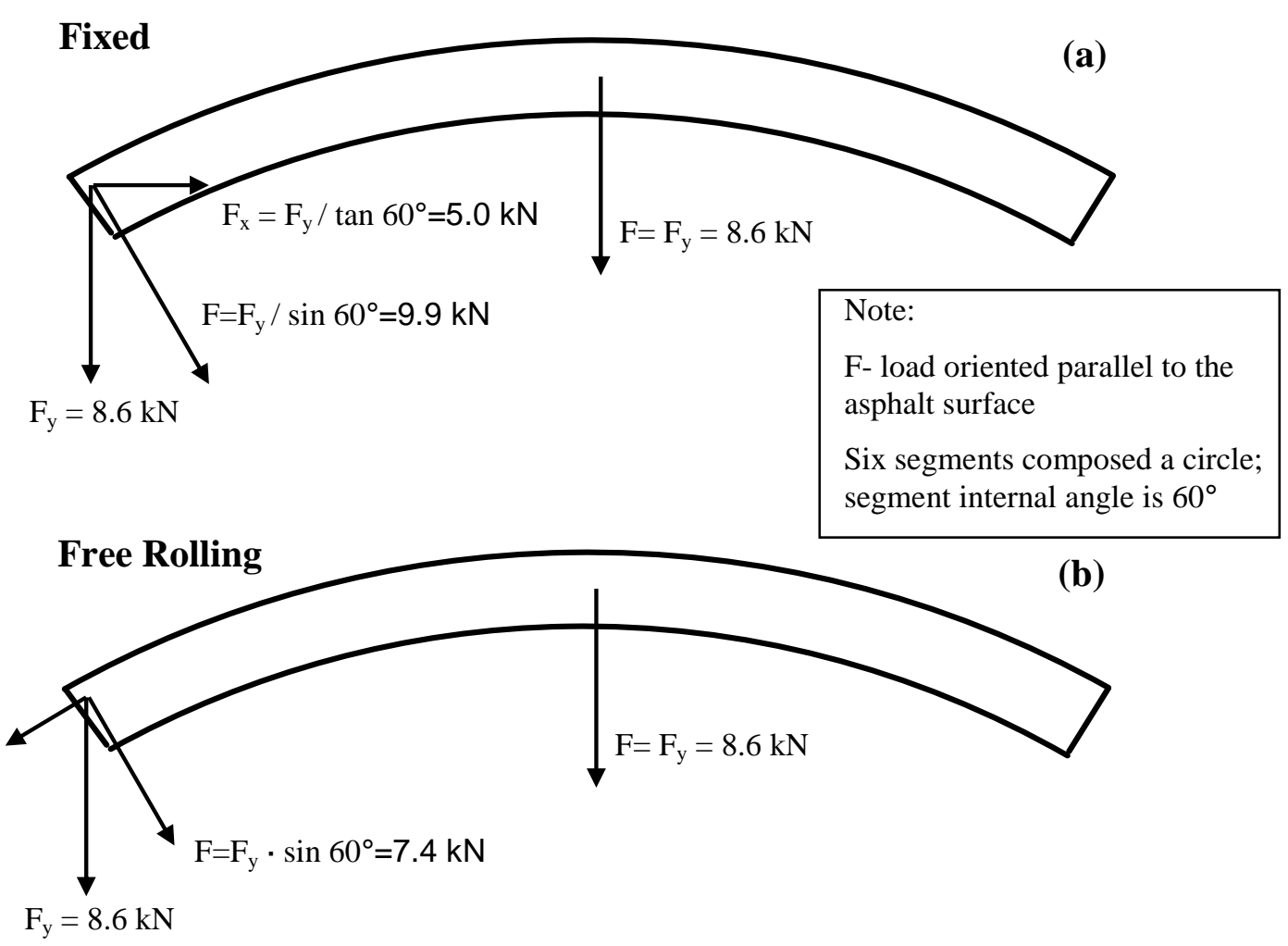

Figure G 3. Roller load distribution during the TPTA samples preparation: (a) roller in the Note: figure not to scale. "fixed" position and (b) free rolling down

As with the friction slabs, the initial amount of mix to be compacted was calculated based on the mold volume and HMA bulk specific gravity $\left(\mathrm{G}_{\mathrm{mb}}\right)$ corresponding to $7 \%$ air voids (or corresponding to $\mathrm{V}_{\mathrm{a}}=22 \%$ for the PFC mixture). However, as opposed to the compaction of specimens for friction testing, somewhat less than the calculated amount of mix was placed into 
the mold. Two objectives had to be balanced during the TPTA specimen preparation: to achieve desired densification and to have a specific thickness of the asphalt layer (76 mm). At the end of the compaction, the asphalt surface had to be even with the top surface of the concrete segment, which could not be reached with the entire amount of mix.

Due to the forklift and mold dimensions, compaction was conducted from the middle of the segment to one side then the other. (The forklift had to drive around the mold from one side to the other.) During compaction the roller rolled from the center down. Compaction was accomplished in one direction in two stages, as shown in Figure G 4. Each of the stages included mixture pre-densification and compaction. During the first stage, only the mixture in the middle part of the mold, from the center to about halfway down each side, was compacted. During the second stage, mixture in the whole segment was compacted. This two stage process was applied in order to prevent the mixture from sliding down from the higher to the lower elevation. (The height difference was about $280 \mathrm{~mm}$, as shown in Figure G 1.)

At the beginning of the first stage (pre-densification of the mixture in the middle part), the mix was placed in the middle part of the mold only and then tamped with a spatula and a hand tamper (with a mass of about $10 \mathrm{~kg}$ ) to prevent loss of material during initial compaction (refer to Figure $\mathrm{G} 4 \mathrm{~b}$ ). A prism from the mixture was formed in such a way that mixture height in areas close to the walls was the lowest (and equal with the wall height). Next (compaction of the mixture in the middle part), two flexible, thin metal sheets were positioned between the roller and compacted mixture (as shown in Figure G 4c). These metal sheets were used to provide a larger contact area between the surface of the mixture and the roller, thus ensuring more uniform load distribution. Using those sheets also reduced shoving of the mixture and heat loss. (Note that the metal sheets were sprayed with an anti-adhesive agent prior to compaction, as shown in 
Figure G 4a.) Rolling was continued until a smooth surface was obtained (Figure G 4d). Note that the metal sheet did not rest fully on the concrete mold. Before each rolling, the metal sheets were raised and the extra mixture resting on the top of the walls was removed (refer Figure G 4e).

At the beginning of the second stage (pre-densification of the mixture in the mold), the mix was placed in the whole volume of the mold and then tamped with a spatula and a hand tamper to prevent loss of material during compaction (refer to Figure G 4f). Again, a prism was formed with the mixture. Next (compaction of the whole unit), thin metal sheets were positioned between the roller and compacted mixture and rolling was initiated. Rolling continued until a smooth surface (with the height equal to the height of the walls of the concrete mold) was obtained and no further densification was observed (see Figure G 4g). Note that the metal sheet is rested fully on the concrete mold. 

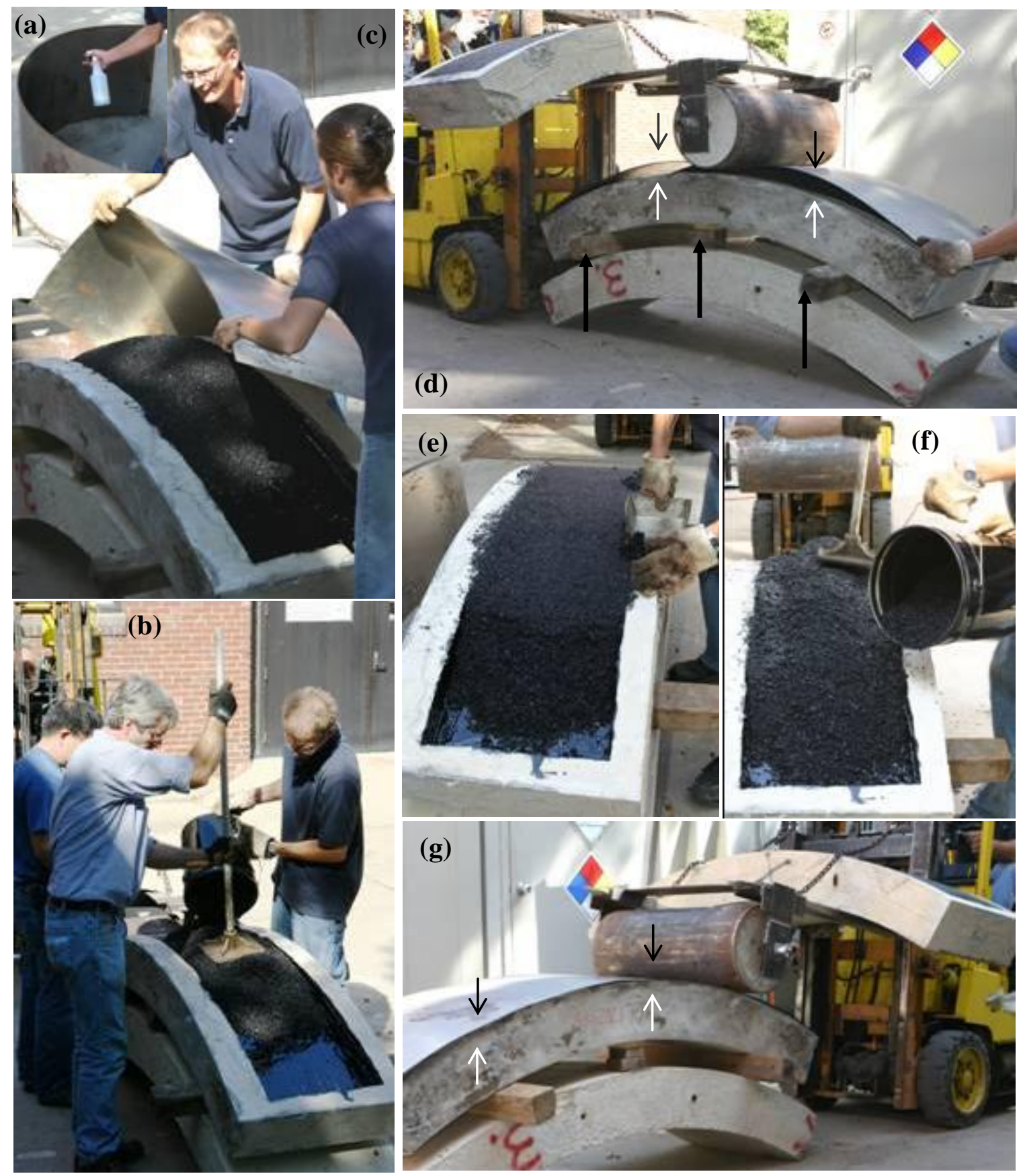

Figure G 4. . Stages of the TPTA segments compaction process: (a) spraying thin metal sheet with an anti-adhesive agent, (b) first stage: pre-densification, (c) specimen before the first stage of compaction, (d) first stage: compaction, (e) removing mixture covering top of the walls, (f) second stage: pre-densification and $(\mathrm{g})$ second stage: compaction

The typical rolling process took about 25 minutes and the mix temperature was monitored during the entire process. This process was developed through trial and error and was 
found to yield an acceptable (smooth) surface profile. Although compaction of the convex sample could not fully simulate field operations, it is believed that the procedure utilized here simulated field compaction to a great extent. It should be noted that due to the heating procedure utilized in this study, only one sample was prepared per day.

Views of the surface texture of the specimens compacted into the concrete molds are shown in Appendix B, in Figures B 12 through B 17.

\section{G.3. Equipment and Testing}

None of the currently existing laboratory methods can fully simulate tire/pavement noise. However, it appears that the Tire/Pavement Testing Apparatus (TPTA) allows investigation of the noise generation phenomenon. It should be noted, however, that the noise propagation phenomenon cannot be studied using this device.

The TPTA device used in this study is unique equipment, which is not covered by any standards. This device is located in an anechoic chamber at the Herrick Laboratories (Purdue Mechanical Engineering Department) and was fabricated in 2001 by the Institute for Safe, Quiet and Durable Highways (SQDH).

The TPTA device (shown in Figure G 5) consists of a fixed drum and a rotating circular plate positioned above the drum. Six curved pavement segments are mounted around the drum. The pavement diameter is about 4.1 meters, and the length of each pavement specimen is approximately $1 / 6$ of the apparatus circumference. Two arms are attached to the rotating plate. A testing wheel is attached to the end of one arm (optionally, wheels could be attached to both arms). During testing, the rotating plate rotates the arms, which causes the wheel (or wheels) to roll along the outside of the specimens. The center of the wheel travels with a maximum speed 
of about $48 \mathrm{~km} / \mathrm{h}(30 \mathrm{mph})$ resulting in about 65 arm rotations per minute (rpm). In other words, the test tire passes over each section of the specimen about once per second. Speed is limited to this value, which is lower than typical highway speed, due to various technical limitations (mainly due to the presence of the large centrifugal forces created at that speed by the rotating $\operatorname{arm})$.

Prior to testing, the wheel assembly is loaded with a force of about $2.67 \mathrm{kN}$ (total force between the tire and pavement), measured when the tire is positioned in the middle of the specimen; this load is approximately equal to the weight of one wheel of a typical passenger car.

It should be noted that a current project is focused on developing an empirical model to predict tire/pavement noise at speeds higher than $48 \mathrm{~km} / \mathrm{h}$ (to predict the value of laboratory measured tire/pavement noise at typical highways speeds). In order to develop this model, a series of tests performed at different speeds is being conducted both in the field and in the lab. In the lab, tests are run at $48 \mathrm{~km} / \mathrm{h}(30 \mathrm{mph})$, at $32 \mathrm{~km} / \mathrm{h}(20 \mathrm{mph})$ and at $16 \mathrm{~km} / \mathrm{h}(10 \mathrm{mph})$. Development of the field noise/speed relationship will be utilized to extrapolate the laboratory developed speed/noise curve to higher speeds. 


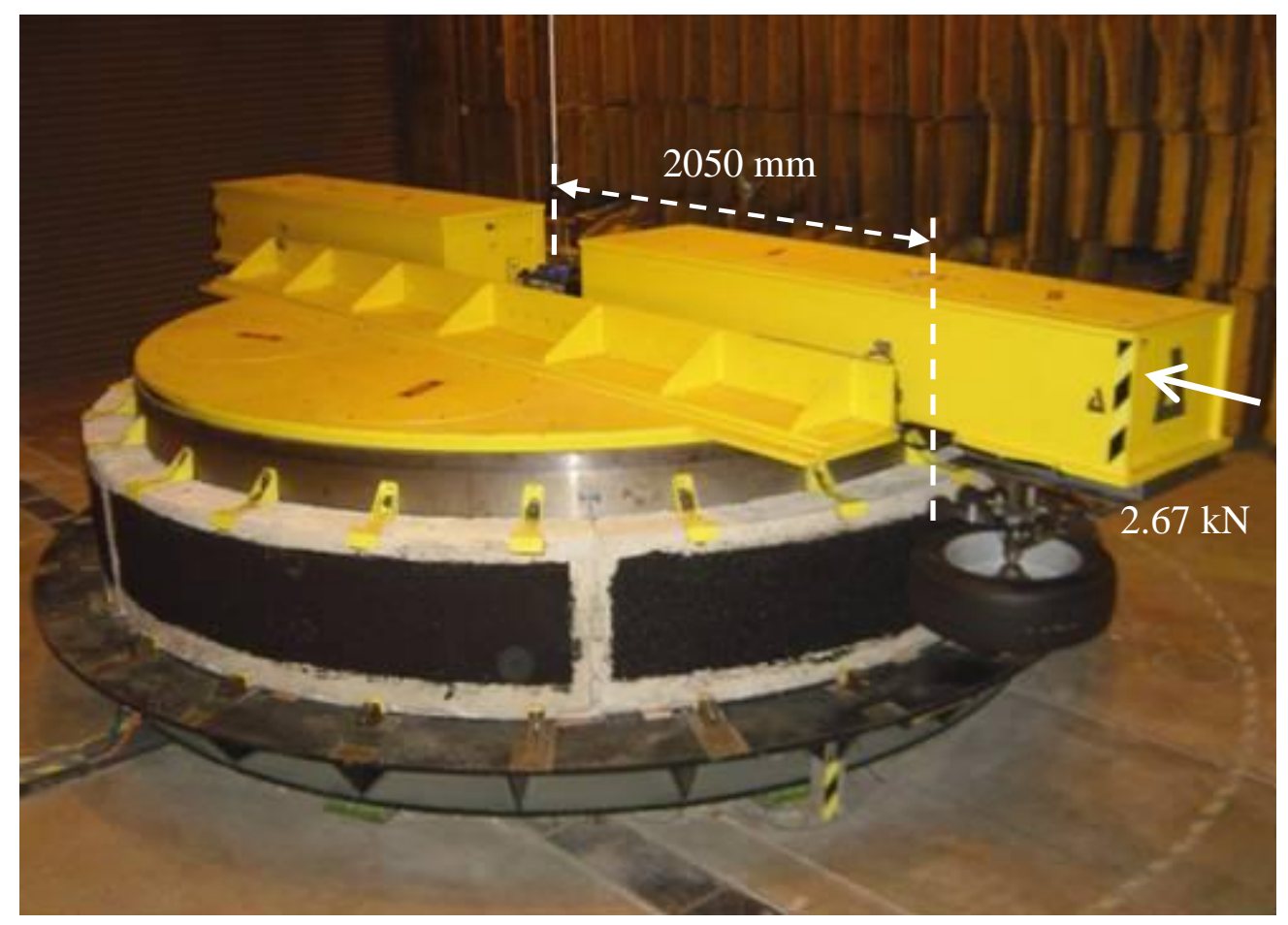

Figure G 5. Tire/Pavement Testing Apparatus (TPTA)

During standard tests, concrete segments with a compacted HMA were positioned vertically in the TPTA device, as shown in Figure G 6. The P225/60R16 97S Radial Standard Reference Test Tire (RSRTT) covered by ASTM F 2493 (2006) was used to roll along the specimen; this tire should not be confused with the Radial Standard Reference Test Tire covered by the ASTM E 1136 (2003) specification. Before the measurements, the tire was conditioned by rolling about the specimens for four hours at a speed of $48 \mathrm{~km} / \mathrm{h}$. Note that the tire conditioning procedure was conducted on other specimens, not tested in this research.

The wheel and specimens were positioned in such a way that the center of the tire rolls along the center of the specimen (refer to Figure G 6). Air pressure in the tire was maintained at a level of $240 \mathrm{~Pa}$. Directly before measurements, the tire was warmed-up by running at a speed of $48 \mathrm{~km} / \mathrm{h}$ along the specimens for 15 minutes. 
It should be noted that data were collected from only one specimen at a time during single operation. (The wheel load was adjusted separately for each specimen.) Moreover, data were collected only from the middle part of the specimen (called the "testing zone"), not the total length. Noise information was not collected from areas before the tire reached the testing zone (called the "approaching zone") and after the tire leaves the testing zone (called the "exiting zone"). Those two areas consist of the concrete wall and HMA mixture. The length of the testing zone was about $106 \mathrm{~cm}$ and this zone was identified in the following way: magnetic triggers were mounted on the drum and on the rotating arm; once the arm and drum were positioned in such a way that the magnetic triggers were close together, the data acquisition system was initiated and started to collect sound data. Those data were then collected continuously for the next 0.08 seconds. Collecting data for this period of time at the speed of 48 $\mathrm{km} / \mathrm{h}$ resulted in the length of the testing zone equal to about $106 \mathrm{~cm}$. The middle of the testing zone was in the middle of the specimen. For tests conducted at lower speeds, the time of data collection was extended to preserve collecting data from the same location during all measurements.

During operation, data from 100 passes of the tire were collected and averaged. It should be noted that typically results stabilized after about 25 passes. 


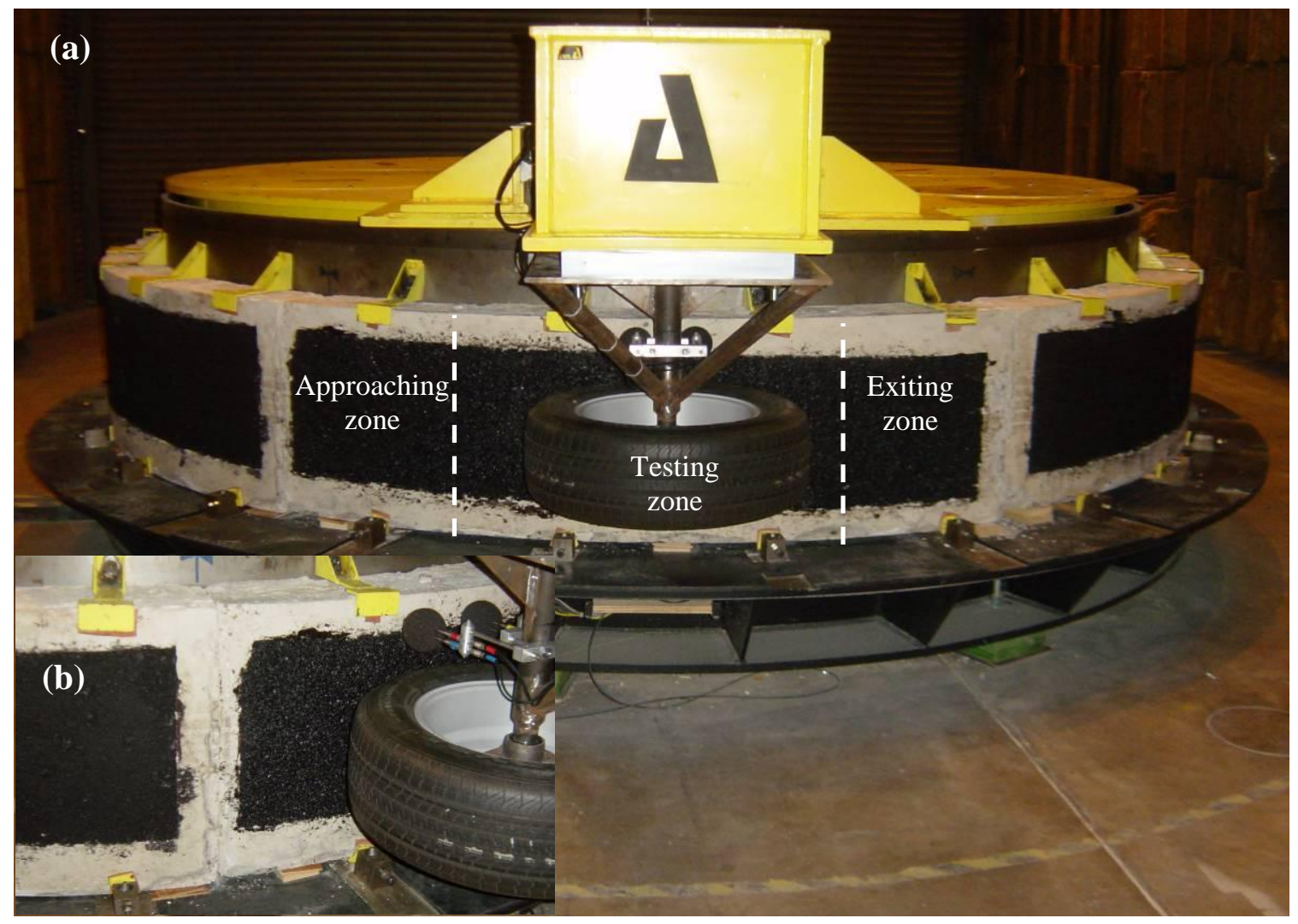

Figure G 6. TPTA during the operation: (a) general view and (b) transition zone between the specimens

The Close Proximity Sound Intensity (CPI) method was utilized to measure the tire/pavement noise in the laboratory. This near-field method is commonly used during field (highway) noise measurements. It should be noted that using this set-up (and using near-field methods in general) the pavement noise propagation phenomenon cannot be deeply investigated.

The measurement procedure followed the draft AASHTO specification [AASHTO OBSI draft (2007)], and the equipment set-up is shown in Figure G 7. The applied procedure is called the On-Board Sound Intensity Method (OBSI) (called also as a CPI method) and includes collecting the sound intensity data from two pairs of intensity probes. Phase-matched microphone pairs, mounted near the test tire, were positioned close to the leading and trailing edges of the contact patch. The center of the probes was located $76 \mathrm{~mm}$ from the pavement and 
$102 \mathrm{~mm}$ above the edge of the tire. In addition, probes were positioned $105 \mathrm{~mm}$ from the center of the hub. The separation between centers of two microphones (for each pair) was $17 \mathrm{~mm}$.

Prior to measurement, each of the microphones was calibrated following the manufacturer's recommendations (Brüel \& Kjær).

The data acquisition system collected information in the following way [Dare et al. 2007]:

For each measurement, A-weighted, narrow-band intensity spectra were collected and averaged over 100 passes of the test tire over the pavement sample with a sampling rate of $12,800 \mathrm{~Hz}$. The narrow-band intensity data resolution was 12.5 $\mathrm{Hz}$ over the frequency range from 12.5 to $5,000 \mathrm{~Hz}$. The intensities from the leading and trailing probes were averaged at each frequency. One-third octave band intensity spectra from 630 to $4,000 \mathrm{~Hz}$ were calculated by summing the narrow-band intensity in each one-third octave band. Overall intensity levels were calculated by adding all of the narrow-band intensities from 500 to $5,000 \mathrm{~Hz}$.
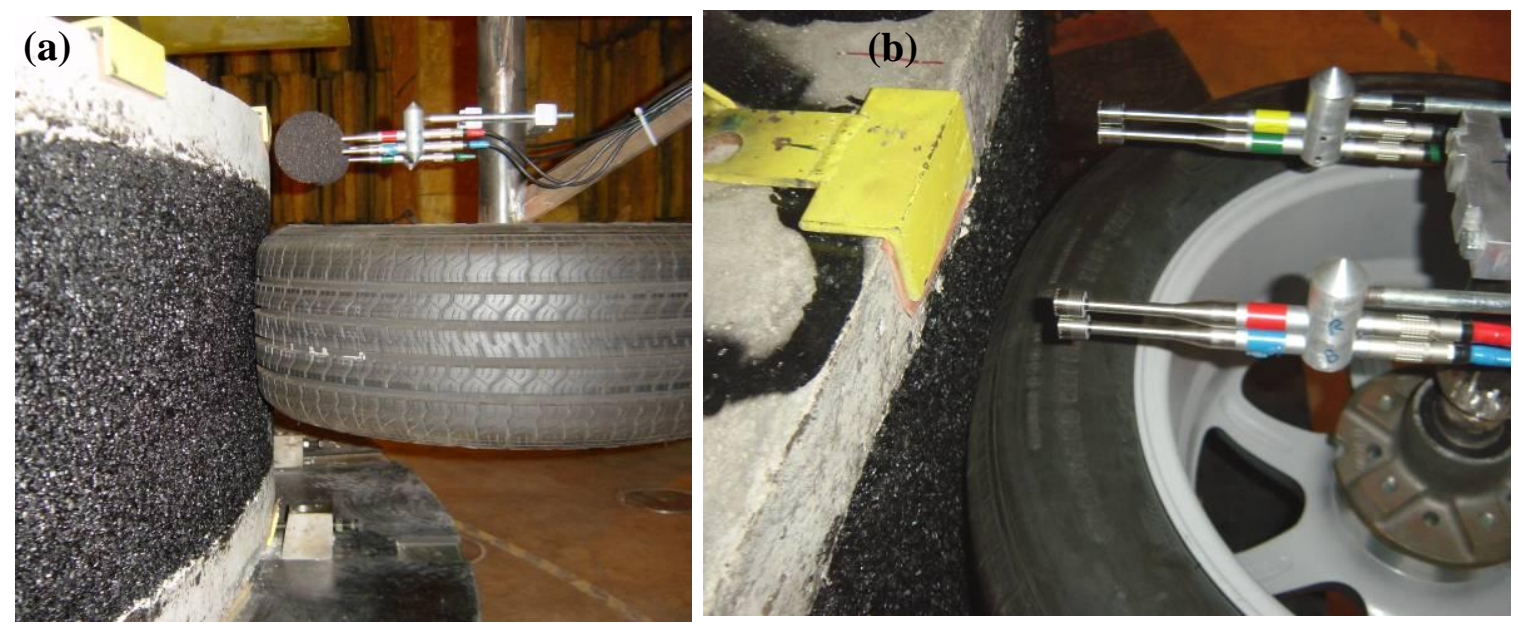

Figure G 7. Microphones set-up: (a) side and (b) oblique view (protective windscreens removed for the picture) 
The specimens were positioned in the TPTA about one to two weeks after sample preparation (each sample was prepared on a different day). Tests were conducted five times, on separate days on potentially different surface condition (SCD) as described below. Each time all six segments were investigated. During the first day of testing (referred to later as surface condition 1 ( $S C D_{-}$1), the specimens' surfaces were in the condition as at the end of the compaction process (specimens were not exposed to tire action, except short trial tests and tire warming-up, or about one hour of TPTA operation). On the second day of testing (referred to later as SCD_2) specimens were tested again. At this time, the specimen surface was not exposed to extensive tire action, however, at the time of testing the tire had already rolled along the surface for about two to three hours. During SCD_1 and SCD_2 testing (first and second day), noise data were collected at speed of $48 \mathrm{~km} / \mathrm{h}$.

Before the third day of tests (SCD_3), specimens were exposed to a longer period of tire action, which potentially could wear off some asphalt coating the aggregate particles in the tire/pavement contact area; during this operation the tire rolled along the surface for about five hours (resulting in about 20,000 wheels passes). During the third day of testing, noise data were collected at three different speeds: at 16, 32 and $48 \mathrm{~km} / \mathrm{h}$.

Non-standard tests conducted on the fourth and fifth day included using two other tires, in addition to the previously used P225/60R16 97S Radial Standard Reference Test Tire. The two tires which were employed were a Uniroyal Tiger Paw (P205/70R15 95S M+S) and a Goodyear Aquatred (P205/70R15 95T M+S) (refer to Figure G 8). The Uniroyal Tiger Paw tire is later referred to as a UTP tire and the Goodyear Aquatred is referred to as the GA tire. Due to differences in the tire dimensions, the centers of those two non-standard tires were positioned about $2.5 \mathrm{~cm}$ above the center of the previously used RSRTT tire. All other set-up 
remained unchanged, and the tires were conditioned before testing in the same way as the tire used in the standard tests. Using those two tires, tests at three different speeds (at 16, 32 and at $48 \mathrm{~km} / \mathrm{h}$ ) were conducted.

The standard test procedure included air temperature measurement, microphone calibration, tire pressure control and adjustment, wheel load adjustment, tire warming-up, positioning of the magnetic triggers, acceleration of the machine to the testing speed and conducting the measurement. After this operation, the machine was stopped and the magnetic triggers were relocated to the next specimen. Again, the wheel load was adjusted, the machine was accelerated to the testing speed and measurements were conducted. This procedure was applied to all six specimens.

It should be noted that all laboratory tests were conducted at a temperature of $20 \pm 3^{\circ} \mathrm{C}$.



Figure G 8. Tires used during the noise tests in TPTA: non standard (a) Uniroyal Tiger Paw (UTP) and (c) Goodyear Aquatred (GA) and (b) standard Radial Standard Reference Test Tire (RSRTT)

Note the directional tred pattern (with water expulsion channels) for GA tire. 
Macrotexture has a great impact on tire/pavement noise. The macrotexture of all six segments was measured using the CTM device. Although this device is designed to operate on a flat surface, it was recognized that this test method could be adopted to testing a convex specimen also. During the standard test, the CTM scans texture on a circular path with a 284 $\mathrm{mm}$ diameter. The radius of the convex specimen used in this study was $2050 \mathrm{~mm}$, thus for a $284 \mathrm{~mm}$ chord of the TPTA specimen $(284 \mathrm{~mm}$ is also diameter of the circle covered by the CTM device during measurement), the difference in TPTA elevations (in this $284 \mathrm{~mm}$ TPTA chord) is about $4.9 \mathrm{~mm}$. Note, that following ASTM E 1845 (2005), the MPD is "the average of all of the mean segment depths of all of the segments of the profile," as discussed in Chapter Two. Thus, analyzing each of the measured segment separately, relative differences in elevations are less significant in MPD determination and it was possible to determine the MPD value for each of the TPTA specimens. This macrotexture was determined after all noise tests were conducted.

\section{G.4. Sample Volumetric Properties}

As discussed previously, the desirable air void content of the specimens is $7 \%$ (and $22 \%$ for PFC). However, due to the compaction technique adopted in this project, this target could not be reached without crushing aggregates. Loss of heat likely played a role. Although it was not verified in this study, it is expected that the air void content would not be uniform either. Partially due to the boundary effect, lower densification is expected in areas close to the concrete segment walls. It also should be noted that based on the visual examination no rutting caused by the wheel action was observed. 
For the purposes of this study, the most important area in which to determine the air content is in the testing zone. Once the tests in the TPTA were completed, three $152 \mathrm{~mm}$ diameter cores were extracted from each segment. As shown in Figure G 9, one core was drilled from the middle of the testing zone (referred to as the "middle" core, $\mathrm{C}_{-} \mathrm{M}$ ) while the other two were extracted from two opposite corners of the testing zone (referred to as left and right cores, C_L and C_R, respectively). Due to difficulties associated with drilling on the non-horizontal surface, these two cores were not located exactly at the ends of the testing zone, but instead were $52 \mathrm{~mm}$ closer to the arc center.



Figure G 9. Location of the cores extracted from the specimens

Note: 1) distances measured on the curved surface and 2) since presented lines are drawn in a plane, there are schematics only (however, line should be drawn on the arc).

Again, a relatively large core diameter $(152 \mathrm{~mm})$ was selected to allow specimens for water permeability tests; however, this testing was not deemed to be necessary and was not conducted. Specific gravity tests were conducted following ASTM D 6752 (2004) using the CoreLok apparatus. Based on this $\mathrm{G}_{\mathrm{mb}}$ and on the previously determined $\mathrm{G}_{\mathrm{mm}}$, the air void content was calculated for each specimen.

As with the lab friction slabs, the air content was higher than expected and had the following average distribution: $17.1 \%$ for SMA, $26.4 \%$ for PFC, $14.0 \%$ for 95SS20F_D, $16.3 \%$ 
for 19SS20F_D, 20.9\% for 95SS20C_D and 23.4\% for 19SS20S_D. The expected air contents for the same mixtures were about 8 to $10 \%$ for all mixtures but PFC, for which about $22 \%$ voids was expected. Differences in the air content values between the three core locations in the same segment were less than about $1 \%$.

The visual examination of the extracted cores revealed random orientation of particles in the cores from both types of specimens.

\section{G.5. Results and Discussion}

During the laboratory noise measurements conducted using the Tire/Pavement Testing Apparatus (TPTA), the influence of mixture composition on the tire/pavement noise was investigated. Series of tests were conducted under various conditions (tire type, speed and surface condition) which could potentially influence the noise generated. Tests were conducted with three different tires: Radial Standard Reference Test Tire (RSRTT), Uniroyal Tiger Paw (UTP) tire and Goodyear Aquatread (GA) tire. Three different speeds were utilized: 16, 32 and $48 \mathrm{~km} / \mathrm{h}$. In addition, for one tire type (RSRTT) running at one speed $(48 \mathrm{~km} / \mathrm{h})$ tests were conducted at different surface conditions (SCD): as constructed (SCD_1), limited exposure to tire action (SCD_2) and after prolonged exposure to tire action, simulating the wear processes taking place during the first few months of the pavement use (SCD_3).

A general comparison of the sound intensity levels (SIL) for tests conducted with the three different tires at a speed of $48 \mathrm{~km} / \mathrm{h}$ is shown in Figure G 10. As expected, the UTP tire was significantly quieter (SIL was between 82 and $91 \mathrm{~dB}(\mathrm{~A})$, depending on the specimen) than the RSRTT and GA tires (84 to $92.5 \mathrm{~dB}(\mathrm{~A})$ ). Moreover, in most cases the GA was louder than the RSRTT. The RSRTT is wider than other two tires, thus it was expected that a higher noise level would be generated with this tire. However, the GA tire has a different tread pattern 
(directional tread pattern) than the RSRTT and UTP; typically tires with such a tread pattern are considered to be louder.

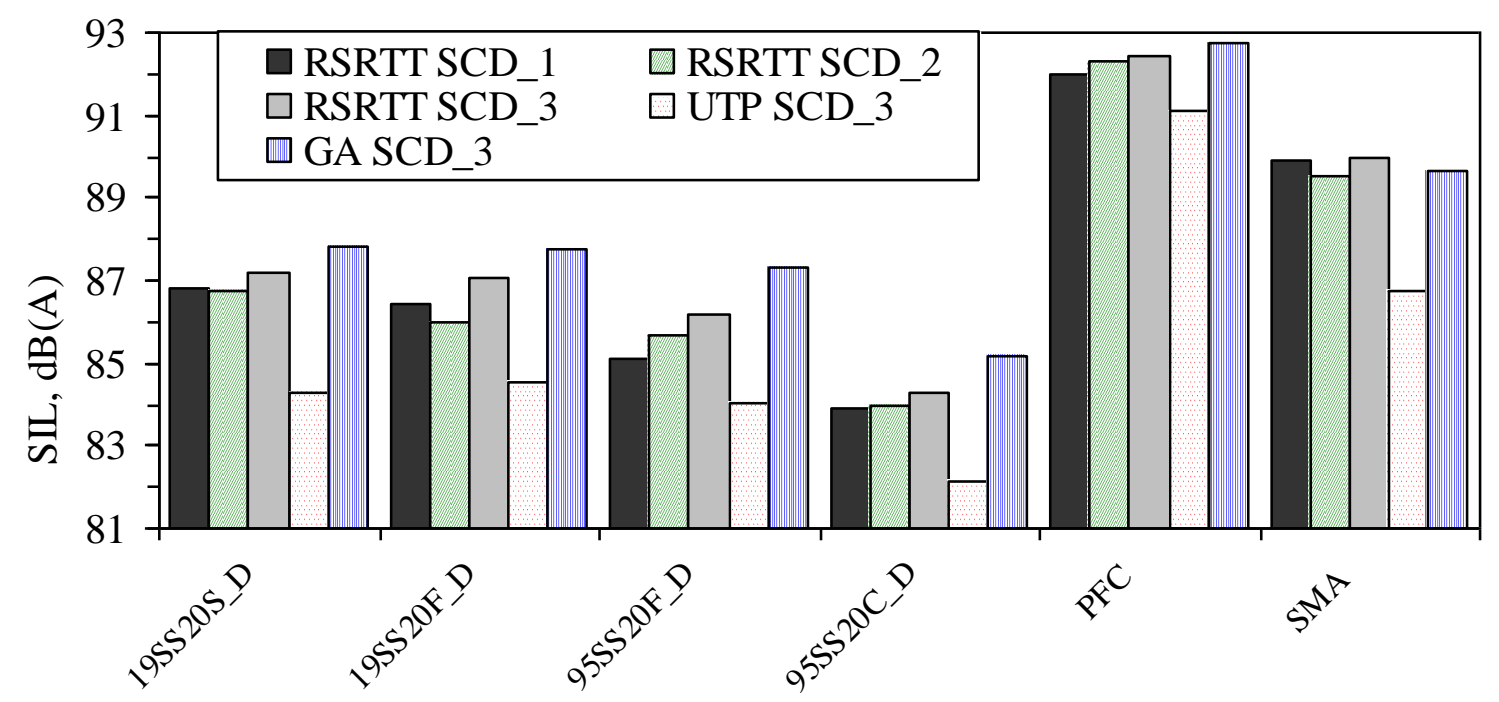

Figure G 10. General comparison of sound intensity levels (SIL) at test speed of $48 \mathrm{~km} / \mathrm{h}$

The general loudness of the pavement specimens (as measured by the generated noise level) was the same for all the tires tested. It was observed that the coarse graded mixture with 9.5 mm NMAS (95SS20C_D) was the quietest and PFC was the loudest. The fine-graded 9.5 mm NMAS (95SS20F_D) was louder by about 2 dB(A) than 95SS20C_D and it was second quietest surface. The SMA surface was the second loudest specimen and mixes with $19 \mathrm{~mm}$ NMAS were located in the middle of the range.

The quietest to loudest order of samples, as presented in Figure G 10, corresponds well with general trends reported in the literature. However, the PFC mixture, which was reported to be the quietest was actually found to be the loudest in the TPTA. In order to investigate this phenomenon, limited texture measurements were conducted and MPD values were calculated for each specimen. These values were as follows: $2.53 \mathrm{~mm}$ for PFC, $1.97 \mathrm{~mm}$ for SMA, $1.77 \mathrm{~mm}$ for 19SS20S_D, $0.98 \mathrm{~mm}$ for 95SS20C_D, $0.88 \mathrm{~mm}$ for 19SS20F_D and 0.44 for 95SS20F_D. 
The high noise level generated on the PFC surface could be potentially related to the high MPD values (although PFC has typically higher MPDs than conventional mixtures, they are generally not as high as observed here). Due to limitations in the available test equipment, texture wave analysis was not conducted. It is likely that additional information about texture wave length and amplitude (not available here) would help to explain the unexpected high noise level generated by the PFC specimen. The observed SIL could not be simply explained by the air content in the specimens: the quietest surface (95SS20C_D) has 20.9\% air but second quietest (95SS20F_D) has the lowest amount of air voids of $14.0 \%$. In addition, the PFC segment with the highest air voids content of $26.4 \%$ was actually the loudest.

As can be further observed in Figure G 10, the noise levels for the SCD_1 and SCD_2 measurements (new and slightly exposed to the tire action, respectively) were similar. However, the noise for SCD_3 was, in most cases, slightly higher. This is, again, expected behavior as the longer exposure to tire wear should result in an increase in the noise level. This phenomenon is most likely related to changes in the surface microtexture caused by the binder wearing from the aggregate surfaces (from those parts of aggregates which are in contact with the rolling tire).

It should be noted that the asphalt specimens tested in this study were significantly quieter than the concrete specimens tested in another study, using the same set-up as for tests with asphalt specimens. The concrete specimens had various surfaces: smooth-finished, ground and grooved using different blade pattern configurations. As shown in Figure G 11, SIL for these concrete segments was between 89.2 and $92.5 \mathrm{~dB}(\mathrm{~A})$ when testing with the UTP tire and between 90.4 and $93.9 \mathrm{~dB}(\mathrm{~A})$ when testing with the GA tire [Dare et al. 2007]. 


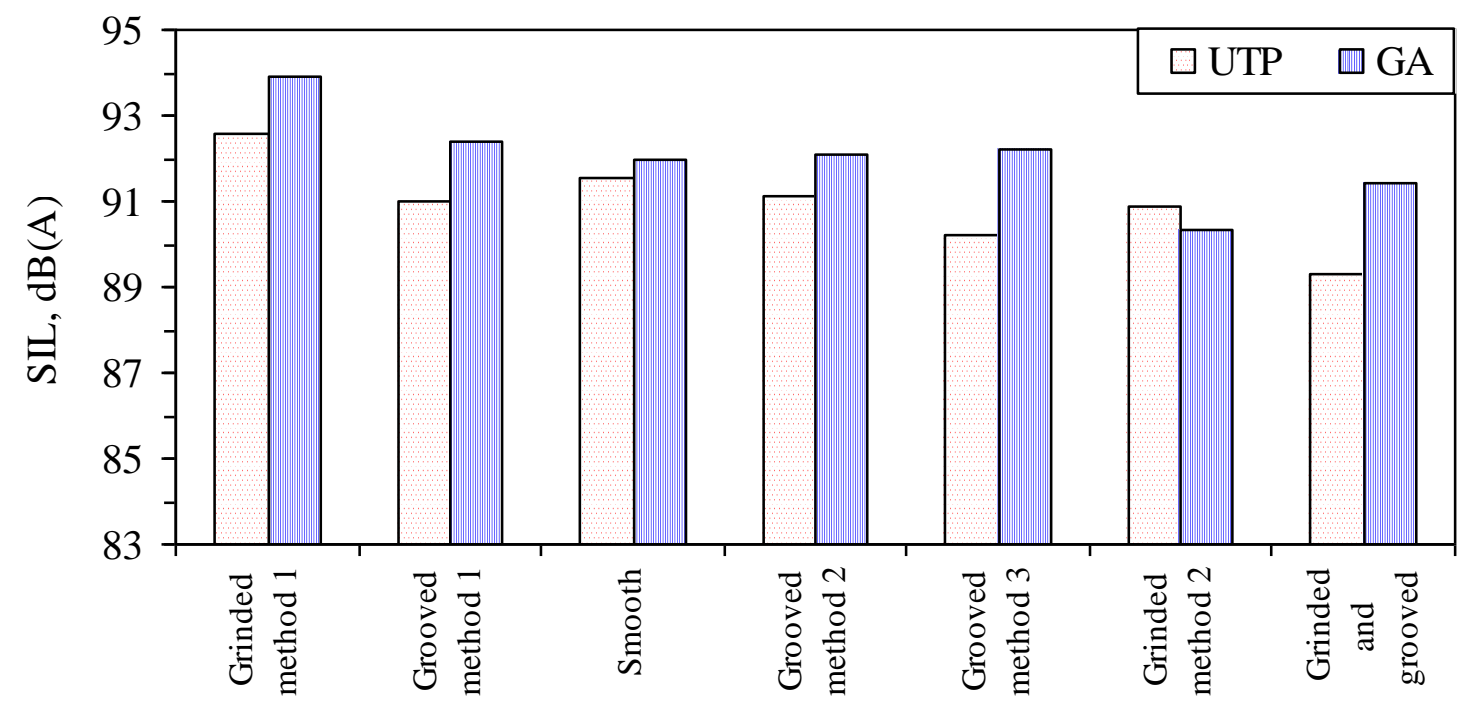

Figure G 11. Sound intensity levels of concrete specimens with various surfaces tested in TPTA [after Dare et al. 2007]

Note: at the time of tests, the RSRTT was approved as a standard tire so only UTP and GA tires were used.

For tests on the asphalt segments, the noise frequency spectra were also determined, as represented by the $1 / 3^{\text {rd }}$ octave band plots shown in Figure G 12 . A frequency spectrum plot shows how much sound energy was presented at a given frequency band. It could be observed that at low frequencies $(500 \mathrm{~Hz}, 630 \mathrm{~Hz}$ and $800 \mathrm{~Hz})$, the PFC surface is the loudest. However, at higher frequencies ( $1250 \mathrm{~Hz}$ and higher), the PFC is quietest or in the middle range of SIL of other specimens. Note that the peak of human hearing sensitivity is in the frequency range of 1 $\mathrm{kHz}$ and $4 \mathrm{kHz}$. In this frequency range, the SIL of the PFC specimen dropped down. The SIL of the quietest surface (95SS20C_D) is continuously within the low range of all frequencies. It could also be observed that the peak noise level was reached at frequencies of about $1250 \mathrm{~Hz}$ for all specimens but the PFC. The PFC peak was located at $630 \mathrm{~Hz}$. At frequencies above $1.6 \mathrm{kHz}$, the SIL of all specimens continued to decrease. The complete frequency spectra plots obtained of different tire/speed combinations are shown in Appendix G in Figures G 15 to 24. 


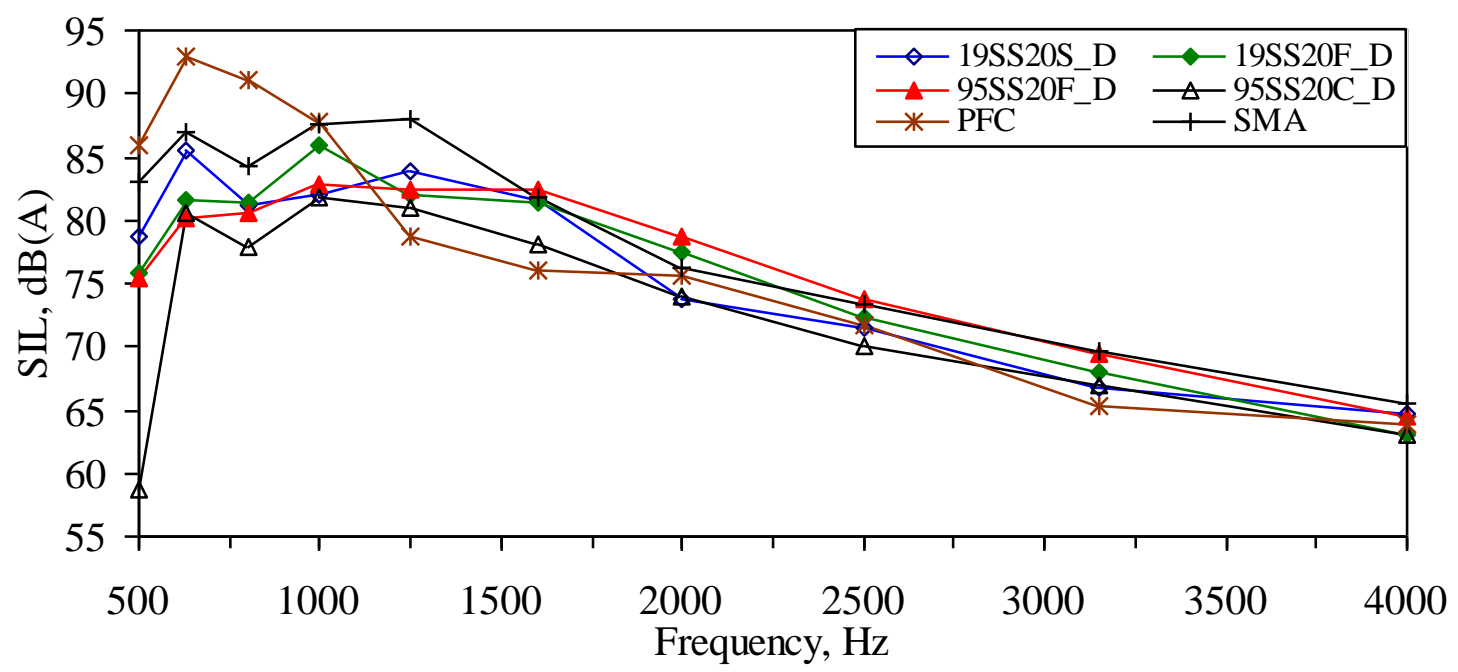

Figure G 12. 1/3 Octave frequency spectra for RSRTT SCD_3, test speed 48 km/h

Results of noise tests conducted at three different speeds are shown in Figure G 13. Due to equipment limitations, during this study, the noise tests were conducted with the maximum speed of $48 \mathrm{~km} / \mathrm{h}$, which is about half of typical highway speed. Since it would be beneficial to be able to predict the noise level at higher speeds based on the laboratory observations conducted at lower speeds, an attempt was made to develop a simple model for this purpose. This was accomplished by fitting (separately for each specimen) a trend line to the result of noise tests conducted at 16, 32 and $48 \mathrm{~km} / \mathrm{h}$. Using this process, logarithmic models have been developed for individual specimens, each approximating the actual data with a high $\left(\mathrm{R}^{2}>0.98\right)$ degree of accuracy. Using this model, the noise prediction at speeds up to $97 \mathrm{~km} / \mathrm{h}$ (or $60 \mathrm{mph}$ ) has been accomplished by extrapolating data acquired at lower speeds. It should be noticed, however, that this approach provides only a rough approximation of the noise level at higher speeds, and the accuracy of prediction was not verified by any of the field tests.

The relationships of the sound intensity level and speed for tests with UTP and GA tires are shown in Figures G 25 to 26. Extrapolation of the sound intensity at higher speeds is also 
shown in those two figures. Generally, the overall prediction of the noise looks consistent, i.e., none of the extrapolation curves cross.

However, it is possible that for the PFC specimen, as the speed increased, the SIL peak would move to lower frequencies (an indication of this could be observed in Figure G 12 and Figures G 15 to 24. Generally, lower frequencies are less annoying to humans. Moreover, it is possible that the proposed extrapolation may not correctly predict the SIL for PFC at $97 \mathrm{~km} / \mathrm{h}$. If this was the case, the actual A-weighted (filtered) noise level would be lower, making the PFC less annoying with respect to noise.

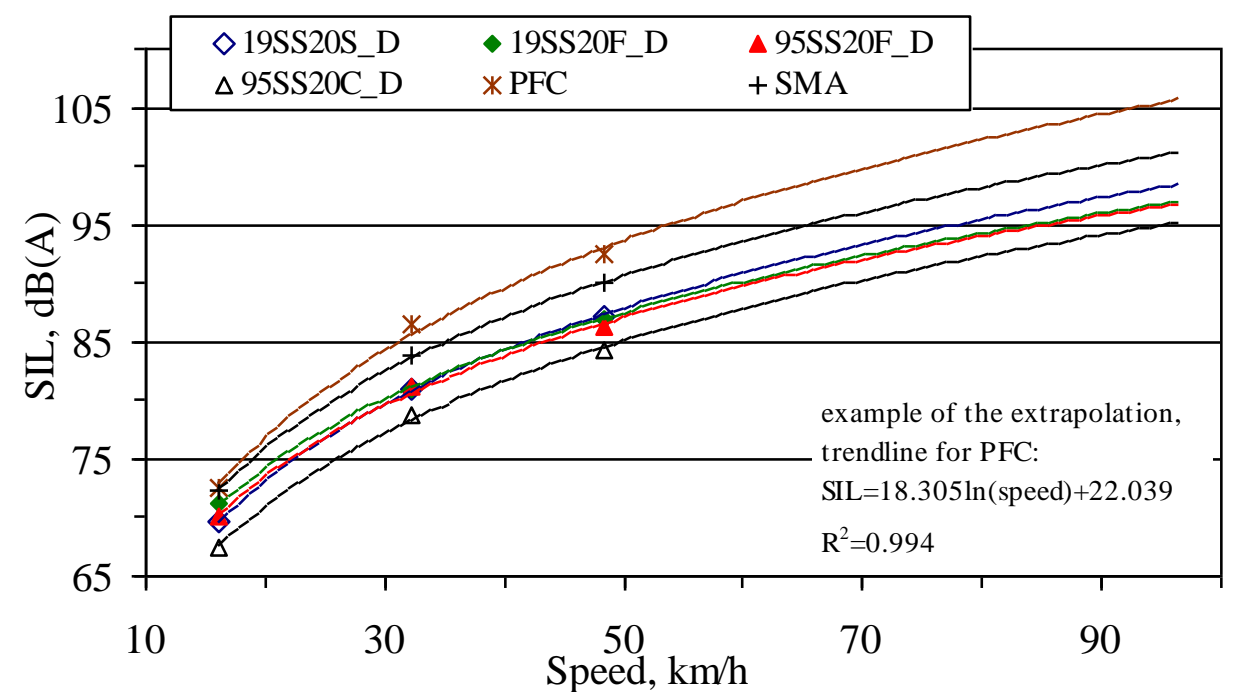

Figure G 13. Sound intensity level vs. speed relationship and SIL extrapolated to $97 \mathrm{~km} / \mathrm{h}$ for tests with RSRTT SCD_3

The overall predicted sound intensity level at $97 \mathrm{~km} / \mathrm{h}$ is presented in Figure $\mathrm{G} 14$ for all three types of tires. It can be observed that, similarly to trends shown in Figure G 10, the GA is louder than the RSRTT, and the RSRTT is louder than the UTP. In addition, the order (from the loudest to the quietest) remains similar to the order presented in Figure G 10. 


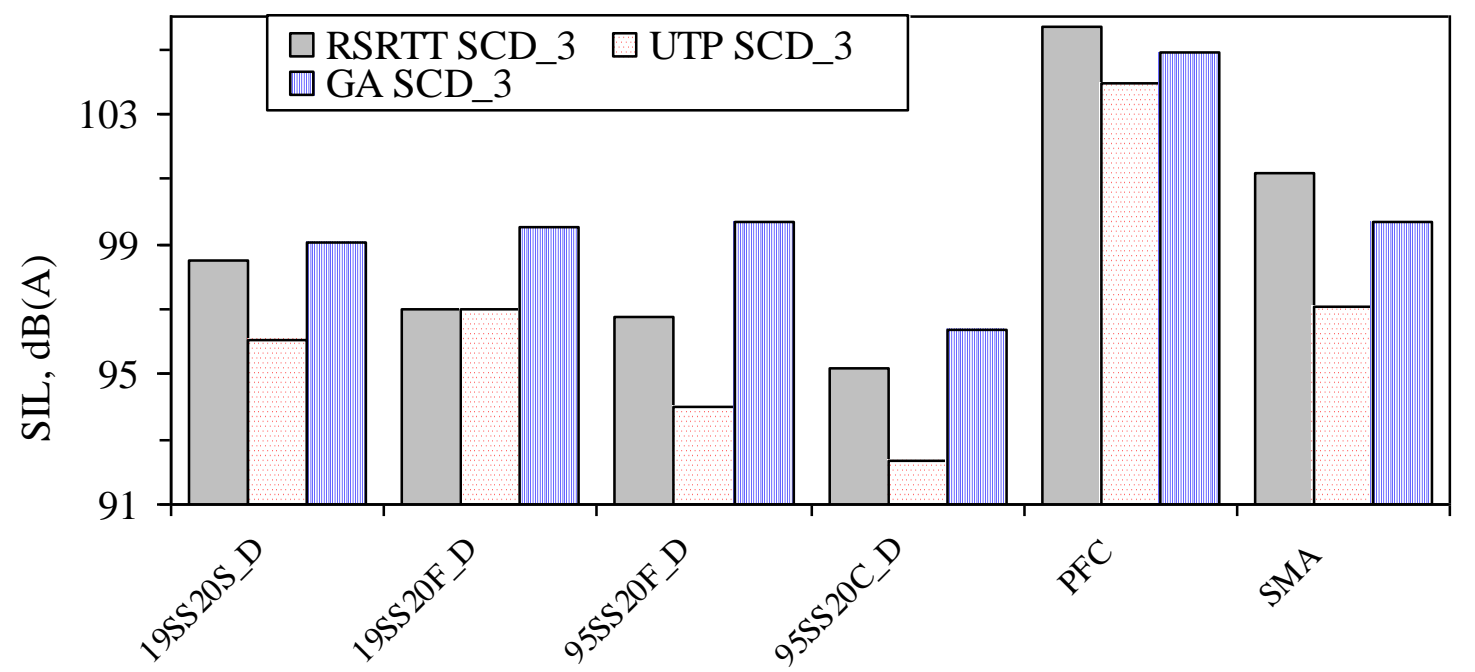

Figure G 14. General comparison of predicted sound intensity levels at test speed of $97 \mathrm{~km} / \mathrm{h}$

\section{G.6. Summary}

Laboratory noise tests were only partially successful, most likely due to difficulties with the compaction of the PFC mixture in the convex mold. However, except for the PFC mixture, laboratory tire/pavement noise measurements suggested that results obtained from the TPTA machine can potentially be used to predict the influence of the mixture composition on the tire/pavement noise without the need of building field test sections. In the tests performed, mixtures with 19 mm NMAS produced higher noise levels than mixtures with $9.5 \mathrm{~mm}$ NMAS. It also was observed that mixtures with the "s-shaped" gradation were louder than the "coarse" or "fine" graded mixtures. 


\section{G.7. Additional Test Results}

Plots of $1 / 3$ octave frequency spectra are presented in Appendix G (Figures G 15 to G 24) for tests with RSRTT conducted at $48 \mathrm{~km} / \mathrm{h}$ (SCD_1 and SCD_2), for same tire but for tests conducted at 16 and $32 \mathrm{~km} / \mathrm{h}$ (SCD_3) and for the UTP and GA tires at 16, 32 and $48 \mathrm{~km} / \mathrm{h}$ (SCD_3).

In addition, the relationships between sound intensity level and speed for tests with UTP and GA tires are shown (refer to Figures G 25 and G 26). For those two tires, the predicted SIL at speed of $97 \mathrm{~km} / \mathrm{h}$ is also presented, separately for each specimen.



Figure G 15. 1/3 Octave frequency spectra for RSRTT SCD_1, test speed 48 km/h 


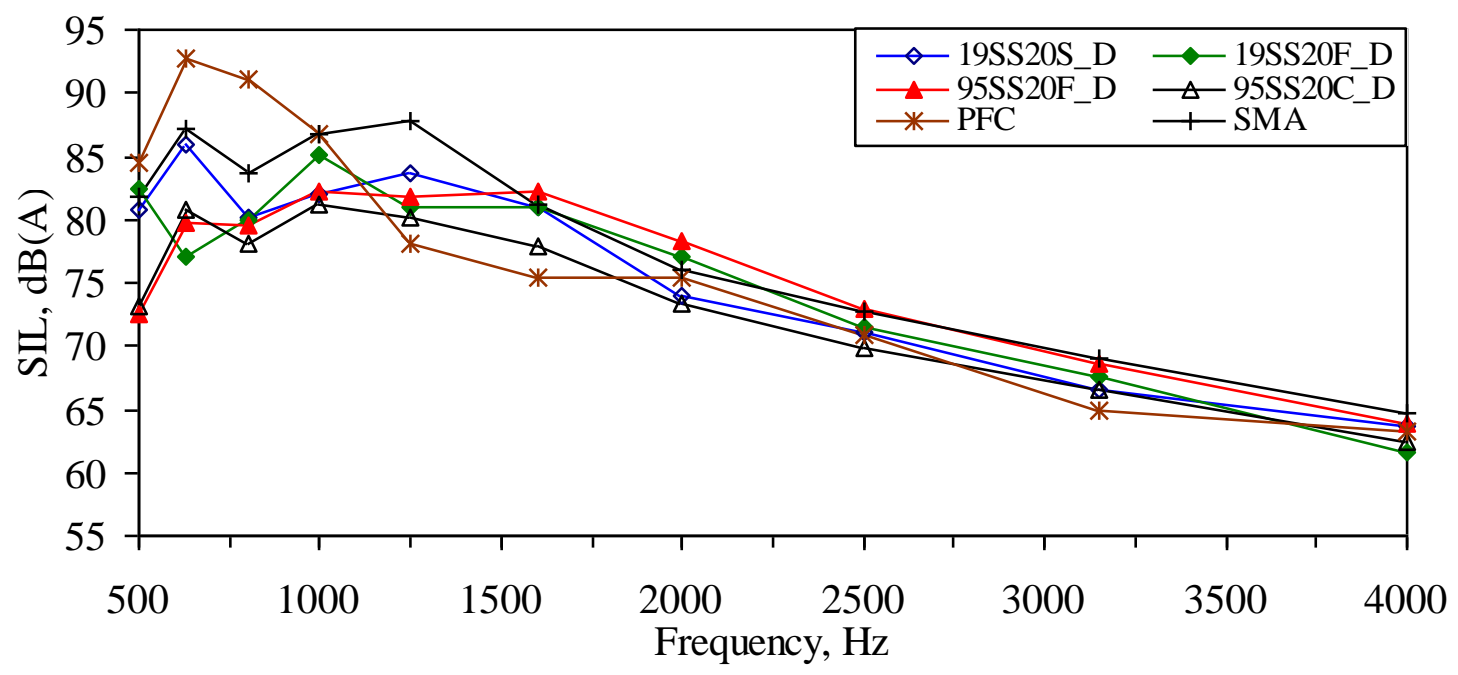

Figure G 16. 1/3 Octave frequency spectra for RSRTT SCD_2, test speed $48 \mathrm{~km} / \mathrm{h}$

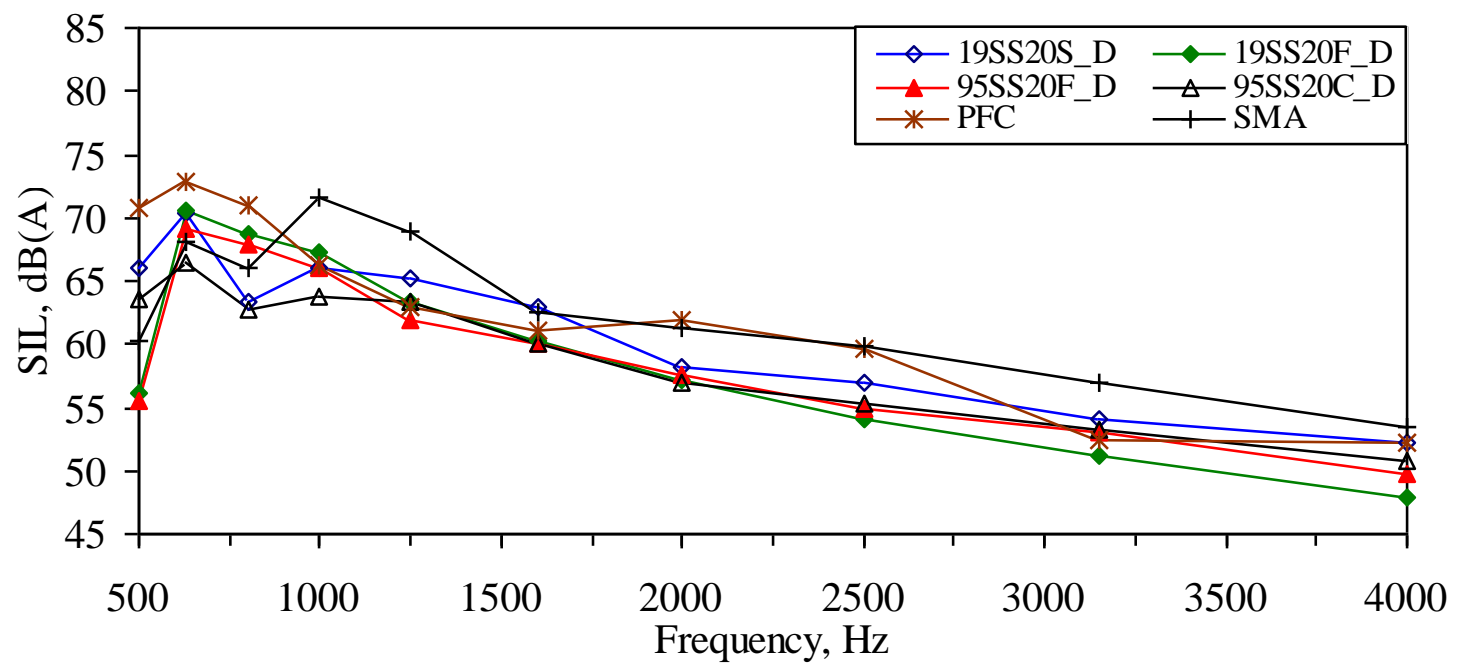

Figure G 17. 1/3 Octave frequency spectra for RSRTT SCD_3, test speed $16 \mathrm{~km} / \mathrm{h}$ 


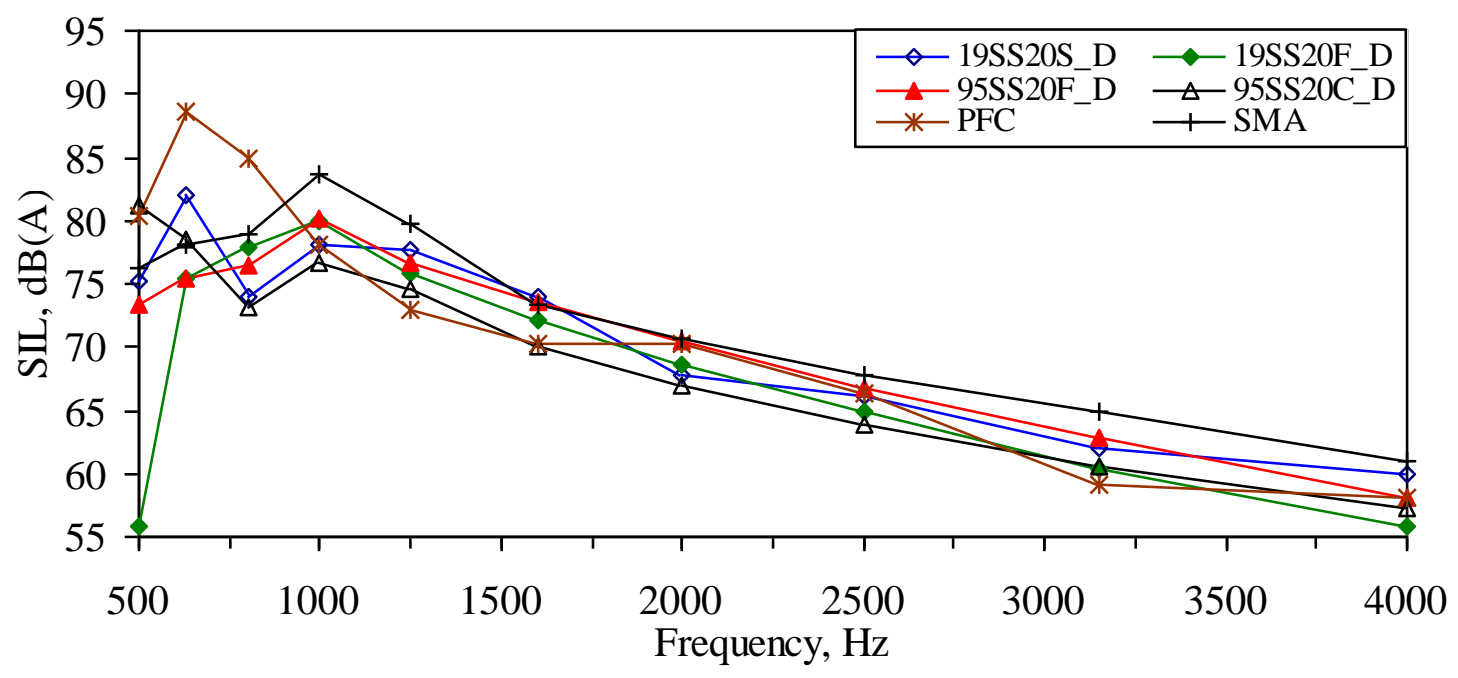

Figure G 18. 1/3 Octave frequency spectra for RSRTT SCD_3, test speed $32 \mathrm{~km} / \mathrm{h}$

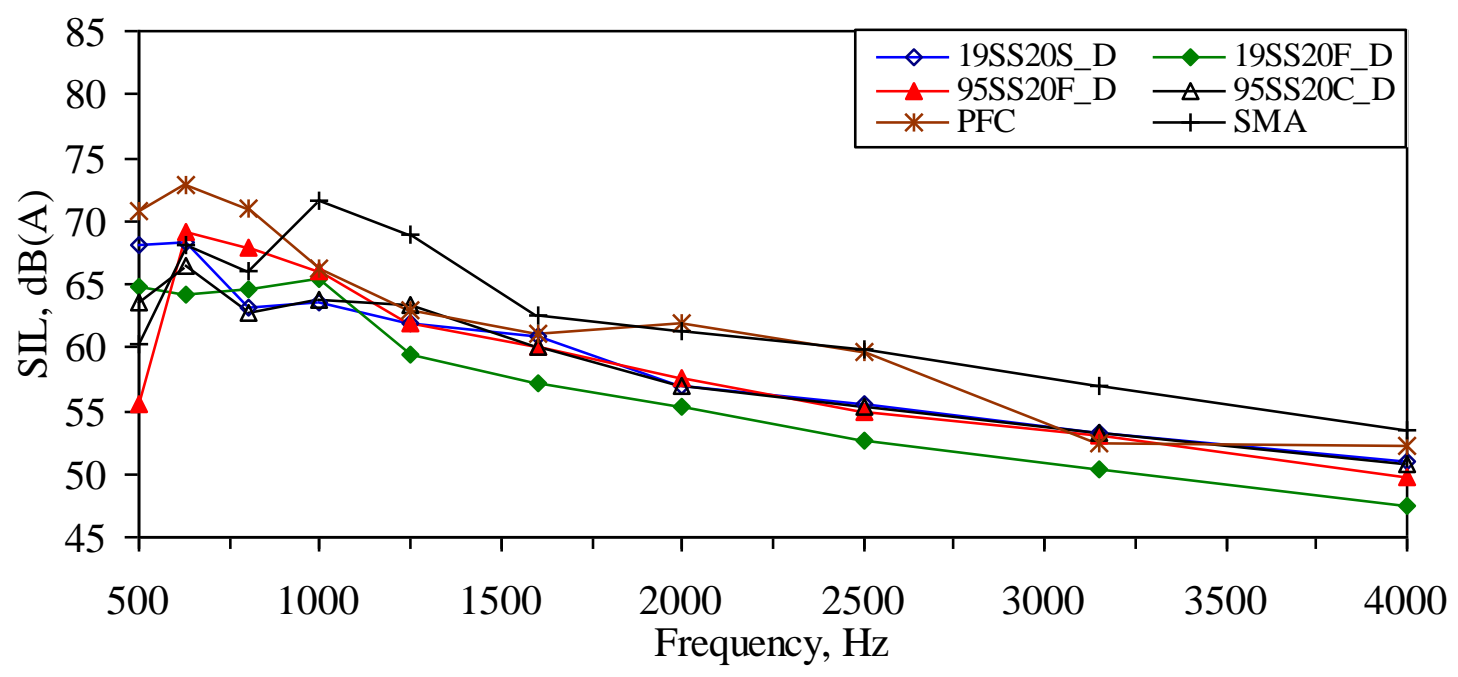

Figure G 19.1/3 Octave frequency spectra for UTP tire, test speed $16 \mathrm{~km} / \mathrm{h}, \mathrm{SCD} 33$ 


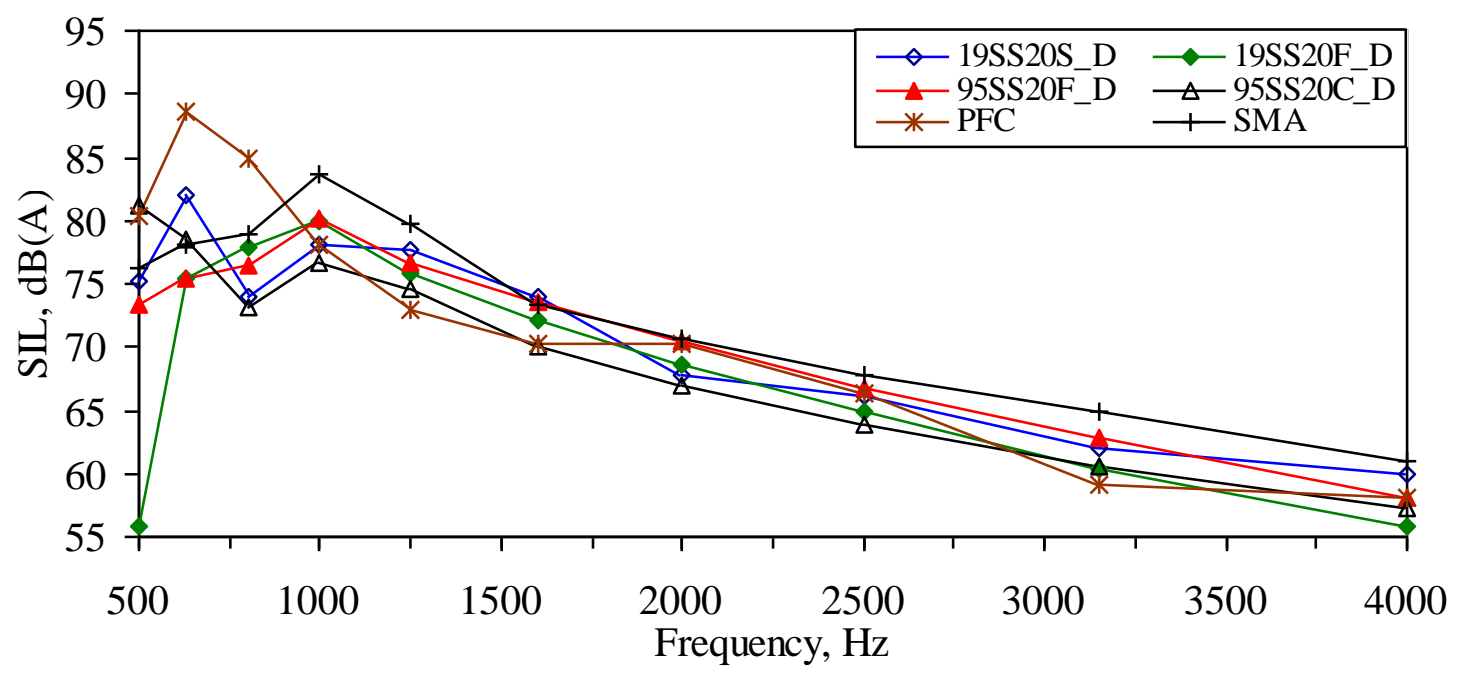

Figure G 20. 1/3 Octave frequency spectra for UTP tire, test speed $32 \mathrm{~km} / \mathrm{h}$, SCD_3

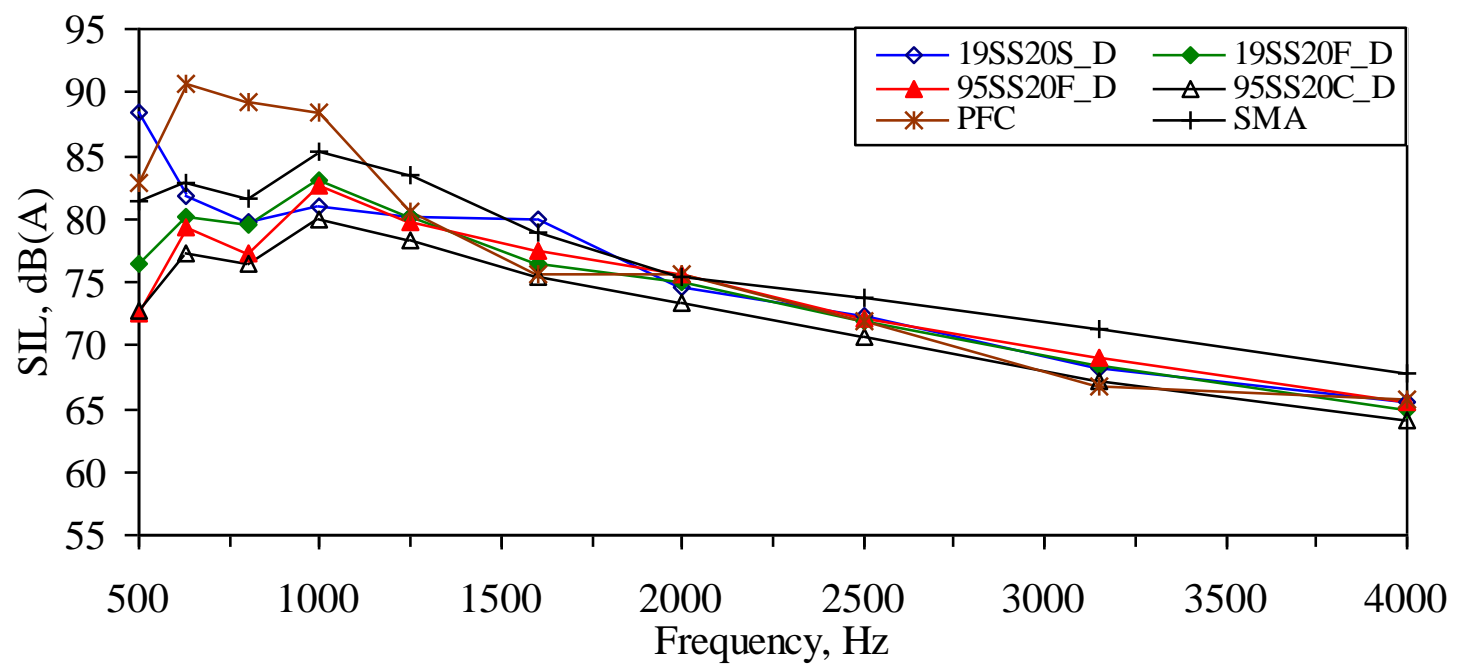

Figure G 21. 1/3 Octave frequency spectra for UTP tire, test speed 48 km/h, SCD_3 


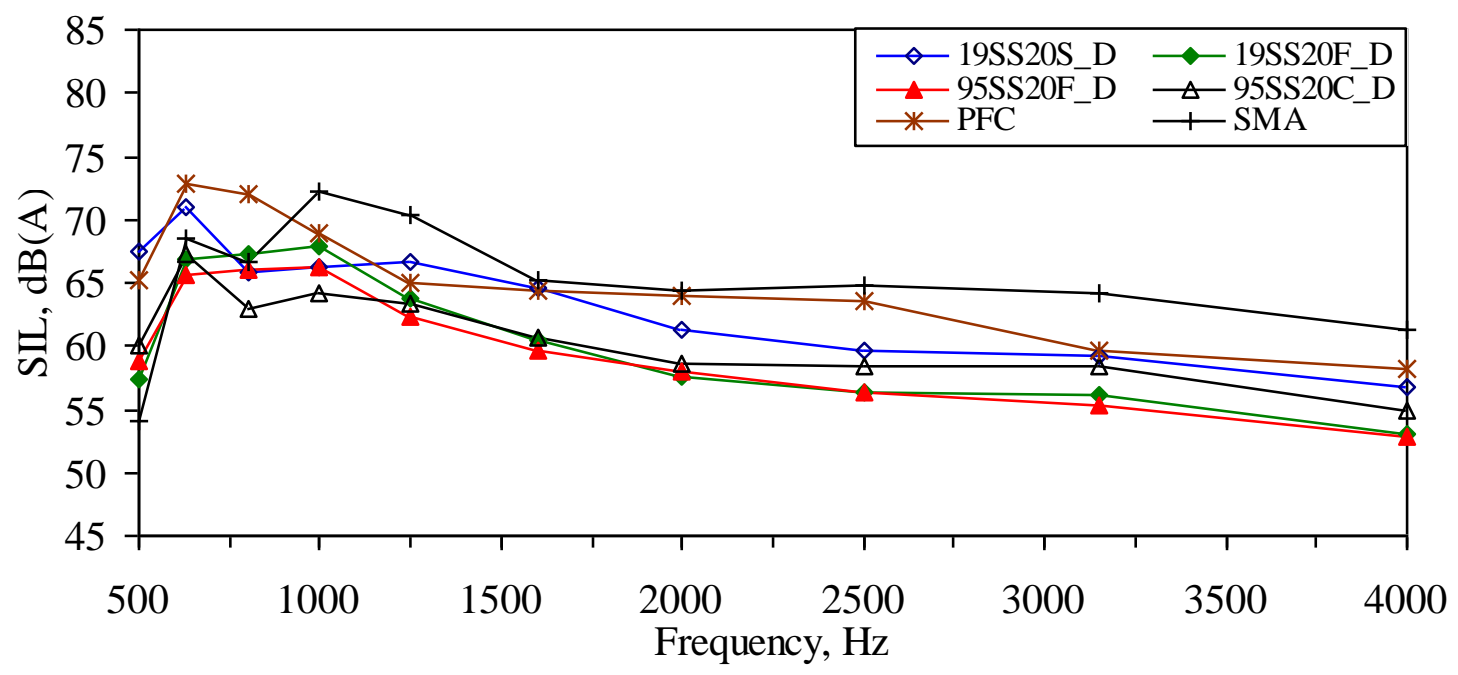

Figure G 22. 1/3 Octave frequency spectra for GA tire, test speed $16 \mathrm{~km} / \mathrm{h}, \mathrm{SCD} \_3$

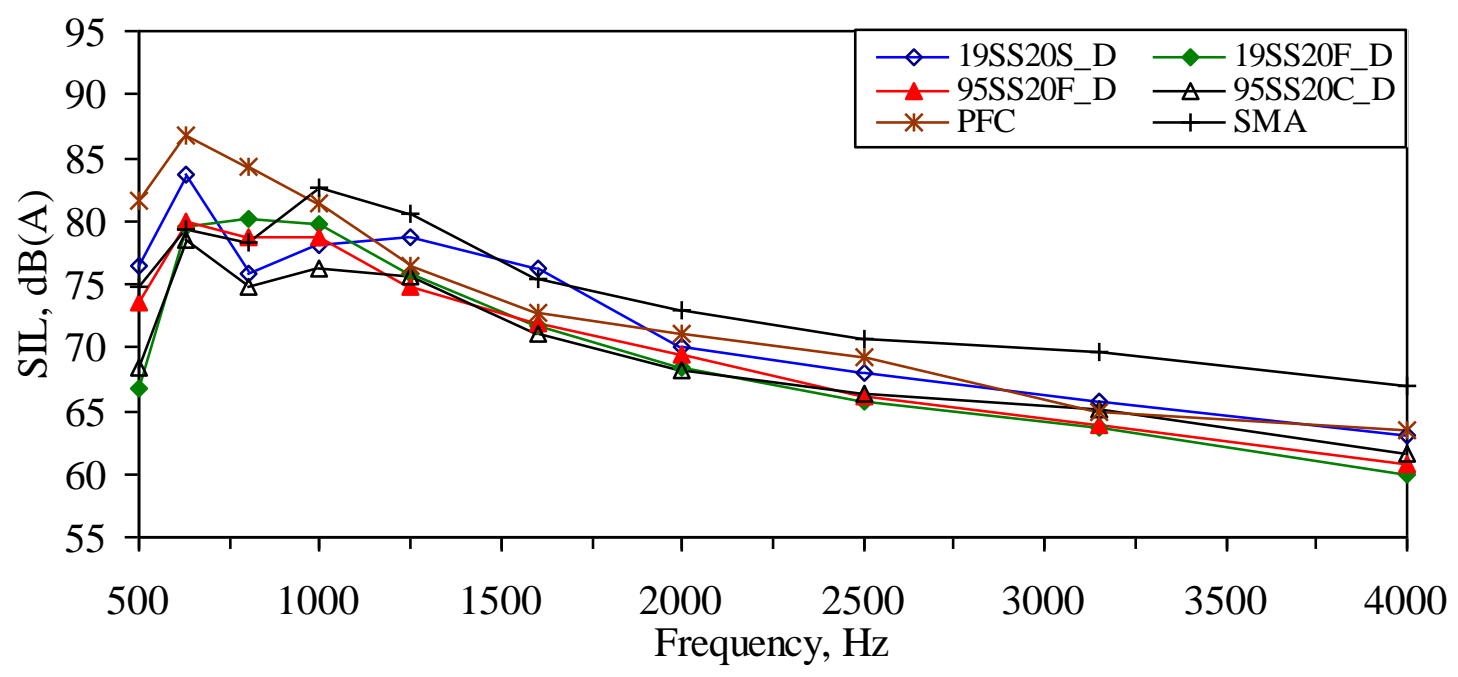

Figure G 23. 1/3 Octave frequency spectra for GA tire, test speed $32 \mathrm{~km} / \mathrm{h}, \mathrm{SCD} \_3$ 


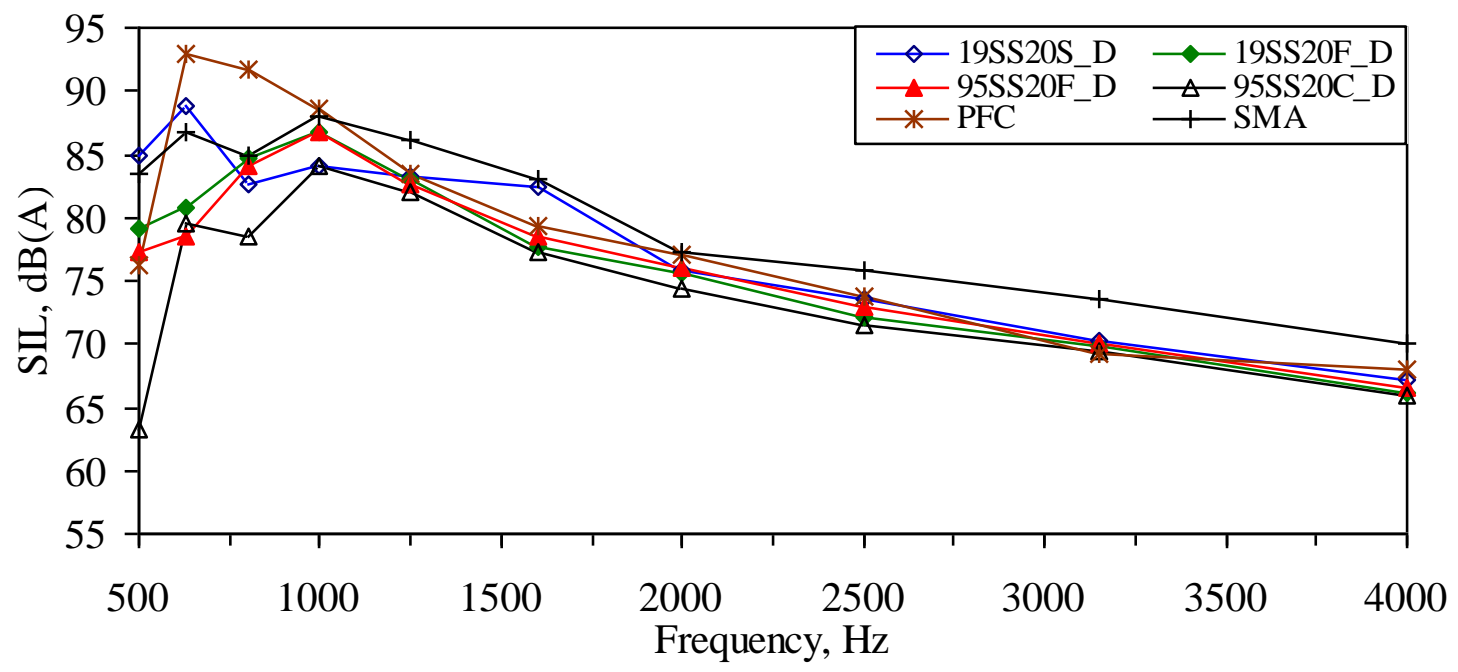

Figure G 24. 1/3 Octave frequency spectra for GA tire, test speed $48 \mathrm{~km} / \mathrm{h}, \mathrm{SCD} \_3$

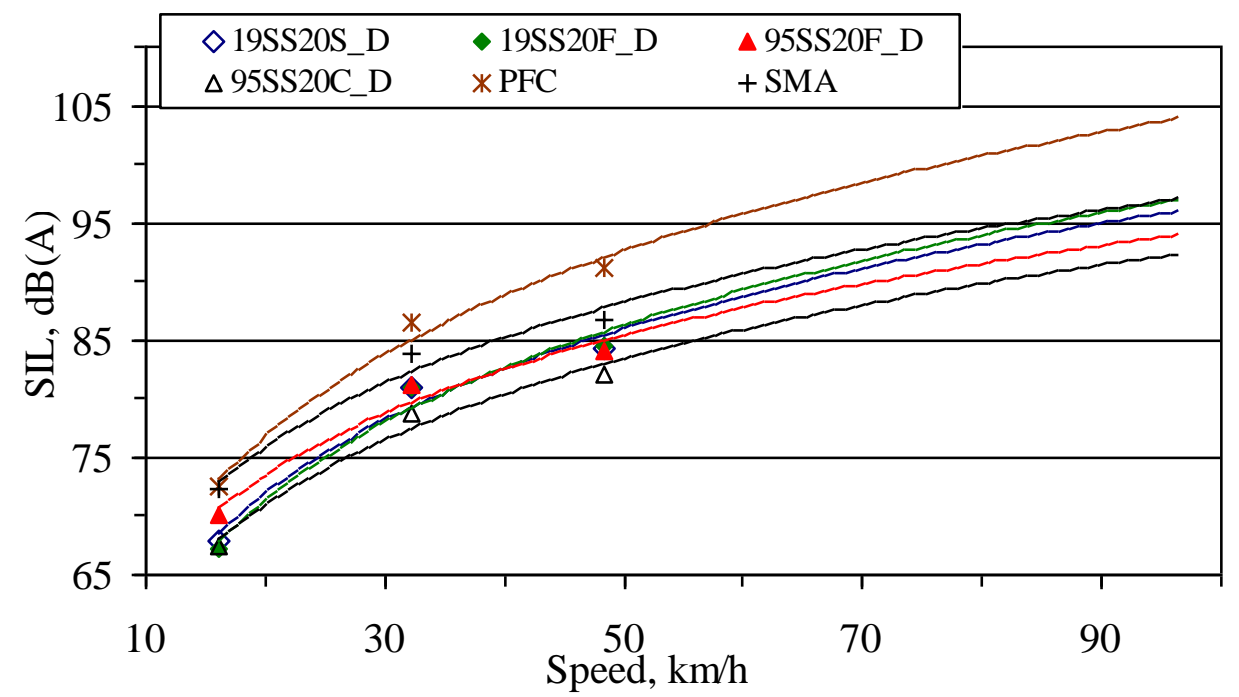

Figure G 25. Sound intensity level vs. speed relationship and SIL extrapolated to $97 \mathrm{~km} / \mathrm{h}$ for tests with UTP tire, SCD_3 


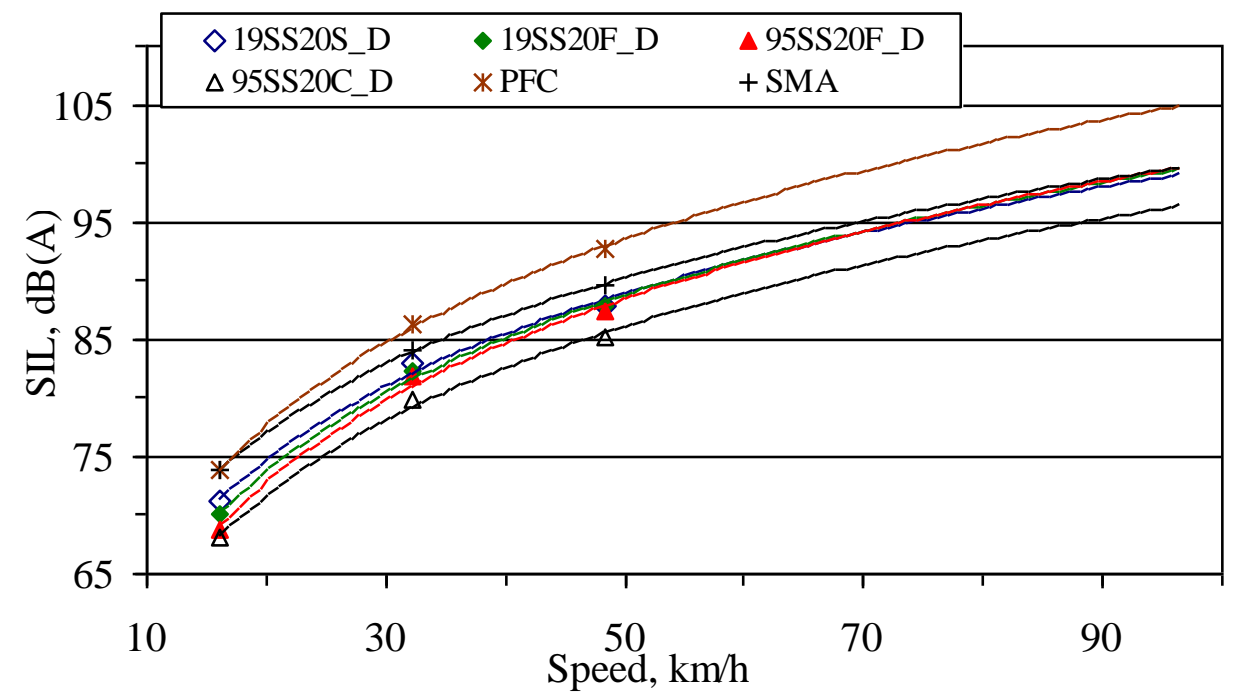

Figure G 26. Sound intensity level vs. speed relationship and SIL extrapolated to $97 \mathrm{~km} / \mathrm{h}$ for tests with GA tire, SCD_3 


\section{Appendix H: Summary of the Field CTM/DFT Measurements}

This appendix contains a summary of the field CTM/DFT tests data. Data are presented in Table $\mathrm{H} 1$ in the following order: section label (and time of test, for PFC, SMA and DGA sections only), age (number of years section is in service), approximate number of vehicle axles passed section at the time of test (\# axle, 10^6), test repetition (R), mean profile depth (MPD) value (for the left, center and right path), dynamic friction $\left(\mathrm{DF}_{20}\right)$ value (for the left, center and right path) and calibrated wet friction (F60) value (for the left, center and right path). 
Table H 1. Summary of the field CTM/DFT measurements

\begin{tabular}{|c|c|c|c|c|c|c|c|c|c|c|c|c|}
\hline \multirow{2}{*}{$\begin{array}{l}\text { Section/Test } \\
\text { Label }\end{array}$} & \multirow{2}{*}{$\begin{array}{l}\text { Age, } \\
\text { Years }\end{array}$} & \multirow{2}{*}{$\begin{array}{c}\# \\
\text { Axles }\end{array}$} & \multirow[t]{2}{*}{$\mathrm{R}$} & \multicolumn{3}{|c|}{ MPD, mm } & \multicolumn{3}{|c|}{$\mathrm{DF}_{20}$} & \multicolumn{3}{|c|}{ F60 } \\
\hline & & & & $\mathrm{L}$ & $\mathrm{C}$ & $\mathrm{R}$ & $\mathrm{L}$ & $\mathrm{C}$ & $\mathrm{R}$ & $\mathrm{L}$ & $\mathrm{C}$ & $\mathrm{R}$ \\
\hline PFC_8_05 & 2 & 22.3 & 1 & 1.45 & 1.52 & 1.47 & 0.68 & 0.66 & 0.64 & 0.46 & 0.45 & 0.44 \\
\hline PFC_8_05 & 2 & 22.3 & 2 & 1.62 & 1.56 & 1.45 & 0.68 & 0.64 & 0.60 & 0.47 & 0.44 & 0.41 \\
\hline PFC_8_05 & 2 & 22.3 & 3 & 1.51 & 1.35 & 1.62 & 0.71 & 0.65 & 0.60 & 0.48 & 0.44 & 0.42 \\
\hline PFC_11_05 & 2.3 & 25.7 & 1 & 1.21 & 1.34 & 1.36 & 0.63 & 0.59 & 0.56 & 0.41 & 0.40 & 0.39 \\
\hline PFC_11_05 & 2.3 & 25.7 & 2 & 1.44 & 1.31 & 1.46 & 0.57 & 0.63 & 0.60 & 0.40 & 0.42 & 0.41 \\
\hline PFC_11_05 & 2.3 & 25.7 & 3 & 1.43 & 1.23 & 1.47 & 0.58 & 0.63 & 0.63 & 0.40 & 0.42 & 0.43 \\
\hline PFC_10_06 & 3.2 & 36.0 & 1 & 1.28 & 1.54 & 1.31 & 0.58 & 0.51 & 0.49 & 0.39 & 0.37 & 0.35 \\
\hline PFC_10_06 & 3.2 & 36.0 & 2 & 1.36 & 1.49 & 1.69 & 0.51 & 0.53 & 0.52 & 0.36 & 0.38 & 0.38 \\
\hline PFC_10_06 & 3.2 & 36.0 & 3 & 1.24 & 1.17 & 1.34 & 0.53 & 0.55 & 0.51 & 0.37 & 0.37 & 0.36 \\
\hline PFC_4_07 & 2.7 & 42.1 & 1 & 1.24 & 1.42 & 1.58 & 0.66 & 0.68 & 0.59 & 0.43 & 0.46 & 0.41 \\
\hline PFC_4_07 & 2.7 & 42.1 & 2 & 1.53 & 1.47 & 1.50 & 0.67 & 0.63 & 0.60 & 0.46 & 0.43 & 0.41 \\
\hline PFC_4_07 & 2.7 & 42.1 & 3 & 1.66 & 1.34 & 1.40 & 0.64 & 0.73 & 0.61 & 0.45 & 0.48 & 0.42 \\
\hline PFC_8_07 & 3 & 45.5 & 1 & 1.81 & 1.51 & 1.51 & 0.59 & 0.67 & 0.67 & 0.42 & 0.46 & 0.45 \\
\hline PFC_8_07 & 3 & 45.5 & 2 & 1.22 & 1.41 & 1.46 & 0.62 & 0.66 & 0.58 & 0.41 & 0.45 & 0.40 \\
\hline PFC_8_07 & $\beta$ & 45.5 & 3 & 1.25 & 1.39 & 1.36 & 0.62 & 0.61 & 0.58 & 0.41 & 0.42 & 0.39 \\
\hline PFC_10_07 & 3.1 & 47.8 & 1 & 1.24 & 1.45 & 1.55 & 0.53 & 0.57 & 0.52 & 0.36 & 0.40 & 0.37 \\
\hline PFC_10_07 & 3.1 & 47.8 & 2 & 1.27 & 1.31 & 1.51 & 0.60 & 0.59 & 0.55 & 0.40 & 0.40 & 0.39 \\
\hline PFC_10_07 & 3.1 & 47.8 & 3 & 1.23 & 1.33 & 1.45 & 0.54 & 0.61 & 0.50 & 0.37 & 0.41 & 0.36 \\
\hline
\end{tabular}




\begin{tabular}{|c|c|c|c|c|c|c|c|c|c|c|c|c|}
\hline \multirow{2}{*}{$\begin{array}{l}\text { Section/Test } \\
\text { Label }\end{array}$} & \multirow{2}{*}{$\begin{array}{l}\text { Age, } \\
\text { Years }\end{array}$} & \multirow{2}{*}{$\begin{array}{c}\# \\
\text { Axles } \\
\end{array}$} & \multirow[t]{2}{*}{$\mathrm{R}$} & \multicolumn{3}{|c|}{ MPD, mm } & \multicolumn{3}{|c|}{$\mathrm{DF}_{20}$} & \multicolumn{3}{|c|}{ F60 } \\
\hline & & & & $\mathrm{L}$ & $\mathrm{C}$ & $\mathrm{R}$ & $\mathrm{L}$ & $\mathrm{C}$ & $\mathrm{R}$ & $\mathrm{L}$ & $\mathrm{C}$ & $\mathrm{R}$ \\
\hline SMA_8_05 & 2 & 22.3 & 1 & 1.17 & 1.17 & 1.14 & 0.77 & 0.75 & 0.68 & 0.49 & 0.47 & 0.43 \\
\hline SMA_8_05 & 2 & 22.3 & 2 & 1.14 & 1.24 & 1.21 & 0.77 & 0.76 & 0.69 & 0.48 & 0.49 & 0.44 \\
\hline SMA_8_05 & 2 & 22.3 & 3 & 1.03 & 1.28 & 0.92 & 0.77 & 0.79 & 0.67 & 0.47 & 0.50 & 0.41 \\
\hline SMA_11_05 & 2.3 & 25.7 & 1 & 1.14 & 0.91 & 0.97 & 0.67 & 0.78 & 0.69 & 0.43 & 0.45 & 0.42 \\
\hline SMA_11_05 & 2.3 & 25.7 & 2 & 1.12 & 1.12 & 1.14 & 0.71 & 0.69 & 0.80 & 0.45 & 0.44 & 0.50 \\
\hline SMA_11_05 & 2.3 & 25.7 & 3 & 1.12 & 1.48 & 1.09 & 0.72 & 0.78 & 0.71 & 0.45 & 0.52 & 0.44 \\
\hline SMA_10_06 & 3.2 & 36.0 & 1 & 0.97 & 1.04 & 1.00 & 0.52 & 0.72 & 0.61 & 0.34 & 0.44 & 0.38 \\
\hline SMA_10_06 & 3.2 & 36.0 & 2 & 0.91 & 1.15 & 1.17 & 0.63 & 0.68 & 0.59 & 0.39 & 0.44 & 0.39 \\
\hline SMA_10_06 & 3.2 & 36.0 & 3 & 1.04 & 1.16 & 1.10 & 0.62 & 0.65 & 0.71 & 0.39 & 0.42 & 0.45 \\
\hline SMA_4_07 & 2.7 & 42.1 & 1 & 1.14 & 1.16 & 1.14 & 0.78 & 0.87 & 0.77 & 0.48 & 0.54 & 0.48 \\
\hline SMA_4_07 & 2.7 & 42.1 & 2 & 1.03 & 1.16 & 1.04 & 0.76 & 0.79 & 0.82 & 0.46 & 0.49 & 0.50 \\
\hline SMA_4_07 & 2.7 & 42.1 & 3 & 1.10 & 1.16 & 1.03 & 0.75 & 0.84 & 0.74 & 0.46 & 0.52 & 0.45 \\
\hline SMA_8_07 & 3 & 45.5 & 1 & 0.80 & 1.28 & 1.03 & 0.74 & 0.78 & 0.69 & 0.42 & 0.50 & 0.43 \\
\hline SMA_8_07 & 3 & 45.5 & 2 & 0.98 & 1.17 & 1.13 & 0.70 & 0.78 & 0.74 & 0.43 & 0.49 & 0.46 \\
\hline SMA_8_07 & 3 & 45.5 & 3 & 0.88 & 1.17 & 1.06 & 0.69 & 0.82 & 0.67 & 0.41 & 0.51 & 0.42 \\
\hline SMA_10_07 & 3.1 & 47.8 & 1 & 1.00 & 1.20 & 1.16 & 0.68 & 0.78 & 0.65 & 0.42 & 0.49 & 0.42 \\
\hline SMA_10_07 & 3.1 & 47.8 & 2 & 0.96 & 1.12 & 1.30 & 0.64 & 0.79 & 0.67 & 0.39 & 0.49 & 0.44 \\
\hline SMA_10_07 & 3.1 & 47.8 & 3 & 0.83 & 1.07 & 1.22 & 0.67 & 0.79 & 0.66 & 0.39 & 0.48 & 0.43 \\
\hline
\end{tabular}




\begin{tabular}{|c|c|c|c|c|c|c|c|c|c|c|c|c|}
\hline \multirow{2}{*}{$\begin{array}{l}\text { Section/Test } \\
\text { Label }\end{array}$} & \multirow{2}{*}{$\begin{array}{l}\text { Age, } \\
\text { Years }\end{array}$} & \multirow{2}{*}{$\begin{array}{c}\# \\
\text { Axles }\end{array}$} & \multirow[t]{2}{*}{$\mathrm{R}$} & \multicolumn{3}{|c|}{$\mathrm{MPD}, \mathrm{mm}$} & \multicolumn{3}{|c|}{$\mathrm{DF}_{20}$} & \multicolumn{3}{|c|}{ F60 } \\
\hline & & & & $\mathrm{L}$ & $\mathrm{C}$ & $\mathrm{R}$ & $\mathrm{L}$ & $\mathrm{C}$ & $\mathrm{R}$ & $\mathrm{L}$ & $\mathrm{C}$ & $\mathrm{R}$ \\
\hline DGA_5_06 & 1.8 & 15.8 & 1 & 0.47 & 0.40 & 0.44 & 0.39 & 0.43 & 0.42 & 0.22 & 0.22 & 0.23 \\
\hline DGA_5_06 & 1.8 & 15.8 & 2 & 0.56 & 0.48 & 0.47 & 0.42 & 0.45 & 0.39 & 0.25 & 0.24 & 0.22 \\
\hline DGA_5_06 & 1.8 & 15.8 & 3 & 0.49 & 0.48 & 0.45 & 0.42 & 0.47 & 0.40 & 0.23 & 0.25 & 0.22 \\
\hline DGA_11_06 & 3.3 & 18.5 & 1 & 0.48 & 0.44 & 0.63 & 0.38 & 0.37 & 0.39 & 0.22 & 0.21 & 0.24 \\
\hline DGA_11_06 & 3.3 & 18.5 & 2 & 0.52 & 0.59 & 0.49 & 0.40 & 0.38 & 0.38 & 0.23 & 0.24 & 0.22 \\
\hline DGA_11_06 & 3.3 & 18.5 & 3 & 0.68 & 0.60 & 0.50 & 0.44 & 0.40 & 0.35 & 0.27 & 0.24 & 0.21 \\
\hline DGA_4_07 & 2.8 & 20.8 & 1 & 0.62 & 0.59 & 0.56 & 0.48 & 0.58 & 0.43 & 0.28 & 0.31 & 0.25 \\
\hline DGA_4_07 & 2.8 & 20.8 & 2 & 0.62 & 0.55 & 0.52 & 0.46 & 0.55 & 0.44 & 0.27 & 0.30 & 0.25 \\
\hline DGA_4_07 & 2.8 & 20.8 & 3 & 0.58 & 0.47 & 0.57 & 0.42 & 0.57 & 0.45 & 0.25 & 0.29 & 0.26 \\
\hline DGA_7_07 & 3 & 22.2 & 1 & 0.53 & 0.53 & 0.55 & 0.48 & 0.54 & 0.49 & 0.26 & 0.29 & 0.27 \\
\hline DGA_7_07 & 3 & 22.2 & 2 & 0.59 & 0.45 & 0.54 & 0.48 & 0.55 & 0.47 & 0.27 & 0.27 & 0.26 \\
\hline DGA_7_07 & 3 & 22.2 & 3 & 0.58 & 0.52 & 0.45 & 0.46 & 0.53 & 0.47 & 0.27 & 0.28 & 0.25 \\
\hline DGA_10_07 & 3.2 & 23.4 & 1 & 0.68 & 0.44 & 0.54 & 0.45 & 0.46 & 0.44 & 0.27 & 0.24 & 0.25 \\
\hline DGA_10_07 & 3.2 & 23.4 & 2 & 0.65 & 0.48 & 0.52 & 0.46 & 0.50 & 0.44 & 0.27 & 0.26 & 0.25 \\
\hline DGA_10_07 & 3.2 & 23.4 & 3 & 0.59 & 0.50 & 0.58 & 0.43 & 0.56 & 0.43 & 0.25 & 0.29 & 0.25 \\
\hline
\end{tabular}




\begin{tabular}{|c|c|c|c|c|c|c|c|c|c|c|c|c|}
\hline \multirow{2}{*}{\begin{tabular}{|l|} 
Section/Test \\
Label
\end{tabular}} & \multirow{2}{*}{$\begin{array}{l}\text { Age, } \\
\text { Years }\end{array}$} & \multirow{2}{*}{$\begin{array}{c}\# \\
\text { Axles }\end{array}$} & \multirow[t]{2}{*}{$\mathrm{R}$} & \multicolumn{3}{|c|}{ MPD, mm } & \multicolumn{3}{|c|}{$\mathrm{DF}_{20}$} & \multicolumn{3}{|c|}{ F60 } \\
\hline & & & & $\mathrm{L}$ & $\mathrm{C}$ & $\mathrm{R}$ & $\mathrm{L}$ & $\mathrm{C}$ & $\mathrm{R}$ & $\mathrm{L}$ & $\mathrm{C}$ & $\mathrm{R}$ \\
\hline HM-IN-1 & 2 & 6.4 & 1 & 0.33 & 0.33 & 0.40 & 0.46 & 0.67 & 0.53 & 0.22 & 0.28 & 0.26 \\
\hline HM-IN-1 & 2 & 6.4 & 2 & 0.44 & 0.38 & 0.45 & 0.50 & 0.69 & 0.57 & 0.26 & 0.30 & 0.28 \\
\hline HM-IN-1 & 2 & 6.4 & 3 & 0.38 & 0.29 & 0.38 & 0.46 & 0.66 & 0.55 & 0.23 & 0.26 & 0.26 \\
\hline HM-IN-1 & 2 & 6.4 & 4 & 0.40 & 0.33 & 0.37 & 0.47 & 0.68 & 0.55 & 0.24 & 0.28 & 0.25 \\
\hline HM-IN-1 & 2 & 6.4 & 5 & 0.42 & 0.29 & 0.47 & 0.49 & 0.71 & 0.60 & 0.25 & 0.27 & 0.30 \\
\hline HM-IN-2 & 1 & 1.1 & 1 & 0.28 & 0.25 & 0.30 & 0.43 & 0.56 & 0.49 & 0.19 & 0.22 & 0.22 \\
\hline HM-IN-2 & 1 & 1.1 & 2 & 0.33 & 0.27 & 0.25 & 0.47 & 0.59 & 0.52 & 0.22 & 0.23 & 0.21 \\
\hline HM-IN-2 & 1 & 1.1 & 3 & 0.33 & 0.27 & 0.30 & 0.50 & 0.61 & 0.49 & 0.23 & 0.24 & 0.22 \\
\hline HM-IN-2 & 1 & 1.1 & 4 & 0.34 & 0.32 & 0.30 & 0.58 & 0.62 & 0.55 & 0.26 & 0.26 & 0.23 \\
\hline HM-IN-2 & 1 & 1.1 & 5 & 0.29 & 0.27 & 0.31 & 0.51 & 0.73 & 0.52 & 0.22 & 0.27 & 0.23 \\
\hline HM-IN-3 & 1 & 1.3 & 1 & 0.35 & 0.28 & 0.29 & 0.54 & 0.58 & 0.54 & 0.25 & 0.23 & 0.23 \\
\hline HM-IN-3 & 1 & 1.3 & 2 & 0.35 & 0.29 & 0.32 & 0.54 & 0.61 & 0.57 & 0.24 & 0.25 & 0.24 \\
\hline HM-IN-3 & 1 & 1.3 & 3 & 0.34 & 0.27 & 0.31 & 0.53 & 0.66 & 0.52 & 0.24 & 0.25 & 0.23 \\
\hline HM-IN-3 & 1 & 1.3 & 4 & 0.33 & 0.33 & 0.30 & 0.58 & 0.57 & 0.61 & 0.25 & 0.25 & 0.25 \\
\hline HM-IN-3 & 1 & 1.3 & 5 & 0.35 & 0.29 & 0.34 & 0.52 & 0.63 & 0.55 & 0.24 & 0.25 & 0.25 \\
\hline HM-IN-6 & 1 & 1.0 & 1 & 0.31 & 0.36 & 0.45 & 0.54 & 0.70 & 0.63 & 0.23 & 0.30 & 0.30 \\
\hline HM-IN-6 & 1 & 1.0 & 2 & 0.33 & 0.39 & 0.41 & 0.54 & 0.73 & 0.63 & 0.24 & 0.32 & 0.29 \\
\hline HM-IN-6 & 1 & 1.0 & 3 & 0.36 & 0.34 & 0.35 & 0.58 & 0.70 & 0.66 & 0.26 & 0.29 & 0.28 \\
\hline HM-IN-6 & 1 & 1.0 & 4 & 0.38 & 0.41 & 0.36 & 0.57 & 0.76 & 0.68 & 0.26 & 0.33 & 0.29 \\
\hline HM-IN-6 & 1 & 1.0 & 5 & 0.34 & 0.37 & 0.37 & 0.63 & 0.82 & 0.63 & 0.27 & 0.34 & 0.28 \\
\hline HM-IN-7 & 1 & 0.7 & 1 & 0.37 & 0.33 & 0.35 & 0.74 & 0.80 & 0.74 & 0.31 & 0.32 & 0.31 \\
\hline HM-IN-7 & 1 & 0.7 & 2 & 0.36 & 0.30 & 0.34 & 0.72 & 0.80 & 0.72 & 0.30 & 0.30 & 0.30 \\
\hline HM-IN-7 & 1 & 0.7 & 3 & 0.30 & 0.35 & 0.41 & 0.68 & 0.76 & 0.67 & 0.27 & 0.31 & 0.30 \\
\hline HM-IN-7 & 1 & 0.7 & 4 & 0.34 & 0.42 & 0.32 & 0.67 & 0.71 & 0.67 & 0.28 & 0.32 & 0.27 \\
\hline HM-IN-7 & 1 & 0.7 & 5 & 0.37 & 0.31 & 0.36 & 0.65 & 0.72 & 0.69 & 0.28 & 0.28 & 0.29 \\
\hline HM-IN-8 & 1 & 1.3 & 1 & 0.35 & 0.34 & 0.41 & 0.67 & 0.76 & 0.68 & 0.28 & 0.31 & 0.31 \\
\hline HM-IN-8 & 1 & 1.3 & 2 & 0.41 & 0.33 & 0.41 & 0.67 & 0.78 & 0.64 & 0.30 & 0.31 & 0.29 \\
\hline HM-IN-8 & 1 & 1.3 & 3 & 0.40 & 0.30 & 0.36 & 0.66 & 0.71 & 0.66 & 0.30 & 0.28 & 0.29 \\
\hline HM-IN-8 & 1 & 1.3 & 4 & 0.39 & 0.33 & 0.31 & 0.61 & 0.73 & 0.62 & 0.28 & 0.30 & 0.26 \\
\hline HM-IN-8 & 1 & 1.3 & 5 & 0.35 & 0.27 & 0.36 & 0.66 & 0.77 & 0.64 & 0.28 & 0.28 & 0.28 \\
\hline
\end{tabular}




\begin{tabular}{|c|c|c|c|c|c|c|c|c|c|c|c|c|}
\hline \multirow{2}{*}{$\begin{array}{l}\text { Section/Test } \\
\text { Label }\end{array}$} & \multirow{2}{*}{$\begin{array}{l}\text { Age, } \\
\text { Years }\end{array}$} & \multirow{2}{*}{$\begin{array}{c}\# \\
\text { Axles }\end{array}$} & \multirow[t]{2}{*}{$\mathrm{R}$} & \multicolumn{3}{|c|}{$\mathrm{MPD}, \mathrm{mm}$} & \multicolumn{3}{|c|}{$\mathrm{DF}_{20}$} & \multicolumn{3}{|c|}{ F60 } \\
\hline & & & & $\mathrm{L}$ & $\mathrm{C}$ & $\mathrm{R}$ & $\mathrm{L}$ & $\mathrm{C}$ & $\mathrm{R}$ & $\mathrm{L}$ & $\mathrm{C}$ & $\mathrm{R}$ \\
\hline HM-IA-1 & 5 & 17.2 & 1 & 0.73 & 0.51 & 0.62 & 0.45 & 0.57 & 0.48 & 0.28 & 0.29 & 0.28 \\
\hline HM-IA-1 & 5 & 17.2 & 2 & 0.61 & 0.45 & 0.69 & 0.46 & 0.62 & 0.51 & 0.27 & 0.30 & 0.30 \\
\hline HM-IA-2 & 15 & 119.7 & 1 & 0.82 & 0.43 & 0.63 & 0.40 & 0.52 & 0.43 & 0.27 & 0.26 & 0.26 \\
\hline HM-IA-2 & 15 & 119.7 & 2 & 0.69 & 0.54 & 0.56 & 0.38 & 0.49 & 0.34 & 0.25 & 0.27 & 0.21 \\
\hline HM-IA-3 & 9 & 15.2 & 1 & 0.62 & 0.41 & 0.38 & 0.24 & 0.39 & 0.26 & 0.18 & 0.21 & 0.16 \\
\hline HM-IA-3 & 9 & 15.2 & 2 & 0.48 & 0.32 & 0.48 & 0.24 & 0.39 & 0.26 & 0.17 & 0.20 & 0.17 \\
\hline HM-IA-4 & 22 & 59.3 & 1 & 1.19 & 0.72 & 0.62 & 0.29 & 0.36 & 0.30 & 0.23 & 0.24 & 0.20 \\
\hline HM-IA-4 & 22 & 59.3 & 2 & 1.21 & 0.95 & 0.73 & 0.34 & 0.54 & 0.36 & 0.26 & 0.34 & 0.24 \\
\hline HM-IA-5 & 15 & 37.7 & 1 & 0.94 & 0.83 & 0.70 & 0.18 & 0.32 & 0.26 & 0.17 & 0.23 & 0.19 \\
\hline HM-IA-5 & 15 & 37.7 & 2 & 0.80 & 1.03 & 1.03 & 0.21 & 0.32 & 0.24 & 0.18 & 0.24 & 0.20 \\
\hline HM-IA-6 & 15 & 25.1 & 1 & 1.22 & 1.11 & 1.23 & 0.65 & 0.75 & 0.64 & 0.43 & 0.47 & 0.42 \\
\hline HM-IA-6 & 15 & 25.1 & 2 & 1.30 & 0.86 & 1.34 & 0.70 & 0.75 & 0.67 & 0.46 & 0.44 & 0.44 \\
\hline HM-IA-7 & 14 & 38.2 & 1 & 0.71 & 0.67 & 0.56 & 0.59 & 0.60 & 0.62 & 0.34 & 0.34 & 0.33 \\
\hline HM-IA-7 & 14 & 38.2 & 2 & 0.65 & 0.53 & 0.61 & 0.63 & 0.63 & 0.65 & 0.34 & 0.32 & 0.35 \\
\hline HM-IA-8 & 11 & 36.7 & 1 & 0.81 & 0.65 & 0.85 & 0.51 & 0.62 & 0.54 & 0.32 & 0.34 & 0.33 \\
\hline HM-IA-8 & 11 & 36.7 & 2 & 0.72 & 0.62 & 0.79 & 0.50 & 0.60 & 0.51 & 0.30 & 0.33 & 0.31 \\
\hline HM-IA-9 & 24 & 32.8 & 1 & 0.28 & 0.45 & 0.15 & 0.27 & 0.43 & 0.29 & 0.15 & 0.23 & 0.13 \\
\hline HM-IA-9 & 24 & 32.8 & 2 & 0.29 & 0.60 & 0.20 & 0.25 & 0.41 & 0.28 & 0.15 & 0.25 & 0.14 \\
\hline HM-IA-10 & 17 & 153.7 & 1 & 1.32 & 1.50 & 1.17 & 0.25 & 0.43 & 0.27 & 0.22 & 0.32 & 0.22 \\
\hline HM-IA-10 & 17 & 153.7 & 2 & 1.29 & 1.26 & 1.25 & 0.26 & 0.44 & 0.29 & 0.22 & 0.31 & 0.24 \\
\hline HM-IA-11 & 10 & 35.9 & 1 & 0.61 & 0.48 & 0.61 & 0.37 & 0.43 & 0.40 & 0.23 & 0.24 & 0.24 \\
\hline HM-IA-11 & 10 & 35.9 & 2 & 0.78 & 0.45 & 0.75 & 0.46 & 0.42 & 0.42 & 0.29 & 0.23 & 0.27 \\
\hline
\end{tabular}

